\title{
Fault Locating, Prediction and Protection (FLPPS)
}

DOE Contract No. DE-FC02-06CH11352

Prepared by Southern California Edison Principal Investigator: Robert J. Yinger

Subcontractors:

KEMA Incorporated: S.S. Venkata

Virginia Polytechnic Institute and State University:

Virgilio Centeno

Submitted: September 30, 2010 


\section{Disclaimer or Warranties and Limitation of Liabilities}

This report was prepared for the Department of Energy by Southern California Edison under contract number DE-FC0206CH11352.

Neither Southern California Edison, Edison International, nor any person working for or on behalf of any of them makes any warranty or representation, express or implied, (i) with respect to the use of any information, product, process or procedure discussed in this report, including merchantability and fitness for a particular purpose, or (ii) that such use does not infringe upon or interfere with rights or others, including another's intellectual property, or (iii) that this report is suitable to any particular user's circumstance.

Neither Southern California Edison, Edison International, nor any person working on behalf of any of them assumes responsibility for any damages or other liability whatsoever resulting from your selection or use of this report or any information, product, process or procedure disclosed in this report. 


\section{Table of Contents}

Section 0: Executive Summary .................................................................. 9

0.1 Phase I - Fault Location, Isolation, and Protection for the Circuit of the

Future 9

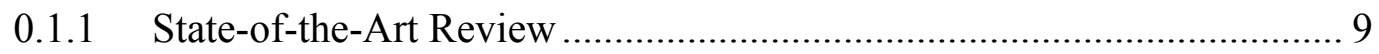

0.1.2 The Circuit of the Future.................................................................... 10

0.1.3 Description of the Circuit of the Future Protection Scheme ................... 10

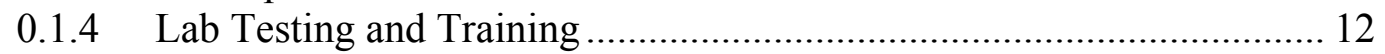

0.1.5 Testing Results ............................................................................... 12

0.2 Phase II - Fault Location, Isolation, and Protection for the Circuit of the

Future with a Fault Current Limiter ........................................................................13

0.2.1 Fault Current Limiter Description....................................................... 13

0.2.2 Fault Current Limiter Testing .............................................................. 14

0.2.3 Fault Current Limiter Field Experience .................................................... 14

$0.3 \quad$ Phase III - Advanced Fault Sensing and Prediction.........................................15

0.3.1 Fault Protection on the System of the Future....................................... 16

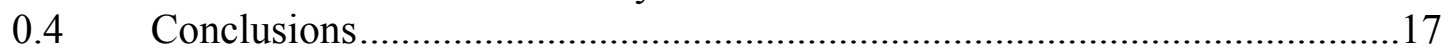

$0.5 \quad$ Recommendations for Further Work ……………......................................19

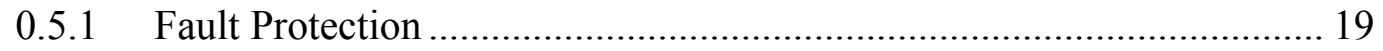

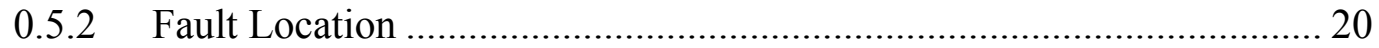

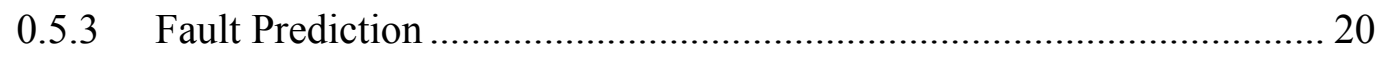

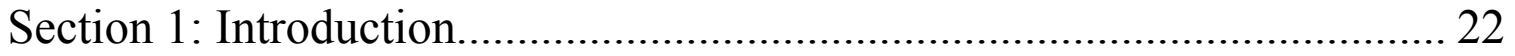

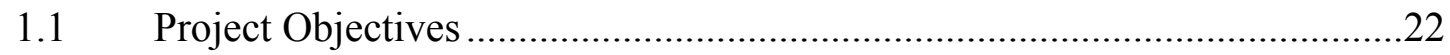

1.2 Project History and Background .............................................................22

1.3 Description of the Circuit-of-the-Future (CoF) .........................................23

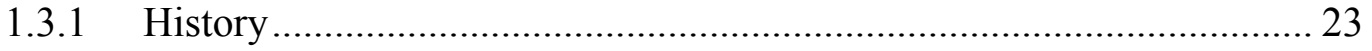

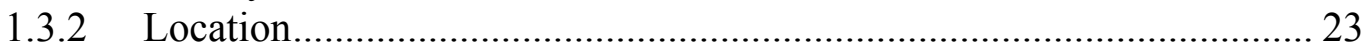

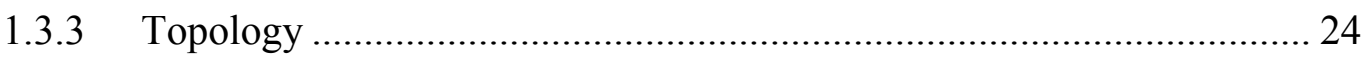

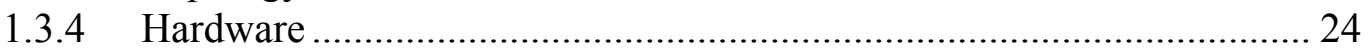

1.3.5 Communications Technologies …………………………………........ 25

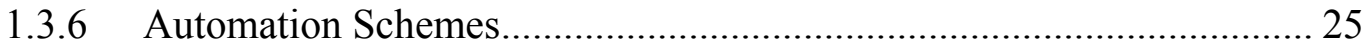

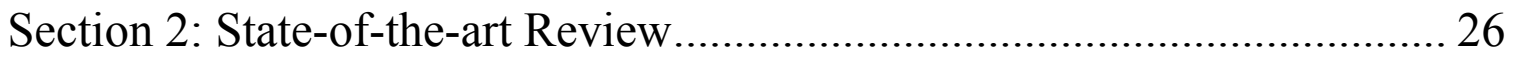

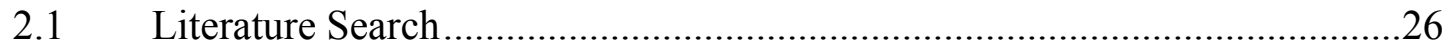

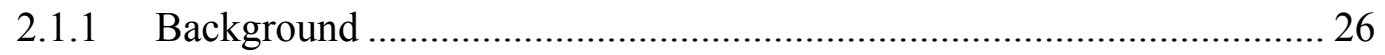

2.1.2 Fault Location and Prediction .............................................................. 26

2.1.3 Recommendations for Fault Location and Prediction............................ 27

2.1.4 Fault Protection (Distribution Automation, High Impedance Faults, and

Fault Current Limiters) ............................................................................ 28

2.1.5 Comments and Recommendations for Fault Protection (Distribution

Automation, High Impedance Faults, and Fault Current Limiters)...................... 30

2.1.6 General Recommendations from the Literature Review ........................ 31

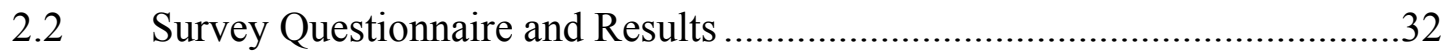

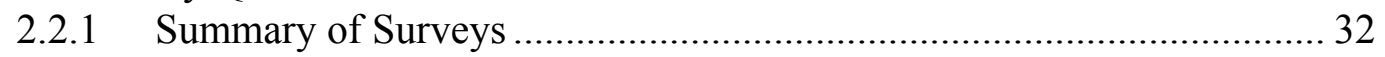

2.2.2 Major Observations from the Survey ………….................................... 33

2.3 Summary of Conclusions from Literature Search and Survey ........................34 
2.3.1 Conclusions:

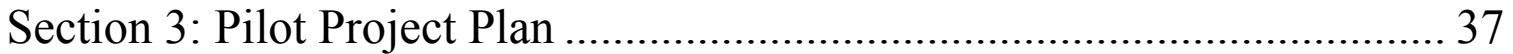

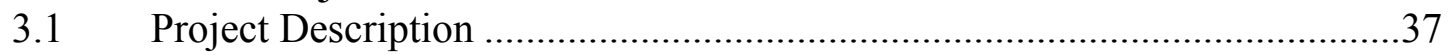

3.2 Schedule

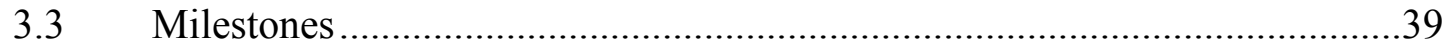

Section 4: Fault Location, Isolation and Prediction for the Circuit of the Future without a Fault Current Limiter ...................................................... 40

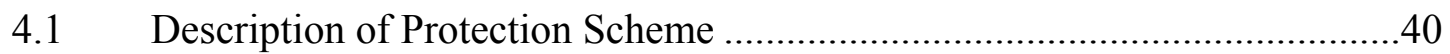

4.1.1 Protection Coordination .................................................................. 41

4.1.2 Operator Controls............................................................................ 43

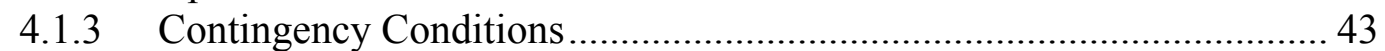

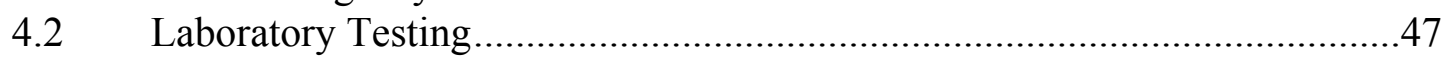

4.2.1 Lab Test Scenarios ………………………...................................... 52

Apply DC Power to RCI/RAR with Simultaneous Loss of Communication ..............53

4.3 Training of Field Personnel .......................................................................53

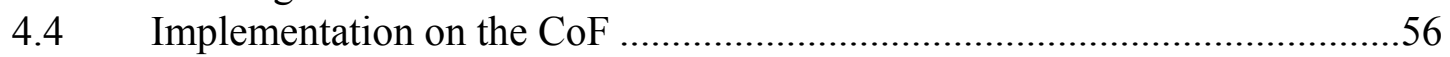

Section 5: Fault Location, Isolation and Prediction for the CoF with a Fault

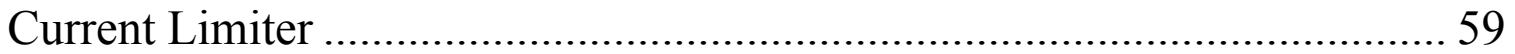

5.1 Zenergy FCL Description and Laboratory Testing.........................................60

5.1.1 Description and Testing of Zenergy Fault Current Limiter ...................... 61

5.1.2 High Voltage Impulse Testing at SSID.................................................. 65

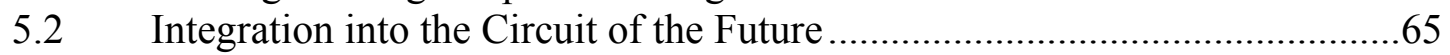

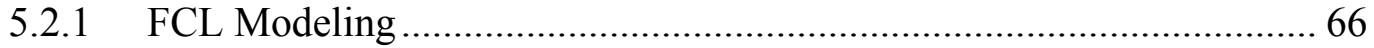

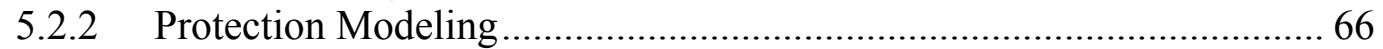

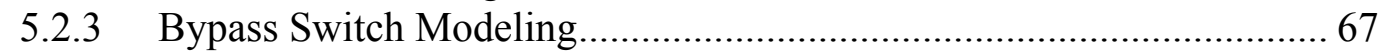

5.2.4 Added Equipment and Controls ........................................................... 72

5.3 Virginia Tech FCL Survey, Models, and Simulations Results.......................72

Section 6: Advanced Fault Sensing and Prediction....................................... 77

6.1 Measures Investigated From the Proposal ........................................................77

6.1.1 Use of Oscillography, Arcing Fault Sensing, Time Domain

Reflectometry, Phasor Measurement and other Technologies to Detect and

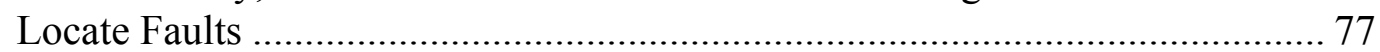

6.1.2 Use Line Sensors for Detection............................................................ 79

6.1.3 Signature Analysis Based on Theory and Tests of Known Bad

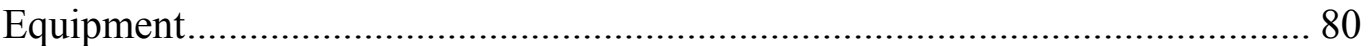

6.2 Other Measures Investigated and not Implemented as Part of the Project ....80

6.2.1 Simultaneous Triggering of all Relays to Record Events ....................... 80

6.2.2 Record Relay Pickups ......................................................................... 81

6.2.3 Record Daily Waveforms................................................................. 81

6.2.4 Investigate and Test A4 Relay Problem............................................... 82

6.3 Measures Actually Implemented as Part of Project........................................82

6.3.1 Measure Power Quality Upstream and Downstream of the FCL........... 83

6.3.2 De-centralized Sensing, Control and Protection and Automation

Functions 
6.3.3 Design and Model System of the Future (SoF) Relay Protection Scheme. 83

Section 7: Evaluation of Results of Pilot Tests .......................................... 90

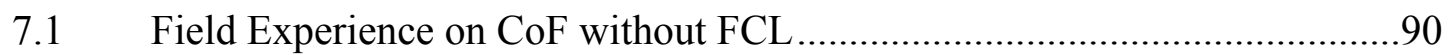

7.1.1 Fault Event - October 21, 2007 .......................................................... 91

7.1.2 Fault Event - December 25, 2007 ……………................................... 92

7.1.3 Fault Event - November 14, 2008 …………......................................... 94

7.1.4 Fault Event - January 30, 2009........................................................... 95

7.2 Field Experience of CoF with FCL........................................................95

7.2.1 FCL Put into Service - March 3, 2009 with no Load and March 6, 2009

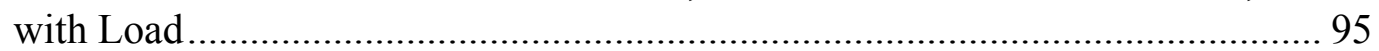

7.2.2 FCL Malfunction Event - March 16, 2009.......................................... 96

7.2.3 FCL Put Back in Service (December 9, 2009 with no load and December

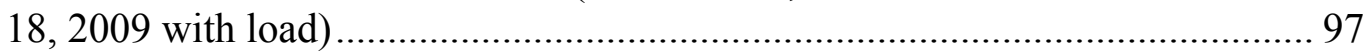

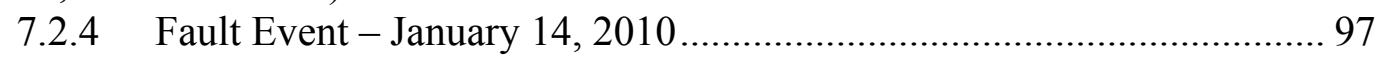

7.3 Analysis of Advanced Fault Sensing and Prediction Measures .....................99

7.3.1 Measure Power Quality Upstream and Downstream from the FCL ...... 99

7.3.2 De-Centralized Sensing, Control and Protection and Automation

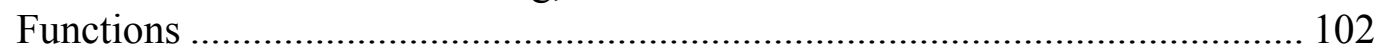

7.3.3 Design and Model System of the Future Relay Protection Scheme .... 103

Section 8: Extrapolation for the Future and for Industry .......................... 104

8.1 De-centralized sensing, control and protection and automation functions...104

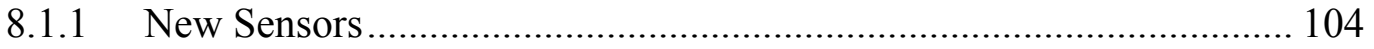

8.1.2 Distributed vs Central Computational Intelligence................................ 105

8.1.3 Distribution System Communications ................................................... 105

8.1.4 Real-Time Analysis and Control....................................................... 106

8.2 Advanced and adaptive protection...........................................................106

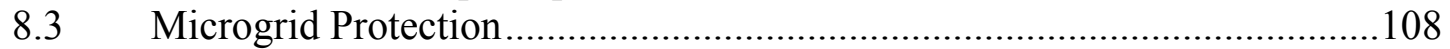

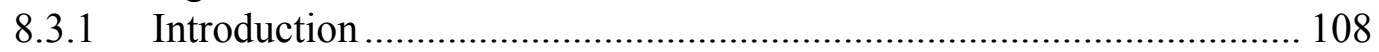

8.3.2 The Case for a New Protection Paradigm ............................................ 109

8.3.3 General Microgrid Protection Philosophy ........................................... 109

8.3.4 Proposed Protection Scheme ............................................................. 111

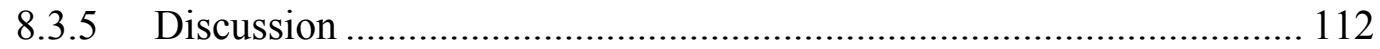

8.4 How to integrate into Smart Grid Build-out? ............................................113

8.5 Technology Transfer, Collaboration and Partnerships ..................................114

Section 9: Project Summary and Conclusions............................................. 116

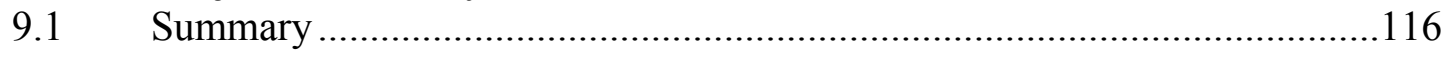

9.1.1 Phase I - Fault Location, Isolation, and Protection for the Circuit of the

Future 116

9.1.2 Phase II - Fault Location, Isolation, and Protection for the Circuit of the

Future with a Fault Current Limiter ............................................................. 117

9.1.3 Phase III - Advanced Fault Sensing and Prediction ........................... 118

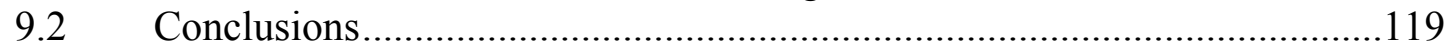

9.3 Recommendations for Further Work …………........................................121

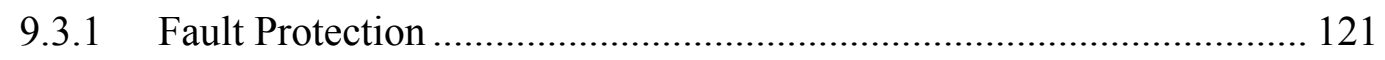




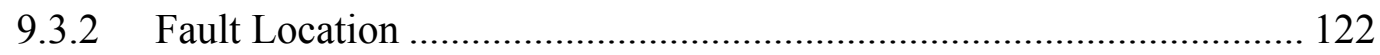

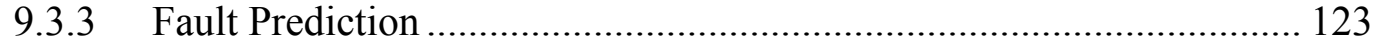

Appendix A: Literature Search and Results ..................................... 124

Appendix B: $\quad$ Survey Questionnaire and Results ................................ 277

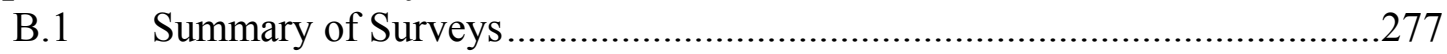

B.1.1 Fault Protection and Sensor Technologies.................................................227

B.1.2 Fault Location and SCADA Technologies ………....................................278

B.1.3 Other Technologies/ Future Work …………..........................................278

B.2 Detailed Responses to the Surveys ........................................................278

B.3 Actual Survey Request Letter Sent to Potential Respondents:.....................293

B.4 Actual Survey Questionnaire Sent to DA Working Group of the IEEE PES T

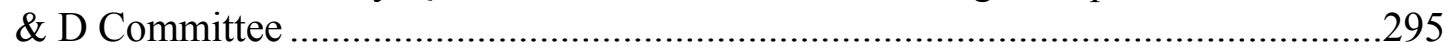

Appendix C: $\quad$ Fault Current Limiter Models ........................................ 301

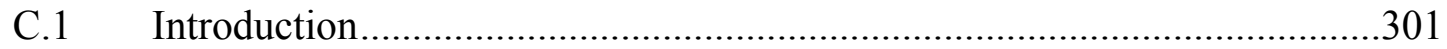

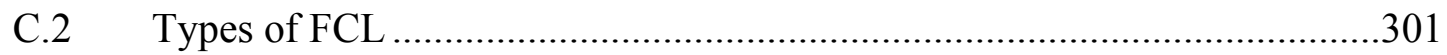

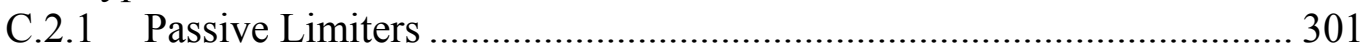

C.2.2 Super conducting limiters ............................................................... 302

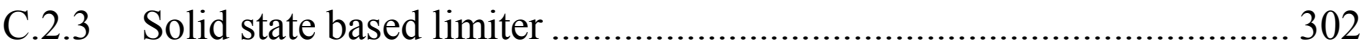

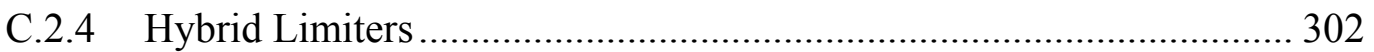

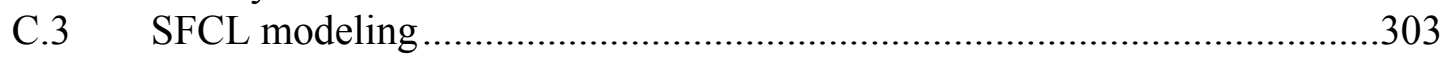

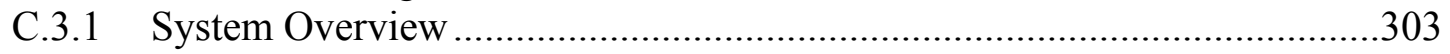

C.3.2 Super conducting fault current limiter .......................................................303

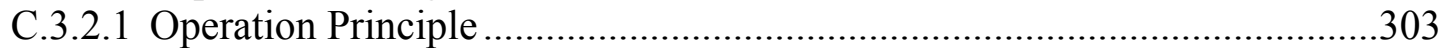

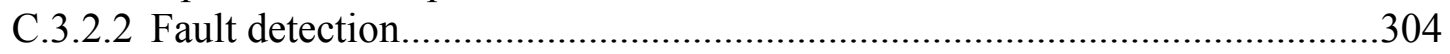

C.3.2.3 SFCL Activation and operational logic ....................................................304

C.3.2.4 Implementation Methodology.................................................................306

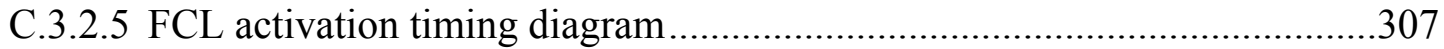

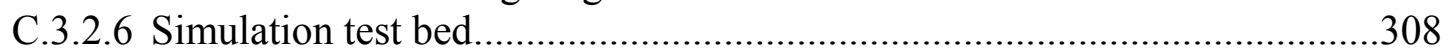

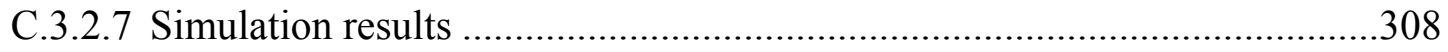

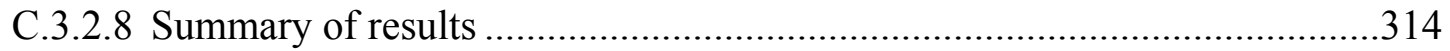

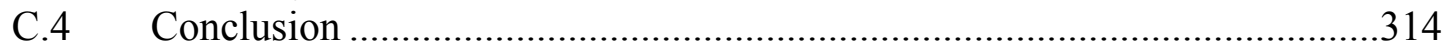

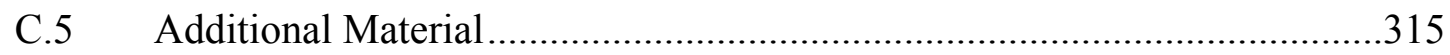




\section{Table of Figures}

Figure 0-1 - General Arrangement of Circuit of the Future ............................................ 11

Figure 0-2 - SEL-2100 Logic Processor and Fiber Optic Communication ....................... 12

Figure 0-3 FCL Components (Courtesy: Zenergy Power Inc.) ……………………….... 13

Figure 0-4 - Voltage Recording from March 16 FCL Resonance Event.......................... 15

Figure 0-5 - SCE's System of the Future (Irvine Smart Grid Demonstration) ................. 17

Figure 1-1 - Circuit of the Future Features …………………….................................. 23

Figure 4-1 - General Arrangement of the Circuit of the Future........................................ 40

Figure 4-2 - SEL-2100 Logic Processor and Fiber Optic Communication ....................... 41

Figure 4-3 - Blocking Scheme Operation ..................................................................... 42

Figure 4-4 - Blocking Operation for Reversed Power Flow.............................................. 46

Figure 4-5 - Circuit of the Future Lab Testing............................................................... 47

Figure 4-6 - Circuit of the Future Lab Testing - Relaying Equipment in Racks .............. 48

Figure 4-7 - Running Lab Test Scenarios ................................................................. 48

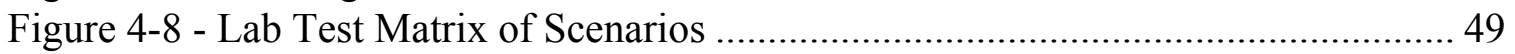

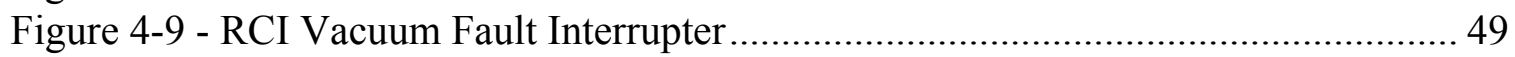

Figure 4-10 - Protective Relay and Controller Cabinet for RCI........................................ 50

Figure 4-11 - Circuit of the Future Protection Elements and Communications .............. 51

Figure 4-12 - Underground Vacuum Switch and Controller Mockup .............................. 54

Figure 4-13 - Overhead Automatic Recloser and Controller Mockup .............................. 54

Figure 4-14 - Training Presentation for Engineers, Maintenance Staff and Operators .... 55

Figure 4-15 - Training Presentation for Engineers, Maintenance Staff, and Operators ... 55

Figure 4-16 - SCE Vice-President Jim Kelly of T\&D Energizing the Circuit of the Future

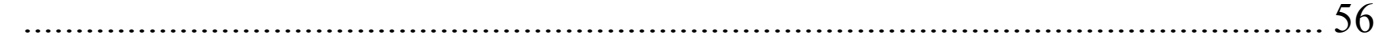

Figure 4-17 - SCE CEO John Bryson at Dedication of Circuit of the Future .................. 57

Figure 4-18 - Press Coverage at Dedication of Circuit of the Future .............................. 57

Figure 4-19 - Press Talking to Operators at Dedication of Circuit of the Future............. 58

Figure 5-1 - Bypass Switch and Fault Current Limiter in Shandin Substations............... 61

Figure 5-2 FCL Components (Courtesy: Zenergy Power Inc.) ....................................... 62

Figure 5-3: Three Phase HTS Saturable Core FCL Design (Courtesy: Zenergy Power

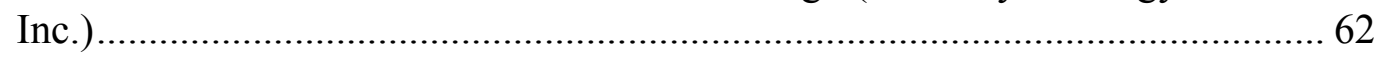

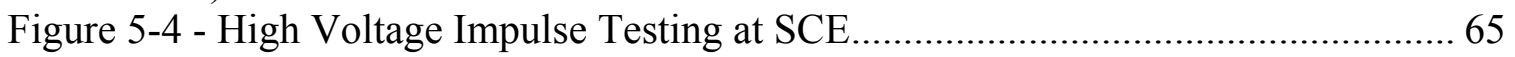

Figure 5-5 - Fault Current Limiter at SCE's Shandin Substation ...................................... 66

Figure 5-6 - Bypassing the FCL under DC Bias (Courtesy: Zenergy Power Inc.)........... 68

Figure 5-7 - Bypassing the FCL without DC Bias (Courtesy: Zenergy Power Inc.)........ 69

Figure 5-8 - PSCAD Representation of the Bypass and Isolation of the FCL (Courtesy:

Zenergy Power Inc.) ...................................................................................... 71

Figure 5-9 - FCL Rms Voltage and Voltage Drop (Courtesy: Zenergy Power Inc.) ....... 72

Figure 5-10: Current and Voltage ratings for DOE sponsored projects ............................ 74

Figure 5-11 - FCL Model Customized for a Superconducting FCL................................. 75

Figure 5-12 - Modeled Fault and Line Current without FCL ………………………..... 76

Figure 5-13 - Modeled Line Current with FCL ............................................................. 76

Figure 6-1 - Phasors of 2nd, 3rd, 4th, and 5th harmonics for a HIF between Sectionalizers

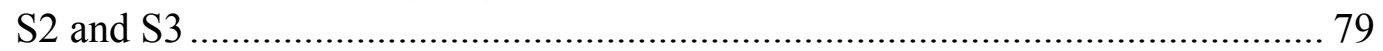

Figure 6-2 - SCE's System of the Future (Irvine Smart Grid Demonstration) ................. 85 


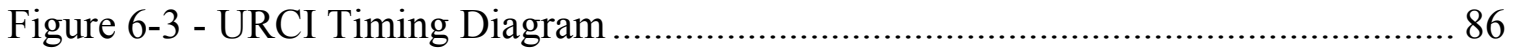

Figure 6-4 - URCI Logic Diagram........................................................................... 87

Figure 7-1 - Circuit Diagram Showing Fault Location................................................... 90

Figure 7-2 - Momentary Fault - RCI-1 - 10/21/2007 8:56:11.536 _................................. 91

Figure 7-3 - Fault Trip - RAR-1 - 10/21/2007 9:10:58.690 ………………………..... 92

Figure 7-4 - Fault Trip - RAR-1 - 10/21/2007 9:11:09.296 ………………………...... 92

Figure 7-5 - Fault - RCI-1 and RCI-2 - 12/25/2007 5:32:43 …................................. 93

Figure 7-6 - Fault Trip - RCI-2 - 12/25/2007 5:32:43 ........................................ 94

Figure 7-7 - Fault - RCI-1 and RCI-2 - 11/14/2008 8:13:34_....................................... 94

Figure 7-8 - Fault Current Limiter and Fault Location................................................... 95

Figure 7-9 - Voltage Recording from March 16 FCL Resonance Event.......................... 96

Figure 7-10 - Fault Current Waveforms Downstream from the FCL - 1/14/2010 ........... 98

Figure 7-11 - Voltage Magnitudes Downstream the FCL - 1/14/2010............................ 99

Figure 7-12 - Normal Voltage Harmonics at Substation ............................................... 100

Figure 7-13 - Normal Current Harmonics Downstream of the FCL ………………...... 100

Figure 7-14 - Substation Voltage Harmonics - First Fault Section .................................. 101

Figure 7-15 - Substation Voltage Harmonics - Second Fault Section.............................. 101

Figure 7-16 - FCL Current Harmonics - First Fault Section ............................................ 102

Figure 7-17 - FCL Current Harmonics - Second Fault Section ....................................... 102

Figure 8-1 - Conceptual Representation of Proposed Protection Scheme....................... 112

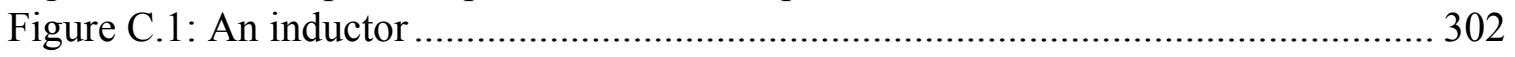

Figure C.2: A solid state based resonant limiter ......................................................... 302

Figure C.3: One-line system diagram of Circuit of Future ............................................ 303

Figure C.4: SFCL Activation and operational logic ....................................................... 305

Figure C.5: SFCL activation timing graph ………………....................................... 307

Figure C.6: Fault and Line current without SFCL....................................................... 309

Figure C.7: Zoomed to show fault and line current without SFCL............................... 309

Figure C.8: Current magnitude and rate of change of current without SFCL ............... 310

Figure C.9: Zoomed to show current magnitude and rate of change of current without

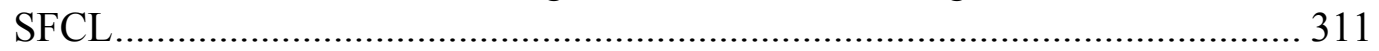

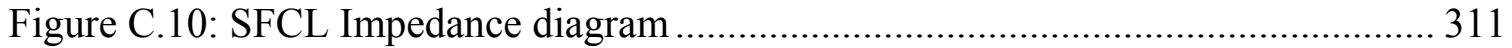

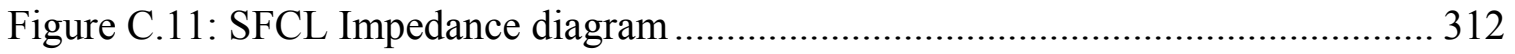

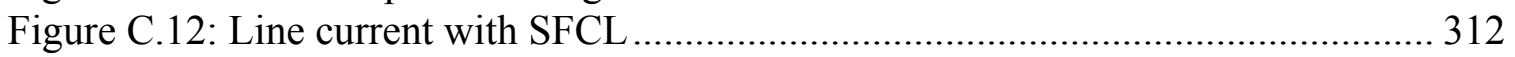

Figure C.13: Zoomed in to show the line current with SFCL ....................................... 313

Figure C.14: Current magnitude and rate of change of current without SFCL .............. 314

\section{Table of Tables}

Table 3-1 - Project Milestones............................................................................................ 39

Table 4-1 - Loss of Communications Actions .................................................................. 45

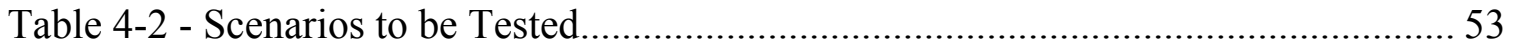

Table C.1: Overview of simulation test parameter ........................................................ 308

Table C.2: Summary of SFCL modeling results........................................................ 314 


\section{Section 0: Executive Summary}

One of the main objectives of this DOE-sponsored project was to reduce customer outage time. Fault location, prediction, and protection are the most important aspects of fault management for the reduction of outage time. In the past most of the research and development on power system faults in these areas has focused on transmission systems, and it is not until recently with deregulation and competition that research on power system faults has begun to focus on the unique aspects of distribution systems. This project was planned with three Phases, approximately one year per phase. Due to some delays encountered with circuit equipment installation and procurement of a fault current limiter, the project actually took place over four years concluding on June 30, 2010.

\subsection{Phase I - Fault Location, Isolation, and Protection for the Circuit of the Future}

The first phase of the project involved an assessment of the state-of-the-art in fault location, prediction, and detection as well as the design, lab testing, and field installation of the advanced protection system on the SCE Circuit of the Future located north of San Bernardino, CA.

\subsubsection{State-of-the-Art Review}

To help understand the trends in the industry and what technologies were being developed in the fault location, prediction and detection areas, a literature search and survey were performed (see Section 2 for more details). The literature search, conducted by Virginia Tech and KEMA Inc., concentrated on more recent developments taking place since 1990. This period covers two important aspects of fault research: a) it encompasses the acceptance and maturing period of digital devices in power system protection functions, and $b$ ) it includes the period of fast development of communications systems that have enhanced the flexibility and area of impact of digital protection systems. This literature review report was supplemented with a survey of utilities, universities, and research organizations to determine which of these techniques are actually being implemented. The survey, sent out jointly with EPRI, was issued to more than 430 different entities in North America and the world as well as the Distribution Automation Working Group of the IEEE. The literature review and industry surveys reveal that system reliability, automation, and customer-focused operation are the main issues driving new developments for distribution system fault location, detection and prediction applications. The key technologies driving these developments are affordable intelligent electronic devices (IEDs), better transducers/sensors, and accessible communication channels. The level of development and acceptance of each of these technologies determines the time frame for the implementation of new developments on

distribution systems. 


\subsubsection{The Circuit of the Future}

The actual Circuit of the Future initial design was based on the collection of a number of ideas from the four SCE Distribution Engineering divisions (described in more detail in Section 4 of this report). These ideas were reduced to a tentative functional design for the advanced protection system to be implemented on the Circuit of the Future. Detailed design work started in 2006 and was supported by this project. Once the detailed design was completed, a lab mock-up of the advanced protection system was assembled. This lab demonstration was used to work out bugs in the design and also to train SCE field personnel on how to operate and troubleshoot the new advanced protection system (early 2007). Once all bugs were worked out and training conducted, the equipment was installed on the actual circuit. Following commissioning testing on the protection system, the circuit was placed in operation on August 14, 2007. A formal dedication of the circuit was held on October 16, 2007 with the SCE CEO including press coverage.

The location for the new Circuit of the Future was chosen in an area where there was new load growth so that at least part of the circuit involved new construction. The location was north of San Bernardino, CA served by Shandin Substation. This new $12 \mathrm{kV}$ circuit would have approximately 2,000 customers and be built with overhead and underground construction. There were also adjacent feeders that could be used for backup after faults. Selection of this circuit allowed detailed design choices to be made and plans formulated for construction.

The present SCE circuit automation scheme uses a single, automated mid-point switch. When a fault occurs, the substation breaker opens and then recloses. If the fault is still present, the substation breaker reopens and the mid-point switch also opens. Then the substation breaker closes for the second time. If the fault is in the first half of the circuit the substation breaker trips, locks out, and the second half of the circuit is restored by the operator through the use of an automated tie switch. If the fault is in the second half of the circuit, the substation breaker closes which restores the first half of the circuit. This method of automation gets many customers back in service quickly, but still causes several operations of the substation breaker and interrupts all customers on the circuit.

\subsubsection{Description of the Circuit of the Future Protection Scheme}

The new feeder automation scheme, with vacuum fault interrupters, will limit the number of customers affected by the fault. Depending on the fault location, the substation breaker might not even trip. Through the use of fast communications (fiber) the fault locations can be determined and the proper fault interrupting switches opened automatically. With knowledge of circuit loadings at the time of the fault, ties to other circuits can be closed automatically to restore all customers except the faulted section. This new automation scheme limits outage time and increases reliability for customers. Figure 0-1 shows the general arrangement of the Circuit of the Future and its protection devices. 


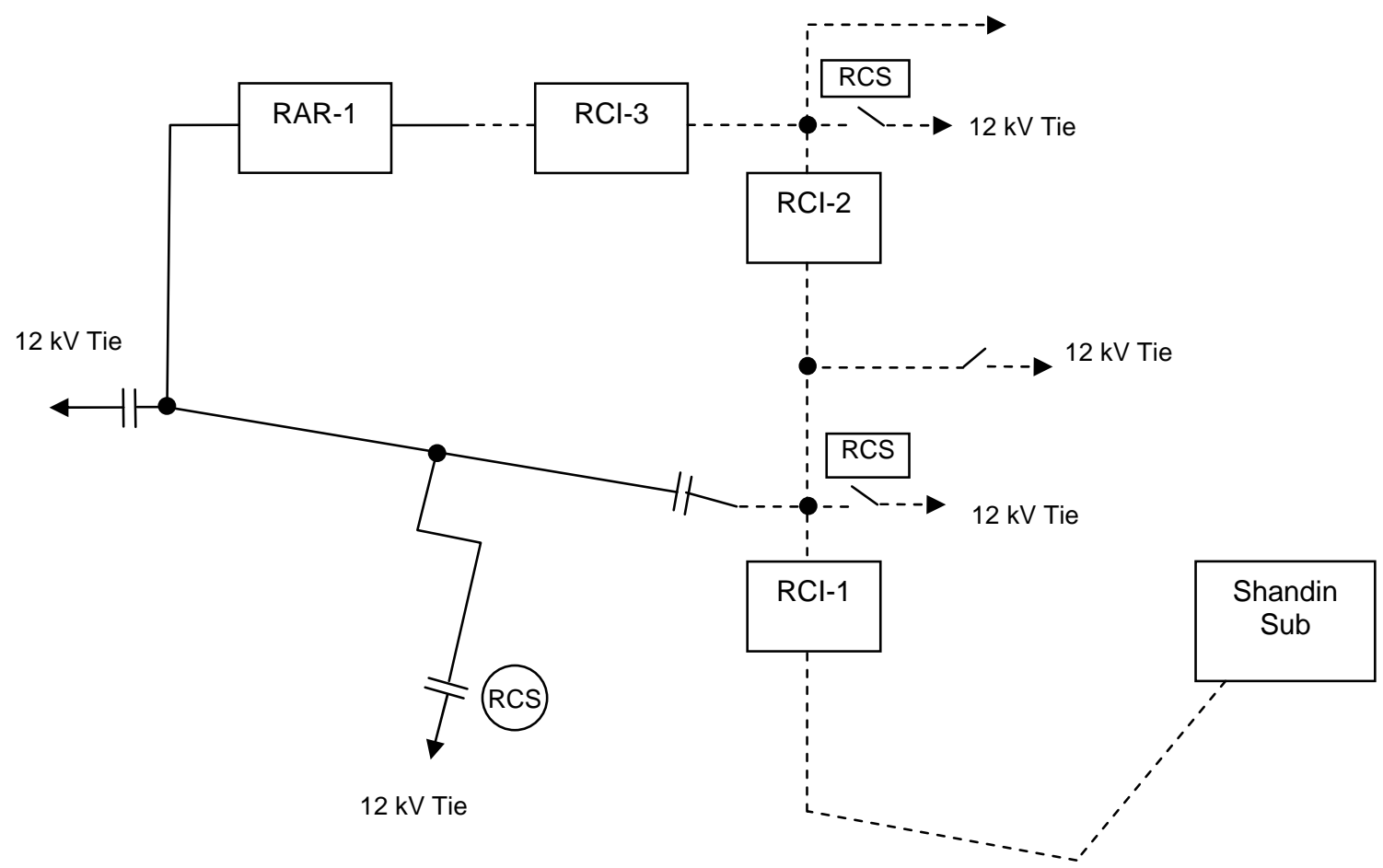

Figure 0-1 - General Arrangement of Circuit of the Future

In order to minimize the number of customers affected by a circuit fault, three Remote Controlled Interrupters (RCIs) and a Remote Automatic Recloser (RAR) are used to isolate the faulted section of the circuit. After a fault, control logic automatically opens the next downstream protective device to facilitate restoration of service to the unfaulted portions of the circuit. This automation function is referred to as an "Iso-Open." A five second delay is incorporated in the automatic opening logic to make it clear that the opening is an automated control operation and not the result of a second fault. For example, a fault between RCI-1 and RCI-2 will trip RCI-1. Five seconds later, control logic opens RCI-2 automatically so that the remotely controlled tie switch between RCI-2 and RCI-3 can be closed, restoring service via a neighboring $12 \mathrm{kV}$ circuit, to all loads beyond RCI-2.

Automation and coordination of the protection is provided by a Schweitzer SEL-2100 Logic Processor located in the control cabinet for RCI-2 and RCI-3. Fiber optic cables connect the SEL-2100 to each RAR and RCI as well as to Shandin substation, as shown in Figure 0-2. RAR-1 is programmed for one automatic reclose after a fault. The RCIs do not automatically reclose. 


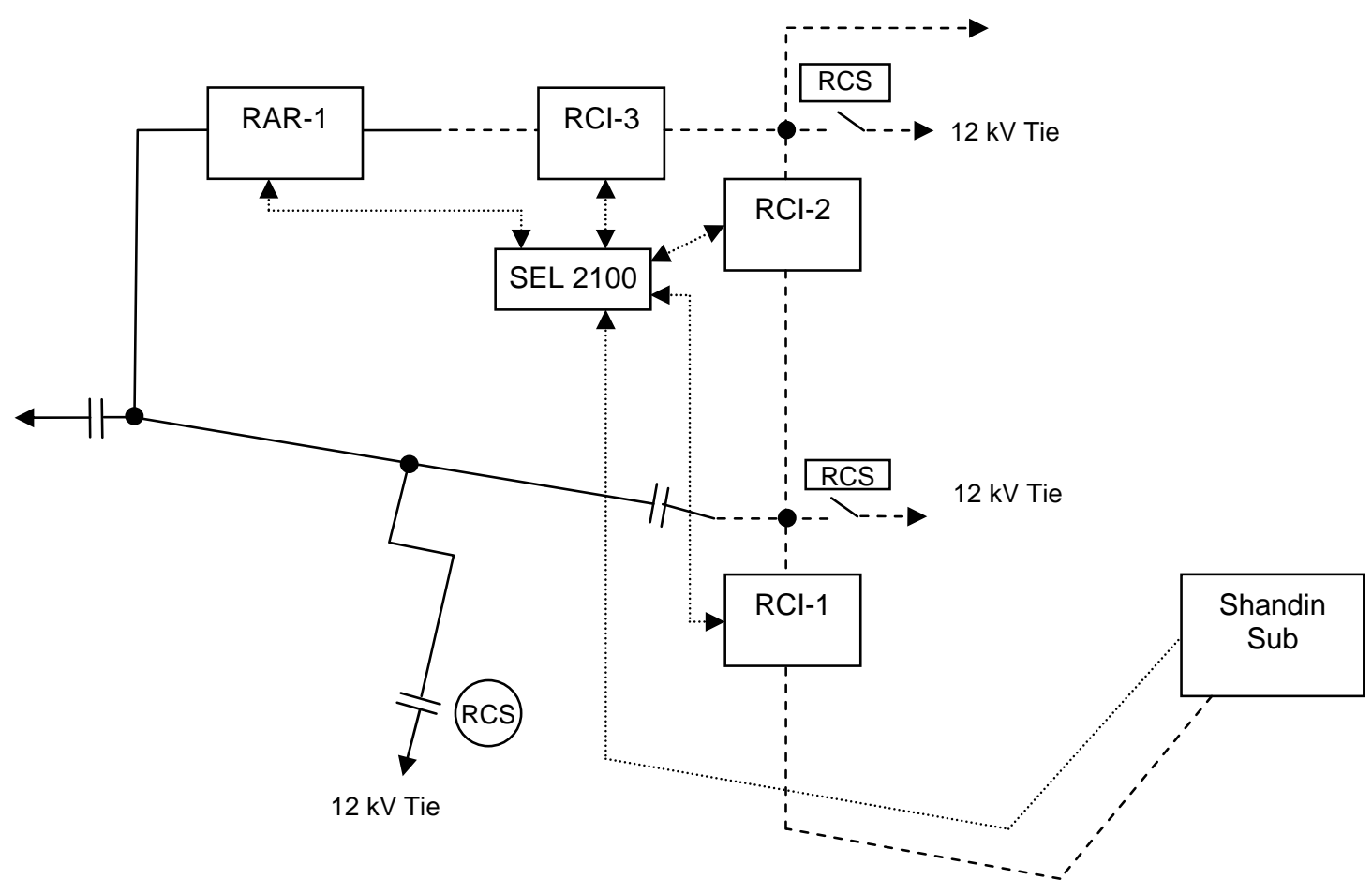

Figure 0-2 - SEL-2100 Logic Processor and Fiber Optic Communication

\subsubsection{Lab Testing and Training}

Preparation for lab testing was begun in late 2006. Testing actually was started in June 2007. The Lab Test Plan objectives included set up and test verification of all identified trip and reclose scenarios for the Circuit of the Future given the advanced protection design.

Since all the field equipment had been set up for lab testing, field personnel were brought in to undergo training on the new system. This training consisted of an overview of the operation of the protection system and more detailed training on the actual equipment and its operation. The goal of this training was to equip operators and maintenance personnel with the skills needed to maintain and troubleshoot the system in case of problems. The operators were also trained in what would happen during and after a fault so they would be able to take proper actions. This training took place at the site of the lab testing setup as well as regional meetings closer to the circuit site.

\subsubsection{Testing Results}

Results from faults experienced on the circuit are described in Section 7 of this report. Four faults were recorded on the circuit. The first one occurred on 10/21/2007 and did not result in tripping of the RCIs. The other three faults resulted in tripping an RCI to isolate the fault without tripping the complete circuit. This demonstrated the goal of only interrupting the faulted portion of the circuit rather than tripping the whole circuit using the substation circuit breaker. 


\subsection{Phase II - Fault Location, Isolation, and Protection for the Circuit of the Future with a Fault Current Limiter}

The second phase of the project involved the selection, modeling, testing and installation of a fault current limiter on the Circuit of the Future. While this project did not pay for the installation and testing of the fault current limiter, it did perform the evaluation of the fault current limiter and its impacts on the protection system of the Circuit of the Future. After investigation of several fault current limiters, the Zenergy superconducting, saturable core fault current limiter was selected for installation.

\subsubsection{Fault Current Limiter Description}

The FCL consists of three major components: An iron core, AC windings, and a high temperature superconducting (HTS) DC winding. The AC winding is on one leg of the iron core and the HTS DC winding is on the other leg. The HTS DC winding serves to bias the magnetic iron core into the saturated condition. The AC line of the FCL is connected in series with the AC line in the power grid in which the fault current is to be controlled. Two iron cores per phase are required, each with the AC coils wound in opposite directions. This is to provide current control during both halves of a single sinusoidal wave cycle as shown in Figure 0-3. Zenergy Power's FCL design takes advantage of an electromagnetic phenomenon - a coil with a magnetically saturated iron core has low ac impedance, while a coil with an unsaturated iron core has high ac impedance. Since the Zenergy FCL is a prototype device and the circuit might be operating for some time without an FCL, provisions were made on the circuit for a bypass switch that would allow the circuit to operate normally with or without the FCL in service. This bypass switch would be able to carry fully rated current as well as expected fault current without the FCL. This bypass switch was located in the substation near the substation switch rack.

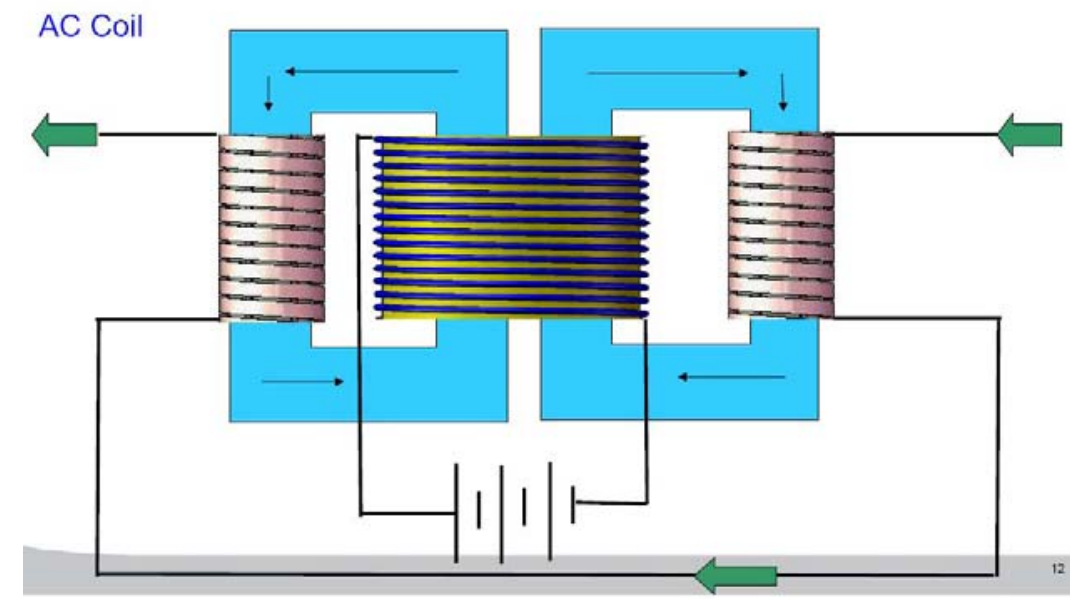

Figure 0-3 FCL Components (Courtesy: Zenergy Power Inc.) 


\subsubsection{Fault Current Limiter Testing}

This fault current limiter, it's testing, and installation is described in more detail in Section 5 of this report. As SCE was investigating the availability of a fault current limiter, research on various types of fault current limiters was conducted by Virginia Tech. Virginia Tech and SCE also built numerical models to be used to evaluate impacts of fault current limiters on the advanced protection system on the Circuit of the Future. Both test measurements and modeling of the FCL indicated that the peak fault current might be limited as much as $20 \%$. Since the peak fault current expected at the substation bus was $23 \mathrm{kA}$, the FCL would be able to limit the fault current to about $18 \mathrm{kA}$ for a fault located at the substation bus. Faults located farther out on the circuit would draw less fault current. Since this is still a significant fault current level, it was determined that the circuit protection would not require any coordination changes. The fault current interrupters on the circuit would also be expected to operate correctly with this small fault current reduction. The modeling studies also showed that problems with coordination of the circuit protection would not be expected to occur until the fault current was limiter to less that $6 \mathrm{kA}$.

\subsubsection{Fault Current Limiter Field Experience}

Because of some testing problems with the Zenergy fault current limiter, installation was delayed until early 2009 with it being put into operation on March 6, 2009. Only one fault was experienced while the FCL was energized, but an unintentional insertion of the FCL impedance also occurred shortly after installation (see Section 7 for more details). A malfunction of the FCL controller caused the DC power supply to the superconducting magnet to be turned off. This inserted the FCL impedance into the circuit while it was in normal operation causing a voltage resonance condition (voltage rise of $3 \%$ - Figure $0-4$ ). While these voltages never reached a point where damage would occur on customer equipment, steps were taken to insure this would not happen again. 


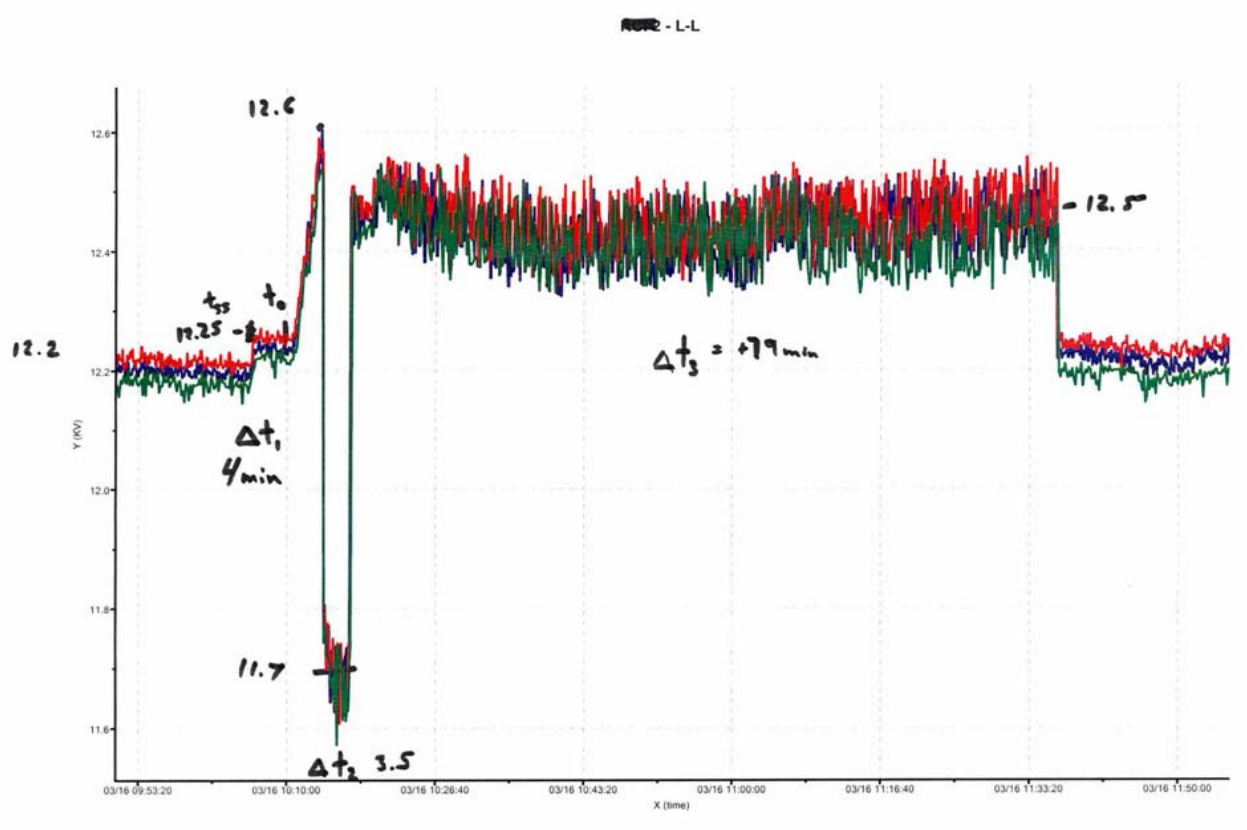

Figure 0-4 - Voltage Recording from March 16 FCL Resonance Event

The FCL was reenergized with load on December 18, 2009. A fault was experienced on the circuit with the FCL in operation on January 14, 2010. The FCL operated properly and reduced the fault current by about $8 \%$, what was expected from tests and modeling. As of the end of the project, the FCL was still in operation on the circuit.

\subsection{Phase III - Advanced Fault Sensing and Prediction}

The third phase of the project involved the exploration of several advanced protection ideas that might be at a state where they could be applied to the Circuit of the Future and elsewhere in the SCE electrical system. Based on the work done as part of the literature review and survey, as well as a number of internal meetings with engineering staff at SCE, a number of ideas were compiled. These ideas were then evaluated for applicability and ability to be applied on the Circuit of the Future in the time remaining for the project. Some of these basic ideas were implemented on the circuit including measurement of power quality before and after the FCL. It was also decided that we would take what was learned as part of the Circuit of the Future work and extend it to the next generation circuit protection for SCE. Also at this time, SCE put in a proposal to the DOE for the Irvine Smart Grid Demonstration using ARRA funding. SCE was successful in obtaining funding for this proposal, so it was felt that exploration of new protection schemes for this Irvine Smart Grid Demonstration would be a good use of the project resources. With this in mind, a protection system that uses fault interrupting switches, high speed communications, and looping two radial distribution circuits would 
have the potential for significant reductions in interruption time and number of interruptions for customers.

\subsubsection{Fault Protection on the System of the Future}

In the Circuit of the Future, fault interrupting switches were connected by fiber optic communications cables so that the location of the fault could be identified by the Logic Processor and the proper switch opened. Since this circuit was a radial design, all customers beyond the opened fault switch would lose power until the tie switch to an adjacent circuit could be closed by a system operator. While this system was able to reduce the number of customers interrupted when a fault occurred, fiber installation was costly and still required actions by a system operator to restore customers located past the faulted section of the circuit.

To address these issues, the design of the advanced protection system to be used on the System of the Future made some changes. To address the cost issue, the fiber optic communications portion of the system would be replaced with a wireless radio system. This radio system needs low latency $(\sim 100 \mathrm{~ms})$, but would not necessarily require high bandwidth. This is because the messages communicated between switches are short. In a move towards standards, the message sent over the wireless radio system is using IP and would conform to IEC61850 GOOSE standards. To fix the issue with operator intervention to restore customers past the fault, two distribution circuits would be looped together (Figure 0-5). With the circuits looped, the faulted section of the loop could be isolated while all other customers would not experience an outage. This protection method would also be able to more easily integrate distributed generation since it can account for bi-directional current flow. Based on radio latency and coverage studies, protection logic simulations, and field circuit constraints, the final circuit protection system is being derived. While this project was not able to complete this work, it is being picked up by the SCE Irvine Smart Grid Demonstration project (DOE ARRA Smart Grid Demonstration project) and is planned for installation on the circuit in 2012. Further information on this project phase is available in Section 6. 


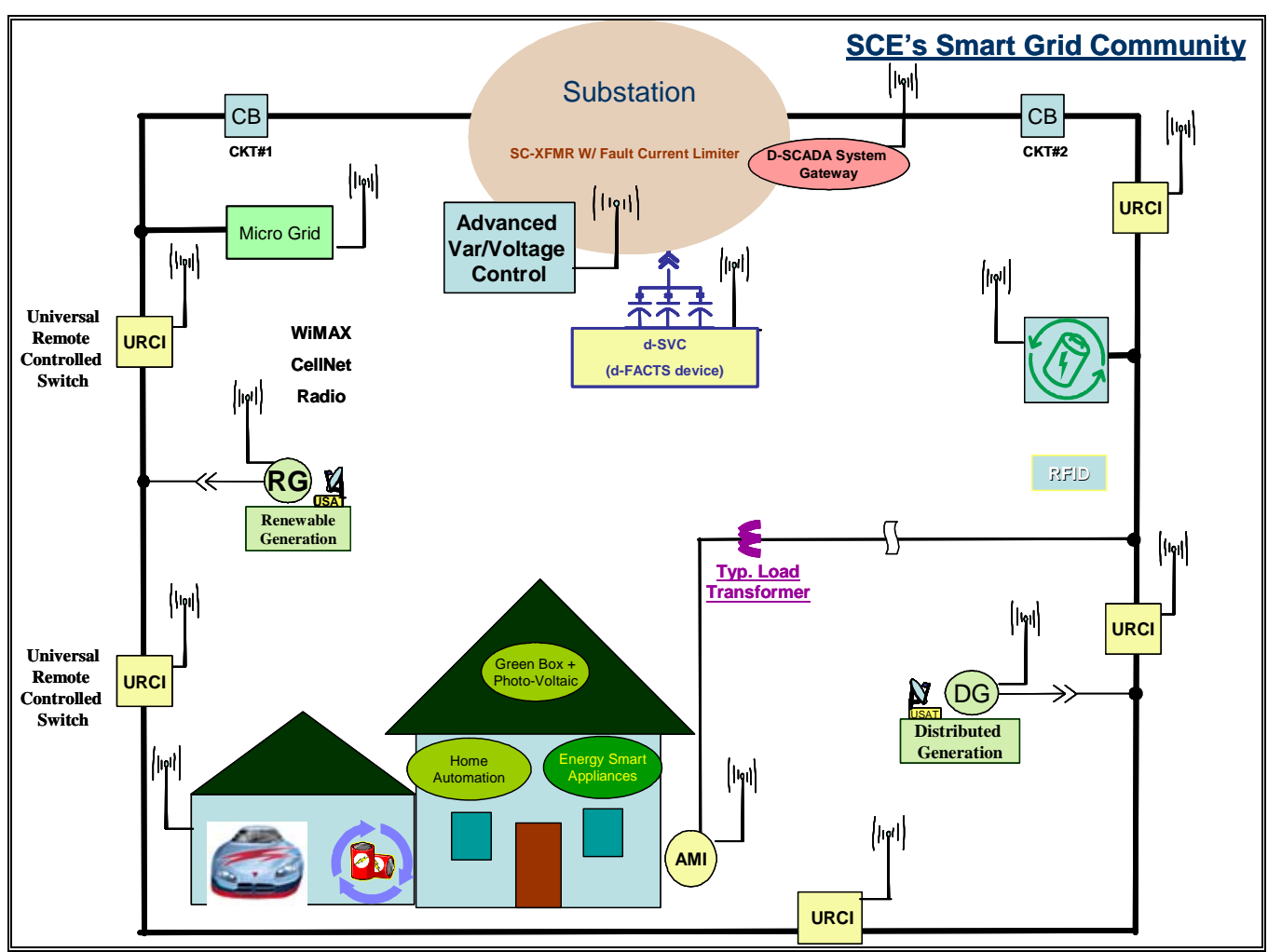

Figure 0-5 - SCE's System of the Future (Irvine Smart Grid Demonstration)

\subsection{Conclusions}

There are a number of drivers that are making the build-out of the Smart Grid necessary. In the distribution area these include the proliferation of distributed generation (e.g. rooftop photovoltaics, battery storage), introduction of electric vehicles and their chargers, and the increased potential of demand response (e.g. smart appliances, air conditioner control). All of these together argue for the need to implement advanced distribution automation and a new generation of advanced Distribution Management Systems (DMS). Advanced protection should be viewed as an integral part of advanced distribution automation. As part of this work, there is a need to define and develop new, advanced distribution automation functions and schemes. Along with these automation systems, new planning methods also need to be developed (see Section 8 for a more detailed discussion).

More advanced computerized relays, cost-effective phasor measurement units, high power electronic control devices, digital communication technologies etc, are continuously evolving to facilitate improved protection of distribution systems. As a result, the monitoring, control and protection of the power systems in real-time is becoming more effective. These new technologies will hopefully allow the systems to become more efficient and reliable. The protection community should seize these opportunities to make power systems safer, more secure, more reliable and less vulnerable to external threats. 
What does this advanced distribution automation system look like? Information and control will be distributed throughout the electrical system in a hierarchical manner. Sensors, remote controlled switches and protective devices will allow integration of fault isolation and system restoration into one function. Microgrids with distributed generation/ storage integrated into utility system can yield higher reliability. Electric vehicles and smart loads will be integrated in a single load management system that conveys pricing signals to the customer. Advanced materials will make possible inexpensive fault current limiting and more efficient transformers.

In this context, advanced DMS applications need to be developed and will include many of the following applications:

- Automatic Fault Location, Prediction, and Protection Systems (FLPPS) and service restoration

- Optimal Volt/VAR Management

- Fast on-line power flow analysis

- Smart Distribution Operations of the Near Future

- Fast short circuit / coordination analysis

- Automatic contingency analysis

- Demand response

- Management of distributed generation and storage

- Automatic vehicle location system (crew management)

Advanced fault location, prediction and protection systems will be an important part of this view of the future. Protection systems that can accommodate distributed generation/ storage, electric vehicle charging, and microgrids will be important as the smart grid moves forward. These protection systems will need more communicating sensors that will allow implementation of advanced protection systems. Lower cost fault interrupting switches and protective relays need to become available so that distribution lines can be broken up into smaller parts, affecting fewer customers when there is a fault. Communications need to be developed with lower costs, higher reliability during disasters, and lower latencies for protection needs. Distributed monitoring and control needs to be introduced in a bigger way than has been done in the past. This will allow control at a substation level so that circuits can do a better job of self monitoring and self healing.

Planning for these advanced systems should be done now, before all of these new generation and load devices are connected to the grid. Through American Recovery and Reinvestment Act (ARRA) projects like the SCE Irvine Smart Grid Demonstration, some of these new concepts can be tested and validated. Because utility planning is usually based on rate case cycles of several years each (three years for SCE), steps need to be taken so that funds are available for smart grid system improvements when the technologies become available and are needed. 


\subsection{Recommendations for Further Work}

Since the primary thrust of this project is on advanced fault protection, location and prediction, the following recommendations are for future work in these three specific areas. These recommendations represent the vision of the investigators based on their experiences with this project and other work they have been involved with. The ideas recommended will hopefully have success in the near future, depending on the pace of technology development.

\subsubsection{Fault Protection}

A lot of creative and new work has been accomplished in this project to advance the state-of-the art of protection systems. The advanced protection scheme designed and implemented for the Circuit of the Future represents the latest state-of-the art technology as described in Section 4. This section describes the details of the new devices installed on the Avanti circuit. The other highlight of the project was the selection the modern Zenergy superconducting fault current limiter. The details of the selection process, installation, lab testing, and field testing results are documented in Sections 5 and 7.

Based on the extensive investigations of this project, a lot of potential exists for advancing the protection schemes for future distribution systems. These schemes will undergo many radical changes due to the incorporation of multitudes of distributed generation/ storage sources and plug-in-hybrid vehicles. These devices will be installed at both primary and secondary distribution levels leading to two-way power flow in the system. Another major change that will occur is the topological change from radial to looped systems. Initially, the primary distribution systems will evolve to looped topology, and then, potentially, to a meshed structure to enhance the reliability, security and efficiency of the smart grid.

Continuous advances in IEDs, signal processing and communication media will determine the progress and penetration of these new developments in distribution system fault location and protection. The increased use of microgrids and distributed generation/ storage sources will change the operation and therefore the methods for fault detection and location in distribution systems. Many systems and algorithms have been proposed and tested to deal with these expected changes in distribution systems but most of these proposed developments depend heavily on the reliability, latency, and cost of available communication channels.

The level of penetration of communication dependent applications will eventually drive the cost to acceptable levels but the reliability and latency will depend more on future technical developments. The time constants required for real-time protection and control application in distribution systems are several orders of magnitudes smaller than today's "human interface" focused applications that drive the present development of communication systems. Protection and control applications require small amounts of information to be transferred reliably, in the shortest possible time, to several locations. This is contrary to most present applications where the speed of communication is 
determined based on the data volume only. New communication systems, developed specifically for the time constants of real-time protection applications, are need to achieve the desired penetration of communication supported protection of distribution systems. Fault detection developments are more affected by the communication latency than fault location or fault prediction developments whose time constants are more in line with present communication systems. To partially cope with the present latency problems, specifically designed prediction and extrapolation algorithms need to be developed to account for the communication channel latency. In addition, detection algorithms requiring external information should be design with fail-safe features that allow them to revert to local operation when the communications signal is delayed or not available. This is a common feature of advanced protection systems presently being tested for transmission.

What do these changes mean to protection? First of all the protection architecture itself has to change. Besides replacing classical electromechanical relays with intelligent digital relays, many of the unintelligent devices such as fuses may have to be abandoned as time passes by. Perhaps the only exception is for protecting single-phase laterals at the end of a feeder. This implies that protection engineers have to change the entire philosophy of relaying. Newer thinking is inevitable and imminent if smart grid goals are to be achieved.

Adaptive protection is another important concept that needs to be implemented for future systems to ensure they can adjust to changes and protect circuits efficiently and effectively.

\subsubsection{Fault Location}

The art and science of fault location has advanced tremendously during the past 20 years. Section 2 provides the latest developments based on the extensive literature search and survey of all stakeholders of the electric utility industry. Even after all these developments, the fault location can not be precisely determined at this stage. As the systems become more and more complex in nature and topology, the location of faults will become a more challenging and arduous task. The investigators strongly believe that protection engineers can take this problem as a challenge and solve it. This requires deploying cost-effective sensors massively at both ends of each line segment, however, short they may be. The data collected from these sensors along with the real-time data provided, will advance fault location, hopefully, in the near future. This will also require developing new algorithms for the design and coordination of protection. In the final analysis, the entire subject of protection design has to be undertaken as a challenging task with no pre-conceived notions involved. The distribution protection for the next 100 years will be radically different from the last 100 .

\subsubsection{Fault Prediction}

With existing technology, data mining has been applied successfully in several locations for fault prediction. Unfortunately these applications have not achieve the level of 
penetration expected based on the level of success achieved by the few existing applications. Part of the limitation is due to the time required to create a significant base of data. The extremely localized nature of the fault phenomena also precludes the direct application of the development techniques to distribution systems in different locations. Additional studies are required in the nature of regional variation of fault data and its applications to fault detection. In addition, there needs to be more development of data collection systems and maintenance of data bases.

While pattern recognition techniques for prediction of faults have made significant strides, these are not sufficient to make future systems resilient and self-healing. What is really needed is a fault prediction device installed at each distribution substation. Its role would be to constantly monitor the system, warn of any impending or brewing problems, and alert the system operators for immediate redial action. This system should also be able to seamlessly correct the problem by itself. In other words this device has to be quite smart, almost matching the operator's intelligence and yet aid him/her in making the system very reliable. 


\section{Section 1: Introduction}

\subsection{Project Objectives}

One of the main objectives of this DOE-sponsored project is to reduce customer outage time. Fault location, prediction, and protection are the most important aspects of fault management for the reduction of outage time. In the past most of the research and development on power system faults in these areas has focused on transmission systems, and it is not until recently with deregulation and competition that research on power system faults has begun to focus on the unique aspects of distribution systems.

\subsection{Project History and Background}

This project was conceived in response to a 2005 Financial Assistance Funding Opportunity Announcement from the Office of Electricity Delivery and Energy Reliability (OEDER) - Electric Distribution Transformation and Gridwise Programs. In their "Electric Distribution Multi-Year Research, Development, Demonstration, and Deployment Technology Roadmap Plan: 2005 - 2009" a need was identified for improved Fault Location, Prediction, and Protection. This project was designed to meet those needs as well as meet Southern California Edison's (SCE) need for a real-world test bed for new distribution technologies. Final contracting, allowing work to begin, was completed in October 2006, nearly 4 months after the originally expected start date.

The overall scope of this effort was built around using the SCE $12 \mathrm{kV}$ Circuit of the Future $(\mathrm{CoF})$ which was conceived and created for the purpose of demonstrating new technologies to be potentially used on future distribution circuits. These new technologies include hardware, sensors, communications, and controls. This circuit, which went into operation in 2007, feeds about 2,000 electricity customers near San Bernardino, CA. It includes fault interrupting switches distributed throughout the circuit and high speed communications to facilitate control and monitoring. Other features of the circuit include: a distributed energy resource interconnection point; a fast-switching, multi-step capacitor bank; a fault current limiter; fiber-based duct bank temperature monitoring; and composite poles. The ultimate goal of the $\mathrm{CoF}$ is to meet the long-term needs of the modern electric distribution system and reduce capital and O\&M costs through improved distribution system reliability, safety, security and efficiency.

- The objective of this DOE project is to better detect and isolate faults on the distribution system so that customer interruptions will be minimized in both frequency and duration thus enhancing the system reliability and enabling distribution automation. Fault anticipation and location is especially important in underground distribution areas where faults are hard to find and damage after 
faults is expensive to repair. These objectives help pave the way to reach the ultimate goal of creating a "Smart Distribution Grid".

\subsection{Description of the Circuit-of-the-Future (CoF)}

The following sections describe a short history of the SCE Circuit of the Future and how it was implemented.

\subsubsection{History}

In March 2004, SCE held an internal competition to develop a new distribution circuit design, which was judged by representatives from the California Energy Commission, the U.S. Department of Energy, Oak Ridge National Laboratory, Electric Power Research Institute and KEMA. The winning design, "SCE's Circuit of the Future," was converted into a detailed design to be built and put into operation.

The Circuit of the Future shown in Figure 1-1 is a live circuit, serving actual customers out of SCE's Shandin Substation in San Bernardino California. The circuit is designed for flexibility allowing for the implementation of a combination of new topology, hardware, communications technologies, and automation schemes.

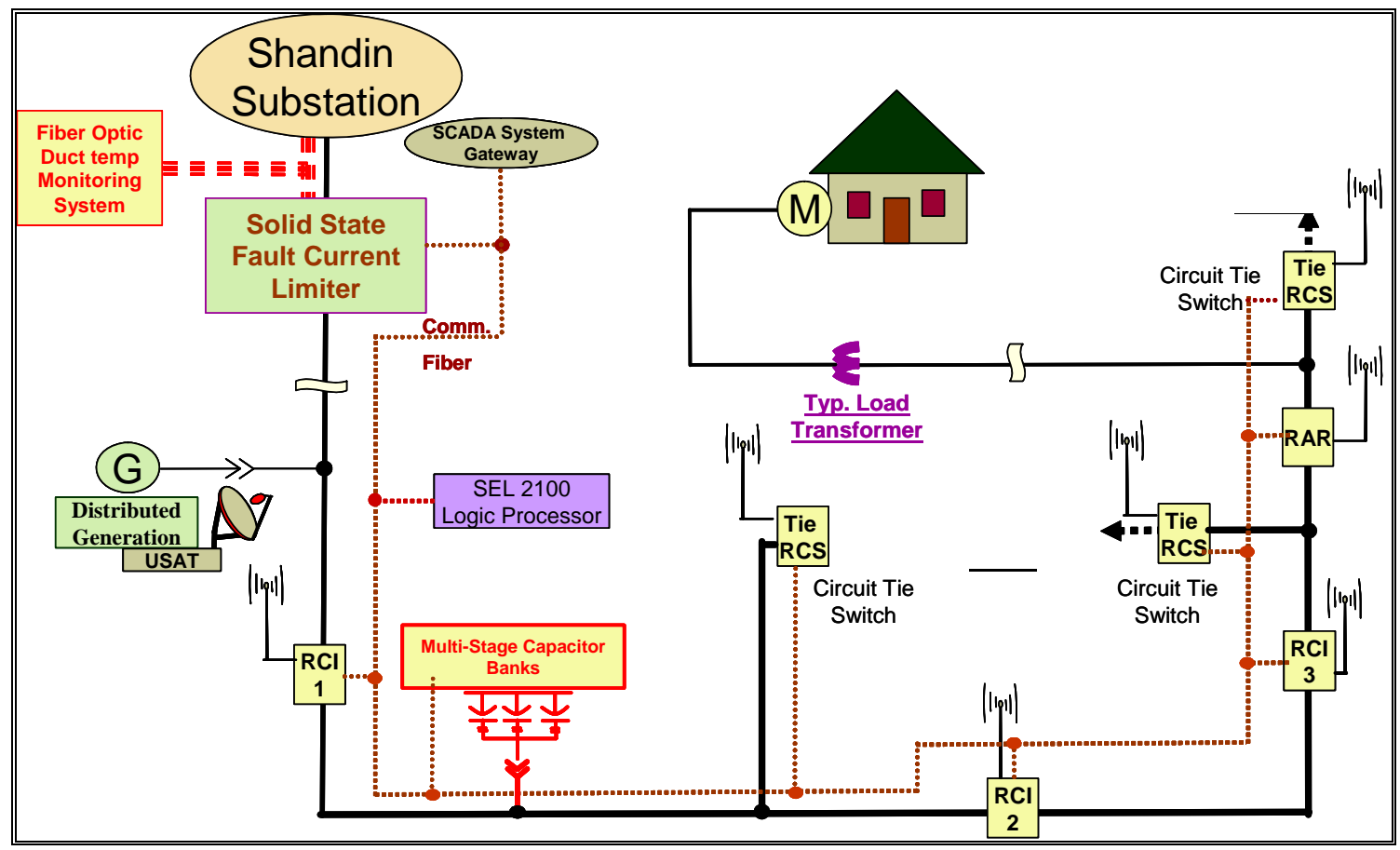

Figure 1-1 - Circuit of the Future Features

\subsubsection{Location}

The location for the new Circuit of the Future was chosen in an area where there was new load growth so that at least part of the circuit involved new construction. The location chosen was north of San Bernardino, CA out of Shandin Substation. This new $12 \mathrm{kV}$ 
circuit would have approximately 2,000 customers and be built with overhead and underground construction. There were also adjacent feeders that could be used for backup after faults. Selection of this circuit allowed detailed design choices to be made and plans formulated for construction. However, it is flexible enough to experiment with other topologies for future application.

\subsubsection{Topology}

A number of circuit topologies were initially considered. These included network designs and looped circuits. As the design phases moved forward, it was realized that almost all of the existing circuits at SCE were radial and the new design would have to integrate smoothly with existing circuits. Because of this limitation, the circuit topology decided on was radial with tie switches to connect to adjacent circuits following faults.

Some consideration was being given to the testing of secondary networks for customers. In this design, a customer would be normally fed from transformers on two circuits out of the same substation. If a problem occurred on one of the circuits, the customer would be fed from the other circuit with no interruption. This concept was planned for testing in a limited manner.

An interconnection point for a distributed generation device would be installed on the circuit. This generation device would be used to supplement the feeder during high load conditions. This interconnection point would also be used to demonstrate the ability of a synchronous generator to supplement reactive power on the circuit. Again, there was adequate flexibility built into the circuit to allow any other DG source to be replaced for future application.

\subsubsection{Hardware}

A range of new equipment was contemplated for testing on the Circuit of the Future. From a structures standpoint, the new circuit used modular, composite poles with molded cross arms in one section. These poles have higher strength than standard wood poles and have much longer life. Composite underground structures for vaults and the use of composite equipment in vaults were both evaluated, but not used at this time. The use of RFID tags was also considered for tracking structures and their characteristics.

At the source end of the circuit, a fault current limiter was installed and tested. This device allowed the use of distribution equipment that is rated at lower current levels on the rest of the circuit. Also in the substation, a fiber optic temperature-measuring device was installed in the getaways for the circuit to evaluate its loading capability.

On the circuit, vacuum fault interrupters were installed in place of switches. This allowed faster isolation of faults while affecting the least number of customers. The device being considered was assembled in a modular manner such that it could be used in overhead, underground, and pad mount locations. Smarter controls attached to these interrupters delivered line loading information as well as fault indications. 
For reactive compensation on the line, transient-free capacitor switching and multi-stage capacitor switching were investigated. Active devices that would reduce line harmonics are also evaluated for application, but not installed as part of this project.

Work with our Customer Service organization was undertaken to determine if there was an opportunity to install remote meter reading or demand response capabilities, but the project went into service before the SCE smart metering project moved to the installation phase.

\subsubsection{Communications Technologies}

Today, most automation of SCE's circuits is done through the use of the UtilitNet peerto-peer packet radio system. This system is well integrated into the SCE operation systems and familiar to all involved. It was used for the basic communications for switch automation and capacitor control. In addition to this radio system, fiber was run to critical information points in the circuit. These points included the vacuum fault interrupters and switches. This high-speed fiber communications allowed the new automation schemes to be implemented (explained below). SCE also investigated broadband over power lines in the circuit for monitoring of equipment as well as remote meter reading, demand response, and other utility applications. This technology was never implemented on the circuit because field trials of the technology in other portions of the SCE system showed it to be problematic.

\subsubsection{Automation Schemes}

The present SCE circuit automation scheme uses a single, automated mid-point switch. When a fault occurs, the substation breaker opens and then recloses. If the fault is still present, the substation breaker reopens and the mid-point switch also opens. Then the substation breaker closes for the second time. If the fault is in the first half of the circuit the substation breaker trips, locks out, and the second half of the circuit is restored by the operator through the use of an automated tie switch. If the fault is in the second half of the circuit, the substation breaker closes which restores the first half of the circuit. This method of automation gets many customers back in service quickly, but still causes several operations of the substation breaker and interrupts all customers on the circuit.

The new feeder automation scheme, with vacuum fault interrupters, will limit the number of customers affected by the fault. Depending on the fault location, the substation breaker might not even trip. Through the use of fast communications (fiber) the fault locations can be determined and the proper fault interrupting switches opened automatically. With knowledge of circuit loadings at the time of the fault, ties to other circuits can be closed automatically to restore all customers except the faulted section. This new automation scheme should limit outage time and increase reliability for customers.

Reactive power compensation was also coordinated for this circuit. Circuit reactive requirements were measured at the substation and the proper number of field capacitor switches commanded to open or close. This better controls circuit voltage and eliminates unnecessary reactive power flows. 


\section{Section 2: State-of-the-art Review}

To help understand the trends in the industry and what technologies are being developed in the fault location, prediction and detection areas, a literature search and survey were performed. The following sections describe the literature search with its recommendations as well as the results of the survey of the industry. The final section describes some conclusions that can be drawn from review of the literature search and survey results.

\subsection{Literature Search}

Work under Phase I of the project included a state-of-the-art review of technologies and methods being researched for fault location, fault prediction, distribution automation, high impedance faults and fault current limiting.

\subsubsection{Background}

The literature search, conducted by Virginia Tech and KEMA Inc., concentrated on more recent developments taking place since 1990. This period covers two important aspects of fault research: a) it encompasses the acceptance and maturing period of digital devices in power system protection functions, and $b$ ) it includes the period of fast development of communications systems that have enhanced the flexibility and area of impact of digital protection systems. The search was based on subject and title search on the IEEE Xplore, Google Scholar, and the Science Citation Index. Papers were summarized by a team of students and facility following pre-established review criteria for each section. The report was divided into sections covering fault location and prediction, fault protection with automation, high impedance faults, and fault current limiters. A summary section presented recommendations for possible implementation on the Circuit of the Future. The details of the literature review are found in the project report entitled "Literature Review of Fault Location, Prediction and Detection for Distribution Systems" prepared by representatives from Virginia Polytechnic Institute and KEMA Inc. included as Appendix A.

\subsubsection{Fault Location and Prediction}

Fault location and prediction can be classified into two main approaches: Signal Analysis and Knowledge Based. Each method offers its own set of requirements, advantages, and disadvantages.

The Signal Analysis method either uses the fundamental frequency $(60 \mathrm{~Hz})$ measurements or high-frequency components. The fundamental frequency approach was first applied using microcontrollers by Takagi in the early 1980s. Since then, different fundamental frequency methods have been derived, and most of them require a detailed modeling of the system, such as accurate line parameters, load data, etc. Some of these 
methods only provide information on the faulted section and not an estimate of the actual fault location. Other methods show an increase in percentage error with higher fault resistance values. However, most of these methods have the advantages of being singleended and not requiring communication channels.

Methods using high-frequency components are mostly based on traveling wave theory. These methods do not have such stringent requirements of accurate line and load modeling. However, multiple reflections and refractions, which are typical of distribution systems with many laterals and load taps, is a concern while using traveling wave based methods. Although quite accurate in operation, most of these methods require communication channels and GPS time synchronization although some singleended methods are also proposed in the literature. The sampling rate requirements of these methods are generally quite high. Not many sufficient amplitude traveling waves are generated when a fault occurs on the system at or near zero-voltage point, thus, the detection of these waves becomes difficult. Some methods using fault-generated high frequency transients are being proposed in the literature. These transients are present even when the fault occurs at low voltage conditions.

Knowledge Based systems use artificial intelligence, fuzzy logic, and neural networks. The methods using artificial intelligence mainly use fuzzy logic or Artificial Neural Networks (ANN) to locate the fault. The feature extraction is a crucial step in the ANNbased scheme. Some of these methods combine information obtained from the network database and the SCADA with heuristic knowledge of operators.

The intent of fault prediction is to determine an imminent failure in a system component early enough to allow for maintenance or replacement of the suspected component. For this report, fault prediction is divided into early detection techniques, data mining, and hidden failures. A large portion of the early detection research concentrates on thermal analysis and other non-invasive techniques for the early detection of damaged components such as transformers, fault arresters, and insulators. Some techniques have been developed to identify current or voltage signals that may indicate imminent component failures. The availability of large fault databases has also sparked the use of a statistical data mining approach to analyze and address the causes of distribution system faults and to investigate the influence of selected factors on distribution system faults. This type of study aims to determine the influence of external factors on the occurrence of some types of faults and the determination of maintenance practices or allocation of resources to reduce the likelihood of a fault. Some of the new interesting concepts developed for transmission systems address the problem of undetected failures in the protection system and their possible failure-magnifying effect after system contingencies. Although developed for transmission systems, these concepts are also applicable to distribution systems.

\subsubsection{Recommendations for Fault Location and Prediction}

Detailed synopses of all articles and papers reviewed are included in the project report entitled: "Literature Review of Fault Location, Prediction and Detection for Distribution 
Systems" authored by Virginia Polytechnic Institute and KEMA Inc as part of this project and included as Appendix A.

1. Implement one of the recommended high-frequency component methods. Highfrequency methods take advantage of the availability of communication channels and include some aspects of GPS time synchronization. This could help gauge the impact of high bandwidth communication systems and time synchronization in distribution systems.

2. Perform a comparison of the three approaches to fault location by selecting and implementing the most promising technique for each method. This could provide very valuable information on the best approach to follow on other existing systems as they evolve into future distribution systems.

3. Implement a differential-signal analysis system with high sampling frequency, time synchronization, and an array of sensitive trigger parameters. The cable splicing detection techniques could serve as the first test for the proposed system. Addition of time synchronization to the thermal information derived from the installed fiber optics may enhance the performance of the proposed system. Implementation of specific fault prediction techniques will depend on the results of the detailed analysis of the collected data.

4. Apply the concept of hidden failures to distribution systems and determine the effect of hidden failures by increasing customer down time. Two aspects of hidden failures could be studied on the system: the current effect of hidden failures in distribution systems and the effects of hidden failures on distribution systems of the future. The availability of high bandwidth communications, advanced IEDs, and the use of fault current limiters facilitate the implementation of a more adaptable protection system. But these increasingly complex systems will become more vulnerable to problems such as hidden failures in the protection elements.

\subsubsection{Fault Protection (Distribution Automation, High Impedance Faults, and Fault Current Limiters)}

Fault detection and protection of most distribution faults is a mature subject with well known and understandable protection algorithms and techniques. Instead of focusing on the enhancement of existing protection algorithms, the fault protection review focuses on the protection of advanced systems where automation, communication and new protective devices will affect the operation of future protection systems. High impedance faults are still not a mature subject, and their protection is reviewed in more detail. The review is divided in three areas: distribution automation, fault detection with focus on high impedance faults, and fault current limiters with some focus on effects on protection systems.

The most common automated functions in distribution systems publications include the following: Volt/Var Control, Fault Location Isolation and Service Restoration (FLIR), Optimal Feeder Reconfiguration, Automated Meter Reading, and Relay Protection Recoordination. The wide spread of fast communication media in the past decade has given rise to the new concept of Advanced Distribution Automation that combines appropriate IT technologies in order to achieve fast and accurate data transmission and processing, for real time operation applications. Four main levels of automation are identified in the 
literature: substation automation, feeder automation, customer automation, and management automation.

High Impedance Faults (HIF) is defined by the IEEE PSRC working group as those that 'do not produce enough fault current to be detected by conventional overcurrent relays or fuses'. HIF typically occur when an overhead line falls in a high impedance surface such as dry earth, concrete or asphalt. Due to the high impedance to ground the fallen conductor fails to draw significant fault current, and it is not cleared by conventional relays resulting in a hazardous situation for persons and property. Unfortunately, very few HIF testing facilities exist, and accurate fault modeling is a big component of HIF research. Many of the proposed methods have only been tested with limited HIF models. The modeling of HIF is a research field of its own, and several models have been proposed for use with common analysis programs. Several mechanical devices have been implemented to force HIF into normal, low impedance, faults to allow clearing of the fault through conventional relays. Most of the proposed techniques focus on electrical devices (relays) for the detection of HIF. Many relays have been proposed and implemented in the past thirty years, and at present, most of the large relay manufacturers offer a HIF relay capable of identifying a very large percentage of the HIF faults.

Several articles provide a necessary background from which to conduct further research into power system performance enhancement via FCLs. While most articles describe the limiters' role in reducing the high current level associated with a fault, each article focuses more precisely on the impact of this technology in the power field. FCLs can be placed within distribution and transmission networks and can offer protection for power systems, as a whole, or particular devices. With the incorporation of a FCL into a protection scheme, there needs to be assurance that the limiter operates quickly enough and can reset in the event of another short circuit. Additionally, ratings requirements must be adjusted to account for the new levels of currents. Overall, the main advantages of current-limiting technology are fast response times, reduction of switchgear ratings, improved transmission capacity, and improved system stability. Although each FCL can satisfy the above requirements, numerous designs are still being tested to maximize the services provided. For example, the costly but effective superconducting fault current limiter requires cooling of a superconductive material in order to maintain the proper operating state and increases in impedance quickly and effectively when heated. This technique is implemented with or without a parallel reactor and/or series capacitor and can be replicated for large-scale applications. Another design calls for solid-state switches and a reactor in a rectifier arrangement. While less expensive and having a slower operation time, this model still offers few losses and can be combined with additional reactors/capacitors as long as proper controls are in place. Tests of threephase and single-phase designs with varying thyristor arrangements show that limiters are capable of handling both common and uncommon fault types without significantly altering the response time and shape. Inductive current limiters are also worth mentioning despite their high cost, weight, and overall size. The screened-core or magnetic shielding-type inductive FCL is an alternative to a purely resistive model and can be likened to a transformer with a variable secondary. A scale up of the Matrix FCL to transmission-level voltages is one design goal whereas operation at $10.5 \mathrm{kV}$ is 
appropriate for the other. Other topics are covered in the review, but they mainly constitute physical properties of superconductors (i.e. thick films, thin films, quenching, V-I characteristics, etc.) and are not as relevant at this time.

\subsubsection{Comments and Recommendations for Fault Protection (Distribution Automation, High Impedance Faults, and Fault Current Limiters)}

According to available publications, the most important issues driving the research and implementation on distribution automation are reliability improvements, reduction of capital and operating costs, innovative customer-focused operation, and connection of distributed generation to distribution systems. Most of these issues are not new but the complexity and cost associated with them puts, at present, an emphasis on the use of technology to address these problems in a coordinated and efficient manner. Adaptive protection combines communication and system automation to improve the performance of the distribution system. Most of the adaptive protection concepts are based on distribution systems, but the use of fault current limiters and advanced communication systems in the distribution systems makes adaptive relaying feasible for the SCE "Circuit of the Future." The existing IEDs, communication channels, and additional signal monitoring can be used to determine the most effective use of automation and adaptive protection in distribution systems.

In the past three decades many algorithms have been proposed for the detection of HIF in distribution and transmission systems. Some of these algorithms have been implemented into commercial devices but most remain in a theoretical or experimental state. Arcing is a common component of HIF, and due to its randomness, asymmetry, and dynamic, its presence is used in many algorithms to detect the presence of a HIF. The knowledge based techniques are the latest application in the evolution of HIF detection and are enabled by the increased computational power of computer relays and the reduced security and dependability of the signal analysis techniques. Although several commercial devices exist for HIF detection, most of these devices detect only up to $85 \%$ of the HIF, and their fault location algorithms are very limited. The use of time synchronization and differential line/cable protection in HIF detection can provide a higher detection rate and more accurate location algorithms, and it could be implemented in the SCE system.

Integration and protection issues are a major concern when dealing with variable impedance devices. The current limiter must be coordinated with the operation of the circuit breakers (current interruption and subsequent reclosing); and at reclosing, once the fault is cleared, the impedance of the FCL should return to zero. A current limiter should operate reliably against a fault whose current is more than its operating point, but FCLs in sound phases or lines should not operate. Also, research has shown that FCLs lower not only the current level but also the rate of rise of the recovery voltage (rrrv), both of which contribute to the burden of a circuit breaker. Thus, the use of a FCL offers the benefit of using a lower capacity the circuit breakers than might be required to handle higher fault duty. The system under consideration should be studied carefully to observe the effects of decreased rrrv with and without the FCL. The severity of the interrupting duty of the circuit breaker will be reduced, so a circuit breaker with a lower interrupting 
performance can be used. The type of current limiter used is important since the effects on the existing protective system vary depending on the type of FCL. For resistive-type FCLs, a first setting method involves the operation start current, ILS, or the current which makes a FCL start its limiting. The upper limit of this current should be the circuit breaker breaking capacity, and the lower limit should be the maximum current on a sound line when a fault occurs elsewhere. This ensures that sound lines are left unaffected by the fault. Also, when a FCL introduces additional impedance to the system, the distance relays (and mho relays) are affected. Since these relays have predetermined operational zones, additional impedance can move operating points outside of the specified zone, which leads to unwanted trips. It is therefore up to a designer to specify a range of the impedance that will sufficiently limit the current yet not affect the response of protective relays or to provide a more accommodating mho characteristic for the relays. For rectifier-type FCL's, care must first be taken to calculate the current traveling through the limiting coil, and once the voltage, frequency, protective relay operation times, and equivalent reactance of the power system at the back of the FCL are known, the limiting coil reactance can be estimated. A circuit breaker breaking capacity limit is also determined based on the ratio (k) of the current through the limiting coil to the fault current magnitude when no FCL is installed (IF) as well as IF itself: ICB $\geq \mathrm{k} \times I F(3)$. In the presence of an FCL, current differential relays operate correctly for all internal faults; however, differential relays cannot discriminate the fault type when a fault occurs near the current limiter. From the protection point of view, the literature review reveals that rectifier-type SFCL's will be easier than resistance-type limiters to introduce into power systems and coordinate with protection schemes.

\subsubsection{General Recommendations from the Literature Review}

\section{Recommendation 1}

Installation of a minimum of two time-synchronized transient recorders in the system that use low-cost optical transducers to obtain some of the system signals. Among the desired characteristics of these devices are:

- GPS synchronization to 1 microsecond

- Minimum of 9 input channels.

- High Sampling rate, over $8 \mathrm{kHz}$

- Minimum of two communication channels

- Large recording media

Recommendation 2

Enable the time synchronization function on the existing SEL relays, and use the relay and transient recorded data to create a fault database.

\section{$\underline{\text { Recommendation } 3}$}

Install the two transient recorders at the two ends of the line that contains the embedded fiber optic for temperature monitoring. Establish this line as our main testing ground. Develop triggering algorithms for common type faults and four incipient faults based of differential relaying principles and the additional information provided by the fiber thermal sensor. 
Recommendation 4

Analyzed the recorded data to develop algorithms to:

a) Detect incipient faults inside our differential protection system.

b) Locate faults inside and outside of our differential protection system.

c) Create a fault library that takes advantage of the time synchronization to provide fault signal delay and propagation information.

\section{$\underline{\text { Recommendation } 5}$}

Take advantage of the existing communication fiber, advanced IED, and requirements of the fault current limiters to study the effect of hidden failures in the protection of distributed systems.

These recommendations from the literature review were analyzed along with other information and what was possible with the SCE's Circuit of the Future to generate what was done for Phases II and III of the project.

\subsection{Survey Questionnaire and Results}

This literature review report was supplemented with a survey of utilities, universities, and research organizations to determine which of these techniques are actually being implemented. The survey, sent out jointly with EPRI, was issued to more than 430 different entities in North America and the world as well as the Distribution Automation Working Group of the IEEE. The summary of the survey results are provided below. Appendix B gives all the details of the original responses.

\subsubsection{Summary of Surveys}

As part of this project, two separate surveys were conducted. The first, sent out questionnaires to over 100 utilities, manufacturers, and universities during the period of June through December, 2008 and received 18 responses (two of these indicated they did not have any research work in progress). These results were divided into three groups:

- Fault Protection and Sensor Technologies

- Fault Location and SCADA Technologies

- Other Technologies/ Future Work

The second survey was composed of 20 multiple choice questions sent out to members of the IEEE T\&D Distribution Automation Working Group during June of 2009. The number of responses to each item is listed to its right. They were grouped in the following categories:

- Feeder Automation

- Line Sensors/Monitoring

- Communications

- Relay Oscillographics/Fault Data

- Advanced Protection Schemes

- Fault Current Limiting

The responses from the IEEE T\&D committee survey were combined under the headings of the first survey and the results are presented below. 


\subsubsection{Major Observations from the Survey}

\subsubsection{Fault Protection and Sensor Technologies}

- Most utilities have the ability to manually sectionalize feeders to speed load restoration and fault isolation. Communications is being connected to switches and reclosers to facilitate restoration. Increasingly, utilities are also putting in logic (both central and local) to automatically do this restoration.

- Voltage and current sensors, along with fault indicators are being installed on feeders to help with fault location. These sensors are being equipped with communications to allow operators to do remote diagnosis as well as permit automatic control.

- Protection schemes to deal with distributed generation are being explored and include interlock or transfer trip of the DG devices when the substation breaker opens. The impacts of inverter-based devices may be less than other types of generators.

- Electronic relays are being installed to replace electro-mechanical devices to allow more data to be collected and better fault location.

\subsubsection{Fault Location and SCADA Technologies}

- The use of circuit models to calculate fault currents at any location on the circuit is increasingly being used. During a fault, the current at the substation breaker is measured and compared with the model to identify potential fault locations. The use of other sensors helps identify the final location.

- Information about outages from Advanced Metering systems ("last gasp" and actual connectivity) is also being used to identify meters that have lost power. Through the use of an Outage Management System, faulty device locations can be identified.

- Monitoring the condition of critical pieces of equipment is helping to estimate when failures might occur. This data, along with detection of momentary relay pickups, is being used to attempt to predict faults. These techniques are new and do not have high accuracy yet.

\subsubsection{Other Technologies/ Future Work}

- A lot of work is being done in the communications area. Most distribution communications is being done through the use of licensed or unlicensed radio (even split between these). There is some use of public wireless networks, but utilities are reluctant to use it for operations because of reliability concerns (will it be there in a disaster when needed most). Fiber is being used for some substations, but it is still too expensive for most distribution installations outside of substations.

- Fault current limiters are being considered for distribution (in substations), but are still too costly and too experimental for widespread use. The biggest benefit of fault current limiting appears to be in the transmission/sub-transmission areas. 


\subsection{Summary of Conclusions from Literature Search and Survey}

The literature review and industry surveys reveal that system reliability, automation, and customer-focused operation are the main issues driving new developments for distribution system's fault location, detection and prediction applications. The key technologies driving these developments are affordable intelligent electronic devices (IEDs), better transducers/sensors, and accessible communication channels. The level of development and acceptance of each of these technologies determines the time frame for the implementation of new developments on distribution systems.

IEDs have been available in power system for more than two decades but in the recent past their application was mostly limited to local use in transmission systems where their cost was justifiable. The increased affordability of IEDs for distribution system applications has opened the door for taking advantage of the three key features of IEDs over previous fault location, detection and prediction devices: a) the ability to send and receive external information and use it to change or enhance their operation, b) the ability to self-test and alarm to determine local status and internal failures, and c) the ability to record and store large amounts of selective information.

In distribution systems, as determined from the literature reviews and surveys, the IED abilities are being applied mostly to: a) accurate fault location for speeding load restoration and fault isolation, b) remote diagnosis for determining status and problems in the fault location and detection devices, and c) collect valuable trend and historical information for data mining aimed at the determination of reliable fault predictors in distribution systems. These applications are part of the observations obtained from the survey as listed in Sections 2.2.2.1 and 2.2.2.2. The recommendations from the literature review on fault location and prediction in Section 2.1.3 are also aimed to take advantage of these abilities of IEDs. Although the surveys and literature review reveal increased use of new transducers/sensors in distribution system their affordability and reliability has not yet achieve the same level as distribution system IEDs.

The advantages of accessible communication channels are present in most of the observations from the survey and recommendations of the literature review. The ability of IEDs to receive and act on external information combined with accessible communication channels is destined to change the operation of fault location, detection and prediction devices and systems. Some of the present applications found in the survey and literature review demonstrate the potential of this combination of technologies for specific applications in fault location, detection and prediction applications; but the survey and literature review also reveal that communication channels applied to distribution system operation have not yet acquire the level of reliability and affordability present in distribution system's IEDs. This lack of reliability of affordable communication channels combined with new requirements of cyber security will delay the main stream implementation of most of the communication enhanced fault location, detection and prediction applications encountered in the literature review and industry surveys. 


\subsubsection{Conclusions:}

a) IEDs are now a common tool in fault detection location and prediction in distribution systems. Some of the algorithms and techniques developed for fault detection and location on transmission systems have found their way into distribution systems. The limitations and operational differences of distribution systems require modification and enhancements to existing algorithms that has led to distribution system specific techniques, algorithms and IEDs.

b) The availability of communication channels is enabling the development and implementation of a wider range of new algorithms and techniques for fault location, detection, and prediction in distribution systems. The wide range of communication options in the distribution system and their variable reliability enhances the possibilities but at the same time limits the development of a utility specific affordable communication network. Adding to the existing limitations, the recent requirements for cyber security that already affect the transmission system application will also have an impact on distribution system applications on fault location, prediction and detection. At the moment the lack of more reliable and affordable communication channels is the bottle neck for applying some algorithms and systems to fault location and detection in distribution systems.

c) Other related external technologies (not derived specifically for utility application) like GPS clocks and fiber optic transducers are also influencing the development of fault location, detection and prediction algorithms and systems. Fiber optics and other transducers/sensors are enabling affordable and reliable non-intrusive metering and distribution voltage and current measurements. Unfortunately the majority of these sensors have not achieved the maturity and reliability required for most IED applications for fault location, detection and prediction. GPS clocks have achieved acceptable affordability and reliability levels for application to fault location, detection and prediction in distribution systems. The availability of GPS synchronized sampling at the distribution level partially reduces the negative impact of the lack of reliable and affordable communication channels for fault location and prediction. GPS clocks may eliminate the requirement of communication channels for fault prediction application through their ability to synchronize measurements.

d) New utility specific technologies such as fault current limiters (FCLs) and distributed generators are changing the operation of the distribution system and affecting the operation of fault detection, location and prediction algorithms and systems. Fault current limiters at the moment lack the maturity and affordability for main stream application to distribution systems but experimental testing such as those perform in SCE Circuit of the Future reveal that they will be a valuable tool in the distribution systems of the future. With enough penetration, FCLs will require changes and enhancements be implemented in the fault location algorithms and the coordination of detection algorithms. To a lesser extent, FCLs may limit the effectiveness of some fault prediction algorithms if their effect cannot be incorporated into existing historical data gathered before the FCLs were implemented. 
Distributed generation is changing the operation of the distribution system and forcing changes into fault location, detection and prediction devices and algorithms. The impact of distributed generation is more immediate and it has already produce changes in fault detection and location techniques. The changes due to distributed generation penetration can be incorporated with relative ease into existing IEDs with the help of communication channels. The degree of availability of reliable communication channels with directly impact the effect of distributed generation on fault location and detection in distribution systems. 


\section{Section 3: Pilot Project Plan}

This project was planned with three Phases, approximately one year per phase. Due to some delays encountered with circuit equipment installation and procurement of a fault current limiter, the project actually took place over 4 years.

\subsection{Project Description}

The first phase of the project involved an assessment of the state-of-the-art in fault location, prediction, and detection as well as the design, lab testing, and field installation of the advanced protection system on the SCE Circuit of the Future located north of San Bernardino, CA. (described in detail in Section 4 of this report). To help understand the trends in the industry and what technologies were being developed in the fault location, prediction and detection areas, a literature search and survey were performed (see Section 2 for more details). The actual Circuit of the future initial design was based on the collection of a number of ideas from the four SCE Distribution Engineering divisions (described in more detail in Section 4 of this report). These ideas were reduced to a tentative functional design for the advanced protection system to be implemented on the Circuit of the Future. Detailed design work started in 2006 and was supported by this project. Once the detailed design was completed, a lab mock-up of the advanced protection system was assembled. This lab demonstration was used to work out bugs in the design and also to train SCE field personnel on how to operate and troubleshoot the new advanced protection system (early 2007). Once all bugs were worked out and training conducted, the equipment was installed on the actual circuit. Following commissioning testing on the protection system, the circuit was placed in operation on August 14, 2007. Results from faults experienced on the circuit are described in Section 7 of this report.

The second phase of the project involved the selection, modeling, testing and installation of a fault current limiter on the Circuit of the Future. While this project did not pay for the installation and testing of the fault current limiter, it did perform the evaluation of the fault current limiter and its impacts on the protection system of the Circuit of the Future. After investigation of several fault current limiters, the Zenergy superconducting, saturable core fault current limiter was selected for installation. This fault current limiter is described in more detail in Section 5 of this report. As SCE was investigating the availability of a fault current limiter, research on various types of fault current limiters was conducted by Virginia Tech. Virginia Tech and SCE also built numerical models to be used to evaluate impacts of fault current limiters on the advanced protection system on the Circuit of the Future. Because of some testing problems with the Zenergy fault current limiter, installation was delayed until early 2009 with it being put into operation on March 6, 2009. More information on the installation, testing, evaluation of the fault current limiter is included in Sections 5 and 7 of this report. 
The third phase of the project involved the exploration of several advanced protection ideas that might be at a state where they could be applied to the Circuit of the Future and elsewhere in the SCE electrical system. Based on the work done as part of the literature review and survey, as well as a number of internal meetings with engineering staff at SCE, a number of ideas were compiled. These ideas were then evaluated for applicability and ability to be applied on the Circuit of the Future in the time remaining for the project. Some of these basic ideas were implemented on the circuit. As part of this section of the project, it was also decided that we would take what was learned as part of the Circuit of the Future work and extend it to the next generation circuit protection for SCE. Also at this time, SCE put in a proposal to the DOE for the Irvine Smart Grid Demonstration. SCE was successful in obtaining funding for this proposal, so it was felt that exploration of new protection schemes for this Irvine Smart Grid Demo would be a good use of the project resources. With this in mind, a protection system that uses fault interrupting switches, high speed communications, and looping two radial distribution circuits would have the potential for significant reductions in interruption time and number of interruptions for customers. On the Circuit of the Future, fiber optic communications was used between the fault interrupting switches. This was found to be too expensive in a retrofit situation (the case of most circuit upgrades in the SCE system). We decided to pursue the use of high-speed radio communications to replace the fiber. This project investigated this protection scheme and built a PSCAD model that would allow testing of the concept. Further information on this project phase is available in Section 6.

\subsection{Schedule}

As mentioned above, this project was implemented in three stages. Each of these stages was to be completed in approximately one year. Due to delays with the fault current limiter, Phase II stretched to almost two years. Here are the three stages:

- Phase I - Testing new protection methods on SCE's Circuit of the Future with networked fault interrupters (2006 - 2007)

- Phase II - Design and testing of protection methods with a fault current limiter (2008 - 2009)

- Phase III - Investigation, design, and testing of advanced fault location and protection methods (2009 - 2010) 


\subsection{Milestones}

A number of project milestones were constructed for this project. The milestones and final scheduled dates are noted in Table 3-1 below:

\begin{tabular}{|c|l|c|}
\hline$\#$ & \multicolumn{1}{|c|}{ Milestone Name } & Date \\
\hline 1 & Complete kickoff meeting and scope definition & $1 / 31 / 2007$ \\
\hline 2 & Complete review of the state-of-the-art practices & $3 / 31 / 2008$ \\
\hline 3 & Complete refining project scope and pilot project plan & $9 / 30 / 2007$ \\
\hline & $\begin{array}{l}\text { Complete fault location and isolation for the Circuit of the Future without a } \\
\text { fault limiter }\end{array}$ & $8 / 31 / 2007$ \\
\hline 5 & $\begin{array}{l}\text { Complete fault location and isolation for the SCE Circuit of the Future with a } \\
\text { fault current limiter }\end{array}$ & $6 / 30 / 2009$ \\
\hline 6 & Complete advanced fault sensing and prediction & $6 / 30 / 2010$ \\
\hline 7 & Complete evaluation of results from the pilot test & $6 / 30 / 2010$ \\
\hline 8 & Complete extrapolation for the future and for industry & $6 / 30 / 2010$ \\
\hline 9 & Submit final report & $9 / 30 / 2010$ \\
\hline
\end{tabular}

Table 3-1 - Project Milestones 


\section{Section 4: Fault Location, Isolation and Prediction for the Circuit of the Future without a Fault Current Limiter}

The second part of the Phase I work included the design, lab testing, and implementation of the advanced protection system on the Circuit of the Future which was placed into operation on August 2007 with a formal dedication taking place on October 2007.

\subsection{Description of Protection Scheme}

Many advanced automation and protection concepts have been incorporated for testing and demonstration purposes on the new Circuit of the Future also called the Avanti 12 $\mathrm{kV}$ circuit out of Shandin substation in the city of San Bernardino. Figure 4-1 shows the general arrangement of the Circuit of the Future and its protection devices.

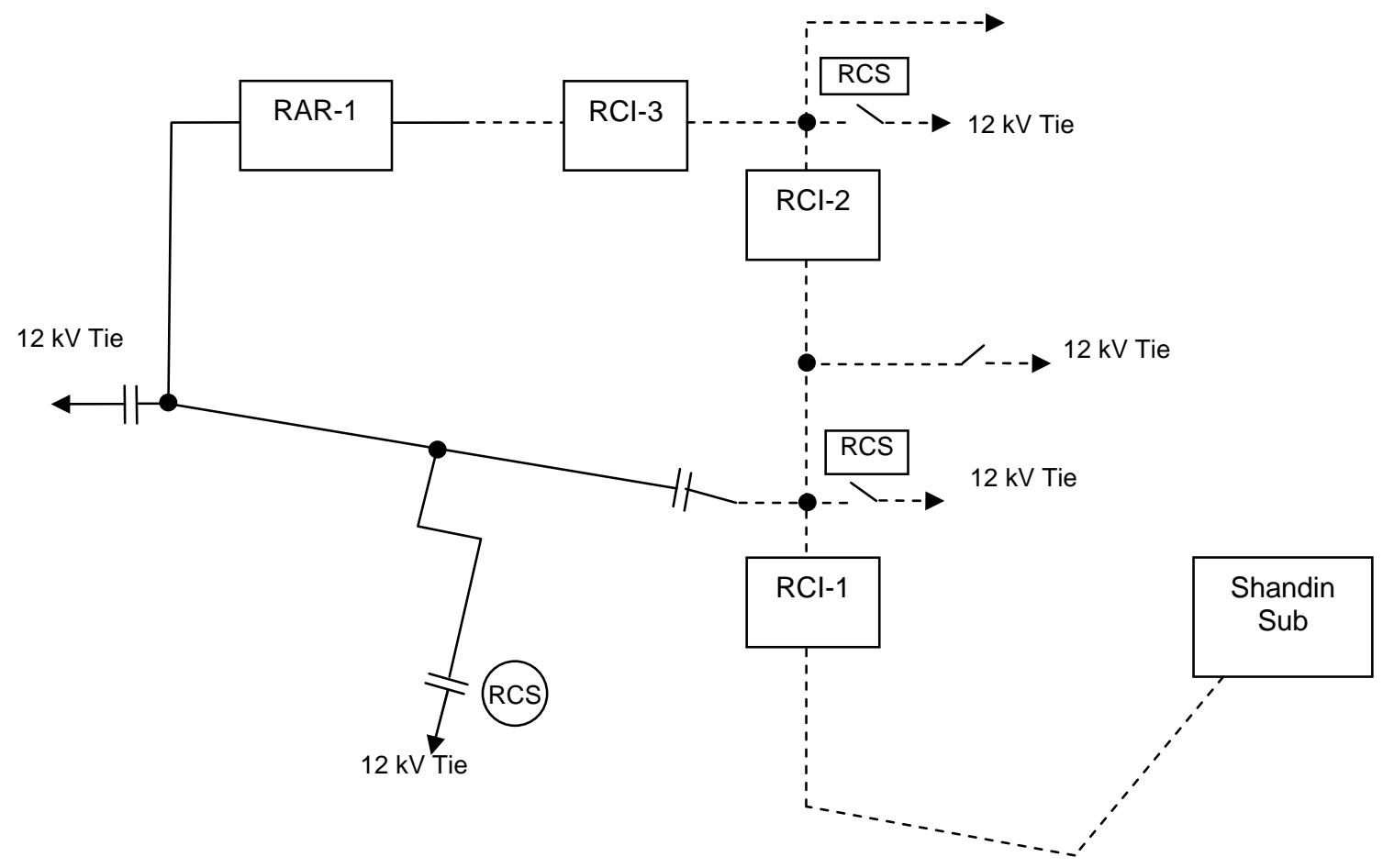

Figure 4-1 - General Arrangement of the Circuit of the Future 
In order to minimize the number of customers affected by a circuit fault, three Remote Controlled Interrupters (RCIs) and a Remote Automatic Recloser (RAR) are used to isolate the faulted section of the circuit. After a fault, control logic automatically opens the next downstream protective device to facilitate restoration of service to the unfaulted portions of the circuit. This automation function is referred to as an "Iso-Open." A five second delay is incorporated in the automatic opening logic to make it clear that the opening is an automated control operation and not the result of a second fault. For example, a fault between RCI-1 and RCI-2 will trip RCI-1. Five seconds later, control logic opens RCI-2 automatically so that the RCS tie between RCI-2 and RCI-3 can be closed, restoring service via the Sweetwater $12 \mathrm{kV}$ circuit, to all loads beyond RCI-2.

Automation and coordination of the protection is provided by a Schweitzer SEL-2100 Logic Processor located in the control cabinet for RCI-2 and RCI-3. Fiber optic cables connect the SEL-2100 to each RAR and RCI as well as to Shandin substation, as shown in Figure 4-2 RAR-1 is programmed for one automatic reclose after a fault. The RCIs do not automatically reclose.

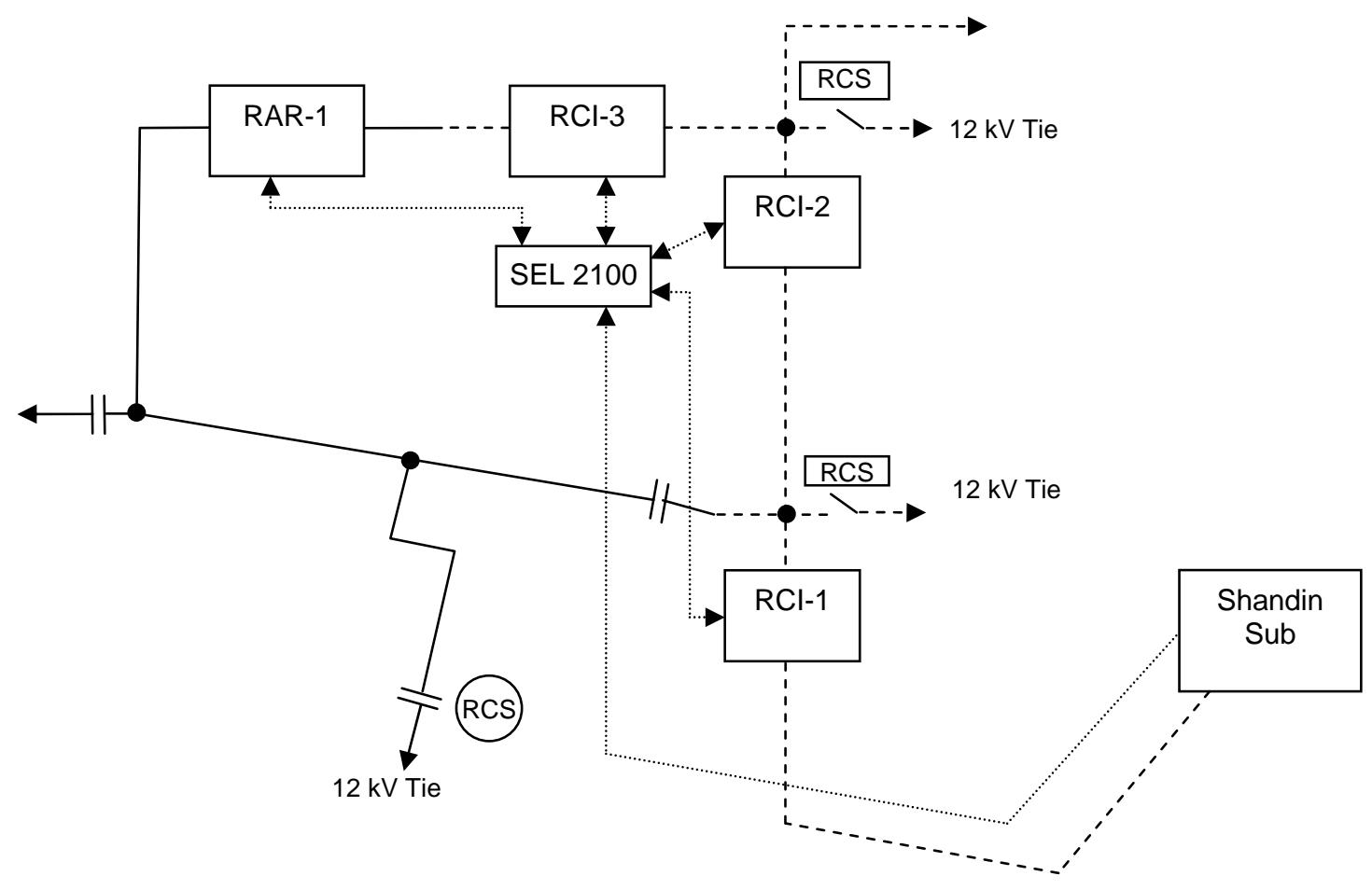

Figure 4-2 - SEL-2100 Logic Processor and Fiber Optic Communication

\subsubsection{Protection Coordination}

The normal protection settings of all three RCIs and RAR-1 have been selected to coordinate with up to $200 \mathrm{E}$ fuses for phase faults and $80 \mathrm{E}$ fuses for ground faults. This was done to allow the installation of new large loads or circuit branches without requiring changes to the settings of the RCIs or RAR. Normal settings for all RCIs and the RAR, except RCI-1, are 600 amps for phase faults and 180 amps for ground faults. RCI-1 
settings are 720 for phase faults and 180 for ground faults to facilitate picking up load of the Northpark or Sweetwater $12 \mathrm{kV}$ circuits if that should become necessary.

With five overcurrent protective devices (RAR, three RCIs, and the substation) in series, fault clearing times for some line sections would become unacceptably long if conventional time/current coordination was used. To avoid this problem, coordination of the Circuit of the Future protection is accomplished by means of a blocking signal system. In this scheme, an RAR or RCI that senses a downstream fault sends a blocking signal, via the SEL-2100, to the next upstream protection device. Receipt of the blocking signal tells the protective device not to trip on its overcurrent protection, but rather to wait for the next downstream device to trip. ${ }^{1}$ Figure 4-3 illustrates how blocking signals are sent for a fault between RCI-3 and RAR-1. ${ }^{2}$ Blocking signals are sent instantaneously whenever the current sensed by an RAR or RCI exceeds its phase or ground pickup current setting. Note that, for the illustrated fault location, RAR-1 does not send a blocking signal because it senses only load current, not fault current. Blocking operation is similar for other fault locations.

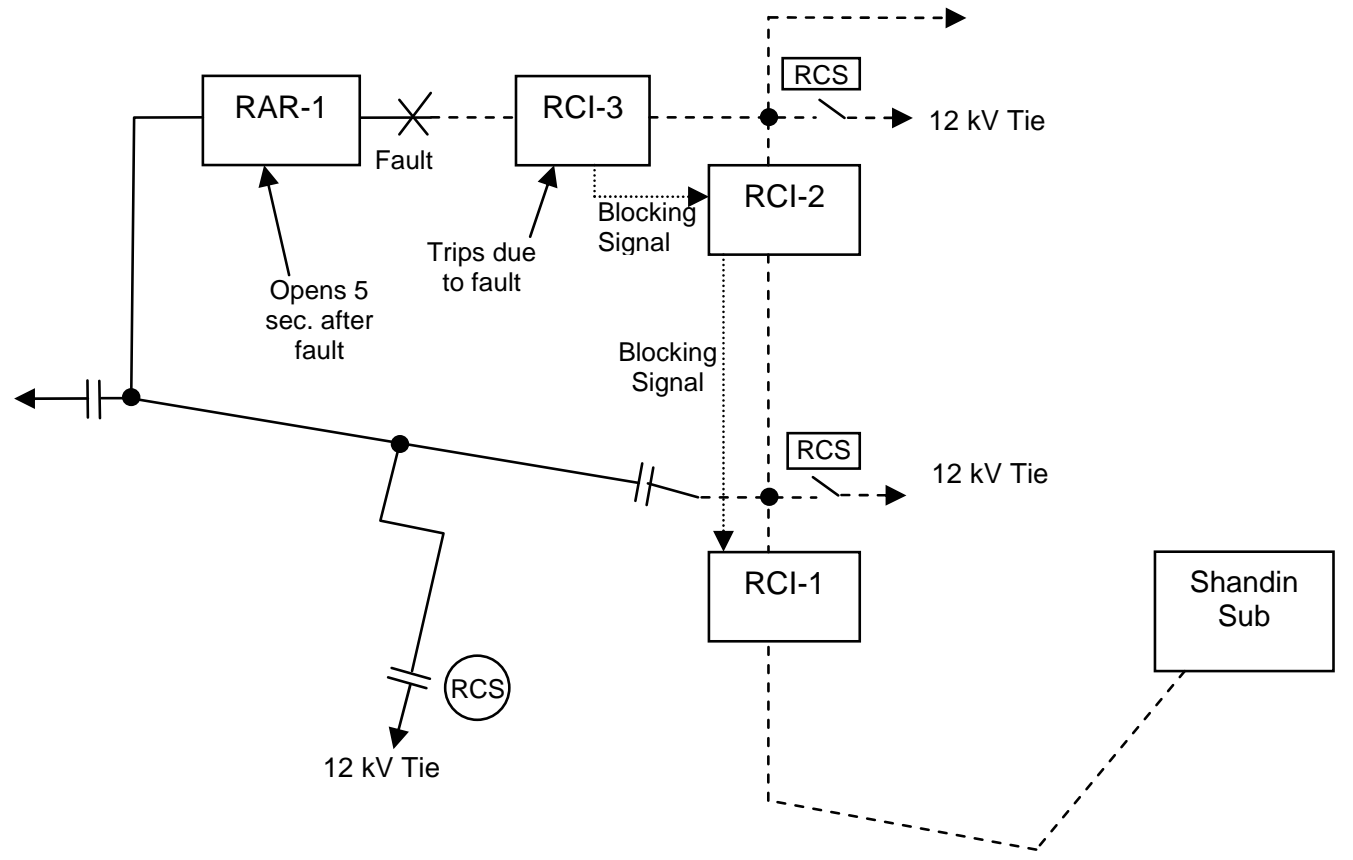

Figure 4-3 - Blocking Scheme Operation

\footnotetext{
${ }^{1}$ The overcurrent relays at Shandin sub are not included in the blocking scheme and are time/current coordinated with the settings of the RCIs and RAR. Therefore, the Shandin relays acts as backup for faults anywhere on the line should the RCIs and RAR not operate as planned.

${ }^{2}$ Figure 4-3 has been simplified in order to illustrate the concept of a blocking scheme. In the actual implementation, an overcurrent pickup signal is received by the 2100 from each protective device that senses fault current. Receipt of this pickup signal causes the 2100 to remove the time-overcurrent (TOC1) enable signal that it is normally constantly sending to the next upstream protective device. Removal of the enable signal effectively blocks the operation of the device.
} 


\subsubsection{Operator Controls}

At each RAR and RCI, local and remote control (via NetComm radio) is provided for the usual Open, Close, Ground enable/disable, and Remote enable/disable control of the devices. This was separate from the advanced protection system being used on this project and was installed to maintain uniformity with controls on other circuits. This was to avoid any confusion for the system operators. In addition, the following special operator controls are provided:

For the RCIs,

- Fault Trip Disable [local or radio control]: The Fault Trip Disabled mode prevents the RCI from tripping for a fault, or automatically opening following a fault (Iso-Open), and is intended for the safety of personnel working in the vault containing the RCI. An Iso-Open command intended for an RCI which is in the Fault Trip Disabled Mode will be redirected by the SEL-2100 to the next operational downstream protective device.

- “2100 Disable” [local or radio control]: When the 2100 is disabled by this means, all of the Circuit of the Future automation functions are turned off and the in-service time-overcurrent units are changed at the RCI's and RAR to maintain coordination to the extent possible without blocking signals. The 2100 can be disabled or re-enabled at any RCI or via the gateway from Shandin substation.

For the RAR,

- “2100 Disable” [radio control only]: When the 2100 is disabled by this means, all of the Circuit of the Future automation functions are turned off. The 2100 can be disabled or re-enabled at any RCI or via the gateway from Shandin substation. Local control of "2100 Disable" is not provided at the RAR because the RAR control does not have a spare operator pushbutton available to be used for this function.

\subsubsection{Contingency Conditions}

In the design of the Circuit of the Future automation and protection, a number of contingency conditions were identified and provisions made to deal with those contingencies. This section describes the methods used for each type of contingency.

\subsubsection{Protection Bypassed or Disabled}

When an RAR is bypassed, line current no longer flows through the RAR's current transformers and the RAR is unable to issue a blocking signal to the next upstream protective device. Thus the RAR automatically becomes inoperative and clearing of faults downstream of the RAR becomes the responsibility of the next upstream device.

When an RCI is placed in the Fault Trip Disabled mode, line current still flows through the RCI's CTs. If not corrected, this situation would permit the RCI to issue an upstream 
blocking signal, preventing backup tripping even though the RCI's own tripping is disabled. To prevent this problem, logic in the SEL-2100 stops transmission of a blocking signal if the RCI that is sending the blocking signal is in the Fault Trip Disabled mode.

\subsubsection{Failure to Trip}

Failure of the SEL-351 relay used in an RAR or RCI automatically results in no blocking signal being sent, so the next upstream device assumes responsibility for clearing faults downstream of the failed relay. However, it is also possible that the relay may operate but the associated RAR or RCI switch fails to trip or fails to interrupt.

Logic in the SEL-2100 senses that this "breaker failure" condition has occurred when,

- A relay trip signal has been received from the failed location for $167 \mathrm{~ms}$ (10 cycles) and,

- The blocking signal (overcurrent pickup) from the failed location has not reset, indicating that fault current is still flowing through the failed location.

When this set of conditions is detected, the SEL-2100 immediately stops blocking the next upstream device (re-enables its TOC1) and also sends it a trip signal, causing the upstream device to trip immediately. Thus, a fault downstream of a failed RAR or RCI is cleared in the normal tripping time plus an additional delay time of 10 cycles. The 10 cycle delay is provided to give the failed switch an adequate opportunity to clear the fault before declaring it failed.

\subsubsection{Loss of Communication}

Successful operation of the blocking coordination scheme depends on the fiber optic communication links between each protective device and the SEL-2100. When and if one of these links fails, the scheme must re-orient itself to function without communication. Each communication link is constantly monitored by the connected SEL-351 protective relay at its end and by the SEL-2100. Thus, the relays and logic processor are aware of the good/bad state of the link and can take action based on that status. The status of the ROK signal at each link terminal provides the information needed to make these decisions.

During communication failures the normal RAR or RCI time-overcurrent functions (TOC1) are disabled and the backup (TOC2) functions are enabled by the relay or the 2100 as needed to maintain coordination. The pickup values and time curves of the backup (TOC2) overcurrent functions are different from the normal (TOC1) values. TOC2 settings for the most downstream devices have lower pickup currents and faster timing than their TOC1 settings (480 phase overcurrent /150 ground overcurrent for RAR-1 and RCI-3); TOC2 settings for the upstream devices are higher and slower (720 phase overcurrent /210 ground current for RCI-1 and RCI-2). 
The following table (Table 4-1) illustrates how the TOC units change and coordination is maintained for various communication link failures. Entries in the "Coord By" columns indicate that coordination between the adjacent protective devices is obtained either by time coordination or by the action of the blocking scheme. The "None" entry indicates that the two adjacent devices are not coordinated for the failure of all communication links, i.e. they "race" and either or both might trip for a fault downstream of RCI-3. A few other conditions involving sequential failures of two or more communication links also result in miscoordination. An Iso-Open intended for an RCI that has lost communication will be redirected by the SEL-2100 to the next available downstream protective device.

\begin{tabular}{|c|l|c|c|c|c|c|c|c|c|}
\hline $\begin{array}{c}\text { Comm. } \\
\text { lost to }\end{array}$ & Sub & $\begin{array}{c}\text { Coord } \\
\text { By }\end{array}$ & $\begin{array}{c}\text { RCI- } \\
\mathbf{1}\end{array}$ & $\begin{array}{c}\text { Coord } \\
\text { By }\end{array}$ & $\begin{array}{c}\text { RCI- } \\
\mathbf{2}\end{array}$ & $\begin{array}{c}\text { Coord } \\
\text { By }\end{array}$ & $\begin{array}{c}\text { RCI- } \\
\mathbf{3}\end{array}$ & $\begin{array}{c}\text { Coord } \\
\text { By }\end{array}$ & $\begin{array}{c}\text { RAR- } \\
\mathbf{1}\end{array}$ \\
\hline None & TOC1 & Timing & TOC1 & Blocking & TOC1 & Blocking & TOC1 & Blocking & TOC1 \\
\hline RCI-1 & TOC1 & Timing & TOC2 & Timing & TOC1 & Blocking & TOC1 & Blocking & TOC1 \\
\hline RCI-2 & TOC1 & Timing & TOC2 & Timing & TOC1 & Timing & TOC2 & Blocking & TOC2 \\
\hline RCI-3 & TOC1 & Timing & TOC2 & Blocking & TOC2 & Timing & TOC1 & Timing & TOC2 \\
\hline RAR-1 & TOC1 & Timing & TOC1 & Blocking & TOC1 & Timing & TOC1 & Timing & TOC2 \\
\hline ALL & TOC1 & Timing & TOC2 & Timing & TOC1 & None & TOC1 & Timing & TOC2 \\
\hline
\end{tabular}

Table 4-1 - Loss of Communications Actions

\subsubsection{Relay or Logic Processor Failure}

Failure of an individual SEL-351 relay at one of the RCI's or the RAR is handled in a manner similar to a communication link failure to that relay. When the relay does not respond to the regular periodic messages sent from the SEL-2100 to the relay, the 2100 assumes that the communication link is bad and makes time-overcurrent function changes to the remaining good relays. No blocking signals can be received from the failed relay and, consequently, the next upstream protective device takes over. Iso-Open commands intended for the failed relay are redirected to the next available downstream device.

Likewise, failure of the SEL-2100 logic processor causes the relays at the RAR and RCIs to assume that they have lost communication to the 2100. Coordination changes to the "ALL" case are described in the Table 4-1 above. Iso-Open commands do not function when the 2100 has failed.

\subsubsection{Reverse Power Flow}

When portions of the Circuit of the Future are picked up from adjacent circuits, e.g. from the tie to the Sweetwater circuit between RCI-2 and RCI-3, it's desirable to have at least some of the automation features continue even though the direction of load current flow may be opposite to normal. For example, power flows from RCI-3 toward RCI-2 when the Sweetwater circuit tie is closed.

To preserve fault coordination under such reverse power flow conditions, the blocking system has been designed to redirect the blocking signals from upstream to downstream (i.e. send the blocking signals in the direction from which the fault current is coming). 
This redirection is accomplished by means of fault-current directional elements in the SEL-351 relays at the RAR or RCIs. ${ }^{3}$ These directional elements determine fault current direction from the phase angle of the fault current when compared to the voltage obtained from open-delta potential transformers at each device location.

When a relay senses fault current in the reversed direction, a "reverse fault" indication is sent to the SEL-2100 along with the blocking signal. The 2100 then redirects the blocking signal to the opposite of the normal direction. For example, if the entire Circuit of the Future were being carried from the Sweetwater circuit through the tie switch between RCI-2 and RCI-3, a fault between RCI-1 and the substation (assumed open) would produce a blocking signal and a Reverse Fault indication at RCI-1 (Figure 4-4). When received by the 2100 , the blocking signal would be directed to RCI-2 to block its operation while RCI-1 clears the fault.

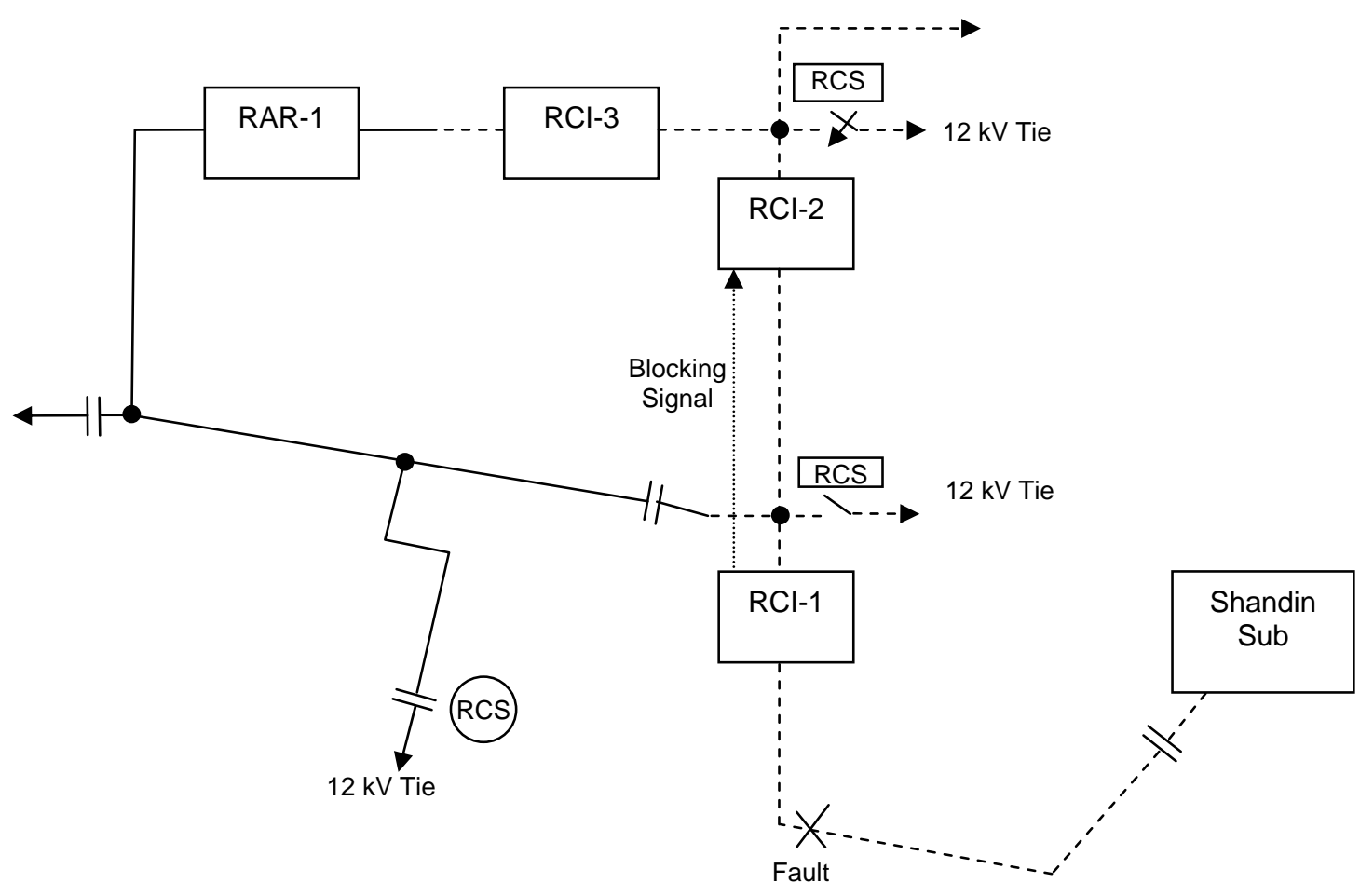

Figure 4-4 - Blocking Operation for Reversed Power Flow

For the same conditions, but with the fault located between RCI-3 and RAR-1, RCI-3 will produce a blocking signal, but no reverse fault indication. This blocking signal will be sent to RCI-2, but will have no effect because RCI-2 does not sense a fault.

\footnotetext{
${ }^{3}$ The directional element does not sense the reversed direction of load current unless the load current magnitude is quite high, more than $100 \mathrm{amps}$. Faults always produce enough current to give a correct directional indication.
} 
Detection of a reverse fault blocks the operation of the Iso-Open automation feature, so that it does not attempt to open the downstream switch automatically. Other automation features such as redirected Iso-Open, Failure to Open, and Communication Failures have, for the sake of simplicity, not been incorporated into the reverse fault logic.

\subsection{Laboratory Testing}

Preparation for lab testing was begun in late 2006. Testing actually was started in June 2007. The photos below show some of the lab testing in progress (Figure 4-5 through Figure 4-10).

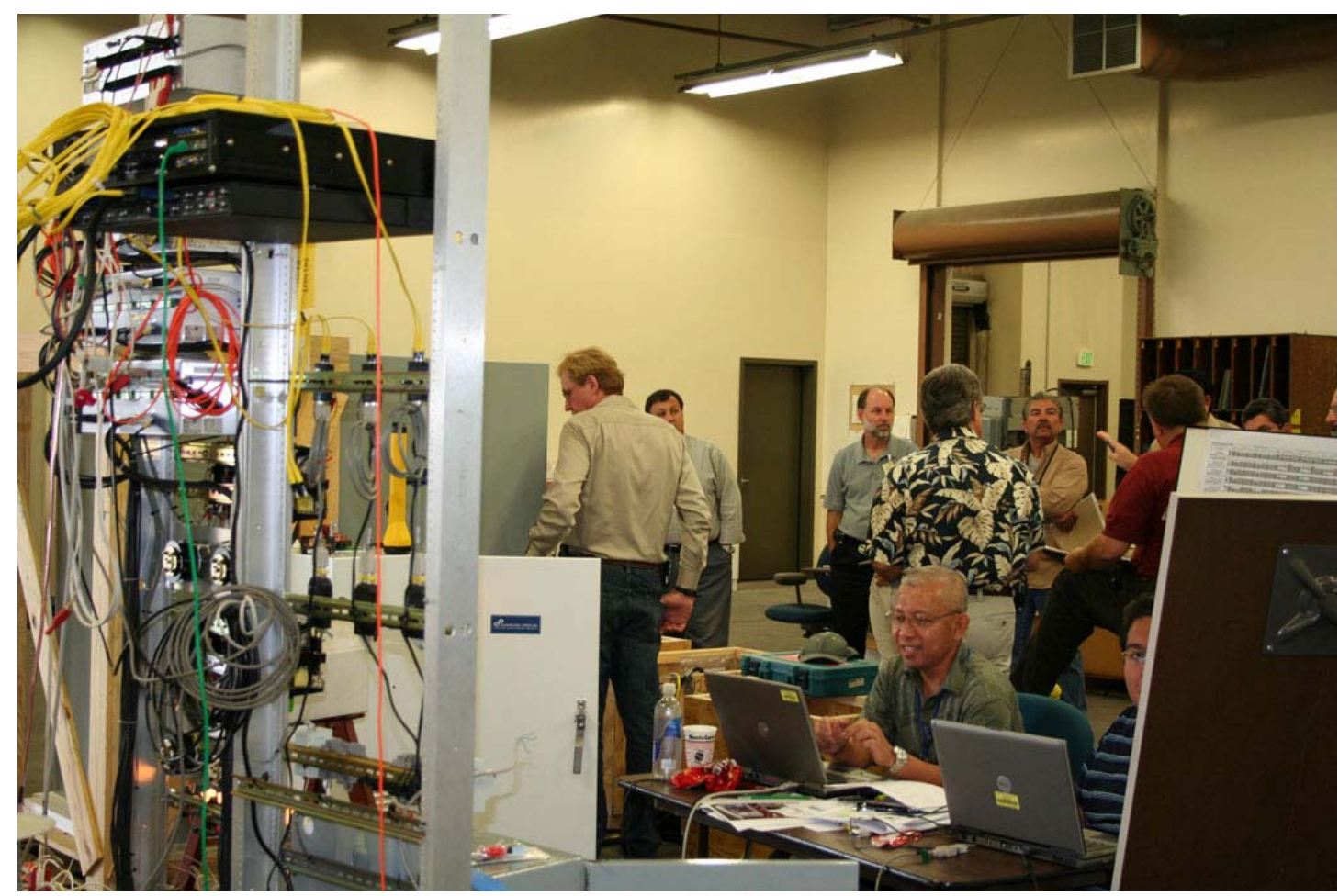

Figure 4-5 - Circuit of the Future Lab Testing 

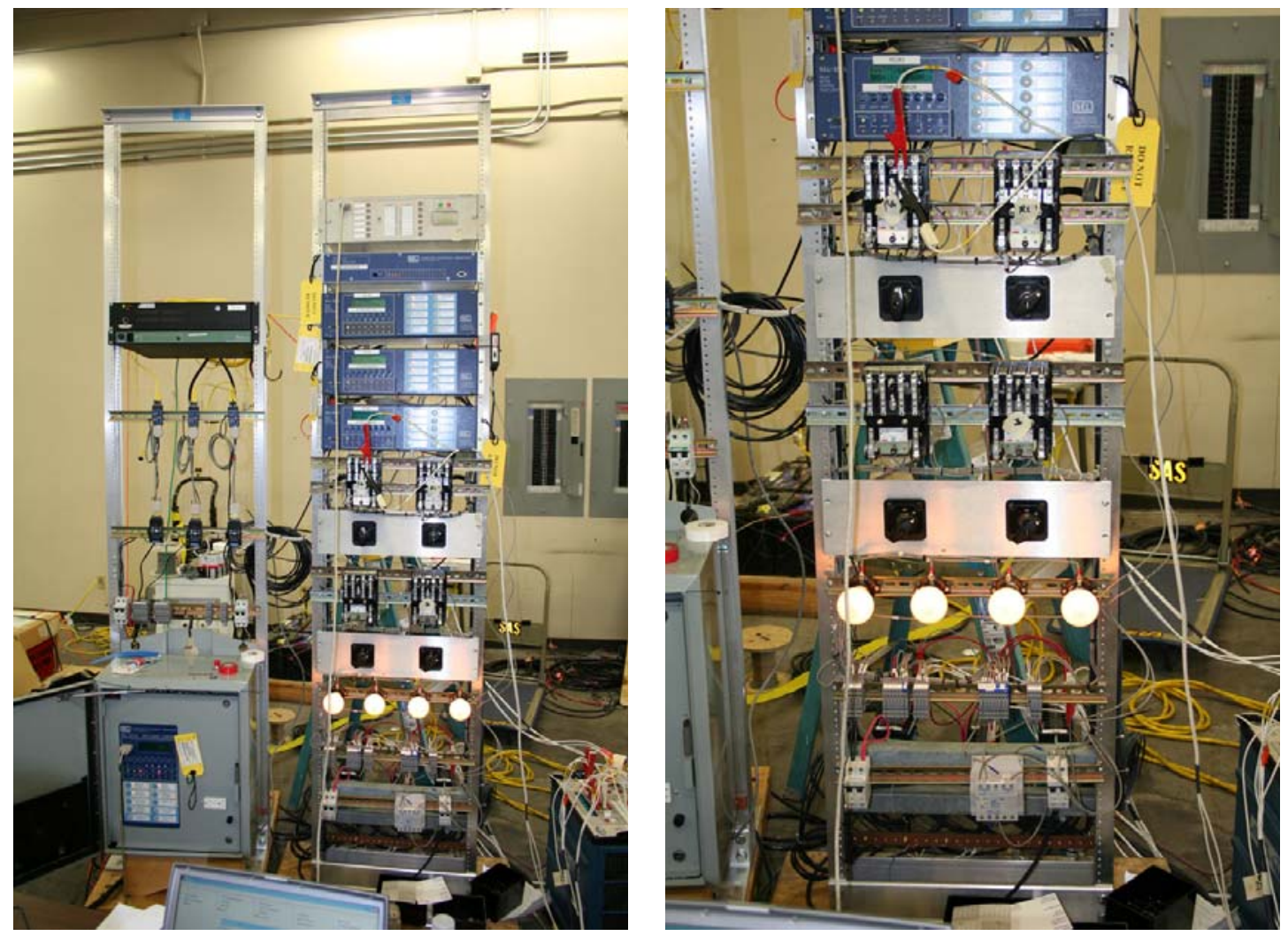

Figure 4-6 - Circuit of the Future Lab Testing - Relaying Equipment in Racks

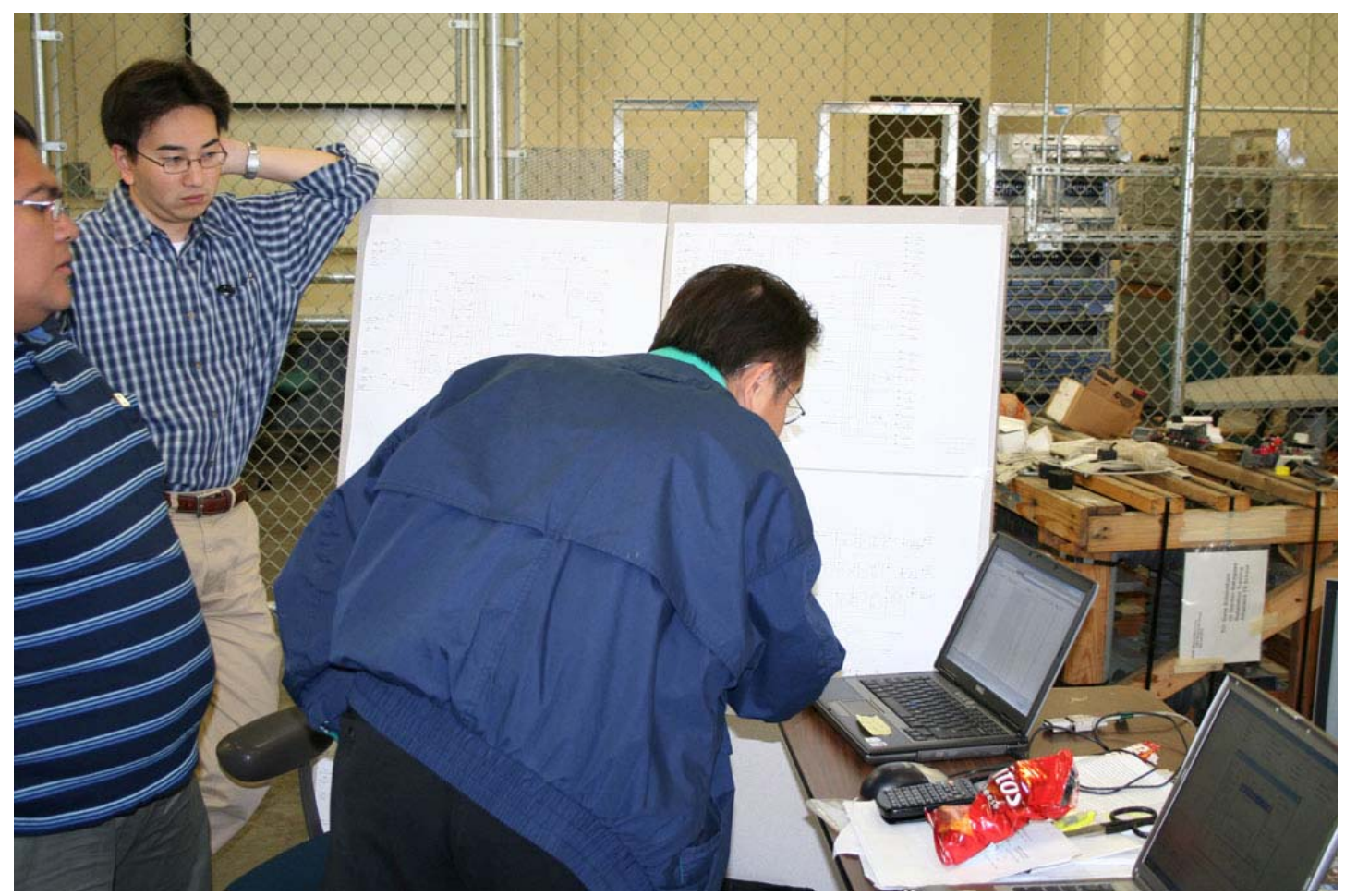

Figure 4-7 - Running Lab Test Scenarios 


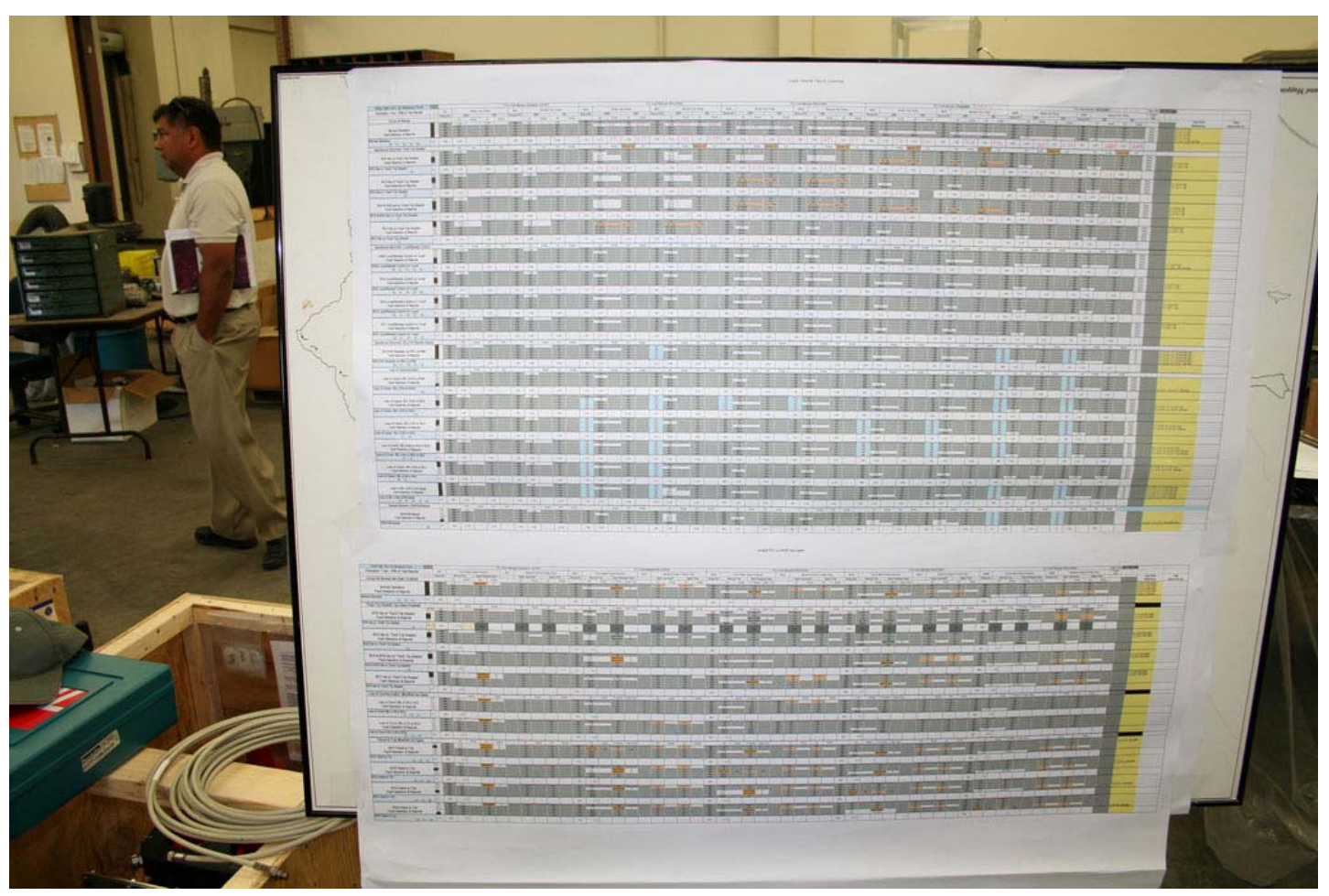

Figure 4-8 - Lab Test Matrix of Scenarios

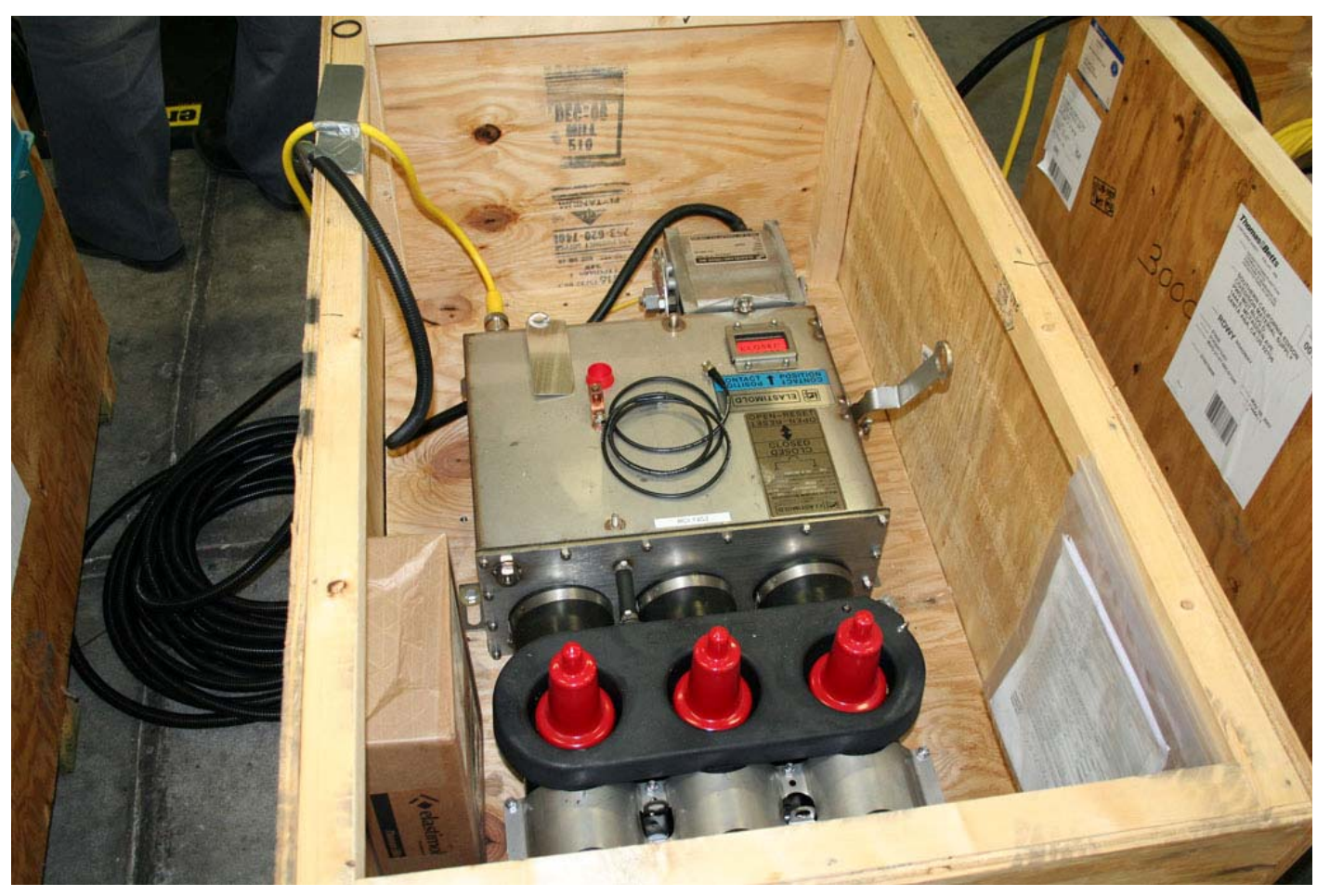

Figure 4-9 - RCI Vacuum Fault Interrupter 


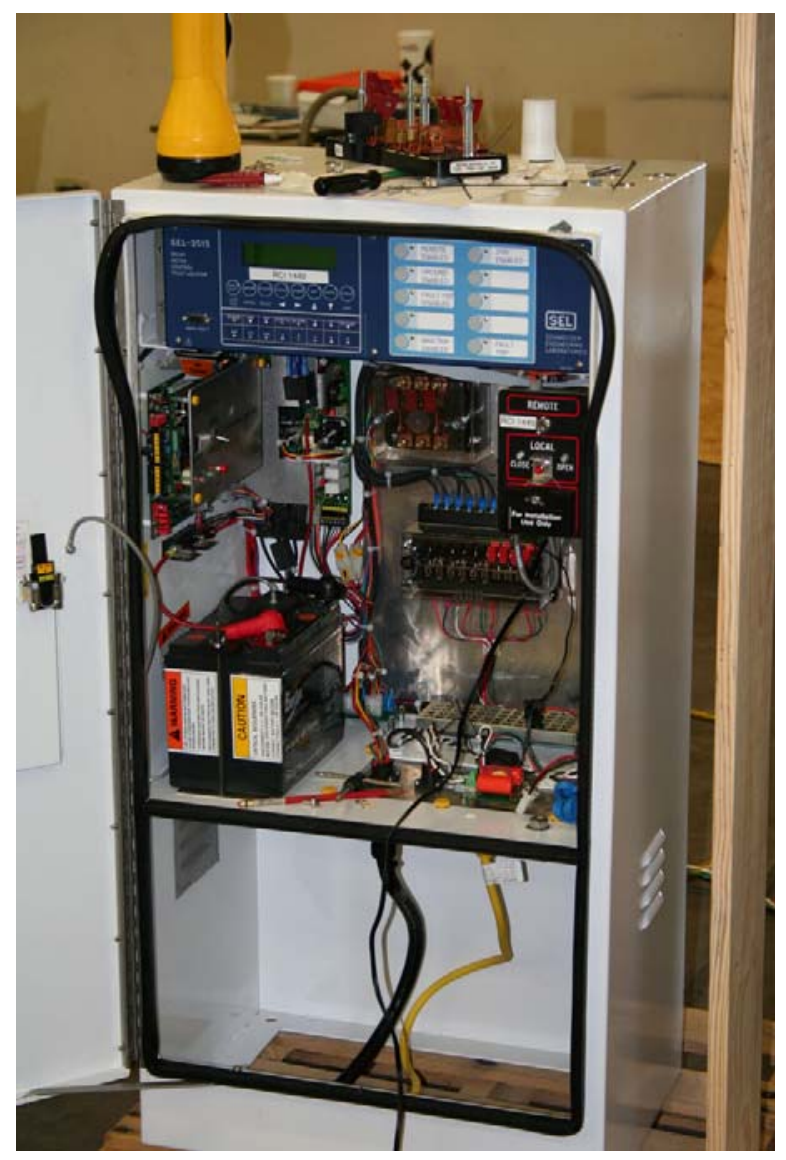

\section{Figure 4-10 - Protective Relay and Controller Cabinet for RCI}

The Lab Test Plan was performed by the SCE Substation Automation Group for the advanced protection and load restoration logic scenarios (Figure 4-11). The primary objective was to set up and test the field relay and restoration system consisting of (1) SEL 2100, (1) SEL 351R and (3) SEL 351S relays. These logical devices were installed on the distribution circuit at RCI-1, RCI-2, RCI-3 and RAR-1. In addition to voltage, primary load, and fault sensing, these devices communicate to each other and Shandin substation via fiber optic and copper communication media. Copper to fiber conversion was accomplished via SEL 2812 and 2829 modules. Communication from the SEL relays to the SEL 2100 was via SEL Mirrored Bits protocol. Communication from the SEL 2100 to the Shandin Substation eLAN server was via DNP serial communications protocol. Standard communications with the existing Distribution Control and Monitoring System (DCMS) was provided through SCE's Netcomm radio system for control and monitoring of the field devices. 


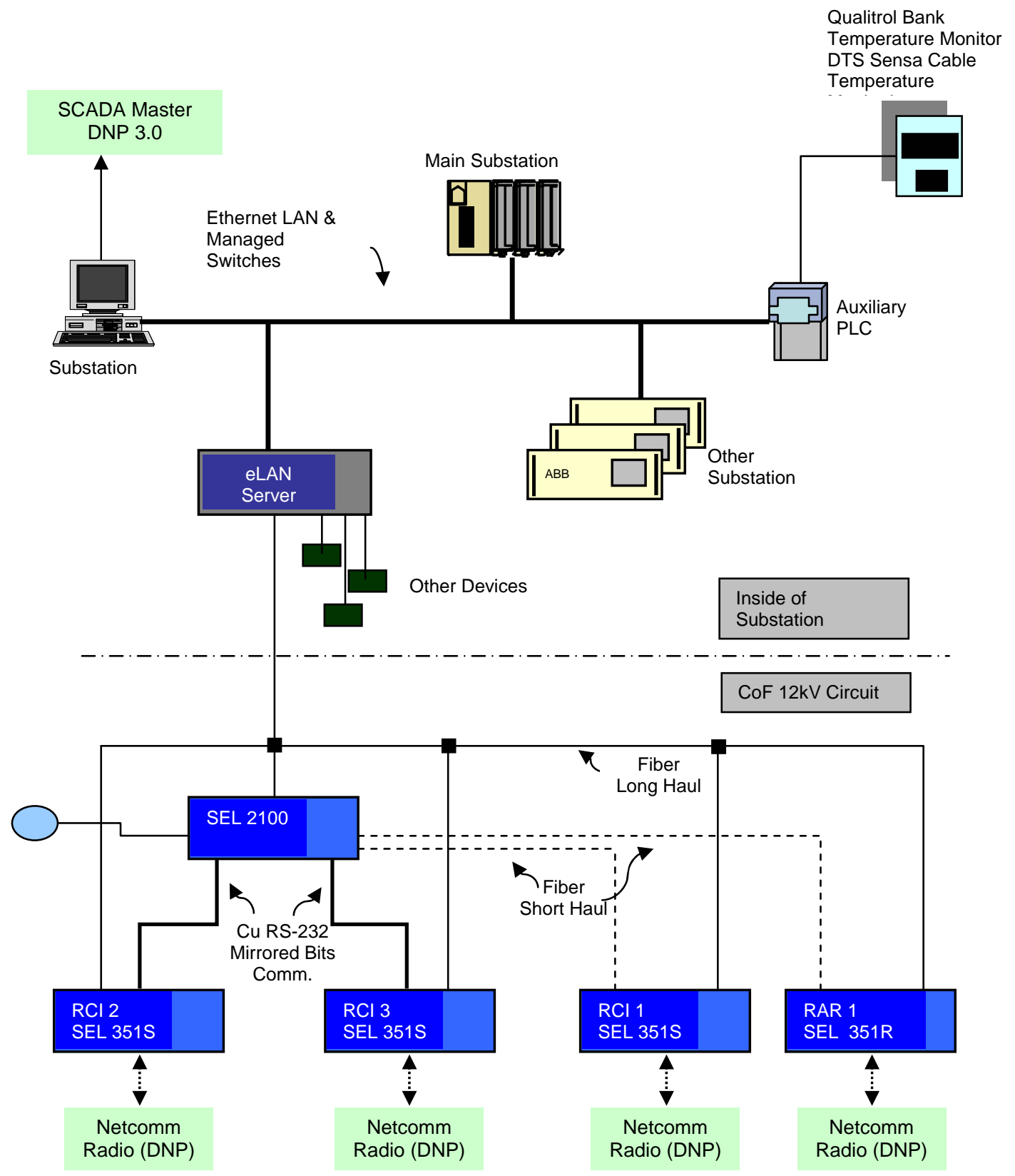

Figure 4-11 - Circuit of the Future Protection Elements and Communications

The Lab Test Plan objectives included set up and test verification of all identified trip and reclose scenarios for the Circuit of the Future given the advanced protection design which includes the following:

- (3) SEL 351S relays located at RC-1, RCI-2 and RCI-3. (1) SEL 351R relay located at RAR-1 and (1) SEL 2100 processor.

- SEL 2401 for integration to Satellite for time synchronization

- Fiber (long and short haul) for control and communication interface between all SEL 351S's, SEL 351R, SEL 2100 and the Shandin Substation eLAN Server. (2) 
SEL 351S's are located in the same location as the SEL 2100 and will be RS-232 copper interfaced.

Additionally, the lab setup was used as a guide for creation of a test plan that was implemented in the field for preliminary and in-service testing of the SEL 351R, SEL $351 \mathrm{~S}$ and SEL 2100 relays in an integrated production environment. (integrated via copper and fiber optic network.) The Lab Test Plan included the Trip and Reclose Scenario Workbook that listed all tests to be performed. Secondary current injection will be based on the primary values provided to a CT ratio of 1000:1. Testing currently is planned for the scenarios of fault current which are listed below.
1. Low Phase Fault Scenario..................1200A
2. Med Phase Fault Scenario...................3000A
3. High Phase Fault Scenario (FO \& F1 only)..6000A
4. Low Ground Fault Scenario................ 540A
5. Med Ground Fault Scenario................1800A
6. High Ground Fault (FO \& F1 only).........3600A

Fault locations will be simulated at (5) locations on the Avanti $12 \mathrm{kV}$ Circuit identified as follows:

1. F0 .......Fault simulated between the Substation and RCI 1

2. F1.......Fault simulated between RCI 1 and RCI 2

3. F2 .......Fault simulated between RCI 2 and RCI 3

4. F3 .......Fault simulated between RCI 3 and RAR 1

5. F4......Fault simulated beyond RAR 1

Timing and blocking results were evaluated and documented. System component and communication failure scenarios were also evaluated under both normal and fault conditions.

\subsubsection{Lab Test Scenarios}

This section summarizes identified system control scenarios and special conditions to be verified as part of this Test Plan. These control scenarios and special conditions will be evaluated under both normal and the simulated fault scenarios identified in the table below (Table 4-2). All lab test scenarios were tested and results were as expected. 


\begin{tabular}{|c|c|}
\hline Scenario & Special Conditions \\
\hline Normal & None \\
\hline \multirow{5}{*}{$\begin{array}{l}\text { Operational Abnormalities } \\
\text { Disable }\end{array}$} & RCI-3 Control on "Fault Trip Disable" \\
\hline & RCI-2 Control on "Fault Trip Disable" \\
\hline & RCI-2 \& RCI-3 Controls on "Fault Trip \\
\hline & Disable" \\
\hline & RCI-1 Control on "Fault Trip Disable" \\
\hline \multirow[t]{5}{*}{ - Local Controls } & RCI-1 Control on "Fault Trip Disable" \\
\hline & RAR-1 REMOTE ENABLED on "Disabled" \\
\hline & RCI-3 Local/Remote Control on "Local" \\
\hline & RCI-2 Local/Remote Control on "Local" \\
\hline & RCI-1 Local/Remote Control on "Local" \\
\hline - SEL2100 Disabled & SEL2100 Disabled via NetComm or RCI/RAR \\
\hline \multirow[t]{5}{*}{ Loss of Communication } & Loss of Comm. from SEL-2100 to RAR-1 \\
\hline & Loss of Comm. from SEL-2100 to RCI-3 \\
\hline & Loss of Comm. from SEL-2100 to RCI-2 \\
\hline & Loss of Comm. from SEL-2100 to RCI-1 \\
\hline & Loss of Comm. from SEL2100 to all devices \\
\hline \multirow[t]{4}{*}{ Interrupters or Recloser Failure } & RAR4 (SEL 351R) Fails to Operate \\
\hline & RCI3 (SEL 351S) Fails to operate \\
\hline & RCI2 (SEL 351S) Fails to operate \\
\hline & RCI1 (SEL 351S) Fails to operate \\
\hline RAR-1 is bypassed & Fault beyond RAR-1 \\
\hline Power Reversal & Reversed Tripping \& Blocking Sequences \\
\hline Power Reversal \& Interrupter/Recloser Failure & $\begin{array}{l}\text { Reversed Tripping, Blocking \& Backup } \\
\text { Sequences }\end{array}$ \\
\hline $\begin{array}{l}\text { Apply DC Power to RCI/RAR with } \\
\text { Simultaneous Loss of Communication }\end{array}$ & Various RCI/RAR Scenarios \\
\hline
\end{tabular}

Table 4-2 - Scenarios to be Tested

\subsection{Training of Field Personnel}

Since all the field equipment had been set up for lab testing, field personnel were brought in to undergo training on the new system. This training consisted of an overview of the operation of the protection system and more detailed training on the actual equipment and its operation. The goal of this training was to equip operators and maintenance personnel with the skills needed to maintain and troubleshoot the system in case of problems. The operators were also trained in what would happen during and after a fault so they would be able to take proper actions. This training took place at the site of the lab testing setup as well as regional meetings closer to the circuit site. The following figures show the training setup and some of the training in progress (Figure 4-12 through Figure 4-15). 


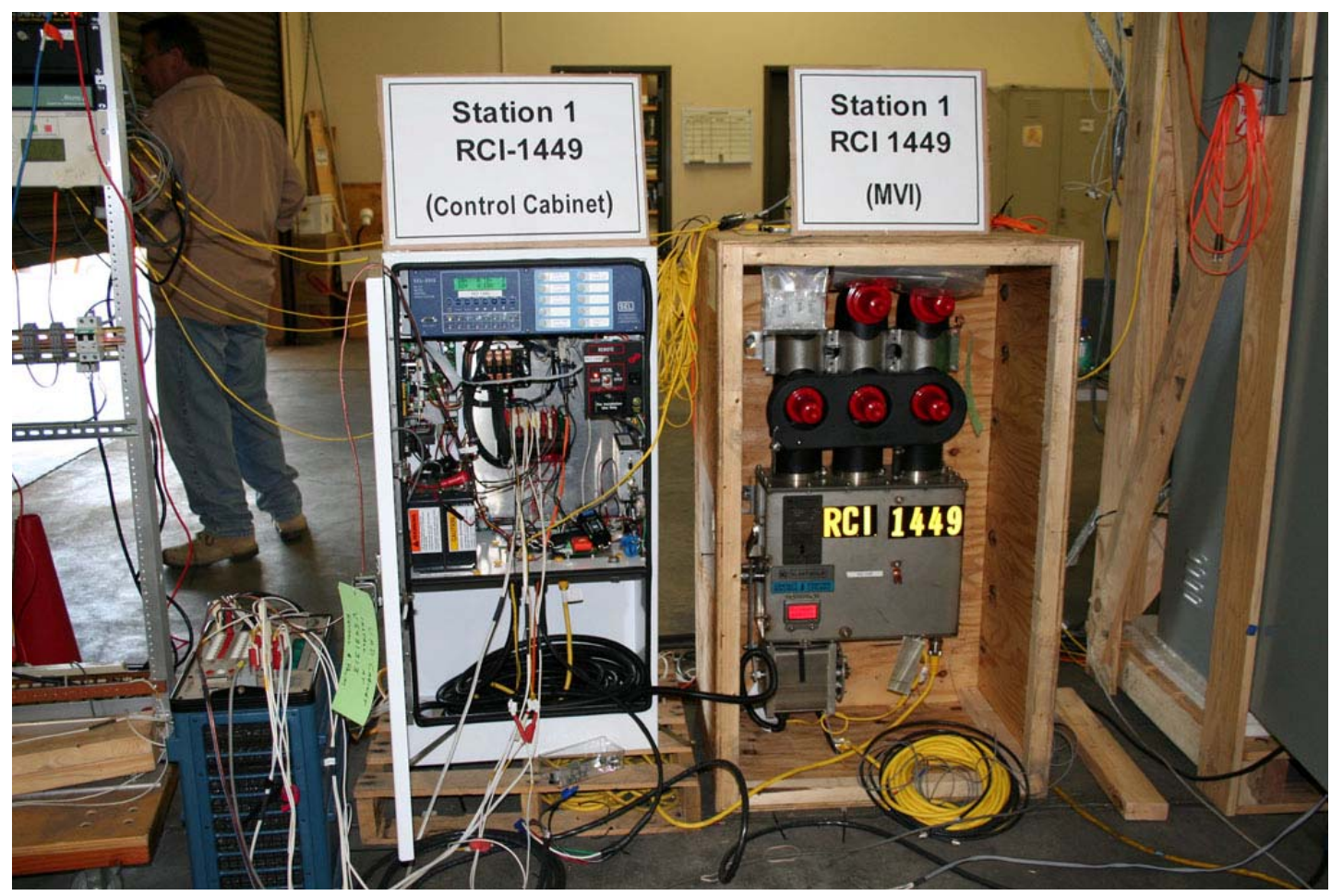

Figure 4-12 - Underground Vacuum Switch and Controller Mockup

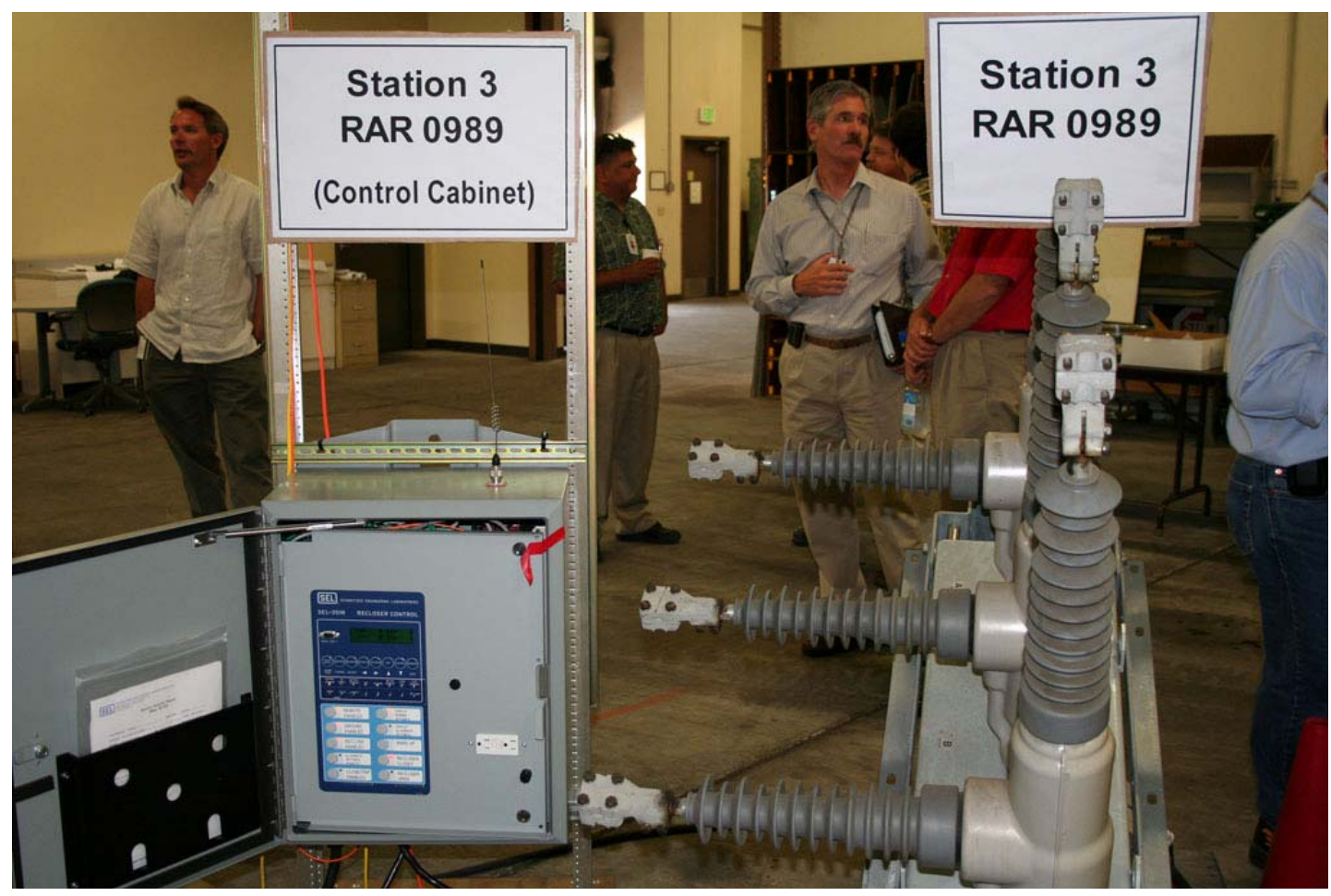

Figure 4-13 - Overhead Automatic Recloser and Controller Mockup 


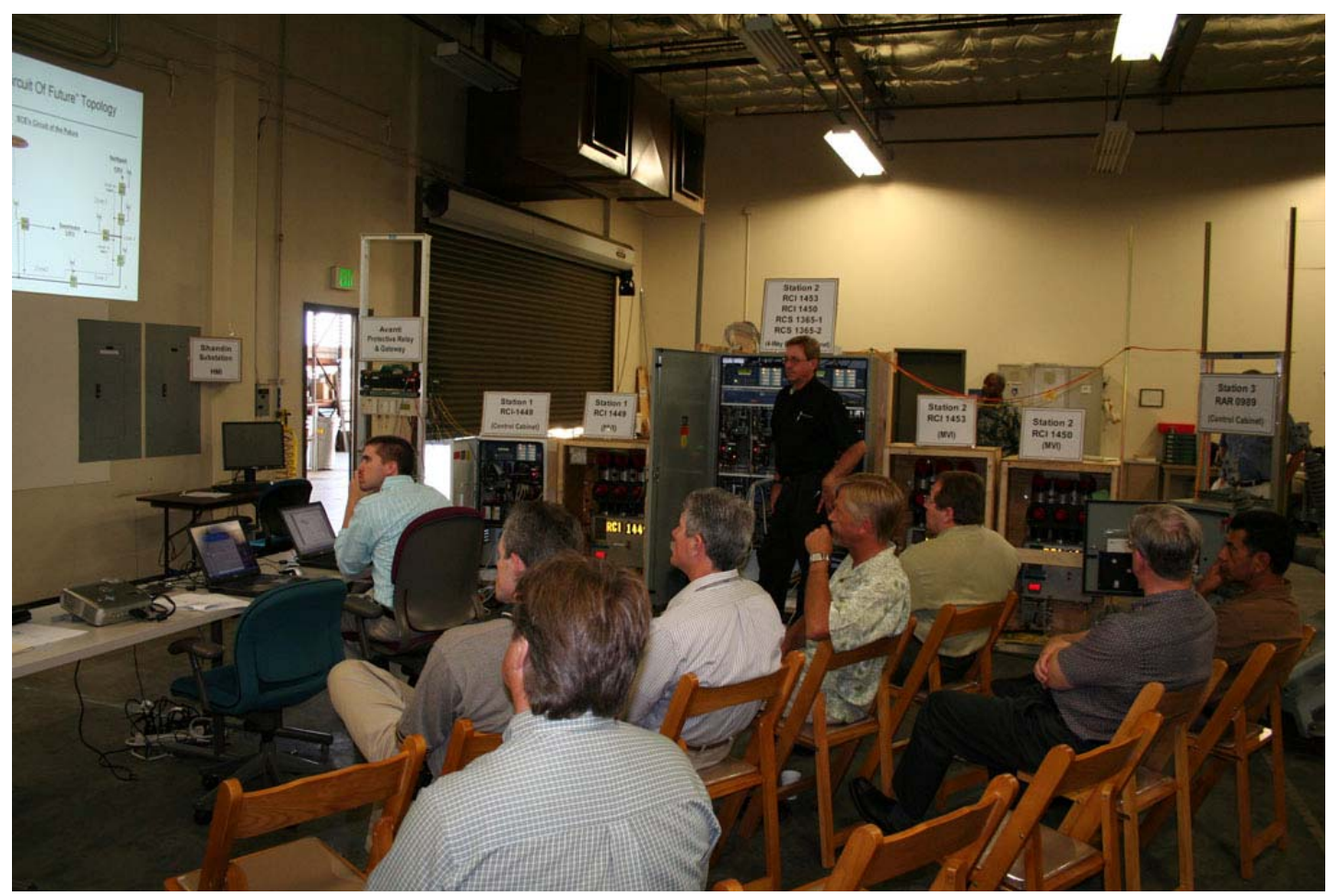

Figure 4-14 - Training Presentation for Engineers, Maintenance Staff and Operators

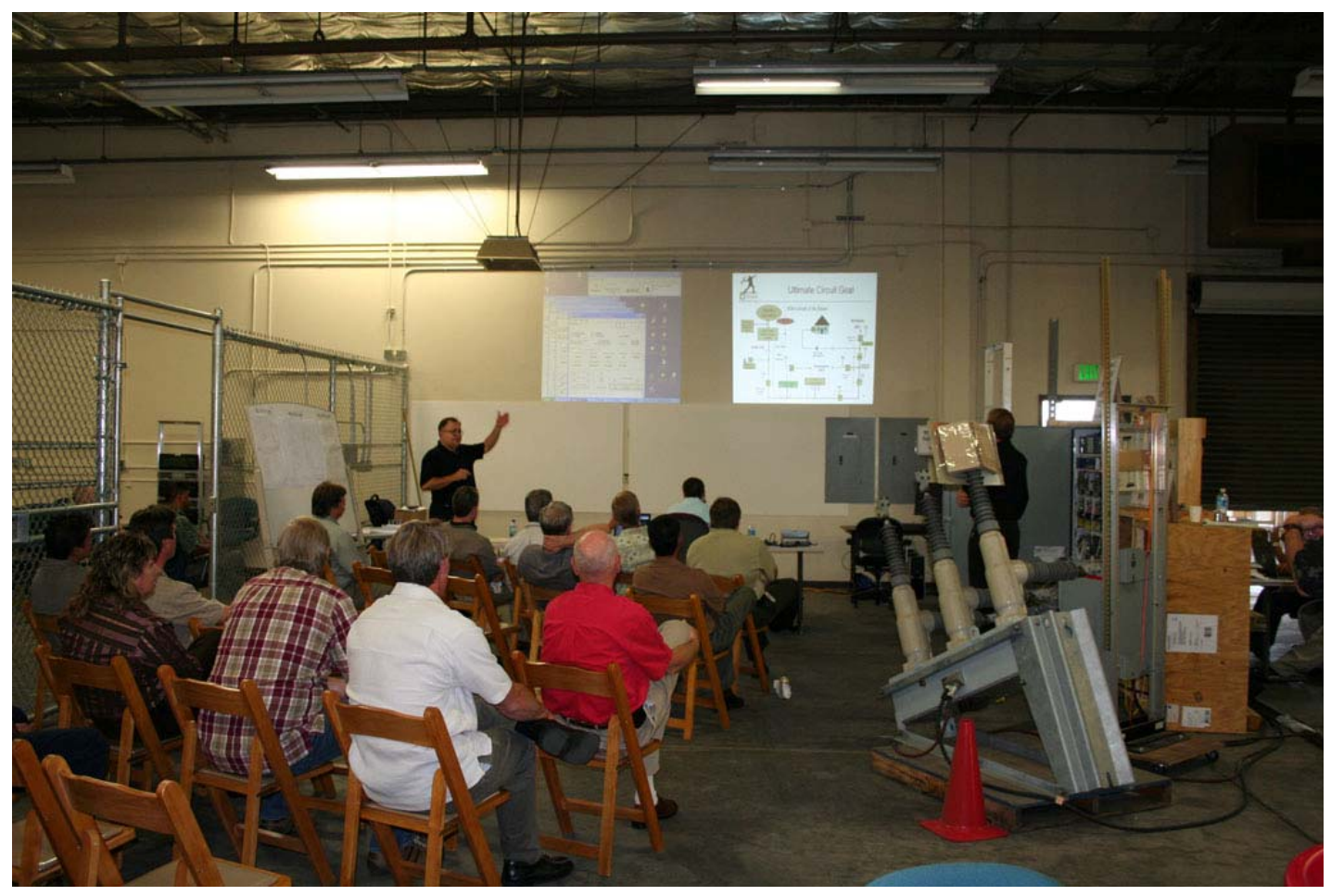

Figure 4-15 - Training Presentation for Engineers, Maintenance Staff, and Operators 


\subsection{Implementation on the $\mathrm{CoF}$}

Circuit equipment, switches and controls were installed during the summer of 2007. After installation was complete, in-service testing was conducted to verify that construction was done correctly. Once this was completed, the circuit was placed in service on August 14, 2007. A formal dedication of the circuit was conducted on October 16, 2007. Some of the pictures from the dedication are included below (Figure 4-16 through Figure 4-19).

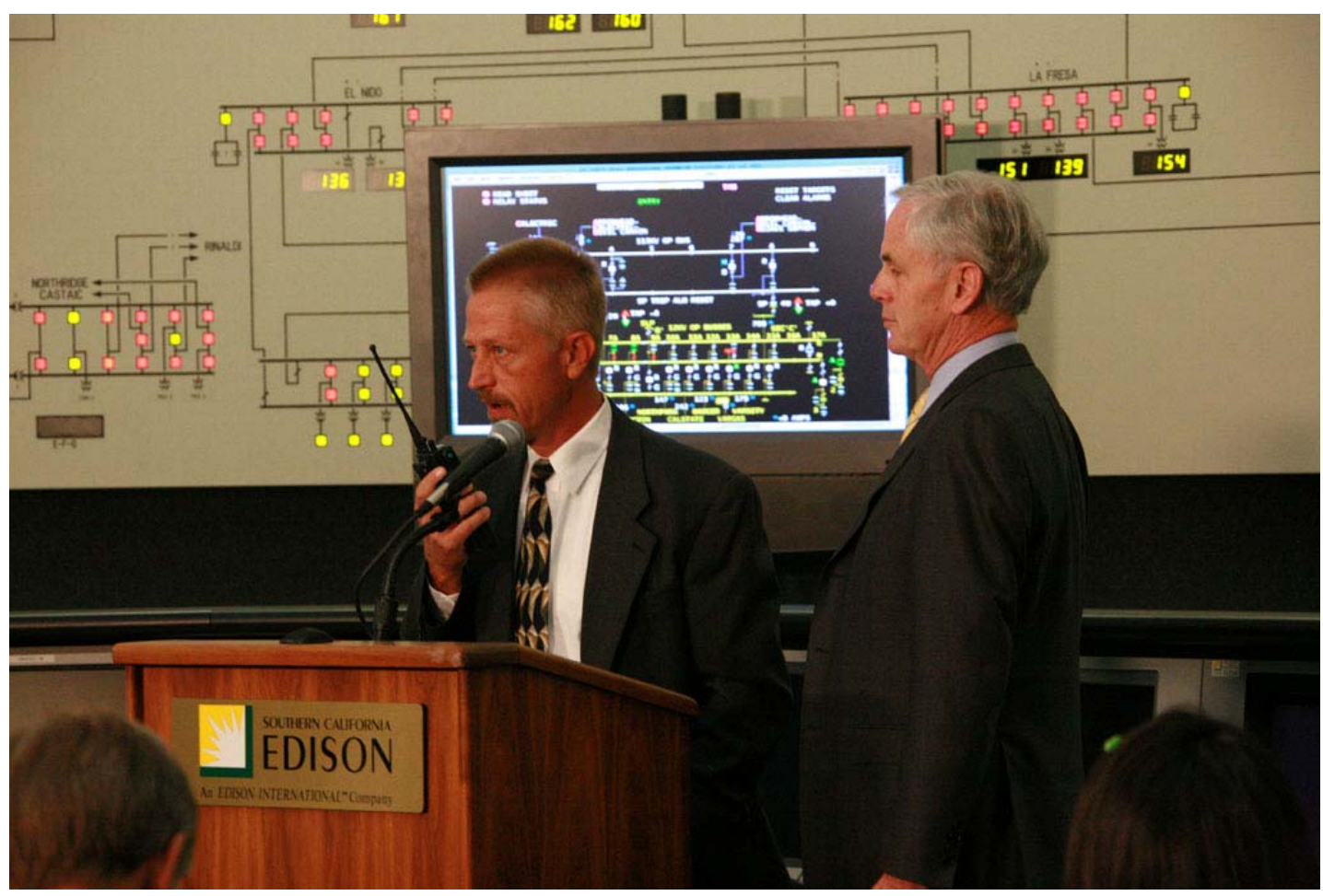

Figure 4-16 - SCE Vice-President Jim Kelly of T\&D Energizing the Circuit of the Future 


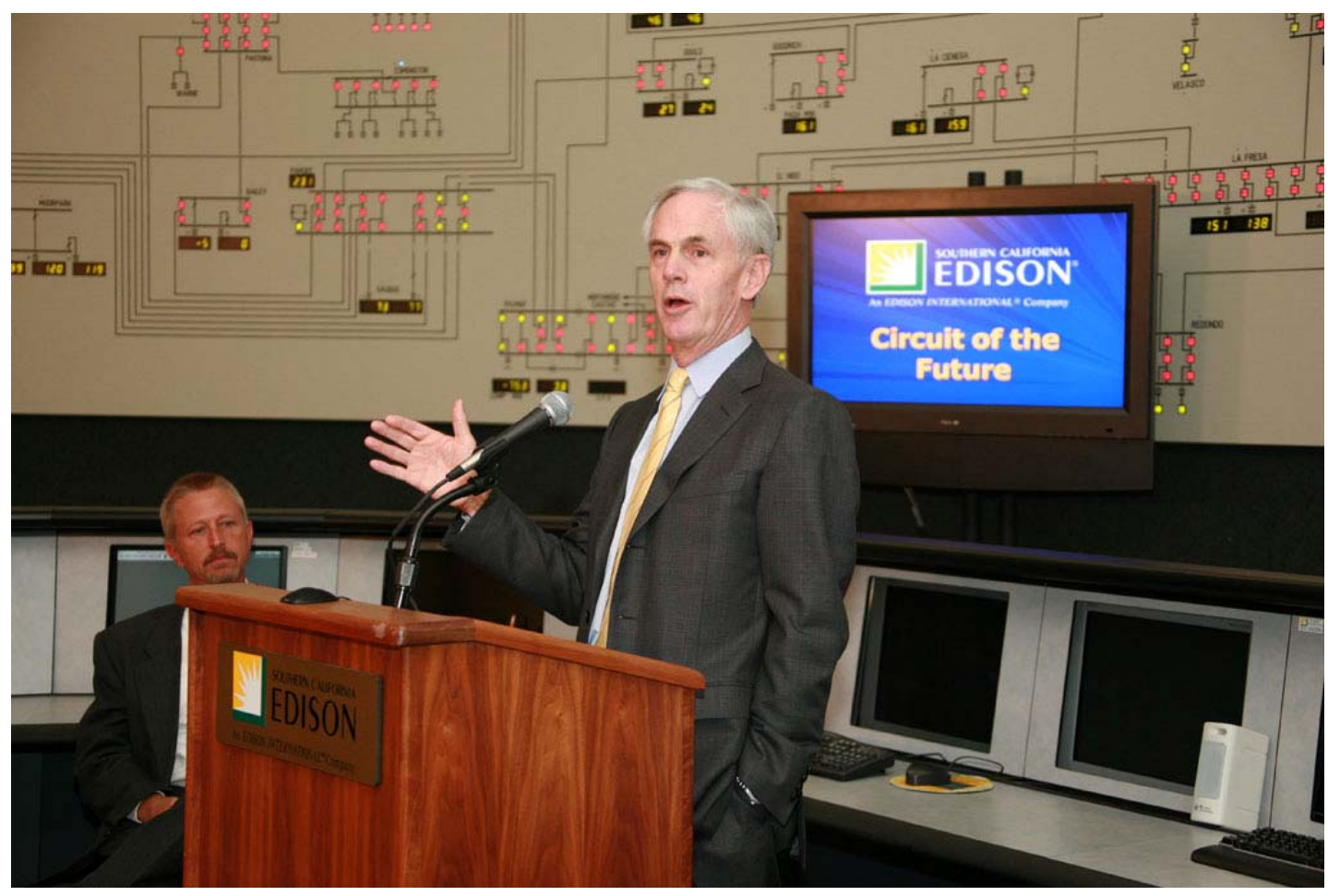

Figure 4-17 - SCE CEO John Bryson at Dedication of Circuit of the Future

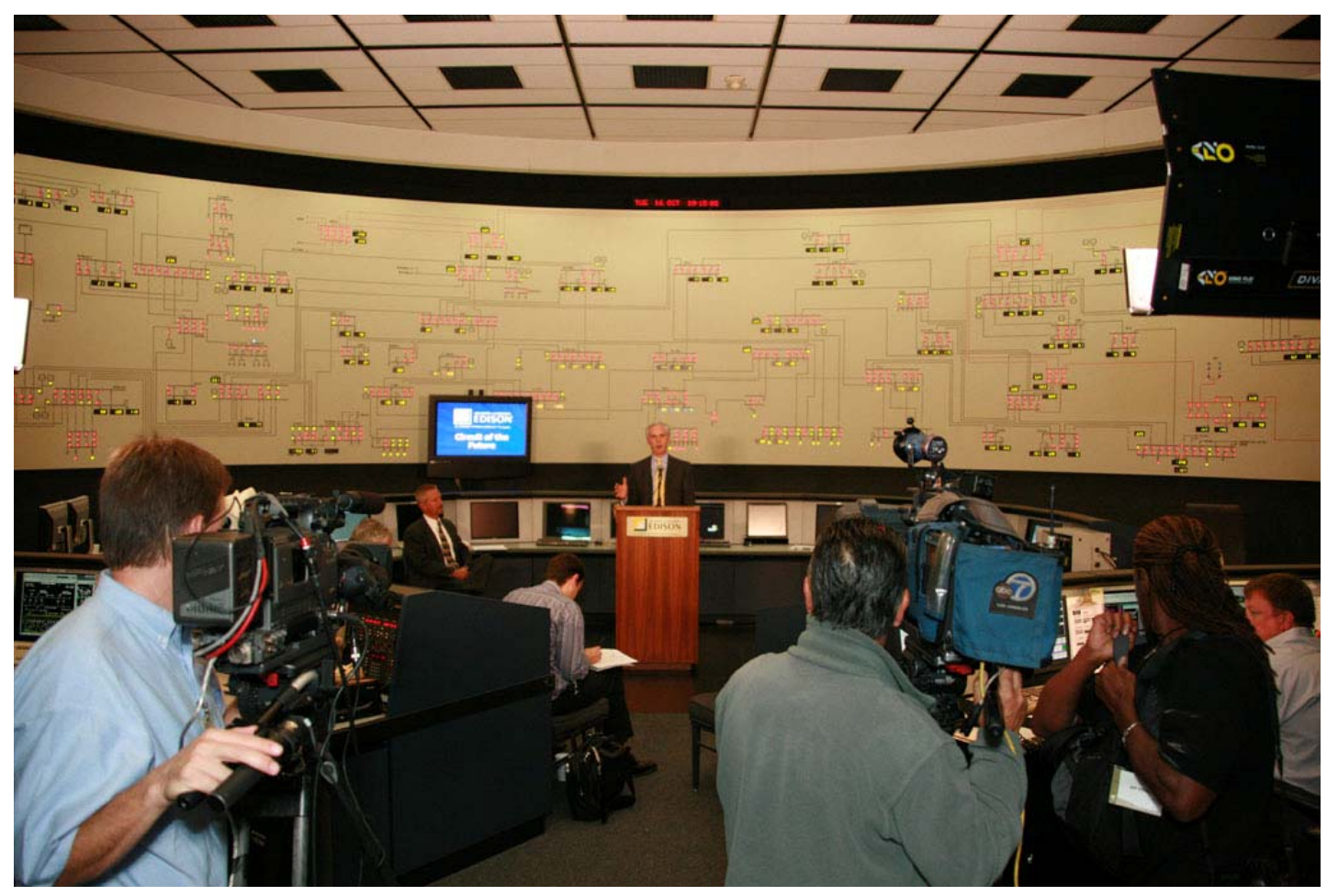

Figure 4-18 - Press Coverage at Dedication of Circuit of the Future 


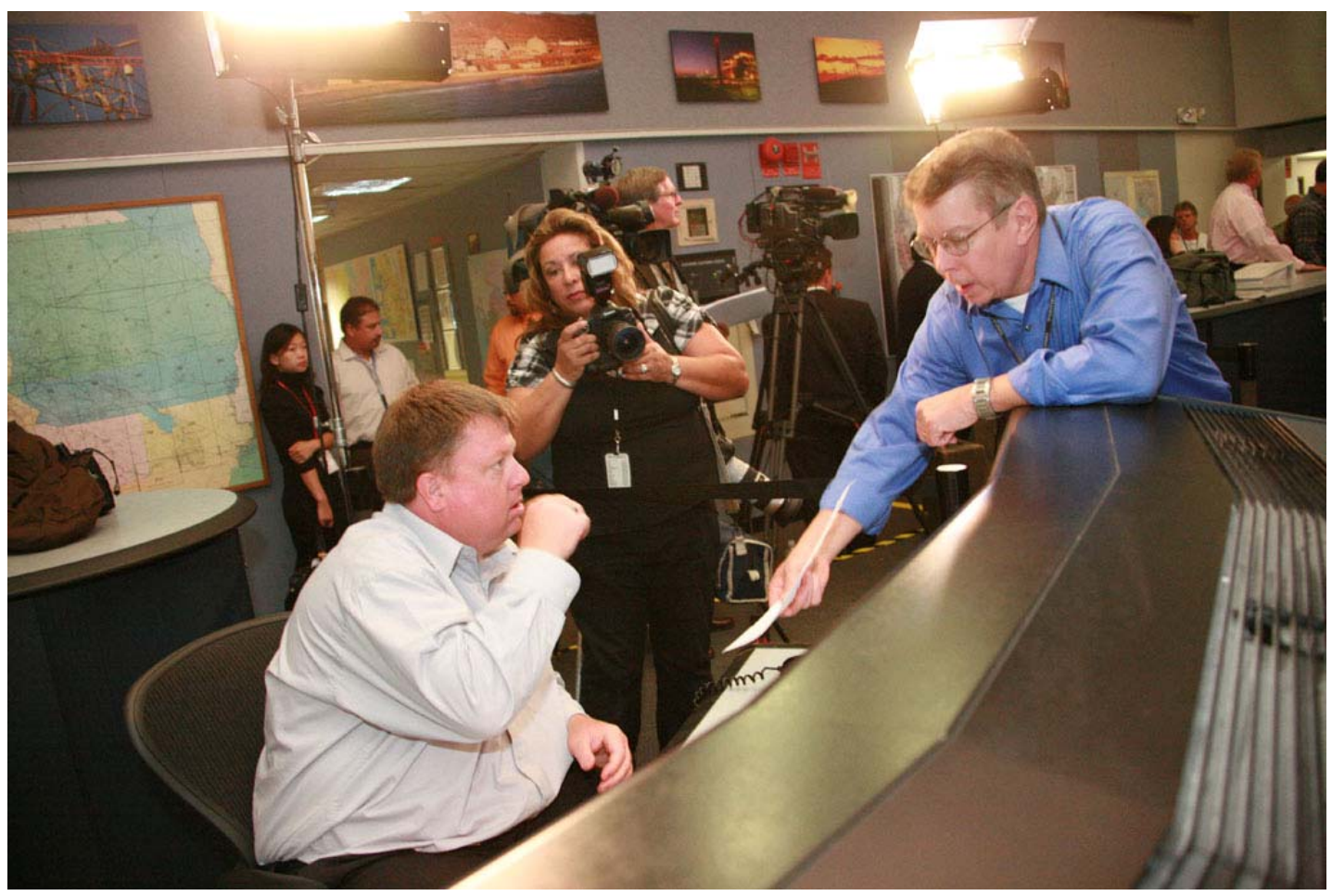

Figure 4-19 - Press Talking to Operators at Dedication of Circuit of the Future 


\section{Section 5: Fault Location, Isolation and Prediction for the CoF with a Fault Current Limiter}

Under Phase II, SCE worked to obtain and install a fault current limiter (FCL) at the head of the Circuit of the Future. The installation of this FCL necessitated the review and modification of the protection scheme that was tested and installed in Phase I. The goal of this new protection scheme is similar to the original scheme with the added benefit of being able to potentially use less expensive equipment because of lowered fault duty. Part of this protection review examined coordination issues because of lower fault current available. Because of the difficulty in obtaining the FCL for the Circuit of the Future, work was also done to model different types of FCLs so that their benefits could be investigated while the actual FCL was being installed. Virginia Polytechnic Institute prepared a PSCAD model for a superconducting FCL that was used with the Circuit of the Future model to evaluate protection needs and FCL behavior.

Fault duty (the currents that would flow during a fault) is growing in most utility systems due to the installation of distributed generation devices. Fault current limiters have the potential to stop the growth of fault duties at the distribution level. High fault duties require the installation of equipment that can withstand high currents and also put higher stresses on components during faults. This higher rated equipment increases costs of construction of the circuits.

Two fault current limiters were considered for installation on the Circuit of the Future. One used solid state switches and was being developed by Silicon Power with funding from EPRI. This device detected faults by looking at the rate of rise and magnitude of the line current to determine if there was a fault. When a fault was detected, current would be shunted to an impedance to clip fault current to a manageable level. Problems and delays with this project caused SCE to select a second fault current limiter being developed by Zenergy as the first FCL installed on the circuit. This second FCL uses a superconducting magnet to saturate an iron core that has windings for each phase. When fault current rises to the point where it drives the iron core out of saturation, the impedance rises in the phase windings, limiting fault current. The device that was selected was essentially an alpha test unit developed by Zenergy to help prove out their design.

In general, fault current limiters are not usually considered for installation in the distribution system due to their cost and limited space for installation. This $12 \mathrm{kV}$ distribution device was viewed as a stepping stone to higher voltage devices that could be installed on the transmission system. The experience gained with the $12 \mathrm{kV}$ device will help utilities and vendors design better devices for higher voltages. 


\section{0}

A more recent concept being considered is the use of superconducting transformers with inherent fault current limiting capabilities for distribution substations $(66 \mathrm{kV} / 12 \mathrm{kV}$ or $115 \mathrm{kV} / 12 \mathrm{kV}$ ). These devices can be installed in space similar to existing transformers and provide the fault limiting capability with little extra cost. SCE is working with Waukesha Transformers as part of an ARRA project to develop and test one of these transformers.

As part of the Circuit of the Future design, a bypass switch was installed in Shandin Substation at the head of the circuit. This bypass switch was designed to ease fault current limiter installation and maintenance. While work was being done on the FCL, the bypass switch would carry all normal circuit current and allow work to be done with the connections to the FCL de-energized. This switch also allowed the FCL to be bypassed in case of problems without interruption to the customers on the circuit. This was deemed necessary because of the developmental nature of the FCL devices.

\subsection{Zenergy FCL Description and Laboratory Testing}

After evaluating the development status and compatibility of several FCLs, SCE selected the Zenergy saturable core FCL for installation on the Circuit of the Future. A $15 \mathrm{kV}$ class prototype device was under development and expected to be available in 2008 for installation. The device was fabricated in early 2008 and sent to the Powertech Labs located in Surrey, British Columbia, Canada for testing. A series of fault current tests and high-voltage dielectric tests were conducted at the lab during the period of October $13-21,2008$. These tests followed the Zenergy test plan dated August 26, 2008. The tests were witnessed by representatives from SCE. Fault current testing was successful with a reduction in fault current of about $20 \%$ at the highest current levels. However, impulse testing caused flashovers and the tests failed. Zenergy subsequently returned the FCL to their facilities and made necessary modifications to pass the impulse tests. The device was then moved to SCE's Shop Services and Instrumentation Division lab facility in Westminster, CA for a repeat of the impulse tests $(110 \mathrm{kV}$ Full Wave Impulse tests and $120 \mathrm{kV}$ Chopped Wave Impulse tests). These repeated tests were conducted on December 15, 2008. The FCL passed these tests and was subsequently transported to Shandin Substation for installation. Installation work was completed and the FCL put into service on March 6, 2009. A picture showing the bypass switch and the FCL is included below as Figure 5-1. 


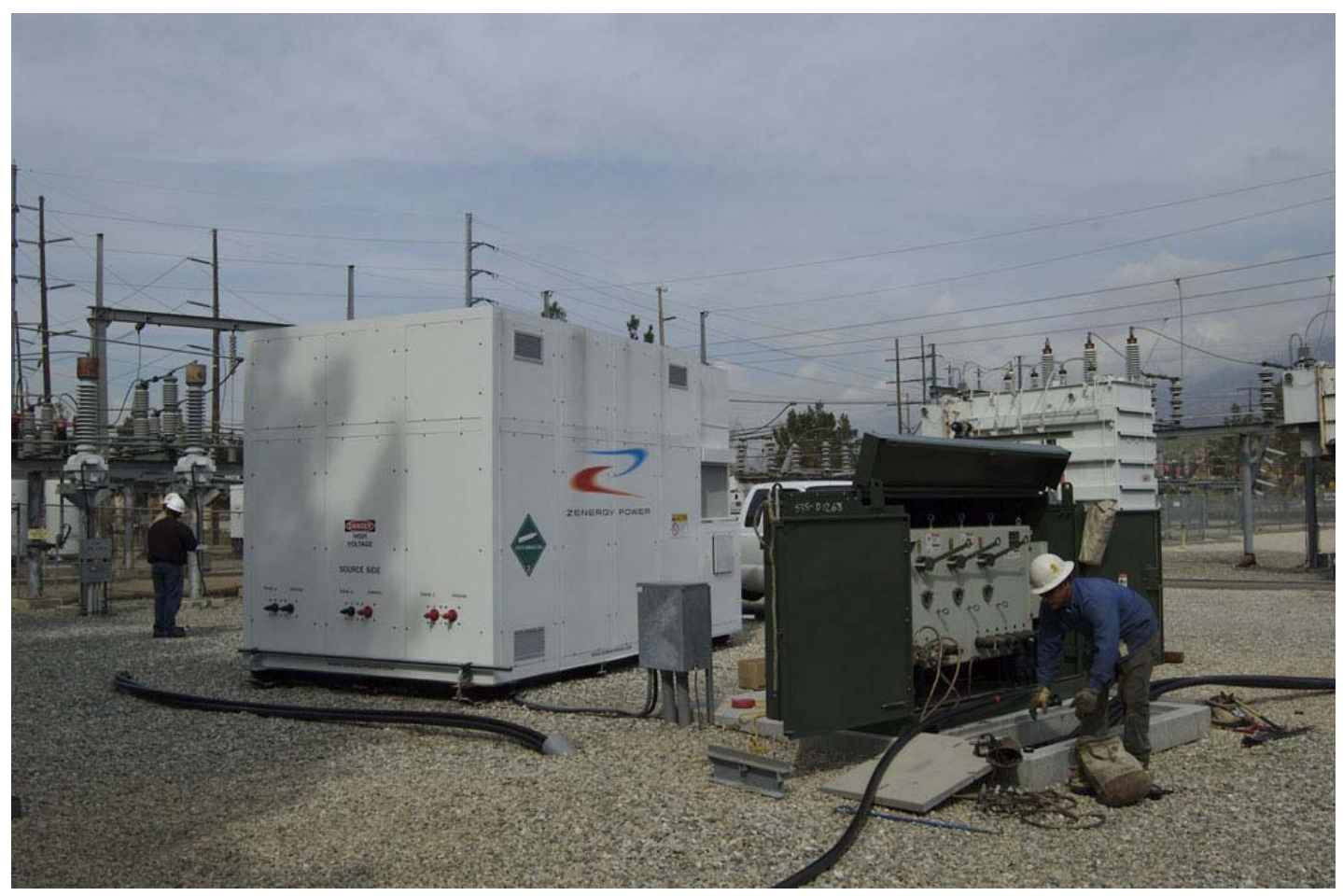

Figure 5-1 - Bypass Switch and Fault Current Limiter in Shandin Substations

\subsubsection{Description and Testing of Zenergy Fault Current Limiter}

The FCL consists of three major components: An iron core, AC windings, and a high temperature superconducting (HTS) DC winding. The AC winding is on one leg of the iron core and the HTS DC winding is on the other leg. The HTS DC winding serves to bias the magnetic iron core into the saturated condition. The AC line of the FCL is connected in series with the AC line in the power grid in which the fault current is to be controlled. Two iron cores per phase are required, each with the AC coils wound in opposite directions, are used to provide current control during both halves of a single sinusoidal wave cycle as shown in Figure 0-3 and Figure 5-3. Zenergy Power's FCL design takes advantage of an electromagnetic phenomenon - a coil with a magnetically saturated iron core has low ac impedance, while a coil with an unsaturated iron core has high ac impedance. 


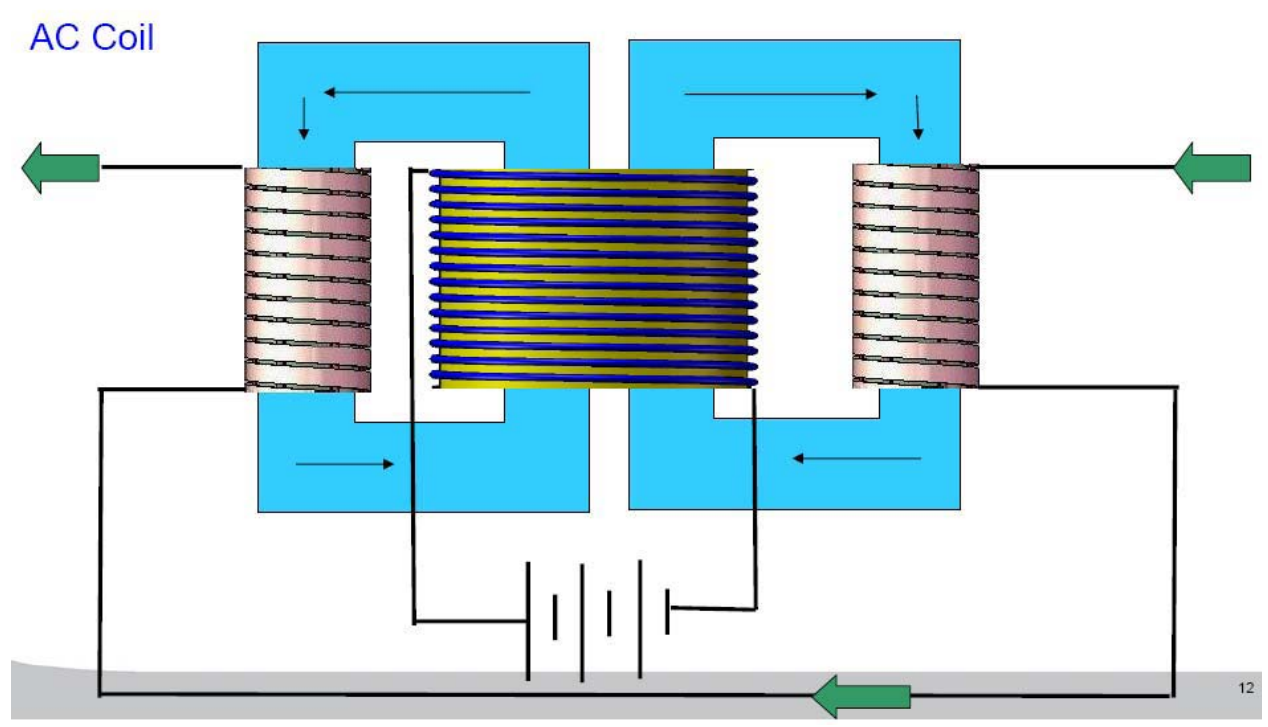

Figure 5-2 FCL Components (Courtesy: Zenergy Power Inc.)

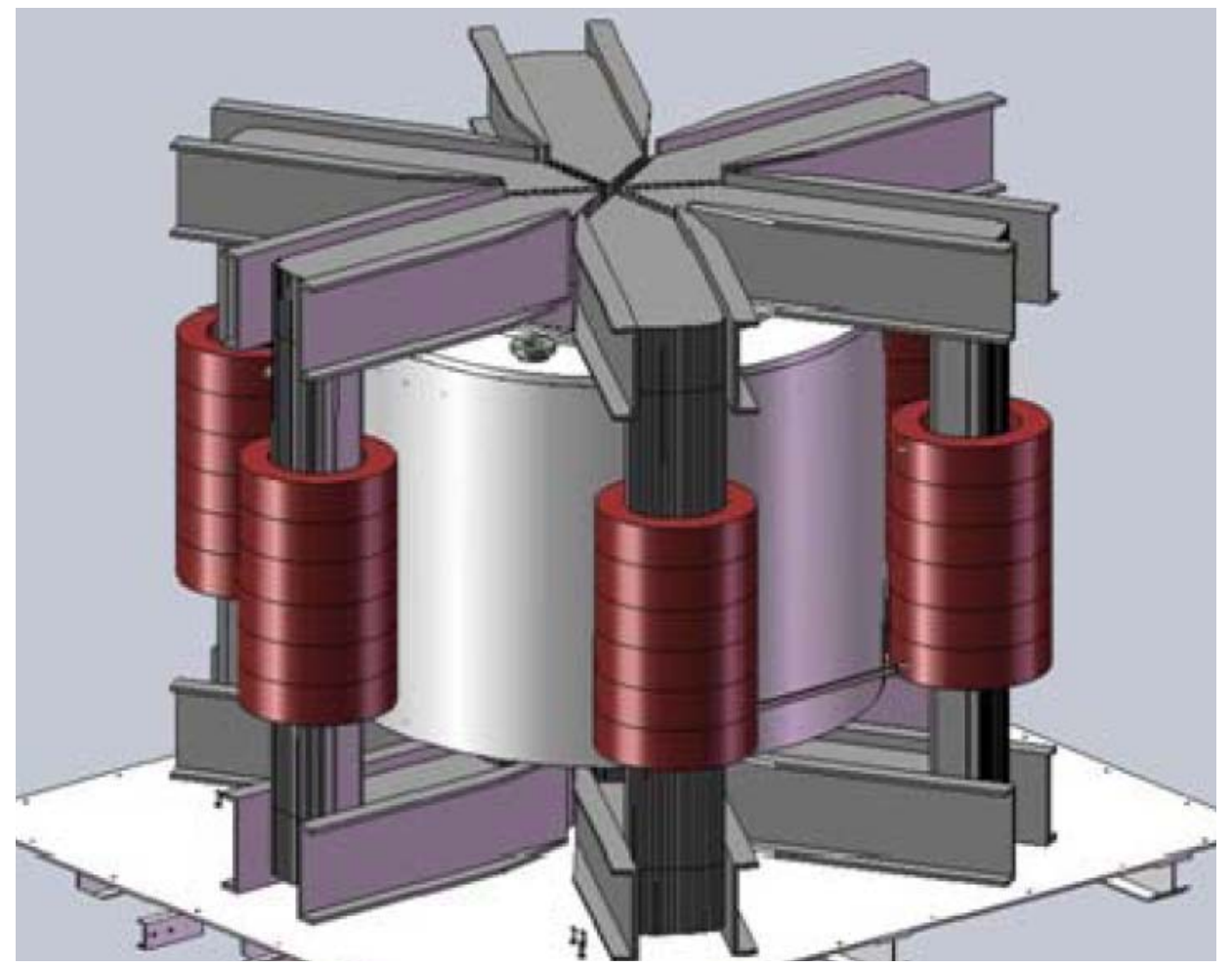

Figure 5-3: Three Phase HTS Saturable Core FCL Design (Courtesy: Zenergy Power Inc.)

The Zenergy Power Inc. FCL is a saturable-core fault current limiter that uses a nitrogen cooled (Cryostat @ 68K) HTS DC coils for reactor biasing. The current FCL was manufactured at the T\&R Electric factory located in South Dakota. The cryostat incorporates a closed-loop compressor system with 2 compressors, for redundant 
operation. The HTS DC coil has 800 turns with a nominal DC bias current of 100 amps. The DC bias current was also tested at 140 amps and 160 amps. The copper AC coils (6 each) were configured for 40 turns each with 3 optional taps available. The FCL was specified to have the following ratings:

- $15 \mathrm{kV}$ Class

- Three-phase

- $110 \mathrm{kV}$ BIL

- AC coil rating of 1,200 Amps continuous AC current. Normal operational insertion impedance of less that $1 \%$ at rated current. This requirement limited the operations to 800 amps based on testing. The 900 Amp $15 \mathrm{kV}$ ESNA connectors, specified by SCE for input and output connections, also limited the actual rating.

- Heat Run Tests could also limit the device rating due to temperature rise.

Initial tests of this design were conducted in December 2007 at the Powertech labs. Areas of design that were found to be improved at the tests conducted in October, 2008 included:

- A closed-loop nitrogen cooling system with two compressors had been installed for redundant operation to eliminate/minimize nitrogen leaks and maintenance. Only one compressor is normally needed for operation.

- Power losses had been reduced at continuous operating current levels. The voltage drop is approximately $1 \%$ or less at an operating current up to 800 amps.

- Voltage drop had been reduced to $1 \%$ or less at continuous operating current levels up to 800 amps. The voltage drop is only $0.3 \%$ at $330 \mathrm{amps}, 0.5 \%$ at 520 amps, and $0.9 \%$ at 750 amps.

- The bracing structure had been altered to use non-metallic components that eliminate any arcing during normal operation; for rated continuous current and fault current levels.

- The reactor coils have been constructed using an Epoxy Cast Coil design.

The FCL was initially configured at the Powertech High Power Lab to conduct Fault Current Tests. Tests on October 14-15, 2008 were conducted at a reduced voltage of $3.76 \mathrm{kV}$. Tests on October 16-17, 2008 were conducted at a nominal voltage of $13.1 \mathrm{kV}$. A total of $\sim 77$ high current tests, including calibration of the test circuit with the FCL bypassed, were conducted. Tests were conducted with the following nominal available current levels:

- Load Current Levels of 300, 500, 800, 1000, and 1200 amps rms

- Fault Current Levels of 3000, 5000, 10,000, 12,500, 15,000, 20,000, and 23,500 amps rms

- DC Bias Current Levels of 100 (typical), 140, and 160 amps DC

- Load Current Durations of 10 cycles, 20 cycles (typical), and 120 cycles

- Fault Current Durations of 20 cycles, 30 cycles (typical), 74 cycles (1 time)

Clamping: The Fault Current test results indicate that the Zenergy FCL has the capability of performing current limiting to $79 \%$ of the available Steady State Short Circuit Duty 
( $21 \%$ clamping at $23.5 \mathrm{kA}$ ). The FCL only limits current to $16 \%$ of the available Steady State Short Circuit Duty at a lower available fault level of $12.5 \mathrm{kA}$. Peak asymmetrical current levels are generally clamped less than this $(<21 \%$ @ 23.5 kA SCD and $<16 \%$ @ $12.5 \mathrm{kA})$.

Insertion Impedance: It was determined that normal continuous operating current levels of $800 \mathrm{amps} \mathrm{AC}$ or less cause no more than 1\% voltage drop across the FCL at $100 \mathrm{amps}$ DC Bias. Voltage drop increases to $2.8 \%$ at 1200 amps AC operating current levels at 100 amps DC Bias. The FCL was found to have slightly lower insertion impedance at higher DC Bias current levels while operating at $~ 1000$ amps AC operating current.

Fault Current Performance: There were no failures noted during the Fault Current tests. No Fault Current tests were conducted with zero DC Bias due to concerns of transformer action possibly affecting the DC circuits. Zenergy will conduct low-current tests at their facility to investigate this issue. The Fault Current test results indicate that the Zenergy FCL has the capability of performing current limiting to $79 \%$ of the available Steady State Short Circuit Duty (21\% clamping at $23.5 \mathrm{kA}$ ). The FCL only limits current to $16 \%$ of the available Steady State Short Circuit Duty at a lower available fault level of $12.5 \mathrm{kA}$. Peak asymmetrical current levels are generally clamped less than this $(<21 \%$ (a) $23.5 \mathrm{kA} \mathrm{SCD}$ and $<16 \% @ 12.5 \mathrm{kA}$ ).

Partial Discharge Tests: Corona levels were originally found to be greater than $400 \mathrm{pC}$ at $19.5 \mathrm{kV}$ L-G when using the Resonant Test Set to energize the FCL. Corona Tests were passed at levels below $100 \mathrm{pC}$ only after the $500 \mathrm{MCM}, 5 / 15 \mathrm{kV}$, Red Insulated HV Jumper Cables rated 90C were changed to Black XLP HV Cables provided by Powertech HV Labs for the purpose of these tests. All subsequent HV tests were conducted with the Black XLP HV Cables. Zenergy intends to change cables to XLP HV Cables to resolve these and other problems identified.

Impulse Tests: $110 \mathrm{kV}$ Full-Wave Impulse and $120 \mathrm{kV}$ Chopped-Wave Impulse tests failed, as found. Flash-Over failures typically occurred on cables at the metal barrier between the Main Reactor Cabinet and the Control Cabinet or the uninsulated tap connections to the Epoxy Cast Coil Reactor Windings. Application of temporary insulation around connections or increasing clearances typically resolved most problems to allow the passing of these tests. C-Phase Epoxy Cast Coil Reactor Winding on the source end of FCL failed impulse tests at the end of the winding to the tap connection. The coil was found to be oriented such that the tap and coil support block were immediately adjacent and arcing appears to have occurred at the interface of the coil to the insulated/taped tap conductor. Zenergy repaired this failure and re-oriented the coil for improved clearances prior to retest.

Turn-to-Turn Tests: The Impulse Test Set was connected for turn-to-turn tests. Tests were begun on C-Phase and curtailed due to impulse test flash over failures at the end of the C-Phase Epoxy Cast Coil Reactor Winding. 


\subsubsection{High Voltage Impulse Testing at SSID}

Due to the problems encountered in passing the Impulse Tests at Powertech, Zenergy made modifications to the FCL. These modifications included changing cables, repositioning the coils to increase clearances, and insulating unused coil taps. With these modifications in place, the FCL was shipped to the SCE Shop Services and Instrumentation Division lab facility in Westminster, CA. and the high voltage impulse tests were repeated $(110 \mathrm{kV}$ Full Wave Impulse tests and $120 \mathrm{kV}$ Chopped Wave Impulse tests) see Figure 5-4. These repeated tests were conducted on December 15, 2008 and all tests were passed. Once these tests were completed, the FCL was shipped to Shandin Sub for installation on the CoF.

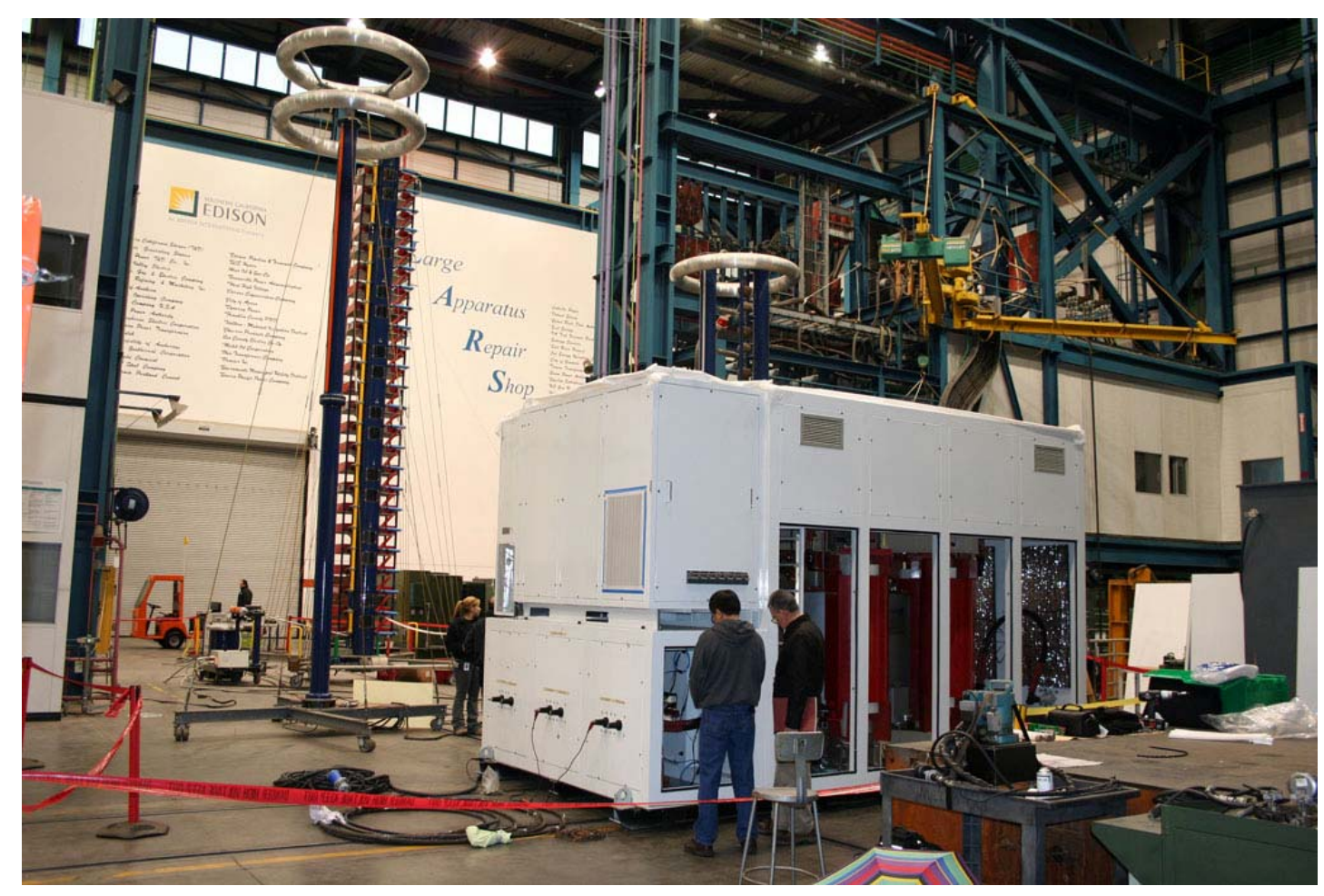

Figure 5-4 - High Voltage Impulse Testing at SCE

\subsection{Integration into the Circuit of the Future}

Since the Zenergy FCL is a prototype device and the circuit might be operating for some time without an FCL, provisions were made on the circuit for a bypass switch that would allow the circuit to operate normally with or without the FCL in service. This bypass switch would be able to carry fully rated current as well as expected fault current without the FCL. This bypass switch was located in the substation near the switch rack. All connections to this bypass switch were accomplished through the use of underground cable and high-voltage elbows. The FCL was situated next to the bypass switch and also connected through the use of underground cable with high-voltage elbow connectors (Figure 5-5). 


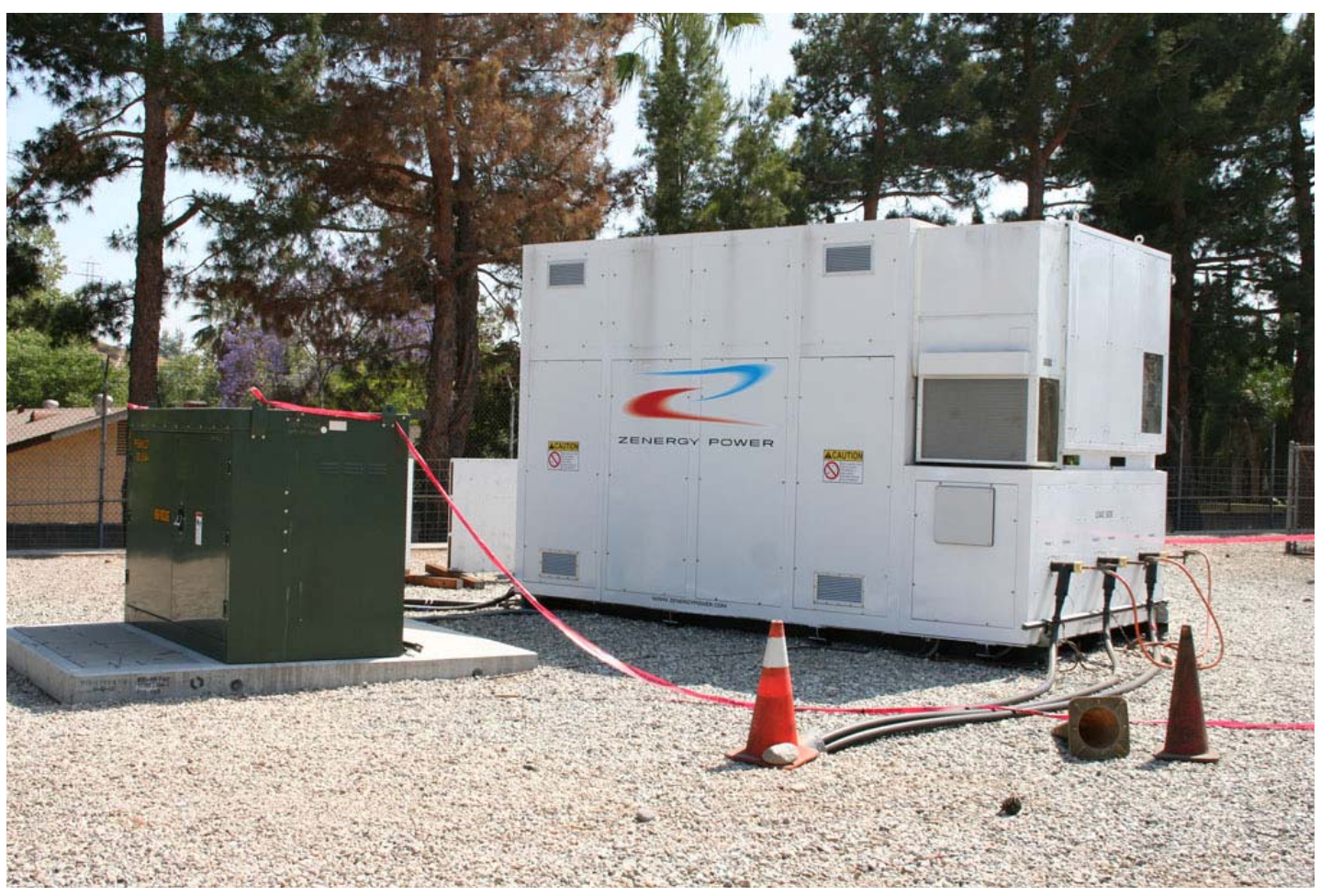

Figure 5-5 - Fault Current Limiter at SCE's Shandin Substation

\subsubsection{FCL Modeling}

Before the FCL was allowed to be put into service on the circuit, several models were constructed to determine if any changes to the circuit protection and equipment were needed. The initial studies were conducted to be sure that the protection system and its coordination would still operate properly. This study was performed through the use of a PSCAD model of the FCL and the circuit. The PSCAD model for the FCL was obtained from Zenergy and the circuit model was constructed by SCE. Both test measurements and modeling of the FCL indicated that the peak fault current might be limited as much as $20 \%$. Since the peak fault current expected at the substation bus was $23 \mathrm{kA}$, the FCL would be able to limit the fault current to about $18 \mathrm{kA}$ for a fault located at the substation bus. Faults located farther out on the circuit would result in less fault current. Since this is still a significant fault current level, it was determined that the circuit protection would not require any coordination changes. The fault current interrupters on the circuit would also be expected to operate correctly with this small fault current reduction. The modeling studies also showed that problems with coordination of the circuit protection would not be expected to occur until the fault current was limiter to less that $6 \mathrm{kA}$.

\subsubsection{Protection Modeling}

An additional set of simulation studies were conducted to determine what the circuit voltages might become if the fault current limiter superconducting coil failed and inserted the full inductance into the circuit. Initial simulations using PSCAD showed the voltage drops to be acceptable and would be mostly corrected by capacitor banks turning on. 


\section{7}

However, after a problem with the FCL on March 16, 2009, the models needed to be revisited. This event (described Section 7) showed the voltages could actually increase under light loading conditions with some of the capacitor banks energized. Working with Zenergy, the PSCAD models were refined and additional simulations were performed. This time, simulation results matched the measured results from the circuit.

Based on the results of the study, a resonant condition can occur if the FCL's DC source is opened with closed shunt capacitors on the circuit. When the DC source is opened, the FCL begins to act like an inductor in a non-faulted state; creating a RLC resonant circuit. The worst case for resonance is when the shunt capacitors are closed and the circuit is lightly loaded. The greater the load current, the greater the voltage drop across the FCL which lessens the resonance problem. Secondly, the FCL is a source of odd order harmonics. However, these harmonics are equal to or less than $0.5 \%$ individual harmonic distortion (IHD) and total harmonic distortion (THD) when the FCL is run at 600A or less. If the FCL is loaded to its maximum steady state limit of $1200 \mathrm{~A}$, the FCL generates approximately $2.5 \%$ THD and IHD.

Based on this study of the resonant conditions, several recommendations were made:

- The bypass switch should be made remotely operable and capable of closing based on a failure signal input from the FCL or loss of communications to the FCL. Disconnect switches should be added to the design, rather than using $600 \mathrm{~A}$ elbow components with parking stands.

- The 1800 kvar capacitor bank should be downsized to a 1200 kvar capacitor bank to minimize voltage rise under resonant conditions.

- More base and off-peak loaded customers should be added to the circuit to help dampen the resonance, if a resonant condition were to occur during off-peak hours.

- The FCL should not be loaded beyond $600 \mathrm{~A}$ in order to keep harmonics below $1 \%$ IHV and THD.

- An event recorder should be programmed to trigger recording on high voltage conditions.

- An additional study is needed to determine how the FCL and the future distribution static VAR compensator (d-SVC) will interact with each other when it is installed.

\subsubsection{Bypass Switch Modeling}

A second modeling effort was conducted to be sure that the bypass switch could be safely opened and closed if the FCL malfunctioned and needed to be bypassed. Again, this study was conducted with the use of PSCAD and determined the voltages across the bypass switch contacts during opening and closing. The study showed that under normal operating conditions, the bypass switch could be opened and closed with no problem since in this condition; there was very little stored energy in the FCL. This is due to the operation of the device in a saturated condition. 
If the FCL superconducting coil shut down and allowed the FCL to move into the current limiting mode, there would be substantially more energy stored in the FCL coils. The models and calculations showed that there was about 173 Joules of energy stored in the FCL under these conditions. A review of the bypass switch rating showed that it was capable of handling up to $16 \mathrm{kA}$ for up to 1 second. This equated to 332 MJoules of energy, much higher than the energy stored in the FCL. Because of this, it was decided there was no problem opening or closing the bypass switch, even in the case where the superconducting coil was de-energized.

The following analysis was done by Zenergy to determine that opening and closing the bypass switch would not cause any problems:

\subsubsection{Shut-down of FCL with DC Saturation.}

The following diagrams (Figure 5-6) show the FCL while operating in the saturation mode with the DC bias in normal condition.
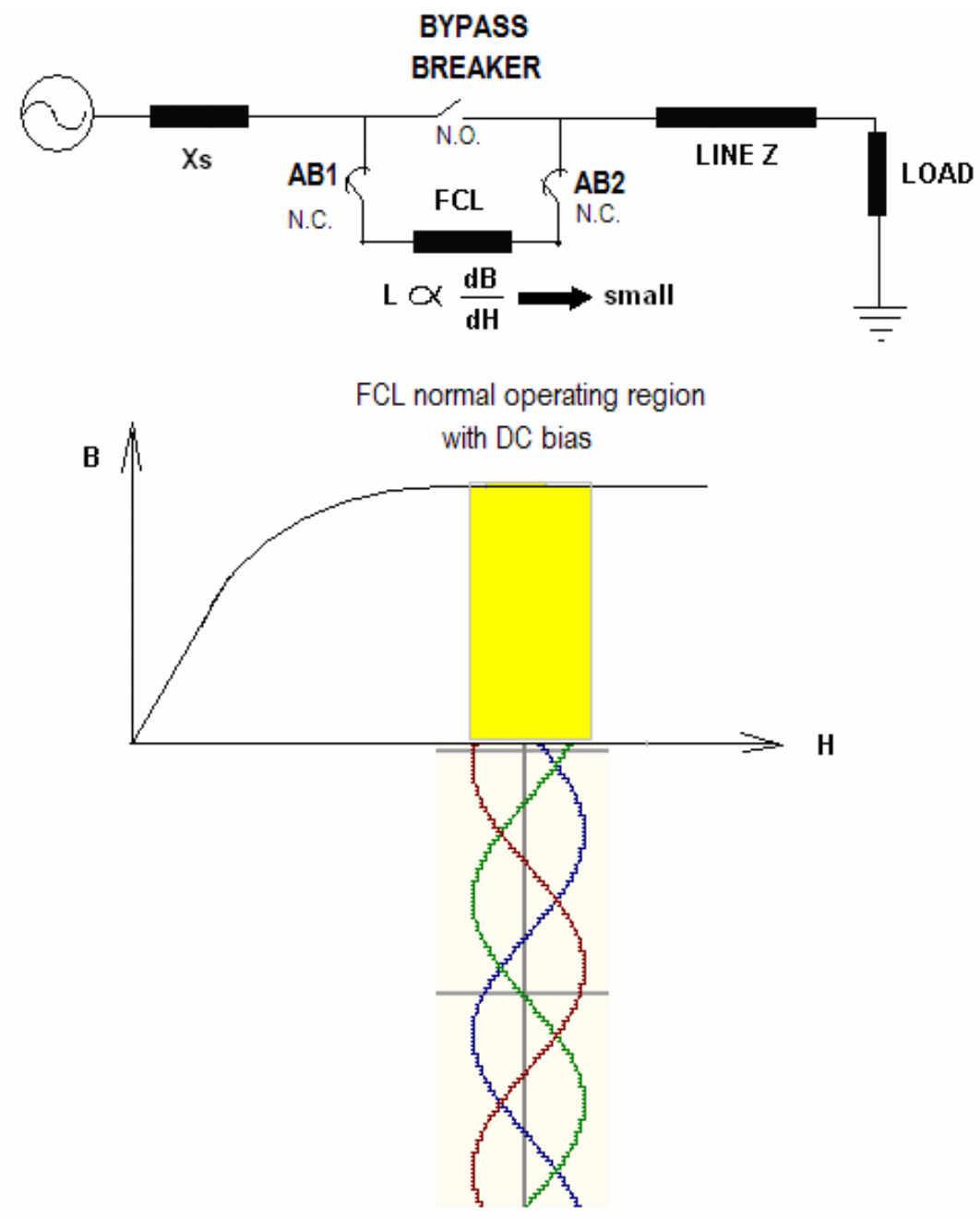

Figure 5-6 - Bypassing the FCL under DC Bias (Courtesy: Zenergy Power Inc.) 
Here we have an extremely low inductance which is proportional to $\frac{d B}{d H}$. Therefore, the energy stored in the AC coil ( $\left.W=\frac{1}{2} L I^{2}\right)$ is small, for which the bypass breaker can be closed and the isolation switches $\mathrm{AB} 1$ and $\mathrm{AB} 2$ can be opened without causing any disturbance to the system. Maintenance work can then be done on the FCL.

\subsubsection{Shut-down of FCL without DC Bias}

The following diagrams (Figure 5-7) show the FCL operating in the unsaturated condition with the DC bias coil disconnected.
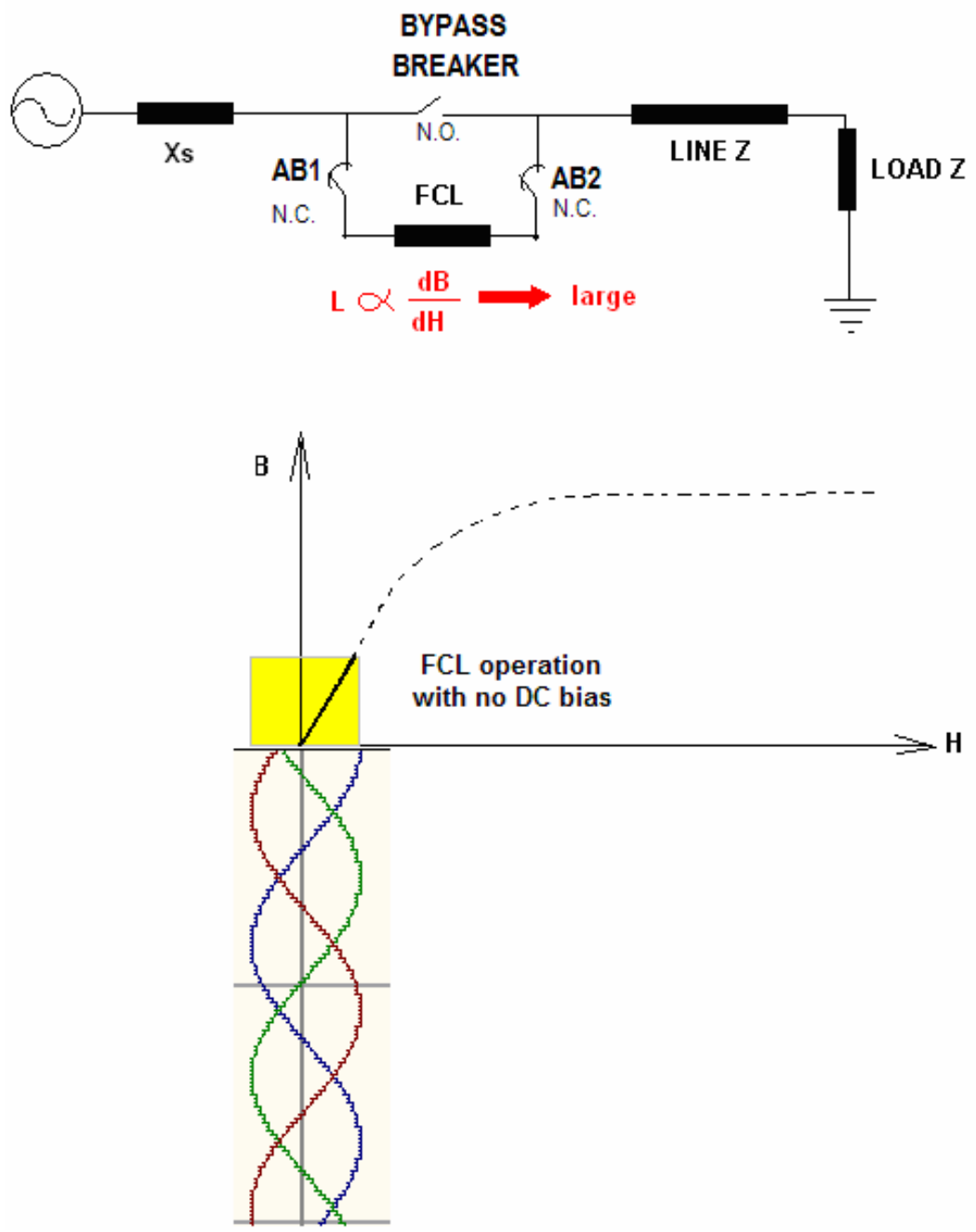

Figure 5-7 - Bypassing the FCL without DC Bias (Courtesy: Zenergy Power Inc.)

Here we have the FCL magnetic core operating on the unsaturated condition, where $\frac{d B}{d H}$ is large. This means operation of the system with a considerable reactance to the 
flow of $60 \mathrm{~Hz}$ current. Once this condition has been detected the FCL has to be bypassed. The energy stored in the FCL AC coils can be calculated as follows:

1. From the coil manufacturer estimation, each $\mathrm{AC}$ coil has an inductance of $60 \mu \mathrm{H}$, with an air core.

2. Now, for an iron core we can assume a permittivity of around 100 , for which the AC coil reactance will be around $60 \mu \mathrm{H} \times 100$ or $0.06 \mathrm{mH}$, or $0.12 \mathrm{mH}$ on the two coils.

3. The stored energy in the FCL AC coils can be calculated as:

$$
W=\frac{1}{2} L I^{2}=\frac{1}{2}\left(0.12 \times 10^{-3} \times 1200^{2}\right)=173 J
$$

This is the amount of energy during the closing of the bypass breaker. As a typical breaker operation, a switching overvoltage will show up on the system as the stored energy in the AC coils is dissipated onto the distribution system. The breaker has to have a breaking capacity to handle the calculated stored energy in the AC coils. The bypass switch used by SCE is the G\&W PRAM43 which is capable of handling 16 $\mathrm{kA}$ symmetrical current for one second or:

1-s MVA_short_circuit $=\sqrt{ } 3(12)(16)=332 \mathrm{MJ}$

This capability is far larger than the energy calculated above.

Once the bypass switch is closed the isolation switches AB1 and AB2 can be opened.

\subsubsection{Isolation versus disconnection of the FCL}

The final questions that need to be answered before the FCL could be put into service were:

- Would it be necessary to disconnect the FCL in the event of a failure or could it simply be bypassed at the disconnect switch?

- Could the isolation switches be switched over at the same time as the bypass switch?

Based on additional modeling, the FCL owner (Zenergy) determined that the FCL should be bypassed and disconnected from the system in the event of a failure condition. The isolation switches (AB1 and AB2 in Figure 5-6 and Figure 5-7) should then be opened. A PSCAD model was set up to study this operation. The model is shown in Figure 5-8. As simulation results show in Figure 5-9, the voltage across the bypass switch and the isolation breakers is small enough that the bypass and isolation operation can be done concurrently if desired. 


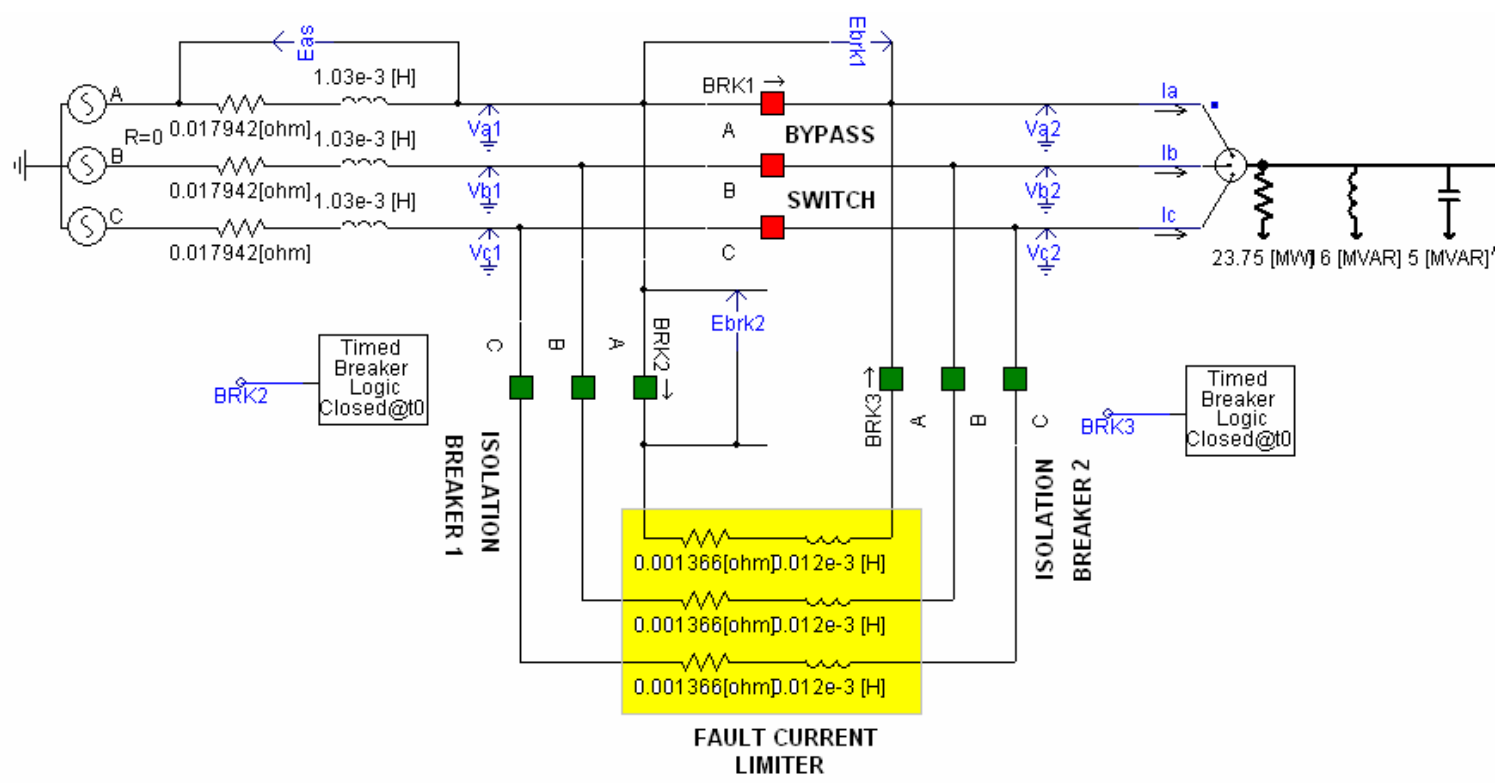

Figure 5-8 - PSCAD Representation of the Bypass and Isolation of the FCL (Courtesy: Zenergy Power Inc.)

The RMS voltage across the bypass breaker is around $240 \mathrm{~V}$ as depicted in Figure 5-9. The voltage drop that persists once the bypass breaker closes at $t=0.5 \mathrm{sec}$ is due to the source impedance and to the breaker contact resistance.

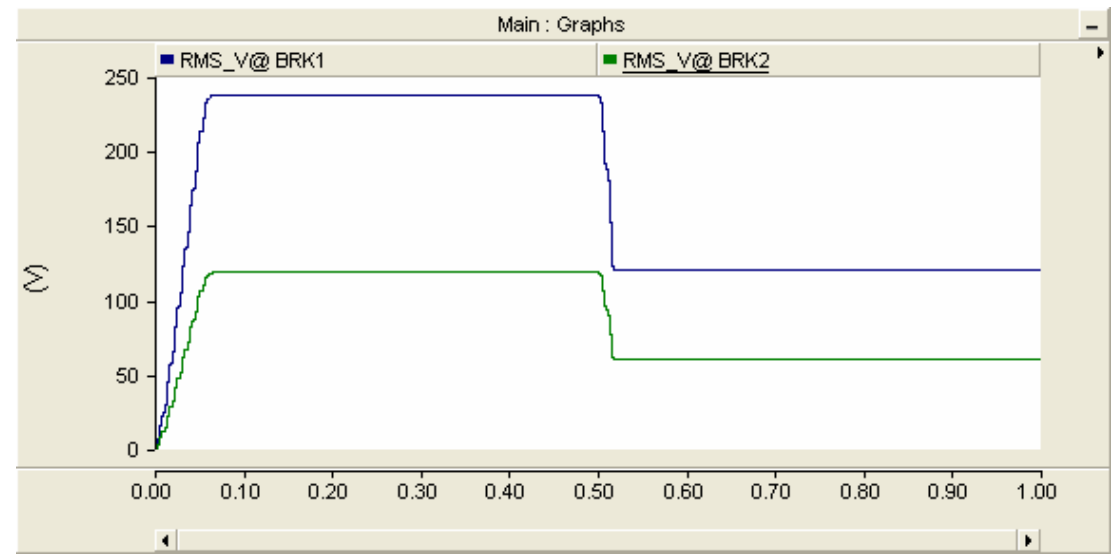

a) Voltage across bypass switch 


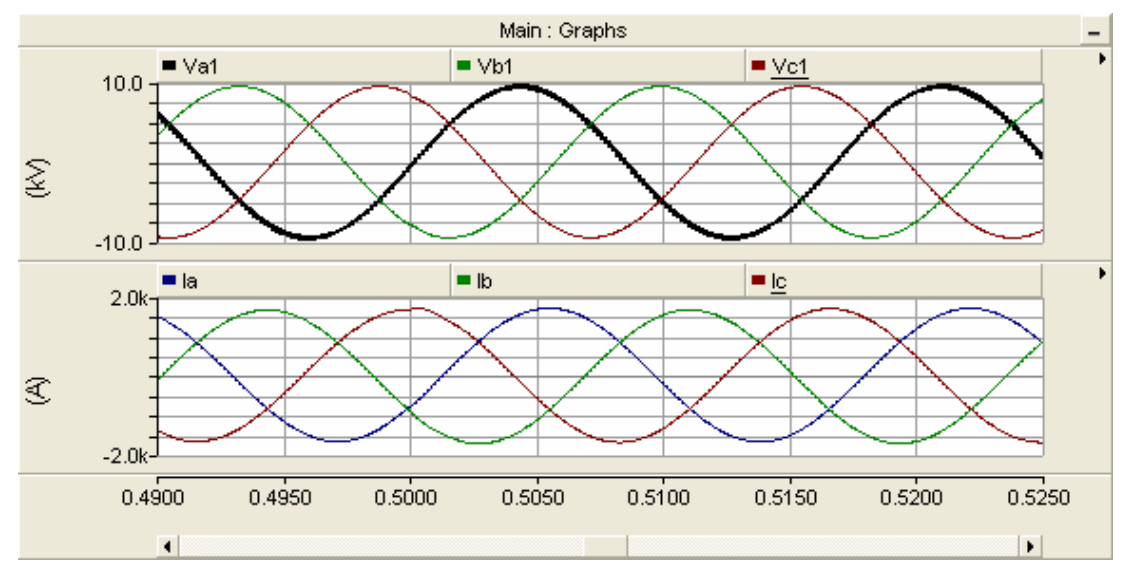

b) Voltage and current waveforms on source side of the FCL

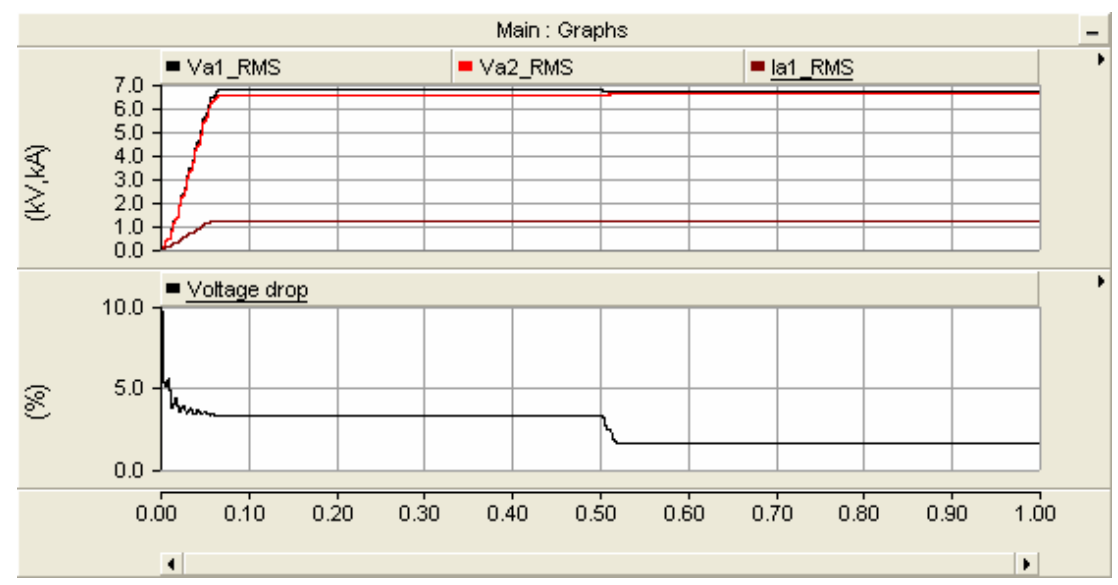

c) FCL RMS voltage and voltage drop

Figure 5-9 - FCL Rms Voltage and Voltage Drop (Courtesy: Zenergy Power Inc.)

\subsubsection{Added Equipment and Controls}

Additional monitoring equipment was added to the circuit to detect power quality and voltage levels on the circuit before and after the connection of the FCL to help confirm these modeled results.

\subsection{Virginia Tech FCL Survey, Models, and Simulations Results}

Since there were significant delays in obtaining models of the Zenergy FCL device, Virginia Tech proceeded to construct an FCL model that could be implemented in PSCAD that did not require details of the actual FCL device. A first step in this work was to perform a literature review of the different FCL technologies that are being worked on. The status and developmental issues of each of these FCL technologies was also noted. The FCL technologies can generally be categorized into 3 broad categories: passive, solid-state, and hybrid. 
Passive FCL devices do not require any external trigger for them to perform their limiting function. The simplest example of this kind of FCL is a series inductor. This technique is well known, low cost, and been used on electrical systems for many years. The disadvantage with this FCL technique is it causes voltage regulation problems as load levels change on the circuit, increased circuit losses and lagging power factor. Simple superconducting FCLs also fit into this category, but do not have the same problems with voltage regulation. Unfortunately, commercial deployment has not been accomplished yet. There is also some concern on how the superconducting FCLs would behave during reclosing situations due to slow recovery after the fault has cleared. The Zenergy FCL that was installed on the Circuit of the Future is also a passive device that uses a superconducting magnet to saturate an iron core inductor. The operation of this device is described in Section 5.1.1.

The second type of FCL is the solid-state limiter. These devices use solid-state switching devices (thyristors, gate turn-off thyristors (GTOs) to quickly insert impedance in the circuit when a fault is detected. These devices can provide significant circuit impedance under fault conditions and also exhibit low impedance under normal circuit operations. The disadvantage to these devices is that they require fast identification of the fault so that the solid-state switching devices can be activated. There are also harmonics introduced into the system due to the switching of the solid-state devices.

Hybrid FCL devices, as the name indicates, use a combination of passive and solid-state switching technologies to perform the current limiting function.

In recent years, most of the work has been focused on FCL technology that uses superconductivity or solid-state switching. This is due to significant cost reductions in superconducting technology and solid-state switching devices. Three of the four FCL devices being funded by the DOE use superconductivity, with the remaining device utilizing solid-state switching technology. The figure below (Figure 5-10) shows where these devices are with respect to voltage and current carrying capability. 


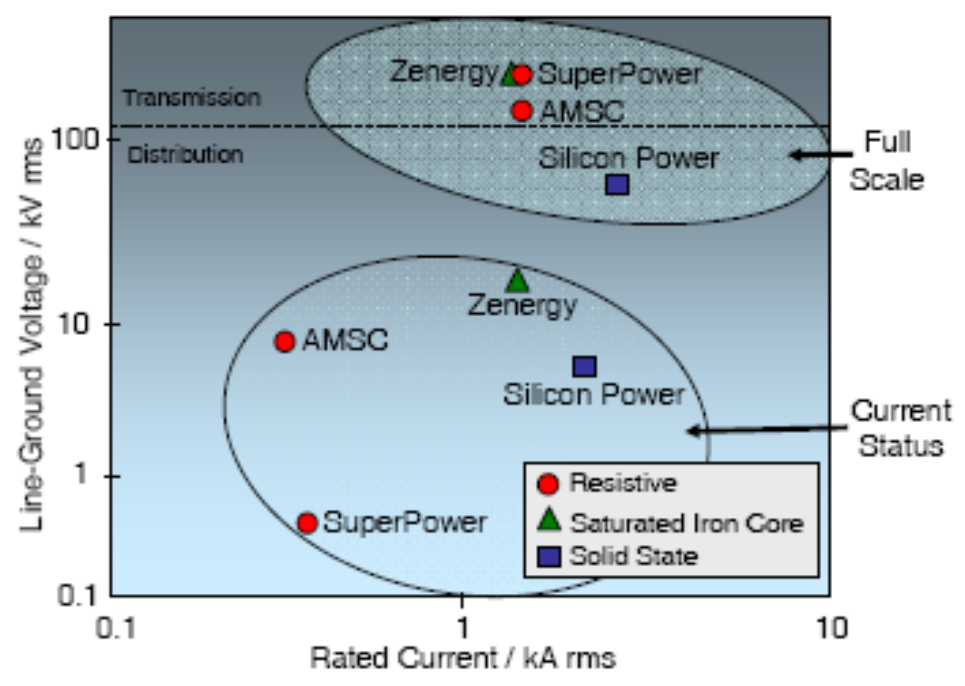

Figure 5-10: Current and Voltage ratings for DOE sponsored projects

(Source: B. M. N. K. Fall, "Fault Current Limiter - R\&D Status and Testing Issues," in 2009 Proceedings of 2009 IEEE Power Engineering Society General Meeting, Calgary, Canada, p. 4)

Virginia Tech developed a PSCAD behavioral model which would mimic the operation of any FCL without having access to proprietary device operational parameter. This would allow the simulation of the operation of a FCL in a circuit without detailed knowledge of the internal operations of the device. The model was designed with a number of settable parameters that allowed the model to be tailored to graphs of the FCL behavior derived from device testing. This model was made up of three main parts: fault detection, insertion of impedance, and removal of the impedance. The fault detection is determined through the use of both current magnitude and current rate of change. This is to prevent false triggering of the FCL. The parameters used for this portion of the model are adjustable so that various kinds of FCLs can be simulated. The second part of the model inserts the impedance into the circuit. This can be done instantaneously or ramped in depending on the type of fault current limiter. The third part of the model removes the impedance after the fault has cleared. Again, the rate of impedance removal is adjustable so that different kinds of FCLs can be simulated. The diagram below (Figure 5-11) shows the various FCL model parts as they were customized for a superconducting FCL. 


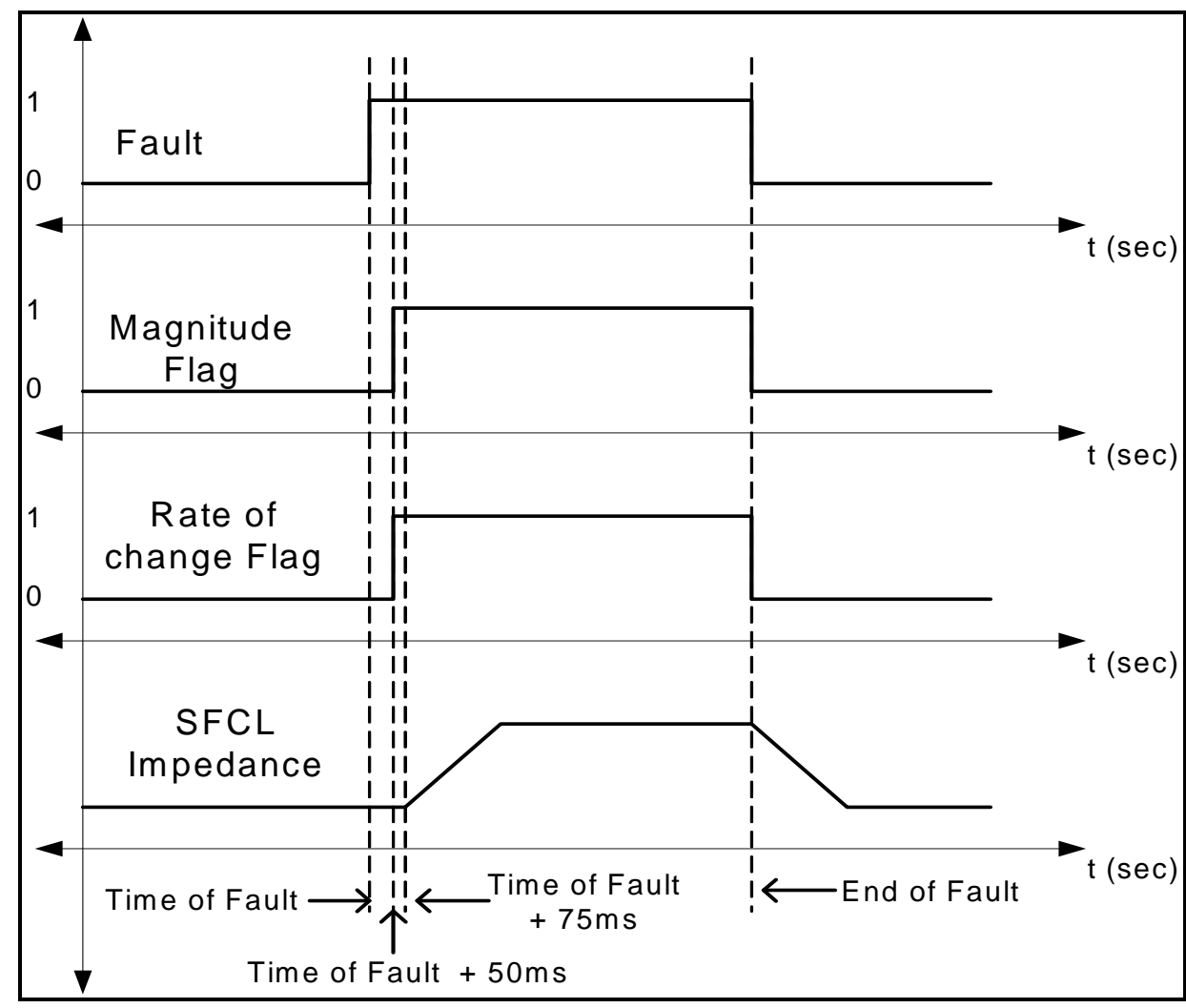

Figure 5-11 - FCL Model Customized for a Superconducting FCL

The model was tested using the Circuit of the Future circuit model and a superconducting FCL. The following figure (Figure 5-12) shows the behavior of the circuit without the FCL in service. In the next figure (Figure 5-13) a fault current limiter is put into the model that ramps the impedance into the circuit and shows fault current limiting. While this model will allow basic studies of the effects of FCLs in a circuit, it still is better to get the actual model from the FCL vendor to properly perform circuit integration studies. 

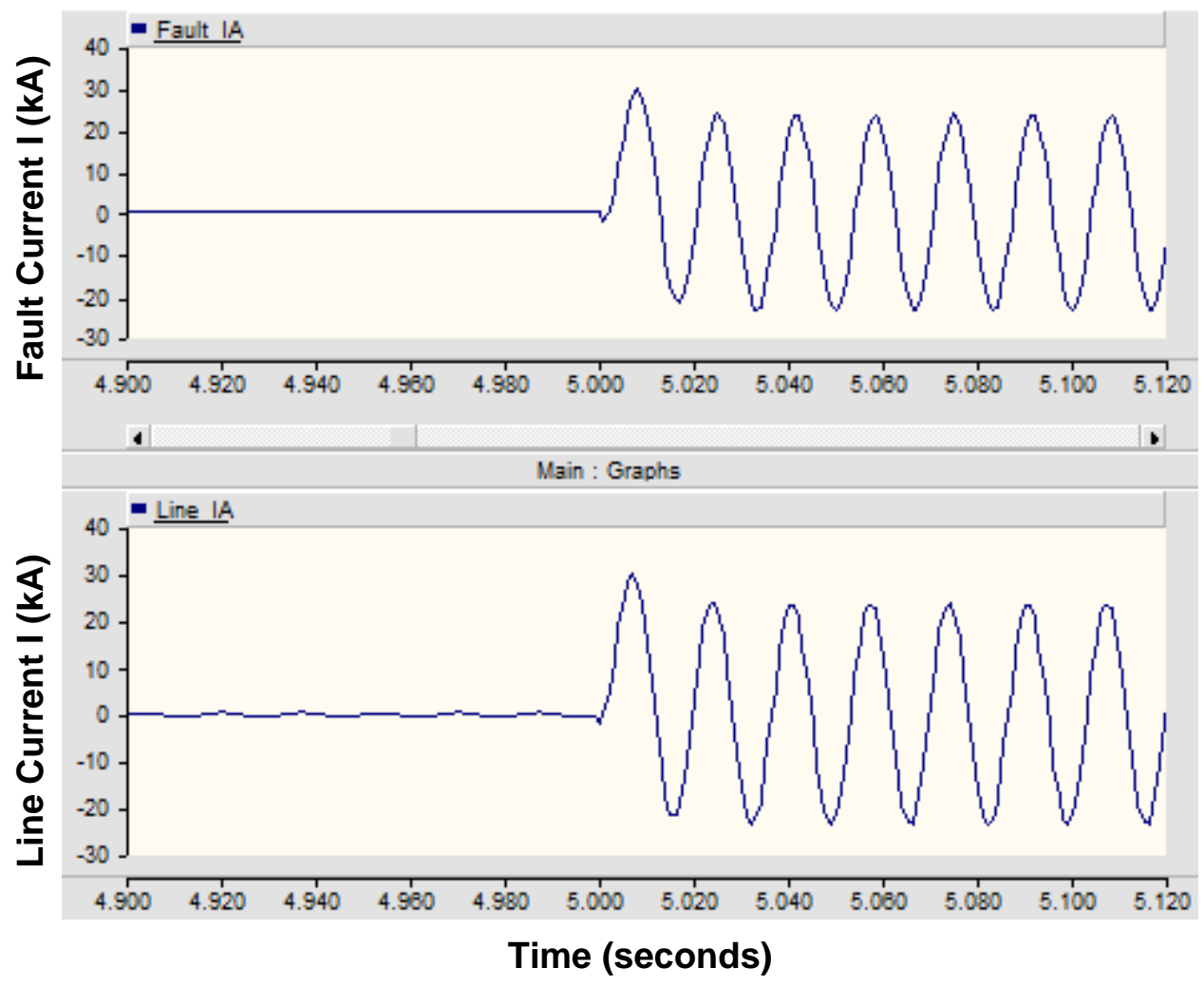

Figure 5-12 - Modeled Fault and Line Current without FCL

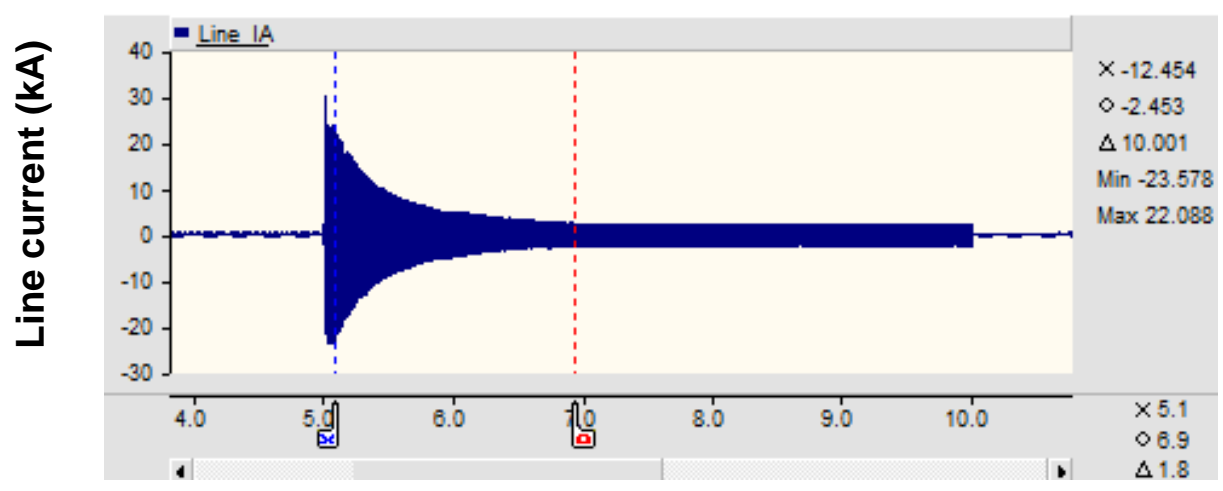

Time (seconds)

Figure 5-13 - Modeled Line Current with FCL 


\section{Section 6: Advanced Fault Sensing and Prediction}

For the third phase of this project, several advanced protection schemes were reviewed and evaluated for implementation on the Circuit of the Future. These ideas came from, the original proposal, the literature survey work, information from various conferences, and internal brain-storming at SCE. Each of the potential technologies and methods were evaluated to see if they could be implemented as part of this project. While a number of these technologies and methods looked promising, many could not be implemented for a number of reasons. The following sections describe the methods and the items selected for further work as part of this project.

Based on work being done by others, time/ funds available on the project, and the team's assessment of which technologies/ methods would be most valuable to the industry, the majority of the efforts were directed to taking the protection system demonstrated on the Circuit of the Future and adapting it to the new System of the Future to be built in Irvine, CA. This project is being partially funded by an ARRA/ DOE Smart Grid Demonstration Grant.

\subsection{Measures Investigated From the Proposal}

In the initial project proposal, a number of new technologies were assessed to determine the most promising areas for this task's research work. Some areas that were investigated include:

- Use of oscillography, arcing fault sensing, time domain reflectometry, phasor measurement and other technologies to detect and locate faults

- Use of line sensors for detection

o BPL coupling electronics as sensor

o Other sensors mounted on or near distribution lines

- Noise analysis of signal from sensors

- Signature analysis based on theory and tests of known bad equipment

\subsubsection{Use of Oscillography, Arcing Fault Sensing, Time Domain Reflectometry, Phasor Measurement and other Technologies to Detect and Locate Faults}

A number of different technologies and methods have been mentioned in the literature for fault location and detection. Oscillography and power quality sensing can give measurements that can be inserted into circuit power flow calculations to help narrow down the location of the fault. Detection of arcing fault signatures can also help determine the location of faults on a circuit if you know enough information on circuit impedances. Both of these techniques rely on very good knowledge of circuit impedances. Unfortunately, SCE does not have detailed circuit models for most of its circuits, so methods that rely on this circuit knowledge are difficult to implement. 
Time domain reflectometry can be used to send a signal down a circuit to determine how far the fault is from the instrument. This is hard to implement on a live electrical circuit. We were unable to find any equipment that could be installed on an operating circuit that would be able to find a faulted section of the circuit.

Another approach investigated was to use synchronized sampling for fault detection and location in distribution systems. In the past two decades use of time synchronized phase angles has been limited to model validation and dynamic monitoring applications in transmission systems. These limitations have been due mostly to the high cost of the phasor measurement units (PMUs), the need for fast communication channels, and the requirement for high precision signal transducers. The recent increase in the computational power in IED's used for distribution applications combined with the increased availability of communication channels and the use of the new generation of fiber optic sensors have made synchronized sampling a more cost effective option for the detection, location, and analysis of faults in distribution systems.

Several algorithms have been proposed in the literature for fault detection and location in transmission systems with synchronized measurements. Most of these methods can be applied to distribution systems; but the real value of synchronized sampling resides in the added information of phase angle difference. In distribution protection, phase angle difference can be applied not only to the detection and location of normal faults but can also be used to develop new algorithms for the detection and location of incipient and high impedance faults (HIFs). As an example of the added information, Figure 6-1 shows a simulation of a HIF in a radial distribution system protected with a re-closer and three sectionalizers. The figure also shows the magnitude and the time synchronized angles of the harmonic phasors computed by the three sectionalizers referenced to the voltage phasor angle. In this example, it can be seen that this specific HIF produces similar angles in the odd harmonic phasors on the buses located before the fault but not on the buses located after the fault. This simulation suggests that proper characterization of the harmonic phase angles can be used to locate HIF in distribution system.

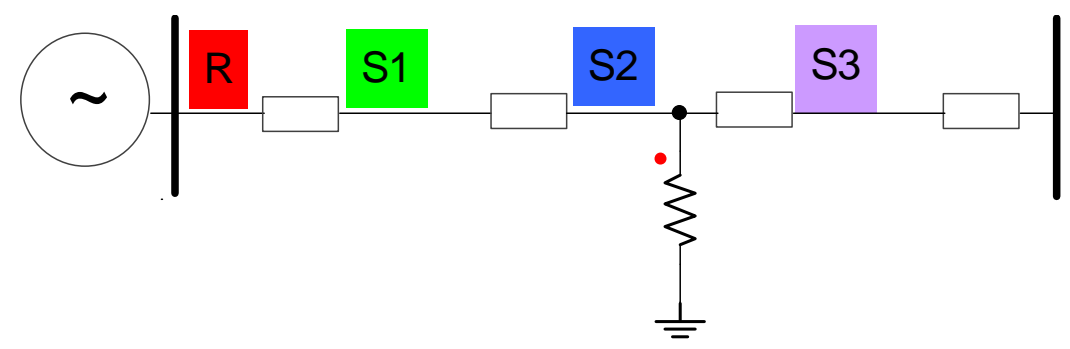




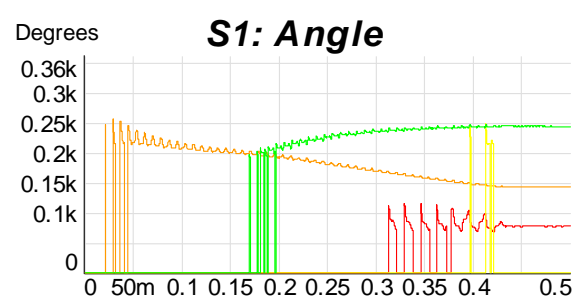

S2: Angle

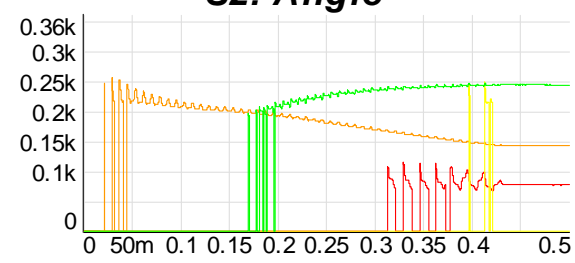

S3: Angle

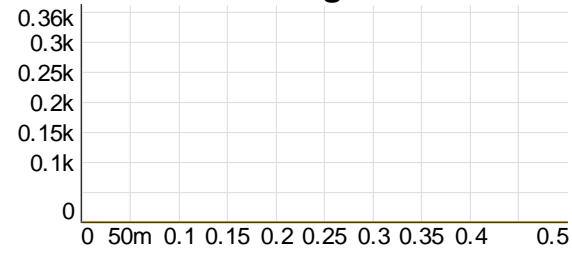

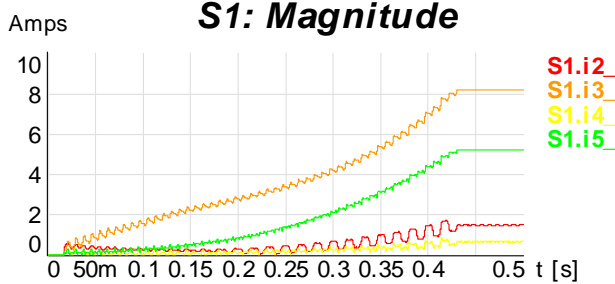

S2: Magnitude

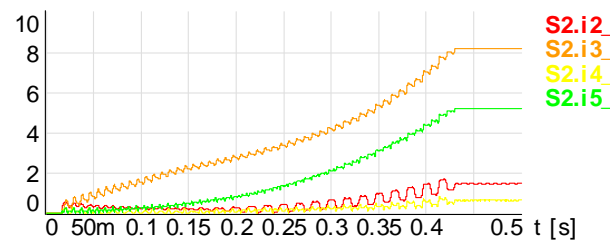

S3: Magnitude

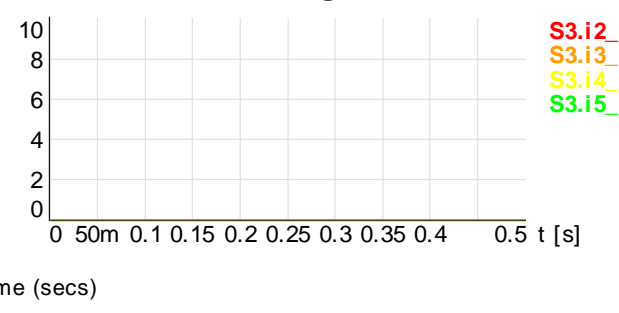

Figure 6-1 - Phasors of 2nd, 3rd, 4th, and 5th harmonics for a HIF between Sectionalizers S2 and S3

This line of study is still in its early stages of development, so SCE was not able to get a system that could be readily installed on the Circuit of the Future for testing during the period of the project.

\subsubsection{Use Line Sensors for Detection}

Various types of sensors can be attached to distribution lines to measure voltage, current, and other electrical parameters. These sensors can measure high-speed transients as well as other signals imposed on the wires (e.g. broadband over power lines (BPL)). Analysis of these signals or changes to these signals can give clues to electrical equipment that is not functioning properly or about to fail.

\subsubsection{Use BPL Coupling Electronics as Sensor}

As part of an internally funded project, SCE performed a small field demonstration and evaluation of broadband communications over power lines at distribution voltages. As part of this project, it was learned that the high voltage signal couplers looked at the noise level over the range of the BPL radio frequency signals. This noise signature was used to help with sending the broadband data. SCE was interested in also using this data as an indication of problems with the distribution equipment such as lightning arrestors, insulators, underground cables, and cable connection elbows. Discussions were held with the vendor of the broadband over power line equipment to see if this data could be made available to this project. While this data was promised, it was never delivered. Because of problems in getting this data, this concept was not investigated further. 


\subsubsection{Other Sensors Mounted on or near Distribution Lines}

While writing this project proposal, one concept was to see if sensors could be devised that would be able to be placed near distribution lines to detect electrical or magnetic field information. This information could be used to detect potential equipment that was beginning to fail. Review of potential products and research work underway did not find any sensors that could be implemented on the Circuit of the Future during the term of the project. Because of this issue, this concept was dropped from the project.

\subsubsection{High-Frequency Noise Analysis from Sensors}

Installation of high-frequency signal sensors on distribution lines can record signals that are different than the normal 60 cycle power. These signals include harmonics and high frequency transients. This concept is very similar to work being done by EPRI under another DOE project. Instead of pursuing this concept, SCE instead participated in project reviews held by EPRI of their project and also presented SCE's project work at these meetings.

\subsubsection{Signature Analysis Based on Theory and Tests of Known Bad Equipment}

This concept would put known bad distribution equipment in a lab and measure the signatures of known bad equipment. These signatures could then be "listened" for on actual distribution systems. It was decided that SCE would not pursue this line of study since it was also similar to the work being done by the EPRI project.

\subsection{Other Measures Investigated and not Implemented as Part of the Project}

As the project progressed, a number of advanced protection techniques were brainstormed through meetings and conversations within SCE and with KEMA and Virginia Tech representatives. The result was a list of advanced protection issues and techniques that seemed worth investigating further. The following section describes these issues and techniques and discusses why they were not pursued by the project. Section 6.3 describes the issues and techniques that actually were implemented as part of the project.

\subsubsection{Simultaneous Triggering of all Relays to Record Events}

Any relay that triggers on a fault will record a short fault record. This concept would have all relays trigger fault records (high speed recordings of the currents and status) simultaneously when a fault was observed by one of the relays. These fault records could be used to better pinpoint the fault location based on the waveforms observed. To make this work properly, all relays would have to be time synchronized using GPS clocks. In addition, fast communication would have to be done between all relays to properly trigger the fault records. One way this could be done is through the use of GOOSE messages over the fiber connections to each relay. 
Some investigation of potential algorithms to use this synchronized data was done. It appeared that the best method would be to plug the fault record data into a model of the circuit. Then the fault location could be pinpointed based on a best fit between the measured data and the circuit model. While the relays on the Circuit of the Future were time synchronized to a single GPS clock, the fiber communications system did not support transmission of GOOSE messages as it stood. We would have had to modify the fiber communications to get this scheme to work properly. In addition, there was security concerns (NERC CIP) expressed about the data channel back to the substation and to the desktop. Because of these issues, this technique was not further pursued.

\subsubsection{Record Relay Pickups}

Based on the work done by EPRI as part of their fault signature library, we looked at what information could be recorded from the existing relays on the circuit without installation of special high-speed recorders. One technique that was discussed was to look for relay "pick-ups" that did not result in actual tripping of the circuit. It was thought that if there were a number of these occurrences, it would be an indication that some circuit element was about to fail or a tree branch was rubbing against an overhead line. If the location on the line causing these "pick-ups" could be identified based on which relays were triggered, the problem might be fixable before an actual, sustained fault occurred.

Some history data was downloaded from several of the Circuit of the Future relays. While we did observe some pick-ups without tripping, there did not appear to be any discernable pattern. We also ran into the problem of routinely retrieving this data from the relays. This is the same problem identified in the previous discussion relating to security and NERC CIP rules. Because of these issues, this technique was not further pursued.

\subsubsection{Record Daily Waveforms}

Another technique that stemmed from the EPRI fault signature work was the concept of taking a waveform capture of each phase voltage and current at the same time every day. Analysis of these waveforms would be conducted to look for changes in the waveforms that might indicate an incipient problem on the circuit. These waveforms would be captured by triggering a fault record recording in each relay at the same time every day.

After review of the Schweitzer relays installed on the circuit it was determined that it would not be possible to do this routine triggering of each relay to make the fault record recording. We also investigated the same concept using an external power quality meter. While the power quality meter concept seemed to be doable, there was no way to routinely download this data from the meters without going to each instrument on a routine basis. This concept was not further pursued because of the difficulty in collecting the data. 


\subsubsection{Investigate and Test A4 Relay Problem}

One problem commonly encountered on distribution circuit protection has been neutral current getting so high (due to phase imbalance and/or third harmonic) that operators are forced to block (disable) the A4 (neutral overcurrent protection) relay to prevent spurious tripping of distribution circuits. This problem can be mitigated to some extent by rebalancing phase loading and changing the A4 relay settings in IED's to only trip on the fundamental component. It is not too difficult to re-balancing phase loading in overhead distribution line areas, but underground circuits are more difficult. Upwards of 100 circuits still have the A4 relay blocked for some portion of the year. Difficulties included the tendency of phase loading to shift during the year, lack of phase identification in the field, the difficulty of changing phase connection for underground circuit segments, the need to change the HMI when the IED was changed, and the inability to do anything about third harmonics in substations with electro-mechanical relays. This latter condition is common on $4 \mathrm{kV}$ circuits. One idea discussed in the past was a variable set point for the A4 relay. A variable set point would have a floor and ceiling value (say 180 and 360 amps for a $12 \mathrm{kV}$ circuit), but in between would self adjust to some value (say $10 \mathrm{amps}$ ) above the measured neutral current value. This self adjusting upward would be slow, and downward fast. In this way, any phase to ground fault that increased neutral current suddenly more than 10 amps would result in a trip. This feature could allow SCE to unblock more relays and avoid blocking others. Obviously, doing it with IEDs would be easier than with electro-mechanical relays. Such a feature might also prove useful by allowing a more sensitive setting for circuits in which a distant high impedance phase to ground fault results in low fault duty.

Discussions with a number of SCE's protection engineers came down to whether there were temporary system conditions where a variable set point ground relay could be fooled. This case might cause the unbalance tracking feature to reduce the minimum trip value, only to have the relay trip falsely when conditions returned to normal. Circuit parallels or temporary circuit rearrangements seem like they might lead to this problem by temporarily reducing the circuit unbalance. An operator control to disable the tracking feature might be a way to avoid this problem for planned conditions, but spontaneous changes like a broken conductor, blown branch-line fuse, or an AR dropping a portion of the line's load could cause the variable set point relay to track downward without giving an operator time to intervene. A better solution might be to use some form of high-impedance arcing fault detection that would allow permanently higher settings on conventional relays. Because of this ongoing discussion as to the applicability of variable set point ground protection relaying within SCE, this concept was not pursued as part of this project.

\subsection{Measures Actually Implemented as Part of Project}

After analysis of the originally proposed concepts and other concepts advanced as the project went forward, tasks were determined for the third phase of the project. It was decided that we would try and get as much data from the fault current limiter installation as well as plan for the next generation distribution circuit protection system. The other 
factor that influenced the decisions for the third phase of the project was the acceptance by DOE of the SCE proposal for the ARRA/Irvine Smart Grid Demonstration project. This project would demonstrate many of the features that are planned for the Smart Grid over the next 5 to 10 years. One of the features to be implemented as part of this project is a new protection system for distribution circuits. This concept is based on the protection work done for the Circuit of the Future as part of Phase I of this project. In the Irvine Smart Grid Demonstration, two radial circuits would be looped together and 4 fault interrupting switches would be inserted. These switches would be connected together through the use of high-speed radio communications. Further discussion on the concept is given in section 6.3 .3 of this report.

\subsubsection{Measure Power Quality Upstream and Downstream of the FCL}

To help verify the operation of the fault current limiter, power quality meters were installed both upstream and downstream of the fault current limiter. These devices were able to look at fault and power quality events to see the effects of the fault current limiter on the circuit. These instruments would also be able to help investigate unusual behaviors caused by the fault current limiter on the distribution circuit including harmonic distortions. The upstream power quality meter was installed in the relay house in the substation at the head of the Circuit of the Future using the potential and current transformers connected to the circuit protective relay. The downstream power quality meter was installed in base of the fault current limiter using potential transformers from the fault current limiter and current transformers installed for this purpose. Both meters were Dranetz PX5's. They were set to capture any voltage or current events that occurred. Waveforms were also captured on a regular basis. The meters were downloaded manually when someone was in the area.

\subsubsection{De-centralized Sensing, Control and Protection and Automation Functions}

As the complexity of systems continue to grow, it is important to allow some of the monitoring data to be consolidated and control decisions to be made at lower levels. On the Circuit of the Future, the high-speed protection decisions were made by the SEL 2100 Logic Processor based on information it obtained from the circuit Remote Controlled Interrupters. These devices send indications of a fault to the Logic Processor and decisions are made to open Interrupters. Once this action is completed, status information is made available to the substation where it is sent to be displayed for operators. This same type of decentralized protection is also being implemented for the Irvine Smart Grid Demonstration project. In the case of the Irvine project, the circuit relays communicate with each other and then independently decide if they need to trip. Status information is then communicated to the substation for display to operators.

\subsubsection{Design and Model System of the Future (SoF) Relay Protection Scheme.}

The advanced protection system installed on the Circuit of the Future was able to reduce the number of customers interrupted when a fault occurred on the circuit. While this project showed that only a section of the circuit would be interrupted, it was costly and 
still required actions by a system operator to restore customers located past the faulted section of the circuit. To address the cost issue, the fiber optic communications portion of the system would need to be replaced with a wireless radio system. This radio system would need to have low latency ( $\sim 100 \mathrm{~ms})$, but would not necessarily require high bandwidth. This is because the messages communicated between switches are short. In a move towards standards, the message sent over the wireless radio system would be using IP and would conform to IEC61850 GOOSE standards. To fix the issue with operator intervention to restore customers past the fault, two distribution circuits would be looped together. With the circuits looped, the faulted section of the loop could be isolated while all other customers would not experience an outage.

In addition to the design of the new advanced protection system, models are being developed to test the system performance under various loads and with distributed generation devices. These models were developed through the use of PSCAD. As part of the DOE-sponsored Irvine Smart Grid Demonstration project, it is planned to take the PSCAD models and move them to a Real-Time Digital Simulator (RTDS) to run the simulations. These simulations will verify operations before the system is installed in the field. The field installation is planned for late 2011 or early 2012.

\subsubsection{Design of Protection System}

The protection scheme to be used on the System of the Future is based on the work done on the Circuit of the Future. In the Circuit of the Future, fault interrupting switches were connected by fiber optic communications cables so that the location of the fault could be identified by the Logic Processor and the proper switch opened. Since this circuit was a radial design, all customers beyond the opened fault switch would lose power until the tie switch to an adjacent circuit could be closed by a system operator. The design of the System of the Future would use two circuits looped together (Figure 0-5) so that when the faulted section of the circuit was isolated through the opening of two fault interrupting switches, only the customers between the switches would lose power. In addition, the communications technology for the System of the Future is planned to be wireless radio to replace the fiber used in the Circuit of the Future. This change was made due to the high cost of fiber installation, especially on underground circuits. The protection method would also be able to more easily integrate distributed generation since it can account for bi-directional current flow. 


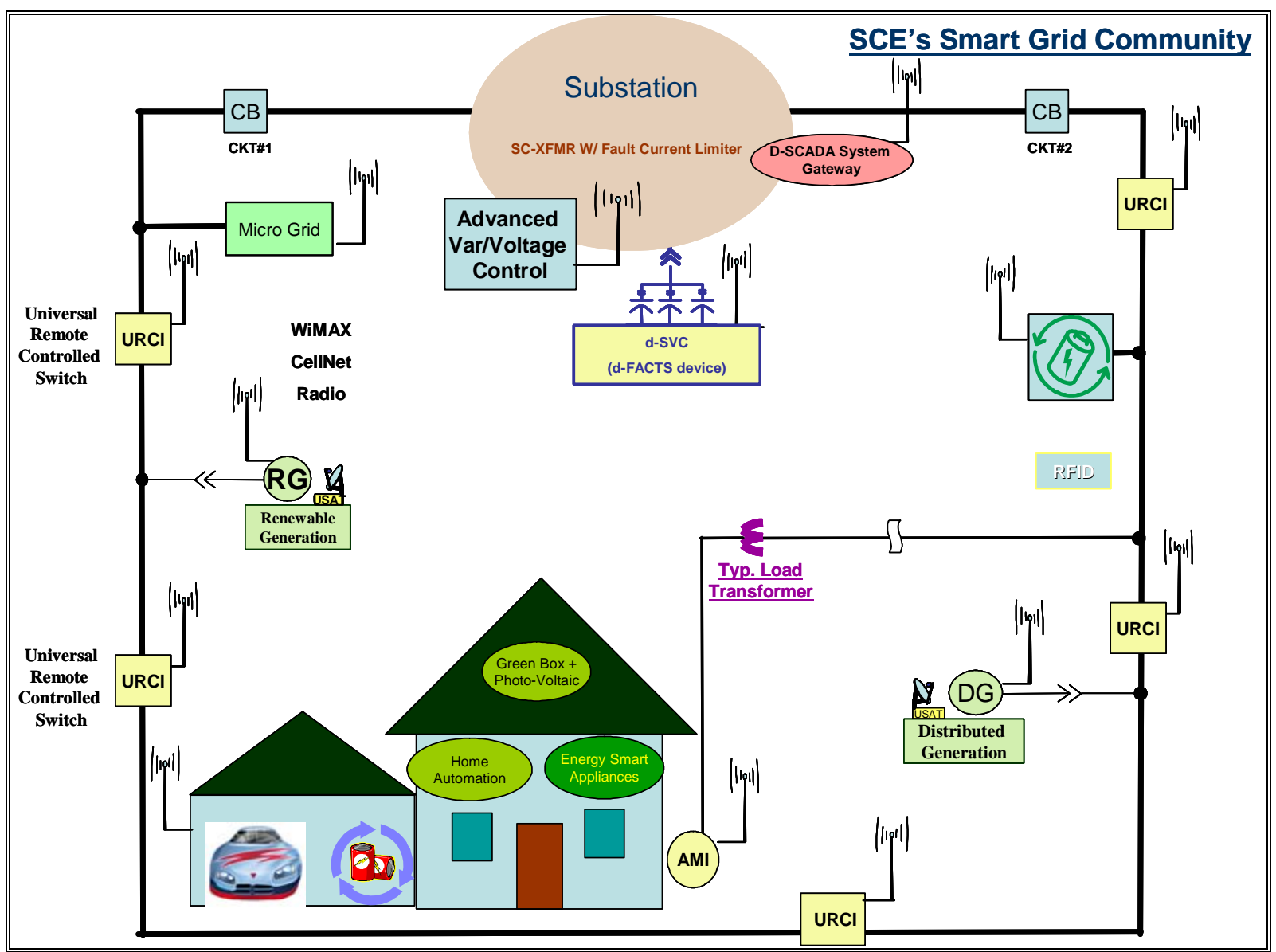

Figure 6-2 - SCE's System of the Future (Irvine Smart Grid Demonstration)

\section{Protection Logic (overall system and in relays)}

The goal of the protection system on the System of the Future is to make the configuration and maintenance of the switch logic as simple as possible. With this in mind, the logic that would reside in all protective relays at the switches is planned to be the same. When configuring the relay, the only thing that would need to be changed would be the information that identified which relay is upstream and downstream from it. While the Circuit of the Future used a central logic processor for the fault location and isolation process, the System of the Future logic is designed to be contained in the relays themselves. The basic concept of the protection system is to use communications to block the operation of fault isolation interrupters that are upstream of the fault so that only the interrupters on either side of the fault operate.

\section{Timing}

Since the main goal of this distribution protection system is to only interrupt the faulted section of the circuit, the protection communications and control needs to operate faster than the substation breaker operates today (500 to $600 \mathrm{~ms}$ ). With this timeline in mind, the protection time line was worked out (see Figure 6-3). 


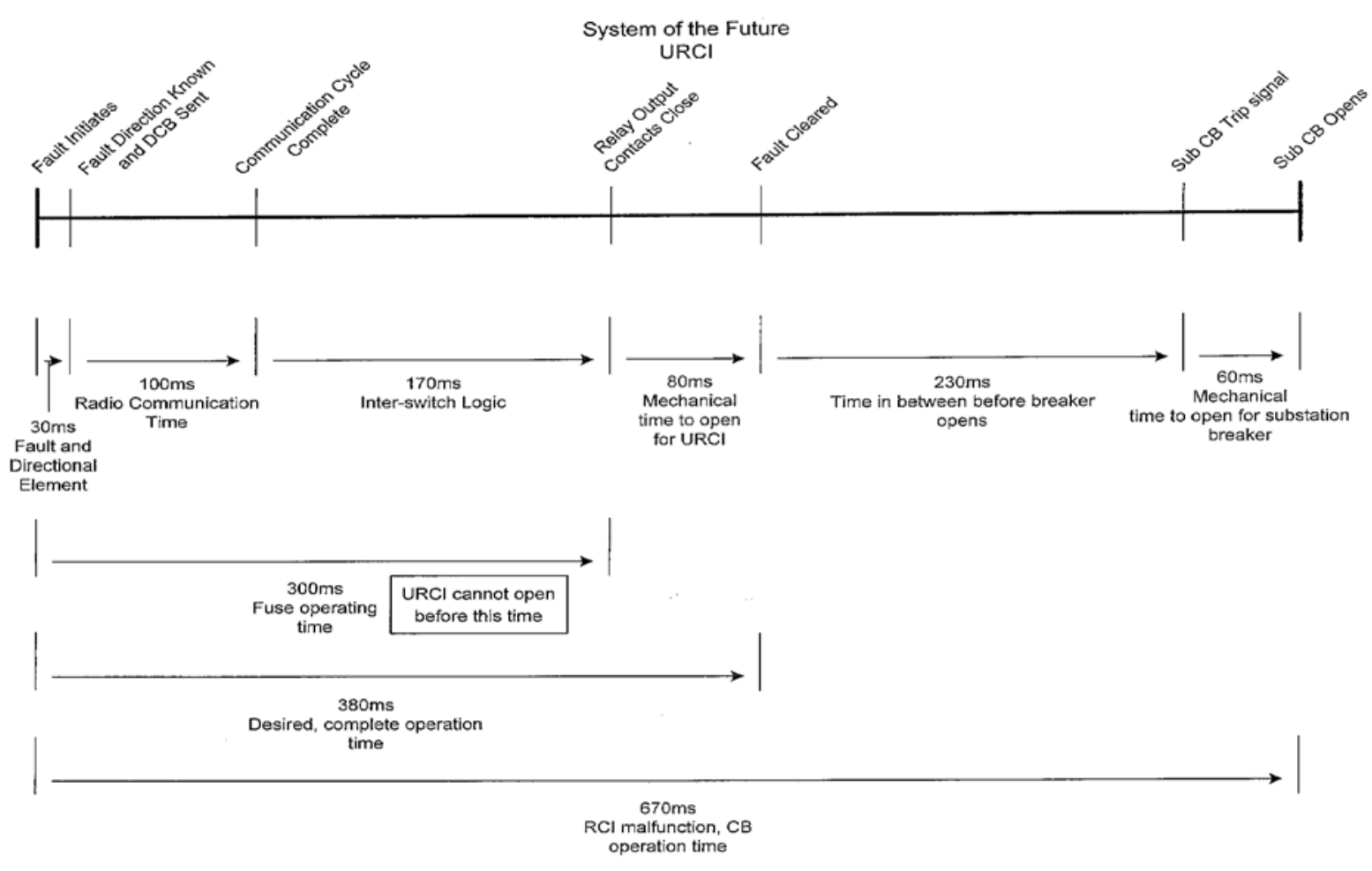

Figure 6-3 - URCI Timing Diagram

Approximately $30 \mathrm{~ms}$ is needed for the fault to be detected and the directional element to determine the direction of the fault (this time can be longer, based on the time/overcurrent curve being used). An additional $100 \mathrm{~ms}$ is allowed for the radio communications to send the blocking signals between the Universal Remote Controlled Interrupter (URCI) relays. Another $170 \mathrm{~ms}$ is allowed for communications retries and execution of the logic in each URCI relay. These times add up to $300 \mathrm{~ms}$ which is the time allowed for circuit branch line fuses to operate. The logic does not want to open URCIs if the fault can be cleared by a branch line fuse. Once this logic is completed, a signal would be sent to the vacuum switch at the relays that need to be opened. It takes about $80 \mathrm{~ms}$ for the switch to actually open (mechanics actuation time). If all operates correctly, the fault would be cleared from the system in about $380 \mathrm{~ms}$.

If for some reason, the URCI protection scheme does not operate properly, another 230 $\mathrm{ms}$ is allowed before the substation circuit breakers are signaled to open and interrupt the fault. Another $60 \mathrm{~ms}$ is allowed after the circuit breaker gets the signal to operate before the fault clears (breaker mechanical operating time). This would clear the fault in $670 \mathrm{~ms}$ in the case of a failure in the URCI protection scheme.

\section{Logic in relay}

The logic in each relay at each URCI is the same. The polarity of the connections of the current transformers at the URCI installation determines which direction is the "forward" and "reverse" for that device. The messages sent from each URCI are IEC 61850 
GOOSE messages containing trip or blocking instructions. The designation of which URCI is upstream or downstream of each URCI needs to be decided when the circuit is designed and this information is programmed into the protective relays. This is done by having a protective relay "subscribe" to messages from a neighboring protective relay. The detailed relay logic is described in the diagram below (Figure 6-4).

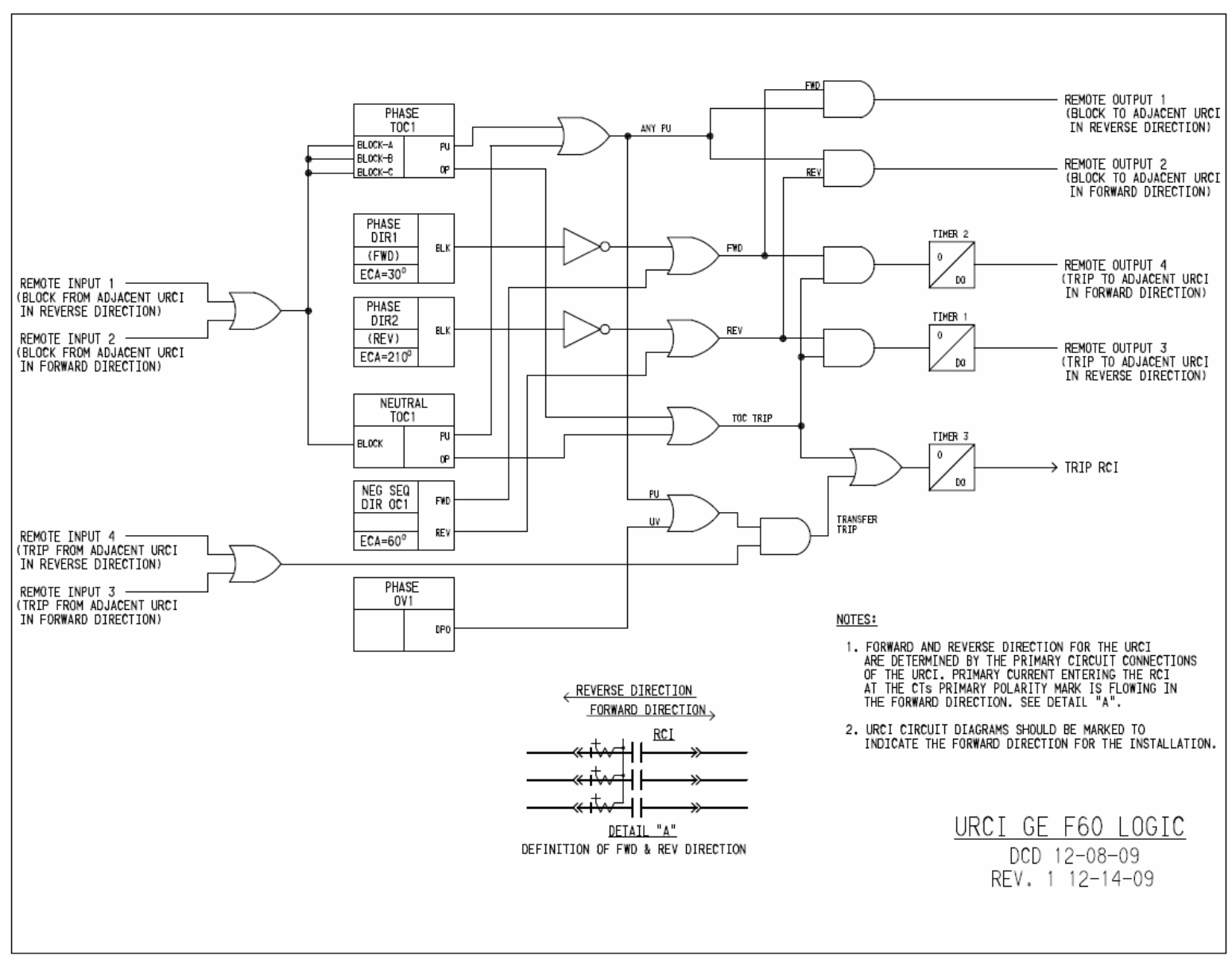

Figure 6-4 - URCI Logic Diagram

The protective relay uses internal logic to determine when a fault occurs and which direction it sees the fault current flowing. The relay senses both phase and neutral timeovercurrent. It also senses the direction of the fault current separately. Another "forward" or "reverse" signal is generated by the Negative Sequence Directional Overcurrent element which is combined with the directional elements to finally determine the direction of the fault current. These outputs are combined to determine whether the blocking signal (GOOSE message) is sent "upstream" or "downstream". When the time-overcurrent element of either the phase or ground relay times out, an operate signal is sent to the vacuum switch in the URCI. This output signal will initiate operation of the breaker unless the phase overvoltage element drops out (voltage is lost) meaning the circuit has lost voltage. A timer element is added to this signal line to be 
sure that the signal is applied to the output of the protective relay for long enough to allow the breaker to operate.

When a blocking signal is received by the relay logic, it stops the phase and neutral overcurrent elements from issuing an "operate" command. This blocking will be in place as long as a blocking signal is received from an adjacent relay. The blocking signal would be received from a GOOSE message coming from an adjacent relay.

Because of different impedances for each of the two ways the current can flow around the circuit loop, the amount current feeding the fault will be different for each direction. The direction with the higher current will trip its protective relay more quickly. To insure that the other relay trips quickly and speeds fault isolation, a signal is sent from the relay that trips first to the second one. This is also a GOOSE message and is held "high" by a timer element to allow enough time for the second relay breaker to trip.

\section{Wireless Radio Communications System}

Part of what helps make this protection system more cost effective is the use of wireless communications in place of fiber optic cables. Since the communications system will be using IEC 61850 GOOSE messages, it needs to be able to handle IP messaging. In addition, the messaging needs to be fast enough to be sure that the GOOSE messages are sent and received in $100 \mathrm{~ms}$ or less. Since GOOSE messages are fairly short, this does not need to be a broadband communications system, but it does need to have low latency. A radio system also needs to have sufficient coverage so that not too many relay radios are required. Relay radios will add latency and possibly make it difficult to meet the timing requirements. The system that is planned to be used in the Irvine Smart Grid Demonstration project operates in the $902-928 \mathrm{MHz}$ unlicensed spread spectrum band. It is an IP system so will handle the messaging properly. As this report is being written, tests are being conducted to verify the coverage in the test area.

\subsubsection{Modeling of the Protection System}

To help work out the relay logic and test protection scenarios, a PSCAD model was developed as part of this project for the two circuits that will be looped together. This model takes into account all major switches, load blocks, and voltage control capacitors. The model, developed by Virginia Tech, will be augmented to include protection elements later this year as part of the Irvine Smart Grid Demonstration project. SCE also has a Real-Time Digital Simulator (RTDS) that allows the connection of real relays to the simulation to determine if they will function properly. This test is planned for next year. The advantage of the RTDS is "real time" simulations that allow the actual relay logic to be tested in many simulated scenarios on the circuit in a very short amount of time.

\subsubsection{Recommended System for Implementation}

Based on radio latency and coverage studies, protection logic simulations, and field circuit constraints, the final circuit protection system is being derived. While this project was not able to complete this work, it is being picked up by the SCE Irvine Smart Grid 
Demonstration project (DOE ARRA Smart Grid Demonstration project) and is planned for installation on the circuit in 2012. 


\section{Section 7: Evaluation of Results of Pilot Tests}

In this section, an analysis of all significant circuit events is discussed. These events include faults on the circuit as well as events related to the fault current limiter.

\subsection{Field Experience on CoF without FCL}

There were four faults recorded during the time the advanced protection system on the circuit was in operation without the fault current limiter. These occurred on: 10/21/2007, $12 / 25 / 2007,11 / 14 / 2008$, and $1 / 30 / 2009$. All of these faults were caused by high winds blowing debris into overhead sections of line and conductors slapping together between RCI-2 and RCI-3 (Figure 7-1).

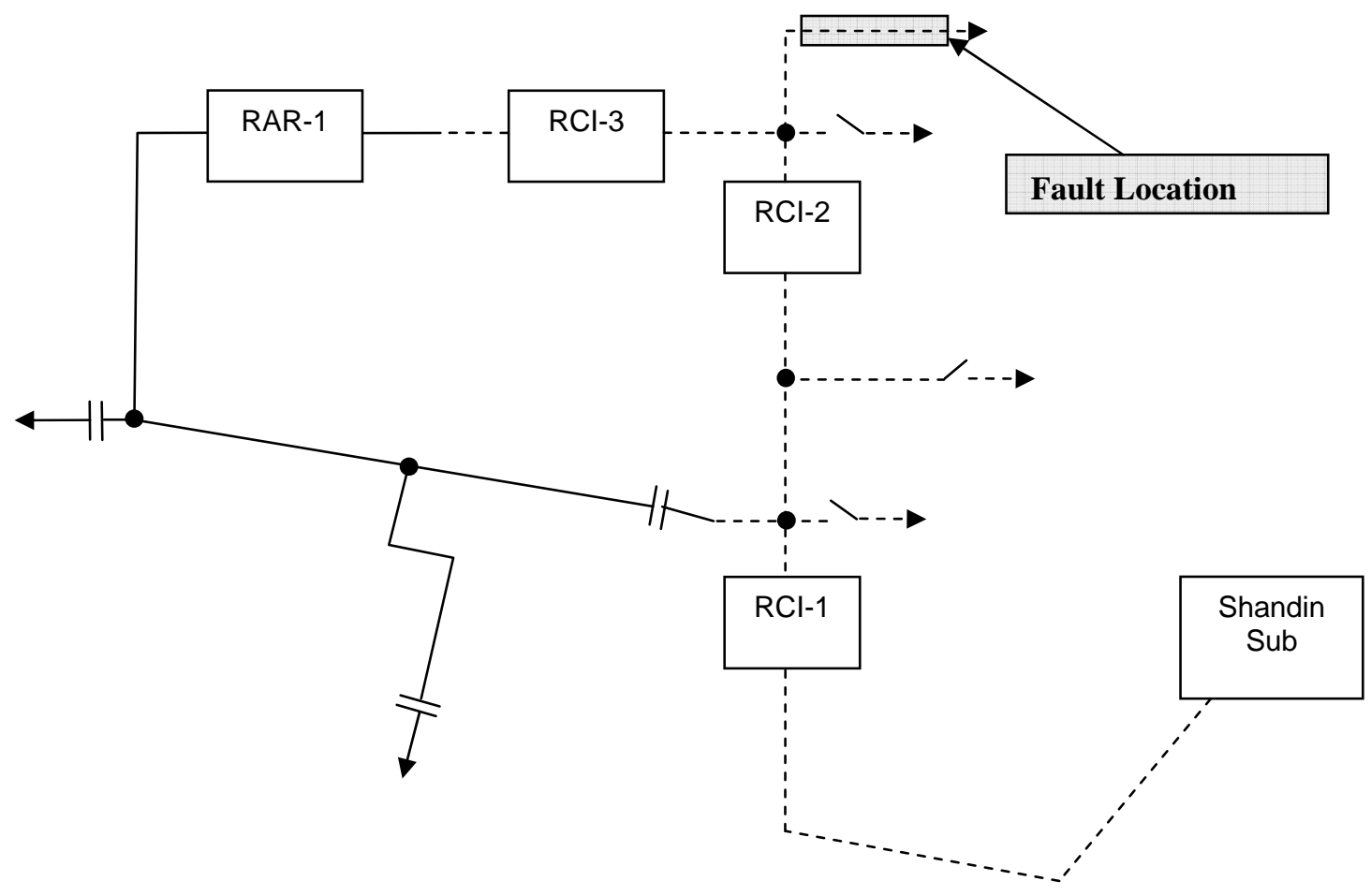

Figure 7-1 - Circuit Diagram Showing Fault Location

The response to a fault on this circuit before the advanced protection system was put in place was for the substation breaker to trip. This would interrupt all customers on the circuit. With the advanced protection system in place, RCI-2 and RCI-1 would report seeing fault current. The logic processor would take this information and select RCI- 2 to open and interrupt the fault. Shortly thereafter, the logic processor would instruct RCI-3 
to open and prepare the back portion of the circuit to be paralleled with an adjoining circuit. The operator would then either remotely operate a tie switch or send a field operator to the site to operate a tie switch. This would energize the portion of the circuit beyond RCI-3. In the end, the only customers out of power were those between RCI-2 and RCI-3.

\subsubsection{Fault Event - October 21, 2007}

This fault event was discovered after examination of the fault records from the circuit remote fault interrupters. From the records, it appears that there was a problem past RAR-1 (the last fault interrupting device on the circuit). At 8:56:11, a very short pickup was observed on RCI-1, but it did not stay long enough for it to trip (Figure 7-2). A few minutes later, all of the RCIs and the RAR observed a fault of about 1500 amps peak resulting in a trip of the RAR at the end of the circuit (Figure 7-3). This indicates that the fault was beyond RAR-1. The RAR then reclosed and another fault was observed at 9:11:08 ( 2500 amps peak) by all RCIs and the RAR. This also resulted in a trip (Figure 7-4). Since there is no record of an outage, the RAR must have reclosed and no more faults were observed. This behavior is common in this area where high winds can cause wires to blow together for a temporary fault. This is an example of an event that is an indication of circuit problems that can be investigated and steps taken to prevent sustained outages.

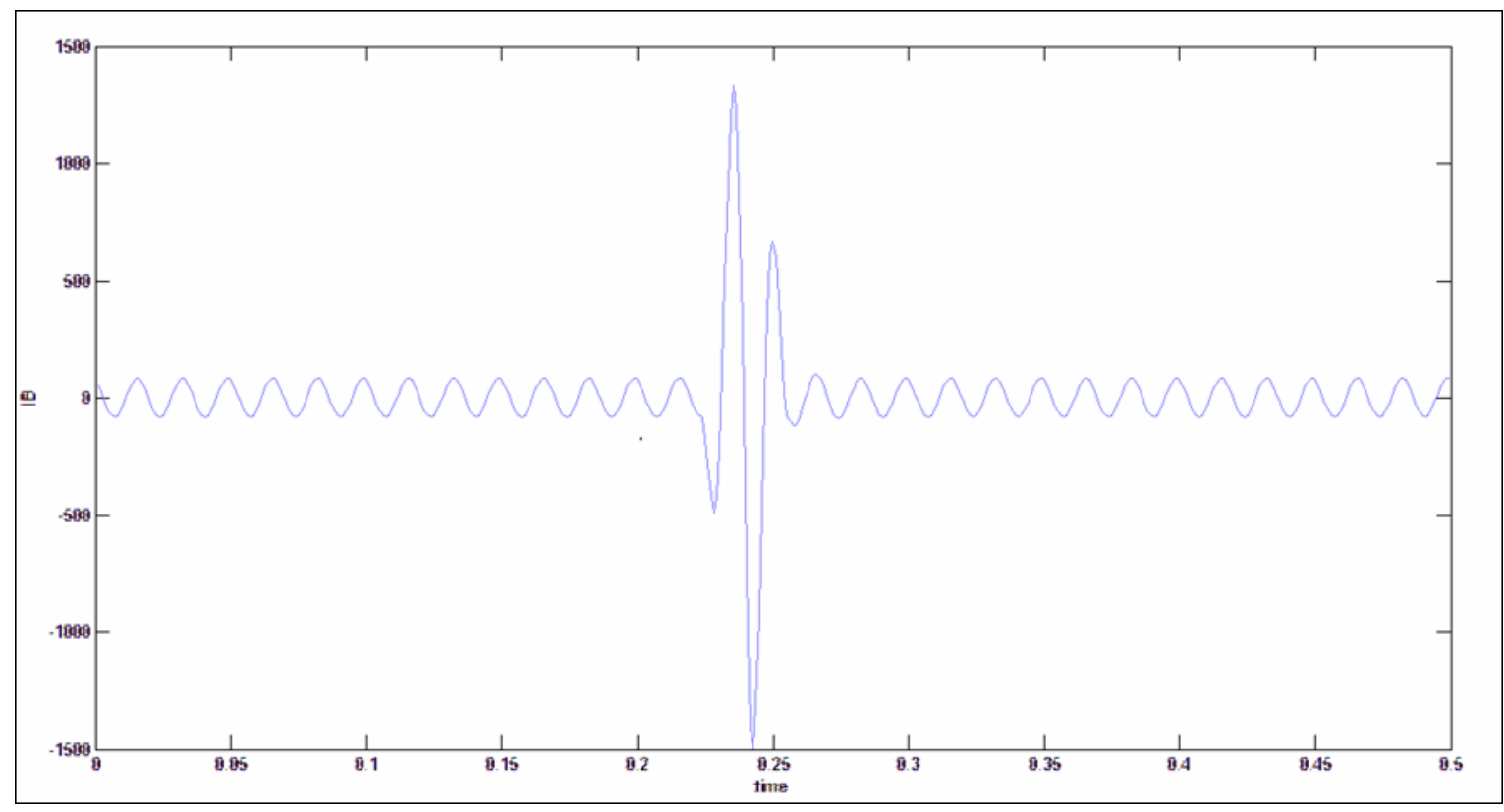

Figure 7-2 - Momentary Fault - RCI-1 - 10/21/2007 8:56:11.536 


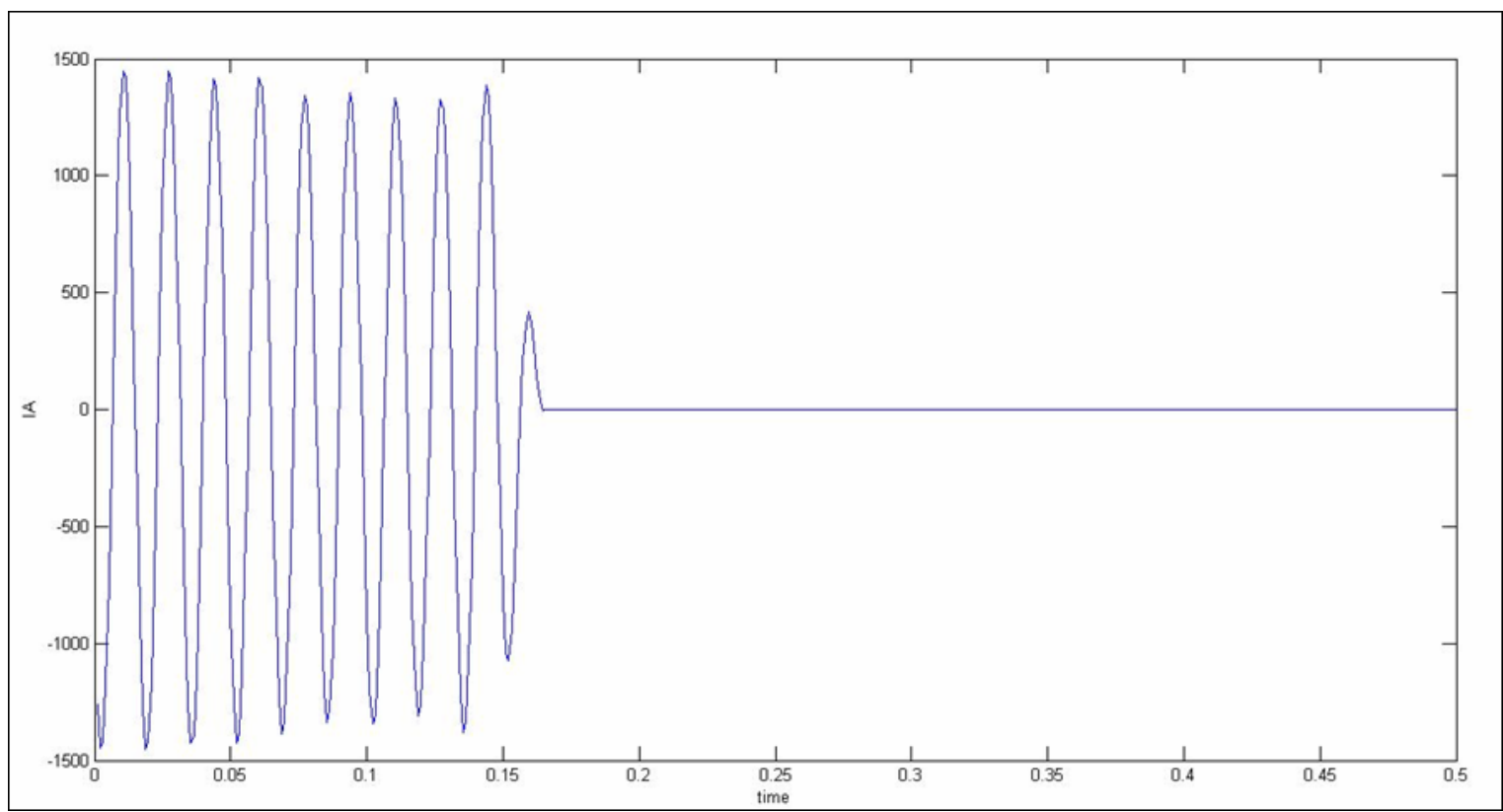

Figure 7-3 - Fault Trip - RAR-1 - 10/21/2007 9:10:58.690

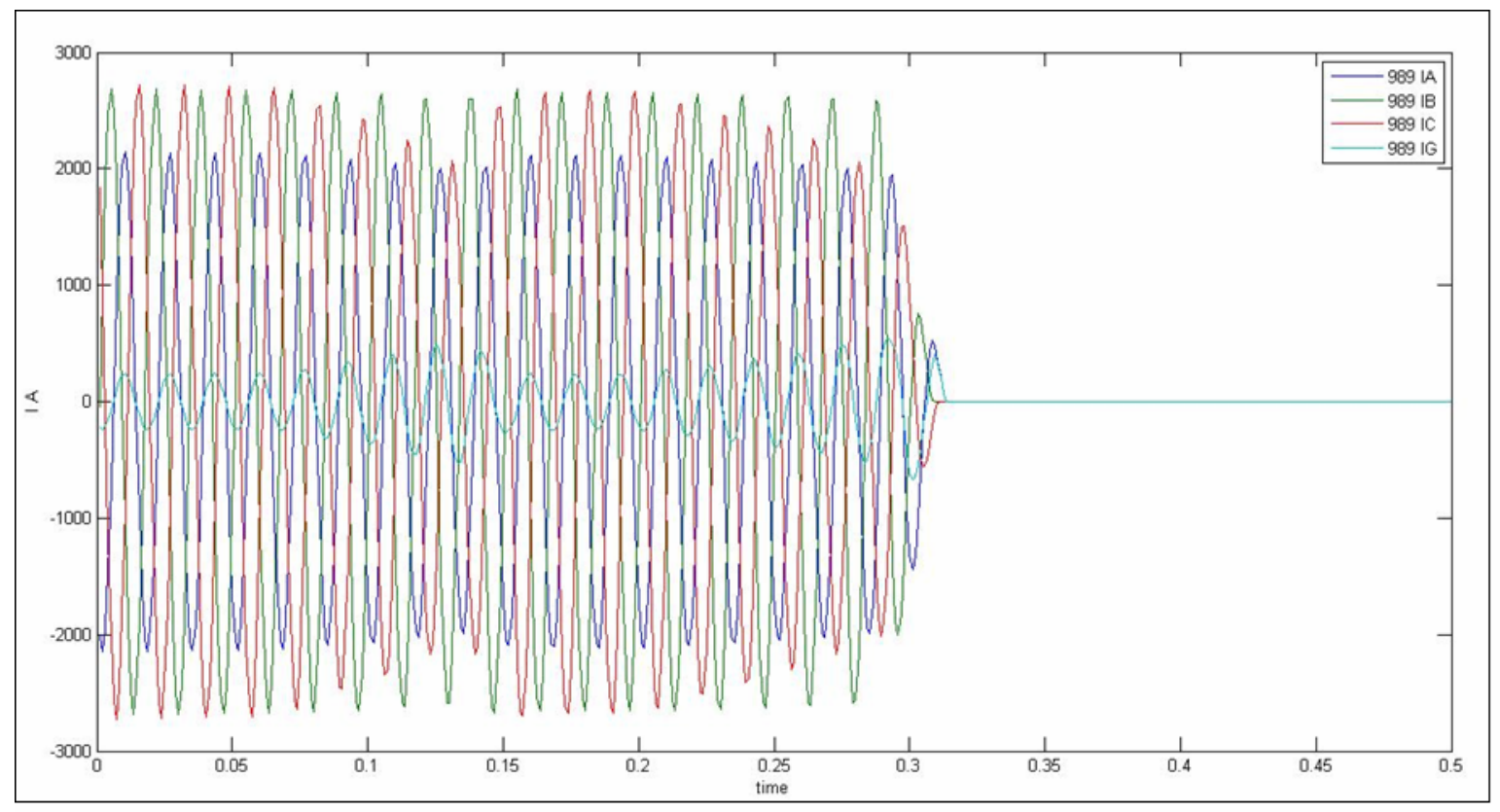

Figure 7-4 - Fault Trip - RAR-1 - 10/21/2007 9:11:09.296

Another event similar to this one occurred on 10/29/2007 which also resulted in a trip and reclose of RAR-1.

\subsubsection{Fault Event - December 25, 2007}

This fault occurred on an overhead section of line and was caused by high winds in the area blowing debris into the line at 5:32:43 with peak fault currents of 3500 amps (Figure 
7-5). It occurred on Christmas day and the protection scheme operated as expected and isolated the fault using RCI-2 (Figure 7-6). After the fault was cleared, the next switch down the circuit (RCI-3) was supposed to automatically open to prepare the end of the circuit to be connected to an adjacent circuit. This second switch did not open as expected. This problem was investigated and determined to be caused by the way voltage sensors were connected to one of the relays. Since there were two RCIs located the same vault, only one set of potential transformers was installed instead of two as originally specified in the design. Since the fault occurred on a spur between the switches, the second switch did not get the proper voltage signal. A second set of potential transformers was installed in early 2008 to prevent a recurrence of this problem. Also, because it was Christmas day, the operator on duty had not been trained in the operation of the circuit so did not know what to do to restore load. This caused the outage to be longer than necessary. This was corrected by additional training in the following months.

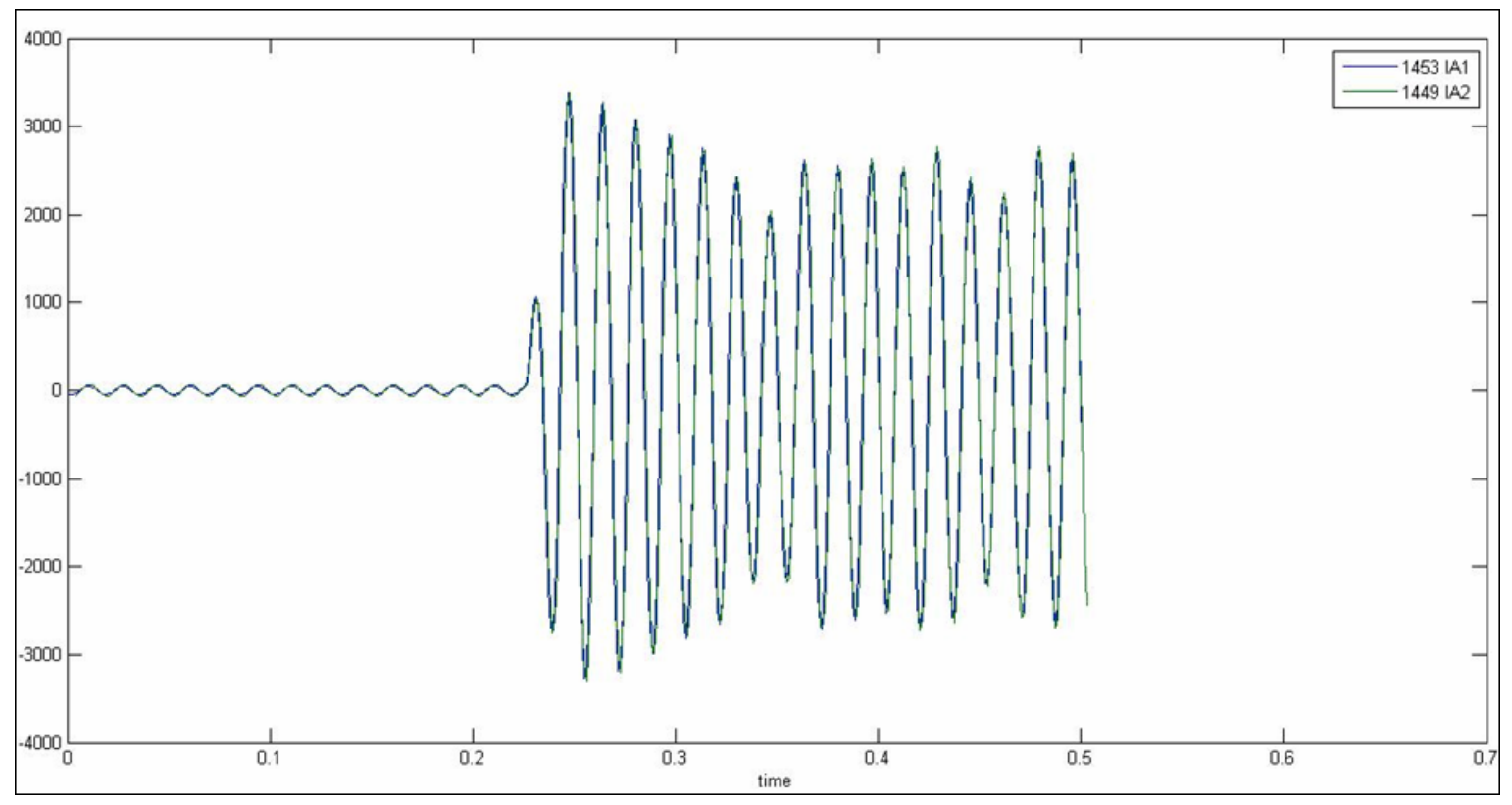

Figure 7-5 - Fault - RCI-1 and RCI-2 - 12/25/2007 5:32:43 


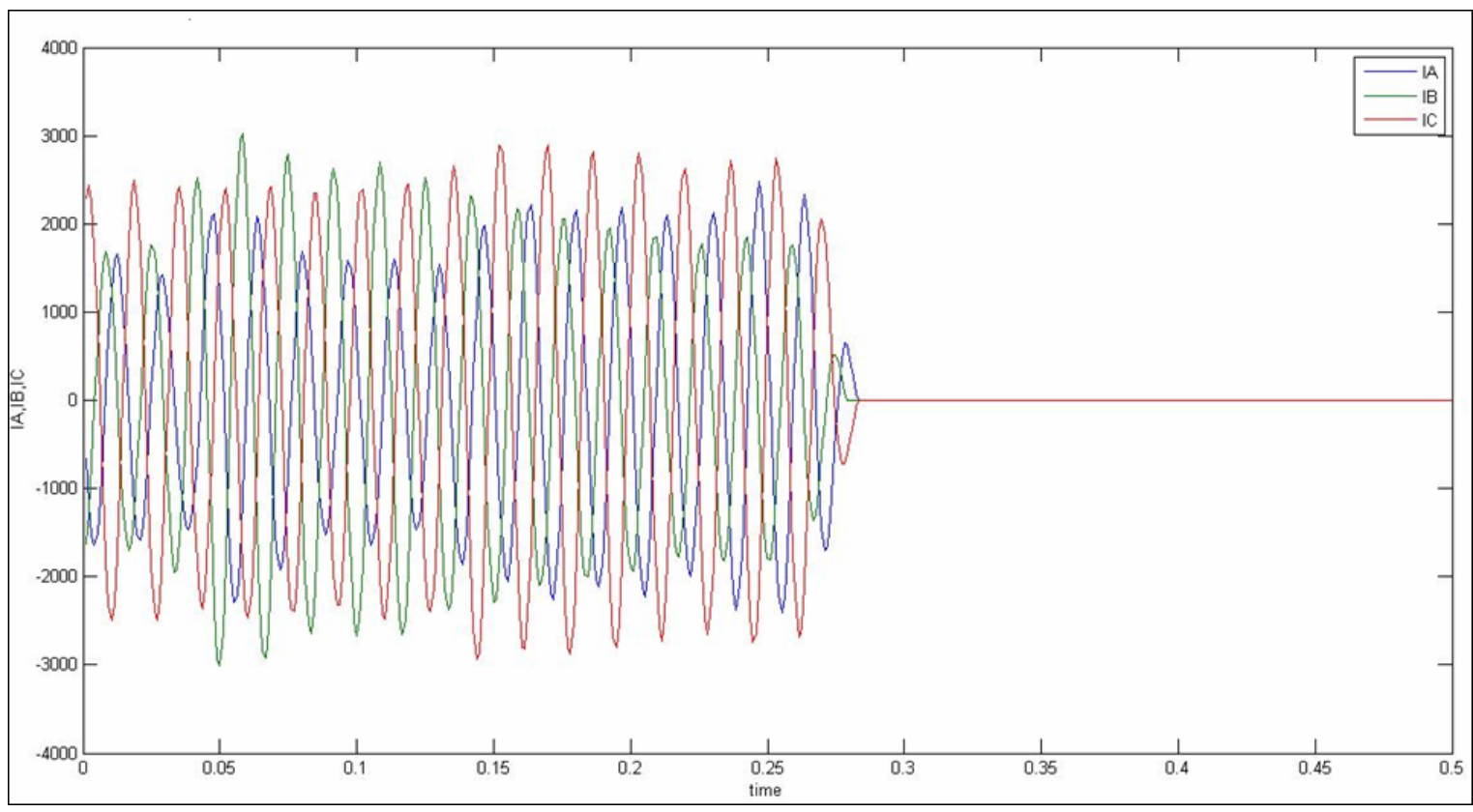

Figure 7-6 - Fault Trip - RCI-2 - 12/25/2007 5:32:43

\subsubsection{Fault Event - November 14, 2008}

This fault event also occurred because of high winds in the area causing debris to blow into the lines. There were numerous relay element pickups with a variety of different fault types (AB, BC, ABC, AG, and BG). The fault current was about 3200 amps for most of the faults (Figure 7-7). In this case the fault isolation worked properly and the downstream RCI-3 opened properly. The operators were able to restore power to most of the customers on the circuit within minutes.

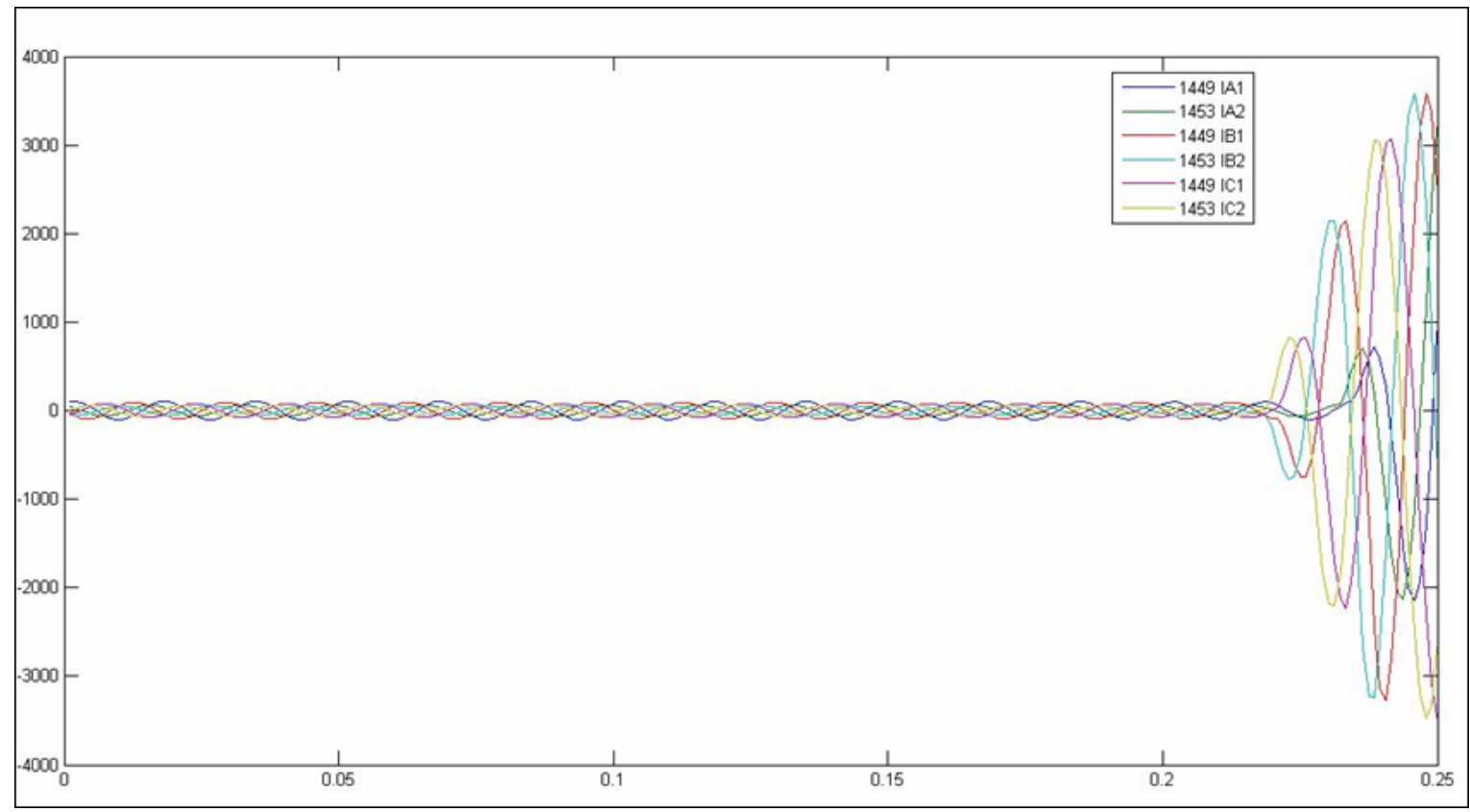

Figure 7-7 - Fault - RCI-1 and RCI-2 - 11/14/2008 8:13:34 


\subsubsection{Fault Event - January 30, 2009}

This fault also occurred on the same stretch of the circuit. In this case, wires blew together because of high winds. These faults caused the C-phase conductor to burn through and fall to the ground. Again the advanced protection scheme worked properly and the fault was isolated and most customers restored quickly.

\subsection{Field Experience of CoF with FCL}

The fault current limiter passed all tests by the end of 2008 and was moved to Shandin Substation for installation. This involved the connection of $12 \mathrm{kV}$ power cables from the bypass switch as well as routing auxiliary power for chilling the superconducting magnet and providing control power. The actual FCL was placed on a temporary foundation of railroad ties due to the temporary nature of the device installation.

As of this report's writing, only one fault has occurred on the circuit with the FCL in service (Figure 7-8). This fault occurred on January 14, 2010.

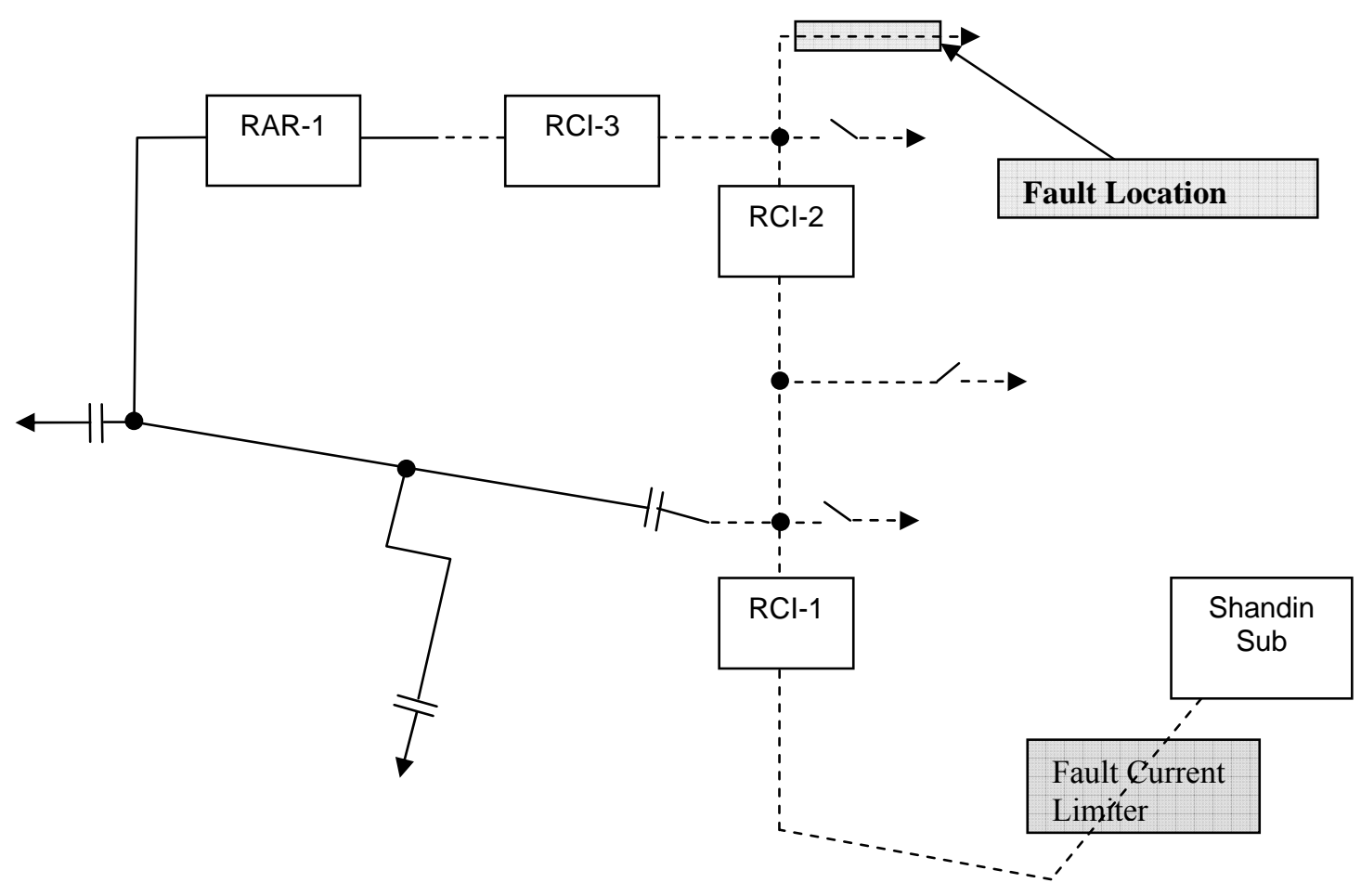

Figure 7-8 - Fault Current Limiter and Fault Location

\subsubsection{FCL Put into Service - March 3, 2009 with no Load and March 6, 2009 with Load}

The Zenergy FCL was initially connected to the circuit by closing the bypass switch on the circuit March 3, 2009 for testing with full voltage. The circuit loads had been transferred to surrounding circuits so that the FCL was isolated with minimal load. On 
March 6, 2009 at 13:30 PST, after verification of proper operation of the FCL, full circuit load was applied for the first time.

\subsubsection{FCL Malfunction Event - March 16, 2009}

At 10:16 am on March 16, the FCL controller reset due to a memory overflow condition. Since the FCL controller was programmed to disengage the DC source to the superconducting magnet upon resetting, the FCL impedance was inserted into the circuit. Over the next 4 minutes the superconducting magnet current dissipated and the magnetic field disappeared. This caused the FCL impedance to be fully inserted into the circuit. This caused a series resonant condition between the FCL and the shunt capacitors located on the circuit with the voltage rising to about $12.6 \mathrm{kV}$ (a rise of about 3\%). At this point, the $1800 \mathrm{kVAR}$ capacitor bank disconnected which interrupted the resonance condition and dropped the voltage to $11.7 \mathrm{kV}$ (about a $4 \%$ reduction from the initial conditions). This caused the $1200 \mathrm{kVAR}$ capacitor bank on the circuit to switch on due to low voltage. This caused the resonance condition to reappear with the voltage rising to 12.4 $\mathrm{kV}$ (about $2 \%$ above the initial conditions on the circuit). The circuit stayed in this resonant condition for approximately 86 minutes until the FCL was manually bypassed by a substation operator. The figure below shows the voltages over the entire event (Figure 7-9). The voltages never reached magnitudes that would cause customer problems, but this problem needed to be resolved.

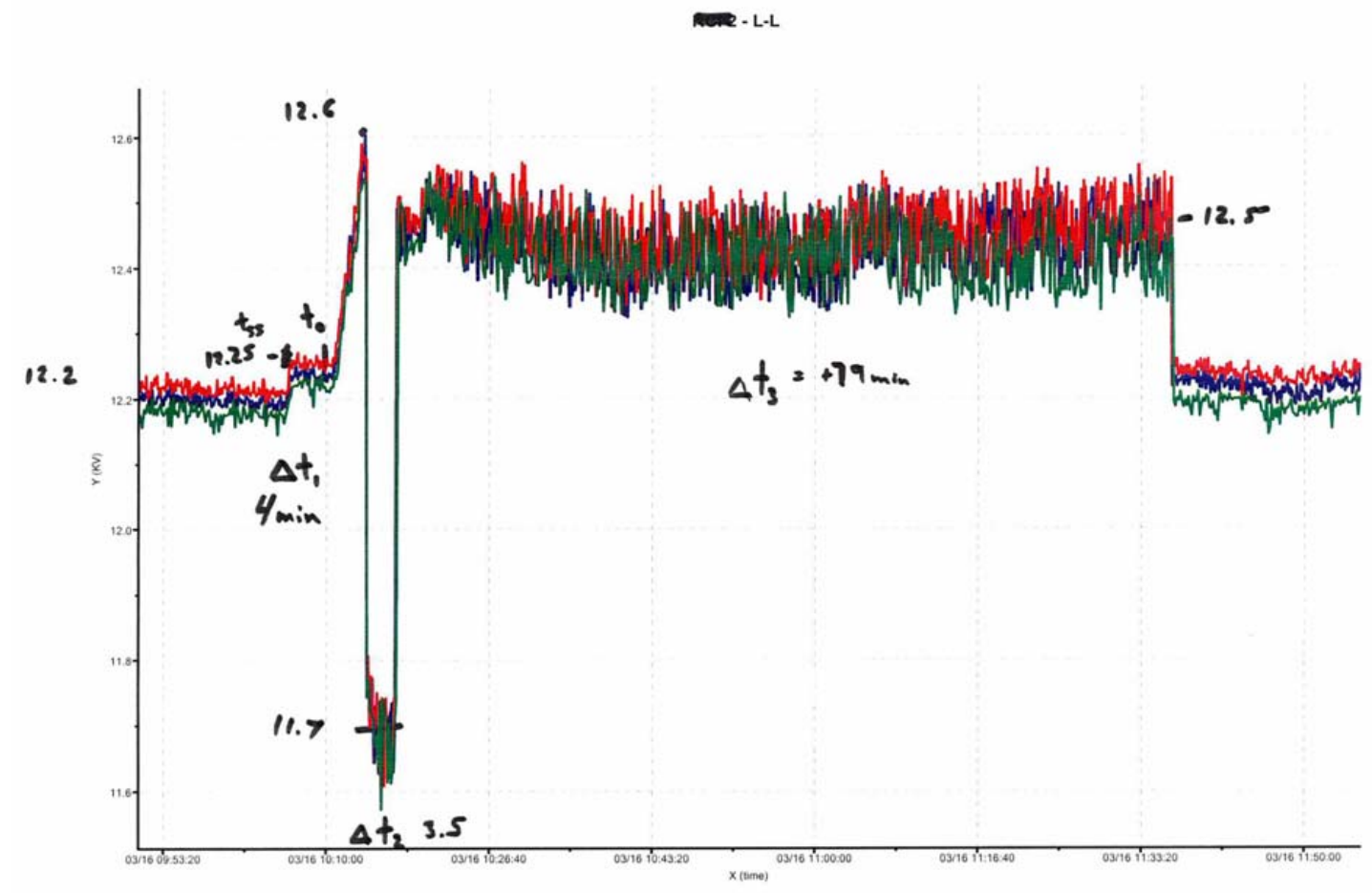

Figure 7-9 - Voltage Recording from March 16 FCL Resonance Event 
Based on the results of a resonance study, it was determined that a resonant condition would occur if the FCL's DC source to the superconducting magnet was opened with closed shunt capacitors on the circuit and light circuit loading. When the DC source is opened, the FCL begins to act like an inductor; creating a RLC resonant circuit. The worst case for resonance is when the shunt capacitors are closed and the circuit is lightly loaded. The greater the load current, the greater the voltage drop across the FCL, reducing the resonance condition. Secondly, the FCL is a source of odd order harmonics when inserted in the circuit. However, these harmonics are equal to or less than $0.5 \%$ individual harmonic distortion (IHD) and total harmonic distortion (THD) when the FCL is run at $600 \mathrm{~A}$ or less. If the FCL is loaded to its maximum steady state limit of $1200 \mathrm{~A}$, the FCL generates approximately $2.5 \%$ THD and IHD.

After detailed analysis of this event, Zenergy and SCE recommended several modifications to prevent further problems.

- SCE should automate the bypass switch so that the FCL could be more quickly bypassed in case of problems.

- Zenergy should replace the controller with an industrial hardened design and correct the software so that if a reset were to occur, the FCL would return to service with the superconducting magnet energized.

- The $1800 \mathrm{kVAR}$ capacitor bank should be downsized to $1200 \mathrm{kVAR}$ to minimize the voltage rise under resonant conditions

- More base and off-peak loaded customers should be added to the circuit to help dampen the resonance, if a resonant condition were to occur during off-peak hours

- The FCL should not be loaded beyond $600 \mathrm{~A}$ in order to keep harmonics below $1 \%$ IHD and THD

- Event recorders should be programmed to trigger recording on high voltage conditions so that event analysis and models could be refined

\subsubsection{FCL Put Back in Service (December 9, 2009 with no load and December 18, 2009 with load)}

After the needed modifications were made to the FCL and the bypass switch, the FCL was placed back into service. Voltage was applied on December 9 and full current applied on December 18. The FCL was observed to be operating normally.

\subsubsection{Fault Event - January 14, 2010}

A fault was experienced on the circuit with the fault current limiter in operation on January 14, 2010. Both power quality monitors on the circuit (one upstream and one downstream from the fault current limiter) recorded the January 14 fault. Preliminary analysis of the fault records and power quality data show the fault current limiter operated correctly limiting fault current by about $8 \%$. This was about what was expected based on the fault current limiter lab testing and the magnitude of the fault current. Additional modeling improvements are being made to better reproduce the current limiter behavior. 
Based on the voltage data recorded by the power quality monitors, the event appears to have evolved from a phase-to-phase fault to a three-phase fault and then a temporary recovery. This was followed by a phase-to-phase fault, then a three-phase fault and then the fault cleared. These events occurred over a period of about 3 seconds. After field inspection, the fault was found to be caused by the $12 \mathrm{kV}$ conductors slapping together in high winds on an overhead section of line located between RCI-2 and RCI-3. The fault current waveforms and voltage magnitudes downstream of the FCL are shown in the graphs below (Figure 7-10 and Figure 7-11).

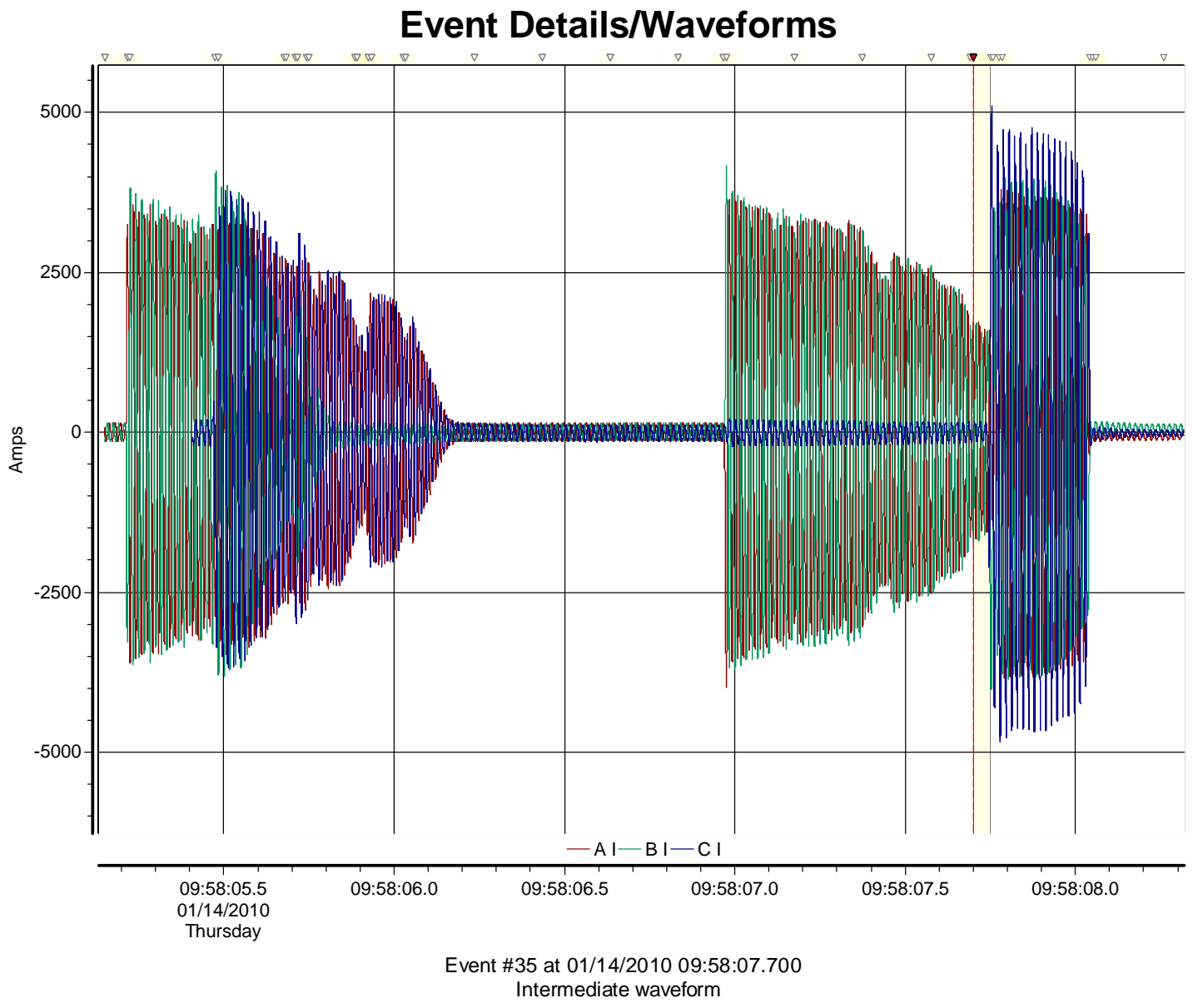

Figure 7-10 - Fault Current Waveforms Downstream from the FCL - 1/14/2010 


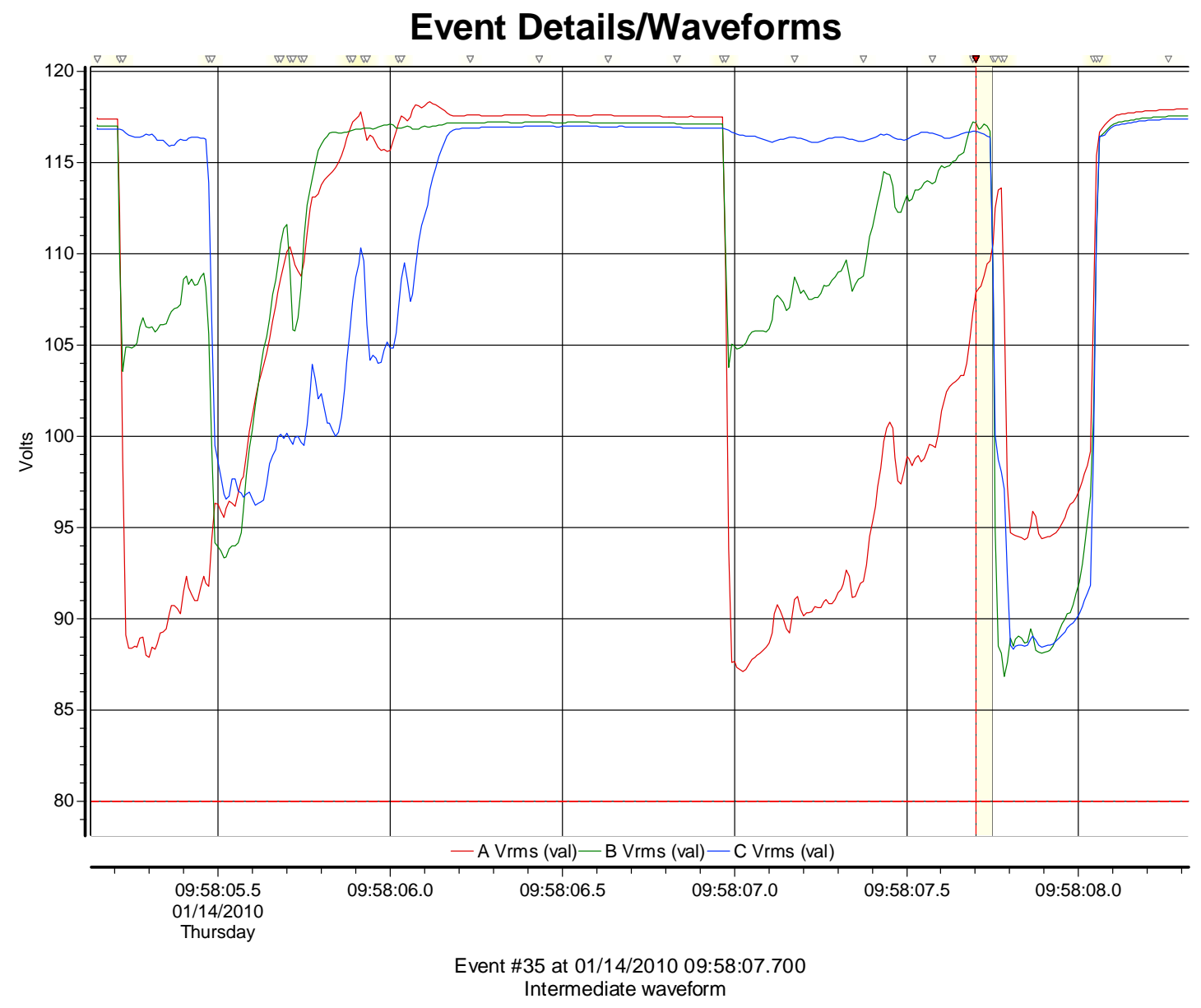

Figure 7-11 - Voltage Magnitudes Downstream the FCL - 1/14/2010

\subsection{Analysis of Advanced Fault Sensing and Prediction Measures}

After analysis of a number of advanced fault sensing and prediction measures, three were selected for further work on this project. The three selected were:

- Measure power quality upstream and downstream of the FCL

- De-centralized Sensing, Control and Protection and Automation Functions

- Design and Model System of the Future Relay Protection Scheme

The following sections will discuss the results of this work in more detail.

\subsubsection{Measure Power Quality Upstream and Downstream from the FCL}

To help verify the operation of the fault current limiter, power quality meters were installed both upstream and downstream of the fault current limiter. These devices looked at fault events to see the effects of the fault current limiter on the circuit. These instruments would also be able to help investigate unusual behaviors caused by the fault current limiter on the distribution circuit including harmonic distortions. Since there was only one fault recorded since the installation of the FCL, information for this section of the report is preliminary. Also, due to the way the power quality meters were configured, 
only voltage harmonics are available on the upstream side of the FCL and only current harmonics are available on the downstream side of the FCL. For the January 14, 2010 fault, there were two closely spaced faults with the second one actually tripping the RCI2. As a reference, harmonics were taken for the time between the two events and used as a reference (Figure 7-12 and Figure 7-13). The substation voltage harmonics are between $1 \%$ and $1.5 \%$ THD. The current harmonics downstream of the FCL were between $4 \%$ and 5\% THD while carrying a load of 95 amps.

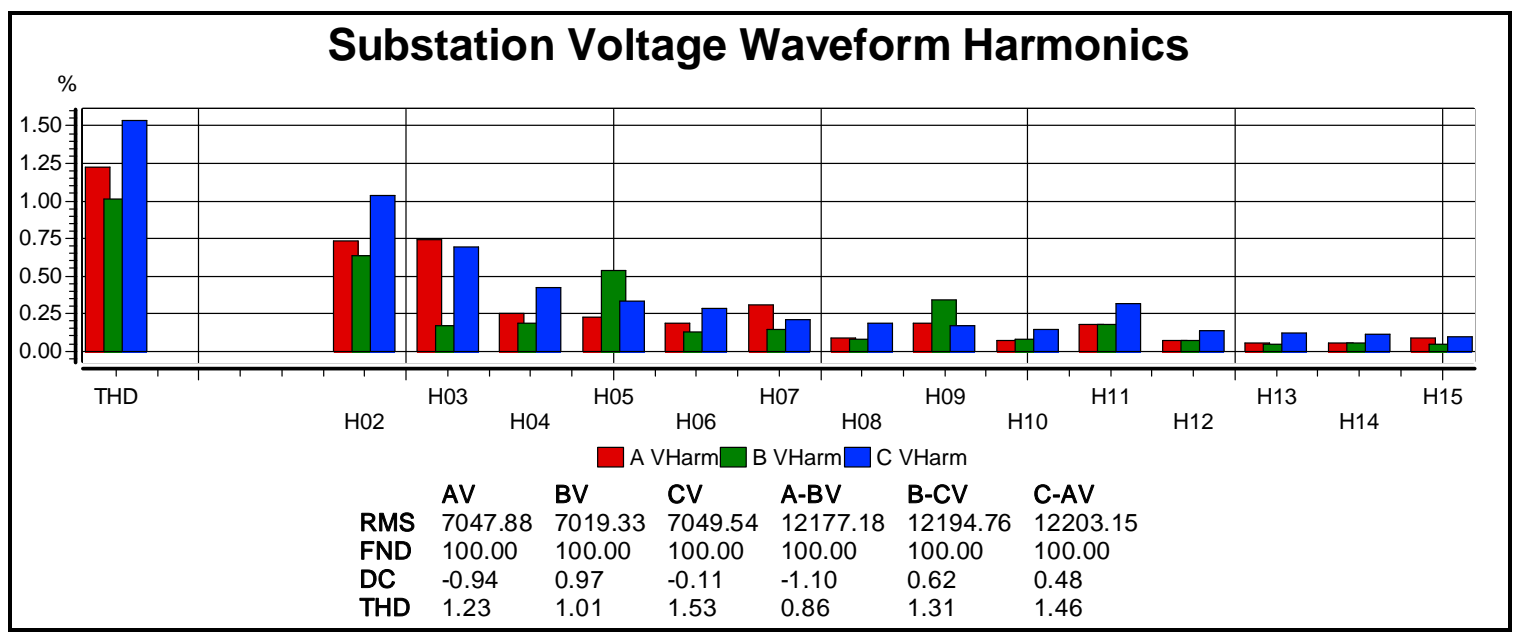

Figure 7-12 - Normal Voltage Harmonics at Substation

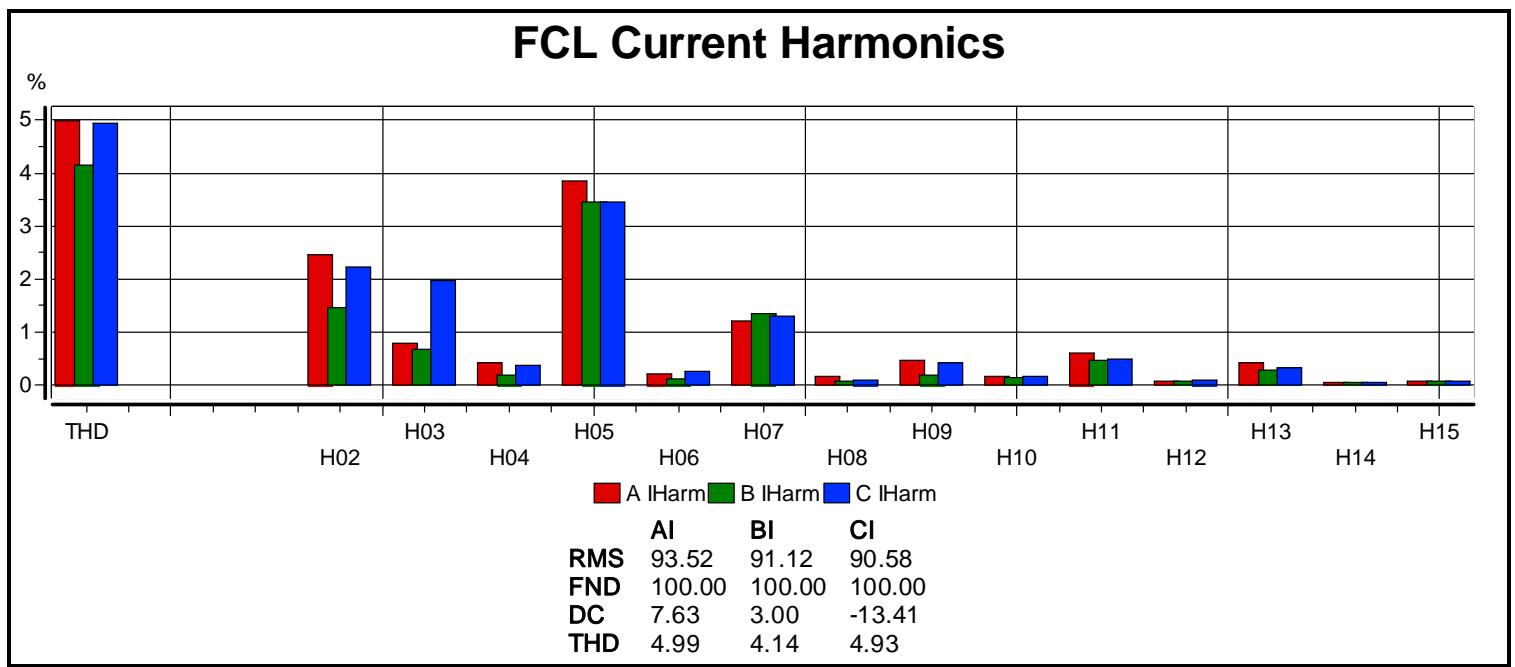

Figure 7-13 - Normal Current Harmonics Downstream of the FCL

There were two sections to the fault (Figure 7-10) and harmonics were calculated for each section. For the voltage harmonics on the substation bus, the harmonics rose to between 2\% and 5\% THD for the first section of the fault (Figure 7-14) and to between $2.5 \%$ and $3 \%$ THD for the second half of the fault (Figure 7-15). This is compared to harmonics in the range of $1 \%$ to $1.5 \%$ for similar condition with no fault. All harmonics seemed to increase during the fault. 


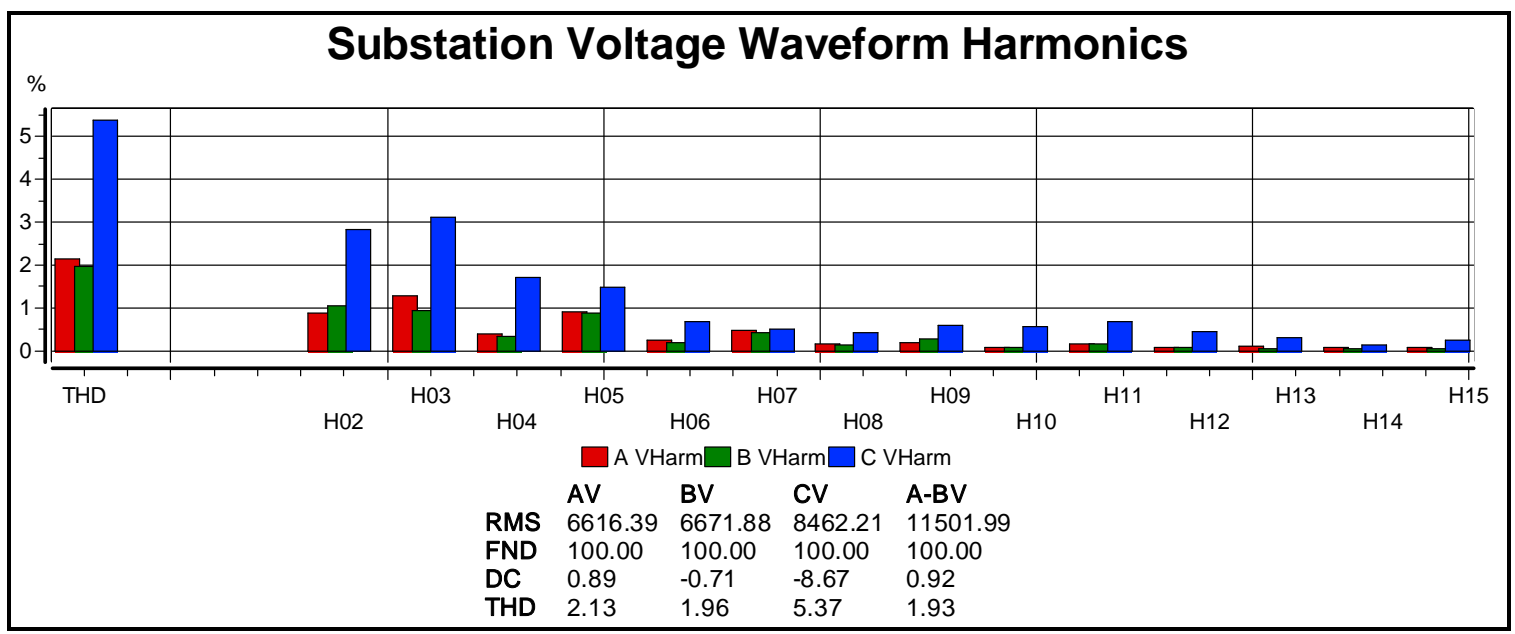

Figure 7-14 - Substation Voltage Harmonics - First Fault Section

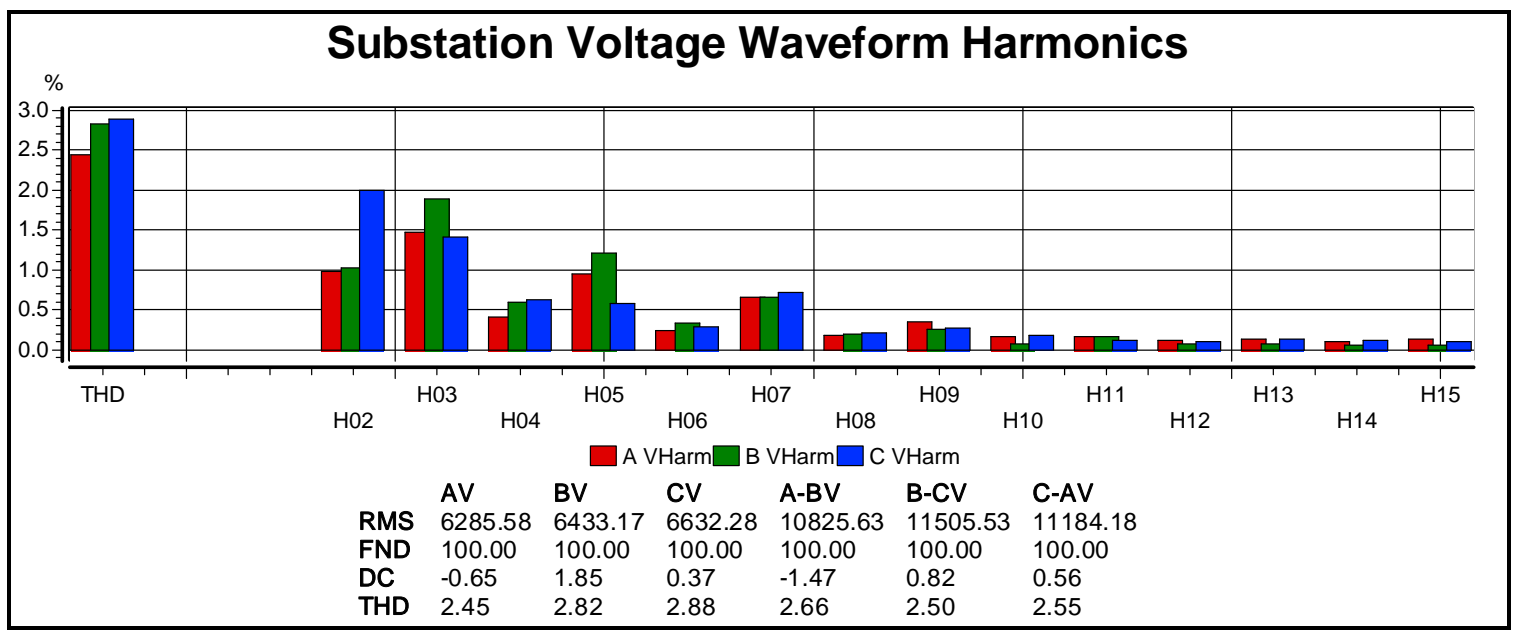

Figure 7-15 - Substation Voltage Harmonics - Second Fault Section

For the current harmonics downstream of the FCL, there was an increase from the steadystate values of $4 \%$ to $5 \%$ to $6 \%$ to $8 \%$ for the first section of the fault (Figure $7-16$ ) and $5 \%$ to $16 \%$ for the second section of the fault (Figure 7-17). Phase $\mathrm{C}$ had a significant amount of $2^{\text {nd }}$ harmonic during the second half of the fault indicating a shift in the voltage zero point due to the fault. 


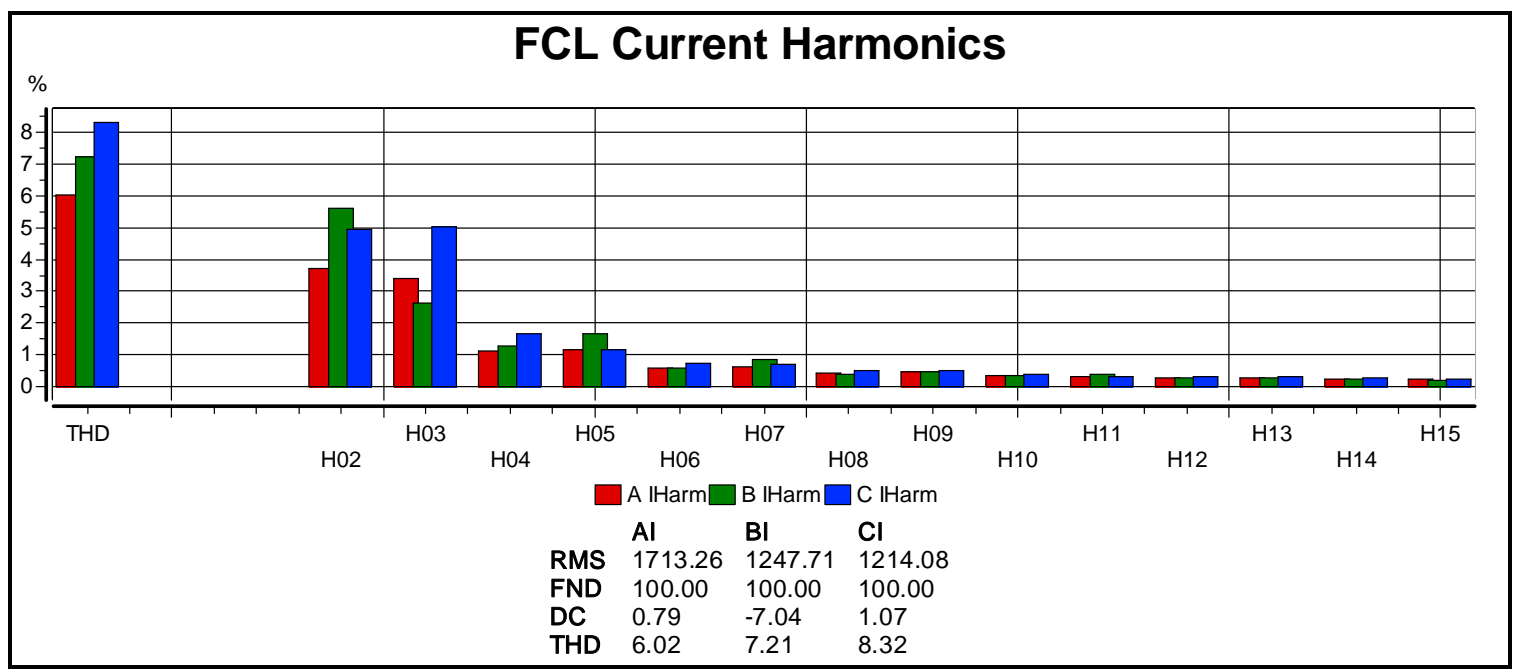

Figure 7-16 - FCL Current Harmonics - First Fault Section

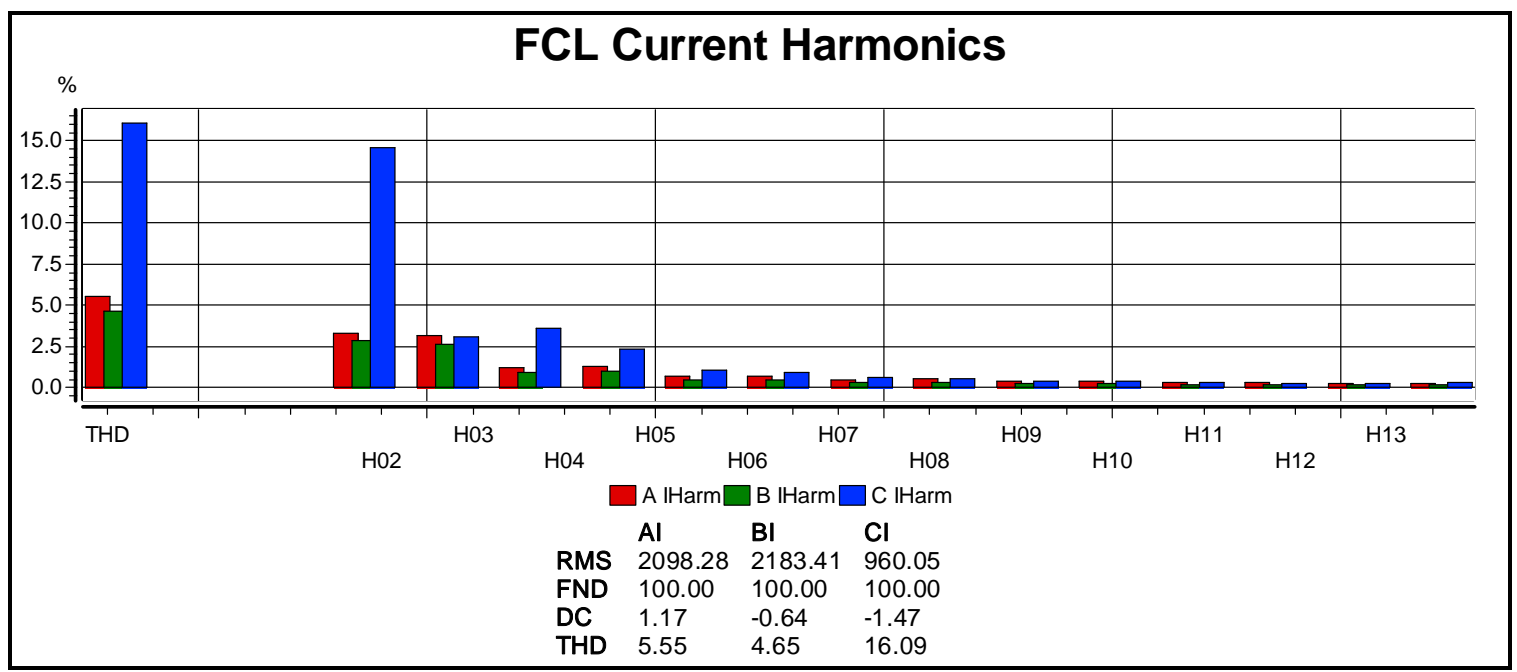

Figure 7-17 - FCL Current Harmonics - Second Fault Section

\subsubsection{De-Centralized Sensing, Control and Protection and Automation Functions}

The Circuit of the Future employed de-centralized sensing and control to implement the advanced protection system. This was made up of sensing using potential and current transformers connected to a Schweitzer 351 relay located at each RCI. This data was communicated through the use of Schweitzer mirrored bits to a Schweitzer 2100 Logic Processor. Each relay communicated its status when a fault was sensed. The Logic Processor executed its pre-programmed logic and then sent trip commands to the proper RCIs. This system worked well and was able to also determine when communications links were lost and correct for them. All status information was then communicated back to a substation gateway where it was sent to the SCE data historian and also made 
available to the system operators. As of the conclusion of this project, the advanced protection system is still in place and operating.

\subsubsection{Design and Model System of the Future Relay Protection Scheme}

Based on what was learned as part of the Circuit of the Future advanced protection system implemented as part of Phase 1, a second generation advanced protection system was designed for the System of the Future/ Irvine Smart Grid Demonstration. This system is designed to again use fault interrupting switches (URCI) on the circuit. The fiber communications used in the Circuit of the Future is being replaced by IP wireless radio communications. The communications protocol is now GOOSE messaging based on the IEC 61850 standard. In addition, two circuits will be looped together so that when the faulted portion of the circuit is isolated; all other customers on the two circuits will still have power.

As part of this project the new protection system was designed (see Section 6.3.3 of this report for the details). This design included the logic that resides in each URCI, communications required, and timing of the protection events. The PSCAD model was constructed so that the dynamic behavior of the circuit with the protection logic could be tested. This project constructed the basic PSCAD model of the circuit, but did not implement the protection logic. At present the PSCAD model is being translated into an RSCAD model that can be run on a Real-Time Digital Simulator (RTDS) that will allow the actual relays/logic to be tested under a wide array of events. This testing will build confidence in the design. Once the design is modeled, a lab mockup will be constructed so that final testing and staff training can be conducted before deployment on the actual circuit. This work is scheduled to be completed in 2012. 


\section{Section 8: Extrapolation for the Future and for Industry}

When the project began in October 2006, the ultimate goal was keeping in mind the country's need to modernize electric distribution over the next 25 years. In fact, we kept this goal in mind throughout the project period and looked for new ideas coming from all parts of the world. Though this proposal addressed the short-term goal of DOE's 2030 Vision, the "Advanced Protection System" was designed to be flexible and accommodate new changes in the industry that are continuously occurring. The project team considered and included new and more efficient sensors, relays, control devices, hardware and software technologies, communications equipment and practices. The project planned to take into account growth and changes in this exciting industry as the project moved forward. Fortunately, SCE's Circuit of the Future had provisions already incorporated into it that help make changes possible within the budgetary bounds. The entire project team took on this challenge using its extensive expertise and experience.

The purpose of this Section is to identify advances that are likely to occur in this field of advanced distribution protection based on the experiences gained in this project. More advanced computerized relays, cost-effective phasor measurement units (PMU), high power electronic control devices, digital communication technologies etc, are continuously evolving to facilitate improved protection of distribution systems over a wide area. As a result, the monitoring, control and protection of the power systems in real-time is becoming more effective. These new technologies will hopefully allow the systems to become more efficient and reliable. The protection community should seize these opportunities to make power systems safer, more secure, more reliable and less vulnerable to external threats. One of DOE's missions is to lead a national effort to help modernize and expand America's electric delivery system to ensure economic and national security. Some of the opportunities for advanced protection for the benefit of the utility industry are identified and described below.

\subsection{De-centralized sensing, control and protection and automation functions}

\subsubsection{New Sensors}

Advances in sensor technology are making new devices available for deployment in distribution systems. These sensors can provide information that was not easily obtainable in the past. If the cost of sensors is low, large quantities can be placed at critical locations in the system. The information available from these sensors can be used 
to implement new applications. The line-post sensor provides two continuous ac voltage outputs that are proportional to the distribution line voltage and current. Connecting the sensor outputs to advanced remote terminal units (RTUs) or smart relays facilitate the recovery of typical power measurements, fault detection data and power quality events.

\subsubsection{Distributed vs Central Computational Intelligence}

Since large amounts of data are expected in smart grid implementations, the computational burden can become very large. Also, there is a possibility of communication bottlenecks due to transmission of large quantities of data. Distributed computational intelligence must be utilized to avoid such bottlenecks. The system can have several concentration points located in strategic areas, probably in substations. Data from specific parts of the system are directed to these concentration points. These devices process the information and make local control decisions. Each device also communicates important information to other distributed devices as well as the central operations and data storage center. This central system receives data from each concentration point and then processes the data to make global decisions for the system. An appropriate balance between local and central computational intelligence is needed for an efficient scheme.

\subsubsection{Distribution System Communications}

Communications is a critical part of the smart grid. The communications will enable the sharing of information between systems that in the past were isolated. Distribution system applications within the smart grid will consist of communication in the following areas:

- Customer - reading of power consumption, voltage and other data from electronic meters at customer premises, as well as sending control information such as pricing to the customer

- Distribution System (Field Area Network) - reading of data from and sending commands to sensors and controllers in the distribution network

- Protection - fast data transfer to response to faults and outages

All of these systems are not expected to generate large data volumes for the foreseeable future (as compared to say telecommunication services), so network technologies that can support effective link rates of $100 \mathrm{kbps}$ to $1 \mathrm{Mbps}$ over a distribution system area should suffice. However, these applications are more appropriately differentiated by their latency requirements. While smart metering requires reliable communications approximately only every hour or less (high latency); any form of closed loop control imposes much more stringent delay budgets. For example, distribution protection requires a latency of $100 \mathrm{~ms}$ or less. The Field Area Network will require latencies in the range of 1 to 10 seconds.

While the use of fiber for all communications would be desirable, costs and issues with implementation make this impossible. For connection to the customer, a combination of radio communications in the unlicensed band or the use of public cellular networks 
would be acceptable. For the field area network, use of licensed (where available) or unlicensed communications would be acceptable. The use of public cell networks for the field area network causes some concerns by the utility industry due to the high reliability communication needs for distribution system control functions. These control functions are most important during emergencies and during disasters, the time when the public cell system is most unreliable. Protections systems offer the greatest challenge for wireless communications networks. Tests done by SCE on several of these systems have turned up problems in either the area of latency or coverage. Systems that meet the latency numbers have had coverage problems and vice versa. Several companies are working to address there problems and should have products ready in the next year. SCE will be testing some of these in their Irvine Smart Grid Demonstration project.

\subsubsection{Real-Time Analysis and Control}

Advanced automation requires faster decisions and thus real-time analysis of distribution systems. A robust distribution state estimator is an example of analysis tools needed for advanced automation. The input data for analysis includes system topology, parameters of different components in the system, status of switches and breakers, and measured data from various points in the system. Since more data can be measured the analysis becomes more complex. The tools should be able to use these data effectively. Realtime analysis will allow faster control of distribution systems. For example, the system can be reconfigured frequently in response to load and other system changes by opening and closing switches to bring it to a new optimal state. Such operation would require more robust switches which can handle multiple opening and closing operations without failing. Solid-state switches could be the answer for some of these applications.

Real-time monitoring and analysis can also lead to faster system restoration following emergencies. Since most of the equipment is expected to operate near capacity, long duration outages can lead to problems due to cold load pickup associated with thermostatically-controlled devices, such as air conditioners. In such cases step-by-step restoration would be needed to avoid stressing the transformers beyond their capability. Real-time monitoring and analysis not only provides the status of equipment loading, but also allows determination of the next step, such as location and time of the next switch to be closed to restore a group of customers. With judicious selection, restoration can be accomplished in a minimum amount of time; thus improving reliability of electricity supply to the customers.

\subsection{Advanced and adaptive protection}

It is well recognized that current distribution systems deserves immediate attention. To quote Horowitz, Phadke and Renz in the $2010 \mathrm{March} /$ April Issue of the IEEE PES Power and Energy Magazine: "The initial focus has been on the fundamental transformation of

distribution system. This in itself a huge technical challenge that will be measured not in years but decades". "While the changes to distribution will be revolutionary, 
transmission will change in an evolutionary manner". These are very profound statements.

Smart distribution grids will be markedly different from their legacy counterparts due to the infusion of new technologies on multiple fronts. Advanced communications, information and control schemes, distributed energy sources including microgrids and new digital or solid state equipment are being introduced at an unprecedented pace. New topologies such as looped and network structures are being adopted to provide increased reliability and efficiencies to customers. In addition, the loads served by the distribution system are changing with more electronic devices, the appearance of electric vehicle chargers as well as an increase in distributed generation and storage devices on the distribution system (e.g. photovoltaic panels and battery devices). New control and protective device development is essential for successful operation of smart primary distribution grids. Newer devices are needed for advanced and adaptive control and protection of these systems. The new loads and distributed generation are already penetrating the system.

Only very rudimentary protection exists in both underground and overhead distribution systems today. The only protection on the radial systems comes from branch line fuses, the primary distribution transformer fuse on the utility side, and the main circuit breakers in the customer premises. No protection coordination is considered between the utility and customers sides. Network protectors and transformer fuses for the distribution network lines are the only protection provided. These distribution systems therefore need a new and revolutionary protection philosophy and design to meet the smart grid performance goals. Therefore what should be the future protection system philosophy for the entire smart distribution grid? The philosophy should consider the following factors:

- Compromise between redundancy versus security

- Simplicity versus complexity

- Reduce response time to align with real-time operation

- New resource skill set for engineers, operators and field crew

The future protection system design should aim at enhancing the following overall system performance measures:

- Customer Satisfaction

- Efficiency

- Reliability

- Cost-effectiveness

- Power Quality

- Cyber security

- Safety

- Customer Engagement \& Demand Response

The following new technologies will influence future protection system design include: 
- Distributed generation and storage (e.g. photovoltaics, wind generators, fuel cells, storage devices)

- New solid-state devices and technologies

- Control devices and smart appliances (loads)

- New smart sensors and meters

- Electro-optic and micro sensors

- Next generation computer and communication networks

The challenges and opportunities for advanced and adaptive protection systems need new generation of models for all the new devices that penetrate the system to accommodate loop and mesh topologies. In addition new methods and analytical tools are needed. These include on-line fault analysis and coordination algorithms. Adaptive protection is the key for success to allow different modes of DG operation, PHEV integration and fast demand response.

The primary objective of this sub-section is to raise issues related the protection of future distribution systems. The traditional protection schemes are not adequate for $21^{\text {st }}$ century power and energy systems. The role and impact of new technologies, sustainable energy sources, communication system and active customer participation require changes to the way protection is implemented.

\subsection{Microgrid Protection}

\subsubsection{Introduction}

One proposed way of integrating high penetration of distributed generation sources is through microgrids and is described in detail in a paper published by one of this project's subcontractors ${ }^{4}$. A microgrid is defined as a low to medium voltage network of small load clusters with distributed generation sources and storage ${ }^{5}$. Microgrids can operate in islanded mode or grid-connected mode. If a microgrid is connected to the system, it is seen as a single aggregate load or source. One of the potential advantages of a microgrid is that it can provide more reliable supply to customers by islanding from the system in the event of a major disturbance. The microgrid protection in islanded operation poses a serious problem. It was shown in ${ }^{6,7}$ that the fault currents for a grid-connected and

\footnotetext{
${ }^{4}$ E. Sortomme, S. S. Venkata and J. Mitra, "Microgrid Protection Using Communication-assisted Digital Relays", IEEE Transactions on Power Delivery, Vol: PP Issue 99, pp. 1-1, Digital Object identifier: 10.1109/TPWRD.2009.2035810, Date of Publication: 11 December 2009.

${ }^{5}$ European SmartGrid Technology Platform, "Vision and Strategy for Europe's Electricity Networks of the Future, EU Commission, Directorate-General for Research, Information and Communication Unit, Brussels, Belgium, 2006.

${ }^{6}$ T. C. Green and J. D. McDonald, "Modeling and Analysis of Fault Behavior of Inverter Microgrids to Aid Future Fault Detection," Proceedings of the IEEE International Conference on System of Systems Engineering, 2007, pp. 1-6.
} 
islanded microgrid are significantly different. Additionally, high penetration of inverter connected distributed generation and storage sources lead to conditions where no standard overcurrent protection methods will suffice. It is clear that protection of microgrids cannot be achieved with the same philosophies that have been used to protect traditional distribution systems. At the very least, a system designed to protect a microgrid should take the following into account bidirectional flow in feeders, looped feeders, and reduced fault levels in islanded operation.

A protection scheme using digital relays with a communication overlay is a potential solution for protection of the microgrid system. A practical system, described in Section 8.3.4, is chosen from ${ }^{7,8}$ to investigate the protection scheme. The increased reliability of adding an additional line to form a loop structure is also explored. A novel method for modeling high impedance faults is demonstrated to show how the protection scheme can protect against them. This is important in microgrid protection not only because a the percentage of high impedance faults on the distribution system is not insignificant ${ }^{9}$, but also because microgrids, in the islanded mode, typically have lower fault currents, and methods of high impedance fault detection will be useful for the detection of these faults.

\subsubsection{The Case for a New Protection Paradigm}

Most distribution systems are operated in radial mode. Most of these radial feeders have switches to connect them to surrounding feeders to restore power to customers after faults where part of the circuit is isolated. Hence the radial structure is preserved. Consequently, in these systems, the protection is designed for radial operation. However, as the penetration of distributed resources increases, these systems will experience two important changes: bi-directional flow in the feeders, and looped operation. Traditional protection schemes for radial operation will no longer be adequate. Nor can one apply traditional protection schemes that are in use even in meshed distribution systems today, because the new protection systems will have to be adaptive. It will need to switch between grid-connected and islanded (as single or multiple islands) modes which causes a change in configuration and fault duty levels. A logical solution that accommodates all these changes is a communication-assisted system.

\subsubsection{General Microgrid Protection Philosophy}

Since a microgrid can operate in a grid-connected mode and in an islanded mode, it is necessary to protect it in both modes of operation. The general philosophy is to find a method that will work equally well in both modes of operation. There are different

\footnotetext{
${ }^{7}$ E. Sortomme, G. J. Mapes, B. A. Foster, and S. S. Venkata, "Fault Analysis and Protection of a Microgrid," Proceedings of North American Power Symposium, 2008, Sept. 2008, pp. 1-6.

${ }^{8}$ Cooper Power Systems, Ed. 3, Electrical Distribution-System Protection, Cooper Industries, 1990.

9 C. G. Wester, "High Impedance Fault Detection on Distribution Systems," General Electric Company, 2008, URL: http://pm.geindustrial.com/faq/documents/f60/ger-3993.pdf [Accessed: Sep. 11, 2009].
} 
philosophies of protecting islanded microgrids. One is to simply trip the entire microgrid offline once the fault is detected during islanding since it is an N-2 failure (two items fail); the first failure being the loss of the feeder. For additional reliability, the faulted line will need to be removed from service and the remaining connected loads and sources will operate as two smaller islands. This will only work if the generation and load in each smaller system match. If higher reliability is required, the feeders can be connected as a loop, so the loss of a feeder or a lateral will not result in service disruption to customers. The higher reliability, however, comes at a higher cost.

Several methods of protecting microgrids have been previously proposed. One scheme is to have each DG source have its own relay and operate without communications ${ }^{10}$. This works well for single line-to-ground faults and line-to-line faults. It relies on the sum of the phase and neutral currents as well as zero sequence currents. However, it fails to detect some high impedance faults. Another proposed scheme is to use a voltage protection scheme ${ }^{11}$. In this case, the phase voltages at the DG source are transformed into the 'dq0' synchronous frame, and then compared against a reference. A voltage drop against the reference initiates switching device tripping. For multiple DG sources, the voltages are compared via an undefined communication link and the lowest relative voltage part is tripped. This method is also ineffective against high impedance faults. An additional protection scheme utilizes standard overcurrent differential protection on each line with backup voltage and frequency protection at each DG source ${ }^{12}$. This scheme is also unable to detect high impedance faults (HIF's). In addition, each of the aforementioned schemes has only been tested on relatively small systems with few buses and undefined distances between distribution lines.

Additionally, it has been proposed that replacing overcurrent relays with directional relays in instances where a problem of directionality exists is possible; but this comes at a high cost. Individual DG's could also be tripped at the first detection of a fault before the distribution relays can operate ${ }^{13}$. The problem with directional relays is that they will also not detect HIF's. Tripping DG sources also reduces the reliability of service to the customer.

It has also been proposed that microgrids could participate in remedial action schemes using synchronized phasor measurements to determine the appropriate islanding and

\footnotetext{
${ }^{10}$ H. Nikkhajoei and R. H. Lasseter, "Microgrid Protection," Proceedings of IEEE Power Engineering Society General Meeting, 2007, June 2007, pp. 1-6.

${ }^{11}$ M. A. Redfern, and H. Al-Nasseri, "Protection of micro-grids dominated by distributed generation using solid state converters," Proceedings of the IET 9th International Conference on Developments in Power System Protection, 2008, pp. 670-674.

${ }^{12}$ H. H. Zeineldin, E. F. El-Saadany, M. M. A. Salama, "Distributed Generation Micro-Grid Operation: Control and Protection," Proceedings of the Power Systems Conference: Advanced Metering, Protection, Control, Communication, and Distributed Resources, 2006, March 2006, pp.105-111.

${ }^{13}$ S. H. Horowitz and A. G. Phadke, Eds., Power System Relaying, 3rd ed. Baldock, Hertfordshire, U.K.: Research Studies, 2008, p. 259.
} 
restoration strategies. These protection schemes however are under development and currently not ready for deployment [9].

\subsubsection{Proposed Protection Scheme}

The protection scheme discussed here utilizes some of the principles of synchronized phasor measurements and microprocessor relays to detect all types of fault conditions including HIF's. It is based on the deployment of digital distribution feeder relays that are currently offered by some of the major manufacturers. These digital relays include standard overcurrent and over/under voltage protection methods. They are programmable and have fiber optic and Ethernet communication links. They are self metering and have oscillographic event reports. By using these relays on the end of each line segment, a very robust protection scheme can be developed. While what is described here uses digital relays, it is conceivable that the use of properly designed sensors and switches will perform adequately for faults encountered in distribution systems, and enable cost-effective protection schemes. The costs can be further controlled by not using independent communication channels, but by 'piggy-backing' on any available channels already deployed in that part of the system. For instance, if 'smart grid' technologies have already been deployed, the corresponding communication channel can be used. The primary protection scheme utilizes a relay that measures absolute current sampled at 16 or higher number of samples per cycle and then transmitted via communication link to the relay on the other side of the line. For distances under 18 miles, the transmission takes less than $0.1 \mathrm{~ms}$ based on the speed of light for signal transmission and several additional microseconds for processing time. This is sufficient for most distribution systems. This means that there is no need to get time-synchronized measurements from both sides of the line for short distribution lines. For lines longer than 18 miles, however, a Phasor Measurement Unit (PMU) may be required. In this way a differential relaying scheme can be successfully created.

The primary protection for each feeder relies on instantaneous differential protection. If absolute values of two samples are found to be above the trip threshold, the tripping signal is sent to the switching device. It is anticipated that these switching devices will benefit from recent advances in switching technologies (such as vacuum interrupters) and higher sampling rates, and it should soon be possible to interrupt currents much faster than the present-day norm of 5 to 10 cycles for distribution breakers. The expected fault currents can vary over a wide range: less than to many times greater than the nominal load current (0.5-20 p.u.). The current transformers will therefore need to have accurate operation over this wide range of fault currents.

In the event of a switching device failure, a backup trip signal will be sent to the adjacent relays on the same bus. This signal is sent after a certain time delay, greater than 0.3 seconds but less than 0.6 seconds, if the measured differential current is still above the threshold. This is the normally accepted practice, but with the advent of high performance relays and breakers the delay could be significantly shorter. If the relay or the communication link fails, this will alert all other connected relays that the differential 
scheme is lost. An alarm will be sent to the distribution control center. The remaining relays will rely on comparative voltage protection until the system is restored. The comparative voltage protection compares the relative rms voltage at each relay with every other connected relay. For voltages less than 0.7 per unit, the relay with the lowest voltage will trip after a 0.6 second but before 0.9 second time delay. This allows the first two schemes to operate. Each DG source is also equipped with undervoltage tripping for voltages less than 0.7 per unit and after one second delay. This protection scheme is depicted in Figure 8-1.

The protection scheme can detect HIF's in two ways. The first way relies on the high sensitivity of the current transformers. As long as the HIF current magnitude is at least $10 \%$ of the nominal current, the HIF will be detected by the differential protection scheme. The other method relies on programming the relays to recognize certain HIF characteristics that have been observed and then tripping when those characteristics are present in the differential current.

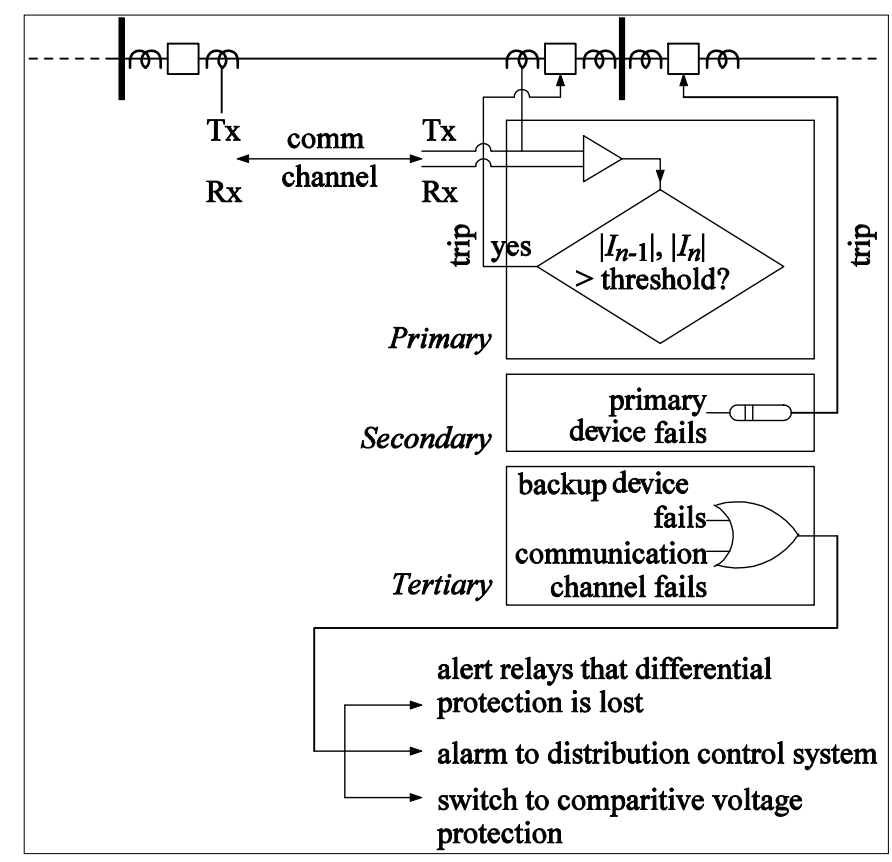

Figure 8-1 - Conceptual Representation of Proposed Protection Scheme

\subsubsection{Discussion}

The proposed protection scheme is clearly able to protect a microgrid in all modes of operation with an improvement in reliability. This is especially true with the loop configuration as the radial configuration usually has generation-load imbalance with the removal of any line due to a fault. However, it is clear that placing these relays and switching devices on each line tap in a distribution system would, in most cases, not be economically justifiable with today's sensor, relay and communications costs. Another method that uses the same principles is to have only sensor units at the buses with a 
communication link to the substation. At the substation, a central controller or logic processor can monitor the current and voltage differences and remotely operate the switching devices. Additionally, any lines that will not experience bi-directional current flow will only need one switching device instead of two, thus further reducing the cost. This scheme will work exactly the same as with multiple relays and for similar faults, similar responses as those previously discussed will be observed. In the event of a communication failure, however, the only protection will be on the sources which will work for all but HIF's. Though even this scheme is still more expensive than traditional protection methods, it is justified if the customers require the additional reliability of islanding. It is hoped that in the future, costs of equipment will be reduced and availability of communications with low latency will be available. This will make a protection scheme like the one described above easily implementable.

\subsection{How to integrate into Smart Grid Build-out?}

There are a number of drivers that are making the build-out of the Smart Grid necessary. In the distribution area these include the proliferation of distributed generation (e.g. rooftop photovoltaics, battery storage), introduction of electric vehicles and their chargers, and the increased potential of demand response (e.g. smart appliances, air conditioner control). All of these together argue for the need to implement advanced distribution automation and a new generation of advanced Distribution Management Systems (DMS). Advanced protection should be viewed as an integral part of advanced distribution automation. As part of this work, there is a need to define and develop new, advanced distribution automation functions and schemes. Along with these automation systems, new planning methods also need to be developed.

What does this advanced distribution automation system look like? Information and control will be distributed throughout the electrical system in a hierarchical manner. Sensors, remote controlled switches and protective devices will allow integration of fault isolation and system restoration into one function. Microgrids with distributed generation/ storage integrated into utility system can yield higher reliability. Electric vehicles and smart loads will be integrated in a single load management system that conveys pricing signals to the customer. Advanced materials will make possible inexpensive fault current limiting and more efficient transformers.

In this context, advanced DMS applications need to be developed and will include many of the following applications:

- Automatic Fault Location, Prediction, and Protection Systems (FLPPS) and service restoration

- Optimal Volt/VAR Management

- Fast on-line power flow analysis

- Smart distribution operations of the near future

- Fast short circuit / coordination analysis 
- Automatic contingency analysis

- Demand response

- Management of distributed generation and storage

- Automatic vehicle location system (crew management)

Advanced fault location, prediction and protection systems will be an important part of this view of the future. Protection systems that can accommodate distributed generation/ storage, electric vehicle charging, and microgrids will be important as the smart grid moves forward. These protection systems will need more communicating sensors that will allow implementation of advanced protection systems. Lower cost fault interrupting switches and protective relays need to become available so that distribution lines can be broken up into smaller parts, affecting fewer customers when there is a fault. Communications need to be developed with lower costs, higher reliability during disasters, and lower latencies for protection needs. Distributed monitoring and control needs to be introduced in a bigger way than has been done in the past. This will allow control at a substation level so that circuits can do a better job of self monitoring and self healing.

Planning for these advanced systems should be done now, before all of these new generation and load devices are connected to the grid. Through American Recovery and Reinvestment Act (ARRA) projects like the SCE Irvine Smart Grid Demonstration, some of these new concepts can be tested and validated. Because utility planning is usually based on rate case cycles of several years each (three years for SCE), steps need to be taken so that funds are available for smart grid system improvements when the technologies become available and are needed.

\subsection{Technology Transfer, Collaboration and Partnerships}

Throughout the project, members of the project team attended conferences and meetings to gather information pertinent to the project and also to make presentations of the project's results. These presentations served to help spread the knowledge acquired as the project progressed and give others the benefits of our experiences. Below is a list of some of the meetings attended by one of the project personnel where presentations are made on the creative and innovative work performed.

- 5/23/2006 - Presentation of the Circuit of the Future and DOE Advanced Protection projects to IEEE T\&D conference, Dallas, TX

- 6/23/2006 - Presentation of the Circuit of the Future and DOE Advanced Protection project to EPRI CEO, Rosemead, CA

- 6/13/2007 - A summary of the DOE Advanced Protection project scope and work to date was presented at the 2007 EPRI PQA/ADA conference in Long Beach, CA. This presentation was made to utilities and vendors that were attending a meeting of the EPRI Fault Anticipator project (DOE funded). 
- 6/14/2007 - Presentation of the Circuit of the Future and DOE Advanced Protection project to EPRI Distribution Projects Meeting, Raleigh, North Carolina

- 10/16/2007 - Dedication of the Circuit of the Future, Alhambra, CA attended by print and TV media

- 8/4/2008 - Presentation of Circuit of the Future and DOE Advanced Protection project status at 2008 EPRI PQA/ADA conference, Cleveland, OH. Also presented project status to a meeting of EPRI's Fault Anticipator project (DOE funded).

- 10/29/2008 - DOE Peer Review - Red Bank, NJ - DOE Advanced Protection project review

- 1/10-14/2010 - Attended the IEEE PES Joint Technical Committee in Orlando, FL. Attended working group meetings related to this project.

- 1 Q2009 - Presentation of the project status at the $6^{\text {th }}$ Annual Workshop in Protection and Control hosted by PG\&E, Mississippi State Univ., and Quanta consulting.

- 2/17/2010 - Presentation on Smart Grid and DOE project to the Northwest Energy Systems symposium on Smart Grid Technology and Applications, University of Washington, WA

- 3/18-20/2009 - Presented project progress at i-PCGRID (Innovations in Protection and Control for Greater Reliability Infrastructure Development) Workshop held from March 18 - 20, 2009 in San Francisco (hosted by PG\&E)

- 6/16/2010 - Present overview of SCE Smart Grid activities and DOE project status to the 2010 EPRI PQ and Smart Distribution conference, Quebec City, Quebec, Canada 


\section{Section 9: Project Summary and Conclusions}

\subsection{Summary}

One of the main objectives of this DOE-sponsored project was to reduce customer outage time. Fault location, prediction, and protection are the most important aspects of fault management for the reduction of outage time. In the past most of the research and development on power system faults in these areas has focused on transmission systems, and it is not until recently with deregulation and competition that research on power system faults has begun to focus on the unique aspects of distribution systems. This project was planned with three Phases, approximately one year per phase. Due to some delays encountered with circuit equipment installation and procurement of a fault current limiter, the project actually took place over four years concluding on June 30, 2010.

\subsubsection{Phase I - Fault Location, Isolation, and Protection for the Circuit of the Future}

The first phase of the project involved an assessment of the state-of-the-art in fault location, prediction, and detection as well as the design, lab testing, and field installation of the advanced protection system on the SCE Circuit of the Future located north of San Bernardino, CA.

To help understand the trends in the industry and what technologies were being developed in the fault location, prediction and detection areas, a literature search and survey were performed (see Section 2 for more details). The literature search, conducted by Virginia Tech and KEMA Inc., concentrated on more recent developments taking place since 1990. This period covers two important aspects of fault research: a) it encompasses the acceptance and maturing period of digital devices in power system protection functions, and b) it includes the period of fast development of communications systems that have enhanced the flexibility and area of impact of digital protection systems. This literature review report was supplemented with a survey of utilities, universities, and research organizations to determine which of these techniques are actually being implemented. The survey, sent out jointly with EPRI, was issued to more than 430 different entities in North America and the world as well as the Distribution Automation Working Group of the IEEE. The literature review and industry surveys reveal that system reliability, automation, and customer-focused operation are the main issues driving new developments for distribution system's fault location, detection and prediction applications. The key technologies driving these developments are affordable intelligent electronic devices (IEDs), better transducers/sensors, and accessible communication channels. The level of development and acceptance of each of these 
technologies determines the time frame for the implementation of new developments on distribution systems.

The actual Circuit of the future initial design was based on the collection of a number of ideas from the four SCE Distribution Engineering divisions (described in more detail in Section 4 of this report). These ideas were reduced to a tentative functional design for the advanced protection system to be implemented on the Circuit of the Future. Detailed design work started in 2006 and was supported by this project. Once the detailed design was completed, a lab mock-up of the advanced protection system was assembled. This lab demonstration was used to work out bugs in the design and also to train SCE field personnel on how to operate and troubleshoot the new advanced protection system (early 2007). Once all bugs were worked out and training conducted, the equipment was installed on the actual circuit. Following commissioning testing on the protection system, the circuit was placed in operation on August 14, 2007. A formal dedication of the circuit was held on October 16, 2007 with the SCE CEO including press coverage.

The new feeder automation scheme, with vacuum fault interrupters, will limit the number of customers affected by the fault. Depending on the fault location, the substation breaker might not even trip. Through the use of fast communications (fiber) the fault locations can be determined and the proper fault interrupting switches opened automatically. With knowledge of circuit loadings at the time of the fault, ties to other circuits can be closed automatically to restore all customers except the faulted section. This new automation scheme should limit outage time and increase reliability for customers.

Results from faults experienced on the circuit are described in Section 7 of this report. Four faults were recorded on the circuit. The first one occurred on 10/21/2007 and did not result in tripping of the RCIs. The other three faults resulted in tripping an RCI to isolate the fault without tripping the complete circuit. This demonstrated the goal of only interrupting the faulted portion of the circuit rather than tripping the whole circuit using the substation circuit breaker.

\subsubsection{Phase II - Fault Location, Isolation, and Protection for the Circuit of the Future with a Fault Current Limiter}

The second phase of the project involved the selection, modeling, testing and installation of a fault current limiter on the Circuit of the Future. While this project did not pay for the installation and testing of the fault current limiter, it did perform the evaluation of the fault current limiter and its impacts on the protection system of the Circuit of the Future. After investigation of several fault current limiters, the Zenergy superconducting, saturable core fault current limiter was selected for installation.

The FCL consists of three major components: An iron core, AC windings, and a high temperature superconducting (HTS) DC winding. The AC winding is on one leg of the iron core and the HTS DC winding is on the other leg. The HTS DC winding serves to bias the magnetic iron core into the saturated condition. The AC line of the FCL is connected in series with the $\mathrm{AC}$ line in the power grid in which the fault current is to be 
controlled. Two iron cores per phase are required, each with the AC coils wound in opposite directions, are used to provide current control during both halves of a single sinusoidal wave cycle. Zenergy Power's FCL design takes advantage of an electromagnetic phenomenon - a coil with a magnetically saturated iron core has low ac impedance, while a coil with an unsaturated iron core has high ac impedance. Since the Zenergy FCL is a prototype device and the circuit might be operating for some time without an FCL, provisions were made on the circuit for a bypass switch that would allow the circuit to operate normally with or without the FCL in service. This bypass switch would be able to carry fully rated current as well as expected fault current without the FCL. This bypass switch was located in the substation near the substation switch rack.

This fault current limiter, it's testing, and installation is described in more detail in Section 5 of this report. As SCE was investigating the availability of a fault current limiter, research on various types of fault current limiters was conducted by Virginia Tech. Virginia Tech and SCE also built numerical models to be used to evaluate impacts of fault current limiters on the advanced protection system on the Circuit of the Future. Both test measurements and modeling of the FCL indicated that the peak fault current might be limited as much as $20 \%$. Since the peak fault current expected at the substation bus was $23 \mathrm{kA}$, the FCL would be able to limit the fault current to about $18 \mathrm{kA}$ for a fault located at the substation bus. Faults located farther out on the circuit would pull less fault current. Since this is still a significant fault current level, it was determined that the circuit protection would not require any coordination changes. The fault current interrupters on the circuit would also be expected to operate correctly with this small fault current reduction. The modeling studies also showed that problems with coordination of the circuit protection would not be expected to occur until the fault current was limiter to less that $6 \mathrm{kA}$.

Because of some testing problems with the Zenergy fault current limiter, installation was delayed until early 2009 with it being put into operation on March 6, 2009. Only one fault was experienced while the FCL was energized, but an unintentional insertion of the FCL impedance also occurred shortly after installation (see Section 7 for more details). A malfunction of the FCL controller caused the DC power supply to the superconducting magnet to be turned off. This inserted the FCL impedance into the circuit while it was in normal operation causing a voltage resonance condition (voltage rise of $3 \%$ ). While these voltages never reached a point where damage would occur on customer equipment, steps were taken to insure this would not happen again. The FCL was reenergized with load on December 18, 2009. A fault was experienced on the circuit with the FCL in operation on January 14, 2010. The FCL operated properly and reduced the fault current by about $8 \%$, what was expected from tests and modeling. As of the end of the project, the FCL was still in operation on the circuit.

\subsubsection{Phase III - Advanced Fault Sensing and Prediction}

The third phase of the project involved the exploration of several advanced protection ideas that might be at a state where they could be applied to the Circuit of the Future and elsewhere in the SCE electrical system. Based on the work done as part of the literature 
review and survey, as well as a number of internal meetings with engineering staff at $\mathrm{SCE}$, a number of ideas were compiled. These ideas were then evaluated for applicability and ability to be applied on the Circuit of the Future in the time remaining for the project. Some of these basic ideas were implemented on the circuit including measurement of power quality before and after the FCL. It was also decided that we would take what was learned as part of the Circuit of the Future work and extend it to the next generation circuit protection for SCE. Also at this time, SCE put in a proposal to the DOE for the Irvine Smart Grid Demonstration using ARRA funding. SCE was successful in obtaining funding for this proposal, so it was felt that exploration of new protection schemes for this Irvine Smart Grid Demonstration would be a good use of the project resources. With this in mind, a protection system that uses fault interrupting switches, high speed communications, and looping two radial distribution circuits would have the potential for significant reductions in interruption time and number of interruptions for customers.

The advanced protection system installed on the Circuit of the Future was able to reduce the number of customers interrupted when a fault occurred on the circuit. While this project showed that only a section of the circuit would be interrupted, it was costly and still required actions by a system operator to restore customers located past the faulted section of the circuit. To address the cost issue, the fiber optic communications portion of the system would need to be replaced with a wireless radio system. This radio system would need to have low latency $(\sim 100 \mathrm{~ms})$, but would not necessarily require high bandwidth. This is because the messages communicated between switches are short. In a move towards standards, the message sent over the wireless radio system would be using IP and would conform to IEC61850 GOOSE standards. To fix the issue with operator intervention to restore customers past the fault, two distribution circuits would be looped together. With the circuits looped, the faulted section of the loop could be isolated while all other customers would not experience an outage. Based on radio latency and coverage studies, protection logic simulations, and field circuit constraints, the final circuit protection system is being derived. While this project was not able to complete this work, it is being picked up by the SCE Irvine Smart Grid Demonstration project (DOE ARRA Smart Grid Demonstration project) and is planned for installation on the circuit in 2012. Further information on this project phase is available in Section 6.

\subsection{Conclusions}

There are a number of drivers that are making the build-out of the Smart Grid necessary. In the distribution area these include the proliferation of distributed generation (e.g. rooftop photovoltaics, battery storage), introduction of electric vehicles and their chargers, and the increased potential of demand response (e.g. smart appliances, air conditioner control). All of these together argue for the need to implement advanced distribution automation and a new generation of advanced Distribution Management Systems (DMS). Advanced protection should be viewed as an integral part of advanced distribution automation. As part of this work, there is a need to define and develop new, advanced distribution automation functions and schemes. Along with these automation systems, new planning methods also need to be developed. 
More advanced computerized relays, cost-effective phasor measurement units, high power electronic control devices, digital communication technologies etc, are continuously evolving to facilitate improved protection of distribution systems. As a result, the monitoring, control and protection of the power systems in real-time is becoming more effective. These new technologies will hopefully allow the systems to become more efficient and reliable. The protection community should seize these opportunities to make power systems safer, more secure, more reliable and less vulnerable to external threats.

What does this advanced distribution automation system look like? Information and control will be distributed throughout the electrical system in a hierarchical manner. Sensors, remote controlled switches and protective devices will allow integration of fault isolation and system restoration into one function. Microgrids with distributed generation/ storage integrated into utility system can yield higher reliability. Electric vehicles and smart loads will be integrated in a single load management system that conveys pricing signals to the customer. Advanced materials will make possible inexpensive fault current limiting and more efficient transformers.

In this context, advanced DMS applications need to be developed and will include many of the following applications:

- Automatic Fault Location, Prediction, and Protection Systems (FLPPS) and service restoration

- Optimal Volt/VAR Management

- Fast on-line power flow analysis

- Smart distribution operations of the near future

- Fast short circuit / coordination analysis

- Automatic contingency analysis

- Demand response

- Management of distributed generation and storage

- Automatic vehicle location system (crew management)

Advanced fault location, prediction and protection systems will be an important part of this view of the future. Protection systems that can accommodate distributed generation/ storage, electric vehicle charging, and microgrids will be important as the smart grid moves forward. These protection systems will need more communicating sensors that will allow implementation of advanced protection systems. Lower cost fault interrupting switches and protective relays need to become available so that distribution lines can be broken up into smaller parts, affecting fewer customers when there is a fault. Communications need to be developed with lower costs, higher reliability during disasters, and lower latencies for protection needs. Distributed monitoring and control needs to be introduced in a bigger way than has been done in the past. This will allow control at a substation level so that circuits can do a better job of self monitoring and self healing. 
Planning for these advanced systems should be done now, before all of these new generation and load devices are connected to the grid. Through American Recovery and Reinvestment Act (ARRA) projects like the SCE Irvine Smart Grid Demonstration, some of these new concepts can be tested and validated. Because utility planning is usually based on rate case cycles of several years each (3 years for SCE), steps need to be taken so that funds are available for smart grid system improvements when the technologies become available and are needed.

\subsection{Recommendations for Further Work}

Since the primary thrust of this project is on advanced fault protection, location and prediction, the following recommendations are for future work in these three specific areas. These recommendations represent the vision of the investigators based on their experiences with this project and other work they have been involved with. The ideas recommended will hopefully have success in the near future, depending on the pace of technology development.

\subsubsection{Fault Protection}

A lot of creative and new work has been accomplished in this project to advance the state-of-the art of protection systems. The advanced protection scheme designed and implemented for the Circuit of the Future represents the latest state-of-the art technology as described in Section 4. This section describes the details of the new devices installed on the Avanti circuit. The other highlight of the project was the selection the modern Zenergy superconducting fault current limiter. The details of the selection process, installation, lab testing, and field testing results are documented in Sections 5 and 7.

Based on the extensive investigations of this project, a lot of potential exists for advancing the protection schemes for future distribution systems. These schemes will undergo many radical changes due to the incorporation of multitudes of distributed generation/ storage sources and plug-in-hybrid vehicles. These devices will be installed at both primary and secondary distribution levels leading to two-way power flow in the system. Another major change that will occur is the topological change from radial to looped systems. Initially, the primary distribution systems will evolve to looped topology, and then, potentially, to a meshed structure to enhance the reliability, security and efficiency of the smart grid.

Continuous advances in IEDs, signal processing and communication media will determine the progress and penetration of these new developments in distribution system fault location and protection. The increased use of microgrids and distributed generation/ storage sources will change the operation and therefore the methods for fault detection and location in distribution systems. Many systems and algorithms have been proposed and tested to deal with these expected changes in distribution systems but most of these 
proposed developments depend heavily on the reliability, latency, and cost of available communication channels.

The level of penetration of communication dependent applications will eventually drive the cost to acceptable levels but the reliability and latency will depend more on future technical developments. The time constants required for real-time protection and control application in distribution systems are several orders of magnitudes smaller than today's "human interface" focused applications that drive the present development of communication systems. Protection and control applications require small amounts of information to be transferred reliably, in the shortest possible time, to several locations. This is contrary to most present applications where the speed of communication is determined based on the data volume only. New communication systems, developed specifically for the time constants of real-time protection applications, are need to achieve the desired penetration of communication supported protection of distribution systems. Fault detection developments are more affected by the communication latency than fault location or fault prediction developments whose time constants are more in line with present communication systems. To partially cope with the present latency problems, specifically designed prediction and extrapolation algorithms need to be developed to account for the communication channel latency. In addition, detection algorithms requiring external information should be design with fail-safe features that allow them to revert to local operation when the communications signal is delayed or not available. This is a common feature of advanced protection systems presently being tested for transmission.

What do these changes mean to protection? First of all the protection architecture itself has to change. Besides replacing classical electromechanical relays with intelligent digital relays, many of the unintelligent devices such as fuses may have to be abandoned as time passes by. Perhaps the only exception is for protecting single-phase laterals at the end of a feeder. This implies that protection engineers have to change the entire philosophy of relaying. Newer thinking is inevitable and imminent if smart grid goals are to be achieved.

Adaptive protection is another important concept that needs to be implemented for future systems to ensure they can adjust to changes and protect circuits efficiently and effectively.

\subsubsection{Fault Location}

The art and science of fault location has advanced tremendously during the past 20 years. Section 2 provides the latest developments based on the extensive literature search and survey of all stakeholders of the electric utility industry. Even after all these developments, the fault location can not be precisely determined at this stage. As the systems become more and more complex in nature and topology, the location of faults will become a more challenging and arduous task. The investigators strongly believe that protection engineers can take this problem as a challenge and solve it. This requires deploying cost-effective sensors massively at both ends of each line segment, however, 
short they may be. The data collected from these sensors along with the real-time data provided, will advance fault location, hopefully, in the near future. This will also require developing new algorithms for the design and coordination of protection. In the final analysis, the entire subject of protection design has to be undertaken as a challenging task with no pre-conceived notions involved. The distribution protection for the next 100 years will be radically different from the last 100 .

\subsubsection{Fault Prediction}

With existing technology, data mining has been applied successfully in several locations for fault prediction. Unfortunately these applications have not achieve the level of penetration expected based on the level of success achieved by the few existing applications. Part of the limitation is due to the time required to create a significant base of data. The extremely localized nature of the fault phenomena also precludes the direct application of the development techniques to distribution systems in different locations. Additional studies are required in the nature of regional variation of fault data and its applications to fault detection. In addition, there needs to be more development of data collection systems and maintenance of data bases.

While pattern recognition techniques for prediction of faults have made significant strides, these are not sufficient to make future systems resilient and self-healing. What is really needed is a fault prediction device installed at each distribution substation. Its role would be to constantly monitor the system, warn of any impending or brewing problems, and alert the system operators for immediate redial action. This system should also be able to seamlessly correct the problem by itself. In other words this device has to be quite smart, almost matching the operator's intelligence and yet aid him/her in making the system very reliable. 


\section{Appendix A: $\quad$ Literature Search and Results}

Literature Review of Fault Location, Prediction and Detection for Distribution Systems

Report to SCE

By

P. Parektar

D. Fan

S. Ming

R. Phillips

K. Dekhane

V. Centeno

S.S. Venkata

$06 / 30 / 2010$ 
Table of Content

Appendix A: Literature Search and Results Error! Bookmark not defined.

A.1. Introduction 131

A.2. Fault Location and Prediction 132

A.2.1 Fault Location 132

A.2.1.1 Signal Analysis Techniques 132

A.2.1.1.1 Fundamental frequency approach 132

A.2.1.1.2 High frequency components approach

A.2.1.2 Knowledge Based Techniques 133

A.2.2 Fault Prediction 133

A.2.2.1 Early Detection Techniques 133

A.2.2.2 Data Mining Techniques 133

A.2.2.3 Hidden Failures $\quad 133$

A.2.3 Comments and Recommendations $\quad 134$

A.2.3.1 Most Promising Publications 134

A.2.3.1.1 Fault location, fundamental frequency

A.2.3.1.2 Fault location, high frequency 135

A.2.3.1.3 Fault location, knowledge based 135

A.2.3.1.4 Fault prediction 136

A.2.3.2 Recommendations 137

A.2.3.2.1 Recommendations of potential focus on fault location: 137

A.2.3.2.2 Recommendations on potential focus on fault prediction: 137

A.2.4 Synopses of Fault Location and Fault Prediction Techniques 139

A.2.4.1 Fault Location, Signal Analysis Techniques 139

A.2.4.1.1 A new fault location algorithm using direct circuit analysis for distribution systems 139

A.2.4.1.2 An intelligent and efficient fault location and diagnosis scheme for radial distribution systems [6] 139

A.2.4.1.3 Accelerated protection of distribution systems with tapped off loads [7] 140

A.2.4.1.4 A mathematical approach for identification of fault sections on the radial distribution systems: voltage sensor [8] 141

A.2.4.1.5 A new fault location algorithm for distribution networks [9] 141

A.2.4.1.6 Automated fault location and diagnosis on power distribution feeders [10] 142

A.2.4.1.7 Fault locating in ungrounded and high-resistance grounded systems [11]143

A.2.4.1.8 Fault locating in ungrounded and compensated systems [12] 144

A.2.4.1.9 A three-phase fault detection algorithm for radial distribution networks [13] 145

A.2.4.1.10 A voltage-based fault location method for radial distribution networks [14] 146

A.2.4.1.11 A technique for estimating locations of shunt faults on distribution lines [15] 148

A.2.4.1.12 Fault location method for distribution networks using distributed parameter line model [16] 
A.2.4.1.13 Accurate Non-iterative Fault Location Algorithm Utilizing Two-End Unsynchronized Measurements [17] 149

A.2.4.1.14 A New Fault Location Algorithm Using Direct Circuit Analysis for Distribution Systems [18] 150

A.2.4.1.15 An Intelligent Fault Location and Diagnosis System On Radial Distribution Systems [19] 151

A.2.4.1.16 A Fault Locator for Radial Subtransmission and Distribution Lines [20] 151

A.2.4.1.17 Calculational Fault Location For Electrical Distribution Networks [21] 152

A.2.4.1.18 Automated Fault Location System for Primary Distribution Networks [22] 153

A.2.4.2 Fault Location, High-frequency Approach 153

A.2.4.2.1 New concept in fault location for overhead distribution systems using superimposed components [23] 153

A.2.4.2.2 A new fault location technique for radial distribution systems based on highfrequency signals [24]154

A.2.4.2.3 A new principle of fault line selection for distribution [25] 156

A.2.4.2.4 A fault transient comparison technique for multi-ended distribution feeders [26] 158

A.2.4.2.5 An accurate fault location technique for distribution lines with tapped loads using wavelet transforms [27]159

A.2.4.2.6 Fault location using traveling wave for power networks [28] $\quad 160$

A.2.4.2.7 Fault location in distribution systems based on traveling waves [29] 160

A.2.4.2.8 Fault section identification and location on a distribution feeder using traveling waves [30] 161

A.2.4.2.9 A new approach to fault location in a single core underground cable system using combined fuzzy logic and wavelet analysis [31] 162

A.2.4.2.10 Accurate fault location technique for distribution system using fault-generated high-frequency transient voltage signals [32]162

A.2.4.2.11 Feature extraction method for high impedance ground fault localization in radial power distribution networks [33] 163

A.2.4.2.12 Application of GPS based fault location scheme for distribution system [34] 164

A.2.4.2.13 A novel fault locator based on the detection of fault generated high-frequency transients [35] 165

A.2.4.2.14 Design of a low voltage cable fault detector [36] 166

A.2.4.2.15 An Alternative Fault Location Algorithm Based on Wavelet Transforms for Three-Terminal Lines [37] 166

A.2.4.3 Fault Location: Knowledge Based Techniques 167

A.2.4.3.1 AI-based methods in practical fault location of medium voltage distribution feeders [38] $\quad 167$

A.2.4.3.2 A network distribution power system fault location based on neural eigenvalue algorithm [39] 168

A.2.4.3.3 Application of a fuzzy set method in distribution system fault location [40] 
A.2.4.3.4 Earth fault distance computation with artificial neural network trained by neutral voltage transients [41]169

A.2.4.3.5 A computerized fault location process for overhead radial distribution feeders [42] $\quad 170$

A.2.4.3.6 Fault section estimation in electrical power systems using Artificial Neural Network (ANN) approach [43] 170

A.2.4.3.7 A new approach to ungrounded fault location in a three-phase underground distribution system using combined neural networks and wavelet analysis [44] 172 A.2.4.3.8 Artificial Neural Network and Support Vector Machine approach for locating faults in radial distribution systems [45] 173

A.2.4.3.9 Fault Diagnosis and Reconfiguration for Multilevel Inverter Drive Using AIBased Techniques [46] 174

A.2.4.3.10 An Unsupervised Learning Method for Comparing the Quality of the Soft Computing Algorithms in Analog Systems Diagnostics [47] 175

A.2.4.3.11 Double Circuit Transmission Line Fault Distance Location using Artificial Neural Network [48] 175

A.2.4.3.12 Fault Classification and Location of Power Transmission Lines Using Artificial Neural Network [49] 176

A.2.4.3.13 Study on Fault Location for High Voltage Overhead Transmission Lines Based on Neural Network System [50] 176

A.2.4.3.14 Performance Evaluation of an Adaptive-Network Based Fuzzy Inference System Approach for Location of Faults on Transmission Lines Using Monte Carlo Simulation [51] $\quad 177$

A.2.4.4 Fault Prediction 178

A.2.4.4.1 Failure analysis in $\mathrm{ZnO}$ arresters using thermal images [52] 178

A.2.4.4.2 Recognition of electrical tree progress in solid insulator [53] 178

A.2.4.4.3 Data mining and analysis of tree-caused faults in power distribution systems [54] 179

A.2.4.4.4 On-Line condition monitoring and diagnostics of power distribution equipment [55] $\quad 180$

A.2.4.4.5 Prediction of weather-related failures of overhead distribution feeders [56] 181

A.2.4.5.6 Analysis of hidden failures of protection schemes in large interconnected power systems [57] 182

A.2.4.4.7 Characterization of transients in transformers using discrete wavelet transform [58] 183

A.2.4.4.8 Getting a clear picture of distortion [59] 184

A.2.4.4.9 Prevention of underground cable splice failures [60] 185

A.2.4.4.10 Most recent developments in surge arrester technology and testing [61]186

A.2.4.4.11 Analysis and prevention of animal-caused faults in power distribution systems [62] 188

A.2.4.4.12 Failure prediction of underground distribution feeder cables [63]

A.2.4.4.13 Transformer failure prediction using Bayesian analysis [64] 190

A.3. Fault Protection 192

A.3.1 Distribution Automation

192

A.3.2 High Impedance Faults 192 
A.3.3 Fault Current Limiters 193

A.3.4 Comments and Recommendations

194

A.3.4.1 Distribution Automation 194

A.3.4.2 High Impedance Faults 194

A.3.4.3 Fault Current Limiters $\quad 195$

A.3.5 Synopses and Historical Overview 196

A.3.5.1 Synopses: Distribution Automation 196

A.3.5.1.1 Advanced feeder automation is here [65] 196

A.3.5.1.2 Interface between distribution automation system and SCADA to minimize the feeder loss [66] 197

A.3.5.1.3 Implementation of network reconfiguration for Taiwan Power Company [67] 198

A.3.5.1.4 New triple-single re-closers improve distribution reliability [68] 199

A.3.5.1.5 Impact of two-stage service restoration on distribution reliability [69] 200

A.3.5.1.6 Coordinated local and centralized control in distribution management systems [70] 201

A.3.5.1.7 Automated solutions for distribution feeders [71] 202

A.3.5.1.8 A multi-objective algorithm for distribution networks restoration [72] 204

A.3.5.1.9 Pilot project to estimate the centralized volt/var control effectiveness [73] 205

A.3.5.1.10 Distribution feeder reconfiguration for operation cost reduction [74] 206

A.3.5.1.11 Network reconfiguration in distribution systems for loss reduction and load balancing [75] 207

A.3.5.1.12 Multi-objective feeder reconfiguration by distribution manager system [76] 207

A.3.5.1.13 Distribution capacitor automation provides integrated control of customer voltage levels and distribution reactive power flow [77] 208

A.3.5.1.14 Adaptive distance protection of a double-circuit line [78] 209

A.3.5.1.15 Prospects for distribution automation at Pacific Gas \& Electric Company [79] 209

A.3.5.1.16 Distribution feeder reconfiguration for loss reduction [80] 210

A.3.5.1.17 Installation of Hydro-Quebec's distribution feeder automation system [81] 211

A.3.5.1.18 An Adaptive Non-communication Protection for Distribution Systems [82] 212

A.3.5.1.19 Implementing Multiagent Systems Technology for Power Distribution Network Control and Protection Management [83] 212

A.3.5.1.20 Impact of automatic control systems of loop restoration scheme on the distribution system reliability [84] 213

A.3.5.1.21 Research of Feeder Automation System Based on Multi-Agent [85] 213 A.3.5.1.22 Outage Avoidance through Intelligence Detection of Incipient Equipment Failures on Distribution Feeders [86] 214

A.3.5.1.23 Feeder Automation System Based on Non-communication Protection and Control Unit [87] 215

A.3.5.2 Historical development, High Impedance Faults 215

A.3.5.2.1 Time domain techniques 215 
A.3.5.2.2 Frequency domain techniques $\quad 217$

A.3.5.2.3 Pattern recognition and knowledge based approaches 218

A.3.5.2.4 High impedance fault modeling and testing $\quad 220$

A.3.5.2.5 Commercial devices 222

A.3.5.2.5 Literature Update 2007-2010 225

A.3.5.2.5.1 Performance of Restricted Earth Fault Protection Scheme in the Presence of Current Transformer Remanence [144] 225

A.3.5.2.5.2 Performance of Novel Neutral Admittance Criterion in MV-Feeder Earth Fault Protection [145] 225

A.3.5.2.5.3 Evaluation of Fault Protection Methods using ATP and MathCAD [146] 226

A.3.5.2.5.4 Network Integrated DSP-based Adaptive High Impedance Ground Fault feeder Protection [147] 226

A.3.5.3 Synopses, Fault Current Limiters 228

A.3.5.3.1 Superconducting fault current limiters [148] 228

A.3.5.3.2 Superconducting fault current limiter [149] 230

A.3.5.3.3 Principle and characteristics of fault current limiter with series compensation [150] 231

A.3.5.3.4 Fault current limiter applications on medium and high voltage power distribution systems [151] 232

A.3.5.3.5 Study of fault current limiters using YBCO thick film material [152] 233

A.3.5.3.6 Discussion of "Fault-current limiter applications in medium-and-high-voltage power distribution systems" [153] 234

A 3.5.3.7 Enhancement of power system transient stability using superconducting fault current limiters [154] 234

A.3.5.3.8 Setting method of specific parameters of a superconducting fault current limiter considering the operation of power system protection [155] 235

A.3.5.3.9 A new model of fault current limiter using YBCO thin film [156] 236

A.3.5.3.10 Stabilization of synchronous generators by superconducting fault current limiter [157] 237

A.3.5.3.11 A nitrogen gas cooled, hybrid, high temperature superconducting fault current limiter [158] 237

A.3.5.3.12 Superconducting current limiters [159] 238

A.3.5.3.13 Stability analysis of a power system with superconducting fault current limiter installed [160] 238

A.3.5.3.14 Application studies of superconducting fault current limiters in electric power systems [161] 239

A.3.5.3.15 Analysis on the impact of HTS cables and fault current limiters on power systems [162] 241

A.3.5.3.16 Reduction of rating required for circuit breakers by employing seriesconnected fault current limiters [163]242

A.3.5.3.17 The influence of superconducting fault current limiter structure on the V-I characteristic [164] 242

A.3.5.3.18 Magnetic-shield type fault current limiter equivalent circuit [165] 243 A.3.5.3.19 Experiment and research on control strategy for a new-type three-phase solidstate current limiter [166] 244 
A.3.5.3.20 Proof-of-concept prototype test results of a superconducting fault current limiter for transmission-level applications [167] 244

A.3.5.3.21 Non-inductive variable reactor design and computer simulation of rectifiertype superconducting fault current limiter [168] 247

A.3.5.3.22 Applications of superconducting fault current limiters in electric power transmission systems [169] 248

A.3.5.3.23 Fault current limiters report on the activities of CIGRE WG A3.16 249

A.3.5.3.24 EMTP RV - based study of solid-state fault current limiter for distribution systems [171] 250

A.3.5.3.25 Development and test of $10.5 \mathrm{kV} / 1.5 \mathrm{kA}$ HTS fault current limiter [172] 254

A.3.5.3.26 State of Art of Fault Current Limiters and their impact on Overcurrent Protection [173] 254

A.3.5.3.27 Using Fault Current Limiter to Minimize Effect of Thyristor Controlled Series Capacitor on Over Reach Problem of Distance Protection [174] 254

A.3.5.3.28 Impact of Solid-State Fault Current Limiters on Protection Equipment in Transmission and Distribution Systems [175] 255

A.3.5.3.29 Minimizing the Impact of Distributed Generation on Distribution Protection System by Solid State Fault Current Limiter [176] 256

A.4. General Recommendations 257

A.4.1 Project Requirements 257

A.4.2 Comments and Recommendations $\quad 257$

A.4.2.1 Recommendation 1258

A.4.2.2 Recommendation 2258

A.4.2.3 Recommendation 3258

A.4.2.4 Recommendation 4258

A.4.2.5 Recommendation $5 \quad 259$

A.5. References 260

A.5.1 Reviews on Fault Location $\quad 260$

A.5.2 Standards on Fault Location 260

A.5.3 Fault Location, Fundamental Frequency Methods 260

[17] J. Izykowski, E. Rosolowski, P. Balcerek, M. Fulczyk and M. M. Saha, "Accurate Noninterative Fault Location Algorithm Utilizing Two-End Unsynchronized Measurements," IEEE Transactions on Power Delivery, Vol. 25, Jan. 2010, pp. 72-80. 261

A.5.4 Fault Location, High frequency based Methods 262

A.5.5 Fault Location, Knowledge Based Methods 263

A.5.6 Fault Prediction 264

A.5.7 Distribution Automation 266

A.5.8 High Impedance Faults 268

A.5.9 Fault Current Limiters 273 


\section{A.1. Introduction}

One of the main objectives of this DOE-sponsored project is to reduce customer outage time. Fault location, prediction, and protection are the most important aspects of fault management for the reduction of outage time. In the past most of the research and development on power system faults in these areas has focused on transmission systems, and it is not until recently with deregulation and competition that research on power system faults has begun to focus on the unique aspects of distribution systems.

To concentrate the review on recent developments, we have limited the literature review to the period from 1990 to the present day. This period covers two important aspects of fault research: a) it encompasses the acceptance and maturing period of digital devices in power systems protection functions, and $b$ ) it includes the period of fast development of communication systems that have enhanced the flexibility and area of impact of digital protection systems. When appropriate, earlier publications were reviewed to establish conceptual bases for later developments.

To limit the search to accessible publications, the search was based on subject and title search on the IEEE Xplore, Google scholar, and the Science Citation Index. The EndNote X.02 software was used to collect and format the lists of references. Paper synopses were performed by a team of students and faculty following pre-established review criteria for each section.

The report is divided into two main sections and two minor sections. This introduction is Section 1 of the report. Section 2 covers the review on Fault Location and Prediction. Section 3 covers Fault Protection and is subdivided into automation, high impedance faults and fault current limiters. Section 4 presents the summary of recommendations and possible implementation on SCE's "The Circuit of the Future," which represents the future of distribution systems. 


\section{A.2. Fault Location and Prediction}

\section{A.2.1 Fault Location}

From the available literature, recent research in fault location can be classified into two main approaches: Signal Analysis and Knowledge Based. Each method offers its own set of requirements, advantages, and disadvantages. In this report, a short overview of the methods, a list of the most promising methods and a synopsis of the most significant papers on fault location are presented [1-3].

\section{A.2.1.1 Signal Analysis Techniques}

For this report, signal analysis techniques are divided into fundamental frequency and high-frequency component methods. Knowledge Based systems use artificial intelligence, fuzzy logic, and neural networks.

\section{A.2.1.1.1 Fundamental frequency approach}

The fundamental frequency approach was first applied using microcontrollers by Takagi in the early 1980s [4]. Since then, different fundamental frequency methods have been derived, and most of them require a detailed modeling of the system, such as accurate line parameters, load data, etc. Some of these methods only provide information on the faulted section and not an estimate of the actual fault location. Other methods show an increase in percentage error with higher fault resistance values. However, most of these methods have the advantages of being single-ended and not requiring communication channels.

Synopses of the papers with the most promising methods and other significant papers using the fundamental frequency approach are listed in Section A.2.4.1.

\section{A.2.1.1.2 High frequency components approach}

Methods using high-frequency components are mostly based on traveling wave theory. These methods do not have such stringent requirements of accurate line and load modeling. However, multiple reflections and refractions, which are typical of distribution systems with many laterals and load taps, is a concern while using traveling wave based methods. Although quite accurate in operation, most of these methods require communication channels and GPS time synchronization although some single-ended methods are also proposed in the literature. The sampling rate requirements of these methods are generally quite high. Not many sufficient amplitude traveling waves are generated when a fault occurs on the system at or near zero-voltage point, thus, the detection of these waves becomes difficult. Some methods using fault-generated highfrequency transients are being proposed in the literature. These transients are present even when the fault occurs at low voltage conditions. 
The synopses of the papers with the most promising methods and other significant papers using the high-frequency approach are listed in Section 2.4.2.

\section{A.2.1.2 Knowledge Based Techniques}

The methods using Artificial Intelligence mainly use Fuzzy logic or Artificial Neural Networks (ANN) to locate the fault. The feature extraction is a crucial step in the ANNbased scheme. Some of these methods combine information obtained from the network database and the SCADA with heuristic knowledge of operators.

The synopses of the papers with the most promising methods and other significant papers using a Knowledge Based approach are listed in section 2.4.3.

\section{A.2.2 Fault Prediction}

The intent of fault prediction is to determine an imminent failure in a system component early enough to allow for maintenance or replacement of the suspected component. For this report, fault prediction is divided into early detection techniques, data mining, and hidden failures.

\section{A.2.2.1 Early Detection Techniques}

A large portion of the research into these techniques concentrates on thermal analysis and other non-invasive techniques for the early detection of damaged components such as transformers, fault arresters, and insulators. Some techniques have been developed to identify current or voltage signals that may indicate imminent component failures.

A.2.2.2 Data Mining Techniques

The availability of large fault databases has also sparked the use of a statistical data mining approach to analyze and address the causes of distribution system faults and to investigate the influence of selected factors on distribution system faults. This type of study aims to determine the influence of external factors on the occurrence of some types of faults and the determination of maintenance practices or allocation of resources to reduce the likelihood of a fault.

\section{A.2.2.3 Hidden Failures}

Some of the new interesting concepts developed for transmission systems address the problem of undetected failures in the protection system and their possible failuremagnifying effect after system contingencies. Although developed for transmission systems, these concepts are also applicable to distribution systems [53].

Due to the limited literature on fault prediction, all the synopses on fault prediction have been placed in Section 2.4.4. 
A.2.3 Comments and Recommendations

A.2.3.1 Most Promising Publications

A.2.3.1.1 Fault location, fundamental frequency

The following methods for fault location based on the fundamental frequency approach seem more promising:

The publication "A new fault location algorithm using direct circuit analysis for distribution systems [5]" presents a fault location algorithm (at the moment limited to single-phase-to-ground faults) using direct circuit analysis for balanced as well as unbalanced distribution systems (three-phase or three/single-phase). Instead of using the symmetrical component analysis technique, which is conveniently applicable only to balanced systems, this method uses direct three-phase circuit analysis techniques which can deal with any unbalance in the system such as unbalanced faults, single-phase lateral loads, etc. The fault location equation has been derived by applying matrix inverse lemma.

"An intelligent and efficient fault location and diagnosis scheme for radial distribution systems [6]" proposes a fault location algorithm which identifies candidate fault locations using an iterative estimation of load current and fault current at each line section. Then a fault diagnosis scheme is used to first identify the fault location among multiple candidate locations using current pattern information specified by the protective device operation and comparing it to the expected one. Then, the method attempts to diagnose the most likely one using interrupted load information.

The publication entitled "Accelerated protection of distribution systems with tapped off loads [7]" presents an accelerated operation scheme for the protection of distribution systems with tapped off loads. In the scheme, the relays are arranged to have two operation modes depending on their locations in the time-grading coordination, which are the fixed time setting and the accelerated operating modes. In the accelerated operation mode, the relay makes tripping decisions by detecting the remote breaker operation, i.e. detecting the breaker operation by determining whether the system is in a balanced operation or not. The ratio signals and superimposed signals (defined in the paper) are used to detect the remote breaker operation, from which it is determined whether a fault is on the protected section. This scheme thus helps to significantly increase the response speed of conventional directional-overcurrent-relay-based protection techniques for systems with tapped off loads.

"A mathematical approach for identification of fault sections on the radial distribution systems: voltage sensor [8]" proposes a new mathematical algorithm for detecting faulted sections and the fault-affected areas. Minimum voltage sensors are installed at various locations at the customer ends where the radial network ends. These sensors measure the three-phase voltages and are set to threshold values to detect whether or not the node loses electrical power. When the voltage sensors detect that the node voltage is lower than its predetermined threshold value, the contact of an alarm device that is associated with this sensor closes to enable operation of the remote fault indicator device for communication purposes. The method uses messages obtained from operated voltage 
sensors (through communication) and topological information of distribution networks to accurately detect fault section(s) and fault-affected area(s) due to single as well as multiple faults. A mathematical algorithm using $\mathrm{C}++$ is developed and employed. A.2.3.1.2 Fault location, high frequency

The following methods for fault location based on the high-frequency approach seem more promising:

"New concept in fault location for overhead distribution systems using superimposed components [23]" presents a novel approach in single-ended fault location for overhead distribution systems based on the concept of superimposed voltages and currents. The superimposed values are simply the differences between the total fault and pre-fault steady-state voltage/current signals. The methodology outlined is based on determining the superimposed values of voltage and current at any assumed fault point from the measured pre-fault and fault values. Using a specified model of the distribution system under consideration, these superimposed voltages and currents are employed to ascertain the voltage at an assumed fault position; this is then back-injected at the assumed fault point in order to check the fault-path currents in the un-faulted phases. Only when the fault point is correct will the healthy phase(s) at the actual fault point in the model attain a near zero value.

"A new fault location technique for radial distribution systems based on high-frequency signals [24]" describes a single-ended fault location technique for radial distribution feeders based on the information provided by the measurements at the substation and the feeder configuration. The proposed method first identifies the fault path, based on the traveling wave information provided by the high-frequency components of the recorded fault transient signals. It then calculates the exact location along the identified path based on the power frequency signals. The phase voltages and currents at the substation are recorded and transformed into modal domain. Then the modal signals are decomposed into their wavelet coefficients (WTC). The WTC of the voltage signal in mode 1 are used for identifying the faulted lateral. The sum of squares of the WTC is used as the identifier for this purpose. The identification of the faulted lateral is based on the voltage signal decomposition in a frequency spectrum from 12 to $25 \mathrm{kHz}$; therefore, voltage transducers with a bandwidth of $50 \mathrm{kHz}$ are needed. Once the faulted lateral is identified, the system is simplified, representing the non-faulted laterals as equivalent impedances. Then the post-fault phasors are extracted from the WTC decomposition of both current and voltage signals at the power frequency scale, and the distance is calculated (using conventional methods).

A.2.3.1.3 Fault location, knowledge based

The following methods for fault location using knowledge based techniques seem more promising:

"AI-based methods in practical fault location of medium voltage distribution feeders [38]" describes a fault location expert system, an application of a distribution management system, which is a part of the integrated control center environment composed of different computer systems and distribution automation. The expert system applies AI-based methods (e.g. object-oriented modeling, blackboard techniques, fuzzy 
reasoning, decision tree, etc.) in incorporating data on external systems in order to support the control center operator in network operation. At present, the expert system is in everyday use in several distribution utilities.

"A network distribution power system fault location based on neural eigenvalue algorithm [39]" describes a new approach to fault location in distribution systems using an Eigenvalue and an Artificial Neural Network based learning algorithm. The main steps of the algorithm are Data acquisition (phase currents; no voltages required) Clarke-Concordia transformation of sample data and eigenvalue approach obtaining eigenvectors and eigenvalues Fault detection (compare fault and pre-fault characteristic eigenvalues) Fault classification (compare characteristic eigenvectors with fault patterns) Fault location/distance calculation (using eigenvalues as neural network inputs)

The applied ANN is of the "feed forward" type, and "error-back-propagation" is considered.

A.2.3.1.4 Fault prediction

The following methods for fault prediction techniques seem more promising:

"Data mining and analysis of tree-caused faults in power distribution systems [50]" proposes a statistical data mining approach to analyze and address the tree-caused fault characteristics according to system configuration and environmental factors using a large outage database. Based on previous work on animal-caused failures [58], the researchers selected six of thirty-three information recorded factors for their data mining. Four measurements are applied to the data set. Actual measurements, to begin, are used to extract information without the regional factors and can be used to allocate trimming resources for each region. Next, normalized measurements are used to provide relative frequency and importance of three faults and to determine the relative importance of this type of fault to help set prevention priorities. Lastly, the relative measurement and likelihood measurement are used to extract more detailed information from the data set. The study found that all six factors are statistically significant except in one region, and season is the least significant factor. The researchers conclude that outage databases can provide quantitative and qualitative information to develop a more effective restoration system and design a more effective prevention strategy.

"Prevention of underground cable splice failures [56]"describes the events recorded in Florida Power Corporation that lead to the identification of the self clearing cable splice fault. The paper also describes the relay hardware and architecture used to implement a sub-cycle overcurrent protection scheme for self-clearing faults that occur in cables due to insulation breakdown. For the basic operation of the scheme the current input to the relay is compared to a fast instantaneous element that responds to faults within $1 / 4$ cycle. When the circuit beaker opens, the self-clearing logic is blocked, assuming the fault has been cleared. If the circuit breaker does not open or if the fault does not last longer than $1 / 2$ cycle, it is assumed that a self-clearing fault has occurred. If the number of faults exceeds the desired value within the time period setting, contacts will close, initiating 
alarming or tripping. This incipient failure detection scheme was successfully integrated into a universal relay platform as an additional function of a universal digital protection scheme intended for distribution feeder protection.

"Analysis of hidden failures of protection schemes in large interconnected power systems [53]": presents a methodology to evaluate the hidden failures' effects based on regions of vulnerability and areas of consequence in protection systems. The authors define hidden failures as "a permanent defect that will cause a relay or a relay system to incorrectly and inappropriately remove a circuit element(s) as a direct consequence of another switching event." A region of vulnerability is defined as the physical area in which the occurrence of an event will trigger the unwanted disconnection due to the hidden failure. These regions are determined by a particular location, dimension, and magnitude depending on the hidden failure mode of the protection system and its location within the power system. The consequences of the unwanted disconnections in the power system are examined and combined with the magnitude of the regions of vulnerability to create an "index of severity." This index identifies the critical protection systems, whose unwanted operations would disconnect power system elements affecting considerably the power system integrity and its capacity to deliver energy.

\section{A.2.3.2 Recommendations}

Although there are very interesting research areas in distribution system fault location and prediction, these recommendations are shaped by the characteristics of SCE's "Circuit of the Future," available communication system, protective relaying, and positive impact on other SCE distribution systems.

A.2.3.2.1 Recommendations of potential focus on fault location:

Implement one of the recommended high-frequency component methods. Highfrequency methods take advantage of the availability of communication channels and include some aspects of GPS time synchronization. This could help gauge the impact of high bandwidth communication systems and time synchronization in distribution systems.

Perform a comparison of the three approaches to fault location by selecting and implementing the most promising technique for each method. This could provide very valuable information on the best approach to follow on other existing systems as they evolve into future distribution systems.

A.2.3.2.2 Recommendations on potential focus on fault prediction:

Implement a differential-signal analysis system with high sampling frequency, time synchronization, and an array of sensitive trigger parameters. The cable splicing detection techniques could serve as the first test for the proposed system. Addition of time synchronization to the thermal information derived from the installed fiber optics may enhance the performance of the proposed system. Implementation of specific fault prediction techniques will depend on the results of the detailed analysis of the collected data. 
Apply the concept of hidden failures to distribution systems and determine the effect of hidden failures by increasing customer down time. Two aspects of hidden failures could be studied on the system: the current effect of hidden failures in distribution systems and the effects of hidden failures on distribution systems of the future. The availability of high bandwidth communications, advanced IED's, and the use of fault current limiters facilitate the implementation of a more adaptable protection system. But these increasingly complex systems will become more vulnerable to problems such as hidden failures in the protection elements. 


\section{A.2.4 Synopses of Fault Location and Fault Prediction Techniques}

Synopses are divided into Signal Analysis Techniques, High-frequency Approach, and Knowledge Based Techniques. Each synopsis consists of a brief summary and, when applicable, comments on a list of implementations, issues, and feasibility.

A.2.4.1 Fault Location, Signal Analysis Techniques

A.2.4.1.1 A new fault location algorithm using direct circuit analysis for distribution systems [5]

Summary: This paper proposes a fault location algorithm using direct circuit analysis for balanced as well as unbalanced distribution systems (three-phase or three/single-phase). Instead of using the symmetrical component analysis technique, which is conveniently applicable only to balanced systems, this method uses a direct three-phase circuit analysis technique which can deal with any unbalances in the system such as unbalanced faults, single-phase lateral loads, etc. The load is modeled as constant impedance, and thus the fault current can be expressed as a function of impedance and measured current by using current distribution rules. The fault location equation has been derived by applying matrix inverse lemma.

Implementation: Simulation results using EMTP (1920 Hz sampling frequency) for a sample distribution system are presented. The results obtained using this method for balanced 3-phase system and an unbalanced 3-phase system are compared with the conventional method using distribution factors of the negative sequence current (procedure described in the paper). Considerable improvement in error, particularly in the unbalanced case, is observed. No actual field testing has been reported in the paper.

Issues:

The paper presents the method for only single-phase-to-ground faults. A phase-to-phase fault procedure needs to be developed.

The load is modeled as constant impedance which may cause errors in certain conditions. The system model for this study has only one source. More results are required to validate the method.

This method requires accurate information about load and line parameters including self inductances and mutual inductances.

The faulted network circuit equations are based on steady-state analysis.

A.2.4.1.2 An intelligent and efficient fault location and diagnosis scheme for radial distribution systems [6]

Summary: This paper proposes a fault location algorithm which identifies candidate fault locations using an iterative estimation of load current and fault current at each line section. This method is similar to the one used in a previous paper [9], but it presents a detailed method on fault diagnosis. The fault diagnosis scheme proposed in this study consists of two steps. It first identifies the fault location among multiple candidate locations using current pattern information specified by the protective device operation 
and comparing it to the expected one. For example, different fault locations have different re-closer actions as well as different current patterns. However, this first step could still end up with more than one location. Then, the second step attempts to diagnosis the most likely one using interrupted load information. For example, different fault locations may have different fault current magnitudes during the operation of the recloser.

Implementation: EMTP simulation results for a sample $22.9 \mathrm{kV}$ feeder with 21 nodes and single-phase and three-phase laterals are provided in the paper. The effects on the results due to use of different load models, different fault resistance values, and load variations are presented. No actual field testing is reported in the paper.

Issues:

This method requires accurate information about load characteristics and line parameters including self inductances and mutual inductances.

The faulted network circuit equations are based on steady-state analysis.

Waveforms recorded by DFRs are needed to do the fault diagnosis if there are multiple fault locations.

Feasibility: Simulation results indicate the estimation errors increase with increases in fault resistance; however, the effect of load variation is small. More results and field testing is required to validate this method's reliability under all operating conditions.

\section{A.2.4.1.3 Accelerated protection of distribution systems with tapped off loads [7]}

Summary: This paper presents an accelerated operation scheme for the protection of distribution systems with tapped off loads. In the scheme, the relays are arranged to have two operation modes depending on their locations in the time-grading coordination, which are the fixed time setting and the accelerated operating modes. In the accelerated operation mode, the relay makes tripping decisions by detecting the remote breaker operation, i.e. detecting the breaker operation by determining whether the system is in a balanced operation or not. The ratio signals and superimposed signals (defined in the paper) are used to detect the remote breaker operation, from which it is determined whether a fault is on the protected section.

This scheme thus helps to significantly increase the response speed of conventional directional-overcurrent-relay-based protection techniques for systems with tapped off loads.

Implementation: Simulation results on an $11 \mathrm{kV}$ multi-section ring-main system with tapped off loads is presented in the paper. Scenarios of single-phase faults, phase-tophase faults and high-impedance faults are studied, and results are reported. The authors also state that this technique can be easily extended to system configurations with more line sections and multiple sources. No actual field testing is reported in the paper.

Issues: 
Results with multiple-source system are required.

Actual field testing is required to validate the performance of the technique for different operating conditions.

Feasibility: The method shows promise. One advantage is that it is a non-communication method. Also, the authors state that this technique can be easily implemented into the existing relay hardware and offers a direct plug-in replacement for the existing relays in service.

A.2.4.1.4 A mathematical approach for identification of fault sections on the radial distribution systems: voltage sensor [8]

Summary: This paper proposes a new mathematical algorithm for detecting faulted sections and the fault-affected areas. Minimum voltage sensors are installed at various locations at the customer end where the radial network ends. These sensors measure the three-phase voltages and are set to threshold values to detect whether or not the node loses electrical power. When the voltage sensors detect that the node voltage is lower than its predetermined threshold value, the contact of an alarm device associated with this sensor closes to enable operation of the remote fault indicator device for communication purposes. The method uses messages obtained from operated voltage sensors (through communication) and topological information of distribution networks to accurately detect fault section(s) and fault-affected area(s) due to single as well as multiple faults. A mathematical algorithm using $\mathrm{C}++$ is developed and employed.

Implementation: Sample results for a one-source, 15-node radial distribution system are presented in the paper. No actual field testing is reported.

Issues:

Multiple-source systems are not considered.

The method provides the faulted section and not the estimate of fault location itself. Actual field testing is needed.

Feasibility: The number of sensors required is less (sensors required only at the load buses) as compared to the conventional methods. The method is effective for both single and multiple faults in the radial system.

\section{A.2.4.1.5 A new fault location algorithm for distribution networks [9]}

Summary: This paper starts with a brief discussion on some general difficulties encountered in determining an accurate estimation of fault location in distribution (MV) networks. It presents a two-step method to determine the distance to a fault in MV networks. First, the equivalent positive and zero-sequence impedance of the network is computed in pre-fault steady-state for all nodes of the network based on existing topology, loads, and feeder parameters. These values represent positive and zero- 
sequence impedances as seen from the substation to a given node of the network. Then, after the fault, the specific fault-loop parameters are calculated depending on the fault type (phase-to-phase or phase-to-ground) and the place of measurements (at the substation or at the faulty feeder). The fault resistance is also included in the fault loop. The fault place is determined by checking a certain set of conditions for consecutive nodes of the network.

The set of conditions to be checked for phase-to-phase and phase-to-ground faults is provided in the paper. The proposed method is represented in Figure A.1.

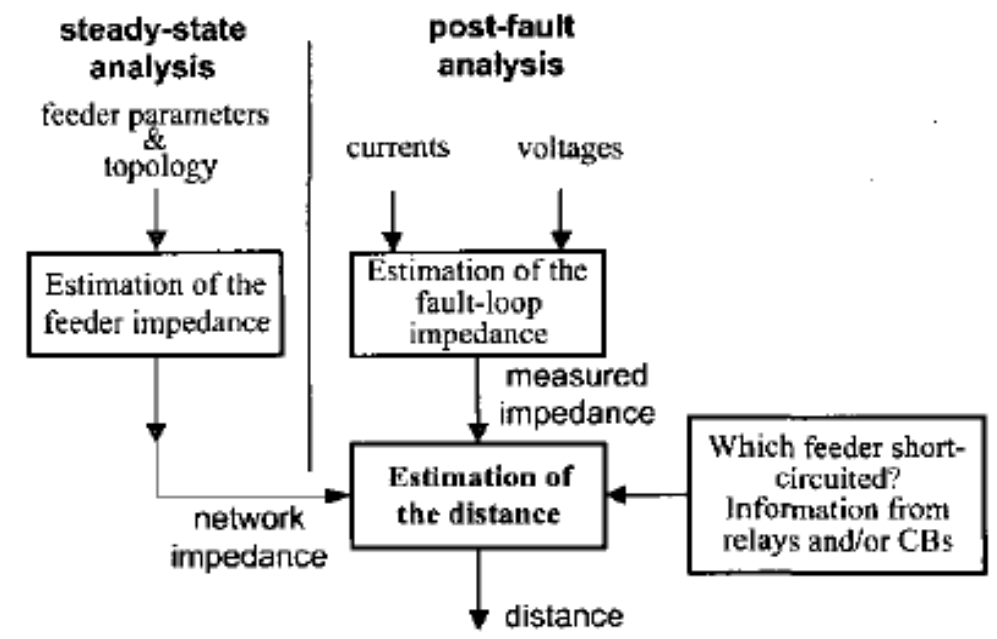

Figure A.1-Flowchart to determine fault location

Implementation: Simulation results using EMPT/ATP are provided for a $10 \mathrm{kV}$ radial cable network. Cable shield is considered as grounded at load points. For the pre-fault parameter estimation, the network is modeled with adequate details; however, some justified simplifications are carried out in post-fault transient analysis. Three-phase and phase-to-ground faults are simulated with different fault resistances and the results (along with errors) are presented. No actual field testing is reported in the paper.

Issues: The pre-fault condition determination requires accurate modeling of network elements as well as the topology.

Feasibility: The method has the advantage of single-ended measurement requirements. More testing is required to validate its reliability.

Related Paper: M. M. Saha, E. Rosolowski, and J. Izykowski, “ATP-EMTP investigation for fault location in medium voltage networks," Presented at the International Conference on Power Systems Transients (IPST), 2005, pp. 1-6.

A.2.4.1.6 Automated fault location and diagnosis on power distribution feeders [10]

Summary: The proposed fault location and diagnosis scheme in this paper involves three sequential processes: signal processing, fault location, and fault diagnosis. This algorithm 
is based on the steady-state analysis of the faulted distribution network. The derived faulted network equation contains three unknowns: fault distance, fault resistance, and fault current. By reasonably assuming an initial fault current, one can calculate the fault distance and fault resistance by separating the faulted network equation into the real part and the imaginary part and then solving the two real equations. Once the calculated fault distance is known, load current during fault is calculated with the assumption of either a constant impedance load model or a dynamic load model, and then the fault current is recalculated and the above process repeated until the calculated fault distance converges. As such, an iteration method is used to locate the fault. For each calculated fault location, a fault region is then estimated based on probabilistic modeling and analysis.

Due to the load taps, multiple fault locations may occur when measurements are available at the substation. By knowing the placement of the various protective devices, such as reclosers and fuses, as well as their operating characteristics, one can apply a fault diagnosis process to prune down and rank the list of multiple faults calculated by the fault location algorithm.

Implementation: It is reported in the paper that preliminary field tests on several distribution feeders using actual disturbance data have been carried out to evaluate the performance of this method. Some results for single-line-to-ground (SLG) and line-toline-to-ground (LLG) faults are provided and the estimated error bounds for the calculated fault distances are also presented.

Issues:

The system model used for this method has only one source.

The faulted network circuit equations are based on steady state analysis.

This method requires accurate information about load characteristics and line parameters including self inductances and mutual inductances.

Feasibility: The method looks promising, particularly because it solves the typical difficulties encountered in distribution fault location, such as load taps, load current during fault, and multiple locations due to laterals. In addition, this method is also reported to be useful in applications that investigate temporary faults that may not result in a blown fuse; thus, it can help in preventing permanent faults.

\section{A.2.4.1.7 Fault locating in ungrounded and high-resistance grounded systems [11]}

Summary: Ground faults that occur in ungrounded and high-resistance grounded systems do not draw enough current to trigger circuit breaker or fuse operation, making them difficult to localize. This paper presents a method to locate ground faults in ungrounded and high-resistance grounded systems. The method couples information from a relay located at a unit substation and inexpensive remote ground-fault detectors to indicate the fault phase, the supply feeder to the fault, an estimate of the fault distance, and the branch circuit or connected load with the fault. A zero-sequence signal generator that operates at a distinct frequency other than the power-line frequency provides a circuit path and 
signal to aid in locating the ground fault. From the signal-generator viewpoint, the fault network behaves like a solidly grounded system. However, from the power-line viewpoint, the system has not changed from being an ungrounded or resistive grounded network.

This ground-fault location system is capable of locating intermittent and multiple faults on the same phase and can detect and distinguish inverted ground faults. The groundfault location system architecture can be represented as

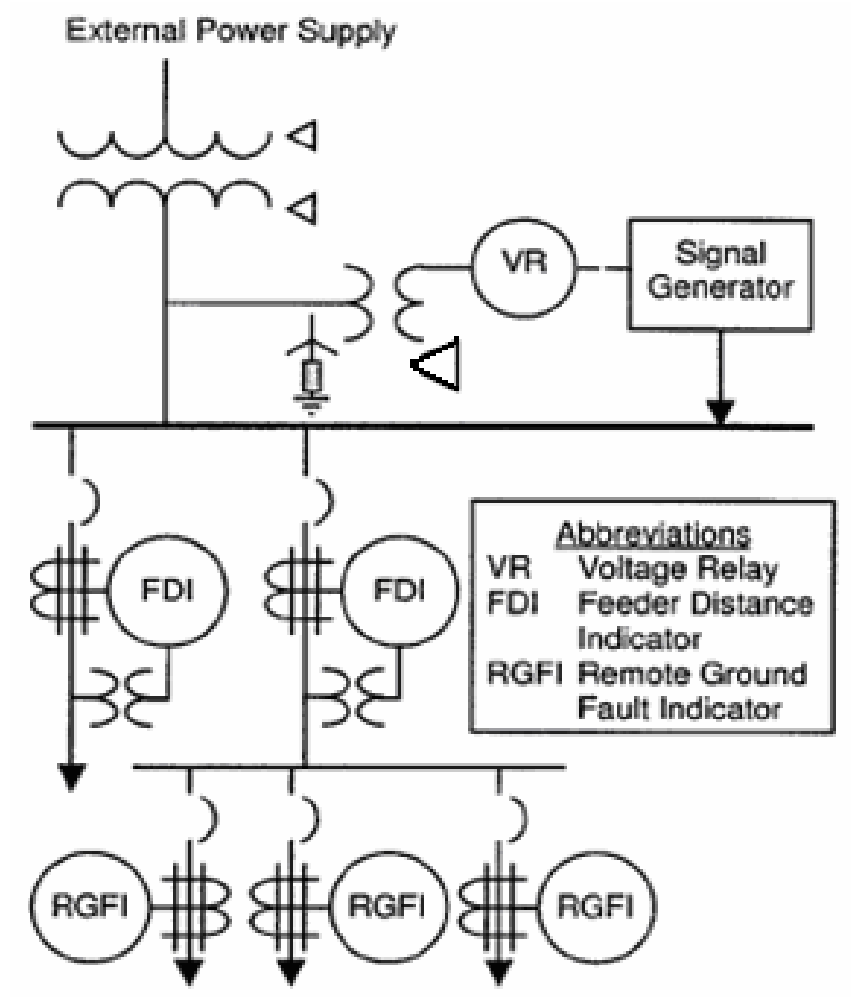

Figure A.2-Ground-fault location system architecture

Implementation: Actual field testing on an industrial manufacturing site with several $480 \mathrm{~V}$ distribution systems is reported in the paper. The first test was conducted on an ungrounded delta-connected system with two loop feeders and two unit substations. A second test was conducted on a high-resistance grounded system with five loop feeders, two tie-lines and four unit substations. The results, along with inferences, are presented in the paper.

Feasibility: One advantage of this ground-fault location system method is that it is capable of locating intermittent and multiple faults on the same phase and can detect and distinguish inverted ground faults.

A.2.4.1.8 Fault locating in ungrounded and compensated systems [12] 
Summary: This paper presents a diagnosis signal injection method for locating ground faults in ungrounded and Peterson-coil grounded systems. A diagnosis signal is injected into the faulted system and is traced in the network to provide the location of the fault. Two separate conditions arise and have been described in the paper, which require different approaches to inject diagnosis signals into the faulted feeder: fault location in live faulted feeders or fault location in de-energized feeders.

The system approach couples information from a relay located at a substation and remote ground-fault detectors to indicate the faulty phase, the supply feeder to the fault, an estimate of the fault distance, and the branch circuit with the fault. A diagnostic signal generator that operates at a distinct frequency other than the power frequency provides a circuit path and signal to help locate the ground fault. The remote ground-fault indicator or hand-held detector is a robust devise that gives indication of a ground fault on a particular circuit. It is sensitive in detecting small diagnostic currents. The direct current high voltage (DCHV) breaks down the fault point again before injecting the diagnosis signal current into the de-energized system.

Implementation: The ground fault location system (with hand-held detectors) is reported to have been applied in various distribution systems in China. These distribution systems include ungrounded systems, Peterson-coil grounded systems as well as high-resistance industrial power systems. The error of fault localization is reported to be less than 10 meters; however, no data or results are presented in the paper as such.

Issues: Even though the authors report high accuracy for this method, no actual data is included in the paper. The systems on which this method was applied are also not described.

\section{A.2.4.1.9 A three-phase fault detection algorithm for radial distribution networks [13]}

Summary: This paper presents an algorithmic approach for finding the location and type of fault based on the three-phase measurements obtained for state estimation. The algorithm detects the fault location and type under loaded and unloaded conditions and also with and without fault impedance.

The state estimator computes the voltage magnitude and phase angle at all the load buses from available real-time measurements. If the data is abnormal or if a protective device operation is noticed, then the data goes to the fault detector where it is compared with normal measurement data and analyzed under two conditions: with negligible fault impedance or with high fault impedance. In the case of negligible fault impedance, the branch flow measurement values until the fault location are expected to be very high while the values beyond the fault location will be close to nil. Then the fault location is that branch beyond which the values are nil. On the other hand, in case of high impedance faults, the healthy branches have normal or low values while the faulty path has high measurement values. The flow measurements that are beyond a threshold value are classified as high values. The fault location is that branch beyond which the high values become low. The detailed flow chart of the algorithm is represented below. 


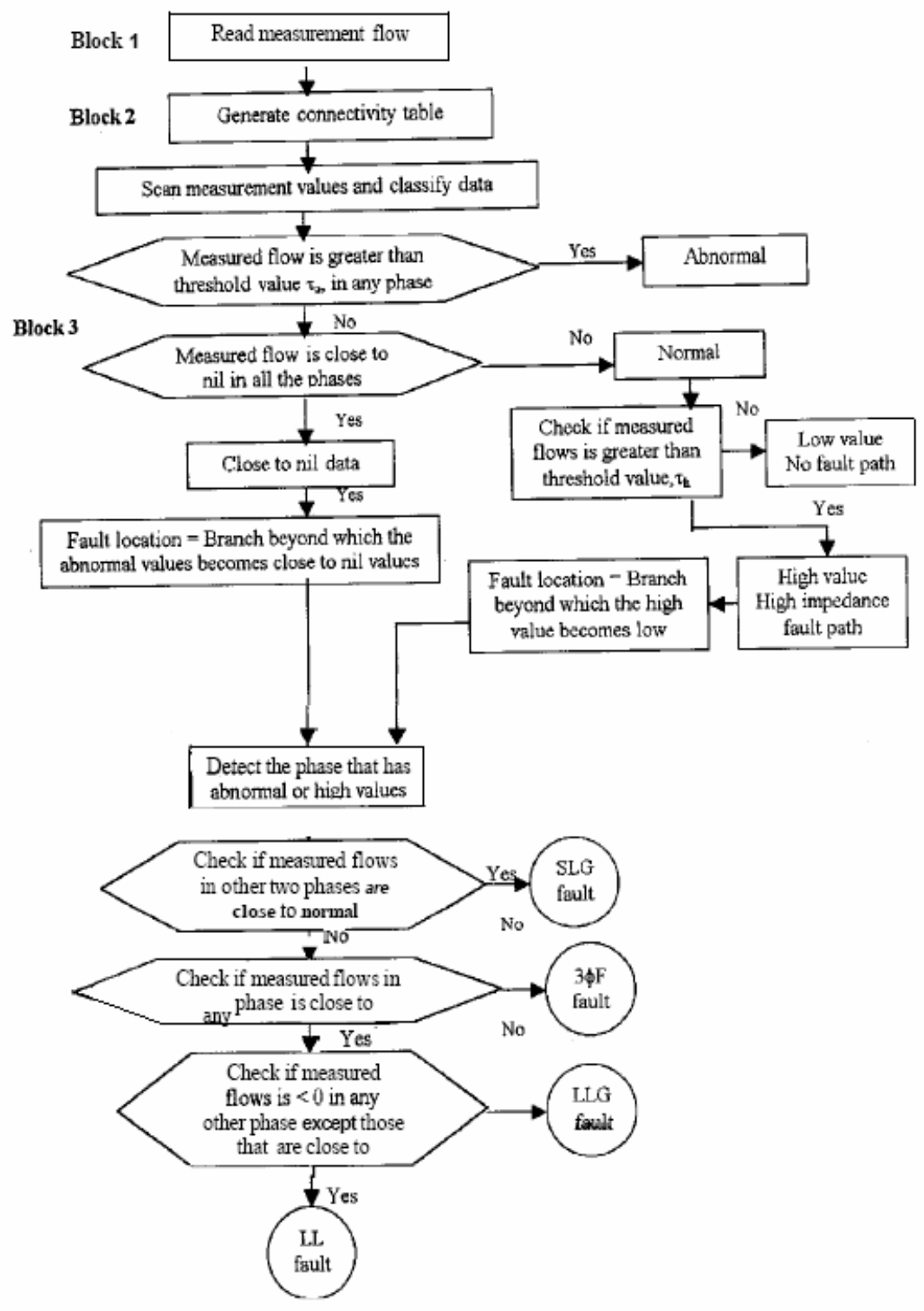

Figure A.3-Algorithm for three-phase fault detection

Implementation: Based on the proposed algorithm a computer program has been developed, and results for a typical 52-node distribution network (with three feeders) are presented. No actual field testing is reported in the paper.

Issues:

Actual field testing results are required to validate its performance.

It provides the faulty branch, not the estimate of actual location of fault on the faulty branch.

A.2.4.1.10 A voltage-based fault location method for radial distribution networks [14] 
Summary: This paper describes a topological method to determine fault location. A conventional method to locate a fault in a radial network consists of placing current detectors along each feeder. The measured currents indicate if the fault is above or below each sensor, thus the fault location can then be determined. This conventional method cannot be used to detect "open circuit" faults. The method proposed in this paper is based on voltage measures, instead of current measures, located in MV/LV stations. The method requires placement at each end of a MV branch a voltage sensor and calculation of a characteristic quantity which will be transmitted to a central point for comparative analysis. The method is divided into two steps: the first one consists of determining whether each sensor is above or below the fault and the second one consists of determining the fault section, from a purely topological method.

The method of determination of sensors located below the fault (active sensors) is based on voltage measures, but it can be slightly different depending on whether the characteristic quantity is computed directly from the phase voltages, positive-sequence voltages, or from the zero-sequence voltages. The method also makes use of the voltage drop due to the fault and not only the voltage itself.

Implementation: Simulation results for a sample $21 \mathrm{kV} / 400 \mathrm{~V}$ radial distribution network (both overhead and underground cable network) are presented for a SLG fault and a broken cable (open circuit) fault with different values of fault resistances. No actual field testing is reported in the paper.

Issues: The method requires a great measuring accuracy to locate a phase-to-earth or phase-to-phase fault.

Feasibility:

The method has the advantage of being able to efficiently locate open circuit faults. In such faults, the measuring precision of the method is not a problem, and the faulty section can be determined very easily.

This method is particularly useful if LV potential transformers are already available. Actual field testing is required to validate the method's reliability under various operating conditions. 
A.2.4.1.11 A technique for estimating locations of shunt faults on distribution lines [15]

Summary: This paper presents a technique for estimating the locations of shunt faults on radial distribution lines using the fundamental frequency components of voltage and current signals measured at the line terminal. When a fault is detected, the fundamental frequency components of the voltage and current phasors are saved. The fundamental frequency components of the voltage and current phasors during the fault are estimated, and fault type is determined online (see Figure A.4). The pre-fault and fault data, along with line and load parameters, are then used in an offline mode to estimate the location of the fault (see Figure A.5). Necessary line and load parameters are obtained from the database.

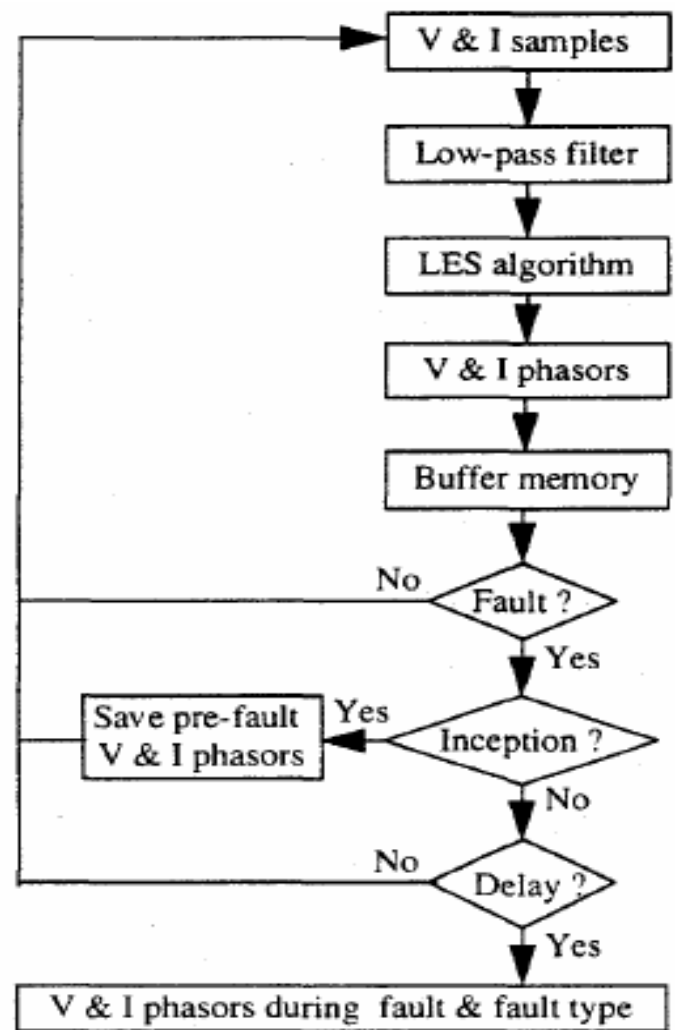

Figure A.4-Data acquisition

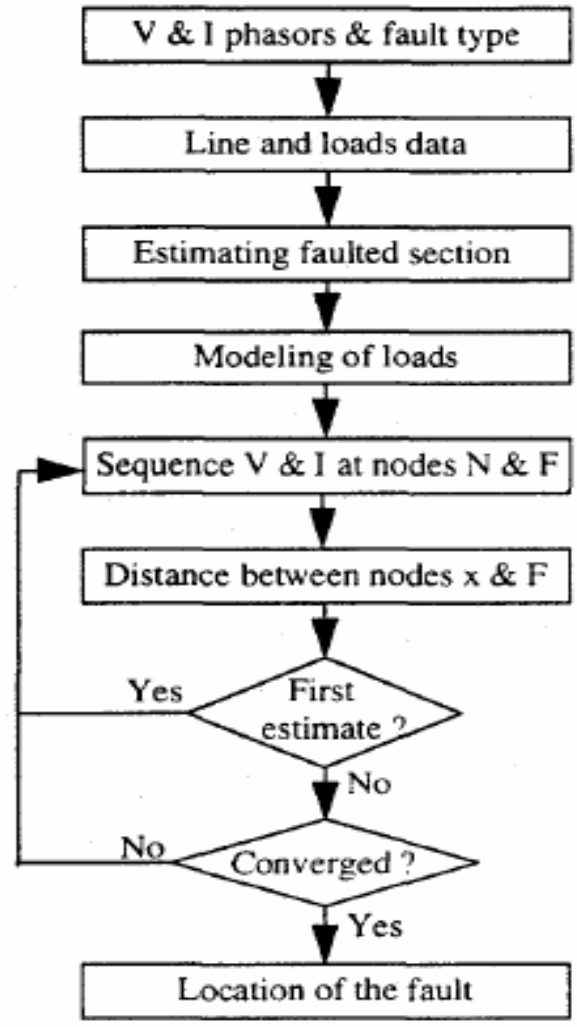

Figure A.5-Estimating fault location

Implementation: Simulation results on a 23-mile-long line of SaskPower distribution circuit are presented using PSCAD/EMTDC. The distribution line consists of sections of different lengths, different types of conductors and single and three-phase tapped loads along its length. Results using several fault types, fault locations, and fault resistance values are presented. No actual field testing is reported in the paper.

Issues:

Increases in error percentage with higher fault resistance values.

Simulation system used is a single-source system.

Accurate database of line and load parameters is required. 
Actual field testing results are needed.

Feasibility: This technique is suitable for non-homogeneous lines, including cables, capacitor banks, and dynamic load taps. The technique also considers auto-reclosing of the lines.

Related paper: R. Das, M. S. Sachdev and T. S. Sidhu, "A fault locator for radial Subtransmission and distribution lines," in IEEE Power Engineering Society Summer Meeting, 2000, pp. 443-448.

A.2.4.1.12 Fault location method for distribution networks using distributed parameter line model [16]

Summary: This paper presents a new fault location technique which uses a distributed parameter line model in its power-frequency-based algorithm. The technique therefore accounts for the line shunt capacitance, which is a major source of error in conventional impedance-based methods, particularly in cable networks. In this technique each line section of the network is modeled by a two-port transmission $[\mathrm{A}, \mathrm{B}, \mathrm{C}, \mathrm{D}]$ matrix.

A pre-estimation of the faulted section is first carried out. Then the path from the relaying point to the head bus of the nominated section is identified, and the laterals and minor networks connected to the path are modeled by equivalent R-L or R-L-C circuits. The main path is modeled by the sequence impedance model for decoupling the three-phase system into three single-phase sequence networks, i.e. zero, positive and negative sequence networks.

Implementation: EMTP simulation results using IEEE 37-bus standard cable network are provided for various fault types (phase-to-earth and phase-to-phase) and fault resistance values. No actual field testing is reported.

Issues:

Accurate line data and network data is required to implement the method. Actual field testing results are needed.

Feasibility: The technique was initially intended for cable networks, but it can easily be applied to overhead or even mixed networks. The method's main features include: it considers line shunt capacitance, it is theoretically independent of fault resistance magnitudes (but simulation results provided in the paper show an increase in error percentage with higher fault resistance values), and it does not need any iterative load flow analysis.

A.2.4.1.13 Accurate Non-iterative Fault Location Algorithm Utilizing Two-End Unsynchronized Measurements [17]

Summary: This paper presents a new two-terminal impedance based fault location algorithm, which takes into account the distributed parameter line model. The algorithm utilizes unsynchronized measurements of voltages and currents from two ends of a line 
and is formulated in terms of the fundamental frequency phasors of symmetrical components of the measured signals. First, an analytical synchronization of the unsynchronized measurements is performed with use of the determined synchronization operator. Then, the distance to fault is calculated as for the synchronized measurements. Simultaneous usage of two kinds of symmetrical components for determining the synchronization operator makes that the calculations are simple, non-iterative and at the same time highly accurate.

Implementation: The redundancy of fault location equations existing under use of twoend unsynchronized measurements is utilized for making the calculations of non-iterative kind, at the same time keeping high accuracy of fault location by taking into account the distributed parameter line model. This is achieved as a result of simultaneous usage of two kinds of symmetrical components.

Feasibility: The developed fault location algorithm has been thoroughly tested using signals of ATP-EMTP versatile simulations of faults on a transmission line. The presented evaluation shows the validity of the developed fault location algorithm and its high accuracy.

\section{A.2.4.1.14 A New Fault Location Algorithm Using Direct Circuit Analysis for Distribution Systems [18]}

Summary: The unbalanced nature of distribution systems due to single-phase laterals and loads gives difficulty in the fault location. This paper proposes a new fault location algorithm developed by the direct three-phase circuit analysis for unbalanced distribution systems. The proposed algorithm overcomes the limit of the conventional algorithm, which requires the balanced system. It is applicable to any power system, but especially useful for the unbalanced distribution systems. Its effectiveness has been proved through many EMTP simulations. The fault location equation has been derived by applying matrix inverse lemma and is relatively simple and easy to be applied for any system regardless of a phase balance condition. Therefore, it can handle not only the transmission systems but also the distribution systems.

Implementation: The suggested direct circuit analysis based-algorithm has been applied to a balanced three-phase system and an unbalanced system. The results are compared with those of the conventional method using distribution factor of the negative sequence current described in the paper. In each case, nine different fault distances varying from 0.1 to 0.9 p.u. by 0.1 -p.u. step and three fault resistances of $0,10,30$, and 50 ohms have been considered. EMTP simulation has been performed, in which 1920-Hz sampling frequency is used. Voltage and current phasors are obtained by the discrete Fourier transform (DFT) having one cycle data window.

Feasibility: The proposed algorithm overcomes the limit of the conventional fault location algorithm based on the sequence circuit analysis, which is the balanced system requirement. The proposed algorithm is applicable to any power system, but especially useful for the unbalanced distribution systems. Its effectiveness has been proved through many EMTP simulations. The fault location algorithm for the phase-to-phase fault in an 
unbalanced system needs to be developed and the effect of load variation or uncertainty needs to be investigated to enhance the practical power and these efforts

\section{A.2.4.1.15 An Intelligent Fault Location and Diagnosis System On Radial Distribution Systems [19]}

Summary: In this paper, an intelligent fault location and diagnosis system is proposed .The proposed system identifies the fault location in a two step procedure? The first step identifies candidate fault location using an iterative calculation of load current and fault current. The second step diagnosis the actual location by comparing the current waveform pattern and the expected operation of the protective devices and comparing the interrupted load with the real load

Implementation: In this paper intelligent fault location and diagnosis system is proposed .Taking voltage and the current samples of the feeder at the substation, it first calculates the possible fault location. In order to enhance the accuracy, updating the voltage and current at each load tap or branching point of the feeder, the fault location is calculated. Since the distribution system is a radial network with many branches multiple fault locations could be computed with measurements available at the substation. Each branch path could result in a candidate fault location by making comparison between the expected operation of protection devices and waveform patterns of current instruments. The load current pattern at the substation before and after the interruption is utilized in identifying the fault location more accurately.

Issues: The uncertainty involved in the load model is a big error source for real application and a further research in this matter is to be proposed.

Feasibility: The system has been developed and tested on many real distribution systems .The transient signals have been obtained by EMTP simulation. The results show the effectiveness of the proposed method with a high accuracy of less than $3 \%$ error

\section{A.2.4.1.16 A Fault Locator for Radial Subtransmission and Distribution Lines [20]}

Summary: This paper presents the design and development of a fault locator that estimates the location of shunt faults on radial sub transmission and distribution lines. Non-homogeneity of lines presence of 'laterals' and capacitor banks and, load taps has been taken into consideration. The fault location technique which uses the fundamental frequency components of the pre fault and fault voltages and currents measured at the line terminal, have been described in the paper by considering a single-phase-to-ground fault. Similar procedure is used for determining the locations of other types of faults.The suitability of the technique, which also takes into account of switch-into-fault and autoreclosing condition, was tested using simulated fault data.

Implementation: When a fault is detected, the fundamental frequency components of the pre-fault and fault voltage and current phasors at node are saved along with the fault type. 
These actions can be taken by a digital relay in real time. The prefault and fault data, along with line and load parameters obtained from a database, are used in an off-line mode to estimate the location of the fault

Issues:

The accuracy of the fault location estimates depends on the accuracy of the input parameters.

The accuracy of the voltage and current phasors depends on the transducers that sample voltages and currents and estimation algorithm.

Accuracy of the load estimates depends on the technique used to estimate the loads at the taps.

Feasibility: The fault locator can be implemented, using available technology, on digital relays with at least two processors. Alternatively, the real-time component of the fault locator can be implemented in a digital relay. The non real-time component of the fault locator can be implemented as a fault analysis package on a personal computer which can be located in a substation for analyzing faults on all lines emanating from that station. Test results indicate that the fault locator is robust and has acceptable accuracy even for fault resistance of $50.0 \mathrm{ohms}$

\section{A.2.4.1.17 Calculational Fault Location For Electrical Distribution Networks [21]}

Summary: The paper discusses the theoretical basis and implementation of Calculational fault location for radially operated electrical distribution networks. The main points of interest in this paper are how current and voltage measurements derived from the substation can be used for post fault analysis when locating the faults on distribution feeders and how fault location should be integrated into the control systems of the network. Networks considered are assumed to be radially operated.

Implementation: An essential task in the fault location is the estimation of the currents and voltages from the measurements derived at the substation during the fault. The prototype system developed samples the measured signals by the rate of $500 \mathrm{~Hz}$. The fundamental components of current and voltage are then estimated using Fouriertransform. This way the desired quantities are derived as phasors, which makes the corrective measures, for instance the load current compensation straightforward. Usually all the data needed for load current compensation is available at the substation. The data transmitted to the higher level includes then the estimated fault current, fault type and the faulty line identification code. The faulty line length calculation is then done in the network control centre using for instance the network data base system.

Issues: The fault location accuracy depends on the variation of the following factors: Measuring system accuracy

Faulty line resistance

Substation transformer impedance

Grid impedance

Grid voltage variation 
Load current active component

Load current reactive component

Feasibility: The integration of calculational fault location into the other control systems of distribution networks was studied using the prototype system composed of disturbance recording units connected to the protective relays and a piece of software running on microcomputer. It was found that as much as possible of the data processing should be done at the substation level. If the faulty feeder has several branches or laterals, the calculation gives several possible fault locations. In this case the calculational fault location should be complemented by remotely read fault current indicators.

\section{A.2.4.1.18 Automated Fault Location System for Primary Distribution Networks [22]}

Summary: This paper presents the development, simulation results, and field tests of an automated fault location system for primary distribution networks. This fault location system is able to identify the most probable fault locations in a fast and accurate way. It is based on measurements provided by intelligent electronic devices (IEDs) with built-in oscillography function, installed only at the substation level, and on a database that stores information about the network topology and its electrical parameters

Implementation: The algorithm combines information about the fault type and load rejection (provided by the DSP module) with the feeder's topology and electrical parameters (stored in the database) to rank them by the most probable ones. Basically, the algorithm follows the rules described below.

- When the fault is permanent, isolated by breaker operation, the algorithm verifies which located points are protected by fuses and ranks them as the less probable ones. - When the fault is permanent, isolated by fuse operation, the algorithm verifies which located points are not protected by fuses and ranks them as the less probable ones. Among those who are protected by fuses, the algorithm ranks as the most probable one the point that is protected by a fuse whose opening would cause an amount of load rejection comparable to the load rejection measured by the IEDs.

Feasibility: The work presents a complete automated fault location system for primary distribution networks and, more specifically, an algorithm for the fault location. This system was installed at an actual substation and its performance demonstrated that it is a powerful tool for the operation and maintenance of the electrical distribution networks. The most important benefits provided by the fault location system proposed in this paper are Downtime reduction, Operational costs reduction, larger profits and Corrective maintenance optimization.

\section{A.2.4.2 Fault Location, High-frequency Approach}

A.2.4.2.1 New concept in fault location for overhead distribution systems using superimposed components [23] 
Summary: This paper presents a novel approach in single-ended fault location for overhead distribution systems based on the concept of superimposed voltages and currents. The superimposed values are simply the differences between the total fault and pre-fault steady-state voltage/current signals. The methodology outlined is based on determining the superimposed values of voltage and current at any assumed fault point from the measured pre-fault and fault values. Using a specified model of the distribution system under consideration, these superimposed voltages and currents are employed to ascertain the voltage at an assumed fault position; this is then back-injected at the assumed fault point in order to check the fault-path currents in the unfaulted phases. Only when the fault point is correct will the healthy phase(s) at the actual fault point in the model attain a near zero value.

Implementation: EMTP simulation results for a sample $11 \mathrm{kV}$ overhead distribution system with load taps, both with and without infeed, commonly found in the UK, are presented in the paper. In order to pose an actual situation, models of CT's, VT's and interface modules are considered. A $4 \mathrm{kHz}$ sampling frequency is used. The effects of fault type and fault location, remote infeed, fault resistance, and load variations are studied; and results are presented. No actual field testing/implementation are mentioned in the paper.

Issues: Actual field testing/implementation results are needed to double-check the method and validate its performance under different operating conditions.

Feasibility: The method shows promise. It gives a high accuracy for various practically encountered system and fault conditions and is robust to large errors in the estimation of load taps and remote source capacity (if present).

A.2.4.2.2 A new fault location technique for radial distribution systems based on highfrequency signals [24]

Summary: This paper describes a single-ended fault location technique for radial distribution feeders based on the information provided by the measurements at the substation and the feeder configuration. The proposed method first identifies the fault path based on the traveling wave information provided by the high-frequency components of the recorded fault transient signals. It then calculates the exact location along the identified path based on the power frequency signals.

The phase voltages and currents at the substation are recorded and transformed into modal domain. Then, the modal signals are decomposed into their wavelet coefficients. The WTC of the voltage signal in mode 1 is used for identifying the faulted lateral. The sum of squares of the WTC is used as the identifier for this purpose. The identification of the faulted lateral is based on the voltage signal decomposition in a frequency spectrum from 12 to $25 \mathrm{kHz}$; therefore, voltage transducers with a bandwidth of $50 \mathrm{kHz}$ are needed. Once the faulted lateral is identified, the system is simplified by representing the non-faulted laterals as equivalent impedances. Then the post-fault phasors are extracted from the WTC decomposition of both current and voltage signals at the power frequency 
scale, and the distance is calculated (using conventional methods). The flowchart of the proposed algorithm follows:

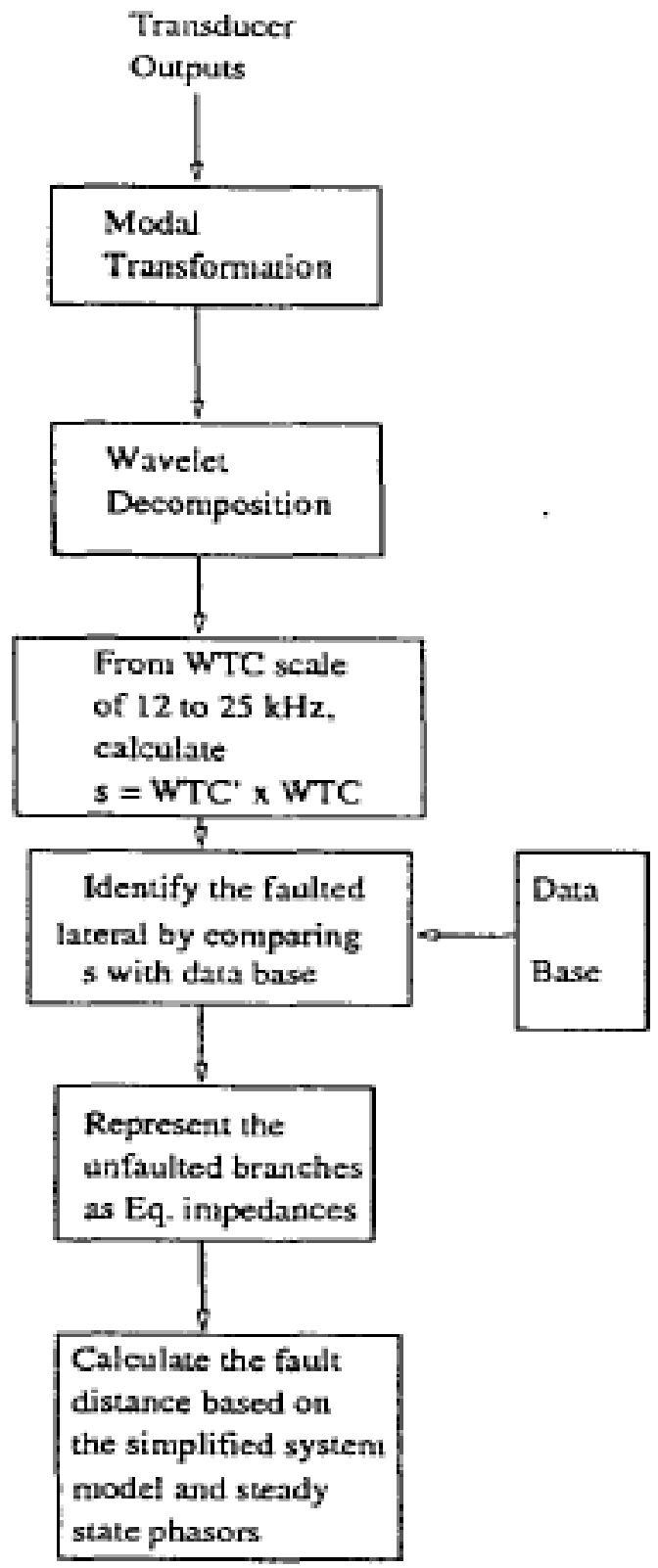

Figure A.6-Fault location algorithm based on high-frequency signals

Implementation: Simulation results (using ATP/EMTP) on a sample radial distribution system (with laterals) are presented in the paper. Various faults are simulated, and the effects of different fault resistance values, influence of incidence angle on the proposed algorithm, and influence of load variation are studied. Results are available. No actual field testing is mentioned in the paper, although there is a mention that the authors obtained network data and fault recordings from a certain industry. 
Issues:

Requires both high frequency and power frequency computations.

What about the case when multiple laterals get identified? (for instance if two fault points have an equal number of equidistant discontinuities between them and the substation).

Feasibility: This algorithm incorporates both high and power frequency computations and needs data available only at substations. It seems promising although field testing is required to validate it more.

\section{A.2.4.2.3 A new principle of fault line selection for distribution [25]}

Summary: This paper presents a method of fault line selection in non-direct ground neutral distribution systems using traveling waves and wavelet transform. The concept of 'modulus maxima' of wavelet transform is utilized to extract the fault-related information from traveling waves. The position of modulus maxima denotes the arrival time of traveling waves, and its amplitude reveals its intensity.

If ' $\mathrm{N}$ ' number of lines are connected to a bus with the same surge impedance ' $\mathrm{Z}$ ' and one line has a SLG fault, the first current traveling wave amplitudes in each of the lines (without bus capacitance) at the bus will be

$$
\begin{aligned}
& i_{H L}=-\frac{2}{N} \times i \\
& i_{F L}=\frac{2 \times N-2}{N} \times i
\end{aligned}
$$

where

$\mathrm{HL}=$ Healthy line

$\mathrm{FL}=$ Faulty line

$\mathrm{i}=$ Initial current traveling wave from the fault point

Some conclusions can then be made:

The amplitude of the first traveling wave in the faulty line and the healthy line are of opposite polarity.

The amplitude in all healthy lines is the same.

The amplitude of the first current traveling wave in the faulty line is usually higher than the one in any healthy line (for $\mathrm{N}=2$, the amplitudes are similar; but the effect of bus capacitance is to increase the amplitude on a faulty line).

Thus, the principle of fault line selection is then to check the first current traveling wave at the bus for each line. The line whose first current traveling wave is of opposite polarity and whose amplitude is also greater than those on other lines is then the faulty line. 
Hence, this boils down to selecting that line whose modulus maxima (as computed from wavelet transform) is of reverse polarity and has larger amplitude than modulus maxima of other lines. Figure A.7 contains the flowchart of this algorithm.

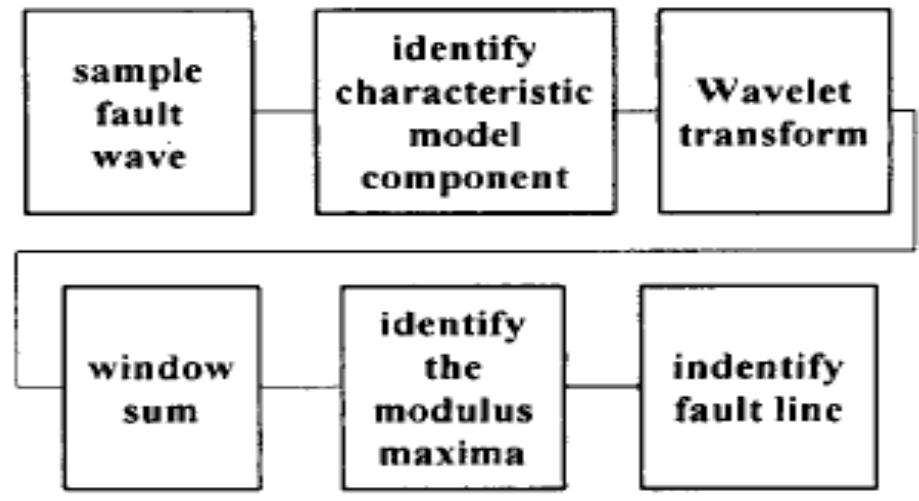

Figure A.7-Algorithm using traveling waves to determine faulty lines

A sample system is then chosen to validate the results using EMTP. Then the effect of various parameters is studied to verify the algorithm for the following conditions: Effect of capacitance: The conclusion is that the bus capacitance has minimal effect on the results. The higher the capacitance value, the higher will be the reflection factor and clearer the results.

Effect of grounding resistance: The conclusion is that the algorithm is minimally affected by effects of grounding resistance. Even for grounding resistance up to $5 \mathrm{k} \Omega$, the algorithm stands useful.

Finally, the authors state that the algorithm has been verified for the cases when the fault line is an open branch, when the load is asymmetrical (10\% asymmetry), in direct-ground neutral system, and arc-suppression-ground neutral system.

Issues:

All $\mathrm{N}$ lines are assumed to be of the same surge impedance ' $\mathrm{Z}$.'

For $\mathrm{N}=2$ case, the 'amplitude criterion' to distinguish the faulty line and healthy line may not be very useful.

Authors state that the algorithm is quite sensitive, so it may detect fault for only a disturbance in the network. (the remedy suggested is to look at the voltages in addition to detecting the current traveling waves; if voltages are asymmetrical in addition to detection of fault by the algorithm, declare a fault; otherwise discard the results of the algorithm).

Implementation: EMPT simulations are provided to validate the results. No actual field implementation/testing are mentioned in the paper. 
A.2.4.2.4 A fault transient comparison technique for multi-ended distribution feeders [26]

Summary: This paper describes a 'fault transient comparison scheme' for multi-ended distribution feeders (teed feeders). Three relays are installed at each teed feeder end, respectively, and currents at each feeder end are measured by CT's locally. The basic elements of the relay are as shown in Figure A.8.

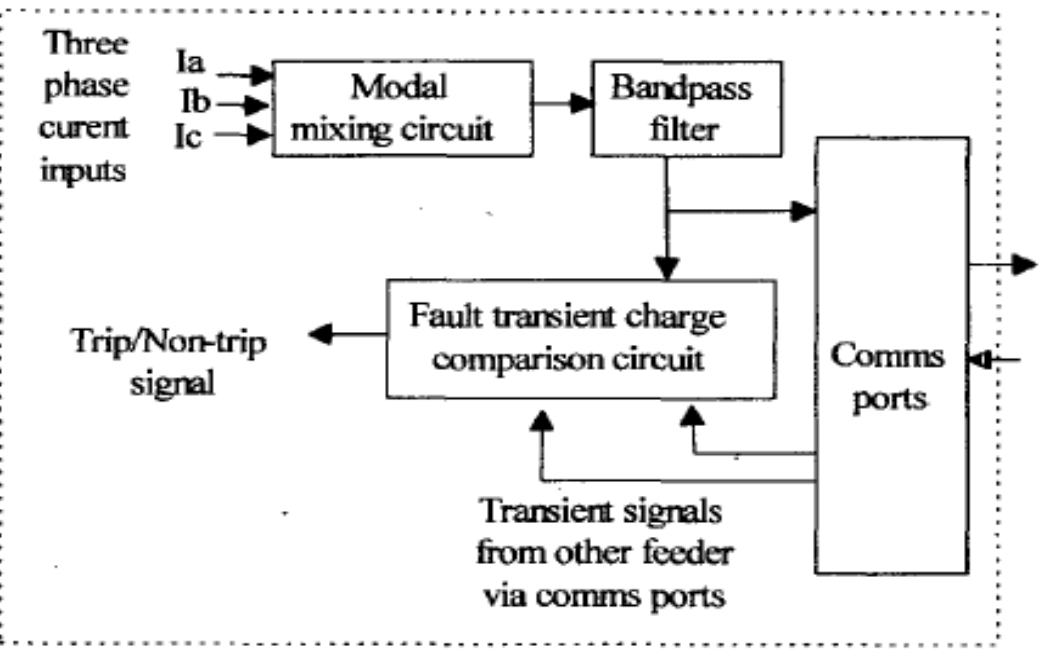

Figure A.8-Distribution feeder relay with a transient fault comparison scheme

The function of a modal mixing circuit is to convert the three-phase signal into a modal signal. The band pass filter extracts the fault current generated fault transient signals from the modal signal. The fault transient signal is then sent to a remote feeder via the communication ports; it is also sent to the fault transient comparison circuit. The differential fault transient comparison circuit is designed to compare all first initiated FT wave state 'signs' received by the feeder-end relays.

Simulation results using EMTP on $33 \mathrm{kV}$ teed feeder configurations are used to demonstrate that for an internal fault, the first initiated fault transient waves at the three feeder ends have the same positive signs (second or later received fault transient reflected waves may have either sign; so comparison is made only using first received transient waves). For external faults, all three feeder ends do not record the same sign of first received traveling waves. Thus, this algorithm can be used to distinguish between internal and external faults in choosing to issue the trip signal accordingly.

Implementation: Simulations on a UK $33 \mathrm{kV}$ distribution teed feeder configuration is reported, but no field implementation/testing is reported in the paper.

Issues: Simulations have not been reported for varying system conditions such as bus capacitance, fault resistance values, etc. 
Feasibility: The method seems promising to differentiate internal faults from external faults on teed feeder configurations. Also, since there are no fault transients during prefault conditions, this scheme only need to transmit fault transient wave states when a fault occurs, thus lowering the requirements for data communication bandwidth as compared to other traditional current phase comparison methods.

A.2.4.2.5 An accurate fault location technique for distribution lines with tapped loads using wavelet transforms [27]

Summary: This paper investigates the application of the wavelet transform in fault location techniques for distribution lines with tapped loads. Fault transient detectors (FTDs) based on a digital wavelet transform are installed at substation bus bars and load terminals to capture the time taken for the first fault-induced traveling wave to arrive and be synchronized with a GPS clock. From the recorded time and the topological structure of the network, fault location can be deduced.

The following flowchart summarizes the components/operation of FTD:

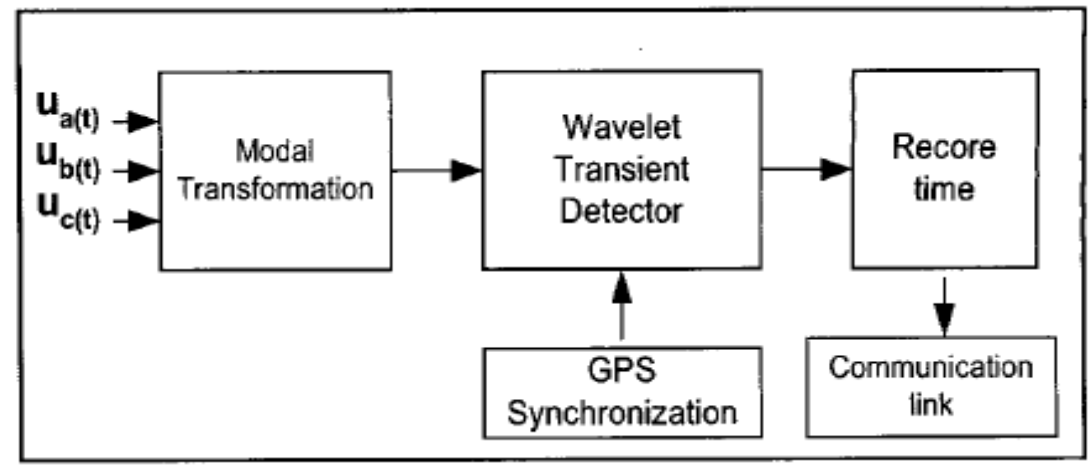

Figure A.9-FTD and fault location technique

One FTD unit is placed at each substation and load terminals. The arrival time of the first voltage traveling wave is recorded by each FTD and marked with the time of a GPS clock. Then, based on the topological structure of the network, the fault location can be determined using the time differences of the individual FTD readings and suitable algorithm. This is very much like the traditional double-ended method of fault location using traveling waves.

Implementation: Simulations are reported for a sample $11 \mathrm{kV}$ distribution line with seven tapped loads using EMTP/ATP, but no actual field trial/testing is reported in the paper.

Feasibility: This method involves placement of a unit of FDR at each load terminal (and also at substations), thus making it a costly affair; however, accurate results are anticipated. 
A.2.4.2.6 Fault location using traveling wave for power networks [28]

Summary: This paper presents a traveling-wave-based fault locator for distribution systems, developed with the goals of reduction in cost and precision in application. Two traveling wave sensors (current and voltage) are developed. The current sensor is installed at the grounded line of capacitive equipment in the network (such as CVT, transformer bushing, etc), and it captures the current traveling waves flowing from the equipment to Earth (This installation does not influence the normal operation of the power network as opposed to the traditional way of capturing traveling waves using an inductive coil in series with the CVT). The voltage signals of the transmission lines are then derived from these current signals. The voltage traveling wave sensor is installed at the zero-sequence winding of a voltage transformer to capture the voltage traveling waves in all three phases. The output of these two sensors (which is a proportionate voltage signal under $50 \mathrm{~V}$ ) is then applied to detect the traveling waves caused by faults using the scheme provided in the paper (polarity of traveling waves can also be detected). The arrival times of the traveling waves are time-tagged using synchronized GPS clocks (arrival time recording scheme is provided in the paper). The developed traveling wave sensors (along with the detection unit and GPS) is installed in every bus in the network, and from the different arrival times of the traveling waves detected at different buses, the fault is located. The fault location system can also be applied to determine the phasors of the lines, thus implementing the function of a PMU.

Implementation: The developed fault locator with traveling wave sensors was tested on a $110 \mathrm{kV}$ power system. The results are presented for two types of faults (high impedance ground fault and low impedance ground fault). Accuracy of less than $120 \mathrm{~m}$ error is reported, and the fault-generated, high-frequency signal is unaffected by the variation of fault type and fault impedance. Whether this was a simulation study or the fault locator unit was tested in a real system is not clear, although the paper does mention briefly in the end that the fault locator has been tested in a real power system (with fault location error of no more than $300 \mathrm{~m}$ ).

Issues:

Simulation results are provided for a $110 \mathrm{kV}$ system and not for lower voltage levels (which are typical of distribution systems).

Noticeable cost-reduction is not quite apparent although the additionally performed function of PMU's is a bonus.

\section{A.2.4.2.7 Fault location in distribution systems based on traveling waves [29]}

Summary: The paper presents a single-ended fault location method for distribution systems based on traveling wave theory. In this method, the current transients are obtained via a current probe with a high pass transfer characteristic which is attached to the secondary circuit of the relay current transformer. The sampling rate is $1.25 \mathrm{MHz}$ with 8-bit resolution. The voltage transients are then deduced from these current transients, and the fault distance is determined using a cross-correlation function. The reflected transient at the bus is cross-correlated with the incident transient, and the peaks 
in the cross-correlation gives the arrival time of the first and second incident waves from which the fault location is determined (procedure is presented in the paper). For singleended case, a longer correlation window had to be used.

Implementation: Simulation results on a $23.8 \mathrm{kV}$ feeder for a three-phase fault occurring on a main feeder are presented, and the complexity induced because of the presence of many sub-feeders is apparent. The results obtained are quite accurate. The method is then field tested on a $23.8 \mathrm{kV}$ Brazilian distribution system, and results are presented.

Issues:

Results/simulations have not been reported for varying system conditions such as bus capacitance, fault resistance values, etc.

Results for faults very close to the substation are also not reported.

The author states that further work is needed to confirm the reliability of the proposed method.

A.2.4.2.8 Fault section identification and location on a distribution feeder using traveling waves [30]

Summary: This paper describes a single-ended approach to determine fault location using traveling waves. The method identifies the faulty section and the probable location of fault by comparing the relative distance of each "peak" in the high-frequency current signals to the known reflection points in the distribution feeder. The probable fault location is then used within a transient power system simulator that models the actual network, and the resulting simulated current waveforms are then cross-correlated against the signal captured on the real network. If the estimated fault location is correct, the highfrequency signals in the simulated waveform will be similar to that of the measured waveforms, and the cross-correlation value will be a high, positive value.

Implementation: Simulation results for a $33 / 11 \mathrm{kV}$ distribution feeder based on UK distribution network were chosen for this method (sampling rate $=1.25 \mathrm{MHz}$ ). No actual field testing is reported. The method uses a technique called "Time Trees" ("Time Trees" give good visual descriptions of how various waveforms are generated at each end of a network, and it is reported in the paper that they are more convenient and faster than running simulations on PSCAD/EMTDC for studies related to traveling waves) to generate signals under several fault conditions. These signals are then cross-correlated (using different lengths of correlation windows) against the waveforms generated using PSCAD/EMTDC simulator, and the values of cross-correlation are monitored and used to decide the fault location. For those points where it becomes difficult to distinguish the incident and reflected peaks in the signals, it is suggested to simulate a fault at several locations on the faulty section (which is already identified from the nature of the waveform) and to cross-correlate the simulated waveforms with the original one.

Issues:

No real field data is used to validate the method. 
Simulations have not been reported for varying system conditions such as bus capacitance, fault resistance values, etc.

A.2.4.2.9 A new approach to fault location in a single core underground cable system using combined fuzzy logic and wavelet analysis [31]

Summary: This paper presents a method for radial distribution cable fault location based on signal processing using wavelet theory and intelligent systems requiring fuzzy logic. In the first stage, a pipe-type single-core cable including core and ungrounded sheath is modeled in LCC support routine of ATP and voltage and current signals are obtained at the sending end. The transient signals are then decomposed into their modal components. In the second stage, useful information is extracted by signal processing using the wavelet transform, and finally the fault position is localized through a fuzzy logic system (details of fuzzy logic system in paper). A fuzzy logic system (FLS) is chosen for this method because it is conceptually easy to understand, flexible, tolerant to imprecise data, based on natural language, and can be built on the experience of experts and blended with conventional control techniques. Four types of faults have been considered: core open circuit, core and sheath open circuit, core with sheath short circuit, and core and sheath through ground short circuit. The squared wavelet coefficients (WTC1) of the first resolution level have been used to detect the number of notches per cycle, and the Haar wavelet is chosen as the mother wavelet with two-scale signal decomposition.

Implementation: Simulations results for an $11 \mathrm{kV}$ pipe-type single-core cable including core and sheath of radial network are presented in the paper. Simulations are done using ATP software. No actual field testing is mentioned in the paper.

Issues:

No actual field test results are provided.

The system considered for simulation is elementary.

Feasibility: More results are required to validate the algorithm.

A.2.4.2.10 Accurate fault location technique for distribution system using fault-generated high-frequency transient voltage signals [32]

Summary: This paper describes a technique for fault location on distribution overhead lines and underground cables. A specially designed fault locator unit is used to capture the high-frequency voltage transient signals generated by faults in the frequency range of 1 to $10 \mathrm{MHz}$. The signals from these are then digitized for processing, and modal mixing transforms are used to extract the aerial mode and ground mode signals. Since at this high frequency range, the busbar impedance is dominated by its capacitance, the reflected wave from the remote busbar will have opposite polarity to that from the point of the fault; therefore, the two waves can be discriminated. Then the traveling time of the highfrequency components is used to determine the fault position. 
The method is reported to be immune to power frequency phenomena such as power swings and CT saturation. It is also reported to be insensitive to fault type, fault resistance, fault inception angle, and system source parameters. The problem of zero voltage inception angle, which is serious for conventional traveling wave techniques, does not affect this technique, which detects the fault arc-generated high-frequency signals (since the high-frequency signals associated with the fault arc do not vary with the point on the wave at which the fault occurs).

Implementation: Simulation results for two $11 \mathrm{kV}$ overhead distribution system configurations (with and without tapped-off loads) are presented in the paper using the EMTP software. Results for fault points close to the busbar and fault points on the line with discrete distributed tapped-off load are presented. Then, simulation results for an 11 $\mathrm{kV}$ aluminum three-core underground cable line are demonstrated, and finally the effects of busbar capacitance on the obtained results are presented. No actual field testing is reported in the paper.

Issues:

A very high sampling frequency is required (20 MHz is used for simulations).

Increases in busbar capacitance deteriorate the obtained results.

Effect of tapped-off load is significant and causes problems in fault position identification.

Feasibility: The method does have the advantage of overcoming the problem of zero voltage inception angles (if a fault occurs at or near zero voltage, the traveling wave produced is very small); however, more actual field results are needed to confirm the reliability of the method.

A.2.4.2.11 Feature extraction method for high impedance ground fault localization in radial power distribution networks [33]

Summary: This paper presents a new approach to localization of high impedance ground faults in compensated radial power distribution networks. The detection and localization system uses broadband $(25 \mathrm{kHz})$ measurements of three-phase voltages and currents at the supplying transformer station. The detection and localization of a ground fault then takes place in three stages: First, the fundamental frequency voltage and current signals are decomposed into the symmetrical components and are used to detect the time instant of a ground fault event by thresholding the zero system; secondly, the transient caused by the ground fault event and a grid model, based on FIR filters, of the feeder is used for feature extraction; and thirdly, this feature extraction serves as input to a neural network which estimates the geographical location of the ground fault. The method can be summarized in the figure on the following page: 


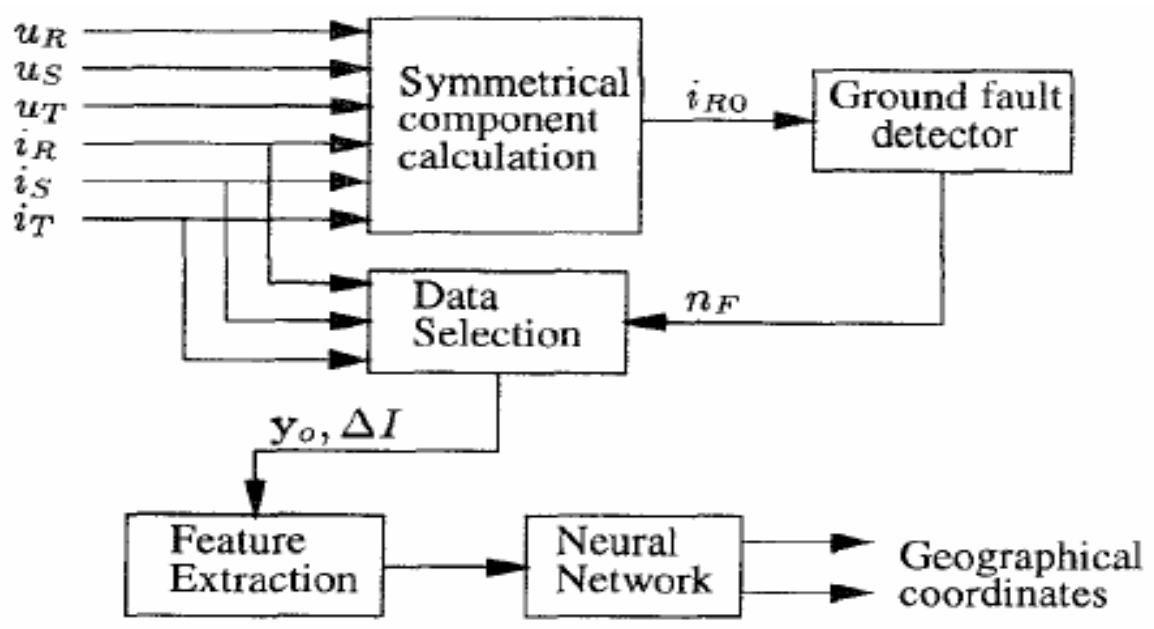

Figure A.10 - Ground fault detection system

Implementation: EMTP simulations on a sample radial feeder are presented. The simulation model includes a total of 1550 meters of cables, two loaded distribution transformers, and three different types of cable distributed on 5 cable segments. The data used for modeling is taken from a utility database. Results for different ground fault locations with fault resistance of $500 \mathrm{ohms}$ are reported. No actual field testing is reported in the paper.

Issues:

Actual field testing is needed.

Sample system used is a single-source system.

A.2.4.2.12 Application of GPS based fault location scheme for distribution system [34]

Summary: This paper presents the application of a transient-based fault location technique to a distribution system using GPS. In the scheme, the proposed locator which contains a specially designed fault voltage transient detection device and a GPS receiver is connected to the power line through a coupling capacitor. The locators are installed at each busbar in the distribution network and are used to capture the fault generated highfrequency voltage transient signals and record the instance (GPS time) when the initial traveling wave generated by the fault arrives. The exact fault location is determined by comparing the instances in time when the fault transient arrives at each locator location. The locator unit is tuned over the range of frequencies from 90 to $110 \mathrm{kHz}$, and thus it effectively acts as a band pass filter to extract a band of high-frequency transient voltage signals from the faulted line.

Implementation: EMTP simulations on a sample $11 \mathrm{kV}$ multi-source distribution system are presented in the paper. A non-linear arc model is used in the system (by detecting the fault-arc-generated transients during voltage zero conditions, the fault can still be detected). Simulation results for different fault locations are presented for different fault 
types, fault resistance, and system voltage conditions. No actual field testing is reported in the paper.

Issues:

The simulation system used is simple and has few reflections/discontinuities.

High accuracy of fault location estimate demands high sampling rate requirements.

Actual field testing is needed.

Feasibility: The scheme is insensitive to fault type, fault resistance, and fault inception angle conditions.

A.2.4.2.13 A novel fault locator based on the detection of fault generated high-frequency transients [35]

Summary: This paper presents a technique of fault location which utilizes specially designed fault locators to capture the fault generated high-frequency voltage or current transient signals from the faulted line/cable. The propagation time of the high-frequency components is then used to determine the fault position.

In the scheme, the fault locator is designed to capture the voltage/current signals of frequencies above $40 \mathrm{kHz}$. Two types of locators are used: the current locator is directly connected to the CT's and the voltage locator is interfaced to the line/cable through coupling capacitors. It is reported that the current-based scheme is suitable for applications involving fault locations in long overhead lines, whereas voltage-based schemes can be used for short-line cables.

The block diagram of the locator unit is shown below.

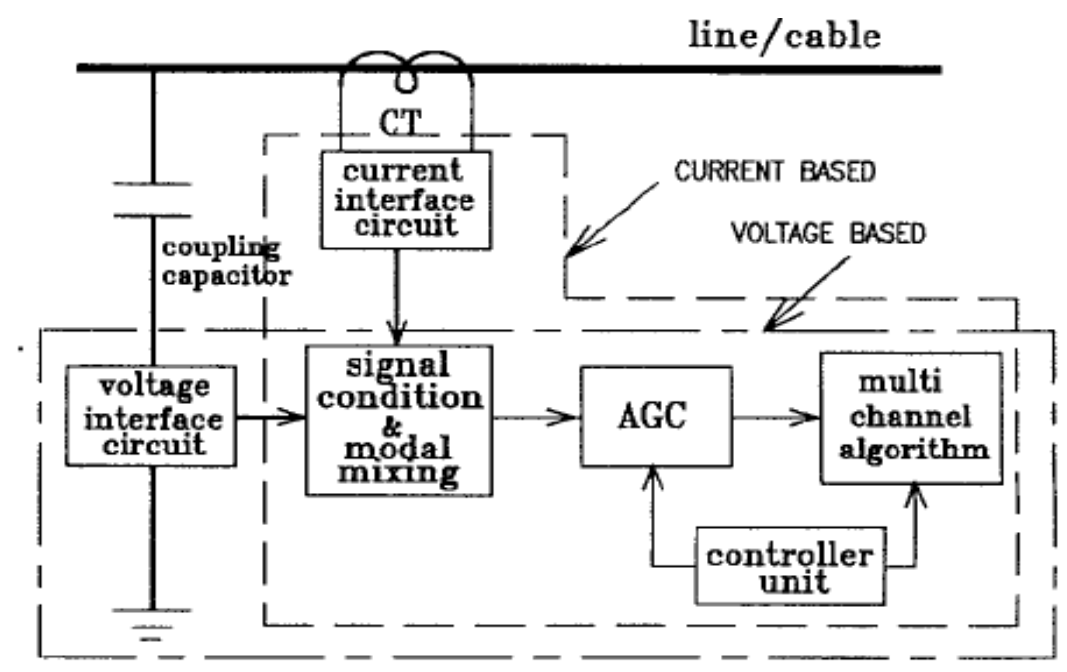

Figure A.11—Fault locator using high-frequency voltage and current signals 
Implementation: EMTP simulation results for a current-based locator for a sample overhead transmission line $(400 \mathrm{kV})$ and a voltage-based locator for a sample underground cable network $(11 \mathrm{kV})$ are presented. In the underground cable case, the voltage-based locator is tuned to center frequency of $3 \mathrm{MHz}$. Results for single-phase-toground fault and faults close to the busbar are presented, and the effect of busbar capacitance is reported. No actual field testing is reported in the paper.

Issues:

For a given fault and system condition, the magnitude of the fault generated highfrequency signal decreases with increasing busbar capacitance. More severe attenuation is shown in the case of a cable.

High sampling rates are required.

A very simple case is used for simulation study. No tapped loads/laterals are considered in the paper.

\section{A.2.4.2.14 Design of a low voltage cable fault detector [36]}

Summary: This paper presents design and construction of a low voltage cable fault location detector. The principle of design is based on the reflection of waves. A pulse is sent into the defective cable, and it gets reflected back. For a broken cable fault, the reflected pulse will have the same phase as the incident pulse, whereas for a short circuit in the cable, the reflected pulse will be out of phase compared to the incident pulse. The time interval of traveling waves is then identified in order to determine fault location and fault type.

The prototype consists of a pulse generator, wave detection circuit, counter circuit, control and processing part, and display unit based on a microcontroller. All are assembled in a compact size. The detector design is divided into two parts, a hardware component and a software component (details in the paper). The proposed detector is able to identify the fault location in a cable of length ranging from 50 to 500 meters.

Implementation: Sample performance results for a cable length of 50 meters up to 500 meters are reported in the paper for both open circuit and short circuit fault cases. No actual field testing is reported in the paper.

Issues:

Complexities of multiple reflections etc. which are typical of distribution systems are not considered for this method.

The amplitude of a reflected wave gets attenuated with increase in cable length, which makes detection difficult after crossing a certain length of the cable.

Actual field test results are needed.

A.2.4.2.15 An Alternative Fault Location Algorithm Based on Wavelet Transforms for Three-Terminal Lines [37] 
Summary: This paper presents a complete fault location algorithm for three-terminal transmission lines based on Wavelet Transforms (WT). In this work, the WT were used to analyze the low and high frequency components of the current and/or voltage signals generated by an electric fault. The methodology uses either traveling waves or impedance calculation methods to locate the fault. The traveling wave method analyses the current and/or voltage high frequency signals extracted by WT to determine the fault location. On the other hand, the fundamental frequency components of the voltage and current signals, also extracted by WT, can be used for the same purpose.

Implementation: The methodology is based on the low and high frequency components of the transient signals originated from a fault situation registered in the terminals of a system. By processing these signals and using the WT, it is possible to determine the time of traveling waves of voltages and/or currents from the fault point to the terminals, as well as to estimate the fundamental frequency components.

Issues:

Concerning the fault location technique based on the fundamental component, the performance was satisfactory with regards to accuracy and speed of response for the tests considered.

Some inaccuracies were observed to faults close to the terminals.

This approach was weakly influenced by the fault impedance, fault type, fault inception angle, fault position and noise at the input signals.

The method showed that it was influenced by mutual coupling effects and fault impedances above $100 \mathrm{ohms}$ and SNR lower than $60 \mathrm{~dB}$.

Feasibility: The algorithm was tested for different fault conditions by simulations using ATP (Alternative Transients Program) software. The results obtained are promising and demonstrate a highly satisfactory degree of accuracy and reliability of the proposed method.

\section{A.2.4.3 Fault Location: Knowledge Based Techniques}

A.2.4.3.1 AI-based methods in practical fault location of medium voltage distribution feeders [38]

Summary: This paper describes a fault location expert system, an application of a distribution management system, which is a part of the integrated control center environment composed of different computer systems and distribution automation. The expert system applies AI-based methods (e.g. object-oriented modeling, blackboard techniques, fuzzy reasoning, and decision trees) in incorporating data on external systems in order to support the control center operator in network operation. At present, the expert system is in everyday use in several distribution utilities.

The fault location expert system comprises modules for event analysis, determining possible fault locations, and switching plans for locating and isolating the fault. The expert system is a part of the fault management entity, which contains additional applications for the automatic management of customers' trouble calls using an 
answering machine, guiding the moving of the working crew using e.g. satellite navigation, and fault reporting. The expert system combines information obtained from the network database (e.g. network data, terrain conditions) and the SCADA (e.g. measured fault current, operation of fault detectors, weather information) together with the heuristic knowledge of operators (e.g. fault sensitivity of network components in different weather conditions) to infer possible fault locations. The operation of the expert system is carried out automatically without any operator actions based on the SCADA information which is transferred via the local area network (LAN). The calculated fault distance is the main information used in the inference. The electrical distance between the feeding point and the fault location is determined by comparing the measured short circuit current and the type of fault (obtained on microprocessor-based relays) with the calculated fault currents of each line-section. The maximum distance is calculated using the zero fault resistance. Because of the uncertainty of fault resistance, the actual fault location is normally a little closer to the feeding point. The possible fault locations are ranked using information on other knowledge and data sources.

Issues: Because of the uncertainty of fault resistance, small errors in fault location estimates are introduced.

Implementation: The expert system is in everyday use in several distribution utilities. Also, mention of testing of this fault location expert system in the pilot utility KoillisSatakunnan Sahko Ltd., having about 1000 distribution substations and $1400 \mathrm{~km}$ of $20 \mathrm{kV}$ feeders, is present in the paper.

A.2.4.3.2 A network distribution power system fault location based on neural eigenvalue algorithm [39]

Summary: A new approach to fault location in distribution systems using Eigenvalue and an Artificial Neural Network based learning algorithm is presented in this paper. The main steps of the algorithm are

Data acquisition (phase currents; no voltages required)

Clarke-Concordia transformation of sample data and eigenvalue approach obtaining eigenvectors and eigenvalues

Fault detection (compare fault and pre-fault characteristic eigenvalues)

Fault classification (compare characteristic eigenvectors with fault patterns)

Fault location/distance calculation (using eigenvalue as neural network inputs)

The applied ANN is of the "feed forward" type, and "error-back-propagation" is considered.

Implementation: Matlab/Simulink simulation results for a sample MV/LV $(60 \mathrm{kV} / 20 \mathrm{kV})$ distribution system consisting of two interconnected sources feeding a load located on a third node are presented in the paper. SLG faults, line-to-line faults, and three-phase fault types are studied. No actual field testing/implementation are reported in the paper. 
Issues:

The system considered is a simple one.

Actual field testing results are needed.

Feasibility: The method shows promise. One advantage is that only current signals are needed (and no voltage signals). The method both identifies the fault type and the fault location and is almost independent of harmonic influence.

Related paper: V. Alegria, L Martins, S. Martins, and J. F. Fernao Pires, “The application of Neural Networks and Clarke-Concordia transformation in fault location on distribution power systems" in IEEE/PES Transmission and Distribution Conference and Exhibition: Asia Pacific, 2002, pp. 2091-2095.

\section{A.2.4.3.3 Application of a fuzzy set method in distribution system fault location [40]}

Summary: A fuzzy-set-based fault location algorithm is presented in this paper. It utilizes the fault related data from fault sensors on the feeders, limited SCADA data from distribution automation systems, and simulation results from short circuit analysis. All this data, information, and knowledge are fuzzy in nature: The measured data may have different degrees of uncertainty; the short circuit calculation results may contain some magnitude of inaccuracy; the operators' experience can tell only the possibility.

The fault location algorithm includes two phases. In the first phase, sensor signals, limited SCADA information, and some heuristic rules are used to form the basic possible faulted zone. The information is deterministic, i.e. crispy. The collection of objects (all line sections on the faulted feeders) is effectively reduced to a much smaller set than that of the original collection. In the second phase, the fuzzy set method is applied to further reduce the size of this set and provide the priority list of possible faulted line sections (The detailed algorithm is presented in the paper).

Implementation: The proposed algorithm was implemented on UNIX and tested on PG\&E distribution feeders. The example feeder used for testing was at Santa Rosa Division of PG\&E, including 495 line sections, 496 node numbers, and 100 switches. There were 20 sensors at the feeder.

Feasibility: The results look promising. However, test result for only one case (one random fault location) is presented in the paper.

Related paper: W.-H. E. Liu and W. Zhong, "A fuzzy set method for fault location identification in power distribution systems," in Proceedings of the 35th IEEE Decision and Control Conference, 1996, pp. 2208-2212.

A.2.4.3.4 Earth fault distance computation with artificial neural network trained by neutral voltage transients [41] 
Summary: This paper presents a novel earth fault distance computation application based on an ANN in a radial power distribution network (with an ungrounded or compensated neutral). It uses the harmonic components of the neutral voltage transients for earth fault distance computation. The benefit of this method is that only one measurement per primary transformer is needed. The results are compared to other ANN's trained by phase current and voltage samples.

Special focus is given to the scaling and adaptation of the input data enabling one single ANN to estimate fault distances in power distribution networks of different sizes. The ANN-type Multilayer Perception, with one hidden layer and trained with the backpropagation algorithm, is used.

Implementation: Both simulation tests and field tests are carried out for comparison with other ANN methods. Together, 18 earth faults are carried out with fault resistances of 0-50 ohms at different line locations.

Issues:

The performance of earth fault location is restricted by the attenuation of the transients. With higher fault resistances (50 ohms), the transients are attenuated; and errors are increased drastically.

The accuracy is also very sensitive to the frequency scaling due to the different network sizes.

Feasibility: The benefit of this method is that only one measurement per primary transformer is needed.

A.2.4.3.5 A computerized fault location process for overhead radial distribution feeders [42]

Summary: This paper describes an automated system for fault location in distribution networks. The method uses information such as feeder configuration, quality of network, type of terrain, human experience, fault statistics, information recorded by protection devices, etc. To deal with uncertainties and inexactness during fault location process, fuzzy set theory is used in the method.

Implementation: Simulation results for a sample $10 \mathrm{kV}$ radial distribution feeder are presented in the paper for three-phase faults and single-phase faults. No actual field testing/implementation are reported in the paper.

Issues:

Requirement to regularly update the data which make membership functions in fuzzy set theory. Actual field testing results are needed.

A.2.4.3.6 Fault section estimation in electrical power systems using Artificial Neural Network (ANN) approach [43] 
Summary: This paper presents an ANN-based approach to estimate the faulty section in a power system by using the information from the protective relays and circuit breakers. The proposed approach is applied to a sample system where bus, transformer, and line protection schemes are considered.

The general procedure of ANN application to fault section estimation used in this method can be represented as

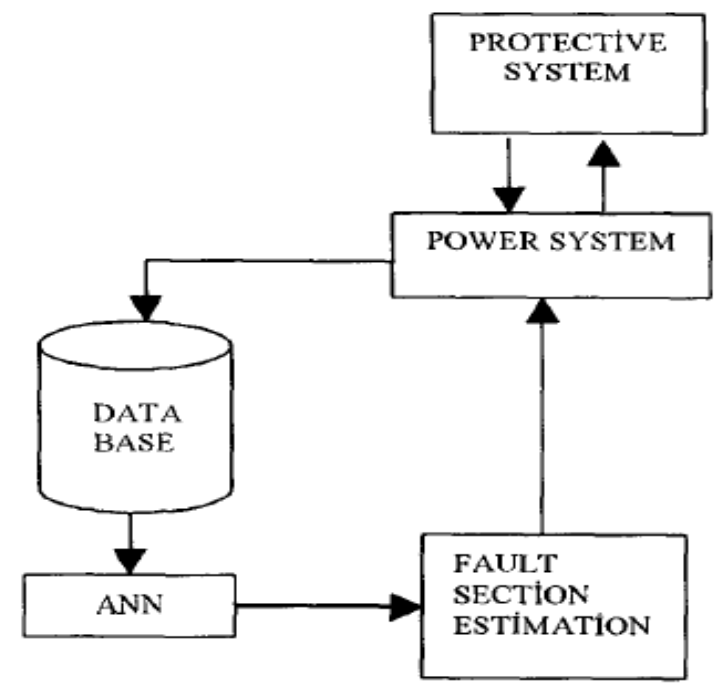

Figure A.12 - ANN used with protection information to estimate fault locations

Relay and circuit breaker status are taken as inputs to the neural network. If the relay has actuated, then the input of the relay is 1 ; otherwise it is 0 . Similarly, if the circuit breaker is open, the input is 1 , otherwise 0 . Output of the neural network depends on the system topology. The output vector has dimensions of the protected sections. If the fault is on the specified section, then the output vector is 1 , otherwise 0 .

The fault scenarios taken into consideration are normal operation and normal tripping, false operation and false tripping, failure of operation and failure of tripping of relays, and circuit breakers. While creating fault scenarios, only one fault at a time is considered because neural networks can make generalization for the multiple faults while learning single faults.

Implementation: A sample 4-bus, 4-line, single-transformer system having 9 protected sections is used for simulation study. Matlab Neural Network Toolbox is used for the same. ANN is trained with a constant learning rate of 0.01 , momentum of 0.8 , and maximum tolerance of 0.001 . Some of the possible fault scenarios in the system are used to generate training pattern sets based on the rule-based structure considering the actuated relays and open circuit breakers, and the output results are the estimated fault sections.

No actual field testing/implementation are reported in the paper. 
Issues: Actual field testing results are needed to validate the method for all operating conditions.

A.2.4.3.7 A new approach to ungrounded fault location in a three-phase underground distribution system using combined neural networks and wavelet analysis [44]

Summary: This paper presents a new offline method for locating ungrounded shunt faults in a practical underground cable distribution system using an advanced signal processing technique based on wavelet analysis to extract useful information from signals. This information is then applied to the ANN's. At first, a new method to analyze power distribution system transient signals is proposed using Wavelet Theory (feature extraction). Daubichies' wavelet is used as the mother wavelet. The sampling frequency is $100 \mathrm{kHz}$. Analysis results show that particular wavelet components can be used as the features to locate the fault in underground distribution system. Then an accurate fault location technique based on ANN is developed. The problem is divided into two parts: firstly, an ANN is employed and trained to classify the faults; secondly, one for each type of fault and faulted phases is used to accurately locate the actual fault position. In this regard, three-layer feed-forward and the Levenberg-Marquardt (LM) learning algorithm is used to adopt the weights and biases to achieve the desired nonlinear mapping from inputs to outputs. Each ANN is tested with a separate set of unseen data, and their performance on the accuracy of results is presented.

The schematic of the fault location technique is represented in Figure A.13 on the following page.

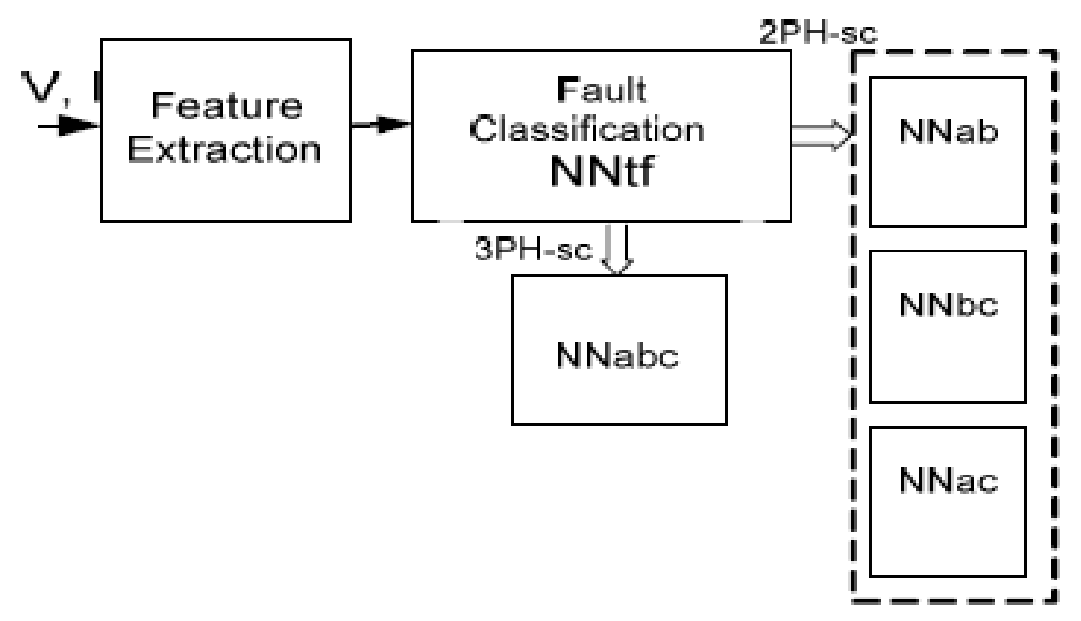

Figure A.13-ANN used to classify and locate faults

Implementation: A practical $11 \mathrm{kV}$ underground power distribution system with a remote source is simulated using EMTP software for simulation study purposes, and results are presented. The faulted current and voltage responses are extracted from the sending end for different faults and fault conditions. The effect of CT's and VT's and hardware errors such as anti-aliasing filters and quantization are taken into account. Effects of fault 
parameter, remote source, load taps, cable length, and external faults are analyzed; and results are presented. No actual field testing/implementation are reported in the paper.

Issues:

Deals with ungrounded faults only

Actual field testing results are needed to validate its performance under real operating conditions.

A.2.4.3.8 Artificial Neural Network and Support Vector Machine approach for locating faults in radial distribution systems [45]

Summary: This paper presents an Artificial Neural Network (ANN) and Support Vector Machine (SVM) approach for locating faults in radial distribution systems. The proposed approach uses measurements available at the substation, circuit breaker, and relay stations. The data is then analyzed using the Principle Component Analysis (PCA) technique, and the faults are classified according to the reactances of their path using a combination of Support Vector Classifiers (SVC's) and Feed-Forward Neural Networks (FFNNs). Two schemes particular to the problem are discussed (details in paper); their schematics are portrayed in upcoming Figures A.14 and A.15.

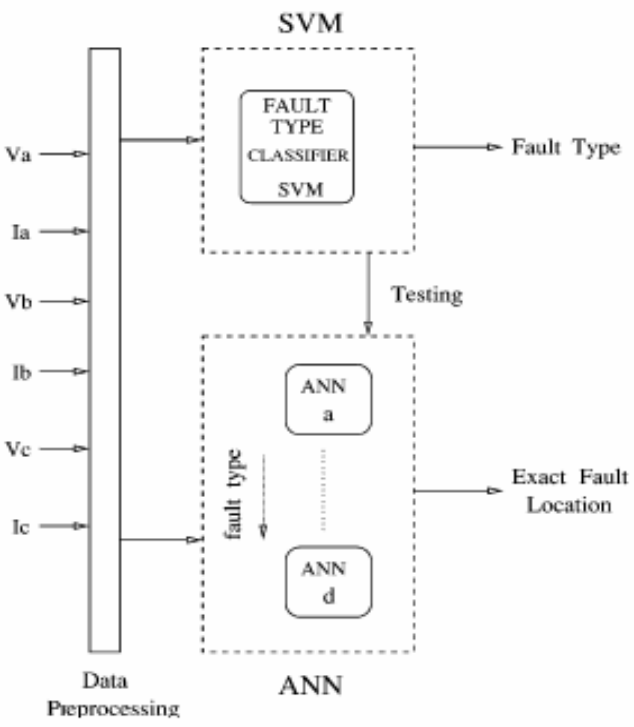

Figure A.14-SVM scheme 1

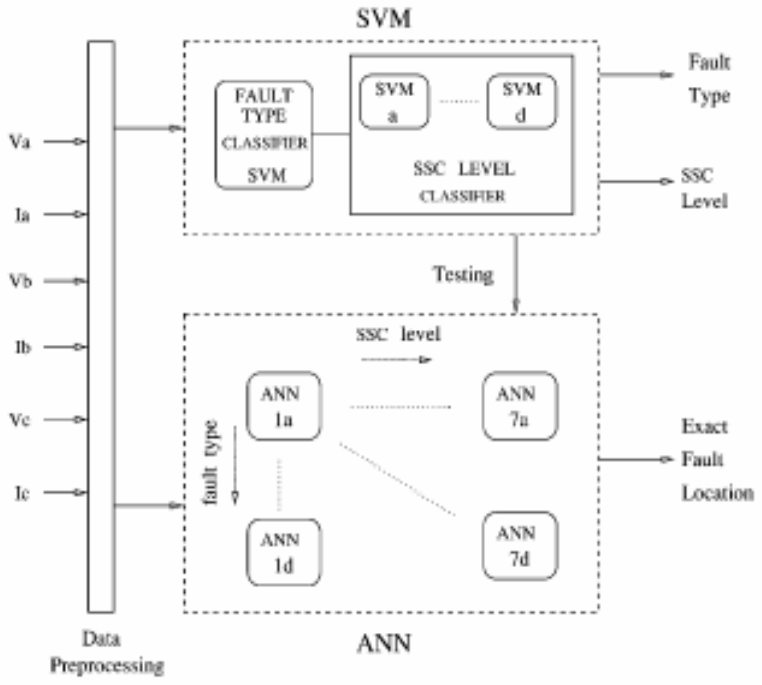

Figure A.15-SVM scheme 2

Implementation: Simulation results using a sample $11 \mathrm{kV}$ practical distribution network consisting of 52 buses with 3 main feeders are presented in the paper. Practical situations, such as protective devices placed only at the substation, all types of faults, and a wide range of varying short circuit levels are considered for this simulation study. No actual field testing/implementation are reported in the paper.

Issues: 
For a change in network configuration, following a contingency, either the ANN has to be retrained or an ANN trained beforehand for the contingency to be put into service (though the paper mentions that if retraining has to be adopted, the time taken would be negligible due to the simplicity and parallelism of the neural network architecture and algorithms).

Actual field testing/implementation results are needed.

A.2.4.3.9 Fault Diagnosis and Reconfiguration for Multilevel Inverter Drive Using AIBased Techniques [46]

Summary: This paper proposes a fault diagnostic and reconfiguration method for a cascaded H-bridge multilevel inverter drive (MLID) using artificial-intelligence-based techniques. Diagnostic signals in the form of output phase voltages of the MILD are used to detect fault and their locations. Due to the nonlinearity factor of the complex system and many switching devices in MLID systems, it is difficult to diagnose an MLID system using a mathematical model. Hence a neural network (NN) classification is used to diagnose the fault in an MLID system. The proposed fault diagnostic system requires about six cycles to clear an open-circuit or short-circuit fault. The experimental results show that the proposed system performs satisfactorily to detect the fault type, fault location, and reconfiguration. To select the valuable principal components the genetic algorithm is also applied.

Implementation: The proposed network is evaluated with simulation test set and experimental test set. The overall classification performance of the proposed network is more than $95 \%$. A reconfiguration technique is also proposed.

Issues:

The fault diagnostic system requires about six cycles ( $100 \mathrm{~ms}$ at $60 \mathrm{~Hz}$ ) to clear the open-circuit and short-circuit faults and about nine cycles to clear the short circuit under loss of SDCS at faulty cell condition.

The clearing time of the proposed system can be shorter than this if the proposed system is implemented as a single chip using a field-programmable gate array or digital signal processor.

The clearing time could be within two cycles if the algorithm is implemented with an embedded single chip

The fuse protecting the SDCS may blow (disconnect the SDCS from an MLID) before the diagnostic system performs fault clearing so that the output phase voltage will be zero.

Feasibility: Output phase voltages of the MLID can be used as diagnostic signals to detect faults and their locations. The proposed fault diagnostic paradigm has been validated in both simulation and experiment. The results show that the proposed fault diagnostic technique performs quite satisfactory. 
A.2.4.3.10 An Unsupervised Learning Method for Comparing the Quality of the Soft Computing Algorithms in Analog Systems Diagnostics [47]

Summary: The paper presents the method of assessing the difficulty of the analog systems' fault detection and location procedure, using the soft computing algorithm. The analysis of learning and testing of data sets (generated for the selected analog system) allows determining the difficulty of the system for the diagnostic procedure. This helps to compare different artificial intelligence and machine learning approaches used to detect, locate and identify faults. The versatile method of the data sets' difficulty based on the graph clustering algorithm is proposed and explained. It is then applied to test two advanced methods: fuzzy logic and rough sets against the sixth order Butterworth low pass filter. The paper is concluded with future prospects of the proposed method.

Implementation: The paper presents the application of the graph clustering method to calculate the difficulty measure of the learning and testing data sets.

Issues:

The complexities of the training data sets are comparable, despite increasing the number of the categories to distinguish.

The main problems with the fault detection depend on the SUT itself, not the resolution imposed by the designer.

The testing data sets seem to be more difficult to diagnose, so the obtained diagnostic results may be worse than expected from the train sets.

Feasibility: The method helps to determine, how difficult the SUT is and relate the diagnostic outcomes of the proposed AI method to this difficulty. Therefore the proposed approach can be used to objectively compare different algorithms.

\section{A.2.4.3.11 Double Circuit Transmission Line Fault Distance Location using Artificial Neural Network [48]}

Summary: Distance relays used for protection of transmission lines have problems of under-reach, over-reach and mal-operation due to high impedance faults. Further the problem is compounded when the distance relays are used for protection of double circuit transmission lines due to effect of zero sequence mutual coupling. This paper presents a single neural network for fault distance location for all the ten types of faults (3 LG, 3 LLG, 3 LL, and 1 LLL) in both the circuits of a double circuit transmission line fed from sources at both the end. This technique uses only one end data and accurate fault distance location is achieved after one cycle from the inception of fault. The proposed Artificial Neural Network (ANN) based Fault Distance Locator uses fundamental components of three phases current signals of both the circuits $\&$ three phase voltage signals to learn the hidden relationship in the input patterns.

Implementation: This paper presents an application of artificial neural network for fault distance location in a double end fed double circuit transmission line for all the ten types 
of shunt faults using only one terminal data considering the effects of mutual coupling, remote source infeed, varying fault type, fault location, fault resistance and fault inception angle.

Feasibility: An improved performance is obtained once the neural network is trained suitably, thus performing correctly when faced with different system parameters and conditions i.e. varying fault type, fault location, fault resistance, fault inception angle, presence of mutual coupling and remote source infeed.

\section{A.2.4.3.12 Fault Classification and Location of Power Transmission Lines Using Artificial Neural Network [49]}

Summary: This paper describes the application of an artificial neural network (ANN) based algorithm with modular structure to the fault classification and location of a singlecircuit high voltage transmission line. The operation of proposed strategy is not dependent on fault inception angle (FIA). A new classification method is proposed for decreasing of training time and dimensions of ANN. Using the proposed method, high accuracy of fault classification is achieved. Fundamental component of pre-fault and post-fault positive sequence component of currents and voltages of three phases have been used as inputs to proposed ANN. The output of the ANN is the estimated fault location. A two machine power system model is simulated by PSCAD/EMTDC to obtain the mentioned voltages and current values. The neural network toolbox of MATLAB is used for training and testing of ANN.

Implementation: Development in personal computers allows us to simulate power electric systems, exactly similar to real systems. In this paper simulation studies have been carried out using PSCAD/EMTDC and MATLAB. A large number of fault samples data have been generated using PSCAD/EMTDC considering wide variations on fault location, fault resistance and fault type. Using these data, fault classification and location is carried out by means of MATLAB that makes use of its "neural network toolbox".

Feasibility: Maximum absolute error for single-phase to ground fault was $0.3324 \%$ and it was $0.1403 \%$ for two-phase, $0.3408 \%$ for two phase to ground and $0.4926 \%$ for three phase fault. The results show valuable operation of proposed ANN based fault locator in estimation of fault location of different types of faults.

\section{A.2.4.3.13 Study on Fault Location for High Voltage Overhead Transmission Lines Based on Neural Network System [50]}

Summary: A distributed \& hierarchical NN (DHNN) system based on the integrated module architecture and hierarchy architecture is proposed in this paper. The DHNN system adequately uses the powerful function of artificial neural networks at aspects of pattern identification, nonlinear approaching, associative memory et al. Its information 
processing mechanism coincides with the processing law of classification sketchinessaccuracy in human biologic NN system. This system not only can deal with the advanced information required by fault location for $\mathrm{HV}$ overhead transmission lines but accurately locate the fault sites. Thus this method for fault location presented in this paper can thoroughly eliminate the disadvantages in other extant fault location methods, such as convergence to the false root or divergence in the procedure of iteration, which result in the great location error in practice.

Implementation: In consideration with the physical architecture and information processing mechanism of the biologic NN system, a DHNN system is presented in this paper, which is modular and hierarchical.

Feasibility: This paper pioneers a new direction to the study and application on fault location. Results from theoretical analyses and simulations by EMTP show that fault location precision of this method can completely satisfy practical requirements. It has been proved that this proposed approach is feasible and effective by theoretical analysis and lots of simulations and tests by EMTP.

\section{A.2.4.3.14 Performance Evaluation of an Adaptive-Network Based Fuzzy Inference System Approach for Location of Faults on Transmission Lines Using Monte Carlo Simulation [51]}

Summary: This paper employs a wavelet multi-resolution analysis (MRA) along with the adaptive-network-based fuzzy inference system to overcome the difficulties associated with conventional voltage- and current-based measurements for transmission-line fault location algorithms, due to the effect of factors such as fault inception angle, fault impedance, and fault distance. This proposed approach is different from conventional algorithms that are based on deterministic computations on a well-defined model to be protected, employing wavelet transform together with intelligent computational techniques, such as the Fuzzy Inference System (FIS), Adaptive Neuro-Fuzzy Inference System (ANFIS), and artificial neural network (ANN) in order to incorporate expert evaluation so as to extract important features from wavelet MRA coefficients for obtaining coherent conclusions regarding fault location.

Implementation: A sample three-phase power system has been simulated using MATLAB software [7]. The line currents are processed using an online wavelet transform algorithm to obtain wavelet MRA coefficients. These coefficients are used for fault location using intelligent techniques like FIS, ANFIS, and ANN.

Issues: This paper does not take into consideration the effect of fault inception angle and fault impedance, which leads to misleading results in the case of high-impedance arcing faults.

Feasibility: A comparative study establishes that the ANFIS approach has superiority over ANN and FIS-based approaches for the location of line faults. In addition, the 
efficacy of the ANFIS is validated through the Monte Carlo simulation for incorporating the stochastic nature of fault occurrence in practical systems. Thus, this ANFIS-based digital relay can be used as an effective tool for real-time digital relaying purposes.

\section{A.2.4.4 Fault Prediction}

\section{A.2.4.4.1 Failure analysis in $\mathrm{ZnO}$ arresters using thermal images [52]}

Abstract: Thermographic inspection is a widely used method to monitor surge arresters, preventing their failure. When an arrester is substituted due to some abnormality detected by means of thermal inspection, no further analysis is done, so that the causes of the problem cannot be evaluated. In addition, there is no study indicating the main causes of failures in Zinc Oxide ( $\mathrm{ZnO})$ arresters or if there is another alternative to the substitution of them. This work presents a study done with $96 \mathrm{kV} \mathrm{ZnO}$ arresters presenting the most common failures detected in substations and their effects on the thermal image.

Summary: Leakage current monitoring and thermal inspections are the most effective techniques for monitoring and predictive maintenance of the $\mathrm{ZnO}$ arrester. But there is no specific methodology for the inspections, and each company has developed its own methodology and parameters for analysis based on their experience. This publication proposes an analysis of arrester failures with a more detailed study of the thermal image and the conditions of operation.

The authors' tested arrester's defects related to thermal overheating by intentionally introducing defects and determining the main effect observed in the thermal profile for each defect. The following observations are obtained from the thermal profile:

Sealing failure: decrease of the temperature peak in the regions of greater heating Internal humidity: a large increase in temperature in distinct regions of the arrester Superficial pollution: greater heating in the proximities of the terminals and downer part of the arrester Varistor degradation: more intense temperature on the region of the group with a degraded varistor.

The authors conclude that for all the tests performed, it was possible to detect variations in the thermal images and profiles for the different sources of failure. The published results are the first step on what the authors hope will be an analysis and diagnostic tool for arrester thermal images.

\section{A.2.4.4.2 Recognition of electrical tree progress in solid insulator [53]}

Abstract: This paper studies the prediction on electrical tree discharge, three types of electrical tree models are made, that is, needle to plane electrode in insulator, needle with void to plane electrode and needle with metal tip to plane electrode in insulator. Partial discharge (PD) data are acquired from PD detector, and then statistical distributions calculated to recognize progress of electrical tree from initial to final stage. The statistical distributions are applied as input data of the classification tools to classify PD sources. The results show good recognition rates of electrical tree models. 
Summary: Electrical tree discharge distinguishes from deterministic breakdown because the energy transfer and the growth of the damaged region cannot be accelerated in time. This publication focuses on the partial discharge (PD) analysis originated from the electrical tree and their distribution characteristics. For the tests, three types of models were used in PD data acquisition, and that data was calculated as a PD distribution. The test system recorded the whole pulse shape and the peak and phase of occurrence for each PD pulse. A time-sequence analysis was used since it is more descriptive of the correspondence between discharges and tree propagation. Distributions were derived from statistical distributions of individual PD events by taking appropriate averages. An adaptive network based fuzzy inference system (ANFIS) was employed for the classification sources. This system achieved a high recognition rate with simplicity of construction and a short learning time.

The paper concludes that the use of different characteristics can discriminate the tree sources and that measuring the time to tree progress can determine prediction of insulation failure for equipment.

A.2.4.4.3 Data mining and analysis of tree-caused faults in power distribution systems [54]

Abstract: In this paper, four different measures: actual measure, normalized measure, relative measure, and likelihood measure are used to data mine the Duke Energy Distribution Outage Database for meaningful data features and to analyze the characteristics of tree-caused distribution faults. This paper also applies statistical techniques to analyze tree-caused faults with respect to several selected influential factors. The results can be used to assist power distribution engineers to provide a more effective fault restoration system and design a more effective tree-fault prevention strategy.

Summary: This paper proposes a statistical data mining approach to analyze and address the tree-caused fault characteristics according to system configuration and environmental factors using a large outage database from Duke Energy. Based on previous work the researchers selected six of thirty-three information factors recorded by Duke Power: circuit ID, weather condition, season, time of day, affected phases, and activated protective device. For this study, Duke Service area is divided into three mutually exclusive subsections, and two or three representative regions are picked from each subsection for a total of seven regions. Outage data is limited to the 1994 to 2002 period.

Four measurements are applied to the data set, and uses for the utility are suggested for each measurement:

Actual measurements are used to extract information without the regional factors and can be used to allocate trimming resources for each region

Normalized measurements are used to provide relative frequency and importance of three faults and determines the relative importance of this type of fault to help set prevention priorities. 
The relative measurement and likelihood measurement are used to extract more detailed information from the data set on weather conditions, season, and time of day, phases affected, and protective devices activated. These measurements can be used for root cause identification of three faults.

Some of the results obtained for the selected factors are

Weather condition: most three faults occur on fair weather but the likelihood of is higher for severe weather

Season: most three faults occur in summer and winter but for winter faults are most likely caused by three faults

Time of day: most three faults occur during daytime but evening faults are most likely caused by three faults

Phases affected: most three faults occur on a single phase but a two or three phase faults is most likely caused by a three fault

Activated devices: line re-closers and circuit breakers are most likely activated by three faults.

The study found that all the six factors are statistically significant except in one region, and season is the less significant factor. The researchers conclude that outage databases can provide quantitative and qualitative information to develop a more effective restoration system and design a more effective prevention strategy.

A.2.4.4.4 On-Line condition monitoring and diagnostics of power distribution equipment [55]

Abstract: The paper presents the results of wireless temperature monitoring of switchgear at a power plant over a two-year period. On-line temperature monitoring helps to coordinate equipment specifications and ratings, determine the real limits of the monitored equipment and optimize facility operations. Using wireless technique eliminates any need for special cables and wires with lower installation costs if compared to other types of online condition monitoring equipment. In addition, wireless temperature monitoring works well under difficult conditions in strategically important locations. Wireless technology for on-line condition monitoring of energized equipment is applicable both as standalone system and with an interface with power quality monitoring system.

Summary: Continuous temperature monitoring will identify potential problem areas that can lead to substantial equipment damage. Correlation between the temperature measurement and load and ambient conditions would allow abnormal conditions to be identified and early alarms to notify operation and maintenance personnel of a potential problem. The real value of the on-line monitoring is not in setting off multiple alarms but in triggering the maintenance events, leading to a true condition-based maintenance, and providing the key answers to implementing the plant assets management.

Wireless continuous temperature monitoring of energized equipment provides true information about the condition of the equipment and provides the following benefits: 
Ease of system application: It can be easily applied to existing power equipment and allows easy installation of the Temperature Monitoring System on existing equipment with short interruption in service.

Equipment integrity: Wireless sensors do not disturb the thermal, dielectric, and mechanical integrity of the power equipment and do not add any additional steps for operation personnel.

System universality: The wireless sensors can be used throughout a facility without any limitation to the type of equipment to be monitored.

Increased equipment reliability: This plan facilitates planned maintenance, decreases downtime, and increases the reliability and availability of power equipment.

Reduced design, construction and maintenance cost: Minimum engineering time is required to integrate the design of new and existing equipment. Simple installation minimizes construction time. Information could be used to initiate condition-based maintenance.

\section{A.2.4.4.5 Prediction of weather-related failures of overhead distribution feeders [56]}

Abstract: This paper presents two methods to predict weather-related overhead distribution feeder failures. The first model is based on linear regression, which uses a regression function to determine correlation between the weather factors and overhead feeder failures. The second method is based on a one-layer Bayesian network, which uses conditional probabilities to model the correlation. Both methods are discussed and followed by tests to assess their performance. The results obtained using these methods are discussed and compared.

Summary: This paper presents two models to predict number of failures related to weather factors. Both models try to capture the trends in the relationship between weather and the number of failures that took place in overhead distribution feeders. The study uses Westar Energy data on overhead feeders' failure from 1998 to 2002 for the distribution system of Manhattan, Kansas. To eliminate outliers, 35 days of data were excluded from the analysis.

The idea on Bayesian network is to use a probabilistic approach to solve uncertainty in the real world. It has the capacity to learn the orders from a large amount of seemingly scattered and intractable data and to extract structure and parameter to predict outputs or make decisions based on probabilities propagation.

To overcome the assumption of randomness in the occurrence of the failures, yearly aggregated data is obtained to make better predictions compared to the monthly aggregated data. Other factors besides weather are included in the model to eliminate the effect of the assumption that trees do not vary a lot and that feeders' condition does not erode much in a five-year period.

The study revealed trends consistent with common experience: an increasing number of failures with increasing wind levels and the expected number of failures in lightning days are clearly above the ones in non-lightning days. 
The authors regard the regression method as a way to regress on probabilities, and the Bayesian network method as a probabilistic approach. Based on their study, the authors prefer the Bayesian network over the regression method since the meaning of the Bayesian network's parameters is straightforward and explainable.

A.2.4.5.6 Analysis of hidden failures of protection schemes in large interconnected power systems [57]

Abstract: Hidden failures in protection systems have been identified as key contributors in the cascading of power system wide-area disturbances. This paper describes a methodology to evaluate the hidden failure's effects based on regions of vulnerability and areas of consequence in protection systems. The mechanisms of the hidden failures as well as the development of the regions of vulnerability from the hidden failures are shown. A number of the regions of vulnerability in a sample 179 test system are represented as physical areas. Two scenarios evaluate the consequences of the unwanted disconnections of the transmission lines caused by hidden failures. The development of the index of severity, which combines the magnitude of the regions of vulnerability and the consequences of unwanted disconnections caused by hidden failures, is proposed. This index would identify the critical protection systems, whose unwanted operations would result in a significant loss of the power system integrity and/or a large cascading event.

Summary: This paper has presented a methodology to evaluate the hidden failure's effects based on regions of vulnerability and areas of consequence in protection systems. The authors define hidden failures as "permanent defects that will cause a relay or a relay system to incorrectly and inappropriately remove a circuit element(s) as a direct consequence of another switching event." Two elements are listed as necessary to have a hidden failure:

A Protection Element Functionality Defect (PEFD) that takes place when the protection devices are unable to perform their designated and expected tasks and may take the form of hardware failures, outdated settings, human errors, and/or negligence.

The logic involved around the PEFD that determines if the failure remains hidden within the protection scheme until another event uncovers the defect.

A region of vulnerability is defined as the physical area in which the occurrence of an event will trigger the unwanted disconnection due to the hidden failure. These regions are determined by a particular location, dimension, and magnitude depending on the hidden failure mode of the protection system and its location within the power system

The paper presents and applies the mechanisms of the hidden failures to a three-zone distance relay. The development of the regions of vulnerability from the hidden failures is derived for the directional comparison blocking scheme, and the region of vulnerability is represented as a physical area in the transmission line used in the example. 
Two scenarios are used to evaluate the consequences of the unwanted disconnections caused by hidden failures. Two transmission lines are taken out of service as due to a permanent three-phase fault inside the region of vulnerability. The consequences of the unwanted disconnections in the power system are examined, and combined with the magnitude of the regions of vulnerability to create an "index of severity." This index identifies the critical protection systems, whose unwanted operations would disconnect power system elements affecting considerably the power system integrity and its capacity to deliver energy.

A.2.4.4.7 Characterization of transients in transformers using discrete wavelet transform [58]

Abstract: This paper presents the characterization of transients resulting from faults in transformers using discrete wavelet transform (DWT). This characterization will aid in the development of an automatic detection method for internal incipient faults in the transformers. The detection method can provide information to predict failures ahead of time so that the necessary corrective actions are taken to prevent outages and reduce down times. The analyzed data are obtained from simulations and experiments for different normal and abnormal operating cases such as external faults, internal short circuit faults, magnetizing inrush, and internal incipient faults. The simulation method and experiment setup are discussed. The experiments and simulations are conducted on a single-phase transformer as an example case. The results of applying the DWT are discussed.

Summary: Differential protection via the terminal behaviors of a transformer is traditionally used for protective purposes, but improvements in protection focus on discrimination between internal short circuit faults and inrush currents. Also important are incipient faults due to deterioration of equipment insulation, and it is important to detect these disturbances and prevent failures ahead of time. Various costly and offline techniques such as dissolved gas analysis and partial discharge analysis have been used to detect incipient faults, but it is advantageous to find an alternative method which utilizes terminal measurements.

Implementation: A Texas A\&M project used the discrete wavelet transform (DWT) to detect and classify incipient faults in transformers. The DWT allows a wavelet to adopt a mother wavelet after numerous low pass and high pass filtering procedures. The parameters for an equivalent circuit representing a transformer with internal faults were calculated using the 2D magneto static solver in ANSOFT's Maxwell Software. Maxwell SPICE was used to study the transformer's terminal behaviors once a model that took into account both the aging phase and the arcing phase was developed. These two stages represented deteriorating insulation.

Experiments were also performed at a test facility using a custom-built, single-phase, $7200 \mathrm{~V} / 240 \mathrm{~V} / 120 \mathrm{~V}, 25 \mathrm{kVA}, 60 \mathrm{~Hz}$ distribution transformer as well as an appropriate load bank and voltage supply. Primary and secondary voltages and currents were 
monitored and acquired in real-time then were compared to the simulation results. The simulation and experimental results were very close, and MATLAB was used to calculate the DWT of the signals, given the following disturbances: magnetizing inrush, external system short circuits, internal short circuits, internal incipient faults, and load changes.

Issues: Classical short circuit methods cannot detect incipient faults by using the terminal behavior of transformers unless a major arcing fault occurs that will be detected by protective devices such as fuse and relay protection. Traditional Fourier analysis fails to describe the eruptions commonly existing in transient processes such as magnetizing inrush and incipient faults, but the wavelet transform can be used for transformer faults.

Feasibility: The DWT was able to distinguish the various types of faults, as was indicated by signal spikes during the fault times and by the presence or disappearance of the primary and differential currents. These results indicate potential for using this method for predictive maintenance and maintaining reliability of transformers.

\section{A.2.4.4.8 Getting a clear picture of distortion [59]}

Abstract: The days of using the square root of three to convert between line-to-line and line-to-neutral voltages and current values are in the past. The proliferation of rectifiedinput power supplies and other non-linear loads, along with the increase in single phase IT equipment loads has resulted in increased distortion and imbalance at the equipment load, the point-of-common- coupling, and even some substation levels. Harmonic distortion and imbalance can have a significant effect on some equipment, especially transformers and motors. Measuring and monitoring these parameters accurately is important when trying to maximize asset usage, prolong the life of the equipment, and prevent catastrophic failures.

Summary: Today's loads are often not balanced. Non-linear loads and rectified-input power supplies have risen from $25 \%$ to $65 \%$ of the total US load from 1985 to 2000 , which has resulted in current and voltage distortion. Single-phase information technology equipment has also led to increased distortion and imbalance at the equipment or load, the point-of-common-coupling and even substation levels. In order to maximize asset usage, care must be taken to accurately measure and monitor the distortion and imbalances in networks.

Implementation: Hardware and software solutions are available. First, hardware is utilized in the form of transducers with ample bandwidth and amplitude accuracy to obtain harmonics and inter-harmonic voltage and current samples. The Nyquist theory states that a signal must be sampled at least twice as fast as the highest frequency component present in order to reproduce the waveform, and anti-aliasing filters should be used for higher frequencies. Current and voltage transformers should offer appropriate linearity in measurements. Software, on the other hand, comes in the form of digital signal processors which optimize DFT and FFT algorithms as well as calculate the positive, negative, and zero sequencing components. Various algorithms exist to calculate imbalance, and alternative systems require different techniques. 
Issues: Magnetic devices, such as transformers and motors, have increased losses when subjected to harmonic voltages, thus reducing the capacity. Motors must be derated because small voltage imbalances can cause phase current unbalances ranging from 6 to 10 times the voltage unbalance. Of course, motors with overcurrent have shorter lives due to excessive heat.

Feasibility: These hardware and software solutions are adequate for providing a clear picture of distorted and imbalanced systems.

\section{A.2.4.4.9 Prevention of underground cable splice failures [60]}

Abstract: The failure of underground medium-voltage cable splices produces many undesired events. When water invades an underground cable splice, a series of selfclearing faults ensues. These self-clearing faults continue until complete splice failure occurs. This paper discusses the nature of medium-voltage cable splice failure in underground installations. In addition, a new methodology is described which will predict the occurrence of cable splice failures before they happen.

Summary: Underground cable splice faults occur over time, and conventional overcurrent protection is ineffective since the fault duration is too short ( $1 / 4$ cycle). Generally due to faulty mechanical splices, these cable splices fail as water invades the spliced connection and breaks down the insulation properties, causing the conductor to arc. Arcing produces heat; this evaporates the water and causes a sudden high vapor pressure inside the cable splice. This event extinguishes the arc and thus interrupts the fault current within $1 / 4$ cycle in a manner deemed "self-clearing." Characteristics of these faults include Faults always occurred on the same phase

Faults were self-cleared within $1 / 4$ cycle

Faults always occurred near a voltage peak

Frequency of fault occurrences increased with time

This paper describes a universal protective relay (UPR) architecture in which a sub-cycle overcurrent protection scheme is fast enough to operate for $1 / 4$ cycle cable splice faults yet has the logic to differentiate from other fault types, including those to be cleared by other protective devices. Three tests have verified the accuracy, security, and reliability of this scheme; they are the virtual test set (VTS), a real-time power system simulator (RTPSS), and a high-power laboratory. Waveforms were generated with VTS to simulate a selfclearing fault while the sub-cycle overcurrent protection scheme was tested with the RTPSS. The high-power lab included a 500 MVA three-phase test generator with voltage transformers up to $69 \mathrm{kV}$ and momentary test capacities up to $150 \mathrm{kA}$. Its procedure was to first establish a load current, then initiate the ground fault, and finally operate the fuse to limit the short circuit current and simulate a self-clearing fault.

Implementation: The UPR integrates functions such as protection, control, metering, and communications into a single device and makes it possible to manipulate input voltage and current samples through different algorithms to obtain quantities such as phasors, 
symmetrical components, and frequency. This protection scheme works by monitoring faults on the feeder and, each occurrence of a self-clearing fault, incrementing a counter. If the counter reaches a preset value within a programmable time period, an output contact closes, providing remote alarming or tripping capability. A flow diagram of this process is found in Figure A.16.

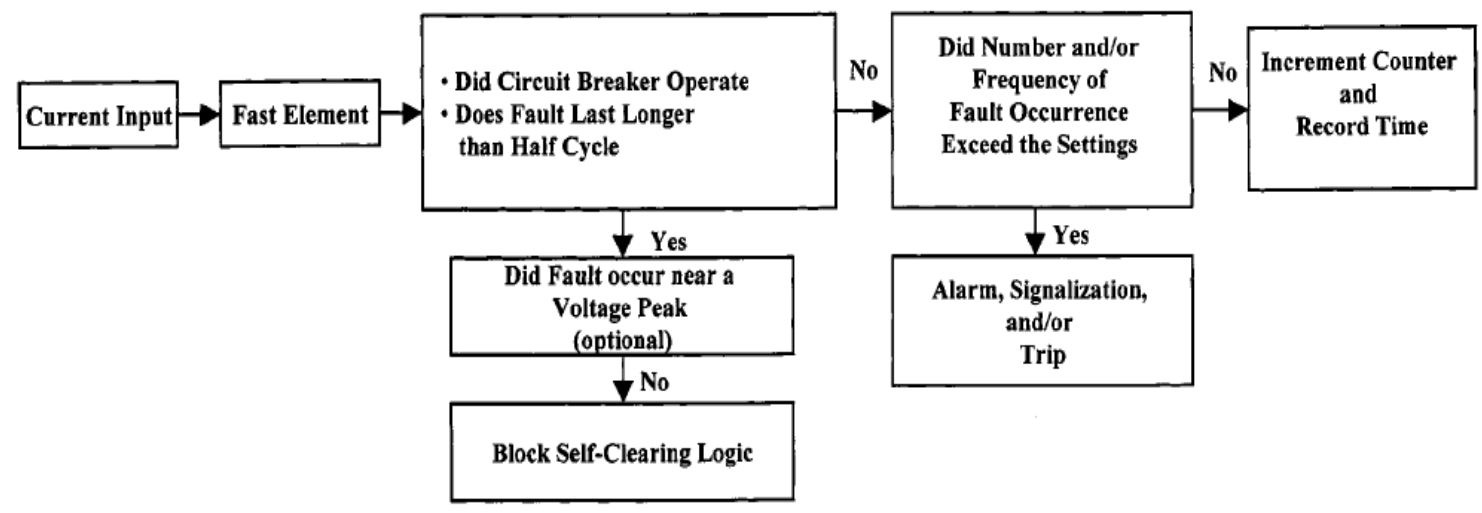

Figure A.16-UPR architecture

Issues: Early detection of the insulation breakdown is required to allow time to locate and repair the failing item before a permanent fault occurs.

Feasibility: The test results indicated proper relay responses with fast element operation followed by the self-clear element, if necessary. The tested relay did not respond to faults cleared by other relays or protective devices, nor for faults which were not initiated near a voltage peak or faults that were not repetitive over some time period. This technique has worked for this case, but there is no guarantee that it will work for all cases.

A.2.4.4.10 Most recent developments in surge arrester technology and testing [61]

Abstract: Surge arresters are used in electric power systems to protect the equipments against overvoltages that may arise from lightning events or from internal switching operations. The technology adopted for the construction of such components has evolved during the years. Silicon-carbide type gapped surge arresters has been replaced by most advanced metal-oxide surge arrester without gaps, that can guarantee better performance and a higher degree of reliability. New developments, presently achieved for distribution range, foresee the replacement of the conventional porcelain housing with a polymeric one, allowing improving the mechanical characteristics and the failure mode behavior. Furthermore, special applications for surge arresters, such as protection of gas insulated substations and prevention of lightning faults in transmissions lines, are now taken into consideration by several utilities. The evolution of surge arrester construction technologies and application requires a continuous revision of relevant Standards and testing techniques. CESI is actively involved in testing surge arresters since the 1960's, through the development and setting up of testing facilities and the participation to the 
major technical and standardization bodies. The paper analyses the most important aspects relevant to surge arrester testing, based on the most recent experience developed in CESI. Particular attention is focused on the short circuit test techniques to address the failure mode and on the ageing test procedures to investigate the long term performance of surge arresters.

Summary: Since the protection against overvoltages is quite important in the design of electrical power systems, it is imperative to have certifiable arresters that confidently meet specified protection standards. In order to achieve these results, we need specific testing procedures to fulfill three basic requirements: proper representation, repeatability, and reproducibility. This paper highlights some of the achievements of CESI in surge arrester testing and specifications, at the light of the evolution of the surge protective devices technology, with particular reference to the metal-oxide type. Tests given to polymeric arresters were the short circuit test (or failure mode test), thermo-mechanical and tightness test, and the accelerated weather ageing test. The article also briefly discussed response times of gas insulated surge arrestors (GIS) as well as the use of metal-oxide surge arresters for overhead transmission lines.

Implementation: First of all, the failure mode test was divided into the preparation phase (test sample is heated) and the short circuit phase. Safety evaluations were based on the extent of ejection of polymeric housing fragments, detachment of the surge protector connections, and fire ignition. The three current types which underwent testing were high current level (HC), medium current level (MC) of 1 to $6 \mathrm{kA}$, and low current level (LC) of 400 to $800 \mathrm{~A}$.

The thermo-mechanical and tightness tests were conducted by heating the samples in ovens at 100 degrees Celsius followed by immersion in cold water and immersion in boiling water. Preconditioning affected the final results of the sealing tests.

Finally, an accelerated ageing process was applied to the sample as it was provided a continuous voltage. Various weathering agents (humidity, rain, surface pollution, solar radiation, and drying periods) influenced the life duration of the polymeric housing and/or of the interface materials and resistor blocks.

Issues:

Polymer arresters have the following advantages over porcelain ones: Better mechanical behavior and a higher safety level in case of failure A lighter construction

Superior performance under polluted conditions, linked with the natural hydrophobic polymeric material and with the possibility to obtain very efficient shed profiles

Feasibility: High voltage surge arresters with low-weight polymeric housing can improve the lightning performance of overhead lines. In terms of protective performance, line arresters must have a lower lightning impulse discharge voltage compared to that of the insulation strings in order to prevent the flashover of the insulation due to a direct stroke to the overhead line. Also, care must be taken to have a 
high enough switching impulse withstand voltage during line re-energization. CESI is still verifying this arrester's abilities in severe conditions, such as wetness, pollution, and temporary overvoltages.

A.2.4.4.11 Analysis and prevention of animal-caused faults in power distribution systems [62]

Abstract: The reliability, security, and quality of power systems are significantly affected by distribution faults. One of the current trends concerning distribution faults is the implementation of more preventive measures instead of reactive measures. In other words, we would like to prevent faults from happening rather than to restore the system after faults occur. Among the different categories of power distribution faults, animalcaused faults are probably the ones that can be prevented most easily and effectively if appropriate control actions are taken. This paper will discuss and analyze animal-caused faults in power distribution systems. The results of an animal fault prevention technique that can effectively reduce these faults will also be presented.

Summary: A variety of events such as animals, winds, trees, lightning, and equipment failures can disrupt electrical services in distribution systems. The primary focus of electric utility reliability programs is to reduce the number of disturbances in the distribution lines. This article explains the relative frequency of animal-related faults in Duke Power Company's distribution systems and how this problem can be minimized. Animals are the second leading cause of distribution fault for Duke Energy (trees are first), and factors affecting animal faults are studied here. Influential factors include weather conditions, season of the year, day of the week, and time of the day.

Consequence factors focus around the number of phases affected and the protection device activated. Care is also taken to calculate SAIFI (ratio of customers interrupted to customers served) and SAIDI (ratio of customer-minutes of interruption to number of customers served). As animal guards were installed in the experiment, the frequency of animal-caused faults diminished as well as the reliability indices SAIFI and SAIDI.

Implementation: To minimize animal-related faults in an area, a power company should install animal guards on the transformers. This type of fault prevention method is a control action fault prevention technique as is trimming trees to prevent tree faults. Other solutions are to provide adequate clearances and adequate insulation.

Issues: There are, on the average each year at Duke Power, 40,000 permanent distribution faults (which cause outages), 30,000 major temporary faults, and 75,000 minor temporary faults that trip line breakers or re-closers (which do not cause outages). Animals can create a momentary outage, voltage sag, or possibly a sustained outage on the distribution circuit.

Feasibility: Introducing animal guards on all transformers could be costly, but it may prove to be a more appropriate solution than having to stop and restart a system. The 
guards are proven to limit phase-to-ground faults, and this, coupled with solutions to move animals away from critical areas, could be viable.

\section{A.2.4.4.12 Failure prediction of underground distribution feeder cables [63]}

Abstract: This paper presents a methodology to determine an age-related reliability index that can be used to compare the relative likelihood of in-service failures among underground distribution feeders. It was developed for the Con Edison $27 \mathrm{kV}$ and 13.8 $\mathrm{kV}$ distribution systems but is sufficiently generic to apply to any group of underground distribution feeders. Each feeder is represented by a combined reliability model of its individual components, including the following:

- $\quad$ Cable Sections: Paper and solid dielectric insulated cables

- Cable Joints: Paper-to-paper, paper-to-solid dielectric and solid dielectric-to-solid dielectric insulated cable joints

- $\quad$ Network Transformers

The parameters of each component model are determined based upon historical failure data according to age. The confidence level associated with the prediction is also determined, and a brief description of the computer program developed and its application to Con Edison's Yorkville $13.8 \mathrm{kV}$ primary distribution network are provided.

Summary: Issues associated with underground distribution feeders such as reliability, testing, and programmed replacement prior to their failure have become priorities of utility management. Optimal budget allocation for preventative programmed cable replacement is a need for utilities, which use probabilistic failure prediction techniques to isolate cable sections that are most likely to fail. For this experiment (Phase 1), failure probability is based on component ages within the feeder. Age-related statistics are available for cables, joints, and transformers that make up the distribution feeder.

Implementation: Failure statistics for transformers, cables, and joints were analyzed from Con Edison data from 1976 through 1988. Quantities of failed components of age 5 years or less for the $27 \mathrm{kV}$ and $13.8 \mathrm{kV}$ feeders were observed as were corresponding quantities for failed components of age greater than 5 years. An engineering judgment was made to use a Weibull Distribution function as the cumulative distribution function (CDF). A Weibull formula for probability of survival is presented as a reliability function, with $\beta$ and $\lambda$ as the respective shape and scale parameters. Variables $\beta$ and $\lambda$ were then calculated to fit each data set.

Issues: Not all units have known lifetimes and not all units were introduced into the system within reasonable time limits, so a modification was made to the units based on the fraction of their life occurring in certain time periods. This procedure allowed for a better approximation in the prediction.

Feasibility: This is not practical seeing that the model does not incorporate other significant stress factors that affect feeder life. These factors include voltage surges, through-faults, loading, environment, ambient temperature, moisture, and installation 
configuration. The authors have identified these shortcomings and have stated a followup paper on Phase 2 will include these items.

\section{A.2.4.4.13 Transformer failure prediction using Bayesian analysis [64]}

Abstract: A new procedure is described for predicting transformer failures to quantify the expected impacts on service reliability. The procedure is designed to get the most out of sparse data by formal incorporation of engineering experience. The method is particularly well adapted to failure frequency forecasts and outage predictions for large, expensive apparatus for which accelerated life testing is not appropriate and for which historical failure data is limited because of an inherent low failure rate. The procedure makes use of a systematic method for combining only the most credible features of engineering models with real world experience and has been referred to as both calibrated engineering analysis and "Combined Analysis" (CA). Bayesian methods are utilized to formalize the statistical aspects of CA. An application example is included which demonstrates how the procedure was used to predict the economics of adding redundant transformer capacity at 20 sing 1 e-transformer substations for the purpose of improving service availability.

Summary: Someone using CA is able to calibrate an incomplete engineering model with historical data and make adjustments using engineering experience and intuition, if so desired. This is due to a Bayesian approach, which uses a heterogeneous combination of related data sets. Certain assumptions are made for the Bayesian method to be valid: statistical data populations have been grouped as much as possible using engineering analysis and judgment, populations share a similar variability, and future component exposure is not to vary much from the database values. Data used for this model include component age-to-failure, significant changes in component exposure, future projections of component usage, and incomplete engineering data and models (from which to establish the new model).

Implementation: The Bayesian CA approach has been applied in a computer program called BALIFE, which predicts outages for a number of $115 \mathrm{kV}$ transformers. Three important random variables modeled in the transformer application are electrical load (current), ambient temperature for every hour, and the influence of the first two variables on the degradation of the transformer. BALIFE first calculates an exposure variable, $t$, and computes the likelihood of a failure to occur at age $t$. The model then uses a fourtask procedure to forecast failure rates using Weibull modeling, Gamma modeling, and Monte Carlo simulations. As a check, the model is projected onto past data to ensure accuracy.

Issues: Difficulties arose in determining the "loss of life" estimation for the transformers, and the predicted summer outages of $77 \%$ substantially overshot the actual failure rate of $45 \%$. Upon better accounting for "loss of life," the model more accurately predicted failure rates for the desired 1970-87 time period. 
Feasibility: BALIFE accurately predicted transformer failures, as was evidenced by the fine fit between modeled and actual data. The model was extended to include customer interruptions over the time interval and was able to conduct cost-benefit analysis with regard to whether it was financially worth it to add a second transformer to the 20 substations. Probabilities for net savings or losses were determined and indicated a 76\% chance that firming the substations would lead to a net loss. Bayesian Combined Analysis is certainly an effective means of predicting failures for equipment with load and exposure variations. 


\section{A.3. Fault Protection}

Fault detection and protection of most distribution faults is a mature subject with well known and understandable protection algorithms and techniques. Instead of focusing on the enhancement of existing protection algorithms, the fault protection review focuses on the protection of advanced systems where automation, communication and new protective devices will affect the operation of future protection systems. High impedance faults are still not a mature subject, and their protection is reviewed in more detail.

The review is divided in three areas: distribution automation, fault detection with focus on high impedance faults, and fault current limiters with some focus on effects on protection systems. Sections A.3.1 to A.3.3 present a brief introduction to the three areas. Sections A.3.4.1 and A.3.4.3 contain synopses on distribution automation and fault current limiters. Section A.3.4.2, due to the large number of publications (common case for specific protection applications) deviates from the synopsis format and presents a historical overview of the high impedance fault research up to the presently available devices.

A.3.1 Distribution Automation

The most common automated functions in distribution systems publications include the following: Volt/Var Control, Fault Location Isolation and Service Restoration (FLIR), Optimal Feeder Reconfiguration, Automated Meter Reading, and Relay Protection Recoordination.

The wide spread of fast communication media in the past decade has given rise to the new concept of Advanced Distribution Automation that combines appropriate IT technologies in order to achieve fast and accurate data transmission and processing, for real time operation applications. Four main levels of automation are identified in the literature: substation automation, feeder automation, customer automation, and management automation.

Section A.3.4.1 contains synopses of the most interesting articles in distribution automation, our recommendation on issues to consider for the SCE distribution system of the future, and an annotated list of references on distribution automation from 1980 to the present.

A.3.2 High Impedance Faults

High Impedance Faults (HIF) are defined by a IEEE PSRC working group as those that 'do not produce enough fault current to be detected by conventional overcurrent relays or fuses' [88]. HIF typically occur when an overhead line falls in a high impedance surface such as dry earth, concrete or asphalt. Due to the high impedance to ground the fallen conductor fails to draw significant fault current, and it is not cleared by conventional relays resulting in a hazardous situation for persons and property.

Unfortunately, very few HIF testing facilities exist, and accurate fault modeling is a big component of HIF research; many of the proposed methods have only been tested with 
limited HIF models. The modeling of HIF is a research field of its own, and several models have been proposed for use with common analysis programs.

Several mechanical devices have been implemented to force HIF into normal, low impedance, faults to allow clearing of the fault through conventional relays. Most of the proposed techniques focus on electrical devices (relays) for the detection of HIF. Many relays have been proposed and implemented in the past thirty years, and at present, most of the large relay manufacturers offer a HIF relay capable of identifying a very large percentage of the HIF faults.

This report presents a short overview of the different signal analysis techniques proposed for HIF detection in section A.3.5.1. Section A.3.5.2 reviews some of the proposed pattern recognition knowledge based techniques that combined artificial intelligence and several detection algorithms to achieve higher reliability. Section A.3.5.3 reviews some of the HIF models used by researchers. Section A.3.5.4 reviews several commercial devices with a short discussion of the methods used by the relays. A.3.3 Fault Current Limiters

Synopses in section A.3.5.3 focus on fault current limiters (FCL's) and range from introductions to specifics of various FCL designs. For the remainder of section A.3.3, any mention of upcoming "items" or "articles" pertaining to FCL literature refers to the synopses in section A.3.5.3. Advantages of current limiters are included throughout the readings as are numerous models and the respective testing of those models. A couple of existing prototypes and design initiatives are also mentioned.

Several articles provide a necessary background from which to conduct further research into power system performance enhancement via FCL's. While most articles describe the limiters' role in reducing the high current level associated with a fault, each article focuses more precisely on the impact of this technology in the power field. Specifically, items 1,2, and 12 offer insight into the need and operation of these devices, albeit pertaining more to superconducting current limiters. Articles 4, 6, 14, and 22 also explain the benefits of current limiters as well as appropriate locations for employing these products. FCL's can be placed within distribution and transmission networks and can offer protection for power systems, as a whole, or particular devices. With the incorporation of a FCL into a protection scheme, there needs to be assurance that the limiter operates quickly enough and can re-set in the event of another short circuit. Additionally, ratings requirements, as discussed in articles 8 and 16, must be adjusted to account for the new levels of currents. Overall, the main advantages of current-limiting technology are fast response times, reduction of switchgear ratings, improved transmission capacity, and improved system stability. Stability information is located in articles 7,10 , and 13 .

Although each FCL can satisfy the above requirements, numerous designs are still being tested to maximize the services provided. For example, the costly but effective superconducting fault current limiter requires cooling of a superconductive metal in order to maintain the proper operating state and increases in impedance quickly and effectively 
when heated. This technique is implemented with or without a parallel reactor and/or series capacitor and can be replicated for large-scale applications (articles 20 and 22). Another design calls for solid-state switches and a reactor in a rectifier arrangement. While less expensive and having a slower operation time, this model still offers few losses and can be combined with additional reactors/capacitors as long as proper controls are in place. Tests of three-phase and single-phase designs with varying thyristor arrangements show that limiters are capable of handling both common and uncommon fault types without significantly altering the response time and shape (articles 19 and 24). Inductive current limiters are also worth mentioning despite their high cost, weight, and overall size. The screened-core or magnetic shielding-type inductive FCL is an alternative to a purely resistive model and can be likened to a transformer with a variable secondary (article 18).

Finally, articles 20 and 25 describe existing models in their testing stages. Matrix fault current limiter (MFCL) test results are available in article 20 (pre-prototype was successful) while a rectifier-type FCL is successfully implemented in article 25 . A scaleup of the MFCL to transmission-level voltages is one design goal whereas operation at $10.5 \mathrm{kV}$ is appropriate for the other.

Other topics are covered in this review, but they mainly constitute physical properties of superconductors (i.e. thick films, thin films, quenching, V-I characteristics, etc.) and are not as relevant at this time.

A.3.4 Comments and Recommendations

A.3.4.1 Distribution Automation

According to available publications [72] [65], the most important issues driving the research and implementation on distribution automation are reliability improvements, reduction of capital and operating costs, innovative customer-focused operation, and connection of distributed generation to distribution systems. Most of these issues are not new but the complexity and cost associated with them puts, at present, an emphasis on the use of technology to address these problems in a coordinated and efficient manner.

Adaptive protection combines communication and system automation to improve the performance of the distribution system. Most of the adaptive protection concepts are based on distribution systems, but the use of fault current limiters and advanced communication systems in the distribution systems makes adaptive relaying feasible for the SCE "Circuit of the future." The existing IED's, communication channels, and additional signal monitoring can be used to determine the most effective use of automation and adaptive protection in distribution systems.

A.3.4.2 High Impedance Faults

In the past three decades many algorithms have been proposed for the detection of HIF in distribution and transmission systems. Some of these algorithms have been implemented into commercial devices but most remain in a theoretical or experimental state. Arcing is a common component of HIF, and due to its randomness, asymmetry, and dynamic, its presence is used in many algorithms to detect the presence of a HIF. The knowledge 
based techniques are the latest application in the evolution of HIF detection and are enabled by the increased computational power of computer relays and the reduced security and dependability of the signal analysis techniques.

Although several commercial devices exist for HIF detection, most of these devices detect only up to $85 \%$ of the HIF, and their fault location algorithms are very limited. The use of time synchronization and differential line/cable protection in HIF detection can provide a higher detection rate and more accurate location algorithms, and it could be implemented in the SCE system.

A.3.4.3 Fault Current Limiters

Integration and protection issues are a major concern when dealing with variableimpedance devices. The current limiter must be coordinated with the operation of the circuit breakers (current interruption and subsequent re-closing); and at re-closing, once the fault is cleared, the impedance of the FCL should return to zero. A current limiter should operate reliably against a fault whose current is more than the breaking capacity, but FCL's in sound phases or lines should not operate.

Also, research has shown that FCL's lower not only the current level but also the rate of rise of the recovery voltage (rrrv), both of which contribute to the burden of a circuit breaker. Thus, the presence of a FCL offers a more severe effect on the circuit breakers than might initially be expected, and any system should be compared with and without a limiter in place to observe the effects of decreased rrrv. The severity of the interrupting duty of the circuit breaker will be reduced, so a circuit breaker with a lower interrupting performance can be used.

The type of current limiter used is important since the effects on the existing protective system vary depending on the type of FCL:

For resistive-type FCL's, a first setting method involves the operation start current, ILS, or the current which makes a FCL start its limiting. The upper limit of this current should be the breaking capacity, and the lower limit should be the maximum current on a sound line when a fault occurs elsewhere. This ensures that sound lines are left unaffected by the fault. Also, when a FCL introduces additional impedance to the system, the distance relays (and mho relays) are affected. Since these relays have predetermined operational zones, additional impedance can move operating points outside of the specified zone, which leads to unwanted trips. It is therefore up to a designer to specify a range of the impedance that will sufficiently limit the current yet not affect the response of protective relays or to provide a more accommodating mho characteristic for the relays.

For rectifier-type FCL's, care must first be taken to calculate the current traveling through the limiting coil, and once the voltage, frequency, protective relay operation times, and equivalent reactance of the power system at the back of the FCL are known, the limiting coil reactance can be estimated. A breaking capacity limit is also determined 
based on the ratio $(\mathrm{k})$ of the current through the limiting coil to the fault current magnitude when no FCL is installed (IF) as well as IF itself:

$I_{C B} \geq \mathrm{k} \times I_{F}$

In the presence of a FCL, current differential relays operate correctly for all internal faults; however, differential relays cannot discriminate the fault type when a fault occurs near the current limiter.

From the protection point of view, the literature review reveals that rectifier-type SFCL's will be easier than resistance-type limiters to introduce into power systems and coordinate with protection schemes.

\section{A.3.5 Synopses and Historical Overview}

\section{A.3.5.1 Synopses: Distribution Automation}

\section{A.3.5.1.1 Advanced feeder automation is here [65]}

Summary: This paper describes the IntelliTeam II automation restoration system introduced by S\&C Electric Company in 2003 that uses distributed intelligence for feeder automation. This automatic power restoration system uses distributed intelligence and peer-to-peer communications to isolate faulted sections and to restore service to unfaulted sections.

The IntelliTEAM II derives its name from the coach and team metaphors. Each team is defined as a line segment bounded by two to eight automated switches, each of which can represent a different power source. Each team has a software coach that when the team is affected by an outage uses real time data to determine a restoration strategy for un-faulted sections. Then the coaches of adjacent teams work together to maximize the system restoration based on user-provided priorities. The coach of a team closes a switch when all appropriate conditions are met, and the coaches of adjacent teams grant their permission. When a switch closes, the IntelliTEAM II system updates available capacity on the feeders used to restore service, ensuring maximum service restoration even at maximum peak load.

The distributed nature of the control algorithms allows for the operation under multiple contingencies. An additional option of contracts negotiated among teams is used to secure resources needed to energize a line segment and to prevent circuit overloading. To implement this system, a wide are communication network is required.

Implementation: The paper claims that since its introduction in 2003, several utilities have taken different strategies for the implementation of IntelliTEAM II in their distribution system. Two cases are described in the paper. The case of ENMAX Power Corporation (Calgary, Alberta, Canada) is presented in detail. ENMAX had the particularity to contain electromagnetic relays that provide only one re-close operation in 
response to a fault feeder. This meant that the fault becomes permanent if the re-close is not good. To solve this problem, ENMAX used a new algorithm. The result on the first phase of the ENMAX project is considered a success. With only $16 \%$ of the system using the system through 11 outage events on the automated feeders, ENMAX has experienced an $8.6 \%$ reduction in the system average interruption duration index and a $1.7 \%$ reduction in the system average interruption frequency index.

The paper presents a second successful case of a rural cooperative in the south eastern United States where high reliability was demanded by a large customer.

A.3.5.1.2 Interface between distribution automation system and SCADA to minimize the feeder loss [66]

Summary: This paper describes the two types of Distribution Automation Systems (DAS) developed by KEPCO (Korean Electric Power Corporation). One is a small DAS that has the functions of remote control, remote data acquisition, and remote setting with simple structure and low cost for small cities and rural areas. This small DAS system has been set up in 177 low-grade business offices in Korea from 1998 to 2001. Another system is a larger DAS for the metropolitan area. It has been tested in three business offices by 2001 and set up in the rest of branch offices from 2002. The large DAS with the structure of duplicate servers and hot standby backup has the functions of feeder automation, simulation, and loss minimization in addition to small DAS functions.

The paper begins with an overview of distribution networks in Korea. Distribution networks in Korea are $22.9 \mathrm{kV}$, multi-point direct grounded systems. The system configuration of small DAS is described in the paper. The main functions of small DAS can be summarized as follows:

Remote supervision: open/close, lock/unlock, remote/local, gas pressure normal/abnormal, battery voltage normal/abnormal, fault indicator (instant mode/permanent mode), open line (phase missing), phase unbalance Remote control: open/close, lock/unlock, battery test, fault indicator reset Remote measurements: current $(A, B, C, N)$, voltage (A-N, B-N, C-N), fault current $(\mathrm{A}, \mathrm{B}, \mathrm{C}, \mathrm{N})$

Remote setting: minimum pickup current (phase, ground), time delay for inrush current and so on

A comparison between small DAS and large DAS is then presented in the paper, along with the configuration of large DAS. Although the basic functions for remote operation of large DAS and small DAS are the same, large DAS can perform the feeder automation to restore feeder faults in 2-3 minutes and, in addition, the simulation function to train system operators and reporting function to edit data. Standard functions of the large DAS for metropolitan areas can be summarized as follows:

Remote supervision: close/open, lock/unlock, open line (phase missing), phase unbalance, fault indicator (instant/permanent), battery status, gas pressure condition Remote control: close/open, lock/unlock, fault indicator reset, battery test 
Remote measurements: current (A,B,C,N), voltage (A-N, B-N, C-N), fault current $(\mathrm{A}, \mathrm{B}, \mathrm{C}, \mathrm{N})$

Remote setting: minimum pickup current (phase, ground), time delay for inrush current, angle of phase unbalance, voltage magnitude low limit

System networking: SCADA -DAS for substation data, NDIS-DAS for distribution network data, large DAS-small DAS for remote control

Other functions: feeder automation for service restoration, simulation for operator training, overload detection and load transfer processing

The interface configuration and data exchange between DAS and SCADA is presented. The loss minimization program is elaborated next. It calculates the loss of each section with load current and calculates the total loss of each feeder and searches the solution of loss minimization of all distribution networks by rearranging switches in the normal open point. Finally, the program presents the control list of switches to change the normal open point for operator. Not only loss minimization, but also limitations of voltage drop and feeder overloads are considered while determining the optimal solution.

Implementation: The small DAS system has been set up in 177 low-grade business offices in Korea from 1998 to 2001. Large DAS system has been tested in three business offices by 2001 and set up in the rest of branch offices from 2002

\section{A.3.5.1.3 Implementation of network reconfiguration for Taiwan Power Company [67]}

Summary: In 2002, Advanced Control Systems (ACS) completed final site acceptance testing of a state-of-the-art, fully integrated DAS for Taiwan Power Corp. This project's objective was to reduce the outage restoration from an average of 58 minutes to less than 20 seconds over a wide distribution network area, while also supporting various optimization functions and simulations. The solution included a fully integrated system including the master, substation RTU's, feeder Rut's, line switches, system software, and communications in a turnkey project. The suite of automation and analysis applications developed by ACS enable any utility to tailor a standard set of products to be implemented as a fully integrated and highly functional real-time operation system. ACS approach integrates a GIS as a source database with the real-time closed loop functions, dispatcher analysis functions, and management functions. The level of automation can be configured to operate automatically or in a dispatcher supervisory mode. The scope of automation can be localized at the district or substation level or expanded globally to the operations control centre at the enterprise level. This paper describes the approach and experience gained in implementing this automatic network reconfiguration from its operation to maintenance.

The paper begins with the description of the used system configuration and the system functions fully integrated into the master station to create a fully integrated DAS. The major justification for this project is to provide feeder fault detection capability to isolate a fault, verify isolation, and restore power to the upstream section of the feeder within 20 seconds. The downstream sections are then to be analyzed for restoration solution scenarios to be implemented either automatically or presented as a set of switching 
instructions for dispatcher approval and implementation. The criteria to be satisfied by feeder switch equipment are reported. The substation monitoring and control operations and the master control center operations are presented next. Specifically, the following DAS applications are implemented in the fully integrated DAS system:

Real-time operational functions such as

Topology processor

Distribution power flow

Fault detection, isolation, and restoration

Capacitor bank control

Outage scheduling

DAS study functions such as

Optimal switching

Protection coordination

Short circuit analysis

Optimal capacitor placement

Dispatcher training simulator

Trouble call analysis

(Details of each function are presented in the paper).

The complexity of data model and its real-time components will drive the accuracy of the solution. A common data model should be developed and maintained from a single source database for all network model-driven applications. ACS uses the GIS as the source database tool in a 4-step.

\section{A.3.5.1.4 New triple-single re-closers improve distribution reliability [68]}

Summary: The new Triple-Single (TS) re-closer revolutionizes distribution protection practices. One such device is the NOVA-TS re-closer system by Cooper power systems, which uses three single-phase NOVA-TS re-closers whose operation is electronically controlled. The paper presents this new control for re-closers, the Triple-Single control, which has three operation modes ( 3 phase $/ 3$ phase, 1 phase $/ 3$ phase and 1 phase $/ 1$ phase). The re-closer operation sequences in each of these three modes are presented in this paper for various combinations of fault types and locations, fault duration (permanent or temporary), and fault current magnitudes.

This paper uses the instructional software developed by Cooper power systems to demonstrate the three modes of operation and the distribution feeder reliability improvements achieved by utilizing a TS re-closer system. Distribution feeder reliability analysis is presented to show the reliability improvements obtained by using the TS recloser control. The inputs for feeder reliability analysis are based on typical data for a distribution feeder: number of customers, number of permanent faults per year, and temporary faults per year on the feeder. The feeder reliability indices SAIFI and MAIFI are calculated for each re-closing mode and are compared. The results demonstrate that distribution reliability is improved by using the new Triple-Single re-closer. 
Implementation: A simplified three-phase distribution feeder is used in the paper to demonstrate the various TS re-closer operations and improvements in feeder reliability. The instructional software developed by Cooper power systems is used for this purpose. It is shown that the Triple-Single demo software is a very useful tool for gaining insight into the reliability improvements that can be achieved by installing a TS re-closer system at the substation. Results are presented for various combinations of fault types and locations, fault duration (permanent or temporary), and fault current magnitudes. The following conclusions are drawn from the case study done in the paper (for the sample three-phase distribution feeder):

The feeder SAIFI can only be improved (67\% for default case) by using the Triple-Single re-closer in 1 phase $/ 1$ phase mode. This improvement is mainly for ground faults on the primary main feeder of the sample system used.

The feeder's overall MAIFI improves significantly ( $62 \%$ for the default case) by using Triple-Single re-closer in either 1 phase/3phase or 1 phase/1 phase mode. This includes improvements for ground faults on the main feeder and the laterals, as well as for phaseto-phase faults on the main feeder.

There is no reliability improvement for three phase faults either for SAIFI or MAIFI. Change in total number of lateral taps on the feeder does not affect the percent reliability improvements obtained from Triple-Single re-closing.

Change in total number of customers on the feeder does not affect the reliability since all the indices are defined on per-customer basis.

\section{A.3.5.1.5 Impact of two-stage service restoration on distribution reliability [69]}

Summary: This paper examines the reliability improvements gained by using a two-stage restoration strategy rather than a single-stage restoration strategy following fault inception. In single-stage strategy, the system is reconfigured only once. In two-stage restoration, a first stage quickly restores a limited set of customers using automated switches, and a later stage restores additional customers using manual switches. The strategies are compared using a predictive reliability assessment algorithm capable of modeling each strategy.

The paper begins with a discussion on the four common methodologies used for distribution reliability assessment and their service restoration modeling capability: network modeling, Markov modeling, analytical simulation, and Monte Carlo simulation. In this paper, the authors chose analytical simulation method to explore the differences between single-stage and two-stage restoration. This paper also examines the question of how upstream restoration and downstream restoration impact system reliability under different restoration strategies. Specifically, the impact of a more complicated two-stage strategy is compared to a simpler single-stage strategy. Additionally, the impact of different levels of feeder automation and operational failures are assessed. The reliability index SAIDI is used as performance measure to compare the switching strategies. The paper discusses qualitative differences between single-stage and two-stage restoration strategies and quantifies the differences between the two methods by applying predictive reliability assessment techniques to a test feeder. 
Implementation: The test feeder used is a 10 MVA peak load, 47 miles of single phase lateral, and a 3.6-mile three-phase trunk, representative of US overhead feeders.

Feasibility: Two primary results are derived from the analysis done in the paper: First, reliability gains of about $8-10 \%$ can be achieved for partially automated feeders with configurations similar to the test feeder by using a two-stage rather than a single-stage restoration strategy. Second, the full potential of targeted feeder automation cannot be quantified by predictive reliability models that only model a single-stage restoration process. Additionally, it is concluded that increasing levels of feeder automation results in an increasing sensitivity of SAIDI to switch POF (Probability of Operation failure). This sensitivity, however, is lower than sensitivities normally encountered for line, cable, and transformer failure rates. Results in this paper indicate that switch POF is a minor consideration in evaluating the reliability impact of switching strategies. Also, as a large percentage of switches on a feeder are automated on a system using two-stage service restoration, marginal gains in SAIDI diminish. A fully automated feeder does have a lower SAIDI than a partially automated feeder, but many partially automated feeders using two-stage restoration is much better from a system reliability standpoint than a few fully automated feeders using single-stage restoration.

A.3.5.1.6 Coordinated local and centralized control in distribution management systems [70]

Summary: This paper discusses the coordinated use of Volt/Var Control (VVC), OFR, traditional functions distribution system planning, and local controllers in distribution system operation for a more effective control of distribution systems. The paper suggests coordination principles for the interaction of local controllers with VVC, OFR, and centralized functions. Guidelines are proposed for selection of possible objective functions for VVC and OFR.

Suggested coordination principles: Allow local controllers to adjust to an optimal solution received from centralized functions to current power system conditions.

Reason: The large size of the distribution system, the lack of real-time measurements, and the use of statistical information limits the periodicity of OFR and VVC execution to minutes or tens of minutes.

The following characteristics should be considered in the local controllers when coordinating operations with centralized functions:

Bandwidth of controllers to determine minimum possible change in settings

Maximum and minimum limits of controllers to restrict operational range of devices

Restrictions on the number of operations in a given period

Restrictions in the time interval between consecutive operations of the same control

device

Time delay between control signal and execution of the action

Validity of settings that cannot be change through the SCADA system 
Under normal conditions, periodicity of execution of VVC occurs a few times in an hour which is much higher than OFR execution that occurs a few times a day. The reason is that VVC works with a subset of the distribution network, and OFR deals with the whole distribution system simultaneously.

Under contingency conditions for the restoration of power, VVC and OFR should be used in consecutive order. The OFR goal for this case is to minimize total de-energized load and to remove significant overloads. The VVC goal is to prevent voltage limit violations and help OFR remove overloads.

Based on the listed principles, the paper gives the following table as recommended objective function pairs for VVC and OFR:

Table 1—Possible VVC and OFR corresponding objectives

\begin{tabular}{|l|l|l|l|l|}
\hline VVC & $\begin{array}{l}\text { Minimize } \\
\text { power losses }\end{array}$ & $\begin{array}{l}\text { Energy } \\
\text { conservation }\end{array}$ & $\begin{array}{l}\text { Maximize } \\
\text { revenue }\end{array}$ & $\begin{array}{l}\text { Keep the system } \\
\text { within constraints }\end{array}$ \\
\hline OFR & $\begin{array}{l}\text { Minimize } \\
\text { power losses }\end{array}$ & $\begin{array}{l}\text { Minimize the worst } \\
\text { voltage drop }\end{array}$ & $\begin{array}{l}\text { Load } \\
\text { balancing }\end{array}$ & $\begin{array}{l}\text { Minimize total de- } \\
\text { energized load }\end{array}$ \\
\hline
\end{tabular}

Implementation: There is no implementation, but the paper presents examples of the applications of the principles to a 20-load 25-line system and to an 80-load and 95-feeder section. Examples are given for objectives of power loss minimization and energy conservation.

\section{A.3.5.1.7 Automated solutions for distribution feeders [71]}

Summary: Utilities are becoming increasingly automated to keep up with the customer load requirements and demands of the new business environment under deregulation. This paper addresses the following two issues with example solutions for overhead feeder automation:

What automation scheme is required?

How are communications implemented?

What automation scheme is required? Presently, utilities employ a variety of techniques to improve reliability such as re-closers, sectionalizers, and load break switches. This paper uses an example of overhead feeders to highlight several feeder automation solutions. The example consists of an overhead feeder with two customers.

Re-closers and the impact on outage minutes: Re-closers must be coordinated with upstream devices such as substation circuit breakers and downstream devices such as fuses. For line fuses, two schemes are frequently deployed: fuse-saving and fuse-clearing. Adding a re-closer to the example overhead feeder, it is shown that customer 2 has the same outage minutes per year whereas customer 1 sees a $50 \%$ reduction in outage minutes per year. 
Sectionalizers and the impact on outage minutes: Sectionalizers are used to further reduce the customer impact due to permanent faults. Typically, they are used on a line downstream from a re-closer or a re-closing relay in the substation. Adding a sectionalizer to the example feeder, it is shown that there is an overall $66.6 \%$ improvement for the outage time of customer 1 and $33.3 \%$ improvement in the outage time of customer 2 (compared to no re-closer or sectionalizer on the feeder), which translates to $33.3 \%$ improvement in outage times for customer 1 and customer 2 (compared to only re-closer being used in the feeder).

Switches at normal open points and the impact on outage minutes: When increased reliability is required and dual feeders are available, it is possible to place a switch at the normal open point (NOP) and restore power following a permanent fault. Two typical implementations are: 1) Remote transfer 2) Load automatic transfer.

Adding an alternate distribution feeder and NOP switch to the example feeder, it is shown that there is $83.3 \%$ improvement in outage times for customer 1 and customer 2 . For selection of proper feeder automation solution for complex systems, intelligent network modeling and optimization are required. For example, intelligent tools to predict items such as outage frequency and duration together with frequency and level of voltage sags at selected points in the distribution network for a given configuration to determine an optimal automation strategy are required, such as the ABB Performance Advantage tool.

How are communications implemented? Integration of automated feeder equipment such as re-closers, switches, or sectionalizers into the DMS system depends on several factors such as: 1) Protocol selected, 2) Automation strategy, 3) Communication medium available.

Communications protocol: For the protocol, DNP 3.0, IEC 870, or ModBus are commonly used for utility automation. ModBus is a widely used protocol due to its simple structure, and it can be implemented in an ASCII or RTU (binary) mode. It is often implemented in RTU mode and interfaced to SCADA Rut's in the field. DNP 3.0 is more complex but offers some nice features such as report by exception and allowing multiple masters.

Automation strategy and communications medium: The automation strategy determines which type of communication system to be used. Two different types, wide-area communications and local communications, are explained in the paper. Selection of the communications approach depends on the number of units to be automated, polling rate, amount of bytes to transmit, and data transport fees, if applicable.

New technologies such as GSM, CDPD, and satellite are becoming available and will allow for new low-cost automation solutions. However, report by exception and slow data throughput will have to be carefully examined. The decision on deploying automation is a complex issue. Detailed analysis is required to select the optimal strategy to fit the utility's and its customer's needs. 


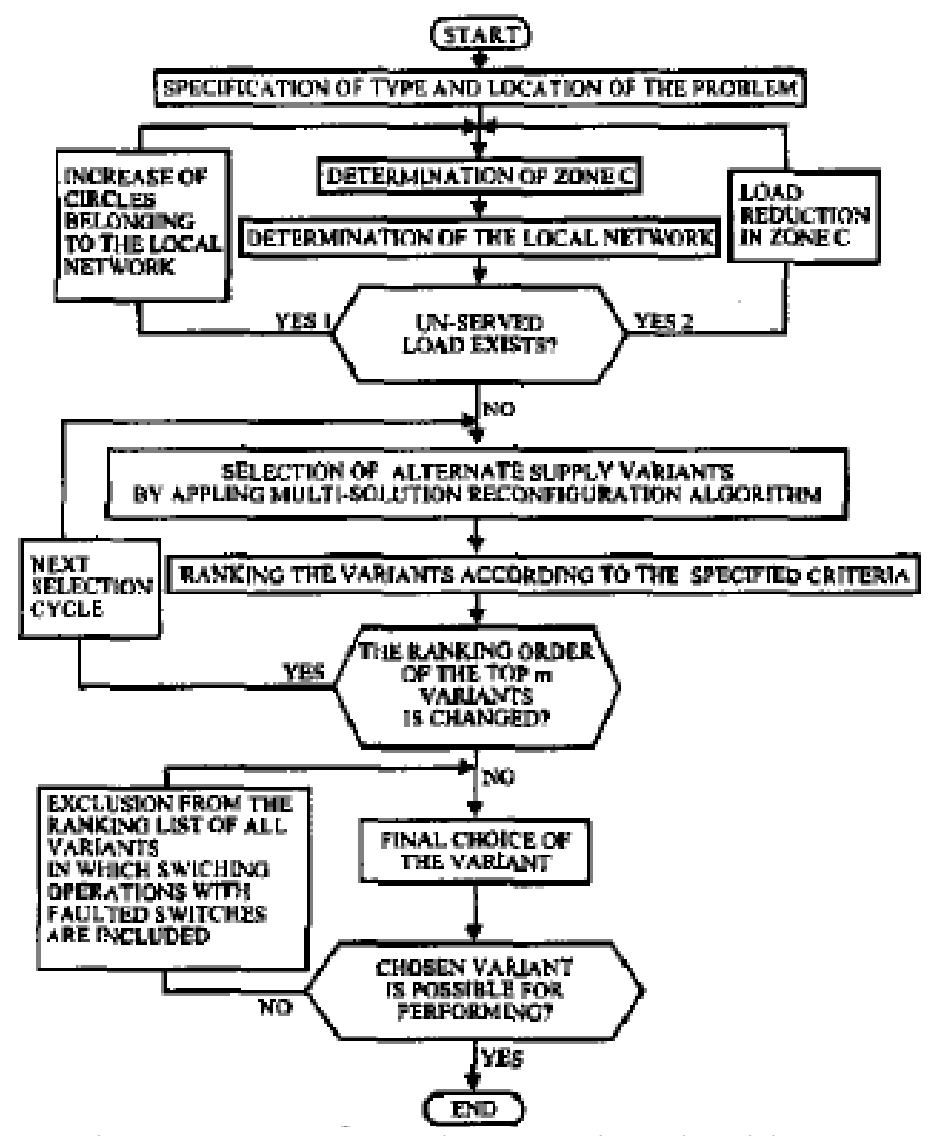

Figure A.17—Network restoration algorithm

Field implementation: No actual field implementation results are presented in the paper.

\section{A.3.5.1.8 A multi-objective algorithm for distribution networks restoration [72]}

Summary: This paper presents a heuristic multi-objective algorithm for determining the restoration plan for a radial distribution network. The algorithm is performed in three steps: 1) The local network is defined to reduce the dimension of the problem, 2) a decision tree based algorithm is used to select variants of alternate supply, and 3) the selected variants are ranked according to a user-specified criterion.

Multi objective algorithm:

The given flowchart describes the proposed algorithm. After identifying the type of problems to be solved by the algorithm, Zone $\mathrm{C}$ is determined as the part of the network affected by the problem. The paper describes the determination of zone $\mathrm{C}$ for the types of problems that can be solved by the algorithm: fault of a transformer, fault of a feeder, overload of a transformer, and overload of a feeder. 
The local network consists of zone $\mathrm{C}$ and the feeders that are able to take over the load of zone $\mathrm{C}$ by closing their normally open switches. The variants of local networks are obtained by topological searches and check on limits satisfaction. The procedure for obtaining the decision tree for the multi-solution reconfiguration algorithm is illustrated in the paper. Ranking of the variants is performed based on a user-specified combination of the following criteria:

Un-served load

Switching cost

Critical reserve of transformers

Critical current-reserve of feeder

Critical voltage drop

Reliability

Mathematical definitions are presented in the paper for each of these criteria.

Implementation: The proposed algorithm is illustrated with three cases for determining restoration plans in a $20 \mathrm{kV}$ network in Yugoslavia containing 170 buses and with a total rated power of 94.5 MVA. The single line diagram of the network is given in the paper. The paper also compares the execution time for three different networks of 170 buses, 440 buses, and 890 buses showing very small difference in execution time for the three networks for two different cases.

A.3.5.1.9 Pilot project to estimate the centralized volt/var control effectiveness [73]

Summary: This paper presents the descriptions and results of studies on the effectiveness of Volt/Var Control for the secondary separate objectives of energy conservation and increased revenue. The paper describes the approach used to determine the VVC effectiveness and presents results in terms of the effectiveness average and total savings. The results of the implemented approach are compared to SCADA archived data to determine the consistency of the results.

Approach for effectiveness calculation: The paper defines the effectiveness of the VVC as the difference between the objective function value for the initial system conditions and the objective function values after the VVC actions have been performed assuming that the load has not changed.

To avoid using the simple approach of comparing the base case real-time-power flow with the results of the power flow simulation after the VVC control, the authors use two real-time power flows from two distribution systems, one with VVC and one without. The power flows are performed in the same day, and the two circuits are chosen based on the correlation of their customer demand. The aim is to eliminate from the effectiveness calculations the effect of natural changes in the customer demand.

The paper presents the following comparison table of the effectiveness of VVC for Energy Conservation using immediate data and SCADA archived data: 
Table 2-VVC results for kWh savings

\begin{tabular}{|l|l|l|l|l|}
\hline Date Test Was Run & $9 / 18$ & $9 / 20$ & $10 / 18$ & $10 / 25$ \\
\hline $\begin{array}{l}\text { Archived Data Saving } \\
\text { kWh }\end{array}$ & 2513.5 & 2675.1 & 3140.5 & 3109.7 \\
\hline Immediate Saving kWh & 2880.7 & 2972.9 & 2718.5 & 2852.5 \\
\hline
\end{tabular}

Implementation: The tests were run at the Northern states Power Company on a networkmodel-based centralized VVC in September/October of 1995 and compared with archived data for the same days. In the test another bus section of the same substation was used to eliminate the effects of the natural changes in customer demand.

\section{A.3.5.1.10 Distribution feeder reconfiguration for operation cost reduction [74]}

Summary: This paper describes a heuristic-based approach to use feeder reconfiguration for the reduction of operating cost. The proposed methodology minimizes the cost of operation over a specific time period instead of a fixed operating point, considers only remote controlled switches, and considers all relevant constraints. The paper also presents a state-of-the-art review of distribution feeder reconfiguration techniques and classifies them as

Composite heuristic and optimization methods

Heuristic based methods

Artificial intelligence based methods

This qualification has been adopted in this report.

Steps of proposed methodology:

Discard from consideration all switches that have reached their limit on their number of operations.

Identify closed switches in the loop formed by closing a normally open switch, and determine the capacity margin of all normally open switches.

Select a candidate open/close switch operation (detailed methodology is described). Procedures are given to identify the candidates and rank them on maximum cost reduction. The highest rank open/close switching operation is selected first.

Run power flow to check for operating constraint violations, and discard the operation if a violation is found.

Check the limit of total number of switching operations; if limit not reached, select the next highest ranked operation, and repeat step 4. If the limit is reached, an optimal solution is assumed.

The authors also developed an algorithm to find the sequence of implementation of the optimal set of switching operations. The paper describes the principles and major steps to implement this algorithm.

Implementation: The algorithms presented in the paper were used to develop the production grade FRECON program that has been successfully tested in PG\&E distribution systems. Results of test performed in two $12 \mathrm{kV}$ PG\&E feeders with 920 
lines and 55 switches. The test was performed for a period of 24 hours and showed that cost reduction can be achieved by two pairs of operations with a total loss reduction of $8.66 \%$.

A.3.5.1.11 Network reconfiguration in distribution systems for loss reduction and load balancing [75]

Summary: This paper describes two new feeder reconfiguration algorithms for the purpose of service restoration and load balancing in a real-time operation environment. The developed methodologies combine optimization techniques with heuristic rules and fuzzy logic for efficiency and robust performance. Many of practical operating concerns of feeder reconfiguration, proper selection of a sequence for implementing switching operations, and coordination with other distribution automation applications are also addressed.

Specifically, this paper presents algorithms for the following functions:

Service restoration in response to a fault by

Isolating the faulted area(s)

Supplying power to the non-faulted out-of-service area(s)

Minimizing the inevitable load shedding

Load balancing in order to relieve the overloads in the distribution system

The paper begins with a mention of the methodology used to develop the proposed reconfiguration algorithms. It develops an Integer Linear Programming (ILP) technique which guarantees a final solution for service restoration problems with the objective of minimizing the number of switching operations. Then, it develops the framework of a service restoration module. The load balancing algorithm is then developed using fuzzy logic reasoning approach (details in paper).

Implementation: A software package FRECON was developed based on the service restoration and load balancing algorithms presented in this paper. It was tested on many of PG\&E's distribution feeders. Two examples of test results are presented in the paper. The test system used consists of two $12 \mathrm{kV}$ feeders with a total load of 4.85MVA along with 920 line sections and 55 switches. Two fault scenarios are considered: a) fault close to the substation on feeder 1 , and $b$ ) fault at the middle of the feeder 1 . The results for service restoration and load balancing algorithms are reported. Test results show that the performance is efficient and robust. A discussion on the implementation issues, such as sequence of implementing the switching operations and protection considerations, follows the test results.

A.3.5.1.12 Multi-objective feeder reconfiguration by distribution manager system [76]

Summary: This paper proposes a two-stage method for load reconfiguration with a multiple objective function. The multi objectives considered are minimization of power loss, balancing of transformer load, minimization of worst voltage drop, minimization of 
service interruption, and balancing of service for important customers. Capacity and protection device constraints are considered in the procedure.

Two-stage approach: In the first stage, all normally open switches are assumed to be closed, and the most critical objective function is selected. A power flow type solution approach is used to solve for the most critical objective function. A preliminary solution is found by opening the switches using the given criteria for the selected most critical objective. A solution method and criteria are given for each of the objective functions. In the second stage, the solution of stage one is improved until a stopping criterion is met. The paper derives a mathematical representation for each of the five objective functions and uses them to create a global objective function where each objective is given a weighting factor. The change in the global objective function is evaluated by simulating the swapping of status of each closed switch and it's adjacent to currently open switch. The swap that yields the greatest decrease in the objective function is performed, and the procedure is repeated until a stopping criterion is met.

Implementation: The method has not been implemented in a real system at the time of publication of the paper, but the results are shown for a sample distribution system consisting of 61 loads, 64 branches ( 28 cables, 36 overhead lines), 3 supply transformers, 7 normally open branches, and 46 important customers. The multi objective optimal solution is compared favorably to results of optimizing each of the objective functions separately.

A.3.5.1.13 Distribution capacitor automation provides integrated control of customer voltage levels and distribution reactive power flow [77]

Summary: This paper presents the control scheme, implementation, and testing of a distribution capacitor automation scheme implemented by Southern California Edison with the aim to reduce energy consumption by reducing customer voltage by $2 \%$.

Control Scheme: A packet radio network is used to collect customer voltage information from voltage sensing meters strategically placed on lower voltage customers. The control scheme objective function is to minimize customer voltage with the primary constraint of maintaining a 114 volt minimum and the secondary constraint of maintaining the reactive power limits. The control algorithm calculates optimal bus voltage a central control processor at the transmission substations uses these values to derive a Subtransmission voltage level for minimum customer voltage and reactive flows.

To determine the effectiveness of the control scheme, the algorithm was cycled on and off every other day during the demonstration period to compare the results to existing control systems. The test showed an average of $3.8 \%$ reduction in customer voltage during the demonstration period.

Implementation: The paper presents results of a demonstration performed at Walnut from January to December of 1993. At the time of publication, SCE had implemented the system in 586 circuit capacitors on 174 circuits served from 20 substations. 


\section{A.3.5.1.14 Adaptive distance protection of a double-circuit line [78]}

Summary: This paper demonstrates that adaptive protection can improve the performance of a relay. The distance protection of a double-circuit line under SLG fault condition is formulated. To achieve correct operation, the relay does not use the measured quantities of the circuit to be protected only, but also the zero-sequence current of the parallel circuit. Such a relay requires extra measuring equipment, and moreover, the zerosequence current of the parallel circuit cannot always be measured. Therefore, another approach is chosen. A correction factor is introduced, set adaptively to the actual power system state. In this way, the appropriate setting of the relay is provided in relation with the actual power system state.

The paper begins with the formulation of the equations for distance protection of a sample double-circuit line under SLG fault condition. The concept of the correction factor to compensate for the mutual coupling of the parallel circuit is introduced. Two categories of changes in the power system state are considered:

Changes in the state of the power system around the line to be protected (such as generator outages, load changes and changes in topology). These changes cause a change in Thevenin impedances.

Changes in the state of the line to be protected (such as double-circuit and single-circuit operation modes). These changes cause a difference in the calculation of the relaying impedance.

Implementation: To demonstrate the behavior of correction factor in a continuously changing power system, the Dutch $400 \mathrm{kV}$ power system is used. The average load on this system is about $800 \mathrm{MW}$ and $4000 \mathrm{MVAR}$. The simulation results for changes in the power system state and in the operation mode of the double-circuit line are reported. To control the impedance that indicates the boundary of the zone to be protected in such a way that optimal selectivity is achieved, a non-linear interpolating algorithm must be used which is able to relate the healthy state power system measurements with an optimal value of this parameter.

Further possibilities: The adaptive scheme presented in this paper can be extended to multi-circuit lines, with possibly different base voltages. Also, it can be possibly extended to protection of multi-terminal lines that are highly sensitive to infeed/outfeed at the terminals.

\section{A.3.5.1.15 Prospects for distribution automation at Pacific Gas \& Electric Company [79]}

Summary: This paper presents a summary of the methodology and results of a study aimed at identifying the distribution automation functions more advantageous to PG\&E. The study was performed in three phases:

Assessments of the needs through a survey of management and technical personnel. 
Development of decision rules and algorithms to quantify the benefits of the needs and recommendation of an implementation plan.

Development of an organizational structure to implement the recommended plan.

The presented methodology classifies the automation functions into three categories: substations automation, feeder automation, and customer interface automation. Only quantifiable benefits are considered in the study, and they are classified as reduction of operation and maintenance cost, and revenue increase. The paper presents tables with the candidate automation functions selected at PGE for each category and their potential economic benefits. The rules and algorithms are not given in the paper, but an appendix presents illustrative examples of some of the benefit equations.

Case studies: Two cases studies were selected.

The city of Vacaville near Sacramento with 32,000 customers, three major substations, and 13 primary feeder circuits

The Jose/Miltipas area with 23,000 customers served by three major substations, with 28 primary feeder circuits

The results of the studies show that the for the city of Vacaville case, only substation automation is economically viable, but additional studies of the benefit-to-cost ratio suggest that feeder automation may be justifiable through unquantifiable benefits. For the Jose/Miltipas case, only substation automation was shown to be justifiable based on the proposed methodology. In general, is concluded from the study cases that substation automation is justifiable in the PG\&E, and feeder automation is not justifiable through the proposed method; but the low implementations risks may make it justifiable through unquantifiable benefits.

\section{A.3.5.1.16 Distribution feeder reconfiguration for loss reduction [80]}

Summary: This paper presents a scheme for determining the open/close state of tie and sectionalizing as a planning or real-time tool to reconstruct primary feeders for loss reduction. A simple example is used to illustrate the proposed procedure.

Method description: The paper defines a "switching option" as the reconfiguration resulting from closing a single tie switch and opening a single sectionalizing while preserving the radiality of the two feeders under consideration. Two objectives are considered for the development of the method:

Minimal computational effort to determine the change is losses from a switching option Criteria to eliminate undesirable switching options

An equation is derived for determining the loss change from a switching option using system information and base case voltages and currents prior to the switching operation. Based on the derived equation and practical assumptions on the phase angle differences, the following criteria are adopted for the elimination of undesirable switching options: "loss reduction can be obtained only if there is significant voltage difference across the 
normally open tie switch and if the loads on the higher voltage drop side of the switch are transferred to the other side."

Implementation: The procedure is illustrated using a three-feeder system. There is no actual implementation of the procedure, and the authors acknowledge that the task of coordinating the scheme developed here and the other related control strategies to attain full benefits from feeder reconfiguration is yet to be accomplished.

A.3.5.1.17 Installation of Hydro-Quebec's distribution feeder automation system [81]

Summary: This paper discusses a pilot project involving feeder automation at HydroQuebec, which was installed in Nov 1984 and commissioned in Feb 1985. Hydro-Quebec generates, transmits, and distributes electricity directly to its customers. With a network of 3000 feeders, it delivers approximately 20,000 MVA to some 2.5 M customers scattered throughout the province of Quebec. The paper begins with a discussion on centralized versus decentralized approaches of automation and some proposed guidelines for automation system design.

The pilot project examined the following aspects in particular:

Technical feasibility of feeder automation as a step towards distribution automation Economic advantages of such a scheme, including the cost and the degree of customer satisfaction in terms of shorter outages

The system design process involved three phases, one for each major component of the system: 1) Remote terminal unit, 2) Communication system, and 3) Master station. Each of these phases and how they were implemented in the pilot project is described in detail in the paper. Then, the testing procedure for a Remote Terminal Unit (RTU) prototype is elaborated. Two categories of tests are defined.

Static tests, including temperature, electric/electromagnetic interference, and vibration, Dynamic tests, including the dynamic behavior of the equipment due to different aspects of normal and abnormal network conditions such as short circuit, capacitive, inductive, loop back load circuit besides the resistive network at different levels of current.

The system cost incurred and the network characteristics are reported.

Field implementation: The system was commissioned in Jan 1985. One master station and 27 Rut's controlling part of the vast distribution network have been officially in operation since Nov 1985 and yielded much valuable experience. From the operator's reports, during the six-month period after operation began, the feeder automation system has successfully reduced outage durations by a factor of nine compared to the traditional maintenance procedure, which relies mostly on customer telephone calls. An average of 3.5 minutes per restoration operation was recorded.

The paper also mentions that a second-generation RTU prototype is already available for production, and a few test projects are planned for the second phase, to be reported in due course. 


\section{A.3.5.1.18 An Adaptive Non-communication Protection for Distribution Systems [82]}

Summary: This paper presents an integrated accelerated protection scheme for distribution systems based on the conventional directional overcurrent (OC) protection. An integrated relay in a distribution substation, connected to both the incoming and outgoing lines associated with the station busbar, is responsible for the protection both line sections. The increase in the operating speed is achieved by identifying the remote circuit breaker through the changes of the local currents and voltages. As a result, a significant increase in the operating speed can be achieved for the relay on the side with the longer definite grading time setting.

Implementation: Extensive tests have been conducted using a Real-time Digital Simulator (RTDS) modeling a typical multi-section distribution system. The increase in the operating speed is achieved by identifying the remote circuit breaker through the changes of the local currents and voltages.

Feasibility: The results prove that the new scheme is able to significantly increase the speed of responses of conventional time grading protection. Furthermore, the scheme not only has good adaptive capacity to source condition change, but also can clear the fault from both ends of the line section especially in single source systems, thereby improving distribution automation.

\section{A.3.5.1.19 Implementing Multiagent Systems Technology for Power Distribution Network Control and Protection Management [83]}

Summary: In this paper, an extended research in possible high-end protection and control methods is presented. The authors intend to show the efficiency of combining modern IT techniques with the equipment provided by distribution automation evolving technology. As a result, a flexible and versatile multiagent system (MAS) is proposed. The effected MAS is fault isolation and power restoration oriented, as shown in the case studies. The structure of the proposed system (software and hardware aspects) is introduced. Both microscopic and macroscopic procedures are revised. Results acquired from computer simulations are evaluated. Conclusions concerning the perspectives of implementing the proposed system are finally presented. The MAS project presented in this paper intends to reinforce self-managing capabilities provided to the network, with adaptive behaviors which are necessary for applications in many different network topologies.

Implementation: A software prototype of the proposed MAS was developed to simulate the first Case of Study. Power line communications technology is implemented. The implementation of the MAS as presented in this work will result in a desirable autonomy, in terms of self-management, of the power distribution network. 
Feasibility: The entire network will become a living organism with self-healing capabilities. The power restoration procedures will become less time consuming. The electrical network will not be forced to sustain successive short circuits, as the traditional "trial and error" methodologies will be obviated. As a result, the diminishing cost of fault management will probably compensate the system's installation initial investment.

A.3.5.1.20 Impact of automatic control systems of loop restoration scheme on the distribution system reliability [84]

Summary: Loop restoration scheme (LRS) is a special feeder automation (FA) scheme, which is used by utilities to improve distribution system reliability. The LRS is controlled and managed by its automatic control system (ACS). The impacts on distribution system reliability indices of implementing LRS mainly depend on the type of its ACS. Two common types of ACS of LRS are presented and used in this study. Successful operation of ACS is dependent on the protection and automatic control functions of switching devices of LRS. Different failure modes of these switching devices can therefore affect the procedure of ACS in fault detecting, isolating and service restoration. The impacts of failure of protection and automatic control functions of switching devices and fuse of lateral distributors on reliability indices are illustrated.

Implementation: The worth of implementing LRS and its ACS type is represented by the reduction in expected customer interruption cost. A distribution test system is utilized to examine the impacts of two common types of ACS of LRS on the distribution system reliability. Selecting the type of ACS of LRS by utilities relies on the desired level of load-point and system reliability improvement.

Feasibility: This study aims to quantitatively assess the impacts of two common types of ACS of LRS on the distribution system reliability. In order to demonstrate the proposed technique, comparative studies and sensitivity analyses are conducted using a distribution reliability test system.

\section{A.3.5.1.21 Research of Feeder Automation System Based on Multi-Agent [85]}

Summary: Feeder automation system based on multi-agent is proposed in this paper after analyzing the shortage of current distribution feeder automation system. Single agent can achieve feeder monitoring and local protection alone. When they cooperate, they can locate the fault line section rapidly and accurately, isolate the fault line section and restore power to un-faulted line sections intelligently. Strategy about fault processing of feeder automation system based on multi-agent is analyzed through experiments done in laboratory. It is proved that feeder automation based on multi-agent is more effective. 
Implementation: With the application of distributed artificial intelligence-DAI technology in software and control areas, a branch of DIA, multi-agent system-MAS is applied in power system. Compared to single intelligent device, more attention is paid to cooperation.

Issues:

For agent is an intelligent unit, traditional feeder terminal unit based on MCU could not be competent to new work whether in speed or processing ability. High performance processor is needed.

Each agent cooperates by exchanging real-time information, which needs high communication demands, such as speed, cost etc.

Feasibility: Feeder automation units are widely distributed, which is unsuited for information integration and enhancement of its intelligence. Multi-agent technology is the ideal method to solve the problem. Single agent can achieve local protection and multiple agents can accomplish more complex tasks by cooperating.

\section{A.3.5.1.22 Outage Avoidance through Intelligence Detection of Incipient Equipment Failures on Distribution Feeders [86]}

Summary: This paper reports the work to date showing the ability to detect and recognize failures, incipient failures, and improper and suboptimal operation of line apparatus. Texas A\&M University is collecting, analyzing, and characterizing high-fidelity current and voltage waveforms in a multi-year project, the goal of which is to discover and take advantage of information "hidden" in waveforms, to recognize early warning signs of incipient apparatus failures and recognize improper or suboptimal equipment operation. This Distribution Fault Anticipation (DFA) project is part of the Electric Power Research Institute's (EPRI) distribution automation roadmap. EPRI-member utilities have instrumented 60 feeders across North America to record high-fidelity data for the project. Tennessee Valley Authority-member utility Pickwick Electric Cooperative has instrumented three feeders and has avoided multiple outages and diagnosed malfunctioning equipment.

Implementation: With the application of distributed artificial intelligence-DAI technology in software and control areas, a branch of DIA, multi-agent system-MAS is applied in power system. Compared to single intelligent device, more attention is paid to cooperation.

Issues:

Detecting low-level parametric changes and signatures requires sensitive monitoring, but sensitive data triggering causes a large volume of "routine" data (e.g., motor starts and other load variations) to be recorded as well.

Locating other types of problems, particularly subtle, incipient failures, can be quite difficult, however. For example another utility in the DFA Users Group has documented 
waveforms produced by incipient winding failure in a customer service transformer. Conditions such as this produce currents limited primarily by contact impedance, winding impedance, etc., not by line impedances. This renders traditional distance- or impedance-based methods useless.

Feasibility: This paper has documented multiple examples of recurrent faults; improper capacitor operation that escalates to cause damage and power quality and reliability problems; and potentially dangerous protective device failure. Other utilities participating in the same project have documented numerous other detectable, recognizable failures and problems that affect service quality and reliability.

\section{A.3.5.1.23 Feeder Automation System Based on Non-communication Protection and Control Unit [87]}

Summary: The paper presents a novel feeder automation system based on noncommunication protection and control unit (PCU) without the need of communication. The system can be adaptive to the changes of distribution network configuration. As a fault occurs on a feeder, Non-Communication Protection at the faulted segment's terminals will operate to isolate the faulted segment. The segments out of service will be restored power supply from back-up source after the operation of Backup Source Automatic Control. Since the existing distribution automation systems cannot meet the reliability requirement, a new system with selective isolation and fast restoration is needed in the distribution networks.

Implementation: The proposed scheme uses a specially designed PCU fed from the primary current and voltage instrument transformers (ITs). The PCU is integrated with non-communication protection and backup source control functions. The PCU extracts the fault information and network operation information.

Feasibility: The system can achieve the aims of local protection, local control and local decision. Any Breaker in the new system only needs to operate once to clear a fault. For one feeder with 6 segments, the longest clearing time is about 1.1 seconds for asymmetrical fault and 2.3 seconds for symmetrical fault, as well as the healthy segments will be restored the service in 3.0 seconds.

\section{A.3.5.2 Historical development, High Impedance Faults}

\section{A.3.5.2.1 Time domain techniques}

Most of the time domain techniques were proposed for electromechanical relays or early digital relays with limited computational power. A few time domain techniques that take advantage of the computational power of new digital devices have been proposed in recent years. 
Proportional relay: The proportional relaying algorithm used the equations for multi grounded distribution systems to derive equations for the calculation of the fault current that is defined in [89] as

$I_{F}=k_{1} \times I_{G}+k_{2} \times I_{N}$

where

$\mathrm{k} 1$ and $\mathrm{k} 2$ are system constants derived from the multi grounded system model

IG is the ground current and

IN is the neutral current.

The idea behind this technique is to increase the sensitivity of the relay by directly calculating the fault current independently of the variations in the load, using measurements and system constants.

Ratio ground relay: The electromechanical ratio ground relay was developed by Westinghouse in the early 1980's in collaboration with Pennsylvania Power and Light (PP\&L). This relay detects open phase conductors and single-phase-to-ground faults independent of fault impedance. The relay uses the ratio of neutral to positive sequence current as a fault indicator based on the concept that the amount of load imbalance is invariant over a small time interval.

An electromechanical relay was implemented with this technique using an operating coil driven by the zero-sequence current and a restraining coil driven by the positivesequence current [90]. The ratio ground relay was tested with favorable results using simulated fault currents from an analog feeder model and actual feeder currents during a staged fault test at PP\&L [91].

Current flicker: In [92], an algorithm is proposed based on the random behavior of arcing faults as determined by the flicker and asymmetry of the arcing fault current. The current flicker is calculated by comparing the positive and negative peaks in one cycle to the previous values. Asymmetry of the fault current is determined by comparing the magnitude of the half-cycle peak current to the magnitude in the previous half-cycle. The detector output is compared with set thresholds to determine if the disturbance is due to an arcing fault. The process is then repeated for the next time window.

Graphical image feature extraction: In [93], a time domain HIF relaying scheme is proposed based on the identification of the shape of the fault trajectory in voltage current plane. Graphical features are extracted from time domain transient signals and compared with stored contour shapes and characteristics to determine the existence of persistent low arcing faults.

Voltage unbalance: This algorithm proposed in [94] is based on the assumption that when a conductor breaks, the primary voltages in the part of the feeder beyond the break point present unbalances which are much greater than those in normal conditions considering the unbalanced loads. Usually, in case of breaking of one phase of a feeder, 
the percentage of negative-sequence as well as of zero-sequence in the phase voltages beyond the breaking point are in the range from 50 to $100 \%$ (with respect to the positivesequence). In case of breaking of two phases of a feeder, the percentages of negative and zero-sequences are both equal to $100 \%$. Comparatively, for distribution systems in normal operation, solely unbalanced loads lead to no more than 5\% negative and zerosequences. Thus, broken or downed conductors are able to be detected by monitoring the negative-sequence and zero-sequence components in the voltages. The shortcoming of this method is being unable to tell high impedance faults from normal broken faults, which disable the warning function in the circumstance of high impedance faults. A.3.5.2.2 Frequency domain techniques

Most frequency domain methods take advantage of the rich harmonic and inter-harmonic content of the arcing associated with high impedance faults.

Second order harmonic: A second-order harmonic algorithm was proposed by researchers in Taiwan in 1985 [95]. This algorithm is based on the assumption that the second-order harmonic current is very small under normal operating conditions but high for arcing currents. Two versions of the method were proposed to detect the presence of a high impedance fault. One uses the amplitude of the magnitude of the second order harmonic, and the other uses the ratio of the second to first order harmonic. No information is available on the implementation of this method in a relay, but the algorithm was compared favorably with other methods using staged faults tests in 1988 [96].

The Texas A\&M Algorithms: Texas A\&M University has conducted some of the most extensive research on high impedance faults since the early 1980's [97-110]. Through this period, several detection algorithms have been developed and tested on their high stage fault facilities. Here are the four most significant frequency domain techniques developed at Texas A\&M:

Energy algorithm: Arcing causes bursts of energy to register throughout the frequency spectrum of the fault currents. The energy algorithm monitors several frequency bands between low order and higher frequency components looking for a significant increase in their magnitude. The algorithm also checks for persistence of the detected components to discriminate arcs against similar signals generated by switching events $[109,110]$.

Randomness algorithm: The randomness algorithm monitors the same set of component energies as the energy algorithm. However, rather than checking for a sudden, sustained increase in the value of the monitored component energy, it looks for a sudden increase in a component energy followed by highly erratic behavior. This type of highly random behavior is indicative of many arcing faults [109, 110].

Arcing phase signature algorithm: This algorithm looks for the occurrence of bursts of arcing current at specific phase angles of the voltage. The algorithm is based on the mechanics of the arcing current that starts when the voltage magnitude is close to its peak 
value and extinguishes when the voltage magnitude becomes zero. Experiments show that this is particularly visible in the high-frequency components of the current [109].

Spectral analysis algorithm: This algorithm is based on the observation that the average Fourier transform of several cycles of an arcing current has an I/f shape that can be used to indicate the existence of a high impedance fault. Due to the effect of the line impedance, the degree of attenuation at higher frequencies is proportional to the distance to the fault, allowing the algorithm to give an indication of the distance to the fault [109].

Kalman filtering approach: Conventional Fourier filtering considers system signatures as steady state or quasi-steady state, but in reality, they are time-varying in nature due to the dynamics of the arcing fault. In this method, the magnitude of the fundamental and harmonic currents is assumed to be formed by a constant and a time varying component. A Kalman filter is proposed to obtain the best estimation of the time variations of system signatures avoiding the errors caused by conventional Fourier filters. The Kalman filter also accounts for the high-frequency decaying component and the dc offset on the fault current. Testing of the algorithm with staged faults showed that the change in low-order, odd harmonics detected by this algorithm is a good indicator of high impedance faults [111].

Wavelet techniques: Wavelets can be used to analyze the transient behavior of signals in both the time and the frequency domain. By selecting the proper wavelet model, different transient events can be determined by looking at the detailed outputs of wavelet filters. Since late 1990, many wavelet-based algorithms have been proposed for the detection of high impedance faults [112-117]. Most of this application combined wavelet with pattern recognition of artificial neural networks for the detection of high impedance faults. Some of these methods are discussed in section 4.

One of the first applications of wavelet analysis was the wavelet filter bank technique. In [118], the author proposed the idea of a wavelet analysis filter bank (WAFB) consisting of a dyadic wavelet transform implemented by two channels of quadratic mirror filters. Extensive simulations showed that WAFB can easily detect the arc phenomenon and discriminate those signals from HIF's from those of a capacitor bank switching operations.

A.3.5.2.3 Pattern recognition and knowledge based approaches

From testing and simulations, researchers and engineers learned that due to the time varying characteristics of HIF, no single algorithm could provide full coverage; and combinations of algorithms need to be used. The first algorithm proposed a simple combination of algorithms, but as more powerful computer relays became more complex, solutions have been proposed. Techniques like pattern recognition, fuzzy logic, artificial neural networks, and genetic algorithms have been proposed and tested for the detection of HIF. In this section we review some of these techniques. 
Pattern recognition: Many pattern recognition techniques have been proposed in the past twenty years to improve the reliability and security of HIF detection systems. In this section we present some samples of the proposed pattern recognition techniques.

In [112], wavelet analysis on the feeder 3 I0 current is proposed with a four-level decomposition tree for the detection of high impedance faults. The algorithm uses Daubachie's-4-mother wavelet to detect the extinction and re-ignitions of the arc current, stressing the importance of the 2nd harmonic spectral component since the arc current might have two extinction and re-ignition events per cycle. The algorithm was successfully tested using simulation and recorded staged faults.

In [119], discrete wavelet transforms and the nearest neighbor rule are used to create a pattern recognition algorithm that discriminates between HIF and capacitor switching.

In [114-120], a secure high impedance fault detector is proposed where an array of different algorithms is combined together with expert system giving the decision logic. The proposed array is shown to enhance the reliability of the detection, and it is claimed to be used in commercial products.

Fractal techniques are proposed in [107]. Using fractal analysis, chaotic properties of high impedance faults can be identified from other transients in a power system. The authors adopted RMS values of currents as input for fractal analysis to distinguish HIF.

Knowledge based techniques:

Neural networks: In the past fifteen years, many algorithms based on artificial neural networks have been proposed by a large number of researchers in combination with wavelet analysis and other signal analysis techniques. In one of the first ANN applications, [121-123] proposes neural networks in combination with the third harmonic magnitude and angle of the positive, negative, and zero-sequence signals. In [124], a back propagation neural network is trained with four 1/4 windows FFT outputs; once trained, the method can discriminate between HIF, load switching, capacitor switching, and arc furnace. In [125], FIR filters are used to obtain the signal for training the neural network to detect HIF's. While in [117], discrete wavelet transforms are used to extract distinctive features of the voltage and current used to train and ANN to discriminate between HIF and switching events. In [126], the third and fifth harmonic components of voltage and current are used to train an artificial neural network to detect and discriminate HIF. In [127], the magnitude of the symmetrical components of the 1st, 2nd, 3rd, and 5th harmonics of the three-phase current signals are used to train the ANN to detect HIF. Two additional ANN's are used to locate the fault, and they are trained using the magnitude of the fundamental voltage, the magnitude of the fundamental third harmonic current, and the angle of the first and third harmonic currents referenced to the respective voltages.

Other techniques: A few fuzzy logic and genetic algorithm approaches have been proposed for HIF detection. In [128], a methodology for HIF detection in radial distribution feeders is proposed based on fuzzy logic reasoning. This method is based on 
the analysis of feeder responses to impulse waves, which are periodically injected in to the feeder. In [129], a fuzzy inference system is proposed to detect high impedance faults. In [130], researchers use DWT to extract features from the signal and a fuzzy inference set with genetic algorithms to detect high impedance faults. In [131], the HIF problem is formulated as an estimation task and a real coded genetic algorithm is used to solve this optimization problem. The method was successfully tested with simulation for tracking harmonics and current angles associated with HIF.

\section{A.3.5.2.4 High impedance fault modeling and testing}

Very few facilities exist for the experimental collection of HIF data under different circumstances and surfaces. One of the best known facilities is located at Texas A\&M and has been used to test many HIF detection algorithms. Unfortunately, most researchers have limited access to these types of facilities, and simulation of HIF is quite difficult due to its diverse and complex arc phenomenon.

Some of the validations of HIF detection algorithms are based on the field test data that have certain limitations due to their specific site and environment. The need for a general and realistic model of HIF is important for HIF detection method research and protective equipment design. In this section, we review some of the staged fault data and HIF models that have been developed and collected over the past two decades.

Staged fault tests: Many staged fault test have been performed around the world to obtain HIF data that is valid for HIF research to the utilities or locations where the tests are performed.

In [96], staged fault tests were performed in Taiwan to obtain HIF data in 1988. The tests were performed in a 12.5 MVA $69 / 11.9 \mathrm{kV}$ feeder with an $800 \mathrm{kVA}$ load. Several tests were performed in the same surfaces and the researchers found that arc was generated in almost all cases, with the same surface fault currents vary with location, and that bare and insulator conductor have different fault currents. The data was used successfully to compare 4 different HIF detection algorithms.

In [92], tests were performed in high voltage laboratory at the University of Manitoba to collect HIF data under controlled conditions in 1994. For these tests, a $25 \mathrm{kVA}$, 240/7200 Volt, 105/3.5 Amp, and 1.5\% impedance, distribution transformer energized from $100 \mathrm{~A}, 208 \mathrm{~V}$, and $60 \mathrm{~Hz}$ was used. One bare and other insulated conductors connected to the high voltage side of the transformer were tested separately by dropping them to a pile of soil placed on a metal pan with the ground return completed through a variable current limiting resistor. The data was used to verify the energy flicker HIF algorithm.

In [112], researchers report on 32 high impedance stage faults in a $20 \mathrm{kV}$ feeder in Spain performed in 1997 and 1998. The tests were aimed to obtain data to study the $64 \mathrm{~N}$ relay alarms and HIF detection algorithms. The faults were staged on different surfaces: soil, 
lawn, sand, gravel, concrete, asphalt, and a tree to obtain information on all possible types of HIF for some of the Spanish utilities.

In 2005, reference [132] describes how TXU Electric Delivery and ABB performed staged fault test to collect HIF data using an active feeder without tripping the feeder and its customers. The tests were performed in different types of surfaces and had to be repeated to overcome unexpected problems caused by the existing protection devices. The data was used by ABB in the research and development of suitable techniques for detecting high impedance faults.

High impedance fault models: Several models have been proposed in the past three decades for HIF, and they have evolved to more realistic models. The first simulations started with a simple model of a resistance connected to the network at the point of the fault [133].

In [134], the simple model is improved by assuming a nonlinear impedance model based on the voltage cycle where the impedance decreases as the voltage increases in magnitude during the negative and positive half cycles. Other researcher improved the model by adding and air gap that helped achieve the reigniting phenomena observed on staged faults.

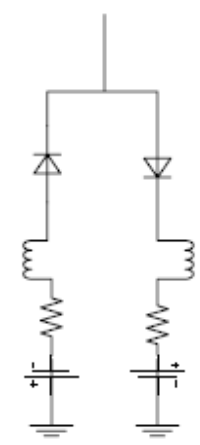

Figure A.18-Advanced HIF model

A more complex model is proposed in [135] where two dc sources, VP and VN, connected in anti-parallel by means of two diodes are used to model the fault. During the positive half cycle, the current flows through the VP terminal and during the negative cycle through the VN terminal, and these voltage values are maintained constant during the simulations. Some researchers improved on this model by adding two resistors in series with the diodes to represent the soil or tree resistance. In [131], the two source arc model is improved by adding two inductors as shown in Figure 18. 


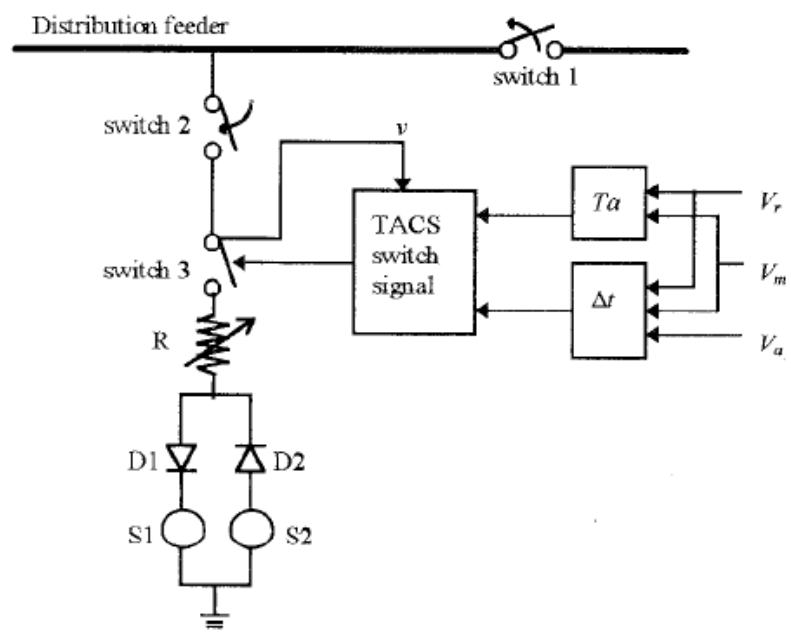

Figure A.19-Complex HIF model

In [118], a model embraces non-linear impedance, time-varying voltage sources, and a controlled switch as shown in Figure A.19. Switch 1 and 2 are conventional timecontrolled switches which isolate the feeder from the load and connect it to the fault path. Switch 3 is a transient analysis of control systems controlled switch which controls the arc re-ignition and extinction.

Another model in [136] is based on experimental data collected by KEPCO and uses two time varying resistances in series to model the HIF. One of the resistance has a periodic characteristic, $\mathrm{R}(\mathrm{t})=\mathrm{R}(\mathrm{t}+\mathrm{T})$. The other resistance has a very large value at the beginning of HIF, and then its value is monotonously decreased in the transient state and becomes zero in the steady state after HIF.

In [137], a HIF due to leaning tree is modeled and verified. The model is divided in two parts: the arc and the high resistance. The dynamic arcing element is simulated using thermal equations. The parameters for the arc and resistance model are determined using the experimental data. Experimental data is used to validate the system transient model. The model is used to test a discrete wavelet transform algorithm for HIF detection.

\section{A.3.5.2.5 Commercial devices}

The HIF detectors can be divided into mechanical and electrical devices. Mechanical devices are less common, and their aim is to convert HIF faults into normal faults to allow their detection and clearance by conventional relays. Electric devices, on the other hand, depend on the analysis of electrical signals using one or more methods to identify high impedance faults. This section presents a review of the mechanical and electrical devices encountered in the literature. The electrical devices and the principles of operation of HIF relays from the three major relay manufacturers are described.

Mechanical devices: Two representatives of the mechanical devices are [138] The Falgard device (invented by Irving Reedy in the mid 80's and later marketed by Alcoa Conductor Accessories) is a pendulum-mounted aluminum rod with hooked ends that is suspended from an under-built neutral conductor. 
The Safety Yoke built by Electric Safety Products, Inc. is mounted to a cross arm or pole. It provides a solid ground fault by intercepting a falling conductor with a grounded spring-loaded bus bar.

Electrical devices: Several HIF relays have been available since the early 1980's. The early devices operated under simple principles and achieve acceptable levels of operation. Most recent devices combine different techniques to differentiate and identify HIF. Most major relay manufacturers have a HIF device.

Ratio ground relay: One of the first electrical devices was the electromechanical "ratio ground relay" developed by Westinghouse in the early 80's in collaboration with Pennsylvania Power and Light. This relay detects open phase conductors and singlephase-to-ground faults independent of fault impedance. The relay uses the ratio of neutral to positive-sequence current as a fault indicator based on the concept that the amount of load imbalance is invariant over a small time interval. The relay was implemented with an operating coil driven by the zero-sequence current and a restraining coil driven by the positive-sequence current [90]. The ratio ground relay was tested with favorable results using simulated fault currents from an analog feeder model and actual feeder currents during a staged fault test at PP\&L [91].

HIFAS: The Nordon Technologies High Impedance Fault Analysis System (HIFAS) measures the third harmonic current phase angle with respect to the fundamental voltage. The operating premise is that the current associated with a HIF produces a third harmonic current with a unique phase relationship to the faulted phase voltage. At the fault location, they will be in phase. The ambient 3rd harmonic phasor is averaged over time and stored for comparison. When a fault occurs, the new 3rd harmonic phasor is vectorially subtracted from the stored value. If the resulting magnitude is above a preset threshold and if the angle matches a preset value for a downed conductor, a HIF is declared. This device is mentioned in the literature in the mid 1990's [139, 140] with some indication that it was used and tested by utilities, but no recent references exist; and no information can be found on the company. It is assumed that several of these devices are still installed, but no new devices are available.

The GE F60 relay: The algorithms used on this relay are based in part on the extensive research performed at Texas A\&M University in the 80's and 90's. F60 analyses harmonic and non-harmonic components of the feeder currents between 30 and $780 \mathrm{~Hz}$ through the use of pattern recognition and artificial intelligence. An expert system with nine different algorithms is employed to detect and classify HIF's. Distinctions are made between downed conductors, persistent arcing, and intermittent arcing. Downed conductors are differentiated from arcing conductors by looking for a loss of load or a high current fault prior to the HIF event [141].

The nine algorithms used for HIF protection in the GE F60 include:

Energy algorithm

Randomness algorithm

Expert Arc Detector (EAD) algorithm 
Load event detector algorithm

Load analysis algorithm

Load extraction algorithm

Arc burst pattern analysis algorithm

Spectral analysis algorithm

Arcing suspected identifier algorithm

The ABB REF 550: This high impedance fault detection systems has three individual algorithms for detecting high impedance faults:

A neural network algorithm: A two layer network is used and trained using backpropagation with an adaptive learning rate.

A wavelet algorithm: Discrete wavelet transform is used for a multi-resolution analysis of current loads and to deliver a load current description as it changes in time at different scales. The algorithm associate large scales to low-frequency components and small scales with high frequencies components.

A high-order statistics algorithm: This algorithm uses the non stationary feature of HIF currents to differentiate them from white noise

Each individual high impedance fault detection algorithm uses various features of phase voltages and currents to respectively detect a high impedance fault with different confidence level. A fault is identified as a high impedance fault once it is detected independently by the algorithms and processed through decision logic [142].

The SEL 450 relay with HiZ: This device uses a sum of difference current, SDI, algorithm to identify signatures of HIF without contamination due to distribution type loads. Through an adaptive tuning process, the relay algorithm learns the ambient noise profile of the feeder by comparing the SDI and the SDI average by memorizing the time and the ratio if the SDI is above the threshold set for the SDI average [143].

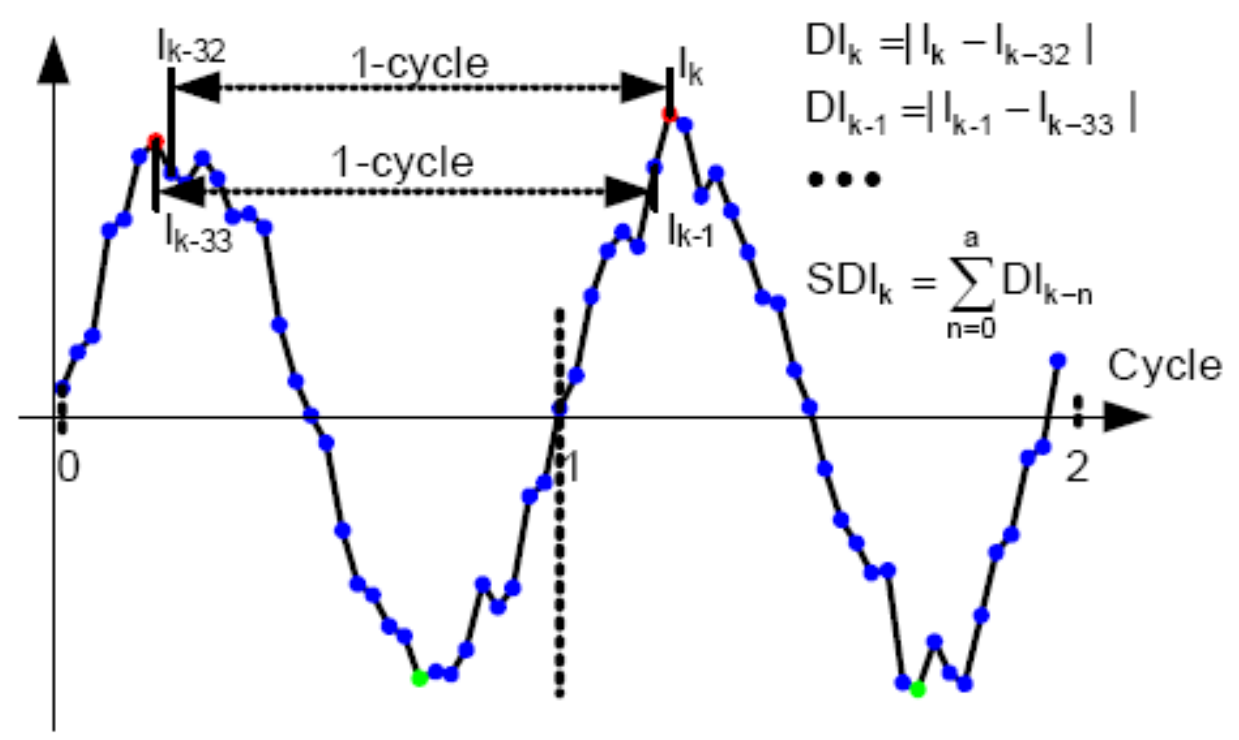

Figure A.20-Illustration of SDI calculation 
The relay algorithm also uses an infinite impulse response filter average to discriminate between switching and fault conditions. A detection logic algorithm uses operating and restraining quantities and counts both temporal and amplitude characteristics of an HIF [143].

\section{A.3.5.2.5 Literature Update 2007-2010}

The initial report was prepared in 2007. This sections presents an update of the literature review on High impedance faults from 2007 to June 2010. This update differs from the previous section and follows a synopsis instead of a narrative description.

A.3.5.2.5.1 Performance of Restricted Earth Fault Protection Scheme in the Presence of Current Transformer Remanence [144]

Summary: In modern power system protection, an accurate transformation of primary short circuit current is vital to ensure correct operation of the high impedance Restricted Earth Fault (REF) protection scheme used for transformer protection. The dc component in the fault current as well as the remnant flux cans causes severe saturation conditions if the current transformer is not selected correctly. Behavior of the current transformer during the transient condition is important as this will determine the stability of the REF protection scheme. This paper discusses how the remanence phenomenon beside the dc component in the fault current can causes severe saturation to the current transformer and affects the stability of the REF protection scheme.

Implementation: REF protection scheme was carried out to analyze the effect of remanent flux on the scheme. A 30MVA 132/11KV power transformer in one of substations located at Meru town was chosen since the REF protection scheme used had experienced a mal-operation during a three phase out of zone fault.

Issues: A careful and thorough calculation of maximum possible out of zone fault current as well as the stabilizing resistor needs to be carried out during pre-commissioning stage to prevent any unwanted maloperation of the REF scheme due to current transformer saturation.

Feasibility: Remanent flux that exists in current transformer can cause severe saturation during a fault condition. Saturation can cause differential protection scheme such as REF to mal-operate due to unbalance current.

\section{A.3.5.2.5.2 Performance of Novel Neutral Admittance Criterion in MV-Feeder Earth Fault Protection [145]}

Summary: This paper describes the fundamentals of the neutral admittance based earthfault protection. Earth-fault (EF) protection is traditionally based on directional residual overcurrent criterion with zero-sequence voltage as the polarizing quantity. An example of such is the $10 \cos (\mathrm{j})$ - criterion that is commonly used in compensated medium 
voltage distribution systems. However, e.g. in Poland, the neutral admittance (Y0) criterion has become popular and is today a standard EF protection function required by the local utilities.

Implementation: Based on the theory and field tests, the admittance calculation should be based on changes in the zero-sequence quantities due to a fault, the novel Y0 characteristic should be used and the parallel resistor should be permanently connected. This maximizes the performance of the $\mathrm{Y} 0$ protection.

Feasibility: The results show that $\mathrm{Y} 0$ protection is a respectable alternative to traditional EF protection functions. Benefits include e.g. good immunity to fault resistance and easy setting principles.

\section{A.3.5.2.5.3 Evaluation of Fault Protection Methods using ATP and MathCAD [146]}

Summary: This paper discusses combining the Alternative Transients Program (ATP) and MathCAD to teach protective relaying and to develop relay algorithms. A power system model is created in ATP with appropriate current and voltage measurements. The simulation output is converted to a COMTRADE format and imported into a detailed relay model implemented in MathCAD. The MathCAD model performs digital filtering calculations, symmetrical components calculations and models relay algorithms based on relay manufacturers published information. Focus here is on differential and ground fault protection for the common two bus, parallel line case. Emphasis is placed on fault detection and localization methods for ungrounded or high impedance grounded systems.

Implementation: This paper will utilize relay models implemented in MathCAD that import COMTRADE files generated from the output of an ATP simulation using ATP Analyzer. The relay models implemented are based on protective algorithm information published by relay manufacturers.

Feasibility: Using two different software packages to develop models requires a thorough knowledge of relay principles. The current flow direction in ATP affects the MathCAD results. While this may be seem obvious, it is easy to include a negative sign in MathCAD for direction as well.

A.3.5.2.5.4 Network Integrated DSP-based Adaptive High Impedance Ground Fault feeder Protection [147]

Summary: This paper presents a practical adaptive strategy for live substation feeder protections with new implementations using state-of-the-art digital signal processing (DSP) technology and modern computer networking technology. This network integrated 
DSP-based feeder protection adapts its trip settings to provide improved selectivity and speed of fault detections for varying power system configurations and loadings. This protection accurately senses and de-energizes downed conductors that often could not be detected by conventional non-adaptive feeder protections. This paper presents the design of this adaptive feeder protection. Several power system faults were studied under varying load and system conditions.

Implementation: paper proposes a practical adaptive strategy for live substation feeder protections with new implementations using state-of-the-art digital signal processing technology and modern computer networking technology.

Feasibility: Results were analyzed to verify the design, to determine improvement over conventional static protections, and to verify correct protection operations. Analysis of the simulated fault tests show that the adaptive protection significantly extends the range of ground fault impedances that can be detected. Field tests were conducted in a substation in Canada to validate this adaptive feeder protection. 


\section{A.3.5.3 Synopses, Fault Current Limiters}

\section{A.3.5.3.1 Superconducting fault current limiters [148]}

Abstract: Three different superconducting fault limiter designs are described in relation to low temperature (metallic) superconductor operating at $4.2 \mathrm{~K}$ and high temperature (ceramic) superconductor at $77 \mathrm{~K}$. A shielded inductive type of limiter is preferred and a brief description of a novel design is given.

Summary: The costs of shutting down a system to replace switchgear are far too high, and the system reliability could be jeopardized; hence, there is a need for fault current limiters. Low-temperature superconductors (LTS) are cooled in liquid helium beneath $2.5 \mathrm{~K}$ while high-temperature superconductors (HTS) are cooled in liquid nitrogen at 77 K. Refrigeration operating costs are 25-100 times reduced by using liquid nitrogen, and refrigeration capital costs are 10 times lower with liquid nitrogen.

As for different types of FCL, the resistive and inductive are stressed. Resistive superconducting fault current limiters (SFCL's) have issues with non-uniform heating and are simpler and require fewer materials than other current limiters. These devices should be able to limit voltage drop within $1 \%$ of the nominal voltage, and a resistive/inductive shunt is appropriate to share heat losses with the superconductor.

The saturated inductive superconducting fault current limiter operates on the principle that, as an inductive device goes into or out of saturation, its impedance changes by a significant amount. To achieve current limiting in both halves of a cycle, two inductive devices (inductors) are acquired per phase. These are driven into saturation by a dc bias field supplied by a superconducting coil. The ac line to be protected is therefore wound around two inductor cores. When a fault current flows, the large mmf produced by the ac winding drives the core out of saturation on one half-cycle, automatically inserting large impedance and limiting the magnitude of the fault current. Limiting on the reverse halfcycle is achieved by the use of the second inductor connected in series with the first and is saturated in the opposite direction. To protect all three phases, six inductors are required. The saturated inductive design is attractive because the superconducting component (the field winding) is external to the circuit being protected. The field winding current is dc, and transition from normal to fault operation and vice versa is gradual and without interruption of the superconducting state of the winding. Opening of a circuit breaker to protect the superconductor is not a requirement of this design, which is thus particularly attractive for overhead transmission networks when it is desirable to have automatic re-closing onto faults.

The shielded inductive SFCL device consists of an iron core, a primary copper winding in series with the circuit to be protected, and a superconducting secondary (cylinder). Because the primary winding in this device is usually wound over the superconducting cylinder, the superconductor is commonly referred to as a magnetic shield or screen, preventing primary winding flux from entering the iron. It may also be visualized as a transformer with a shorted secondary winding with a single turn. For normal operation, 
the ampere-turns in a coil are balanced by the induced current in the superconductor and the SFCL behaves as a low-impedance device. During a fault situation, however, the ampere turns balance is destroyed, flux enters the iron, and high impedance is inserted in the circuit to be protected.

In accordance with transformer theory, the relative magnitude of the superconductor resistance will determine whether the limiter impedance is predominantly reactive or resistive. The shielded inductive design requires superconducting properties that are achievable with HTS materials operating at $77 \mathrm{~K}$. With correct design, it has the potential to have a very short recovery time, thus not always requiring switchgear for protection of the limiter. Current may be limited to near the maximum nominal operating current; hence, switchgear ratings could be substantially reduced. Normal operating voltage across the limiter is $4 \%$ of the normal rated line voltage.

The shielded inductive type incorporating HTS discs may be considered the most practical, using near-term state-of-the-art technology and operating at $77 \mathrm{~K}$. Operating with bulk liquid nitrogen would make the device virtually maintenance free and lifetime expectancy should be in excess of 30-40 years. Of course, technology has progressed considerably since the release of this paper.

Table 3-Comparison of superconducting FCL types 


\begin{tabular}{||l|c|c|c||}
\cline { 2 - 4 } \multicolumn{1}{c|}{} & Resistive & Saturated & Shielded \\
\hline Self activating & yes & yes & yes \\
\hline Fail safe & yes & yes & yes \\
\hline Operating temp & $1.2-77 \mathrm{~K}$ & $4.2-30 \mathrm{~K}$ & $77 \mathrm{~K}$ \\
\hline Relative wt. for same rating & 0.1 & 1 & $\leq 1$ \\
\hline Switching on time & $\leq 1 \mathrm{~ms}$ & no delay & «lms \\
\hline SCFCL Recovery time after fault & seconds & no delay & «lms possible \\
\hline $\begin{array}{l}\text { Circuit breaker opening } \\
\text { absolutely necessary }\end{array}$ & yes & no & no \\
\hline Shunt impedance rcquired & probably & no & no \\
\hline $\begin{array}{l}\text { Operating voltage drop SCFCL } \\
\text { to line voltage \% }\end{array}$ & $<1 \%$ & $-4 \%$ & $<1 \%$ \\
\hline Relative operating losses & 1 & -2 & $\leq 1$ \\
\hline Current leads required & yes & yes & no \\
\hline High voliage bushings required & yes & no & no \\
\hline $\begin{array}{l}\text { Relative cost estimate based on } \\
\text { Ref [9] (includes operating loss) }\end{array}$ & $0.4-0.8$ & 1 & 0.5 \\
\hline
\end{tabular}

\section{A.3.5.3.2 Superconducting fault current limiter [149]}

Summary: Two schematics show a single-loop system with and without a FCL and discuss the difference. The purpose of the FCL is to limit excessive currents for $50 \mathrm{~ms}$ or so. Broadly, in resistive FCL's the superconducting element carries the main current (normal or fault), whereas, in inductive FCL's it does not. Conceptually, the resistive type is the simplest to envisage; a superconducting section of the power line switches from zero resistance to a significant resistance when either the critical current or the critical magnetic field is exceeded within the material. In the screened core design, the superconducting element is a cylinder which, in effect, forms the single turn secondary of an iron-core transformer, which has part of the power line as its primary. In its superconducting state, this cylinder effectively screens the iron core from the primary, and a low inductance is in the line. However, when the current (and hence the magnetic field) increases above a certain level, the superconductor becomes normal, and the line impedance increases substantially to that of an iron-core primary, thus limiting the current. A variant of this, sometimes called the air-gap FCL, has a superconducting disc 
inserted into a thin air-gap in the iron core. When superconducting, this disc prevents the core from forming a complete magnetic circuit, and again, the line impedance is low. However, when the disk becomes normal, the magnetic loop is completed, and the impedance becomes that of an iron-core inductor.

In the saturated-core FCL, two iron cores (one for each half of the cycle) are saturated by the magnetic field produced by a superconducting coil wrapped around each core. The main power line is wound around both cores, and when the current becomes high enough, the cores are driven out of saturation and the impedance rises, limiting the current. The advantage of this concept is that it does not require the superconductor to become normal to operate. However, it requires approximately twice as much iron (two cores). The principle disadvantage of all inductive FCL's is the amount of iron that is needed to make a practical, high power device. Indeed, the bulk and cost of the iron alone probably will make FCL's based on this design large, heavy, and prohibitively expensive.

An effort was also made to discuss the heating effects of LTS and HTS devices.

A.3.5.3.3 Principle and characteristics of fault current limiter with series compensation [150]

Abstract: A fault current limiter with series compensation, which is composed of a compensation capacitor and a limiting reactor in series, is proposed. A solid-state switch connected in parallel with the capacitor controls either the ordinal series compensation or fault current limitation. A feasibility study of the current limiter by simulation analysis is presented and the effectiveness of the current limiter is evaluated from the viewpoints of transient stability improvement and device capacity. The current limiter is a useful protection device for large, high power transmission systems.

Summary: This paper opens by describing a need to have quick system protection. Typical breakers operate in 2 or more cycles, but a fault current limiter is even quicker. An FCL with series compensation counterbalances the series inductive reactance of a transmission system via series capacitors. This combination also serves to increase power transmission capacity by preventing the initial generator acceleration (after a fault occurrence) and providing additional decelerating energy at the time of fault protection.

The FCL with series compensation is achieved with a series compensating capacitor having a quick operating bypass switch in parallel and a current limiting reactor connected in series to the capacitor. An overvoltage protecting device and another bypass switch, which backs up the other switch, are also connected in parallel to the capacitor.

The FCL with series compensation has the following distinctive characteristics which conventional current limiters do not have:

Quick current limiting by the solid-state switch control

No power loss in the solid-state switch 
Simultaneous current limiting and capacitor protection

Improved device use rate by series compensation

Improved transmission capacity and system stability

Two circuit parameters, the limiting rate $\alpha$ of the fault current and the compensation degree $\mathrm{k}$ of the power system series reactance, are then discussed.

Next, an electro-magnetic transients program (EMTP) transient analysis simulation shows the effect of adding a FCL to a system with regard to current limitation and timing as well as overloading of the current limiter and transient stability. The results show that the FCL can improve system stability by quick current limiting at the beginning of a fault and series compensation after fault clearing.

Final discussion relates to device capacity of the FCL (limits of the reactor and series capacitor), improvement of system stability and transmission capacity, and estimation of losses through the capacitor and switch. The FCL is more efficient than the current limiter with the current interrupting portion of the solid-state switch.

A.3.5.3.4 Fault current limiter applications on medium and high voltage power distribution systems [151]

Abstract: Electronically triggered fault current limiters have been used in the medium and high voltage power distribution system, and they are used to limit the available short circuit current to a lower level, so that the under-rated switchgear can be operated safely. However, to insure a safe and proper usage of those devices, there are a few major operating parameters needed to be considered: 1) selection of trigger level, 2) selection of di/dt, 3) effect of the capacitor bank and stray capacitances, 4) coordination between these devices and phase o/c relays at the faulted breaker. This paper will examine these major operating parameters and evaluate the performance of the current limiters under various simulated fault conditions, and develop application guidelines for current limiters.

Summary: This paper begins by introducing the concept of a fault current limiter as two parallel elements: the main conductor to service the normal load and the current limiting fuse, which drops the current within a quarter to half a cycle when the fault occurs. Two of the main historic problems with FCL's have been false trips and coordination with protective elements although EMTP simulations show that FCL's can be effective.

The paper then presents the equation of an asymmetrical short circuit current. Relations between asymmetrical and symmetrical fault currents are then derived.

Furthermore, operating parameters of the current limiter are developed. The trigger level is a value defined as the maximum short circuit contribution permitted from a major power source, so that the total overall fault current superimposed from all short circuit contributions will not exceed the momentary and interrupting ratings of the switchgear. 
Switchgear sizing and transformer inrush are considerations that must be accounted for when determining a trigger level.

Next, the di/dt in $\mathrm{kA} / \mathrm{ms}$ is a value defined as the minimum fault current change rate limit to prevent nuisance current limiter operation. The nuisance tripping is essentially caused by high-frequency transients such as switching transients. Also, lightning strikes on exposed (bare) overhead lines can cause nuisance tripping as well. A relationship to determine the time derivative of the current as it relates to the system angular frequency is presented, too.

Simulation of a typical distribution system shows that two bus-tie FCL's are able to lower fault currents enough to maintain the switchgear rating.

FCL's can be used to expand the capacity of a power distribution system, but other factors must be considered: location of FCL, essential loads (which are not to be shut off), transformer inrush current, coordination between FCL and phase overcurrent relays, and presence of a parallel current limiting reactor.

\section{A.3.5.3.5 Study of fault current limiters using YBCO thick film material [152]}

Abstract: A small scale inductive fault current limiter has been designed, modeled and tested. The device outlined in this paper utilizes a simple ferrite E-core arrangement. Electromagnetic analysis is used to predict its performance and this has been subsequently compared with experimental results. The merits of several different arrangements of the components of the fault current limiter are then outlined. Melt processed thick film YBa2Cu307-6 (YBCO) rings have been fabricated. The thick films are in planar form; screen printed and fired on yltria stabilized zirconia (YSZ) substrates. The characteristics of the superconducting rings have been studied and compared in the fault current limiter (FCL).

Summary: Some inductive screened FCL types have utilized cylinders of both thick film and bulk BSCCO 2212; BSCCO 2223 silver clad wires have also been used. Inductive designs have incorporated bulk YBCO rings. Some resistive designs have employed thick film YBCO deposited by pulsed laser deposition on planar polycrystalline yltria stabilized zirconia (YSZ) substrates. YSZ is stable up to very high temperatures and can be made up to relatively large sizes. In terms of fault current limiter design, planar thick films possess the following beneficial features: good heat loss characteristics due to their large surface area; the YSZ substrates provide mechanical stability for the superconducting films; the thick film components can be stacked up and individual ones replaced if necessary. By changing the layout of the superconductors and winding so as to break the magnetic paths through the core, the leakage reactances and hence the insertion loss of the FCL can be reduced. Increasing the number of superconducting rings has the effect of reducing the leakage reactance and increasing the trigger current.

However, if a large area of superconductor exists or if many rings are used to increase the trigger current, the impedance during the faulted state may be too low. Thus, there is a tradeoff between faulted state impedance, number of rings, and trigger current. 
A.3.5.3.6 Discussion of "Fault-current limiter applications in medium-and-high-voltage power distribution systems" [153]

Summary: This article pertains to material discussed in [137]. The fault-current limiters commonly available in this country and developed under Electric Power Research Institute (EPRI) Project RP 1142 have no di/dt sensing. Also, the definition of the trigger level stated in Section III of the paper as the maximum short circuit contribution permitted from a major power source so that the total overall fault current superimposed from all short circuit contribution will not exceed the momentary and interrupting ratings of the switchgear, is incorrect and misleading. Trigger level is simply the threshold level of instantaneous current expressed in kilo ampere crest, which, maintained for a certain time delay, will operate the limiter. Different trigger levels can be set on a FCL.

A 3.5.3.7 Enhancement of power system transient stability using superconducting fault current limiters [154]

Abstract: Transient stability investigations consist of studying the rotor oscillations of generators (electro-mechanic oscillations, $0.1-2 \mathrm{~Hz}$ ) after the occurrence of a fault of large amplitude, e.g. short circuit. The goal is to indicate if the generators are capable to stay synchronous after a fault has occurred. The fault duration is one of the most important factors to be determined. In fact, the shorter the fault, the more the maintaining of synchronization can be guaranteed. Now in case of a fault, a fault current limiter has an extremely fast current transition in comparison to electro-mechanic time constants. This implies a quasi-instantaneous elimination of the fault through a limitation of the current and consequently a better ability to maintain the synchronization of the system. We recall that in a classic system, the elimination of a fault, by opening a circuit breaker, is carried out in two or three cycles in the best case. We have here studied a simple, radial electric network configuration with a machine and an infinite network. The study covers simulations of a fault that can occur in a network and the consequences of the recovery time of the fault current limiter.

Summary: First, this paper introduces and describes conditions of stability, discussing the need for equilibrium between the consumed and produced power in the system. During a fault, the voltage drop causes a corresponding drop of the power transmitted to the network, and therefore the generators accelerate (greater mechanical power than electrical power) and acquire an increase of kinetic energy which is larger with longer fault duration. After the fault has been eliminated and if the power system is capable to suppress this excessive kinetic energy, it will find a stable state of operation.

A simulated power system of a generator supplying power to an infinite network via a transformer and two parallel transfer lines follows. The fault is applied midway across one of the overhead lines. The generator's mechanical behavior is described by the swing equation while its electrical part is given a seven-winding model in state space representation. The step-up transformer at the generator is assumed ideal with negligible inductances in primary and secondary windings for each phase. The $50 \mathrm{~km}$ overhead line 
models have self-inductance of $1.17 \mathrm{mH} / \mathrm{km}$ and mutual inductance between the phases of $224 \mathrm{uH} / \mathrm{km}$. Since the superconducting FCL's are resistive type, they are assumed mainly resistive for superconducting and normal operation. The FCL consists of a superconducting material in parallel with a bypass with constant resistance so that the superconductor transports the main part of the current in the superconducting state and so does the bypass when the superconductor enters the normal state. A temperature and current dependent relation is used to describe voltage-current characteristics for the superconductor.

Simulation results show the generator angle going to infinity without any FCL's in place, but with FCL's, a noticeable increase in the stability margin and a return to equilibrium of the internal generator angle exists. Problems exist with the recovery time of the superconductor (i.e. cooling time) being 3 seconds, which is too long for resetting and becoming available for additional protection. Superconducting FCL's are able to increase system stability due to their fast transition from low to high impedance. For a meshed system, a larger number of FCL's would be necessary, but more research must be done to coordinate these units and optimize their positions.

A.3.5.3.8 Setting method of specific parameters of a superconducting fault current limiter considering the operation of power system protection [155]

Abstract: We have developed an EMTP model of a resistance-type and a rectifier-type for super-conducting fault current limiter (SFCL) and defined their parameters which a user specifies when he plans to introduce an SFCL into a power system. Using these models, we propose the setting method of these parameters so as not to affect the response of protected relays when SFCL's operate.

Summary: This paper attempts to show the coordination necessary between SFCL's and protective relays.

First, EMTP models of resistance-type SFCL's and rectifier-type SFCL's are developed. For a resistance-type SFCL, the superconductor quenches immediately after the fault current exceeds the operation start current and changes its resistance.

A table is presented to clarify the different ways in which SFCL's can be attached to systems as well as the advantages of each setup. For example, SFCL's can be installed to combine two power systems, as part of trunk transmission lines, as protection for other superconducting equipment, or as part of feeders in distribution systems.

Given a model power system, parameters are specified for the SFCL. Current differential relays are used as primary protection, and distance relays serve as back-up protection at each end of the transmission lines. SFCL's are set to operate when a fault causes a current which is more than the breaking capacity, but SFCL's in sound phases or sound lines are not to operate. The operation start current is set to have an upper limit as the breaking capacity and a lower limit as the maximum current in the sound line when a fault occurs. For a resistance-type SFCL, resistance of the SFCL is set in accord with the 
mho relay and current differential relay. For a rectifier-type SFCL, estimation of the limiting coil inductance and relay coordination are also covered.

\section{A.3.5.3.9 A new model of fault current limiter using YBCO thin film [156]}

Abstract: Resistive fault current limiters which consist of an YBCO film and a normal conducting film were constructed. The YBCO film was connected by indium wires to the normal conducting film in parallel at intervals of about $10 \mathrm{~mm}$ to increase the allowed voltage across the fault current limiter during the current-limiting operation. Current limiting experiments show that an allowed voltage across the fault current limiter during current-limiting operation of over $13 \mathrm{~V} / \mathrm{cm}$ was achieved. Moreover, by combining in parallel 2 sets of 3 serially connected fault current limiters fabricated using $100 \mathrm{~mm} \times 10$ nun YBCO film, a rated power of over $20 \mathrm{kVA}$ is obtained.

Summary: The studies of FCL using high-Tc superconductors mainly focus on the magnetic shielding type using Bi-compound superconducting thick films and the resistive type using $\mathrm{Y} 1 \mathrm{Ba} 2 \mathrm{Cu} 307-\mathrm{x}$ (Y BCO) thin films. A magnetic shielding type FCL basically consists of a normal conducting coil, a superconducting cylinder, and an iron core. When a current in the normal conducting coil exceeds a certain level, the inductance increases as a result of entry of magnetic fluxes into the inner region of the superconducting cylinder, and the fault current is reduced. Recently, a test of this type of FCL with a rated power of over 1 MVA has been reported. However, this type of FCL is liable to be large and heavy because of the iron core. In comparison, although the rated power of resistive FCL's is smaller than that of the magnetic shielding type at the present time, no iron core is required and a fast recovery time is expected. Therefore, it seems appropriate that the resistive type FCL using YBCO thin films is more suitable for making practical FCL's.

To realize a practical FCL, the rated power of resistive FCL's must be increased. For this purpose, there are three points to consider:

Fabrication of large and high quality YBCO films, Improvement of the allowed voltage per unit length across an FCL during currentlimiting operation

Connection in series and parallel of FCL's made by one YBCO film

Improvement of the allowed voltage is very important, because the total length of the YBCO film to make an FCL is estimated as the rated voltage divided by the allowed voltage per unit length across the FCL. The joule heating increases with decreasing resistance of the FCL. Nevertheless, the resistance of an YBCO film is reduced by deposition of gold or silver on the YBCO strip to avoid local excessive heating at an early stage of the current-limiting operation (hot-spot formation).

To enlarge the rated power of a resistive FCL using an YBCO film, a new design of resistive FCL, which consists of an YBCO film and a normal conducting film, was proposed. The basic idea of this design is to divert excess current from the YBCO film to the normal conducting film. On the basis of this idea, a FCL was fabricated using an approximately 1-pm thick YBCO film on a 100-mm-long, 18-mm-wide LaAlO3 substrate 
and an approximately 200 -nm-thick gold film on a 100 -mm-long, 30 -mm-long AIN substrate. This FCL reduced an intended 910 A peak short circuit current to a 400 A peak current in a two-cycle load test. The voltage drop across the FCL was over $107 \mathrm{~V}$ peak, and an allowed voltage per unit length of over $13 \mathrm{~V}$ peak/cm was achieved.

Furthermore, the current-limiting properties of a combined FCL, by combining in parallel 2 sets of 3 serially connected FCL's, were measured. These FCL's were made using about 1-pm-thick YBCO film on a 100-mm-long, 10-mm-wide LaAlO3 substrate and about 400-nm-thick nickel film on a 100-mm-long, 30-mm-long AIN substrate. This combined FCL has a $100 \mathrm{~A}$ rms current carrying capacity, and an allowed voltage of over $200 \mathrm{~V}$ rms has been achieved in a two-cycle load test.

It is concluded that there is no serious problem with increasing the rated power of the FCL by series and parallel connections of FCL's.

A.3.5.3.10 Stabilization of synchronous generators by superconducting fault current limiter [157]

Abstract: This paper presents an analysis of the effects of superconducting fault current limiter on enhancement of generator transient stability and suppression of turbine shaft torque oscillations as well as limiting of fault current during short circuit faults. The analysis is performed in terms of instantaneous values using EMTP (ATP). It is concluded that the high resistance type fault current limiter is effective for the three purposes stated above.

The abstract sufficiently covers the material within this paper for the purposes of this project.

A.3.5.3.11 A nitrogen gas cooled, hybrid, high temperature superconducting fault current limiter [158]

Abstract: All concepts for fault current limiters employing high temperature superconductors (SCFL) have the disadvantage of significant AC losses caused by the superconductor carrying a continuous land current. Therefore, in the presented paper it is investigated what electrical and thermodynamic requirements have to be fulfilled by a superconductor if the fault current limiter is of a hybrid type. Assuming the availability of $\mathrm{n}$ suitable design for a superconductor used in a delayed reacting resistive limiting element in parallel with a fast acting load switch, the concept of a hybrid fault current limiter is introduced. The concept is based on a novel fast acting mechanical switch for several $\mathrm{kA}$ at medium voltage level to commutate the rising fault current to the superconducting element within several hundred microseconds after fault detection. It is found that a critical current density not higher than $1.5 \mathrm{kA}$ per centimeter square is adequate for the proposed system. The superconductor carries only the fault current during switching and thus the total losses of the hybrid system are only 4 percent of the losses of a conventional SCFL, which offers high economical benefits. Cooling of the 
superconductor by gaseous nitrogen is feasible, and presents simplicity and flexibility of operation.

Summary: By connecting a mechanical switch that carries the continuous current during normal operation in parallel to the superconducting (SC) element, a hybrid-type SFCL is proposed. In such a concept, the SC element operates only for a few milliseconds after the fault occurs; at normal operation it is permanently in no-load condition. The losses are only thermal conduction losses through the leads and radiation losses to the dewar.

\section{A.3.5.3.12 Superconducting current limiters [159]}

Summary: This paper introduces the need for SFCL's as well as the programs which are currently developing this product. ABB has a shielded iron core design, GA has a resistive/electronic one, and EA Technology has a resistive/inductive one. There is also a Siemens resistive thin film approach. All models apply HTS. The various geometric forms are tube, coil, rod, and thin film, respectively.

Table 4-Brief list of superconducting FCL programs

\begin{tabular}{|c|c|c|c|c|c|c|c|c|c|}
\hline Country & Organization & Type & $\begin{array}{c}\text { SC } \\
\text { Forms }\end{array}$ & $\begin{array}{l}\text { Nominal } \\
\text { Voltage } \\
(\mathbf{k V})\end{array}$ & $\begin{array}{l}\text { Current } \\
\text { (A rms) }\end{array}$ & $\begin{array}{c}\text { Fault Current } \\
\text { Before/After } \\
\text { (A rms) }\end{array}$ & $\begin{array}{l}\text { Power } \\
\text { Rating } \\
\text { (MVA) }\end{array}$ & $\begin{array}{c}\text { Year } \\
\text { Achieved }\end{array}$ & Status \\
\hline Switzerland & ABB & $\begin{array}{l}\text { Shielded } \\
\text { iron core }\end{array}$ & \begin{tabular}{|l} 
Bi-2212 \\
rings
\end{tabular} & 0.48 & 130 & & 0.1 & 1993 & Completed \\
\hline Switzerland & ABB & $\begin{array}{l}\text { Shielded } \\
\text { iron core }\end{array}$ & $\begin{array}{l}\mathrm{Bi} \cdot 2212 \\
\text { rings }\end{array}$ & 10.5 & 70 & $60,000 / 700 / 250$ & 1.2 & 1997 & Completed \\
\hline Switzerland & ABB & $\begin{array}{l}\text { Shielded } \\
\text { iron core }\end{array}$ & $\begin{array}{l}\text { Bi-2212 } \\
\text { rings }\end{array}$ & 8.3 & 200 & $13,200 / 4,300 / 1,400$ & 1.6 & 1999 & Completed \\
\hline U.S.A. & GA & $\begin{array}{l}\text { Inductive/ } \\
\text { electronic }\end{array}$ & $\begin{array}{l}\text { Bi-2223 } \\
\text { Ag tape }\end{array}$ & 2.4 & 80 & $3,000 / 1,600 / 1,233$ & 7.2 & 1995 & Completed \\
\hline U.S.A. & GA & $\begin{array}{l}\text { Inductive/ } \\
\text { electronic }\end{array}$ & $\begin{array}{l}\mathrm{Bi}-2223 \\
\mathrm{Ag} \text { tape }\end{array}$ & 12.5 & 1,200 & $20,000 / 9,000 / 4,000$ & 25 & 1999 & $\begin{array}{l}\text { Continual } \\
\text { Testing }\end{array}$ \\
\hline UK & EA Technology & $\begin{array}{l}\text { Resistive/ } \\
\text { inductive }\end{array}$ & $\begin{array}{l}\mathrm{Bi}-2212 \\
\text { rods }\end{array}$ & 11.0 & 400 & $37,000 / 12,000$ & 7.5 & 1999 & Completed \\
\hline $\begin{array}{l}\text { Germany, } \\
\text { Canada }\end{array}$ & \begin{tabular}{|l} 
Siemens, \\
Hydro-Quebec
\end{tabular} & $\begin{array}{l}\text { Resistive thin } \\
\text { film }\end{array}$ & YBCO & 0.77 & 135 & $666 / 108$ & 0.1 & 1999 & Completed \\
\hline $\begin{array}{l}\text { Germany, } \\
\text { Canada }\end{array}$ & $\begin{array}{l}\text { Siemens, } \\
\text { Hydro-Quebec }\end{array}$ & $\begin{array}{l}\text { Resistive thin } \\
\text { film }\end{array}$ & YBCO & & & & 1.0 & & $\begin{array}{l}\text { Being } \\
\text { Planned }\end{array}$ \\
\hline Japan & Toshiba, TEPCO & $\begin{array}{l}\text { Resistive } \mathrm{Cu} \\
\text { wire }\end{array}$ & LTS NbTI & 6.6 & 2,000 & & & 1998 & Completed \\
\hline $\begin{array}{l}\text { France } \\
\text { EDF }\end{array}$ & GEC & Thin film & LTS NbTI & 36 & 210 & & & 1995 & Completed \\
\hline
\end{tabular}

A.3.5.3.13 Stability analysis of a power system with superconducting fault current limiter installed [160]

Abstract: As a process of developing high temperature superconducting fault current limiter (SFCL), the stability of a power system in which SFCL's were installed was analyzed. For the investigation into the effect of SFCL's to a power system, we have proposed a simple model power system that had SFCL circuits. The modeling parameters of SFCL are obtained by experiment of a prototype SFCL, which are $440 \mathrm{~V}$ class and a shielding type model. This electric circuit was solved for transient 
performance by numerical methods. In case the SFCL's are installed in a power system, it can effectively protect synchronization both in a symmetrical three-phase fault and a single-phase line to ground fault by maintaining synchronism of the synchronous machines for a long time. By this analysis, we found a quantitative effect of SFCL's to a power system. Limiting fault currents means not only an improvement of circuit breaker abilities but also a protection of synchronism. So its synchronism protection property must be considered for a design of superconducting fault current limiters.

Summary: This paper begins by modeling the elements of a simple power system in a transient state analysis. The synchronous generator is 2-pole, $220 \mathrm{~V}, 3$-phase, Yconnected, $60 \mathrm{~Hz}$, and rated at $3 \mathrm{MW}$. Relationships between the rotor angle, angle velocity, electrical torque, mechanical torque, moment of inertia, and flux are then developed. The model power system has a generator, three single-phase SFCL's, and three load resistors of 72 ohms. As for the SFCL's, under normal conditions, the currents induced in the superconducting tubes result in a magnetic flux cancellation (with no voltage drop in the terminals), but under a fault condition, the current in the superconducting tube exceeds the critical current so that the magnetic flux in the core abruptly increases due to the superconductor presenting a flux-flow resistance (causing a voltage drop).

Simulations were completed for a three-phase fault (worst-case scenario) and single-lineto-ground faults. For the three-phase fault without SFCL's, the PD controller could not overcome the rapid change in mechanical torque (resulting from the increase in electrical torque), and the machine went out of order within 3 seconds. The fault current increased to 3 times the normal current. With SFCL's in place, the synchronous angular velocity only had 3\% breakaway from synchronism (was $7 \%$ before), and the mechanical torque was able to settle at a value within 3 seconds that was $60 \%$ of the value of the previous result.

The SFCL improved the stability of a power system by restraining the change of angular velocity. For a SLG fault, the variation of the steady-state point was very small, and the SFCL's could more effectively protect synchronism of the power system. A comparison of degrees of synchronism of each scenario was made via observing that SFCL's effectively prevent load angles from breaking away.

A.3.5.3.14 Application studies of superconducting fault current limiters in electric power systems [161]

Abstract: In power systems, superconducting fault current limiters (SFCL's) can limit the prospective short circuit currents to lower levels, so that the underrated switchgears can be operated safely. This paper presents a detailed theoretical analysis of improving power system stability by using of SFCL's. Electromagnetic Transient Program (EMTP) simulation results based on a model system also show that SFCL's are effective for enhancing the transient stability of electric power systems by restraining the generator rotors from accelerating after faults and improving power quality. This paper outlines the basic application guidelines for using superconducting fault current limiters in different 
voltage level power systems. In order to insure the safe and proper usage of SFCL's, some future considerations on the interaction between SFCL's and power systems are presented as well.

Summary: Traditionally, the most common ways to limit high-level fault currents are uprating of switchgear and other equipment, splitting the power grid and introducing higher voltage connections (AC or DC), using current-limiting fuses or series reactors or high-impedance transformers, and using strategies like sequential network tripping. However, these methods create problems such as loss of power system safety and reliability, high cost, and increasing power losses. This paper shows that by using an SFCL which is capable of consuming the excessive accelerating generator power, the stability limit of the system can be increased; and the stability region after the fault can be enlarged.

A review of short circuit currents explains that an asymmetrical fault current is composed of the symmetrical short circuit and the exponentially decaying dc offset. The maximum asymmetrical current appears within the first half-cycle if the fault occurs at the zero voltage crossing point, so the onset of SFCL's must be available to limit the max fault currents within milliseconds.

It is shown that the power angle characteristic of a generator can be expressed by the electric potentials and the relative angles of other generators.

The power system model consists of a generator supplying power to a network via transformers and two parallel transmission lines. A three-phase fault is applied to one of the lines. Power characteristics are determined for the generator with and without consideration of the SFCL. By setting the derivative of power with respect to rotor angle equal to zero, the power limit of the generator is found for the 2 cases. Without a SFCL, the electrical power from the generator to the system drops to zero, and the driving mechanical power becomes much greater than the opposing electrical power; thus, the rotor begins to accelerate and may lose synchronism. With a SFCL, an extra resistance is in place to increase the electrical power and consume the kinetic accelerating power. The decelerating torque will force the generator rotor back toward the equilibrium point or reach an acceptable steady-state operating point. The power swing is damped, and the generator shaft torque oscillations are suppressed. Essentially, the power characteristic moves upward and the power angle shifts left so that the power limit is increased and the stability region is enlarged (better transient stability).

Another finding is that SFCL's, especially when placed at bus-ties, can give lower voltage dips, which helps to improve power quality (aids with reliability of supply).

Additionally, suggestions are given for selecting different types of SFCL's. For high voltage power systems, reactance-type SFCL's should have carefully selected inductances that cooperate with the current protective devices. These units have reactances that are magnetically coupled to the network to limit fault current. Resistive- 
type SFCL's can restrain the fault current and consume electrical power, as mentioned above.

Benefits of SFCL's placed at strategic points include the following: separate buses can be tied together without a large increase in the fault duty on either bus, a large transformer can be used to meet increasing demands on a bus without circuit breaker upgrades, a low impedance transformer can be used to maintain voltage regulation at the new power level, and $\mathrm{I} 2 \mathrm{t}$ damage to the transformer is limited.

Concluding remarks indicate the need to consider other factors before employing SFCL's: coordination between SFCL's and protective devices, recovery characteristics and open-close-open duty, inrush currents of large transformers, and operation with other FACTS devices.

A.3.5.3.15 Analysis on the impact of HTS cables and fault current limiters on power systems [162]

Abstract: Power system studies on potential applications of superconducting cables and fault-current limiters and their impact on utility networks were performed by digital models developed in ATP (Alternative Transients Program). Simulation results from lightning overvoltage and fault analyses on $132 \mathrm{kV}$ cable and 3-phase faults on $20 \mathrm{kV}$ current limiter are reported.

Summary: Since the impact of high temperature superconductors (HTS) cables is not a primary concern, skip ahead to the FCL discussion.

Simulations are carried out to reproduce two different lightning phenomena:

Back flashover: lightning strikes the top of the tower, generating an overvoltage that causes a discharge across the insulating string

Shielding failure: lightning directly strikes the conductor avoiding the shielding offered by the ground wire

Positive polarity lightning flashes exhibit only one impulse current while negative polarity ones, which are more likely, can have multiple impulse currents.

Explanation of the operating principles of resistive SFCL's is followed by a discussion of the effects. By increasing the resistive component of the circuit impedance seen by circuit breakers, the power factor gets higher values; this means smaller phase angles between voltage and current and hence an easier and safer operation of circuit breakers, with a reduction of transient overvoltages (TRV). SFCL requirements center upon resistance level since too low of resistance values could not limit the fault current below acceptable values while too high of resistance values could not allow the opening of the circuit breaker during fault conditions. The SFCL operation needs to be coordinated with the operation of the circuit breakers (current interruption and subsequent re-closing) and at re-closing, once the fault is cleared, the resistance of the SFCL should be back to zero. 
For the simulation, the critical current value is set at least greater than $1.5 \mathrm{pu}$ of the network nominal current to prevent false operation. The SFCL is placed in 2 different configurations: single feeder and two feeders in bus-tie connection. A 3-phase fault is applied since it would result in higher currents than would other disturbances. The adoption of a properly designed SFCL in a configuration similar to the one in this paper leads to the advantage of a more meshed network during normal operation and a fast separation of the faulted feeder from the rest of the network during short circuit conditions.

A.3.5.3.16 Reduction of rating required for circuit breakers by employing seriesconnected fault current limiters [163]

Abstract: Calculation was made to examine the reduction of the rating required for circuit breakers by using series-connected resistive and inductive fault current limiters (FCL's) under various fault locations. In the model of the inductive FCL, a capacitance $\mathrm{Cp}$ consisting of a coil stray capacitance and a necessary additional capacitance was taken into account. The insertion of the resistive FCL and the inductive FCL with $\mathrm{Cp}=100 \mathrm{nF}$ into the power system proved to decrease the severity of the interrupting duty so that a lower-rated circuit breaker can be used. However, the introduction of the inductive FCL with $\mathrm{Cp}=10 \mathrm{nF}$ was found to lessen the fault current, while raising the rate of rise of the recovery voltage. From the variation of the breaking condition, the limiting impedance required to allow a circuit breaker to perform a satisfactory interruption is discussed.

Summary: The paper begins by discussing effects of FCL's on power systems such as reduction of voltage sags, stability improvement, and reduction in peak electrical and mechanical torque of a generator. By reducing the current level, FCL's are believed to ease the interrupting burden on circuit breakers, which depends on the interrupting current and the transient recovery overvoltage appearing across the contacts of a circuit breaker. For this paper, a fault occurring at a distance close to the terminals of the FCL is discussed first since it produces the maximum fault current that a circuit breaker must interrupt. Also, in order to assess the TRV, a fault further away from the FCL is observed.

The results of the study indicate that in either of the fault locations, the insertion of the resistive FCL reduces not only the current but also the rate of rise of recovery voltage. On the other hand, whereas the presence of an inductive FCL lessens the current, the behavior of the rrrv depends mainly on the magnitude of the capacitance $\mathrm{Cp}$. The insertion of a resistive FCL and an inductive FCL is shown to reduce the severity of the interrupting duty of the circuit breaker so that a circuit breaker with a lower interrupting performance can be used.

A.3.5.3.17 The influence of superconducting fault current limiter structure on the V-I characteristic [164] 
Abstract: Superconducting Fault Current Limiters use the natural ability of rapid shifting from the normal to the resistive state of superconductor due to their critical current value exciding. This special feature of superconducting materials enables designing of electrical devices with parameters that cannot be achieved by using conventional materials. The voltage dependence on the structure of superconducting inductive fault current limiter in a superconducting state has been described in this paper. This dependence has enabled elaboration of computer software for parameters optimizations of SFCL primary winding. This dependence in a resistive state is also described. The analysis was carried out using FLUX2D software based on a finite element method.

Summary: This paper begins with a discussion of how SFCL's operate. During normal operation, the resistance of the superconducting secondary winding is equal to zero, and the magnetic flux does not penetrate the iron core because the superconducting tube acts as a magnetic screen. When the fault occurs, the increasing current exceeds the critical value of the HTS element, then the resistance of the secondary winding is reflected into the circuit, and the magnetic flux penetrates the iron core increasing the impedance of the limiter. The voltage on the limiter depends mainly on the structure of the limiter, its geometrical dimensions, the number of the primary winding turns, and the distance between the windings and the size of the core.

This paper goes into great detail with regard to the structure of a FCL affecting its performance, but we will not be designing a FCL, so the results are not significant for this project.

\section{A.3.5.3.18 Magnetic-shield type fault current limiter equivalent circuit [165]}

Abstract: In order to investigate how a superconducting fault current limiter (SFCL) can enhance the performance of a power system, an accurate circuit model of the device needs to be introduced in power system simulators. In this paper, we present a finiteelement numerical model to calculate the time evolution of the voltage across a magneticshield-type SFCL, when it is connected to an external circuit. The calculation of the voltage is carried out by using the energy conservation law, and requires the calculation, at any instant, of the current density distribution inside the superconducting tube and magnetization distribution inside the ferromagnetic core of the device. These distributions are determined by means of two coupled equivalent electric and magnetic circuits, whose topology and components are obtained through the spatial integration of quasi-static form of Maxwell equations. Comparisons between numerical and experimental results are shown.

Summary: A general model for the performance of a magnetic shield-type SFCL is proposed. The complex model as well as the simplified version indicates similar results of performance enhancements of a power system with a SFCL in place. The model is based on an equivalent circuit approach, where the circuit components, characteristics, and topology are obtained through the integration of quasi-static form of Maxwell equations. 
A.3.5.3.19 Experiment and research on control strategy for a new-type three-phase solidstate current limiter [166]

Abstract: According to many Chinese patents, a new-type three-phase solid-state current limiter topology and its characteristics together with the principle are presented here to satisfy with the actual requirements of the three phase electric power system. In order to switch off the transmission line as soon as possible, there me there modes of control strategy presented here. The fixed time made is also developed to meet certain case, like delaying time protection. The corresponding control function is given, too.

Experimental results illustrate that the topology and control method for this new-type three-phase solid-state current limiter is effective, and agree well with the design, and have a good future in the industrial application as well.

Summary: This paper presents a practical and economical topology for a three-phase solid-state current limiter. A single-phase bridge-type current limiter is first discussed, and to get three phases, a current limiter with twelve thyristors and three reactors would be needed. The three-phase topology instead uses eight thyristors and a single limiting reactor.

The rest of the paper discusses how the current limiter operates in 3 phases via a coupling transformer and a control circuit. Also, this FCL does not affect the whole system in normal operation.

There are three control modes to switch off the fault line. One mode is inverting control where the rectifier is on inverting mode to send the energy in the reactor back to the power network when detecting the fault. Another mode is the freewheeling mode, and the final mode is a combination of the previous two (hybrid mode). Hybrid mode is more practical and reliable. The effects of limiting the current in the different modes are presented graphically.

Part of the paper discusses how to model the transfer function of the FCL, so that the control is better understood.

This technology is an improvement upon the traditional FCL.

A.3.5.3.20 Proof-of-concept prototype test results of a superconducting fault current limiter for transmission-level applications [167]

Abstract: SuperPower, Inc. and Nexans Superconductors GmbH have partnered to develop a superconducting fault current limiter for transmission level applications. The device employs SuperPower's Matrix Fault Current Limiter (MFCL) technology and BSCCO-2212 bulk material manufactured by Nexans' melt cast processing technique. The development program has been underway since June 2002 and is divided into a series of prototype demonstrations of progressive capability, culminating in a Beta prototype that will be installed at a host utility on a $138 \mathrm{kV}$ line. This paper describes the test results of the first major program milestone, a proof-of-concept prototype at 
distribution level voltage. The test results show the expected current limiting performance of the MFCL technology to provide fast current limiting and dynamic resistance development before the first peak of the fault. The fault current evenly distributes among the elements of the MFCL array, which employs a resistive-inductive approach for limiting the current. The results also show the excellent electric field performance of the BSCCO-2212 material.

Summary: The MFCL employs modular features that enable the scale-up to transmission voltage levels of $138 \mathrm{kV}$. It also allows for redundancy in the system so that reliability improves should any individual component fail.

Each current-limiting matrix element includes a parallel electrical arrangement of a superconductor R and an inductor L. A most important feature of the MFCL design is the passive way a magnetic field is generated to "trigger" the quenching of the superconducting elements in the current-limiting matrix.

The objective of the proof-of-concept was to demonstrate that the device reacts rapidly at the onset of the fault and starts to limit the increase in short circuit current immediately. In an actual power system, this reduces the first peak of prospective fault current to reduce stress on power system components. The reduction in fault current by the third cycle allows the conventional circuit breakers in the system to safely interrupt at a reduced current level.

Test results for the system are displayed in Table 5, Table 6, and Table 7.

Table 5-Pre-prototype design requirements

\begin{tabular}{lc}
\hline \multicolumn{1}{c}{ Property } & Magnitude \\
\hline Line-to-line voltage & $15 \mathrm{kV}$ \\
Phase-to-ground voltage & $8.66 \mathrm{kV}$ \\
Load Current & $800 \mathrm{Arms}$ \\
Overcurrent allowance & $20 \%$ \\
Prospective Fault Current (Symmetrical) & $10 \mathrm{kA}$ \\
Limited Fault Current (Symmetrical) & $8 \mathrm{kA}$ \\
Prospective Fault Current (Asymmetrical) & $25 \mathrm{kA}$ \\
Fault duration & $3 \mathrm{cycles}$ \\
Cryostat Operating Temp. Range & $74 \mathrm{~K}$ to $77 \mathrm{~K}$ \\
\hline
\end{tabular}

Table 6-Summary of applied faults 


\begin{tabular}{lcccc}
\hline $\begin{array}{c}\text { Input } \\
\text { Voltage } \\
\text { (Vrms) }\end{array}$ & $\begin{array}{c}\text { Fault } \\
\text { Duration } \\
\text { (Cycles) }\end{array}$ & $\begin{array}{c}\text { Asymmetric } \\
\text { First Peak } \\
\text { (kA) }\end{array}$ & Cryostat Temp & $\begin{array}{c}\text { Faults } \\
\text { Applied }\end{array}$ \\
\hline 2400 & .5 to 3 & 17 to 27 & $77 \mathrm{~K}$ Open Bath & 9 \\
4160 & .5 to 3 & 19 to 23 & $77 \mathrm{~K}$ & 6 \\
4160 & 3 & 17 to 25 & $74 \mathrm{~K}$ & 3 \\
8660 & .5 to 3 & 19 to 23 & $77 \mathrm{~K}$ & 5 \\
8660 & 3 & 17 to 25 & $74 \mathrm{~K}$ & 3 \\
\hline
\end{tabular}

Table 7-Summary of current limiting performance

\begin{tabular}{cccc}
\hline $\begin{array}{c}\text { Input } \\
\text { Voltage }\end{array}$ & $\begin{array}{c}\text { First Peak } \\
\text { Current (kA) }\end{array}$ & $\begin{array}{c}\text { Avg. First Peak } \\
\text { Limiting }\end{array}$ & $\begin{array}{c}\text { Limiting at 3 } \\
\text { Cycle }\end{array}$ \\
\hline 2400 & 23.6 & $73.4 \%$ & $44.4 \%$ \\
2400 & 27.2 & $68.6 \%$ & $39.2 \%$ \\
4160 & 23.4 & $82.6 \%$ & $52.3 \%$ \\
8660 & 25.6 & $83.7 \%$ & $55.9 \%$ \\
\hline
\end{tabular}

Current waveforms are provided in Figure A.21 and Figure A.22.

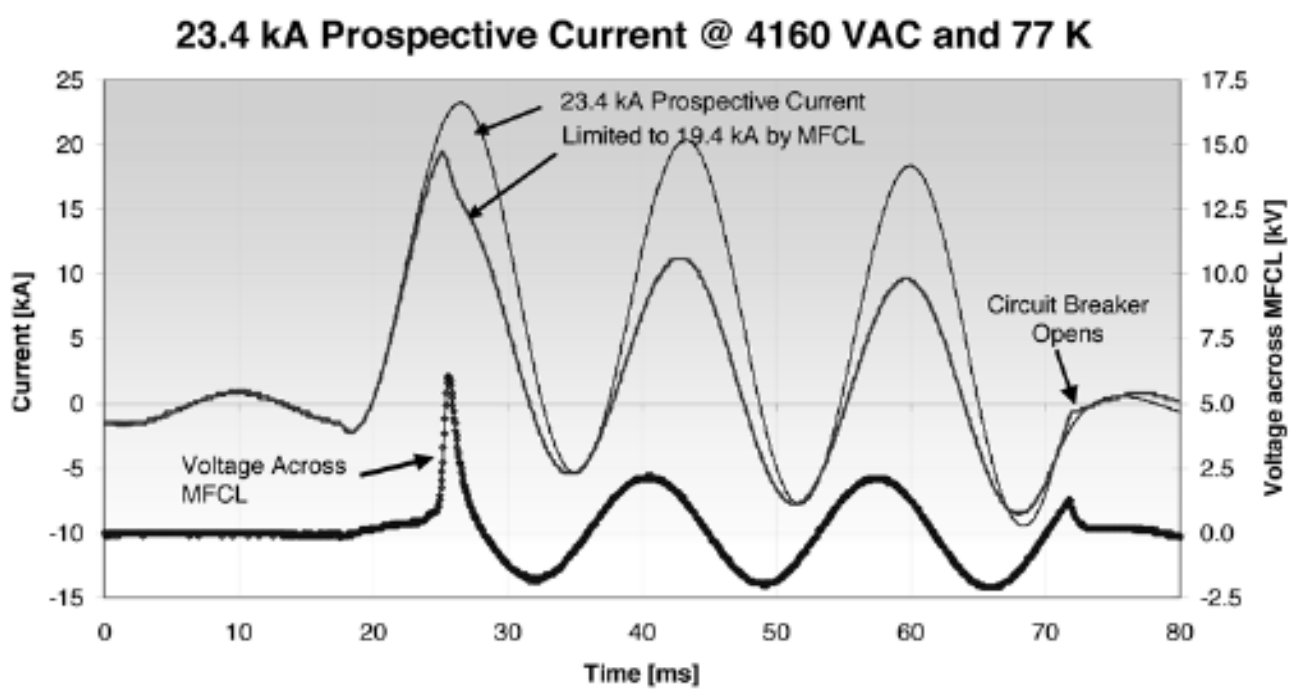

Figure A.21-MFCL achieves $83 \%$ first peak limiting at 4160 VAC 
25.6 kA Prospective Current @ 8660 VAC and 74 K

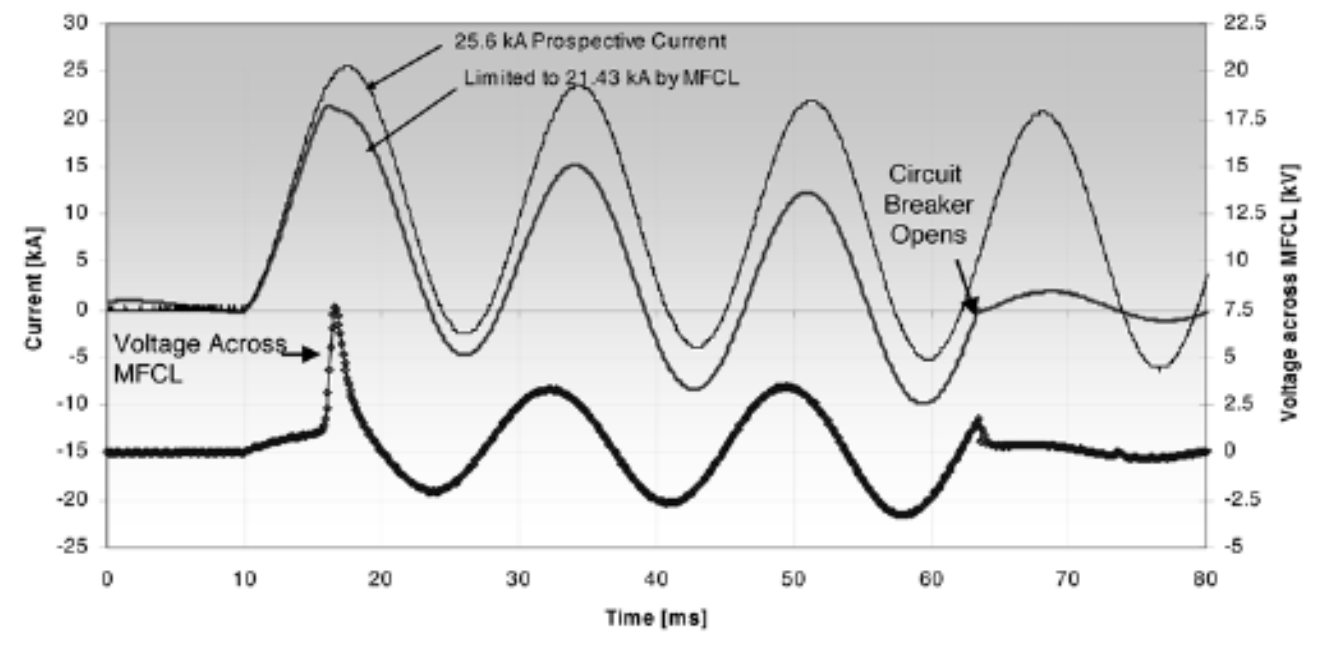

Figure A.22-MFCL achieves $84 \%$ first peak limiting at 8660 VAC

Since this design proved successful, the actual prototype is now being built.

A.3.5.3.21 Non-inductive variable reactor design and computer simulation of rectifiertype superconducting fault current limiter [168]

Abstract: A rectifier type superconducting fault current limiter with non-inductive reactor has been proposed by the authors. The concept behind this SFCL is that the high impedance generated during superconducting to normal state of the trigger coil limits the fault current. In the hybrid bridge circuit of the SFCL, two superconducting coils: a trigger coil and a limiting coil are connected in anti-parallel. Both the coils are magnetically coupled with each other and could have the same value of self inductance so that they can share the line current equally. At fault time when the trigger coil current reaches a certain level, the trigger coil changes from superconducting state to normal state. This super to normal transition of the trigger coil changes the current ratio of the coils and therefore the flux inside the reactor is no longer zero. So, the equivalent impedance of both the coils is increased and limits the fault current. We have carried out computer simulation using PSCAD/EMTDC and observed the results. Both the simulation and preliminary experiment shows good results. The advantage of using a hybrid bridge circuit is that the SFCL can also be used as a circuit breaker.

Summary: Basic coil design for a $6.6 \mathrm{kV} / 1 \mathrm{kA}$ rated non-inductive reactor was carried out. Two configurations of the non-inductive reactor for rectifier-type SFCL were proposed. Coaxial coil arrangements and bifilar winding arrangements were compared. The bifilar winding arrangement was superior and had a high impedance ratio (normal operating mode and current limiting mode), but the coaxial arrangement was selected in the point of the insulation. 
A.3.5.3.22 Applications of superconducting fault current limiters in electric power transmission systems [169]

Abstract: The introduction of new generating facilities by independent power producers and increasing load demand can result in fault-current over-duty on existing transmission system protective equipment. Conventional solutions to fault current over-duty such as major substation upgrades, splitting existing substation busses or multiple circuit breaker upgrades could be very expensive and require undesirable extended outages and result in lower power system reliability. Less expensive solutions such as current limiting reactors may have unwanted side effects, such as increase in system losses, voltage regulation problems or possibly could compromise system stability. This paper discusses the benefits of superconducting Fault Current Limiters (FCL's) which can be economically competitive with expensive conventional solutions. Superconducting FCL's are invisible in normal operation and do not introduce unwanted side effects. The performance of a particular type of limiter, the Matrix Fault Current Limiter (MFCL) is presented and examples are provided on how it could relieve fault current over-duty problems. The use of this device in a particular application in the American Electric Power (AEP) $138 \mathrm{kV}$ transmission grid is also discussed.

Summary: The matrix fault current limiter is targeted to address fault current over-duty problems at the transmission voltage level of $138 \mathrm{kV}$ and higher. Conventional solutions to this problem are first expressed in this report:

Construction of new substations

Bus splitting

Multiple circuit breaker upgrades

Current limiting reactors

High impedance transformers

Sequential breaker tripping.

Some of these solutions are costly, may fail to supply loads during normal conditions, introduce voltage drops and power losses, add unnecessary delays, and affect zones that were not originally impacted by the fault.

A pre-prototype MFCL device has successfully demonstrated current limiting [153]. The device achieved the ratio of first peak to prospective current as $84 \%$, and this number fell to $56 \%$ by the third cycle. This fast response and significant limiting by the third cycle (when a breaker would typically open) are as desired.

MFCL is expected to be cost competitive with all of the solutions except for current limiting reactors and sequential breaker tripping, which have additional problems with reliability and life-cycle costing.

While MFCL has been demonstrated for medium voltage systems, it has yet to prove its worth when scaled-up to transmission level voltages. The impact on protection schemes and the length of the recovery period must be taken into account before attempting this addition. 


\section{A.3.5.3.23 Fault current limiters report on the activities of CIGRE WG A3.16 [170]}

Abstract: The paper presents an overall view on the activities of the CIGRE Working Group (WG) A3.16 which focuses on the interaction between different fault current limiting technologies and protection systems in both MV and HV systems. Beside state of the art of fault current limiters (FCL) and a general classification of different types of FCL's, the paper presents the results of a survey which shows an increased need for short circuit current limitation in the voltage level of 110 to $145 \mathrm{kV}$. The limiting behaviors as well as potential applications of fault current limiters are described and some basic ideas presented showing the influence of FCL devices on protection schemes. The paper concludes with a report of a recent field test of a fault current limiter installed in a distribution network in Germany.

Summary: This paper begins by addressing the need for FCL's to lower currents so that switchgear can be sized smaller. Only fault current limiters for medium and high voltage systems are considered since FCL's are not used in low voltage networks. Passive FCL's make use of an already initially high source impedance both at normal and at fault conditions whereas active FCL's bring about a fast increase of the source impedance at fault conditions only. Suggestions are made for alternatives to FCL's, but these have been covered in other papers.

A 2003 survey shows an increasing need for short circuit current limitation especially in $\mathrm{HV}$ level of 110-145 kV.

The terms associated with a FCL before, during, and after the fault, such as recovery time, action time, fault duration, peak value of follow current, etc., are then defined.

Next, the paper suggests selecting FCL's that comply with existing protective elements. Criteria to consider are measuring, pick-up (distinguishing between load and fault current), processing (decision to trip $\mathrm{CB}$ ), and coordination. A figure is also provided to suggest possible locations of FCL's in a power system.

Feasibility: Much of the rest of the paper discusses the resistive SFCL that was installed in the $10 \mathrm{kV}$ systems in Germany in 2004. In the laboratory, the SFCL limited fault current levels to $7.2 \mathrm{kA}$ from $18 \mathrm{kA}$, better than the expected result of $8.7 \mathrm{kA}$. In the field, though, this limiter has yet to encounter a short circuit condition to merit its use. Once it does successfully operate in the field, the device will be removed from the power system and placed under further laboratory testing.

The concluding remark is that with deregulation of transmission and generation, there is a higher likelihood of short circuits; therefore, the need for FCL's exists, but the technology simply is not there yet. 
A.3.5.3.24 EMTP RV - based study of solid-state fault current limiter for distribution systems [171]

Abstract: A Fault Current Limiter (FCL) can reduce the fault current in a feeder system to a desired level and permit continuous power to be fed to loads. An EMTP RV based study to limit fault currents in a distribution system using a Solid-State Fault Current Limiter (SSFCL) is presented in this paper. For demonstration purposes, two types of distribution systems have been considered: a single-source radial system and a multiple source distribution system with a bus-tie. Simulations have been done for different types of faults to show that the SSFCL is effective for limiting fault currents.

Summary: With the development of high capacity (in respect of voltage and current) fast-acting, multi-operation GTO thyristor switches, the application of a solid-state fault current limiter in the distribution systems is becoming attractive for distribution networks. A full-wave bridge rectifier is the most common type of solid-state fault current limiter (SSFCL). For this study, the fault current limiting system is implemented by inserting a SSFCL downstream to the transformer of the single-source radial system and bus-tie in a multiple-source distribution system. Since GTO thyristors are rated at roughly $9 \mathrm{kV}$, a step-down series-connected transformer is used to lower the bridge-side operating voltage, thus allowing a single GTO thyristor to be used to form a valve. This reduces the capital and operating costs but introduces an added cost for the transformer.

This study uses 3 single-phase limiters as opposed to a three-phase limiter so that singlephase faults will not affect each phase.

In a power system, the most common faults are the asymmetric faults. A single-phase-toground fault occurs most frequently followed by the 2-phase fault. Results for these fault types are provided in Figure A.24, Figure A.25, Figure A.26, and Figure A.27 below.

Note that the fault current is quickly limited (within $5 \mathrm{~ms}$ ) for both the radial network and the nested system. This means a lower capacity circuit breaker can be used at the bus-tie location. Overall, these are promising results. 


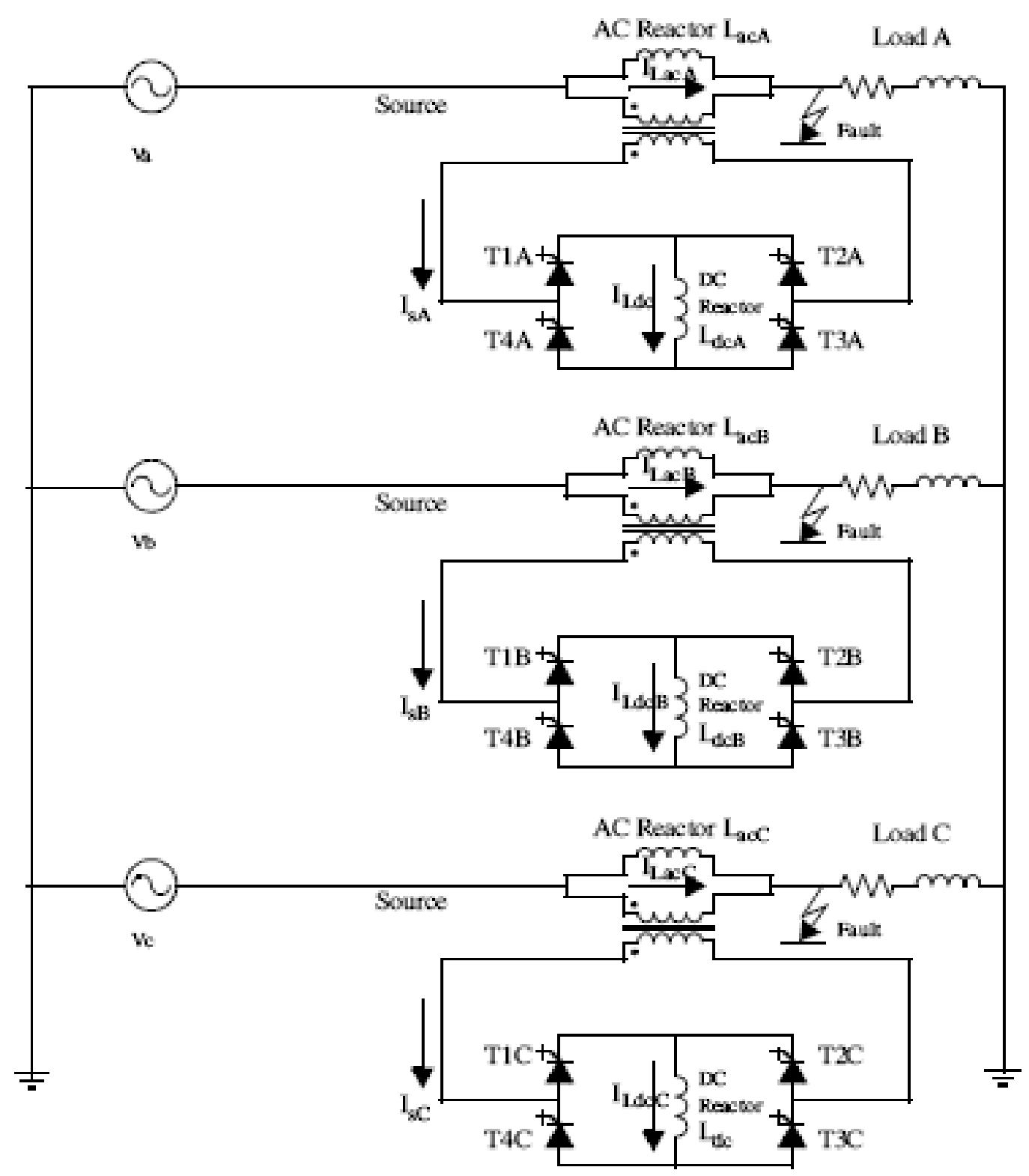

Figure A.23—Proposed 3-phase bridge-type SSFCL

In a power system, the most common faults are the asymmetric faults. A single-phase-toground fault occurs most frequently followed by the 2-phase fault. Results for these fault types are provided in Figure A.24, Figure A.25, Figure A.26, and Figure A.27 below.

Note that the fault current is quickly limited (within $5 \mathrm{~ms}$ ) for both the radial network and the nested system. This means a lower capacity circuit breaker can be used at the bus-tie location. Overall, these are promising results. 

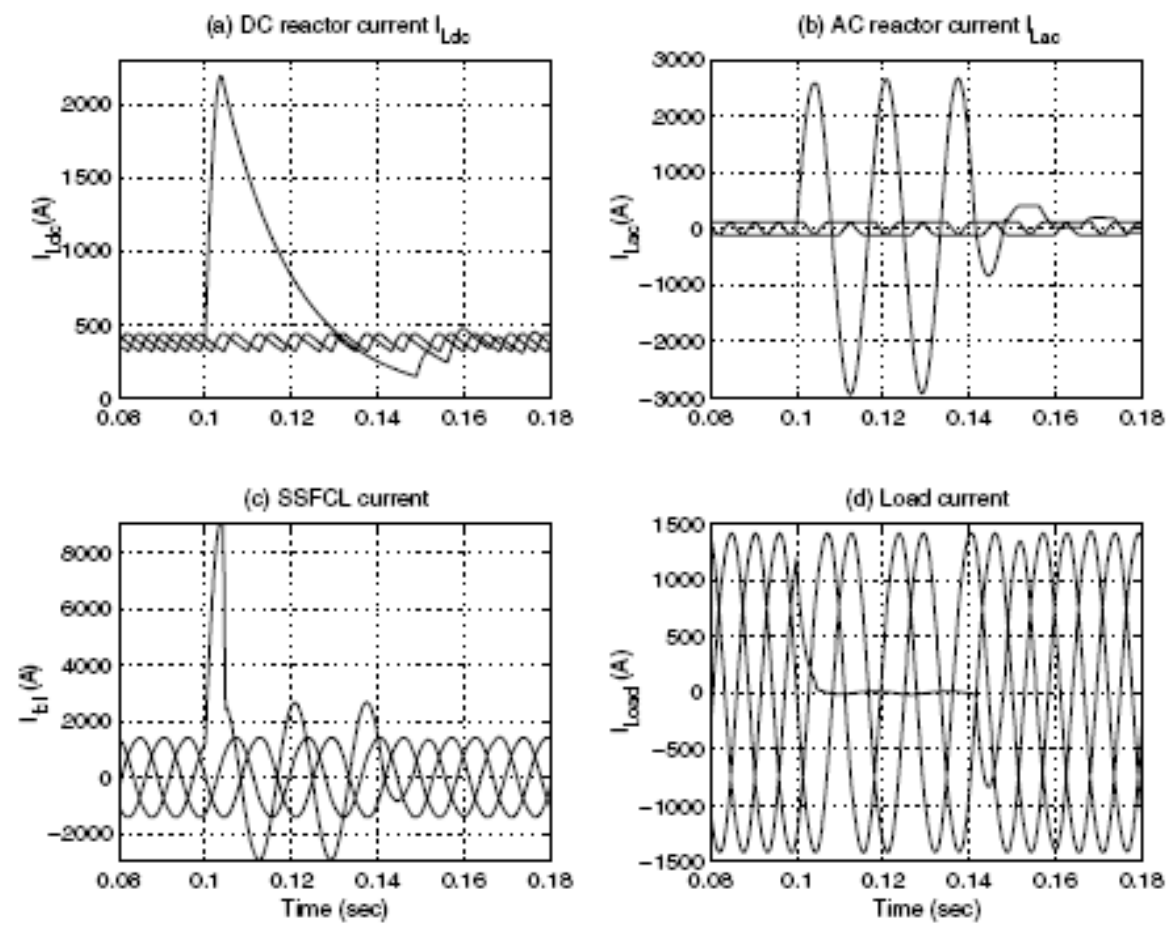

Figure A.24-SSFCL in $11 \mathrm{kV}$ radial line with single source (1-phase-to-ground)
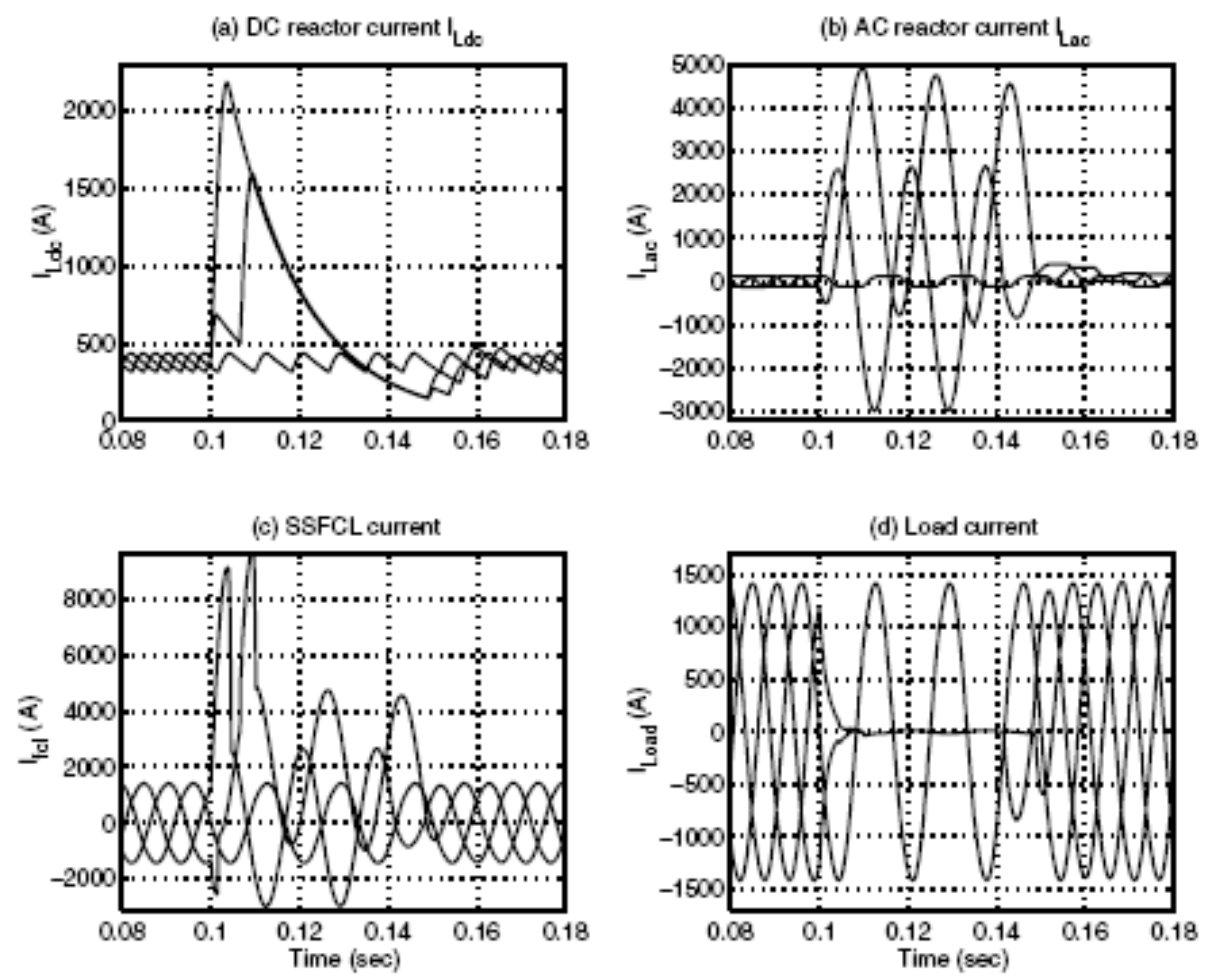

Figure A.25-SSFCL in a $11 \mathrm{kV}$ radial line (2-phase-to-ground fault) 

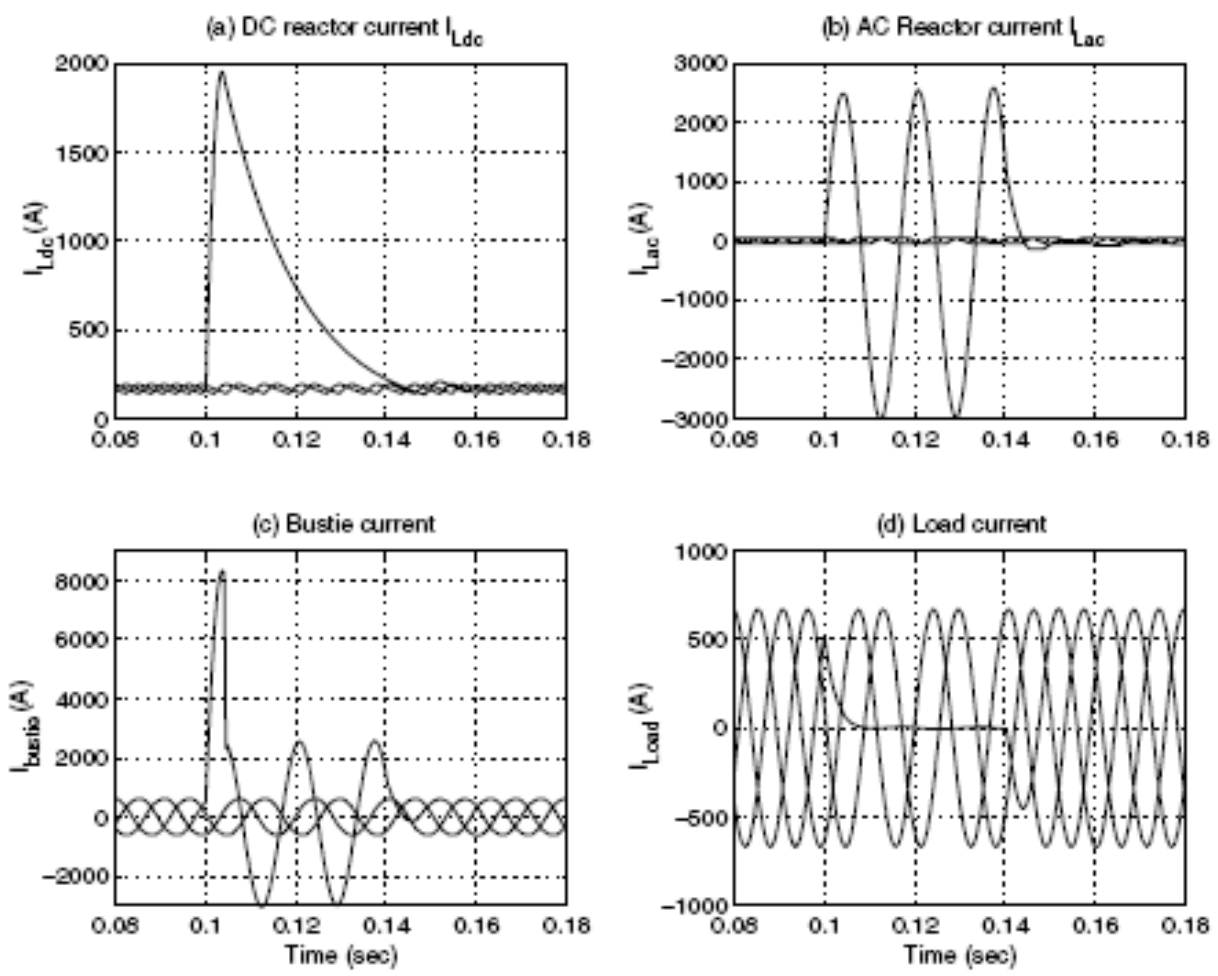

Figure A.26-SSFCL in $11 \mathrm{kV}$ bus-tie with single source (1-phase-to-ground)
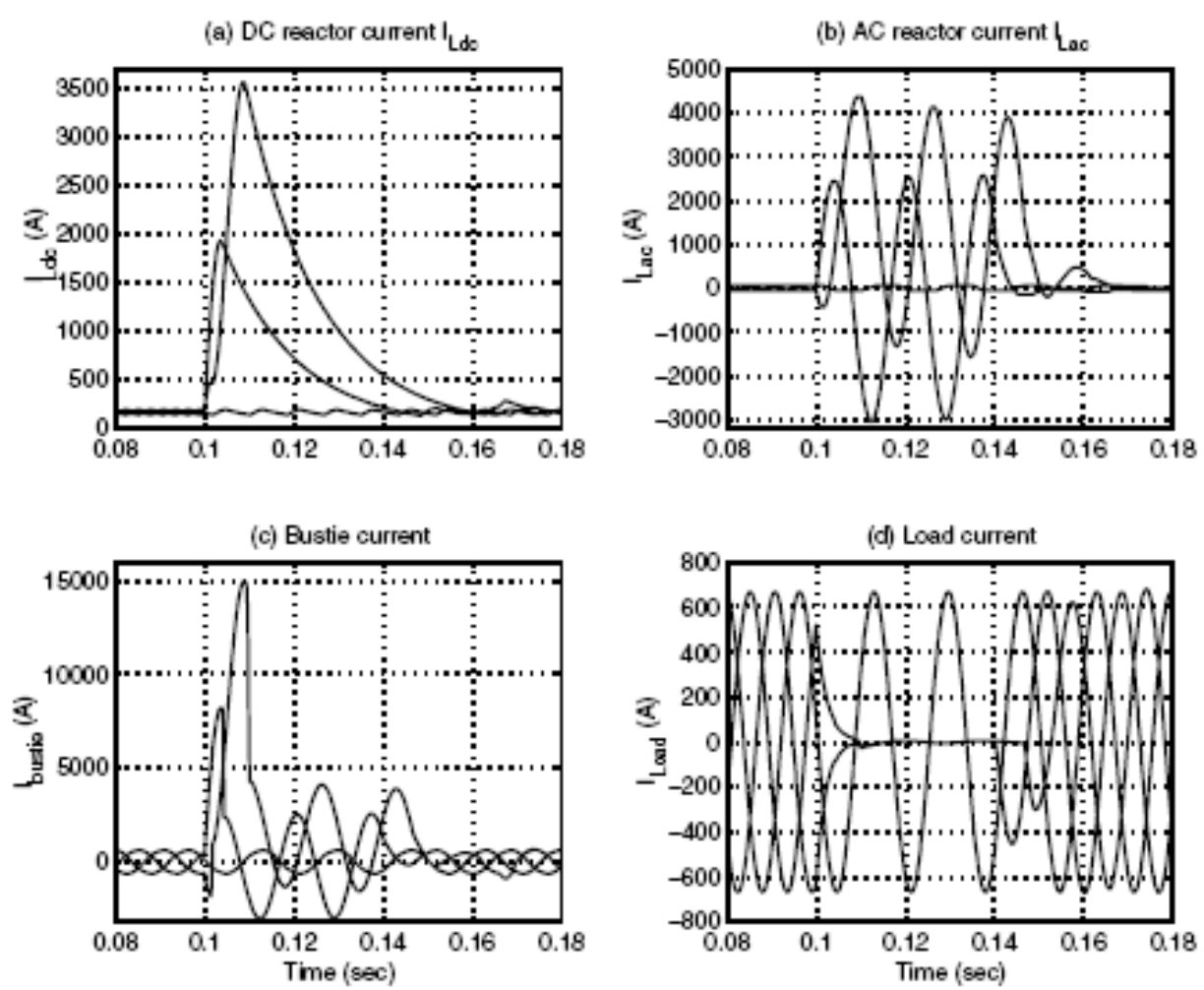

Figure A.27-SSFCL in an $11 \mathrm{kV}$ bus-tie line (2-phase-to-ground fault) 
A.3.5.3.25 Development and test of 10.5 kV/1.5 kA HTS fault current limiter [172]

Abstract: Superconducting Fault Current Limiter (SFCL) is an attractive appliance for modern electrical power system. A $10.5 \mathrm{kV} / 1.5 \mathrm{kA}$ three-phase HTS fault current limiter was developed by IEE, CAS. This improved rectifier-type SFCL with HTS coil of 6.25 $\mathrm{mH}$ is going on a demonstrated long-term reliable operation in a real $10.5 \mathrm{kV}$ substation located in Hunan, China. In a three-phase-to-ground short circuit test of grid, the prospective fault current of $3.5 \mathrm{kA}$ was limited to $635 \mathrm{~A}$ at the pre-setup short circuit point successfully.

Summary: This is another example of a FCL in operation although it too employs the more costly superconducting equipment. The performance specifications are provided in the abstract.

\section{A.3.5.3.26 State of Art of Fault Current Limiters and their impact on Overcurrent Protection [173]}

Summary: Due to increasing fault current levels in transmission and distribution level, considerable attention is given towards developing Fault Current Limiter (FCL) devices in recent years. Most of the FCL technologies are in their initial stages of deployment and are being assessed before their use become more widespread. As the objective of FCL is to limit the fault current, it will have impact on protection system. This paper gives an overview of the different FCL technologies, improvements made in FCL devices and assesses their impacts on over-current relay protection. An analysis on Over-current relay operating time and therefore co-ordination has been presented based on the results obtained from a network splitting model developed using PSCAD/EMTDC.

Implementation: The network splitting models are modeled in PSCAD/EMTDC which was further used to assess the impact of FCL on over-current relay pickup and therefore co-ordination. Change in relay operating time was observed with and without FCL.

Feasibility: The benefits of utilizing FCL in Transmission and distribution networks can be appreciated. It protects the existing infrastructure from increasing fault current levels and gives more flexibility to design the networks.

A.3.5.3.27 Using Fault Current Limiter to Minimize Effect of Thyristor Controlled Series Capacitor on Over Reach Problem of Distance Protection [174]

Summary: FACTS series devices such as Thyristor Control Series Capacitor (TCSC) are used to improve the power transfer capability of long transmission lines. These series connected FACTS devices inject a series voltage with the line and change the line impedance and cause the impedance seen by distance relay to be lower or higher than the 
actual line impedance and the distance relay either over reaches or under reaches. In this paper, a new method is proposed to minimize the impact of TCSC on distance relay. In this method each TCSC is equipped with the Variable Impedance Fault Current Limiter, when fault is occurred, this Fault Current Limiter enters in the system and by injecting series impedance, minimizes the impact of TCSC on distance relay. The simulation results verify the suggested method.

Implementation: Then FCL is used to minimize the over reach problem of distance protection due to TCSC capacitive impedance. This method is simple and does not need to replace the existing relay with adaptive relay. Numerical simulation using PSCAD/EMTDC shows the operation of the proposed coordination method.

Issues:

This method does not have any effect on under reach problem of distance protection due to TCSC.

Feasibility: In the presence of TCSC the relay coordination encounters problems. A novel method is presented to minimize the over reach problem using FCL. Numerical simulations have shown the effectiveness of the new method.

\section{A.3.5.3.28 Impact of Solid-State Fault Current Limiters on Protection Equipment in Transmission and Distribution Systems [175]}

Summary: Solid-state fault current limiters (SSFCLs) offer a number of benefits when incorporated within transmission and distribution systems. SSFCLs can limit the magnitude of a fault current seen by a system using different methods, such as inserting large impedance in the current path or controlling the voltage applied to the fault. However, these two methods can introduce a few problems when SSFCLs are used in a system along with other protection equipment such as protective relays and sensors. An experiment was designed and implemented to evaluate the behavior of the protective relays in a mimic distribution system with a SSFCL. This paper introduces the details of the experiment and the result shows that the distorted current and voltage waveforms resulting from the action of the SSFCL disturb the protective equipment.

Implementation: An experiment using the SEL-311C distance relay to evaluate the impact of SSFCL in a mimic transmission and distribution system was implemented. The goal of this experiment is to compare the performances of the distance relay under two different operating conditions; namely, without and with the SSFCL. 
Feasibility: Different algorithms to control the SSFCL are being investigated in ongoing research work to enable operation of standard protective equipment and the SSFCL in transmission and distribution systems.

A.3.5.3.29 Minimizing the Impact of Distributed Generation on Distribution Protection System by Solid State Fault Current Limiter [176]

Summary: In this paper, a DG with appropriate rating is connected to the different places of distribution network and recalculates the fault current in each section after modeling the distribution network in PSCAD/EMTDC. The impact of DG on distribution protection system is studied comprehensively. Then the proposed SSFCL is connected in series with DG where the effect of SSFCL on limiting fault current is investigated through computer simulations. The results indicate the effectiveness of proposed SSFCL by minimizing the problems associated with the existing DG in distribution system.

Implementation: An experiment using the SEL-311C distance relay to evaluate the impact of SSFCL in a mimic transmission and distribution system was implemented. The goal of this experiment is to compare the performances of the distance relay under two different operating conditions; namely, without and with the SSFCL.

Feasibility: The effectiveness of propose SSFCL in protection problems mitigation is determined and examined in the test system. Simulation is accomplished in PSCAD/EMTDC. The proposed method is fully validated on test system. 
A.4. General Recommendations

A.4.1 Project Requirements

The DOE solicitation requirements for a fault locating/prediction/protection system were to enable all the following functions:

Compatible with industry standards

Integration with existing electronic controls, devices, or relays

Compatible with existing switching equipment

Sensors that capture current and voltage signals without distortion

Recognition of direction of current

Time synchronized phase angles

Signature recognition for prediction of imminent equipment failure

In response to the DOE requirements, our proposal listed the following items for a Fault Location, Prediction, and Protection System (FLPPS):

Microprocessor relays, solid-state sectionalizers and re-closers, and bi-directional devices Phasor measurement technology for distribution networks and the use of GPS

Signature analysis of real-time monitored data

Distributed intelligence architecture

Peer-to-peer communications technologies

Power flow models

Interface to Outage Management Systems, with ties to Geographical Information System (GIS)

Coordination with system restoration schemes such as RAS

\section{A.4.2 Comments and Recommendations}

Since FLPPS is a real installation, items 1 to 3 of the requirements and items "a" and "e" of the proposed system are already included on the existing technical requirements for any equipment installed in the system. Required items 4 and 5 and proposed item "g" are partially met by the installed sensors in the existing IED's but probably require expansion to the use of optical sensors for both voltage and current.

Items 6 to 7 in the DOE solicitation and "b" and "c" of our proposal require the use of specialized IED's at some locations in the system. The existing SEL 351 relay provides some of the required functionality including time synchronization but with some limitations.

Our research plan to DOE promised to lead to the following:

Develop the appropriate signature analysis and FLPPS algorithms for the most efficient and effective fault localization, prediction and service isolation and restoration. The algorithms and analyses will involve the use much of the data to be collected by various "low cost sensors." 
Develop the integrating infrastructure for using the FLPSS analysis methodology and fault location and prediction algorithms to integrate with the OMS, Distribution Automation and DSCADA systems.

Develop the detailed experimental plan for testing such integrated methodology for FLPPS on the SCE's Circuit of the Future.

Develop the data collection plan to know what data to collect.

Develop data analysis plan to know what information to be extracted from the collected data.

Execute the pilot project by following the experimental plan and data collection plan. Analyze the collected data to extract the appropriate information on the efficiency and effectiveness of the FLPPS.

Extrapolate the results to estimate the impacts of such FLPPS on the industry.

\section{A.4.2.1 Recommendation 1:}

Installation of a minimum of two time-synchronized transient recorders in the system that use low-cost optical transducers to obtain some of the system signals. Among the desired characteristics of these devices are:

GPS synchronization to 1 microseconds

Minimum of 9 input channels.

High Sampling rate, over $8 \mathrm{kHz}$

Minimum of two communication channels

Large recording media

\section{A.4.2.2 Recommendation 2:}

Enable the time synchronization function on the existing SEL relays, and use the relay and transient recorded data to create the fault database required for the project.

\section{A.4.2.3 Recommendation 3:}

Install the two transient recorders at the two ends of the line that contains the embedded fiber optic for temperature monitoring. Establish this line as our main testing ground. Develop triggering algorithms for common type faults and four incipient faults based of differential relaying principles and the additional information provided by the fiber thermal sensor.

\section{A.4.2.4 Recommendation 4:}

Analyzed the recorded data to develop algorithms to detect incipient faults inside our differential protection system.

Locate faults inside and outside of our differential protection system. 
Create a fault library that takes advantage of the time synchronization to provide fault signal delay and propagation information.

\section{A.4.2.5 Recommendation 5:}

Take advantage of the existing communication fiber, advanced IED, and requirements of the fault current limiters to study the effect of hidden failures in the protection of distributed systems. 


\section{A.5. References}

\section{A.5.1 Reviews on Fault Location}

[1] R. Horazio Díaz and T. Máximo López, "Fault location techniques for electrical distribution networks: a literature survey," Proceedings of the 5th IASTED International Conference, June 2005, pp. 311-317.

[2] M. Kezunovic, Fault location. Wiley Encyclopedia of Electrical and Electronics Engineering, John Wiley \& Sons Inc, 1999.

\section{A.5.2 Standards on Fault Location}

[3] C37.114 IEEE Guide for Determining Fault Location on AC Transmission and Distribution Lines, sponsored by the Power System Relaying Committee, IEEE Power Engineering Society, IEEE Guide for Determining Fault Location on AC Transmission and Distribution Lines, IEEE Std C37.114-2004, pp. 1-36.

\section{A.5.3 Fault Location, Fundamental Frequency Methods}

[4] T. Takagi, Y. Yamakoshi, M. Yamaura, R. Kondow, and T. Matsushima, "Development of a new type fault locator using the one-terminal voltage and current data," IEEE Transactions on Power Apparatus and Systems, vol. PAS-101, Aug. 1982, pp. 2892-2898.

[5] M.-S. Choi, S.-J. Lee, D.-S. Lee and B.-G. Jin, "A new fault location algorithm using direct circuit analysis for distribution systems," IEEE Transactions on Power Delivery, vol. 19, Jan. 2004, pp. 35-41.

[6] S. Lee et al.,"An intelligent and efficient fault location and diagnosis scheme for radial distribution systems," IEEE Transactions on Power Delivery, vol. 19, Apr. 2004, pp. 524-530.

[7] Z.-Q. Bo, X.-Z. Dong and B. R.-J. Caunce, "Accelerated protection of distribution systems with tapped off loads," IEE Proceedings on Generation, Transmission, and Distribution, vol. 151, July, 2004, pp. 461-468.

[8] C. Wang, H. Nouri, and T. S. Davies, "A mathematical approach for identification of fault sections on the radial distribution systems: voltage sensor," in Electrotechnical Conference, 2000, pp. 882-886.

[9] M. M. Saha, E. Rosolowski, and J. Izykowski, “A new fault location algorithm for distribution networks," in Proceedings of the 8th IEE International Conference on Developments in Power System Protection, 2004, pp. 168-171. 
[10] J. Zhu, D. L. Lubkeman, and A. A. Girgis, "Automated fault location and diagnosis on electric power distribution feeders," IEEE Transactions on Power Delivery, vol. 12, Apr. 1997, pp. 801-809.

[11] T. Baldwin, F. Renovich, L. F. Saunders, and D. Lubkeman, "Fault locating in ungrounded and high resistance grounded systems," IEEE Transactions on Industry Applications, vol. 37, July-Aug. 2001, pp. 1152-1159.

[12] H. F. Zhang, Z. C. Pan, and Z. Z. Sang, "Fault locating in ungrounded and compensated systems," in Proceeding of the 8th IEE International Conference on Developments in Power System Protection, 2004, pp. 469-472.

[13] D. Thukaram, H. P. Khincha, L. Jenkins, and K. Visakha, "A three-phase fault detection algorithm for radial distribution networks," in IEEE Region 10 Conference on Computers, Communications, Control and Power Engineering, 2002, pp. 1242-1248.

[14] P. Bastard, L Garcia-Santander, X. Le Pivert, I. Gal, and E. L. Parra, "A voltagebased fault location method for radial distribution networks," in IEEE 5th International Conference on Power Systems Management and Control, Apr. 2002, pp. 216-221.

[15] R. Das, M. S. Sachdev, and T. S. Sidhu, "A technique for estimating locations of shunt faults on distribution lines," in IEEE Conference Proceedings on Communications, Power, and Computing-WESCANEX 95, 1995, pp. 6-11.

[16] S. Jamali and V. Talavat, "Fault location method for distribution networks using distributed parameter line model," in Proceedings of the 8th IEE International Conference on Developments in Power System Protection, 2004, pp. 216-219. [17] J. Izykowski, E. Rosolowski, P. Balcerek, M. Fulczyk and M. M. Saha, "Accurate Noninterative Fault Location Algorithm Utilizing Two-End Unsynchronized Measurements," IEEE Transactions on Power Delivery, Vol. 25, Jan. 2010, pp. 72-80.

[18] M.-S. Choi, S.-J. Lee, D.-S. Lee and B.-G. Jin, "A new fault location algorithm using direct circuit analysis for distribution systems," IEEE Transactions on Power Delivery, vol. 19, Jan. 2004, pp. 35- 41.

[19] B.-G. Jin et.al. "An intelligent fault location and diagnosis system on radial distribution systems," IEEE Transactions on Power Delivery, vol. 19, Apr. 2004, pp. 524532.

[20] R. Das, M. S. Sachdev, and T. S. Sidhu, "A fault locator for radial subtransmission and distribution lines," Proceedings of the IEEE Power Engineering Society Summer Meeting, 2000, pp. 443-448.

[21] M. Lehtonen, S. Pettissalo, and J.-H. Etula, "Calculational fault location for electrical distribution networks," in 3rd International Conference on Power System Monitoring and Control, 1991, pp. 38-43. 
[22] E.C. Senger, G. Manassero, C. Goldemberg, and E. L. Pellini, "Automated fault location system for primary distribution networks," IEEE Transactions on Power Delivery, vol. 20, Apr. 2005, pp.1332-1340.

A.5.4 Fault Location, High frequency based Methods

[23] R. K. Aggarwal, Y. Aslan, and A. T. Johns, "New concept in fault location for overhead distribution systems using superimposed components," IEE Proceedings on Generation, Transmission, and Distribution, vol. 144, May 1997, pp. 309-316.

[24] F. H. Magnago and A. Abur, "A new fault location technique for radial distribution systems based on high-frequency signals," in Proceedings of the IEEE Power Engineering Society Summer Meeting, 1999, pp. 426-431.

[25] S. Qianli, D. Xinzhou, S. Shenxing, S. Bin, and H. Jiali, "A new principle of fault line selection for distribution," in Proceeding of the 7th IEE International Conference on Developments in Power System Protection, 2001, pp. 379-382.

[26] H. Li, Z. Q. Bo, B. Caunce, and S. Potts, "A fault transient comparison technique for multi-ended distribution feeders," in IEE 7th International Conference on Developments in Power System Protection, 2001, pp. 153-156.

[27] H. Nouri, C. Wang, and T. Davies, "An accurate fault location technique for distribution lines with tapped loads using wavelet transform," in IEEE Porto Conference Power Tech Proceedings, 2001, pp. 4.

[28] Z. Xiangjun, K. K. Li, L. Zhengyi, and Y. Xianggen, "Fault location using traveling wave for power networks," in IEEE Industry Applications Conference- 39th Industry Applications Society Annual Meeting, 2004, pp. 2426- 2429,.

[29] D. W. P Thomas, R. J. O Carvalho, and E. T. Pereira, "Fault location in distribution systems based on traveling waves," in IEEE Bologna Power Tech Conference Proceedings, 2003 , pp. 23-26.

[30] H. Hizman, P. A. Crossley, P. F. Gale, and G. Bryson, "Fault section identification and location on a distribution feeder using traveling waves," in IEEE Power Engineering Society Summer Meeting, 2002, pp. 1107-1112.

[31] J. Moshtagh and R. K. Aggarwal, "A new approach to fault location in a single core underground cable system using combined fuzzy logic and wavelet analysis," in Proceedings of the 8th IEE International Conference on Developments in Power System Protection, 2004, pp. 228-231.

[32] Z. Q. Bo, G. Weller, and M. A. Redfern, "Accurate fault location technique for distribution system using fault-generated high-frequency transient voltage signals," IEEE 
Proceedings on Generation, Transmission, and Distribution, vol. 146, Jan 1999, pp. 7339.

[33] K. J. Jensen, S. M. Munk, and J. A. Sorensen, "Feature extraction method for high impedance ground fault localization in radial power distribution networks," in

Proceeding of the IEEE International Conference on Acoustics, Speech, and Signal Processing, 1998, pp. 1177-1180.

[34] Z. Q. Bo, G. Weller, F. Jiang, and Q. X. Yang, "Application of GPS based fault location scheme for distribution system," in Proceedings of the International Conference on Power System Technology, 1998, pp. 53-57.

[35] Z. Q. Bo, A. T. Johns, and P. K. Aggarwal, "A novel fault locator based on the detection of fault generated high-frequency transients," in Proceedings of the 6th IEE International Conference on Developments in Power System Protection, 1997, pp. 197200.

[36] S. Potivejkul, P. Kerdonfag, S. Jamnian, and V. Kinnares, "Design of a low voltage cable fault detector," in Proceedings of the IEEE Power Engineering Society Winter Meeting, 2000, pp. 724-729.

[37] M. da Silva, D.V. Coury, M. Oleskovicz, E. C. Segatto, "An alternative Fault Location Algorithm Based on Wavelet Transforms for Three-Terminal Lines," IEEE Power and Energy Society General Meeting - Conversion and Delivery of Electrical Energy in the 21st Century, 200, pp. 1-7.

\section{A.5.5 Fault Location, Knowledge Based Methods}

[38] P. Jarventausta, P. Verho, M. Karenlampi, and J. Partanen, "AI-based methods in practical fault location of medium voltage distribution feeders," in Proceedings of the International Conference on Intelligent Systems Applications to Power Systems, 1996, pp. 164-169.

[39] L. Sousa Martins, J. F. Martins, C. M. Alegria, and V. Fernao Pires, "A network distribution power system fault location based on neural eigenvalue algorithm," in IEEE Proceedings of the Power Tech Conference, 2003, pp. 23-26.

[40] W. Zhong and W. H. E. Liu, "Application of a fuzzy set method in distribution system fault location," in IEEE International Symposium on Circuits and Systems, 1996, pp. 617-620.

[41] S. Hanninen and M. Lehtonen, "Earth fault distance computation with artificial neural network trained by neutral voltage transients," in Proceedings of the IEEE Power Engineering Society Summer Meeting, 2001, pp. 1187-1192. 
[42] A. Marusic and S. Gruhonjic-Ferhatbegovic, "A computerized fault location process for overhead radial distribution feeders," in IEEE Mediterranean Electrotechnical Conference, 2006, pp. 1114-1117.

[43] Z. E. Aygen, S. Seker, M. Bagnyanik, F. G. Bagnyanik, and F. G. Ayaz, "Fault section estimation in electrical power systems using artificial neural network approach," in IEEE Transmission and Distribution Conference, 1999, pp. 466-469.

[44] J. Moshtagh and R. K. Aggarwal, "A new approach to ungrounded fault location in a three-phase underground distribution system using combined neural networks and wavelet analysis," in Canadian Conference on Electrical and Computer Engineering, 2006, pp. 376-381.

[45] D. Thukaram, H. P. Khincha, and H. P. Vijaynarasimha, "Artificial neural network and support vector machine approach for locating faults in radial distribution systems," IEEE Transactions On Power Delivery, vol. 20, Apr. 2005, pp. 710-721.

[46] S. Khomfoi and L. M. Tolbert, "Fault Diagnosis and Reconfiguration for Multilevel Inverter Drive Using AI-Based Techniques," IEEE Transactions on Industrial Electronics, Vol. 54, No. 6, Dec 2007, pp. $2954-2968$.

[47] P. Bilski, "An Unsupervised Learning Method for Comparing the Quality of the Soft Computing Algorithms in Analog Systems Diagnostics," 16th International Conference - Mixed Design of Integrated Circuits and Systems - June 25-27, 2009, pp. 499 - 504.

[48] A. Jain, A.S Thoke and R. Patel, "Double Circuit Transmission Line Fault Distance Location using Artificial Neural Network," 2009 World Congress on Nature \& Biologically Inspired Computing, 2009, pp. 13-18.

[49] M. Tarafdar Hagh, K. Razi and H. Taghizadeh, "Fault Classification and Location of Power Transmission Lines Using Artificial Neural Network," The 8th International Power Engineering Conference, IPEC, 2007. pp 1109 - 1114.

[50] X. Lin, Peng Mao, H. Weng, B. Wang, Z. Q. Bo and A. Klimek, "Study on Fault Location for High Voltage Overhead Transmission Lines Based on Neural Network System," Intelligent Systems Applications to Power Systems, ISAP, 2007, pp. 1-5.

[51] M. J. B. Reddy and D. K. Mohanta, "Performance Evaluation of an AdaptiveNetwork-Based Fuzzy Inference System Approach for Location of Faults on Transmission Lines Using Monte Carlo Simulation," IEEE Transactions on Fuzzy Systems, Vol. 16, No. 4, Aug 2008, pp. 909-919. A.5.6 Fault Prediction 
[52] E. T. Wanderley Neto, E. G. da Costa, M. J. A. Maia, and T. V. Ferreira, "Failure analysis in $\mathrm{ZnO}$ arresters using thermal images," in IEEE/PES Transmission \& Distribution Conference and Exposition: Latin America, 2006, pp. 1-5.

[53] S. H. Park, K. J. Lim, and S. H. Kang, "Recognition of electrical tree progress in solid insulator," in 8th International Conference on Properties and Applications of Dielectric Materials, 2006, pp. 575-578.

[54] X. Le, C. Mo-Yuen, and L. S. Taylor, "Data mining and analysis of tree-caused faults in power distribution systems," in IEEE/PES Power Systems Conference and Exposition, 2006, pp. 1221-1227.

[55] A. Livshitz, B. H. Chudnovsky, and B. Bukengolts, "Online condition monitoring and diagnostics of power distribution equipment," in IEEE/PES Power Systems Conference and Exposition, 2004, pp. 646-653.

[56] Y. Zhou, A. Pahwa, and S. Das, "Prediction of weather-related failures of overhead distribution feeders," in International Conference on Probabilistic Methods Applied to Power Systems, 2004, pp. 959-962.

[57] D. C. Elizondo and J. De La Ree, "Analysis of hidden failures of protection schemes in large interconnected power systems," in Proceedings of the IEEE Power Engineering Society General Meeting, 2004, pp. 107-114.

[58] K. L. Butler-Purry and M. Bagriyanik, "Characterization of transients in transformers using discrete wavelet transforms," IEEE Transactions on Power Systems, vol. 18, May 2003, pp. 648-656.

[59] R. P. Bingham, "Getting a clear picture of distortion," in Proceedings of the IEEE Power Engineering Society Summer Meeting, 2002, pp. 927-929.

[60] N. T. Stringer and L. A. Kojovic, "Prevention of underground cable splice failures," IEEE Transactions on Industry Applications, vol. 37, Jan. /Feb. 2001, pp. 230239.

[61] M. De Nigris et al., "Most recent developments in surge arresters technology and testing," in Proceedings of the International Conference of Power System Technology, 1998, pp. 194-201.

[62] C. Mo-yuen and L. S. Taylor, "Analysis and prevention of animal-caused faults in power distribution systems," IEEE Transactions on Power Delivery, vol. 10, Apr. 1995, pp. $995-1001$.

[63] R. M. Bucci, R. V. Rebbapragada, A. J. McElroy, E. A. Chebli, and S. Driller, "Failure prediction of underground distribution feeder cables," IEEE Transactions on Power Delivery, vol. 9, Oct. 1994, pp. 1943-1955. 
[64] E. M. Gulachenski and P. M. Besuner, "Transformer failure prediction using Bayesian analysis," IEEE Transactions on Power Systems, vol. 5, Nov. 1990, pp. 13551363.

A.5.7 Distribution Automation

[65] D. M. Staszesky, D. Craig, and C. Befus, "Advanced feeder automation is here," in IEEE Power and Energy Magazine, 2005, pp. 56-63.

[66] B. N. Ha and H. H. Lee, "Interface between distribution automation system and SCADA to minimize the feeder loss," in IEEE/PES Transmission and Distribution Conference and Exhibition: Asia Pacific. 2002, pp. 2096-2099.

[67] G. L. Ockwell, "Implementation of network reconfiguration for Taiwan Power Company," in Proceedings of the IEEE Power Engineering Society General Meeting, 2003, pp. 2434.

[68] L. Haijun, S. Hari, and D. Walder, "New triple-single reclosers improve distribution reliability," in Rural Electric Power Conference, 2001, pp. D2/1-D2/6.

[69] R. E. Brown and A. P. Hanson, "Impact of two-stage service restoration on distribution reliability," IEEE Transactions on Power Systems, vol. 16, pp. 624-629, Nov. 2001.

[70] I. Roytelman and V. Ganesan, "Coordinated local and centralized control in distribution management systems," IEEE Transactions on Power Delivery, vol. 15, Apr. 2000, pp. 718-724.

[71] D. G. Hart, D. Uy, J. Northcote-Green, C. LaPlace, and D. Novosel, "Automated solutions for distribution feeders," IEEE Computer Applications in Power, vol. 13, Oct. 2000, pp. 25-30.

[72] D. S. Popovic and R. M. Ciric, "A multi-objective algorithm for distribution networks restoration," IEEE Transactions on Power Delivery, vol. 14, July 1999, pp. 1134-1141.

[73] I. Roytelman, B. K. Wee, R. L. Lugtu, T. M. Kulas, and T. Brossart, "Pilot project to estimate the centralized volt/VAr control effectiveness," IEEE Transactions on Power Systems, vol. 13, Aug. 1998, pp. 864-869.

[74] Z. Qin, D. Shirmohammadi, and W. H. E. Liu, "Distribution feeder reconfiguration for operation cost reduction," IEEE Transactions on Power Systems, vol. 12, May 1997, pp. 730-735. 
[75] M. E. Baran and F. F. Wu, "Network reconfiguration in distribution systems for loss reduction and load balancing," IEEE Transactions on Power Delivery, vol. 4, Apr. 1989, pp. 1401-1407.

[76] I. Roytelman, V. Melnik, S. S. H. Lee, and R. L. Lugtu, "Multi-objective feeder reconfiguration by distribution management system," IEEE Transactions on Power Systems, vol. 11, May 1996, pp. 661-667.

[77] B. R. Williams, "Distribution capacitor automation provides integrated control of customer voltage levels and distribution reactive power flow," in Proceedings of IEEE Power Industry Computer Application Conference, 1995, pp. 215-220.

[78] A. G. Jongepier and L. van der Sluis, "Adaptive distance protection of a doublecircuit line," IEEE Transactions on Power Delivery, vol. 9, July 1994, pp. 1289-1297.

[79] D. L. Brown, J. W. Skeen, P. Daryani, and F. A. Rahimi, "Prospects for distribution automation at Pacific Gas \& Electric Company," IEEE Transactions on Power Delivery, vol. 6, Oct. 1991, pp. 1946-1954.

[80] S. Civanlar, J. J. Grainger, H. Yin, and S. S. H. Lee, "Distribution feeder reconfiguration for loss reduction," IEEE Transactions on Power Delivery, vol. 3, July 1988, pp. 1217-1223.

[81] L.-Q. Nguyen and B. Paradis, "Installation of Hydro-Quebec's distribution feeder automation system," IEEE Transactions on Power Delivery, vol. 3, Oct. 1988, pp. 19351941.

[82] D. Yuan, N. Zhang, X. Dong, Z Q Bo and A Klimek, "An Adaptive Noncommunication Protection for Distribution Systems," 42nd Universities Power Engineering Conference, UPEC, 2007, pp. 257 - 261.

[83] I. S. Baxevanos and D. P. Labridis, "Implementing Multiagent Systems Technology for Power Distribution Network Control and Protection Management," IEEE Transactions on Power Delivery, Vol. 22, No. 1, Jan 2007, pp. 433 - 443.

[84] S. Kazemi, M. Fotuhi-Firuzabad, M. Sanaye-Pasand and M. Lehtonen, "Impact of Automatic Restoration Scheme on the Distribution System Reliability," IET Generation, Transmission \& Distribution, Vol. 3, Issue 3, Dec 2008, pp. 891 - 902.

[85] Li Le-le, Xie Zhi-Yuan and Li Na, "Research of Feeder Automation System Based on Multi-Agent," IEEE third conference on Industrial Electronics and Applications, ICIEA 2008, pp 538-541

[86] John S. Bowers, Ashok Sundaram, Carl L. Benner and B. Don Russell, "Outage Avoidance through Intelligent Detection of Incipient Equipment Failures on Distribution 
Feeders," IEEE Power and Energy Society General Meeting - Conversion and Delivery of Electrical Energy in the 21st Century, 2008 IEEE, pp. 1-7.

[87] X. Dong, S. Shi, B. Wang, W. Kong and Z. Bo, "Feeder Automation System Based on Non-communication Protection and Control Unit," IEEE Power and Energy Society General Meeting, 2009, pp. 1-7.

A.5.8 High Impedance Faults

[88] WG Members, "High impedance fault detection technology," D15 WG Report of Power System Relaying Committee, 1996, pp. 1-12.

[89] J. Carr, "Detection of high impedance faults on multi-grounded primary distribution systems," IEEE Transactions on Power Apparatus and Systems, vol. PAS100, Apr. 1981, pp. 2008-2016.

[90] H. Calhoun, M. T. Bishop, C. H. Eichler, and R. E. Lee, "Development and testing of an electro-mechanical relay to detect fallen distribution conductors," IEEE Transactions on Power Apparatus and Systems, vol. PAS-101, June 1982, pp. 1643-1650.

[91] R. E. Lee and M. T. Bishop, "Performance testing of the ratio ground relay on a four-wire distribution feeder," IEEE Transactions on Power Apparatus and Systems, vol. PAS-102, Sept. 1983, pp. 2943-2949.

[92] A. F. Sultan, G. W. Swift, and D. J. Fedirchuk, "Detecting arcing downed-wires using fault current flicker and half-cycle asymmetry," IEEE Transactions on Power Delivery, vol. 9, Jan. 1994, pp. 461-467.

[93] A. M. Sharaf and S. I. Abu-Azab, "A smart relaying scheme for high impedance faults in distribution and utilization networks," in Canadian Conference on Electrical and Computer Engineering, 2000, pp. 740-744.

[94] E. C. Senger, W. Kaiser, J. C. Santos, P. M. S. Burt, and C. V. S. Malagodi, "Broken conductors protection system using carrier communication," IEEE Transactions on Power Delivery, vol. 15, Apr. 2000, pp. 525-530.

[95] C. L. Huang, H. Y. Chu, and M. T. Chen, "Analysis and detection algorithms of high impedance fault in primary distribution systems," National Science Council of Taiwan. Dec. 1985.

[96] C.-L. Huang, H.-Y. Chu and M.-T. Chen, "Algorithm comparison for high impedance fault detection based on staged fault test," IEEE Transactions on Power Delivery, vol. 3, Oct. 1988, pp. 1427-1435.

[97] B. M. Aucoin and B. D. Russell, "Distribution high impedance fault detection utilizing high-frequency current components," IEEE Transactions on Power Apparatus and Systems, vol. PAS-101, June 1982, pp. 1596-1606. 
[98] M. Aucoin, "Status of high impedance fault detection," IEEE Transactions on Power Apparatus and Systems, vol. PAS-104, Mar. 1985, pp. 637-644.

[99] M. Aucoin and B. D. Russell, "Detection of distribution high impedance faults using burst noise signals near $60 \mathrm{~Hz}$," IEEE Transactions on Power Delivery, vol. 2, Apr. 1987, pp. 342-348.

[100] C. J. Kim and B. d. Russell, "Classification of faults and switching events by inductive reasoning and expert system methodology," IEEE Transactions on Power Delivery, vol. 4, July 1989, pp. 1631-1637.

[101] B. D. Russell, "Computer relaying and expert systems - new tools for detecting high impedance faults," Electric Power Systems Research, vol. 20, Dec. 1990, pp. 31-37.

[102] C. J. Kim and B. D. Russell, "A learning method for use in intelligent computer relays for high impedance faults," IEEE Transactions on Power Delivery, vol. 6, Jan. 1991, pp. 109-115.

[103] C. J. Kim and B. D. Russell, "High-impedance fault detection system using an adaptive element model," IEE Proceedings on Generation, Transmission, and Distribution, vol. 140, Mar. 1993, pp. 153-159.

[104] B. D. Russell and C. L. Benner, "Performance of high-impedance fault-detection algorithms in long-term field trials," Electric Power Systems Research, vol. 31, Nov. 1994, pp. 71-77.

[105] A. V. Mamishev, B. D. Russell, and C. L. Benner, "Analysis of high impedance faults using fractal techniques," IEEE Transactions on Power Systems, vol. 11, Feb. 1996, pp. 435-440.

[106] B. M. Aucoin and R. H. Jones, "High impedance fault detection implementation issues," IEEE Transactions on Power Delivery, vol. 11, Jan 1996, pp. 139-148.

[107] A. V. Mamishev, B. D. Russell, and C. L. Benner, "Analysis of high impedance faults using fractal techniques," IEEE Transactions on Power Systems, vol. 11, Feb. 1996, pp. 435-440.

[108] A. C. Depew, J. M. Parsick, R. W. Dempsey, C. L. Benner, B. D. Russell, and M. G. Adamiak, "Field experience with high-impedance fault detection relays," in Annual Conference for Protective Relay Engineers, 2006, pp. 6.

[109] M. Aucoin, B. D. Russell, and C. L. Benner, "High impedance fault detection for industrial power systems," in Conference Record of the IEEE Industry Applications Society Annual Meeting, 1989, pp. 1788-1792. 
[110] B. D. Russell and C. L. Benner, "Arcing fault detection for distribution feeders: security assessment in long term field trials," IEEE Transactions on Power Delivery, vol. 10, Apr. 1995, pp. 676-683.

[111] A. A. Girgis, W. Chang, and E. B. Makram, "Analysis of high-impedance fault generated signals using a Kalman filtering approach," IEEE Transactions on Power Delivery, vol. 5, Oct. 1990, pp. 1714-1724.

[112] A. Lazkano, J. Ruiz, E. Aramendi, and L. A. Leturiondo, "A new approach to high impedance fault detection using wavelet packet analysis," in 9th International Conference on Harmonics and Quality of Power, 2000, pp. 1005-1010.

[113] D. C. T. Wai and Y. B. Xia, "A novel technique for high impedance fault identification," IEEE Transactions on Power Delivery, vol. 13, July, 1988, pp. 738-744.

[114] Y. Xia, L. Qi, and D. T. W. Chan, "DSP implementation of a wavelet analysis filter bank for high impedance fault detection," in Proceedings of the International Conference on Energy Management and Power Delivery, 1998, pp. 417-421.

[115] S.-J. Huang and C.-T. Hsieh, "High-impedance fault detection utilizing a Morlet wavelet transform approach," IEEE Transactions on Power Delivery, vol. 14, Oct. 1999, pp. 1401-1410.

[116] C.-H. Kim, H. Kim, Y.-H. Ko, S.-H. Byun, R. K. Aggarwal, and A. T. Johns, "A novel fault-detection technique of high-impedance arcing faults in transmission lines using the wavelet transform," IEEE Transactions on Power Delivery, vol. 17, Oct. 2002, pp. 921-929.

[117] Y. Ming-Ta, G. Jhy-Cheng, J. Chau-Yuan, and K. Wen-Shiow, "Detection of high impedance fault in distribution feeder using wavelet transform and artificial neural networks," in International Conference on Power System Technology, 2004, pp. 652-657.

[118] W. David Chan Tat and Y. Xia, "A novel technique for high impedance fault identification," IEEE Transactions on Power Delivery, vol. 13, July, 1998, pp. 738-744.

[119] T. M. Lai, L. A. Snider, and E. Lo, "Wavelet transform based relay algorithm for the detection of stochastic high impedance faults," Electric Power Systems Research, vol. 76, May 2006, pp. 626-633.

[120] C. L. Benner and B. D. Russell, "Practical high-impedance fault detection on distribution feeders," IEEE Transactions on Industry Applications, vol. 33, May/June 19997, pp. 635-640.

[121] A. M. Sharat, L. A. Snider, and K. Debnath, "A neural network based back error propagation relay algorithm for distribution system high impedance fault detection," in 
1st New Zealand International Two-Stream Conference on Artificial Neural Networks and Expert Systems, 1993, pp. 321-324.

[122] A. M. Sharaf, L. A. Snider, and K. Debnath, "A neural network based relaying scheme for distribution system high impedance fault detection," in 1st New Zealand International Two-Stream Conference on Artificial Neural Networks and Expert Systems, 1993, pp. 321-324.

[123] A. W. Sharaf, L. A. Snider, and K. Debnath, "A third harmonic sequence ANN based detection scheme for high impedance faults," in Canadian Conference on Electrical and Computer Engineering, 1993, pp. 802-806.

[124] K. Jae-Ho, S. Jae-Chul, R. Chang-Wan, P. Chan-Gook, and Y. Wha-Yeong, "Detection of high impedance faults using neural nets and chaotic degree," in Proceedings of the International Conference on Energy Management and Power Delivery, 1998, pp. 399-404.

[125] T. M. Lai, E. L. Snider, C. H. Cheung, and K. W. Chan, "High impedance faults detection using artificial neural network," in 6th International Conference on Advances in Power System Control, Operation, and Management, 2003, pp. 821-826.

[126] M. M. Eissa, G. M. A. Sowilam, and A. M. Sharaf, "A new protection detection technique for high impedance fault using neural network," in Large Engineering Systems Conference on Power Engineering, 2006, pp. 146-151.

[127] A. S. Bretas, L. Pires, M. Moreto, and R. H. Salim, "A BP neural network based technique for HIF detection and location on distribution systems with distributed generation," in Computational Intelligence, Vol. 4114, 2006, pp. 608-613.

[128] P. R. S. Jota and F. G. Jota, "Fuzzy detection of high impedance faults in radial distribution feeders," Electric Power Systems Research, vol. 49, Apr. 1999, pp. 169-174.

[129] F. Ruz and J. A. Fuentes, "Fuzzy decision making applied to high impedance fault detection in compensated neutral grounded MV distribution systems," in 7th IEE International Conference on Developments in Power System Protection, 2001, pp. 307310 .

[130] M.-R. Haghifam, A.-R. Sedighi, and O. P. Malik, "Development of a fuzzy inference system based on genetic algorithm for high-impedance fault detection," IEE Proceedings-Generation, Transmission, and Distribution, vol. 153, May 2006, pp. 359367.

[131] N. Zamanan, J. Sykulski, and A. K. Al-Othman, "Arcing high impedance fault detection using real coded genetic algorithm," in 7th IASTED International Conference on Power and Energy Systems, 2007, pp. 35-39. 
[132] M. Carpenter, R. R. Hoad, T. D. Bruton, R. Das, S. A. Kunsman, and J. M. Peterson, "Staged-fault testing for high impedance fault data collection," in 58th Annual Conference for Protective Relay Engineers, 2005, pp. 9-17.

[133] R. E. Lee and R. H. Osborn, "A microcomputer based data acquisition system for high impedance fault analysis," IEEE Transactions on Power Apparatus and Systems, vol. PAS-104, Oct. 1985, pp. 2748-2753.

[134] D. C. Yu and S. H. Khan, "An adaptive high and low impedance fault detection method," IEEE Transactions on Power Delivery, vol. 9, Oct. 1994, pp. 1812-1821.

[135] A. E. Emanuel, D. Cyganski, J. A. Orr, S. Shiller, and E. M. Gulachenski, "High impedance fault arcing on sandy soil in $15 \mathrm{kV}$ distribution feeders: contributions to the evaluation of the low frequency spectrum," IEEE Transactions on Power Delivery, vol. 5, Apr. 1990, pp. 676-686.

[136] S. R. Nam, J. K. Park, Y. C. Kang, and T. H. Kim, "A modeling method of a high impedance fault in a distribution system using two series time-varying resistances in EMTP," in Proceedings of the IEEE Power Engineering Society Summer Meeting, 2001, pp. 1175-1180.

[137] N. I. Elkalashy, M. Lehtonen, H. A. Darwish, M. A. Izzularab, and A.-M. I. Taalab, "Modeling and experimental verification of high impedance arcing fault in medium voltage networks," IEEE Transactions on Dielectrics and Electrical Insulation, vol. 14, Apr. 2007, pp. 375-383.

[138] J. Tengdin, R. Westfall, and K. Stephan, "High impedance fault detection technology," in Report of Power System Relaying Committee, 1996, pp. 1-12.

[139] E. A. Atwell, A. W. Shaffer, D. I. Jerrings, and J. R. Linders, "Performance testing of the Nordon high impedance ground fault detector on a distribution feeder," presented at Rural Electric Power Conference, 1990, pp. C6/1-C6/7.

[140] J. Reason, "Relay detects downed wires by fault-current harmonics," Electrical World, vol. 208, issue12, Dec. 1994, pp. 58-59.

[141] G. Multilin, "F60 feeder management relay UR series instruction manual," 2003, pp. 8.1-8.4.

[142] R. Das and S. A. Kunsman, "A novel approach for ground fault detection," in 57th Annual Conference for Protective Relay Engineers, 2004, pp. 97-109.

[143] D. Hou, "Detection of high-impedance faults in power distribution systems," SEL-Inc, White Paper 2006. 
[144] K. Jalal, A. Jalil, A. Halim, A. Bakar, W. Norliza, W. Mahadi, F. Hani and M. Salleh, "Performance of Restricted Earth Fault Protection Scheme in the Presence of Current Transformer Remanence," 2nd IEEE International Conference on Power and Energy, Power Con., Dec 2008, pp. 549 - 552.

[145] A. Wahlroos and J. Altonen, "Performance of Novel Neutral Admittance Criterion in MV-Feeder Earth-Fault Protection," 20th International Conference on Electricity Distribution, CIRED 2009, June 2009, pp. 1-4.

[146] L. V. Dusang jr. and Brian K. Johnson, "Evaluation of Fault Protection Methods using ATP and MathCAD," IEEE Canada Power and Energy Conference, 2008, pp. 1-8.

[147] L. Wang, F. Chen, G. Allen, H. Cheung, T. Mander and R. Cheung, "Network Integrated DSP-based Adaptive High Impedance Ground Fault Feeder Protection," Power Engineering Society General Meeting, IEEE Power Engineering Society General Meeting, June 2007, pp. 1-7.

A.5.9 Fault Current Limiters

[148] F. Mumford, "Superconducting fault current limiters," BEC ALSTHOM Engineering Research Centre, 1995, pp. 1-7.

[149] Rowley, Andrew, "Superconducting fault current limiters," EA Technology Core Research programme, 1995, pp. 1-3.

[150] S. Sugimoto, J. Kida, H. Arita, C. Fukui, and T. Yamagiwa, "Principle and characteristics of a fault current limiter with series compensation," IEEE Transactions on Power Delivery, vol. 11, Apr. 1996, pp. 842-847.

[151] A. Wu and Y. Yin, "Fault current limiter applications on medium and high voltage power distribution system," IEEE Transactions on Industry Applications, vol. 34, Jan. /Feb. 1998, pp. 179-185.

[152] D. J. Moule, P. D. Evans, T. C. Shields, S. A. Foulds, J. P. Price, and J. S. Abell, "Study of fault current limiter using YBCO thick film material," IEEE Transactions on Applied Superconductivity, vol. 7, June 2007, pp. 1025-1028.

[153] Das, J.C., "Discussion of 'Fault-current limiter applications in medium and high voltage power distribution systems," IEEE Transactions on Industry Applications, vol. 34, July/Aug. 1998, pp. 638.

[154] M. Sjostrom, R. Cherkaoui, and B. Dutoit, "Enhancement of power system transient stability using superconducting fault current limiters," IEEE Transactions on Applied Superconductivity, vol. 9, June 1999, pp. 1328-1330.

[155] H. Kameda and H. Taniguchi, "Setting method of specific parameters of a superconducting fault current limiter considering the operation of power system 
protection," IEEE Transactions on Applied Superconductivity, vol. 9, June 1999, pp. 1355-1360.

[156] H. Kubota, Y. K. Arai, M. Yamazaki, and H. Yoshino, "A new model of fault current limiter using YBCO thin film," IEEE Transactions on Applied Superconductivity, vol. 9, June 1999, pp. 1365-1368.

[157] M. Yagami, T. Murata, and J. Tamura, "Stabilization of synchronous generators by superconducting fault current limiter," in Proceedings of IEEE Power Engineering Society Winter Meeting, 2000, pp. 1394-1398.

[158] M. Steurer, H. Brechna, and K. Frohlich, "A nitrogen gas cooled, hybrid, high temperature superconducting fault current limiter," IEEE Transactions on Applied Superconductivity, vol. 16, Mar. 2000, pp. 840-844.

[159] Leung, Eddie, "Superconducting fault current limiters," IEEE Power Engineering Review, Aug. 2000, pp. 15-18.

[160] S. Lee, C. Lee, T. K. Ko, and O. Hyun, "Stability analysis of a power system with superconducting fault current limiter installed," IEEE Transactions on Applied Superconductivity, vol. 11, Mar. 2001, pp. 2098-2101.

[161] L. Ye, L. Z. Lin, and K.-P. Juengst, "Application studies of superconducting fault current limiters in electric power systems," IEEE Transactions on Applied Superconductivity, vol. 12, Mar. 2002, pp. 900-903.

[162] L. Martini, L. Bigoni, G. Cappai, R. Iorio, and S. Malgarotti, "Analysis on the impact of HTS cables and fault current limiters on power systems," IEEE Transactions on Applied Superconductivity, vol. 13, June 2003, pp. 1818-1821.

[163] E. Calixte, Y. Yokomizu, H. Shimizu, T. Matsumura, and H. Fujita, "Reduction of rating required for circuit breakers by employing series-connected fault current limiters," IEE Proceedings on Generation, Transmission, and Distribution, vol. 151, Jan. 2004, pp. 36-42.

[164] Janusz Kozak et al., "The influence of superconducting fault current limiter structure on the V-I characteristic," IEEE Transactions on Applied Superconductivity, vol. 14, June 2004, pp. 811-814.

[165] M. Fabbri, A. Morandi, F. Negrini, and P. L. Ribani, "Magnetic-shield-type fault current limiter equivalent circuit," IEEE Transactions on Applied Superconductivity, vol. 14, Sept. 2004, pp. 1966-1973.

[166] Z. Zhao, Z. Lu, and W. Yao, "Experiment and research on control strategy for a new-type three-phase solid-state current limiter," in 20th Annual IEEE Applied Power Electronics Conference and Exposition, 2005, pp. 1778-1781. 
[167] X. Yuan, K. Tekletsadik, L. Kovalsky J. Bock, F. Breuer, and S. Elschner, "Proof-of-concept prototype test results of a superconducting fault current limiter for transmission-level applications," IEEE Transactions on Applied Superconductivity, vol. 15, June 2005, pp. 1982-1985.

[168] T. Hoshino, I. Muta, T. Nakamura, K. Salim, and M. Yamada, "Non-inductive variable reactor design and computer simulation of rectifier type superconducting fault current limiter," IEEE Transactions on Applied Superconductivity, vol. 15, June 2005, pp. 2063-2066.

[169] L. Kovalsky, X. Yuan, K. Tekletsadik, A. Keri, J. Bock, and F. Breuer, "Applications of superconducting fault current limiters in electric power transmission systems," IEEE Transactions on Applied Superconductivity, vol. 15, June 2005, pp. 2130-2133.

[170] Schmitt, Heino, "Fault current limiters report on the activities of CIGRE WG A3.16," in IEEE Power Engineering Society General Meeting, 2006, pp. 1-5.

[171] V. K. Sood and M. Salam, "EMTP RV-based study of solid-state fault current limiter for distribution systems," in IEEE Power India Conference, 2006, pp. 1-6.

[172] D. Hui et al., "Development and test of $10.5 \mathrm{kV} / 1.5 \mathrm{kA}$ HTS fault current limiter," IEEE Transactions on Applied Superconductivity, vol. 16, June 2006, pp. 687690.

[173] S. Orpe and Nirmal-Kumar C. Nair, "State of Art of Fault Current Limiters and their Impact on Overcurrent Protection," IEEE Power and Energy Society General Meeting, 2009, pp. 1-5.

[174] S. A. A Shahriari, M. Hosseini Abardeh, A. Yazdian Varjani and M. Mohamadian, "Using Fault Current Limiter to Minimize Effect of Thyristor Controlled Series Capacitor on Over Reach Problem of Distance Protection," IEEE/PES Power Systems Conference and Exposition, 2009, pp. 1-6.

[175] Y. Feng, Johnson, O. Saadeh, J. C. Balda1, H. A. Mantooth and M. Schupbach, "Impact of Solid-State Fault Current Limiters on Protection Equipment in Transmission and Distribution Systems," IEEE PES Transmission and Distribution Conference and Exposition, 2010, pp. 1-6.

[176] S. A. A. Shahriari, M. Abapour, A. Yazdian and M. R. Haghifam, "Minimizing the Impact of Distributed Generation on Distribution Protection System by Solid State Fault Current Limiter," IEEE/PES Transmission and Distribution Conference and Exposition, 2010., pp. 1-7. 


\section{Appendix B: Survey Questionnaire and Results}

This literature review report was supplemented with a survey of utilities, universities, and research organizations to determine which of these techniques are actually being implemented. The survey was sent to more than 430 different entities in North America and the world as well as the Distribution Automation Working Group of the IEEE.

\section{B.1 Summary of Surveys}

As part of this project, two surveys were conducted. The first, sent out questionnaires to over 100 utilities, manufacturers, and universities during the period of June through December, 2008 and received 15 responses (two of these indicated they did not have any research work in progress). These results were divided into three groups:

- Fault Protection and Sensor Technologies

- Fault Location and SCADA Technologies

- Other Technologies/ Future Work

The second survey was composed of 20 multiple choice questions sent out to members of the IEEE T\&D Distribution Automation Working Group during June of 2009. The number of responses to each item is listed to its right. They were grouped in the following categories:

- Feeder Automation

- Line Sensors/Monitoring

- Communications

- Relay Oscillographics/Fault Data

- Advanced Protection Schemes

- Fault Current Limiting

The responses from the IEEE T\&D committee survey were combined under the headings of the first survey and the results are presented below. Major observations from the surveys were as follows:

\section{B.1.1 Fault Protection and Sensor Technologies}

- Most utilities have the ability to manually sectionalize feeders to speed load restoration and fault isolation. Communications is being connected to switches and reclosers to facilitate restoration. Increasingly, utilities are also putting in logic (both central and local) to automatically do this restoration.

- Voltage and current sensors, along with fault indicators are being installed on feeders to help with fault location. These sensors are being equipped with 
communications to allow operators to do remote diagnosis as well as permit automatic control.

- Protection schemes to deal with distributed generation are being explored and include interlock or transfer trip of the DG devices when the substation breaker opens. The impacts of inverter-based devices may be less that other typed of generators.

- Electronic relays are being installed to replace electro-mechanical devices to allow more data to be collected and better fault location.

\section{B.1.2 Fault Location and SCADA Technologies}

- The use of circuit models to calculate fault currents at any location on the circuit is increasingly being used. During the fault, the current at the substation breaker is measured and compared with the model to identify potential fault locations. The use of other sensors helps identify the final location.

- Information about outages from Advanced Metering systems ("last gasp" and actual connectivity) is also being used to identify meters that have lost power. Through the use of an Outage Management System, faulty device locations can be identified.

- Monitoring the condition of critical pieces of equipment is helping to estimate when failures might occur. This data along with detection of momentary relay pickups is being used to try and predict faults. These techniques are new and do not have high accuracy yet.

\section{B.1.3 Other Technologies/ Future Work}

- A lot of work is being done in the communications area. Most distribution communications is being done through the use of licensed or unlicensed radio (even split between these). There is some use of public wireless networks, but utilities are reluctant to use for operations because of reliability concerns (will it be there in a disaster when needed most). Fiber is being used for some substations, but is still too expensive for most distribution installations outside of substations.

- Fault current limiters are being considered for distribution (in substations), but are still too costly and too experimental for widespread use. The biggest benefit of fault current limiting appears to be in the transmission/sub-transmission areas.

\section{B.2 Detailed Responses to the Surveys}

\section{B.2.1 Fault Protection and Sensor Technologies}

0 Advanced protection for distribution systems

o Integration of adaptive protection with distribution automation

o Communication approaches for monitoring systems and/or protection system implementation 
o Sensors for monitoring on distribution systems

o Sensors for fault location and/or indication

\section{B.2.1.1 Questionnaire Results}

Company 7 (utility):

1) Advanced protection for distribution systems:

No projects currently.

2) Integration of adaptive protection with distribution automation:

We do not presently have a project to automatically locate faults on the distribution system but we have been using a manual process on both our distribution and sub-transmission systems with very promising results. Products: MehtaTech digital fault recorder, SEL relays, Cooper Form 6 (and earlier) and GridSense LineTrackers.

3) Communication approaches for monitoring systems and/or protection system implementation:

Our communications plan is to leverage the AMI communications infrastructure as much as possible for use with the Smart grid. Products: We are testing several of the major AMI meter manufacturer's system and also looking at alternative communications backhaul using various communications options such as cellular, BPL and WiMax.

Company 9 (utility): Respondent will start a joint study (fall 2008) with an equipment manufacturer to evaluate how the new protection technologies can be applied to the distribution system to improve actual protection schemes. This work will involve new MV equipment concept and new protection logic possible through the latest protection controllers. First results are to be available in 2009/2010. Some possibilities to detect high impedance faults are available in newest generation of substation numerical protection relays. Although the project is not yet structured, respondent may want to try this feature to evaluate how it performs. There is no schedule yet for this one.

Company 10 (consultant): Their Response consists of a 127 page PDF titled "Value of Distribution Automation Applications" submitted to the California Energy Commission (CEC)

Company 11 (utility):

Advanced protection for distribution systems:

1) The IDMS Short Circuit Analysis application will calculate on a real-time basis the maximum short circuit current available for a phase to ground or phase to phase fault at any point modeled in the electric distribution system.

2) The IDMS Short Circuit Protection Coordination application checks coordination of protective devices with the next upstream protective device.

Integration of adaptive protection with distribution automation:

In the Distribution SCADA/Distribution Automation initiatives, respondent utilizes micro-processor based protection relays to manage the protection and coordination of the electric distribution circuits. The IDMS short circuit analysis and protection coordination tools will support adaptive relay setting changes to 
ensure effective coordination of the electric distribution circuit as the circuit configuration dynamically changes

Sensors for fault indication or fault location:

In the Distribution SCADA/Distribution Automation initiatives, respondent utilizes Distribution Line-Post Sensors for Distribution line fault detection. A fault condition is reported on a per phase basis when line current exceeds a pre-set magnitude threshold for a pre-set duration of time.

\section{Company 12 (research group):}

Implementation of advanced fault detection technologies, in the context of advanced distribution system protection with DG:

It will assess whether special consideration is required when implementing advanced protection technologies into systems with DG as well as how multi-functional relays can be used to facilitate the integration of DG, particularly when there are a number of DG on a given feeder. At the first stage, we have proposed to partner with ENMAX and analyze the impact of DG on their IntelliRuptor system.

\section{Company 14(utility):}

Advanced protection of distribution systems:

We have improved fault detection and better coordination using microprocessor based feeder breaker relays. We have attempted fault location but had mixed results.

Integration of adaptive protection with distribution automation:

We have piloted this type of protection, but do not have it system wide.

\section{Company 15 (utility):}

Advanced protection of distribution systems:

Respondent is developing protection schemes required to protect the integrity of the Western Interconnection. Remedial Action Scheme (RAS) devices are in place and are currently being tested. Each scheme has specific requirements related to timing and back up power supplies and redundant protection. In addition, respondent has an additional scheme known as Overload Scheme (OLS), which is used for local respondent area grid protection. Respondent has been replacing obsolete electromechanical relays with modern microprocessor based relays. In 2005, a projected was initiated to replace the obsolete relays on the transmission lines based on reliability, failure rate, parts, and maintenance issues. So far, $40 \%$ of obsolete relays have been replaced with the microprocessor relays. Integration of adaptive protection with distribution automation:

Respondent has installed a decentralized application of Feeder Automation System Technology (FAST) in 2007. FAST is an automatic switching scheme that will restore service following an outage event. This approach uses a switching logic programmed in the controller of the field switch where automatic switching occurs. Respondent is installing and testing a centralized application of FAST where a programmed switching logic is installed on the centrally located SCADA Master. Upon a system disturbance, a master system takes information 
from the remote site, such as analog voltage/current and status indications/alarms, and will perform the appropriate action.

Sensors for monitoring on distribution systems:

Respondent is installing fiber optic cables with the power conductors to transmit real time temperature data which will be used to calculate a real time ampacity for the conductors. This will allow for maximum equipment utilization, static, and ultimately, dynamic ratings for conductors. New hardware and software technology is being developed to measure the condition and performance of distribution equipment. This technology is utilizing sensors to provide diagnostic information to facilitate increase capacity utilization of transformers. Respondent has installed power quality instruments in substations to collect data and monitor disturbances in the system. These instruments will provide information to indicate a fault and potentially have a way to anticipate a fault by identifying short duration faults in the system. Respondent is currently targeting the installation of 1.5 field SCADA devices on every $12 \mathrm{kV}$ circuit. Typically, 1.5 switches per circuit are comprised of a mid-point SCADA switch and a SCADA tie switch that is shared with an adjacent circuit.

Company 17 (utility):

Integration of adaptive protection with distribution automation:

Pilot projects are proceeding in decentralized and distributed intelligence systems that enable fault isolation and restoration to alternative sources based on available system capacity. S\&C IntelliTeam with SCADA-mate switches and Cooper Cybectech SMP controllers with reclosers are the DA focus areas at this time. Centralized DA control/overlay is also being studied for potential DA application and enhancement. All DA schemes have the requirement of interfacing with SCADA.

\section{B.2.1.2 June 2009 Survey of the Distribution Automation Working Group of the IEEE T\&D Committee (number of responses listed to the right of items)}

\section{$\underline{\text { Feeder Automation }}$}

1. Types of distribution line devices used to interrupt and sectionalize faults?
a) Hydraulic reclosers
10
b) Automatic line sectionalizers
10
c) Electronic reclosers
13
d) SCADA-controlled load break switches
13
e) Remotely controlled vacuum interrupters
f) Network protectors
g) Load break switches

2. What are approaches for which automatic switches are currently applied?
a) Centrally-controlled sectionalizing scheme via SCADA 
b) Normally-open tie points 9

c) Distributed control strategy with SCADA supervision $\quad 10$

d) Local control strategy with no communications 10

e) None 1

f) Non-closing AR scheme 1

g) Distributed control strategy w/o SCADA but with comm. 1

3. Key issue with deployment of automated switches?
a) SCADA Integration
b) Cost Justification 9
c) Lack of Communications 7
d) Additional Maintenance 5
e) Value in terms of benefit-cost ratio 1
f) Additional operation
g) Changing circuitry for load rolls
h) Redundant Feed capability
i) Standard and procurement
j) Figuring out the basics with new personnel
k) Reliability

4. What approaches are being considered for future automation schemes?

a) Centrally-controlled sectionalizing scheme via SCADA 13

b) Normally-open tie points 4

c) Distributed control strategy with SCADA supervision 9

d) Local control strategy with no communications 3

e) Combination of distributed and centralized 1

\section{Line Sensors/Monitoring}

5. What types of sensors do you use on your distribution feeders?
a) Line voltage sensors
b) Power quality monitors
c) Line current sensors
d) Fault current indicators
e) Conductor temperature monitors
f) Remote controlled switches \& breakers
2

6. What types of sensors are you planning/considering for use on your distribution feeders?
a) Line voltage sensors
b) Power quality monitors
c) Line current sensors
d) Fault current indicators with communication 14
e) Conductor temperature monitors 1
f) Other
g) On-going study 
7. What type of fault location techniques do you currently use on your distribution circuits?

a) Fault current indicators on overhead 12

b) Fault current indicators on cable 9

c) Fault current indicators with communications 4

d) Fault location embedded in relay 7

e) Fault indicators embedded in automated switches 7

f) Fault location based on SCADA input 6

g) Custom application 1

8. What distribution field equipment do you monitor for equipment diagnostics? (circle all that apply)
a. Capacitor banks
4
b. Regulators
3
c. Reclosers
9
d. Customer distribution transformers 1
e. Automated switches 8
f. None 4
g. Circuit breakers 1

\section{Advanced Protection Schemes}

9. Do you perform any kind of fault anticipation/ prediction on your distribution circuits?

a) Yes 3

b) No

If yes, what do you do?

i. Relay element pickup monitoring 1

ii. Periodic sampling of voltage/current waveforms 2

iii. Power quality monitoring 3

iv. Participated EPRI Fault Anticipator pilot 1

10. Have you implemented (or plan to implement) a fast feeder restoration scheme based on dedicated high-speed communications?

a) Yes 6

b) No $\quad 7$

If yes, what communication media?
i. Fiber
3
ii. Radio
4
iii. Copper
0

11. Have you implemented any special schemes for distributed generation protection based on high-speed or dedicated communications?

a) Yes 
b) No

If yes, what is the nature of this scheme?

$\begin{array}{ll}\text { i. Interlock between DG and substation } & 4 \\ \text { ii. Direct transfer trip } & 1\end{array}$

12. Have you implemented (or plan to implement) any other special purpose protection schemes involving high-speed communications for distribution systems?

a) Yes 3

b) No 10

If yes, what is the nature of this scheme?

i. Fast Wireless (GOOSE messaging protocol) on looped circuit topology

ii. Fiber for Directional Control Blocking scheme

\section{B.2.2 Fault Location and SCADA Technologies}

o Automatic fault location for distribution systems

o Advanced monitoring applications

- Data management, processing and archiving

- Disturbance monitoring for fault location, fault prediction, and equipment diagnostics

- Equipment diagnostics

\section{B.2.2.1 Questionnaire Results}

Company 3 (utility): Using advanced fault locating software throughout the medium voltage distribution feeders and developing significant enhancements and extensions.

\section{Company 4 (vendor):}

The TWACS communication technology, which uses the electric energy delivery network as its physical communication medium, is currently used by many utilities for Automatic Meter Reading and other utility functions and has the following unique characteristics:

1) All electric metering transponders can be reached by the TWACS communication system.

2) The TWACS communication path to the customer premises is the same as the phase, feeder, and bus serving the feeder electric energy delivery path to the customer premises. Hence an outage at the customer also de-energizes the metering transponder and the transponder cannot respond to an outbound command.

3) These characteristic features can be used to identify outages. Transponders responding to polling commands are assumed connected to an energized part of the electric circuit. The ones which do not respond are assumed to be connected to part of the circuit that is de-energized. This isolation is due to the disconnecting action of a protective device. 
4) The protective device works in a selective coordinated fashion with other protective devices following a hierarchical order. This method is used by utilities to isolate faults and to minimize the effects of a fault on the total network.

5) By selecting a few metering transponders beyond each protective device for polling, the outage mapping function can be accomplished through TWACS communication and polling.

Provide automatic fault location for distribution systems. Identify $100 \%$ of solid faults (i.e. the vast majority of all main line faults)

- Provide advanced monitoring applications/diagnostics to assess outages/faults and geographically map such events to the show the extent of an outage.

- A device which continuously monitors all currents for all phases at all feeders and provides intelligence to capture a fault and identify the feeder and phase(s) where the fault occurs.

- The System will be able to detect any solid fault that causes a protective device on the medium voltage to trip. This would include down to a low-voltage fault that would cause the high-side fuse of the service transformer to blow.

- Autonomous notification to the Master Station System to start Polling to quickly identify outages.

- The System will generate alarm messages that identify the specific feeder and phase(s). These alarms will trigger the System to ping to a canned group of meters for each feeder/phase identified in the alarm message.

- The overall time required to complete the outage assessment is expected to be within two to three minutes, depending on the system configuration and size.

- Additional desirable Goals / Next Phase

- Identify network switching events and autonomously update the system's communication path to all affected end-points (meters).

\section{Company 5 (utility):}

1) On 11-kV distribution systems we use vacuum circuit breakers for fault interrupter. We do not use fault current limiters on $11-\mathrm{kV}$ systems. The old electrometrical relays are being replaced by numerical relays which record the fault current of the circuit. Communicating protective relays \& advanced fault locator are still not adopted. 2) For $66 / 132 / 220 / 400-\mathrm{kV}$ transmission systems, numerical relays with fault locator are being adopted by M/s GETCO which has facility of disturbance recording for fault analysis. We are using equipment diagnostic kits for power factor measurement of transformers, CTs and VTs, Circuit breaker analyzer, thermo vision camera, leakage current monitoring of surge arresters, Sweep frequency response analysis etc. for condition monitoring of EHV class equipments.

Company 7 (utility):

1) Advanced data management and data processing for distribution monitoring systems, especially related to disturbance monitoring for fault location, fault prediction, and equipment diagnostics: 
We are still in the process of getting automated data collection installed and still using manual data collection/management/processing. As we expand our data collection capabilities we will need to implement more advanced systems, this is on our radar screen and will be addressed at the appropriate time.

2) Data warehousing of circuit information for advanced monitoring applications: We have just implemented several of the SAP suites of applications along with a new SCADA, OMS and GIS system. We are still in the process of optimizing these systems and are planning on integrating the AMI/SG data into them. In addition, we have taken a preliminary look at some of the Distribution Management Systems that are available and are planning on evaluating these in the near future. Products: The vendor that we are using for the AMI data warehousing is OSIsoft and we will be following up with them to try and integrate their system with our SCADA and OMS systems. We have also been participating in AMI@SAP.

Company 9 (utility): Respondent has its own fault location project based on voltage signature from dispersed monitors. This innovative approach to locate faults is made through a Voltage Drop-based Fault Location (VDFL) technique using remote powerquality measurement devices. This technique accurately locate faults on feeders using few decentralized PQ sensors ( 3 to 5 per feeders), providing voltage drops signatures, that could be already be part of another system such as Advanced Distribution Automation or Automatic Metering Infrastructure. VFDL is a good example of integrating an application into these advanced systems at low cost. Collaboration has been agreed between EPRI and respondent on this project. Tests are being made combining respondent and EPRI's approaches on fault location. First results will be available in 2009.

Company 10 (consultant): Their Response consists of a 127 page PDF titled "Value of Distribution Automation Applications" submitted to the California Energy Commission (CEC)

Company 11 (utility):

Automatic fault location for distribution systems:

Fault location is a future application development initiative for the respondent's IDMS project. The initial approach will be to utilize data from digital relays that are being installed on electric distribution feeder breakers and line reclosers.

Other advanced monitoring applications, such as equipment diagnostics based on monitoring:

1) The respondent's IDMS Unbalanced Load Flow analysis will be utilized to determine the power measurement parameters at each point modeled in the electric distribution system and to identify line and equipment overloads. 2) The respondent's IDMS Optimal Volt/Var Loss Management application recommends circuit reconfiguration to reduce distribution losses by a quantifiable amount with the capability, if enabled, direct Distribution SCADA control actions to controlled devices to achieve the recommended configuration. 
3) The respondent's IDMS Power Quality application utilizes harmonic content measurements to identify potential circuit conditions such as voltage distortion in excess of IEEE 519 limits, blowing of capacitor fuses from excess current due to harmonic current flow, conductor overload, voltage unbalance, etc.

4) The respondent's IDMS Contingency Analysis application analyzes various single contingencies based on the present state to determine if the contingencies can be remedied or if there will be loss of customer service for a substantial period of time.

Company 12 (research group): Advanced fault location technologies have shown promising initial results, enabling location of faults to within 100 `s of meters, even on lines of greater than $20 \mathrm{~km}$. However, the impact of DG on the accuracy of these methods has yet to be determined. In this project, the respondent investigates how DG could impact their fault location approach. If complications are encountered the development of mitigating solutions will be pursued.

\section{Company 13 (utility):}

Automatic fault location for distribution systems:

The Distribution Management System (DMS) has a fault location application that is integrated with the distribution system Supervisory Control \& Data Acquisition (SCADA), but it is currently being used in the manual method until it can be automated. Microprocessor (i.e. SEL) relays are required to determine an expected distance from the source, but fault current is retrieved when necessary and then CYME studies are run to determine approximate location. This is predominately a manual process.

Other advanced monitoring applications, such as equipment diagnostics based on monitoring:

By the second quarter of 2009, the DMS SCADA system will monitor all distribution Intelligent Electronic Devices (IED's) such as reclosers, switches, capacitor banks, and voltage regulators. The DMS will monitor status values such as open, close, and alarms; analog values such as voltage, current, watts, Vars, and VA; and remote control.

\section{Company 15 (utility):}

Automatic fault location for distribution systems:

Under the Advanced Metering Infrastructure (AMI) program, respondent will be installing smart meters that will interface with the outage management system (OMS) to use real time and historical information, including last gasp notification, to precisely assess an outage size and location. There are many events that occur in the system that are not faults but are picked up by power quality instruments. Those specific events are being examined and researched in the goal of fault anticipation. Respondent is researching the characteristics of a fault that last for two or less transient cycles and developing an algorithm to correlate that location as a possible prediction of a future fault. Other advanced monitoring applications, such as equipment diagnostics based on monitoring: 
Under the Condition Based Maintenance (CBM) program, respondent is developing hardware and software technologies that will measure the condition and performance of transformers and circuit breakers in T\&D substations to make better maintenance decisions. Diagnostic analytics will be used to detect potential problems before they occur. Respondent is working with a company in a pilot project to detect electromagnetic emissions or failure signatures caused by arcing in faulty equipment on the distribution system. The instrument detects an arc source and captures data that is analyzed to identify equipment that may potentially fail in the near future. Testing and data gathering has been conducted and locations of potentially faulty equipment have been identified. Respondent is investigating the locations of the faulty equipment. The company is also evaluating the cost effectiveness and reliability of this equipment and the associated data analysis methods.

Company 17 (utility):

Automatic fault location for distribution systems:

AMI interface to outage engine. Monitoring to enable the following real-time SCADA information.

Other advanced monitoring applications, such as equipment diagnostics based on monitoring:

GE M2 Hydran monitors being installed on select transformers + temperature monitoring to enable the following real-time SCADA information: Fault Gas reading $(\mathrm{H} 2, \mathrm{CO}$, etc.), Moisture in oil, top oil temperature, winding current reading - all three phases, winding hot spot reading - all three phases, insulation aging - all three phases, moisture content of cellulose winding insulation, bubbling temperature of insulating oil.

\section{B.2.2.2 June 2009 Survey of the Distribution Automation Working Group of the IEEE T\&D Committee (number of responses listed to the right of items)}

\section{$\underline{\text { Relay Oscillographics/Fault Data }}$}

1. Do you perform data archiving of your relay and transient recording information?
a) Yes
8
b) No
5

2. If you perform data archiving, what is the main application for the archived data?
a) Planning
b) Post-mortem analysis
13
c) Improvement of relay settings/practices
6
d) Check for proper equipment operation
e) Training

3. What other information from automated switches is of value? (circle all that apply) 
a) Fault location 11

b) Switch open/close status 12

c) Power quality 11

d) Momentary operations $\quad 12$

e) Metering 11

f) Oscillography 8

\section{B.2.3 Other Technologies/ Future Work}

\section{B.2.3.1 Questionnaire Results}

Company 7 (utility):

1) Sensors for monitoring on distribution systems:

As part of our AMI pilots, we are looking at installing some additional line monitoring to assist in determining system losses (both technical and nontechnical). The issue that we are having is that for determining losses we will need to have monitoring devices that are capable of metering accuracy. Most of the devices that we presently have out there are relay accuracy or less. Products: Presently we are using substation protective device controls (SEL \& Cooper) along with devices (LT40 LineTrackers from GridSense) out on the distribution lines. For our sub-transmission system we use MehtaTech digital fault recorders. We are also looking into MetPOD from FieldMetrics for both substation exit and line monitoring.

2) Sensors for fault indication or fault location:

We have mostly installed multi-functional sensors that are capable of determining several system conditions (voltage, load current, fault current, power factor, etc.) and as such monitoring and fault indicating would be one and the same.

Company 9 (utility):

Data Management:

Nowadays, several manufacturers offer intelligent electronic devices (IED) or intelligent controllers to improve network performance. In order to achieve these goals, the actual distribution system infrastructure (especially remotely controlled IED) shall be used to gather as much information as possible related to network, equipment and product (i.e. power quality) to improve the distribution system overall performance. Respondent is conducting several projects to qualify and quantify the type of data that can be gathered from major distribution equipment including substation digital protection relays and Distribution reclosers. A paper was presented at the IEEE /PES GM in 2008.

Telecommunications:

A study by respondent's Telecommunication department is undergoing to evaluate the most appropriate telecommunication technologies to apply to the several Distribution automation applications in the telecommunication environment. 
Company 10 (consultant): Their Response consists of a 127 page PDF titled "Value of Distribution Automation Applications" submitted to the California Energy Commission (CEC).

Company 11 (utility):

Communication approaches for monitoring systems and/or protection system implementation:

In the Distribution SCADA/Distribution Automation initiatives, the respondent utilizes MAS Communications (licensed $952-926 \mathrm{MHz}$ radio system) to support deterministic system-wide communications with distribution substations and distribution line devices

Advanced data management and data processing for distribution monitoring systems, especially related to disturbance monitoring for fault location, fault prediction, and equipment diagnostics:

The respondent's Distribution Feeder Fault Anticipation project is an EPRIsponsored multi-year project that is conducted by Texas A\&M, and utilizes digital monitoring to anticipate failures and faults before they cause outages or other reliability problems in the electric distribution system

Data warehousing of circuit information for advanced monitoring applications: The respondent's IDMS Data Archiving will support future data mining techniques to effectively manage, verify and quantify the benefits of the future Green Circuits

Sensors for monitoring on distribution systems: In the Distribution SCADA/Distribution Automation initiatives, the respondent utilizes Distribution Line-Post Sensors for Distribution line monitoring. The linepost sensor facilitates detection per phase line voltage, current, Megawatts and Megavars. The sensor outputs are used to calculate MVA and power factor on a per phase basis.

Company 13 (utility):

Communication approaches for monitoring systems and/or protection system implementation:

A licensed and unlicensed radio system is used for communicating with IED's.

Respondent is researching other communications options.

Data warehousing of circuit information for advanced monitoring applications:

Respondent is using OSI Soft Inc. PI Processbook and PI DataLink for data warehousing of circuit information.

Sensors for monitoring on distribution systems:

Reclosers and motorized gang operated switches will monitor current and voltage levels. Power Quality meters will monitor feeders. Manual fault locators are placed on circuits suspected of having problems.

Company 14 (utility):

Sensors for monitoring on distribution systems:

Tried Lyndsey current/voltage sensors

Sensors for fault indication or fault location: 
Tried Lyndsey current/voltage sensors

Company 15 (utility):

Communication approaches for monitoring systems and/or protection system implementation:

Respondent is installing a pilot project in 2008 with a scope of 5000 Smart Meters in a particular community. In residential communities, the smart meters can communicate the location of a fault faster than the current "call-in" method where SCADA is not present. Smart meters will also have the ability to communicate its time based energy consumption and demand data which can be utilize to monitor system demand and resource availability. Respondent uses SCADA that has a communication infrastructure that operates on a $900 \mathrm{MHz}$ channel. A SCADA switch would send status and analog data to the remote terminal unit (RTUs), which then converts the data to digital data, and sends it to the supervisory control station via a $900 \mathrm{MHz}$ channel. In return, the supervisory station is capable of communicating control commands to the SCADA switch via the same medium. Advanced data management and data processing for distribution monitoring systems, especially related to disturbance monitoring for fault location, fault prediction, and equipment diagnostics:

Respondent is using data from PQ instruments to predict future faults by analyzing patterns of events leading up to a fault. The data is loaded into an application developed by respondent and EPRI to perform applications such as coding system disturbances, analyzing specific customer/system issues when prompted by a user, identifying and supporting additional monitoring sites, and generating a monthly and yearly analysis of the distribution system performance. Respondent is developing a new OMS/DMS tool that will provide more detailed information about customers affected by outages and automatically calculate reliability statistics for the outage. The new OMS will be capable of interfacing with smart meters to utilize real time and historical information to precisely assess outage size and location. This will expedite the determination of outage causes and eliminate the confusion that arises when multiple faults occur. The new DMS tool will allow scenario analysis, and it will generate switching orders to support planned or unplanned outages.

Company 16 (vendor): Currently designing and modeling a Saturable core High temperature superconducting Fault current Limiter

Company 17 (utility):

Communication approaches for monitoring systems and/or protection system implementation:

Utilize $900 \mathrm{MHz}$ Spread Spectrum radio systems interfacing with SCADA. Mesh networks and directional communication schemes with preference for mesh dynamic routing. Data warehousing of circuit information for advanced monitoring applications: 
Utilization of AMI products for time interval loading and voltage information as well as momentary/sustained outage reporting storage of this information for circuit studies and importing into various applications.

Sensors for monitoring on distribution systems:

Implementing remote monitoring and control of distribution capacitors using radio or digital cellular communications.

Sensors for fault indication or fault location:

Fault detection devices manufactured by Cooper Power Systems and Schweitzer are in the process of being field tested. Fault indication is provided via digital cellular interface.

\section{B.2.3.2 June 2009 Survey of the Distribution Automation Working Group of the IEEE T\&D Committee (number of responses listed to the right of items)}

\section{$\underline{\text { Communications }}$}

1. What type of communications technologies do you currently use to exchange information with distribution circuit elements (switches, capacitor banks, fault indicators, etc.)?
a) Phone (Modem)
6
b) Cell
c) RF unlicensed
13
d) RF licensed
e) Fiber
f) Power line carrier
g) Broadband over power line
h) Satellite systems
i) Pager
j) Microwave

2. What type of communications technologies are you planning to use to exchange information with distribution circuit elements in the near future?
a) Phone (Modem)
b) Cell
c) RF unlicensed
d) RF licensed
e) WiMax
f) Fiber
6
g) Power line carrier
h) Broadband over power line
i) Satellite systems
j) Ongoing study

3. What types of devices in use are time synchronized (by GPS clocks or other means? (circle all that apply)

a) Relays 
b) Transient recorders 3

c) Revenue meters 3

d) Phasor measurement units 3

e) None 5

f) Other 1

g) RTU 3

h) Line sectionalizers 1

\section{Fault Current Limiting}

4. What issues related to high fault currents do you have on distribution systems?
a) Exceeding circuit breaker rating
b) Voltage sag
5
c) Equipment damage
d) Contribution of distributed generation
e) None
f) Fire hazard
g) Exceeding fuse cutout ratings

5. Do you use or plan to use fault current limiters for distribution systems?
a) Yes
b) No
If yes, where in the system and at what voltage?
i. At the beginning of the $12-\mathrm{kV}$ feeder
ii. 24-kV bus tie
iii. Subtransmission
iv. On the network systems
v. On the $34.5-\mathrm{kV}$ side of the distribution transformer 1

\section{B.3 Actual Survey Request Letter Sent to Potential Respondents:}

Southern California Edison (SCE) and the Electric Power Research Institute (EPRI) have both received funding from the DOE to work on new technologies for advanced fault location, prediction and detection for distribution systems. As part of this project, we would like to know what advanced techniques you are developing, using or plan to use in the near future.

SCE's DOE project focuses on implementing advanced protection systems on our Circuit of the Future in 2007 - 2009. This project involves the use of fast fault location and isolation using vacuum fault interrupters and communicating protection relays. A second phase (2008 - 2009) will look at what changes need to be made to the protection scheme when a fault current limiter is installed upstream of the circuit. The final phase (2008 2009) will implement an advanced fault location and isolation system based on literature research and promising technologies determined through this survey. SCE's partners on this project are KEMA and Virginia Tech. 
EPRI's DOE project focuses on development of a National Disturbance Library that can be used for development of advanced algorithms and techniques for intelligent monitoring applications. The project is collecting monitoring data from utilities from around the country and developing a library of events and monitoring results that include documentation of effects and the electrical system characteristics. The project includes documentation of existing approaches for intelligent monitoring applications.

This survey will compile a listing of various projects and developments being undertaken by utilities, vendors and research organizations. Please help us by providing a summary of your work and contact information for projects or products in the following categories (please include information even if the project is in the planning stages):

- Advanced protection for distribution systems

- Integration of adaptive protection with distribution automation

- Automatic fault location for distribution systems

- Other advanced monitoring applications, such as equipment diagnostics based on monitoring

- Communication approaches for monitoring systems and/or protection system implementation

- Advanced data management and data processing for distribution monitoring systems, especially related to disturbance monitoring for fault location, fault prediction, and equipment diagnostics

- Data warehousing of circuit information for advanced monitoring applications

- Sensors for monitoring on distribution systems

- Sensors for fault indication or fault location

Any observations, thoughts, or experiences with these technologies are welcome. We will incorporate the information you send for the project to guide the definition of the state-of-the-art. As a token of our appreciation, we will provide you the results of this survey. To protect your confidentiality needs, SCE/EPRI/KEMA/Virginia Tech will not identity the responses by company; however, a list of the companies responding to this request will be included in the summary report. If a company prefers not to be named, please indicate this and your request will be honored.

Please send your responses to S.S. (Mani) Venkata at KEMA as early as possible. His contact information is provided below:

S. S. (Mani) Venkata, Ph. D., P. E.

KEMA Inc.

Mobile: 520-820-8005

Tel: $\quad 520-797-1161$

Fax: $\quad 520-797-1161$

E-mail: ss.venkata@kema.com mailto:ss.venkata@kema.com 
We thank you for your participation in this survey and will forward the survey summary to you within 6 weeks of the closing of this survey.

Sincerely
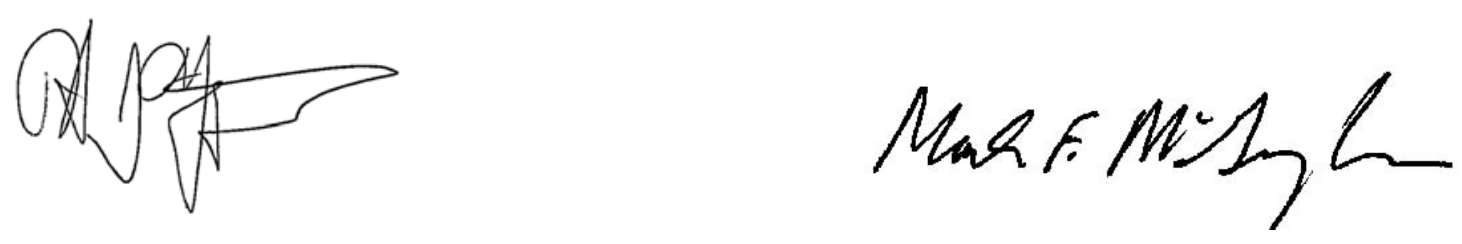

Robert J. Yinger

Mark McGranaghan

Project Manager,

Transmission and Distribution Business Unit

Southern California Edison

Director,

Distribution, PQ, and Intelligrid

Electric Power Research Institute

626-302-8952

\section{B.4 Actual Survey Questionnaire Sent to DA Working Group of the IEEE PES T \& D Committee}

Feeder Automation

1. Types of distribution line devices used to interrupt and sectionalize faults?

(Circle all that apply)

h) Hydraulic reclosers

i) Automatic line sectionalizers

j) Electronic reclosers

k) SCADA-controlled load break switches

1) Remotely controlled vacuum interrupters

m) Other

2. What are approaches for which automatic switches are currently applied? (circle all that apply)

h) Centrally-controlled sectionalizing scheme via SCADA

i) Normally-open tie points

j) Distributed control strategy with SCADA supervision

k) Local control strategy with no communications

l) None

m) Other

3. Key issue with deployment of automated switches? (circle all that apply)

1) SCADA Integration

m) Cost Justification

n) Lack of Communications

o) Additional Maintenance

p) None 
q) Other

4. What approaches are being considered for future automation schemes? (circle all that apply)

f) Centrally-controlled sectionalizing scheme via SCADA

g) Normally-open tie points

h) Distributed control strategy with SCADA supervision

i) Local control strategy with no communications

j) None

k) Other

\section{Line Sensors/Monitoring}

5. What types of sensors do you use on your distribution feeders?
g) Line voltage sensors
h) Power quality monitors
i) Line current sensors
j) Fault current indicators
k) Conductor temperature monitors
1) None

m) Other

6. What types of sensors are you planning/considering for use on your distribution feeders?

h) Line voltage sensors

i) Power quality monitors

j) Line current sensors

k) Fault current indicators with communication

1) Conductor temperature monitors

m) None

n) Other

7. What type of fault location techniques do you currently use on your distribution circuits? (circle all that apply)

h) Fault current indicators on overhead

i) Fault current indicators on cable

j) Fault current indicators with communications

k) Fault location embedded in relay

1) Fault indicators embedded in automated switches

m) Fault location based on SCADA input

n) Other

8. What distribution field equipment do you monitor for equipment diagnostics? (circle all that apply)

a. Capacitor banks

b. Regulators 

c. Reclosers
d. Customer distribution transformers
e. Automated switches
f. None
g. Other

\section{Communications}

9. What type of communications technologies do you currently use to exchange information with distribution circuit elements (switches, capacitor banks, fault indicators, etc.)? (circle all that apply)

k) Phone (Modem)

1) Cell

m) RF unlicensed

n) RF licensed

o) Fiber

p) Power line carrier

q) Broadband over power line

r) Satellite systems

s) Other

t) None

10. What type of communications technologies are you planning to use to exchange information with distribution circuit elements in the near future? (circle all that apply)

k) Phone (Modem)

1) Cell

m) RF unlicensed

n) RF licensed

o) WiMax

p) Fiber

q) Power line carrier

r) Broadband over power line

s) Satellite systems

t) Other

11. What types of devices in use are time synchronized (by GPS clocks or other means? (circle all that apply)

i) Relays

j) Transient recorders

k) Revenue meters

1) Phasor measurement units

m) None

n) Other 
12. Do you perform data archiving of your relay and transient recording information?
c) Yes
d) No

13. If you perform data archiving, what is the main application for the archived data?

f) Planning

g) Post-mortem analysis

h) Improvement of relay settings/practices

i) Check for proper equipment operation

j) Training

k) Other

14. What other information from automated switches is of value? (circle all that apply)

g) Fault location

h) Switch open/close status

i) Power quality

j) Momentary operations

k) Metering

1) Oscillography

m) Other

\section{Advanced Protection Schemes}

15. Do you perform any kind of fault anticipation/ prediction on your distribution circuits?

c) Yes

d) No

If yes, what do you do?

i. relay element pickup monitoring

ii. Periodic sampling of voltage/current waveforms

iii. power quality monitoring

iv. other

16. Have you implemented (or plan to implement) a fast feeder restoration scheme based on dedicated high-speed communications?

c) Yes

d) $\mathrm{No}$

If yes, what communication media?
i. Fiber
ii. Radio
iii. Copper
iv. Other 
17. Have you implemented any special schemes for distributed generation protection based on high-speed or dedicated communications?

c) Yes

d) No

If yes, what is the nature of this scheme?

18. Have you implemented (or plan to implement) any other special purpose protection schemes involving high-speed communications for distribution systems?

c) Yes

d) $\mathrm{No}$

If yes, what is the nature of this scheme?

\section{Fault Current Limiting}

19. What issues related to high fault currents do you have on distribution systems?

h) Exceeding circuit breaker rating

i) Voltage sag

j) Equipment damage

k) Contribution of distributed generation

1) None

m) Other

20. Do you use or plan to use fault current limiters for distribution systems?

c) Yes

d) No

If yes, where in the system and at what voltage? 


\section{Appendix C: $\quad$ Fault Current Limiter Models}

The increasing demand for electric power has put great pressure on our generation system and consequently the transmission and distribution systems. With extra loads being added to existing lines, fault current levels have exceeded the maximum shortcircuit ratings of switchgears upstream. As a consequence, there is an increased mechanical and electrical stress on power system components like generators, transformers, transmission lines and loads. Also, excessive fault currents are often accompanied by dangerous electric arcs that can be hazardous to the equipment and people. Fault current limiters are unique devices that not only reduce the levels of fault currents but also enhance power system stability. This report gives an overview of the fault current limiter, and some of the available technologies. A rather new kind of fault current limiter known as the superconducting fault current limiter has been researched and results have been presented.

\section{C.1 Introduction}

Fault current limiters are power devices that can limit prospective fault currents to a lesser value. In principle, an ideal FCL has:

- Instantaneous detection of faults

- Rapid reduction of a fault current to a desirable percentage of original current

- Virtually in existent during steady state and can operate up to the $15 \mathrm{kV}$ distribution voltage class. This means that there must be zero voltage drop and impedance.

- Capable of handling multiple faults within a short time frame

- Auto recovery of the device so that no human intervention is required

- Compact, lightweight package that is reliable, maintenance free and economical to deploy and manufacture.

\section{C.2 Types of FCL}

In the past, many approaches to the FCL design have been conducted ranging from the very simple to complex designs. However, they have been very slow in responding to faults and take in the order of a few seconds to limit lethal fault current. Below are a few kinds of FCL. A detailed description can be found in the mid term report.

\section{C.2.1 Passive Limiters}

The simplest of all kinds of fault current limiter is the inductor. The current limiting strategy is achieved by inserting impedance $Z=j \omega L$. Now since current cannot change instantaneously in an inductor, current is therefore limited. Figure C.1 shows an inductor. 


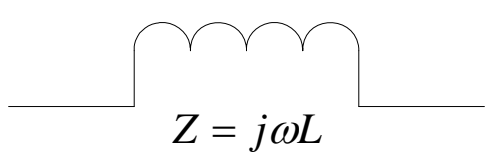

Figure C.1: An inductor

\section{C.2.2 Super conducting limiters}

Super conducting limiters are one of the latest kinds of limiters using the properties of electrical wires when cooled to $0 \mathrm{~K}$. Most of the current research has been directed towards this kind of limiter. Below are a few pros and cons:

- Current limiting potential by $50 \%$ and very fast in response

- Virtually no voltage drop across the line, BUT

- High cooling losses lead to regular maintenance

- Economical cooling techniques still under development

- Reliability is still questionable

We will explore the modeling of this limiter in greater detail during the later part of this report.

\section{C.2.3 Solid state based limiter}

These kinds of limiters typically use power electronic switches to do the current limiting. There are various configurations in which these limiters can be designed by using inductors, capacitors and thyristor or SCR. One of the possible configurations has been shown in Figure C.2.

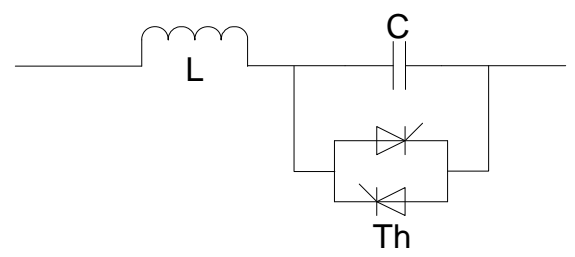

Figure C.2: A solid state based resonant limiter

\section{C.2.4 Hybrid Limiters}

These types of limiters use a combination of solid state switches and conventional mechanical switches to achieve current limitation. They are capable of handling multiple faults repeatedly and with the advent of high power switches, they can be used to protect up to the $15 \mathrm{kV}$ voltage class.

A longer list of FCL's is presented in the Appendix A of this report. 


\section{C.3 SFCL modeling}

\section{C.3.1 System Overview}

The system that Virginia Tech is working on is the "Circuit of the Future" distribution system which is operated by Southern California Edison (SCE) as shown by the one line diagram in Figure C.3. This is the Avanti 08 system. The key parameters are:

- Roughly 30 busses

- $12.5 \mathrm{kV}$ system voltage at $60 \mathrm{~Hz}$

- 21 loads connected with each load rated at roughly $350 \mathrm{kVA}$

- Single source of power from a $115 / 12.5 \mathrm{kV}$ transformer

- A network consisting of underground and overhead lines.

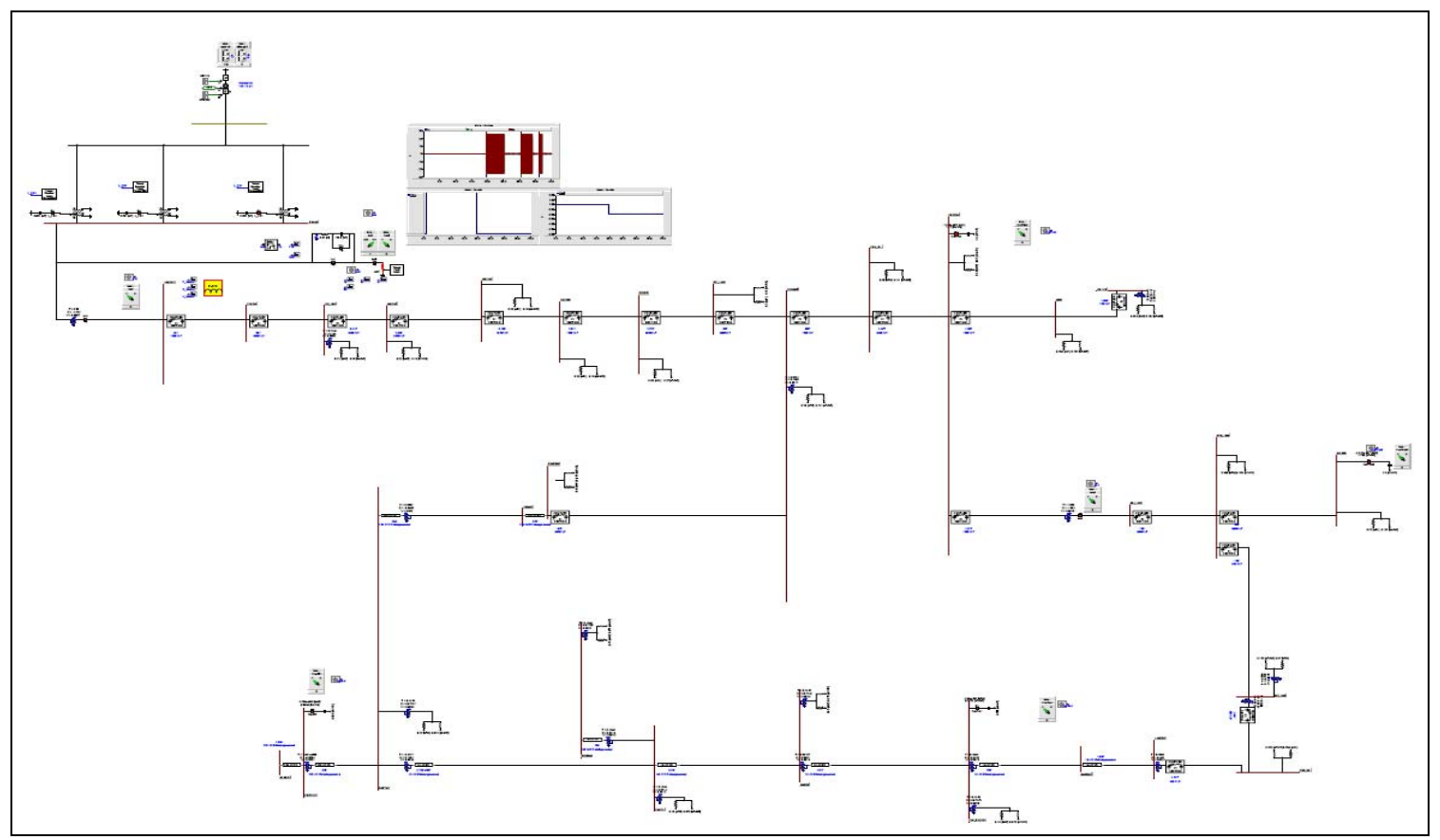

Figure C.3: One-line system diagram of Circuit of Future

\section{C.3.2 Super conducting fault current limiter}

In this section we will present a detailed description on the modeling of super conducting fault current limiter.

\section{C.3.2.1 Operation Principle}

The basic idea of a super conducting fault current limiter, here after known as SFCL, is as follows,

At $t<T_{\text {FAULT }}$, the impedance of the SFCL is close to $0 \Omega$ 
At $t>T_{\text {FAULT }}$, the impedance of the SFCL rises sharply within a few seconds.

From a simulation and modeling perspective, an SFCL is simply a variable resistor. However, the important thing to model from a simulation standpoint is the automatic detection of fault. By automatic detection, we mean that there is no external trigger or relay to the SFCL to start its operation. The triggering of SFCL is purely a function of the line quantities and its appropriate manipulation to predict a fault situation.

\section{C.3.2.2 Fault detection}

As noted earlier, fault detection is perhaps the single most important ingredient in modeling a SFCL. There are essentially two ways to automatically detect faults:

The easiest method to check for a fault situation is to monitor the magnitude of line current. The other alternative is to check for the rate of change of current. Although, either one of the above approaches can be used, however each of them has certain limitations which are as follows:

- If only the magnitude is used as a detection technique, the SFCL might be falsely triggered in cases when there is an inrush current due to heavy loads coming online, which is not a fault by any means.

- If only the rate of change of current is used, we might not necessarily have a fault condition.

\section{C.3.2.3 SFCL Activation and operational logic}

In order to model a robust FCL triggering mechanism, a systematic approach has been taken to design which is shown in Figure C.4. 


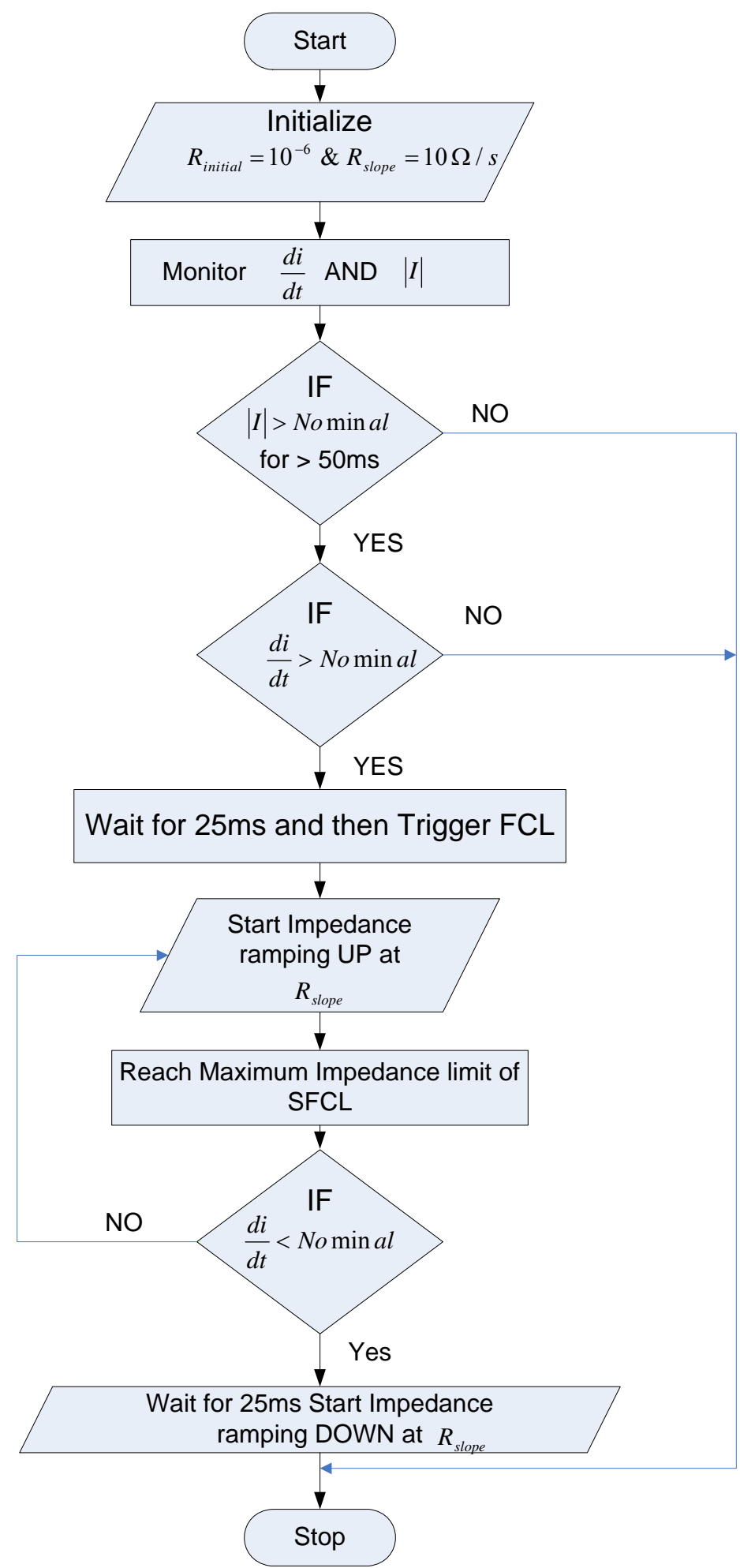

Figure C.4: SFCL Activation and operational logic

\section{Explanation of the flowchart (figure 4):}


Before starting simulation, the SFCL is initialized with an initial impedance value $R_{\text {initial }}$ and a ramp rate $R_{\text {slope }}$. The simulation is then started and current magnitude AND the rate of change of current is monitored during the entire simulation time. After achieving some steady state operating point a three phase-to-ground fault is inserted into the system external to the SFCL. As soon as the fault is inserted, the magnitude of the line current jumps up instantaneously and achieves a new steady state value as long as the fault is inserted. The same thing holds true for the rate of change of current. However, in order to make sure that there is indeed a fault in the system and we are not falsely triggering the $\mathrm{SFCL}$, a $50 \mathrm{~ms}$ ON delay timer is used. Along with checking for the magnitude, the rate of change of current is also monitored. A fault will change the rate instantaneously. Once again, a $25 \mathrm{~ms}$ ON Delay timer is used to make sure there is a fault.

After setting the necessary flags, the SFCL impedance is ramped up at a rate specified by the user. As long as the fault is present, the impedance will keep increasing till a certain value which is a function of the physical properties and design of the SFCL. After the fault is cleared a $25 \mathrm{~ms}$ delay is used and the SFCL impedance will be ramped down to the initial impedance. This cycle takes place whenever there is a fault.

\section{C.3.2.4 Implementation Methodology}

In this section the implementation methodology is presented in order to achieve the functionality as per figure 4 flowchart.

\section{Magnitude:}

There are two ways to monitor a magnitude:

Take the root mean square (RMS) of the line current

Take the phasor value of the current and extract the magnitude

Any one of the above ways is acceptable. Since, $\operatorname{PSCAD}^{\circledR}$ has a built-in function for RMS, option 1 has been used.

\section{Rate of change:}

From basic three phase circuits we know that in steady state, the area covered under a curve by a sinusoid over one cycle should equate to zero or very close to zero. However, if the area under the curve is NOT equal to zero we can conclude that there might be an impending fault situation.

PSCAD $^{\circledR}$ has a built-in integration function which will check for the rate of change of current and the area under the curve.

\section{SFCL impedance ramping}

Programming the impedance ramping in the PSCAD ${ }^{\circledR}$ environment was perhaps the most challenging task. In general the equation for a ramp is given as follows: 


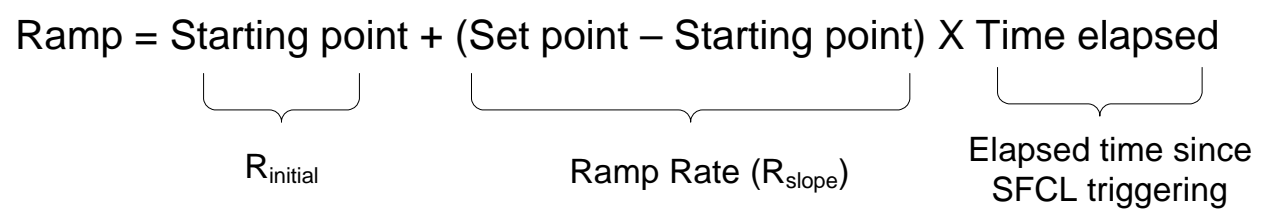

From (C.1) we see that the impedance of the SFCL increases linearly with time. The ramp rate is user configurable which is a function of the SFCL physical properties and design.

At this point it must also be noted that the impedance is clamped to a final value, after which it will not increase any further. This is to ensure that the SFCL is modeled more realistically.

\section{C.3.2.5 FCL activation timing diagram}

Figure C.5 shows the SFCL activation timing diagram from the time the fault occurs to the time the SFCL impedance is actually ramped up and down.

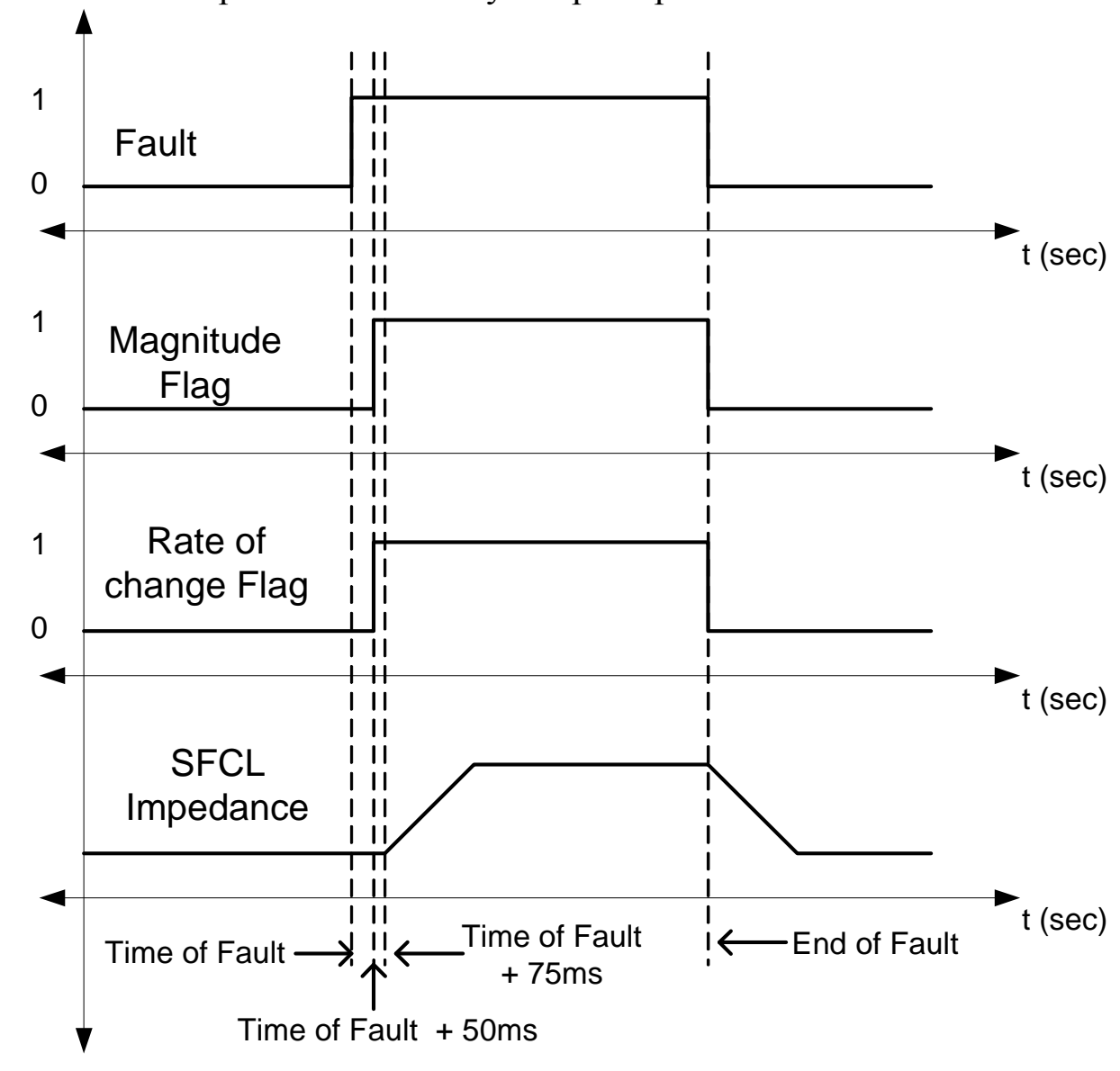

Figure C.5: SFCL activation timing graph 
In Figure C.5 we see that it takes about $75 \mathrm{~ms}$ after the time of fault for the SFCL to activate. This is because, while simulating, it is important that we confirm there is indeed a fault and also to avoid false triggering. At the end of the fault, the impedance is slowly ramped down to steady state value.

It must be noted that the time of SFCL activation is user configurable.

\section{C.3.2.6 Simulation test bed}

The simulation test parameters used are as per the following:

Table C.1: Overview of simulation test parameter

\begin{tabular}{|l|l|}
\hline Parameter & Value \\
\hline Total simulation time & $20 \mathrm{sec}$ \\
\hline Time of fault & $5 \mathrm{sec}$ \\
\hline Duration of fault & $5 \mathrm{sec}$ \\
\hline SFCL placement & Beginning of distribution system \\
\hline Fault insertion & $1022 \mathrm{ft}$ from SFCL \\
\hline Type of fault & Three phase-to-ground \\
\hline Sampling rate & $1 \mathrm{kHz}$ \\
\hline
\end{tabular}

\section{C.3.2.7 Simulation results}

In this section, simulation results are presented along with their explanations. First, simulation results without the SFCL are presented and then with the SFCL are shown.

\section{Results without SFCL}

\section{A) Prospective fault current and line current:}

Figure C.6 shows the waveforms for the fault and line current without the SFCL. 


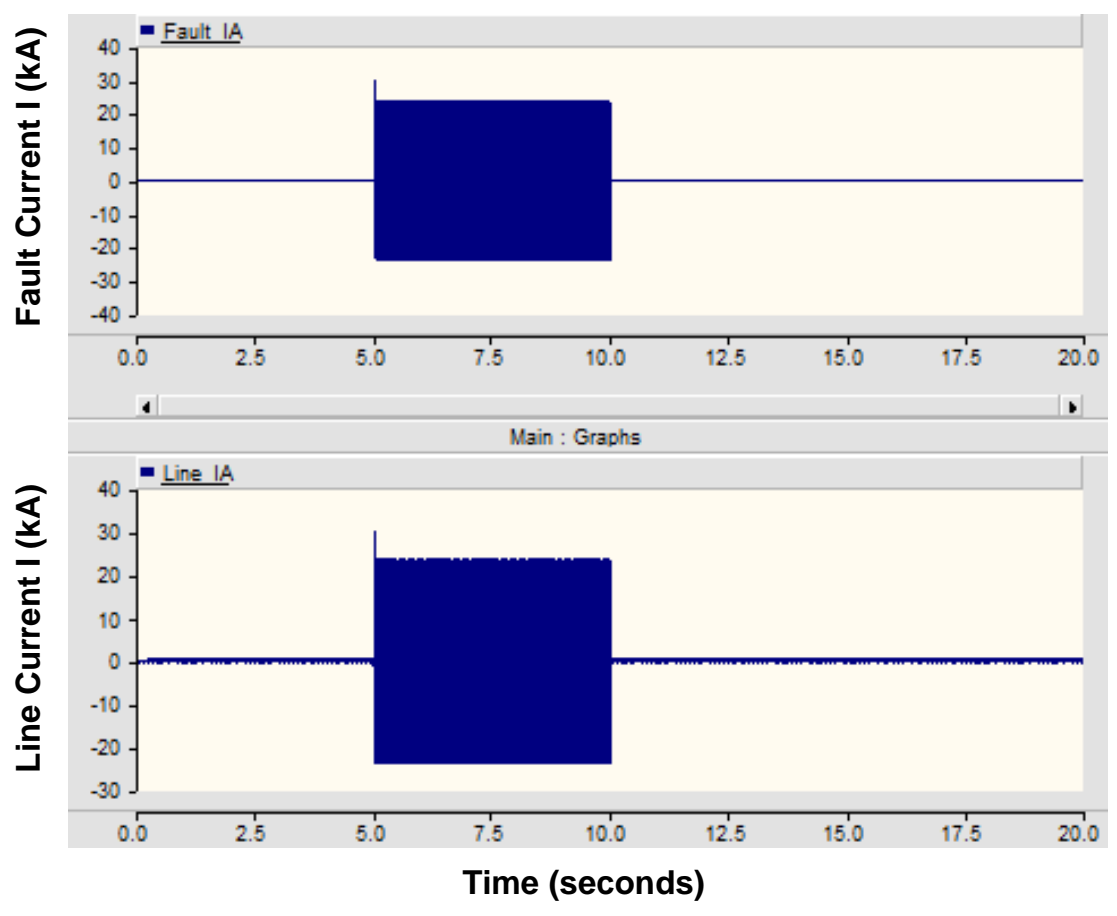

Figure C.6: Fault and Line current without SFCL.

It can be seen that in steady state the fault current is zero. The nominal line current is about $410 \mathrm{~A}$. However, the moment a fault is inserted into the system, the line current jumps to $29.7 \mathrm{kA}$ before stabilizing to the faulted state current of $23.2 \mathrm{kA}$. This is about 56 times the nominal operating current. Figure C.7 shows a zoomed in view for the first couple of cycles after the fault is inserted.

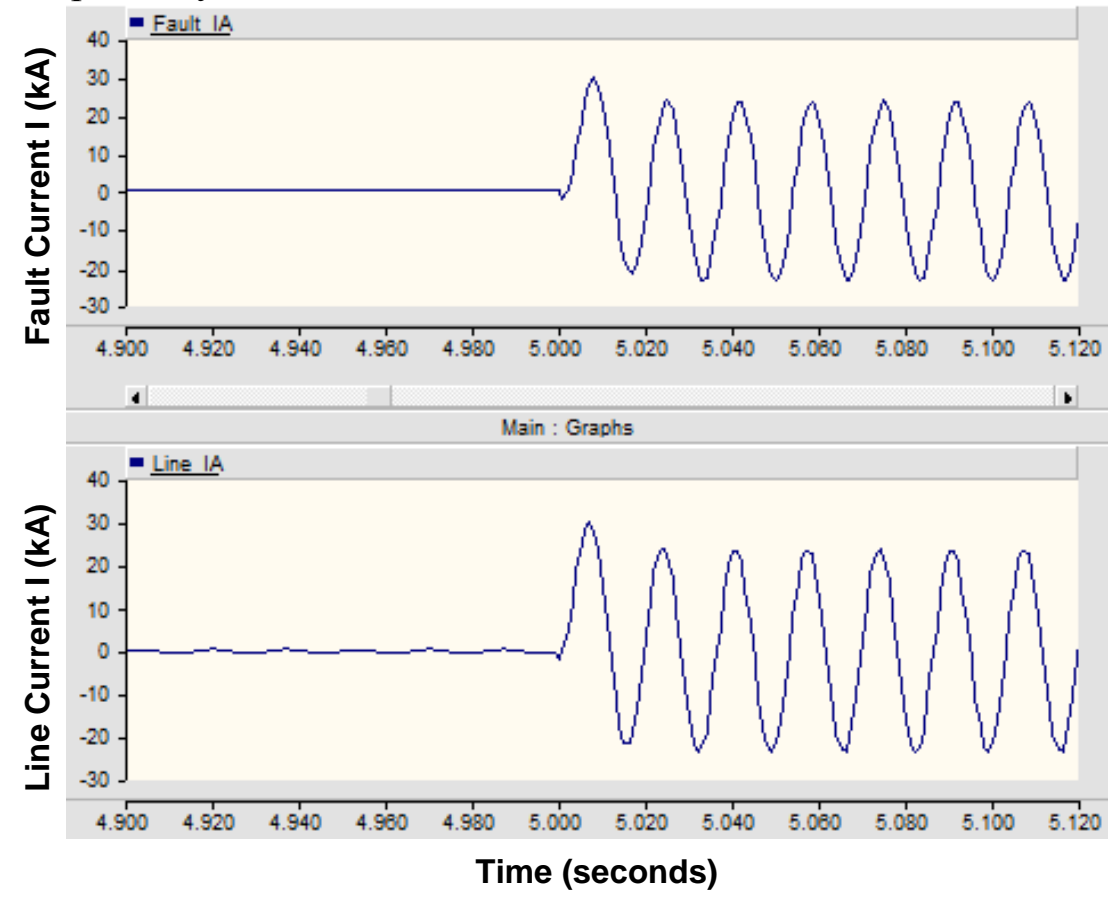

Figure C.7: Zoomed to show fault and line current without SFCL. 


\section{B) Prospective current magnitude and rate of change of current without SFCL}

Figure C.8 shows the waveforms for the current magnitude and rate of change of current

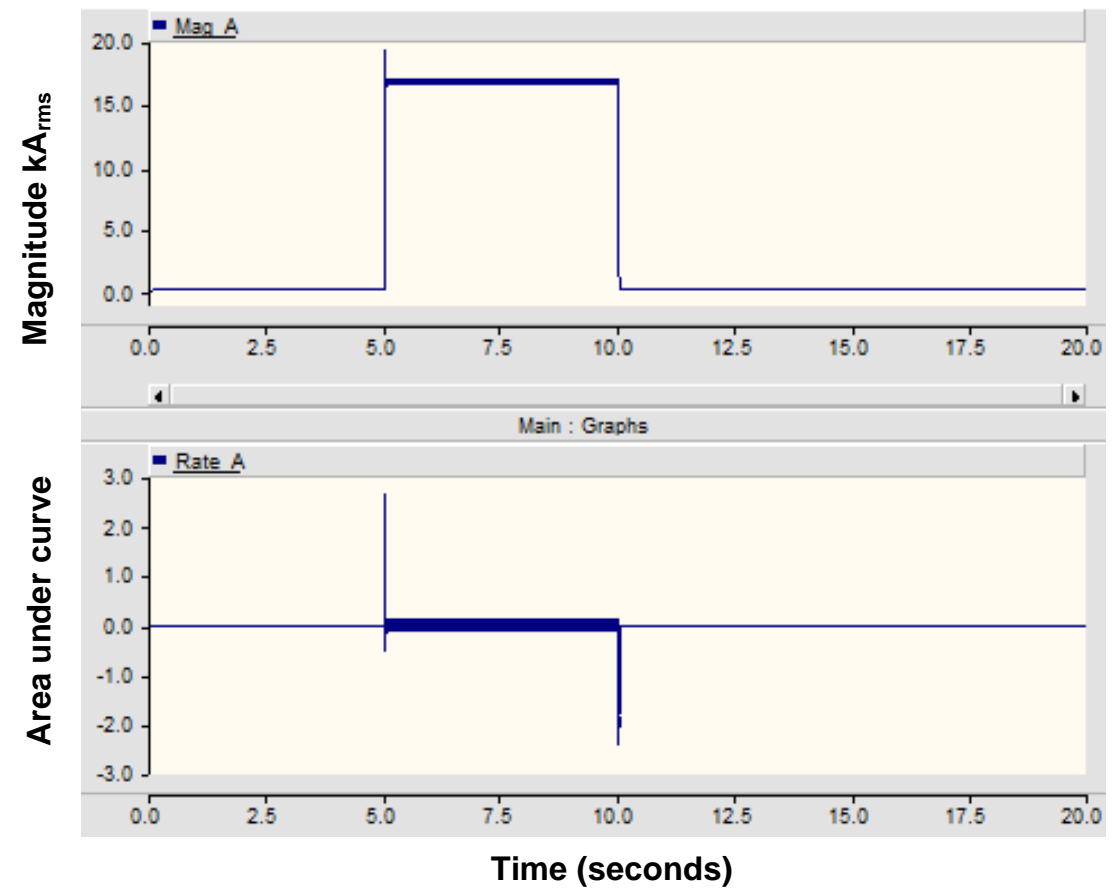

Figure C.8: Current magnitude and rate of change of current without SFCL

From Figure C.8 it can be inferred that the steady state magnitude is about 290A (not clearly shown in the graph). However, the moment a fault is inserted into the system, the current magnitude jumps roughly $19 \mathrm{kA}_{\mathrm{rms}}$ before stabilizing to the faulted state RMS current of $16.9 \mathrm{kA}_{\mathrm{rms}}$.

Also, from Figure C.8, the area under the curve, which is a function of the rate of change of current, is also zero. But when a fault is inserted, the rate changes instantaneously before settling down to a faulted state value. We would expect the area under the curve to equate to zero during the faulted state, however, we see that there is a small ripple during the faulted time. This is because, the area under the curve is being calculated from the RMS value and from Figure C.9, and there is small ripple in the current magnitude also which is reflected in the area calculations. 


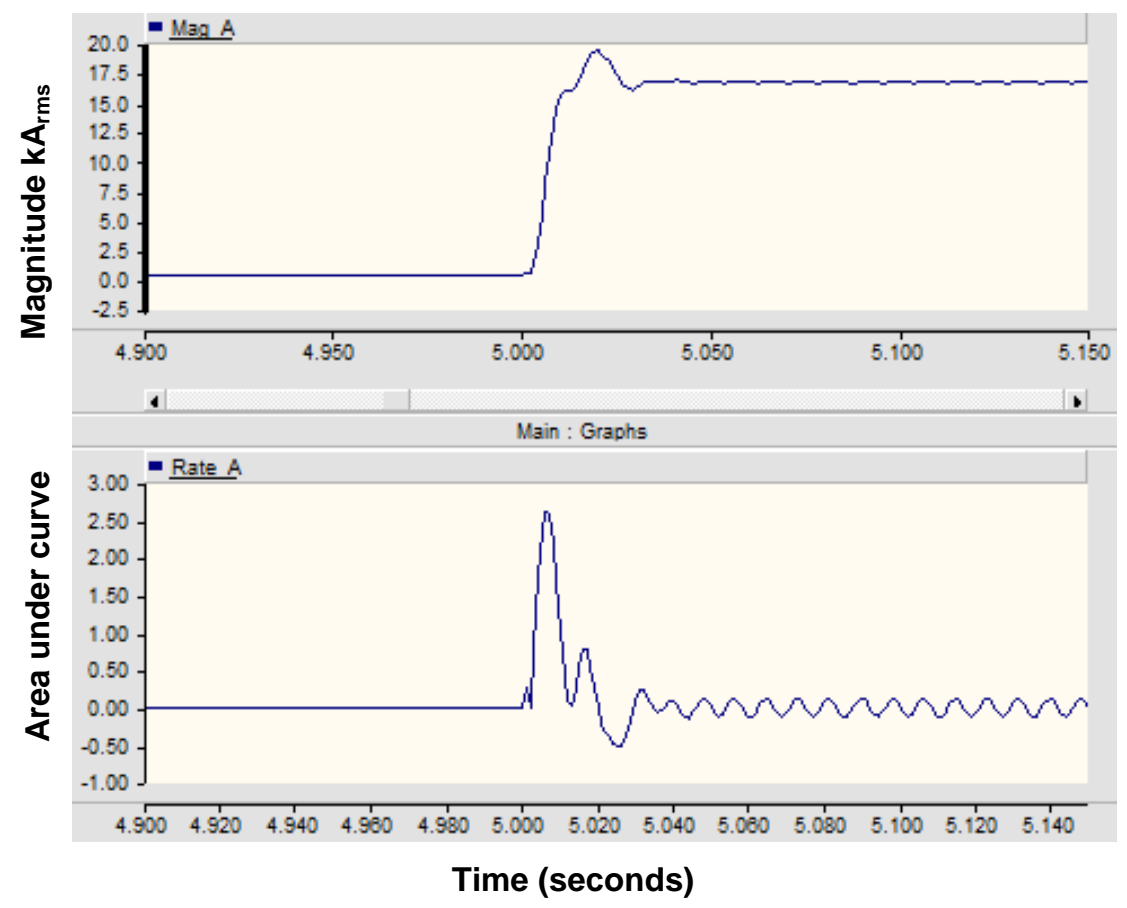

Figure C.9: Zoomed to show current magnitude and rate of change of current without SFCL

\section{Results of SFCL impedance}

In section C.3.2.3, it was noted that the impedance of the SFCL will be ramped up steadily when the fault occurs as a user specified rate. Similarly it will be ramped down once the fault is removed. Figure C.10 shows the SFCL impedance diagram.

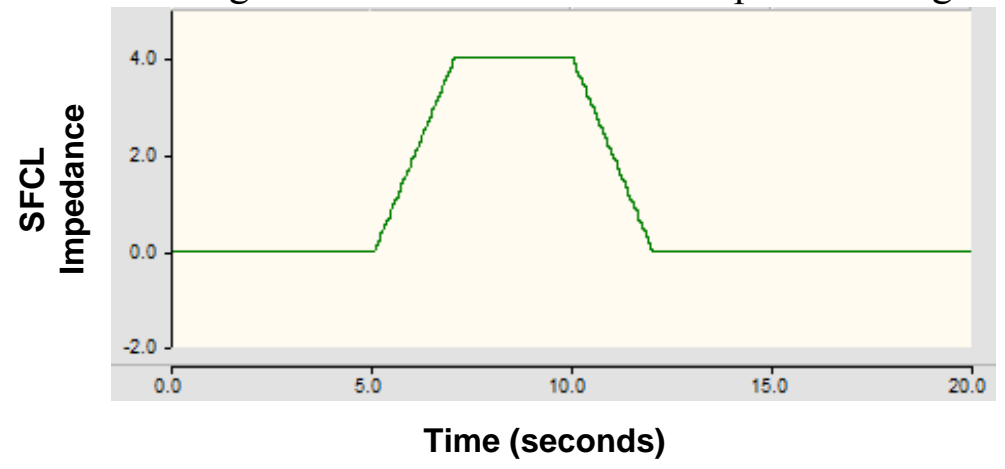

Figure C.10: SFCL Impedance diagram

An important note in the figure is that the impedance of the SFCL is clamped at a certain value even if the fault is still present in the system. This impedance limit is a function of the physical properties and design of the actual SFCL. This limit is also user settable.

Figure C.11 shows the timing of FCL triggering. The expected delay is $75 \mathrm{~ms}$ $(50 \mathrm{~ms}+25 \mathrm{~ms})$. However, from Figure C.11, the delay is roughly $82 \mathrm{~ms}$. This is mainly due to the fact of the simulation time step in PSCAD®. A shorter time step has shown to reduce this delay, however, there are PSCAD ${ }^{\circledR}$ limitations to this. This extra delay of 
$7 \mathrm{~ms}$ can be compensated by either reducing the delay in current magnitude or the rate of change of current.

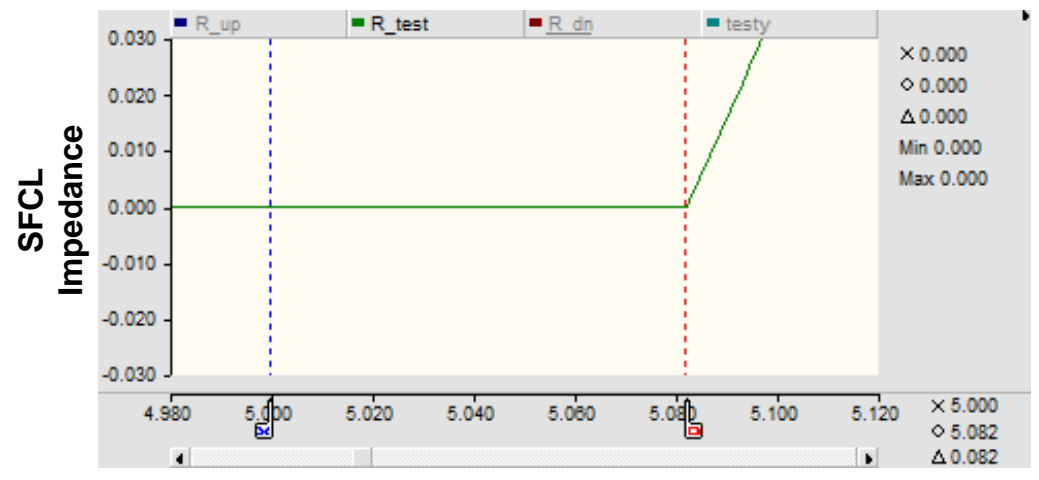

Time (seconds)

Figure C.11: SFCL Impedance diagram

\section{Results with SFCL:}

\section{A) Line current:}

Figure C.12 shows the line current with the SFCL in operation.

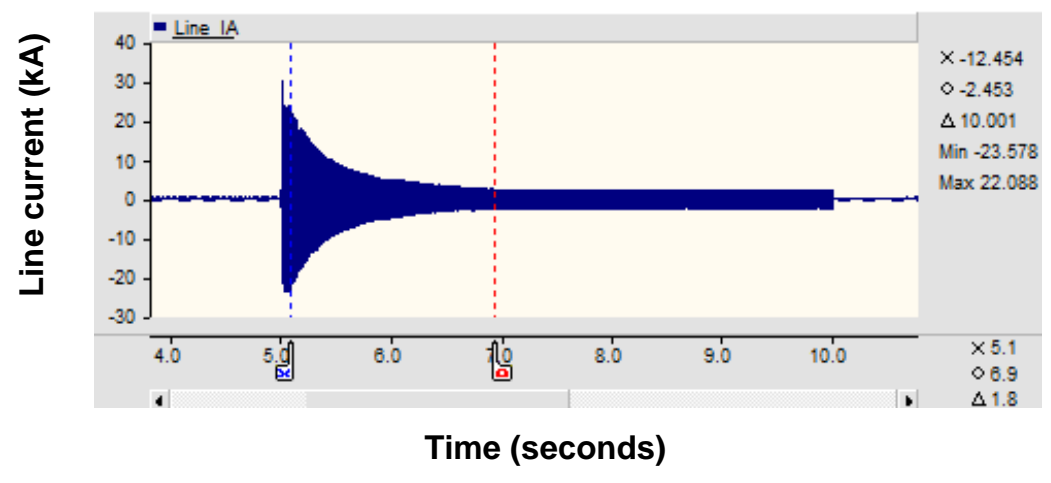

Figure C.12: Line current with SFCL

From Figure C.12, it can be seen that after the fault is inserted, the SFCL starts acting after a set delay and an acceptable line current (based on user) is achieved within 1.8 seconds of the fault. This value can be adjusted by changing the impedance ramp-up rate. The steady state faulted line current achieved by this FCL is about $2.3 \mathrm{kA}$. This is about 6 times the nominal current and a $90 \%$ reduction in current if the SFCL would not have been used. Although, achieving 90\% limitation in real life is nearly impossible, but this would be a good illustration example. Figure C.13 shows a zoomed in view of the line current. 


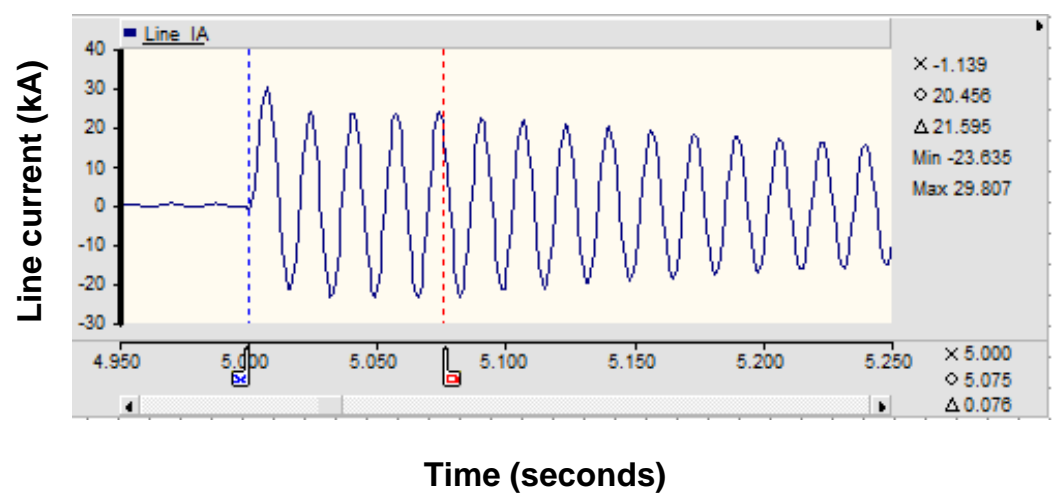

Figure C.13: Zoomed in to show the line current with SFCL

By looking at Figure C.13, it can be seen that the fault current is NOT limited for the first $75 \mathrm{~ms}$. This is as per expectation, because there is a $75 \mathrm{~ms}$ delay before which the SFCL is triggered. After $75 \mathrm{~ms}$, the line current starts to reduce as long as the fault is present.

\section{Current magnitude and rate of change of current}

Figure C.14 shows the current magnitude and area under the curve graphs. As with the line current, the current magnitude also starts to decrease when the SFCL impedance is inserted. The current magnitude gradually decreases until the steady state faulted magnitude it achieved. This is found to be roughly $1.6 \mathrm{kA}_{\mathrm{rms}}$.

Similar arguments can be applied to the rate of change of current. During the steady faulted state the area is close to zero which we expect. The reason for small ripple was explained earlier in this section. 


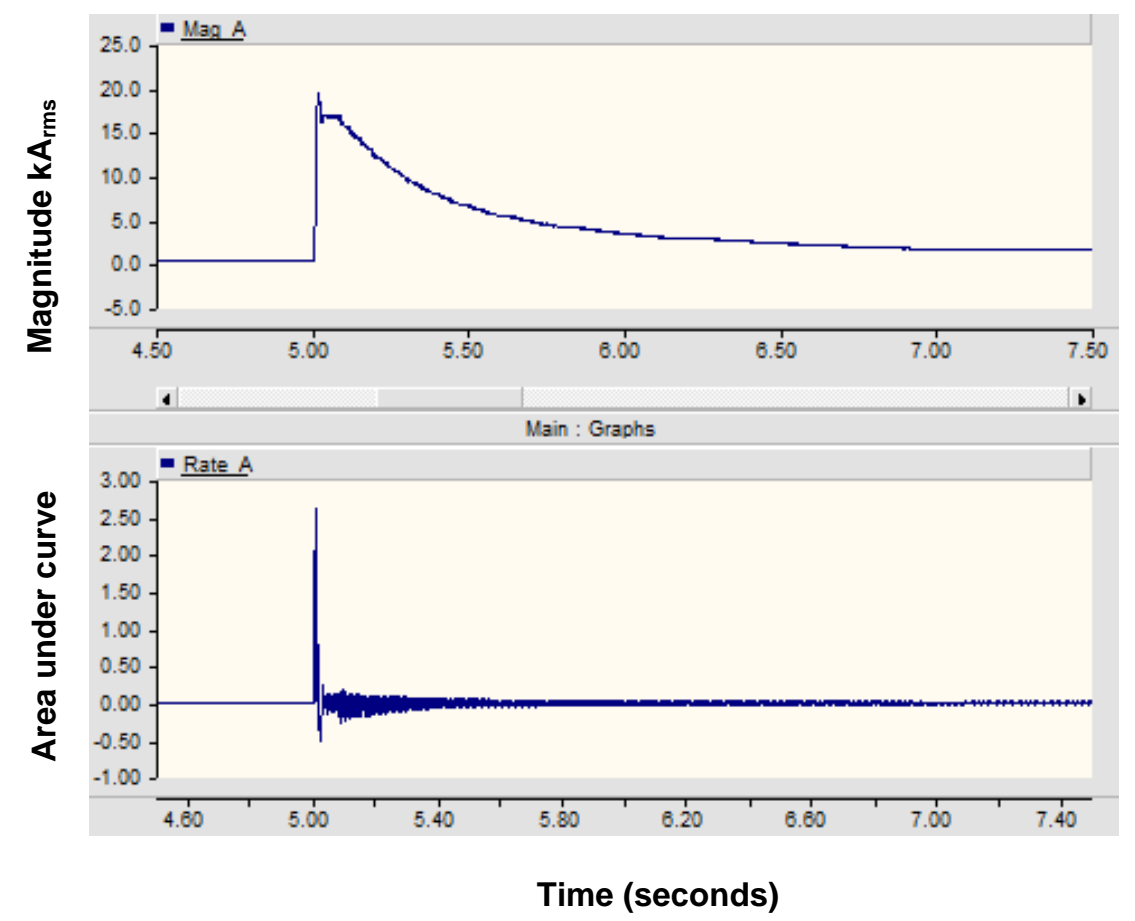

Figure C.14: Current magnitude and rate of change of current without SFCL

\section{C.3.2.8 Summary of results}

Table C.2 summarizes the results that were obtained in the SFCL modeling exercise:

Table C.2: Summary of SFCL modeling results

\begin{tabular}{|l|c|c|c|}
\hline Parameter & Pre fault & \multicolumn{2}{|c|}{ During fault } \\
\hline & & Without SFCL & With SFCL \\
\hline Line current $(\mathrm{kA})$ & 0.41 & 23.2 & 2.3 \\
\hline Current magnitude $\left(\mathrm{kA}_{\mathrm{rms}}\right)$ & 0.29 & 16.9 & 1.6 \\
\hline Time limit current $(\mathrm{ms})$ & \multicolumn{3}{|c|}{1.8} \\
\hline
\end{tabular}

\section{C.4 Conclusion}

In this report we have explored the need for an FCL and the different kinds of FCL that have been researched and tested by various other universities, scholars and corporations. Special emphasis was given on the superconducting fault current limiter in this report. Key fault detection methods and SFCL activation logic was explained in a detailed manner. Industry standard transient analysis software like PSCAD ${ }^{\circledR}$ was used to run simulations and check the response of the SFCL. Results of fault and line currents with and without FCL were presented and discussed. From the report, it can be concluded that the SFCL is very effective in limiting the fault current and the reduction can be user controlled by changing SFCL impedance values which give the user better option to do a system study. Overall, the simulation design of the SFCL was a success and worked as per expectations. 


\section{C.5 Additional Material}

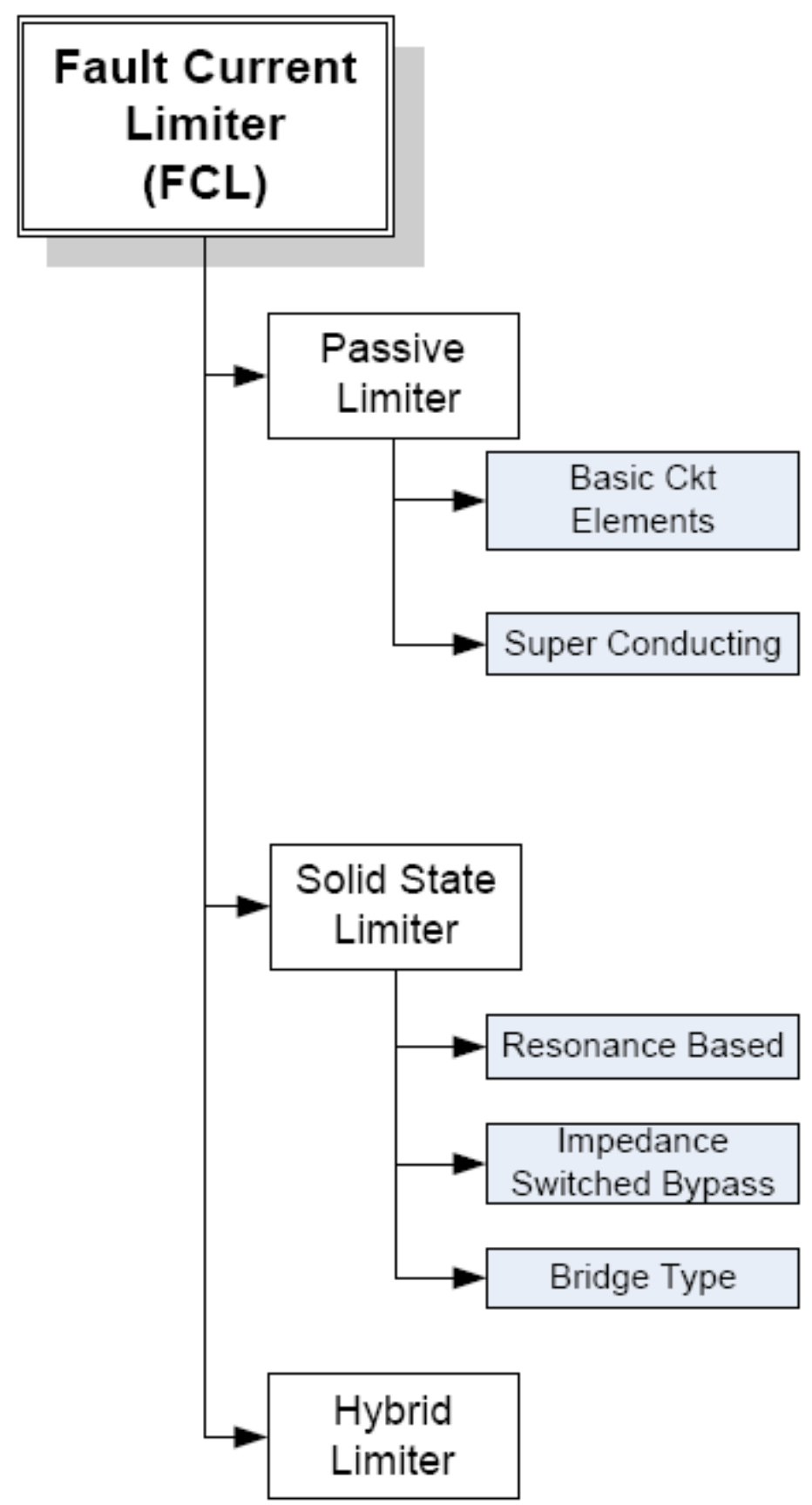




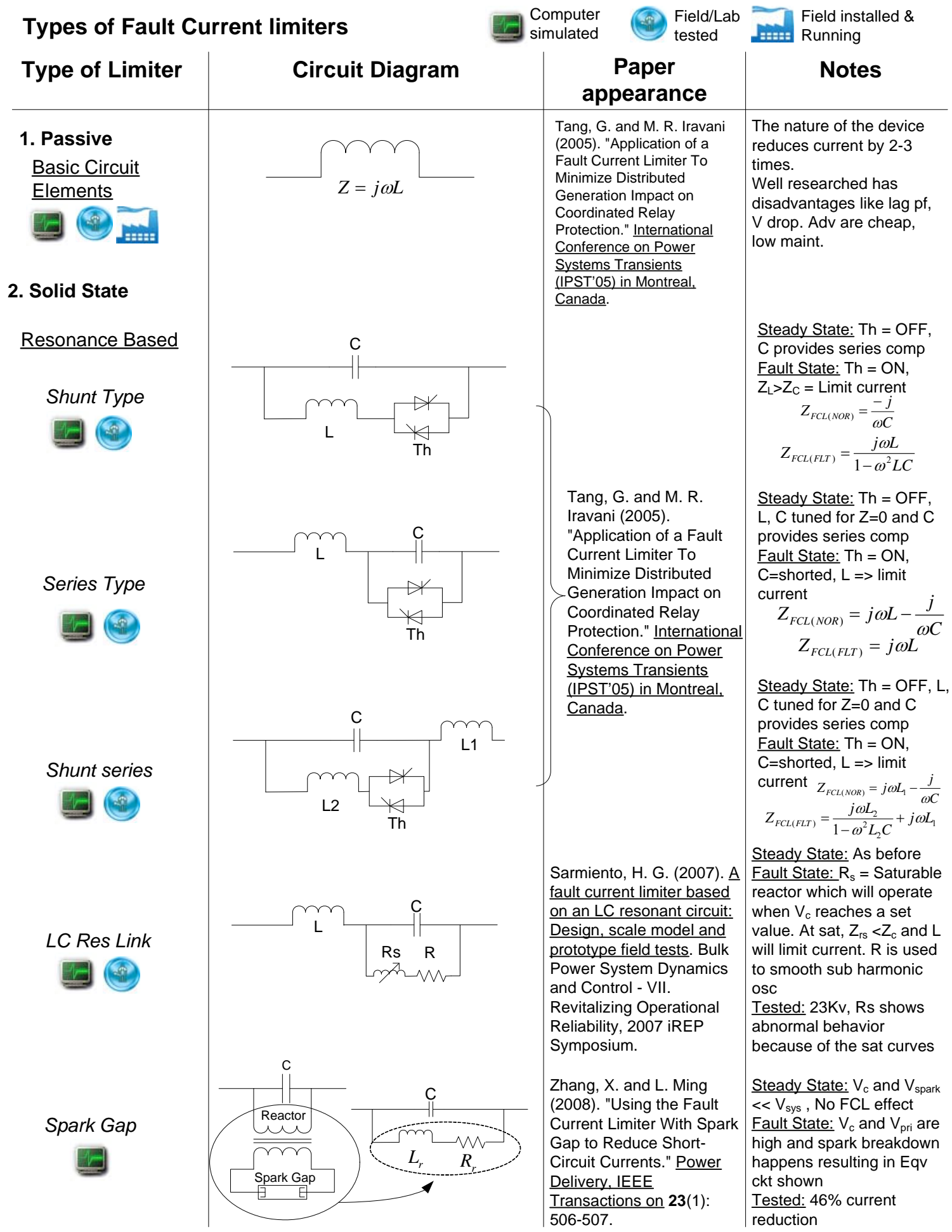




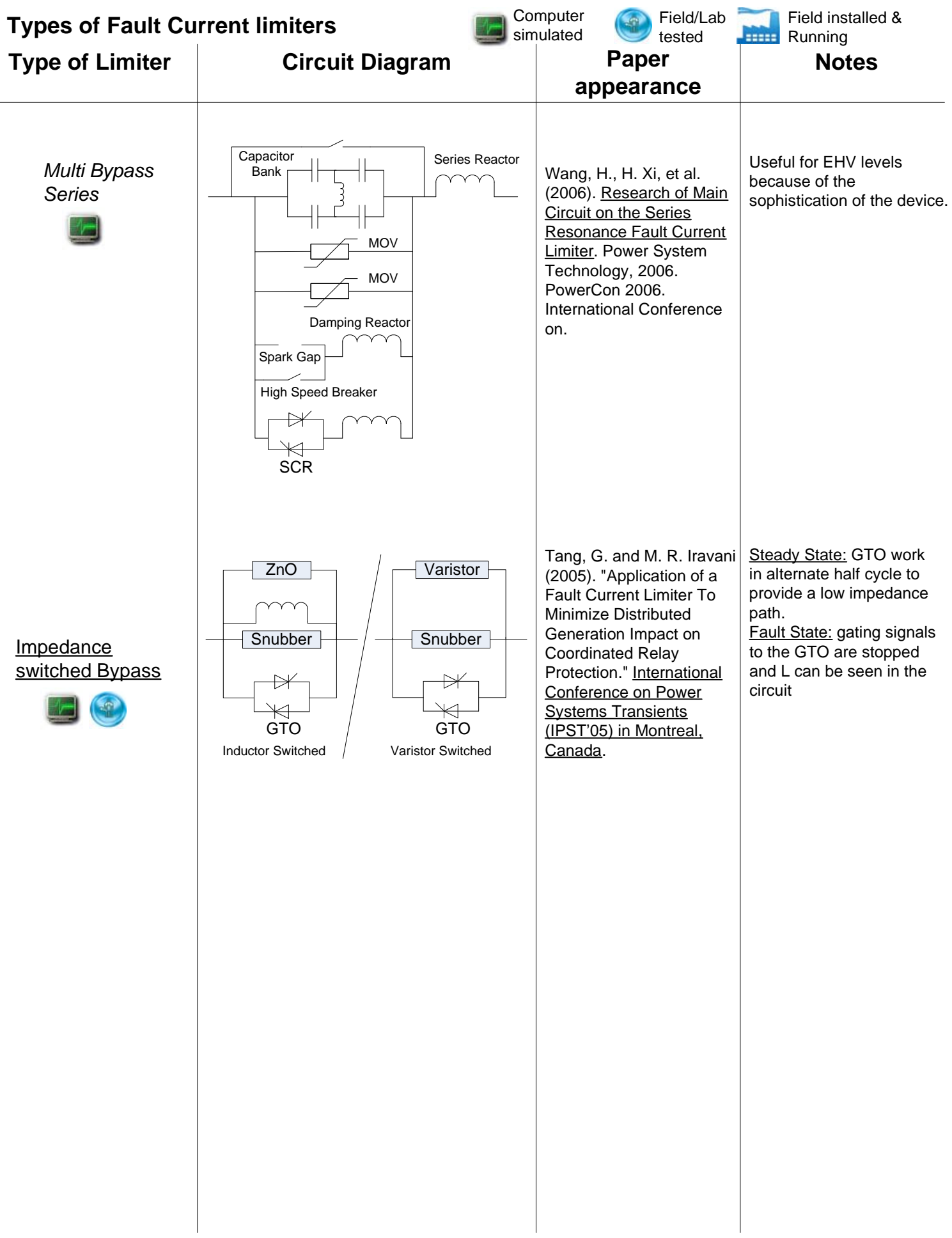




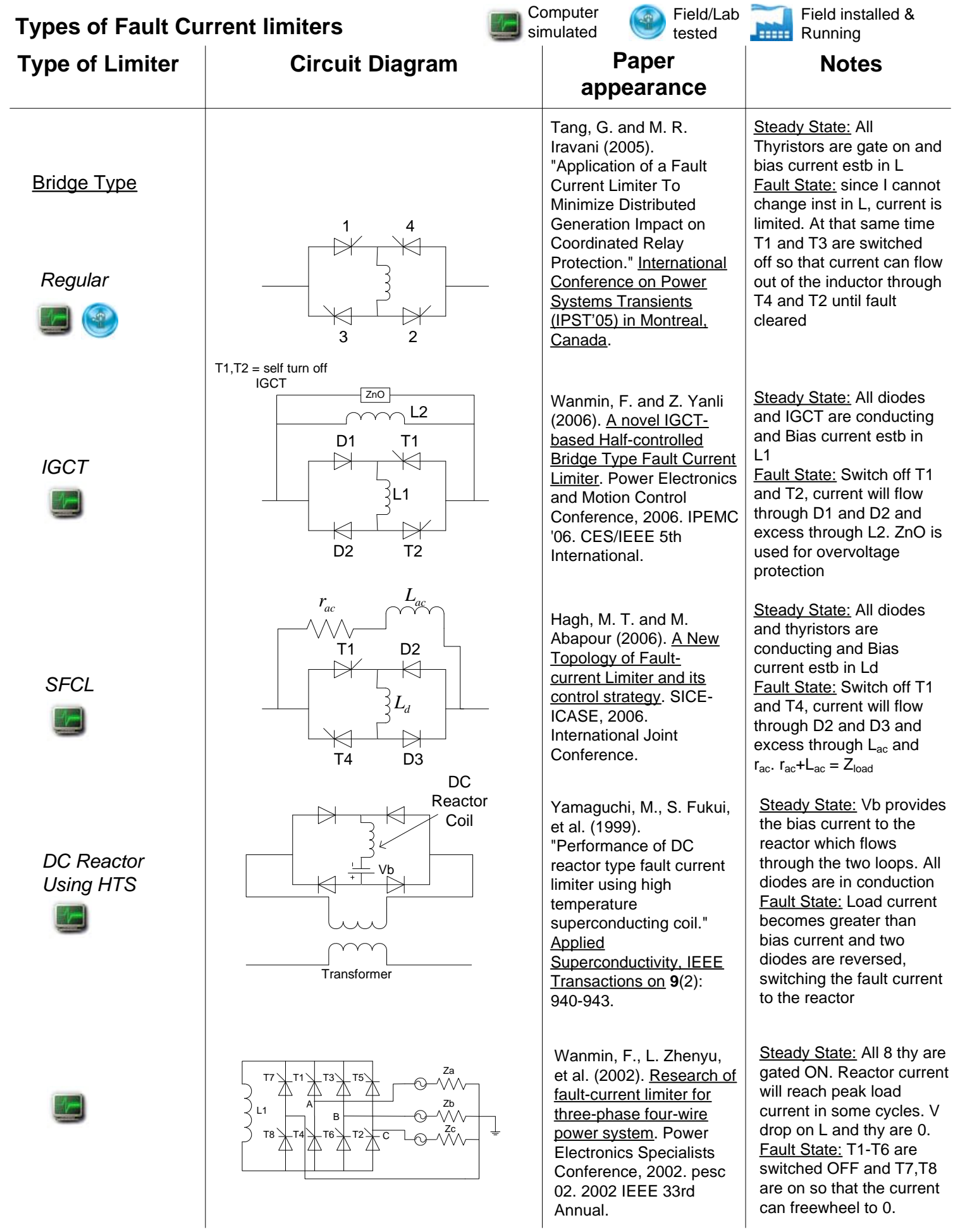




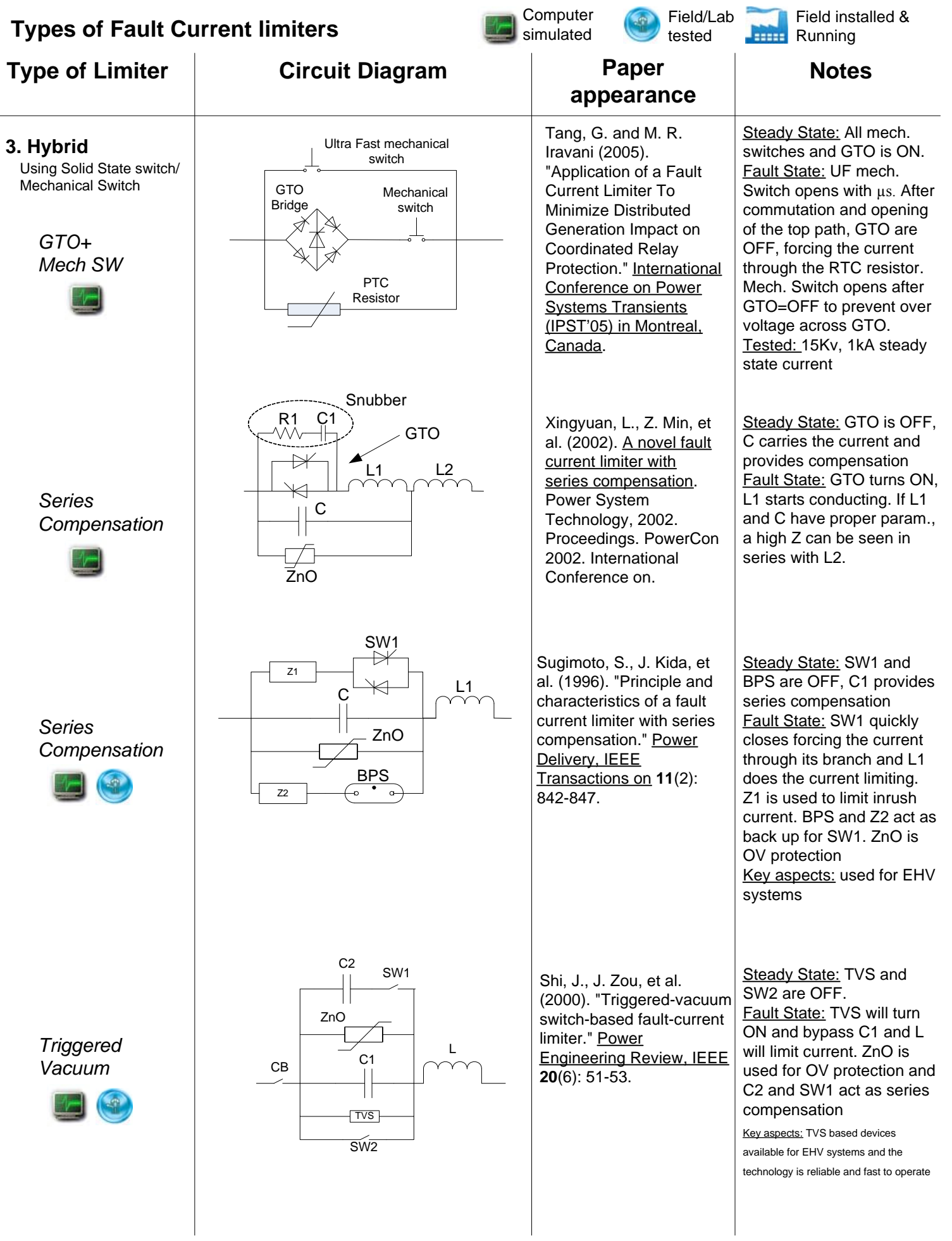

